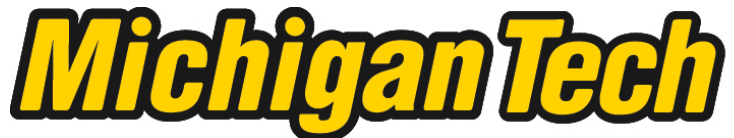 \\ Michigan Technological University Create the Future Digital Commons @ Michigan Tech
}

Sulfoxides as an intramolecular sulfenylating agent for indoles and diverse applications of the sulfide-sulfoxide redox cycle in organic chemistry

\author{
Parag V. Jog \\ Michigan Technological University
}

Follow this and additional works at: https://digitalcommons.mtu.edu/etds

Part of the Chemistry Commons

Copyright 2005 Parag V. Jog

\section{Recommended Citation}

Jog, Parag V., "Sulfoxides as an intramolecular sulfenylating agent for indoles and diverse applications of the sulfide-sulfoxide redox cycle in organic chemistry", Dissertation, Michigan Technological University, 2005.

https://doi.org/10.37099/mtu.dc.etds/36

Follow this and additional works at: https://digitalcommons.mtu.edu/etds

Part of the Chemistry Commons 


\title{
SULFOXIDES AS AN INTRAMOLECULAR SULFENYLATING AGENT FOR INDOLES AND DIVERSE APPLICATIONS OF THE SULFIDE-SULFOXIDE REDOX CYCLE IN ORGANIC CHEMISTRY
}

By

PARAG V. JOG

\begin{abstract}
A DISSERTATION
Submitted in partial fulfillment of the requirements

for the degree of

DOCTOR OF PHILOSOPHY

(Chemistry)
\end{abstract}

MICHIGAN TECHNOLOGICAL UNIVERSITY

2005 
This dissertation, "Sulfoxides as an Intramolecular Sulfenylating Agent for Indoles and Diverse Applications of the Sulfide-Sulfoxide Redox Cycle in Organic Chemistry", is hereby approved in partial fulfillment of the requirements of the degree of DOCTOR OF PHILOSOPHY in the field of Chemistry.

Department of Chemistry

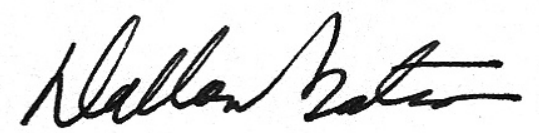

Dissertation Advisor: Prof. Dallas K. Bates

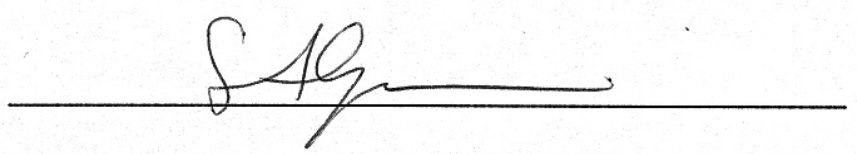

Department Chair: Dr. Sarah A. Green

Felwery 23, 2005

Date 


\section{Acknowledgements}

I would like to present my sincere gratitude to my research advisor Dr. Dallas K. Bates for his patience, help and understanding during this study. His guidance both inside laboratory and in general life is invaluable and everlasting. I thank Dr. Urnezius for not only to be on my graduate advisory committee but also for all his help and some good discussions about research and life in general. I am thankful to Dr. Richard E. Brown for his guidance and time during the computational work. I would like to acknowledge Dr. Donald Lueking, Dr. Pushpa Murthy and Dr. Shiyue Fang for being on my graduate advisory committee.

The help and understanding of all the staff of chemistry department which include Dean Seppala, Don Wareham, Ramona Johnson, Rita Smith, Michelle Saxton, Pamela Long, Lorri Reilly, Kelley Smith, Celine Grace, Denise Laux and Aparna Pandey is highly appreciated. Special thanks to Jerry Lutz and Shane Crist for their help with instrumental and computer related needs respectively. I also appreciate the help of David Lepse and Amanda Binoniemi (MTU Library staff) for making me knowledgeable in doing library related things.

I am thankful to the Department of Chemistry for providing financial assistance throughout this study.

I thank my current and former labmates (Kexue Li, Xiaofen Li, Geetha Boreddy, Anil Ekati, Jody Scheffler, Peter Klassen and Joe Lamberson) for giving some memorable years in my life. In addition, I greatly appreciate the friendship and help of all my friends including Yuth, Soumya and many others-the list of which is too long to be included. 
I would like to thank my wife Sonali for her help, patience, understanding and importantly, for her love during my graduate studies. Lastly, I pay sincere gratitude to my parents and in-laws for their support and encouragement during my life at Michigan Tech. 


\begin{abstract}
This dissertation involves study of various aspects of sulfoxide chemistry. Specifically designed t-butyl and propanenitrile sulfoxides tethered to indole-2-carboxamide were used as a source of intramolecular sulfenylating agents to synthesize novel indolo[3,2-b]1-5-benzothiazepinones which are structurally analogous to the other biologically active benzothiazepinones. This study reveals that the intramolecular cyclization of sulfoxide follows an electrophilic sulfenylation (Sulfoxide Electrophilic Sulfenylation, SES) reaction pathway. Evidence of the absence of sulfenic acid as a transient reactive intermediate in such intramolecular cyclization is also provided.

In another study, sulfoxide was used as a "protecting group" of thioether to synthesize 8-membered, indole substituted, thiazocine-2-acetic acid derivative via Ring Closing Metathesis (RCM). Protection (oxidation) of inert (to RCM) sulfide to sulfoxide followed by RCM produced cyclized product in good yields. Deprotection (reduction) of sulfoxide was achieved using Lawessons Reagent (L.R.). Application of the sulfide-sulfoxide redox cycle to solve the existing difficulties in using RCM methodology to thioethers is illustrated.

A new design of a "molecular brake", based on the sulfide-sulfoxide redox cycle is described. N-Ar rotation in simple isoindolines is controlled by the oxidation state of the proximate sulfur atom. Sulfide [S(II)] shows "free" [brake OFF] N-Ar rotation whereas sulfoxide displayed hindered [brake ON] N-Ar rotation. The semi-empirical molecular orbital (PM3) calculations revealed concerted pyramidalization of amidic nitrogen with $\mathrm{N}-\mathrm{Ar}$ rotation.
\end{abstract}




\section{Table of Contents}

Thesis approval page

Acknowledgements

ii

Abstract

iv

Table of contents

$\begin{array}{ll}\text { Unifying Theme of Research } & 1\end{array}$

$\begin{array}{ll}\text { Chapter } 1 \text { Introduction } & 6\end{array}$

Part A: Sulfoxides as an intramolecular sulfenylating agent $\quad 6$

Part B: Sulfoxide as a "protecting group" $\quad 22$

Part C: Sulfoxide as a "molecular brake" 33

Chapter 2 Sulfoxides as an intramolecular sulfenylating agent 51

"Intramolecular Sulfoxide Electrophilic Sulfenylation (SES) of 2- and 3- Indole Carbanilides: Formation of Indolo[3,2-b]-1,5-benzothiazepinones”

Introduction $\quad 54$

Results \& Discussion $\quad 57$

Experimental Section $\quad \mathbf{7 1}$

Chapter 3 Sulfoxide as a “protecting group” $\quad 89$

“Simple Thiazocine-2-acetic acid Derivatives via Ring Closing Metathesis” $\begin{array}{ll}\text { Introduction } & \mathbf{9 0}\end{array}$

Results \& Discussion $\quad 92$

Experimental Section $\quad 93$

Chapter 4 Sulfoxide as a "molecular brake” 95

“A Redox-Mediated Molecular Brake: Dynamic NMR Study of 2-[2(Methylthio)phenyl]isoindolin-1-one and S-Oxidized Counterparts" Introduction

Results \& Discussion

96

Experimental Section (see Appendix 4)

Future Directions

Appendix 1

Supplemental Information of Chapter 2

Appendix 2

Supplemental Information of Chapter 3 


\section{Appendix 3}

Additional Supplemental Information of Chapter 3

\section{Appendix 4}

Experimental Section \& Supplemental Information of Chapter 4

\section{Appendix 5}

Step by step guide to convert and analyze NMR spectra from VNMR to gNMR (V4.1.0)

Step by step guide to perform PM3 Semi-empirical 


\section{Unifying Theme}

This dissertation is a combination of efforts in understanding different aspects of sulfoxide chemistry in chemically varying substrates. The chosen sulfoxide substrates are chemically diverse in nature, however, each substrate is specifically designed to suit the requirements of the intended study. Each study is included as a separate chapter (Chapters 2, 3 and 4) and hence the chronological order of compounds and references is restricted to each chapter and is not continued from one chapter to another. Background about principles and techniques used in each study is explained in the first chapter (Chapter 1). The following brief discussion is intended to give an idea of studied aspects of sulfoxide chemistry.

Sulfoxide is an important functional group in organic chemistry. To date numerous applications of sulfoxides in organic synthesis have been disclosed and further research is in progress. Our research group has an impact in specific reaction of sulfoxides, namely Sulfoxide Electrophilic Sulfenylation (SES) reaction. In the 1980's, our research group developed SES reaction to synthesize novel $N, S$-heterocycles, which commonly exist as substructures of more complex biologically active compounds. In this reaction, sulfoxide is used as an intramolecular sulfenylating agent. Mechanistically, during this reaction, sulfoxide is activated thermally (heating in a high boiling solvent) or electrophilically (by an electrophilic species) to produce $N, S$-heterocycle. Both electrophilic and thermal activation is mechanistically different and the electrophilic activation mechanism is wellknown. However, the mechanism of thermal activation is still under study mainly because it takes place in absence of any other chemical reagent. We had proposed a mechanism of thermal activation of sulfoxides involving electrophilic addition of 
nucleophile at sulfur producing sulfonium salt as a reactive intermediate. As opposed to our proposed mechanism, other studies (discussed later) involving thermal activation of sulfoxides proposed existence of sulfenic acid (R-S-OH) as a reactive intermediate in intramolecular cyclization reactions of sulfoxides. Both sulfonium salt and sulfenic acid are extremely reactive, hence difficult to isolate species. Their presence in the reaction mixture is usually derived by the identity of by-products obtained from the reaction. However, they share one thing in common; they both originate from a sulfoxide functional group. We have shown earlier that methyl and ethyl sulfoxides follow the SES reaction pathway (sulfonium salt as reactive intermediate), for thermal activation of $t$ butyl sulfoxides, however, the sulfenic acid pathway is more common in literature. Synthesis of benzisothiazolones has been achieved by intramolecular cyclizations of $t$ butyl sulfoxides and it was argued that these proceed via sulfenic acid. However, enough evidence to prove the presence of sulfenic acid was not provided. Hence, it is unclear whether or not intramolecular cyclizations of $t$-butyl sulfoxides generate sulfenic acid under thermal activation reaction conditions. Chapter 2 of this dissertation resolves some of these mechanistic issues regarding the presence of sulfenic acid as a reactive intermediate in intramolecular cyclization of sulfoxides. We employed thermal and electrophilic activation of appropriately designed t-butyl sulfoxides and achieved synthesis of benzothiazepinones (Figure 1), a novel heterocyclic system which was synthesized earlier in our research laboratory from corresponding ethyl sulfoxides.
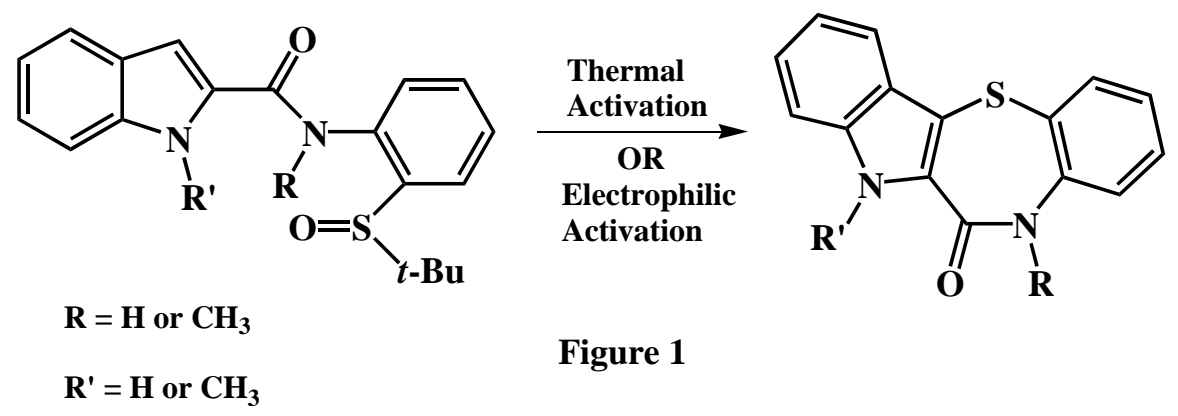

Figure 1 
Importantly, we were able to prove that the t-butyl sulfoxides do not generate sulfenic acid during the intramolecular cyclization process under the employed reaction conditions.

This study emphasizes the importance of alkyl (t-butyl in this case) sulfoxides as intramolecular sulfenylating agents. In addition, it should be noted that if the sulfoxide is suitably substituted, it can be used to resolve some of the important mechanistic issues.

In our research laboratory, Sulfoxide Electrophilic Sulfenylation (SES) has also been applied to synthesize novel fused large ring heterocycles via ring expansion of sulfonium salt of sulfoxides. For a specific ring expansion study, we needed to synthesize an 8membered ring sulfoxide substrate (A, Figure 2). We designed a new protocol to synthesize this novel 8-membered N,S-heterocycle via Ring Closing Metathesis (RCM) reaction in sulfide $[\mathrm{S}(\mathrm{II})]$ oxidation state, with eventual oxidation of sulfide to sulfoxide A in mind.

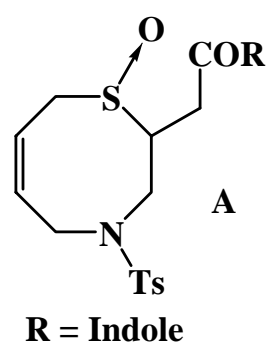

Figure 2
However, all our attempts to get successful RCM reaction with sulfide met with complete failure under varied reaction conditions. The main reason of such failure was the $\mathrm{Ru}-\mathrm{Sulfur}$ coordination between sulfide substrate and the Ru-based (Grubbs $2^{\text {nd }}$ generation) catalyst. To prevent such coordination, we envisioned sulfoxide as a "protecting group" for such thioethers. Indeed sulfoxide gave good yields of a cyclized product under standard RCM reaction conditions. Protection (oxidation) of sulfide and deprotection (reduction) of sulfoxide was achieved by conventional reagents, $m$-CPBA and Lawessons Reagent (L.R.) respectively. We were able to synthesize this 8 -membered ring $N, S$-heterocycle with sulfur in sulfide oxidation 
This concept is novel as it is the only example of using sulfoxide as a "protecting group" for thioether, which fails to produce the cyclized product via RCM.

The sulfide-sulfoxide redox cycle is a well-known process with various procedures available to achieve both, sulfide oxidation and sulfoxide reduction. Application of this relatively simple and well studied concept to solve existing difficulties in using RCM with thioethers is the most important outcome of this research (explained in Chapter 3).

Besides change in electronic effects caused by sulfide oxidation, steric effects also play a crucial role. Due to an increase in steric hindrance with the presence of oxygen in sulfoxides, molecular motions around the sulfur center would be affected. The understanding of the steric bulk of sulfoxide oxygen and the ease with which sulfidesulfoxide redox cycle can be achieved (reversibility, in other words) is extremely important. It is this sulfide-sulfoxide redox cycle which is the basis of our design of the "molecular brake" (Chapter 4).

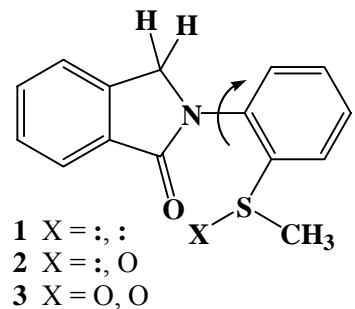

Figure 3

With the help of Dynamic NMR (DNMR) spectroscopy, we were able to show that the rate of N-Ar rotation in simple isoindolines (Figure 3) can be controlled with the oxidation state of proximate sulfur atom.

Sulfide [S(II)] shows "free" rotation [brake "OFF" mode] around the N-Ar bond, whereas sulfoxide displayed "hindered" rotation [brake "ON" mode]. Design of the molecular brake based on sulfide-sulfoxide redox cycle is one of the diverse applications of this redox chemistry. This molecular brake design can be a very useful component of the molecular machine, a molecular analog of any machine that we use in our everyday life. Further details about the design and its advantages will be discussed in Chapter 4 . 
This dissertation is a combination of various approaches in understanding the diverse applications of sulfoxides in organic chemistry. Although, all of the systems (Chapters) do not relate to each other directly, they all have a common thread, the presence of sulfoxide and its diverse applications. 


\section{Chapter 1}

This Chapter includes a detailed introduction, along with some literature background of all the subsequent Chapters (Chapters 2, 3 and 4) that are included in this dissertation. In addition, some of the basic principles of the techniques used in following chapters $(2,3$ and 4) are also covered. The Chapter is divided in three parts A, B and C and these notations are limited only to this Chapter. In addition, the compound numbers, schemes and figures are restricted to each part and are not continued from one part to another.

Part A: Sulfoxides as an intramolecular sulfenylating agent.

Part B: Sulfoxide as a protecting group.

Part C: Sulfoxide as a "molecular brake".

\section{Part A: Sulfoxides as an intramolecular sulfenylating agent}

As a part of our continuing efforts to synthesize novel $N$ - and $N, S$-heterocycles, we have developed an intramolecular sulfenylation reaction, Sulfoxide Electrophilic Sulfenylation (SES) reaction. In this method, sulfenylation is carried out intramolecularly by a sulfoxide moiety. This reaction takes place under the normal Pummerer reaction conditions and was initially called "Interrupted Pummerer reaction".

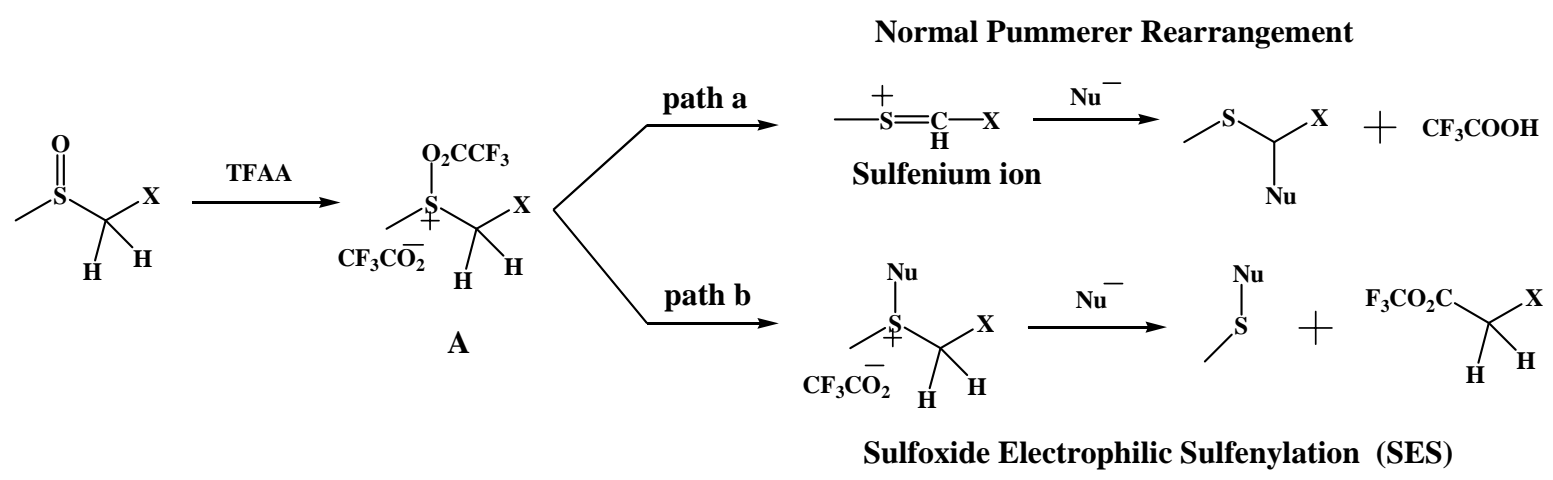

Scheme 1 
The mechanism of this reaction is shown in Scheme 1. In the Pummerer reaction, sulfoxide bearing an alpha-hydrogen is treated with anhydride, acyl halide, inorganic acids or other activating species. This converts the oxygen atom of sulfoxide into a better leaving group by forming species such as $\mathbf{A}$. This species then looses a proton from the adjacent carbon producing sulfenium ion (path a, Scheme 1). Nucleophile then attacks the carbon producing alpha-substituted sulfide. Our research group has developed a method in which the path of the normal Pummerer reaction can be altered by varying sulfoxide substituent. Under identical reaction conditions as Pummerer reaction, sulfoxide bearing an alkyl (methyl, ethyl, etc.) group can follow path b in Scheme 1. During this path, nucleophile attacks at sulfur in species A, followed by alkyl group displacement to produce the $S$-heterocycle.

SES is a very attractive method to synthesize novel $N, S$-heterocycles. Conventional methods to carry out sulfenylation involve the use of a sulfide/positive halogen source or thiol/halogen ${ }^{1}$, which results in polyhalogenated or polysulfenylated products when more than one unsubstituted site is available. SES products are always monosulfenylated because of the use of intramolecular sulfoxide functionality and relatively mild reaction conditions (explained later) compared to conventional methods.

\section{History of Sulfoxide Electrophilic Sulfenylation (SES) reaction:}

Our research group has synthesized a variety of $N, S$-heterocycles using SES methodology. One of the earliest example ${ }^{2}$ of SES reaction is shown below (Scheme 2). Activation of ethyl sulfoxide using Vilsmeier-Haack reagent $\left(\mathrm{POCl}_{3}, \mathrm{DMF}\right)$ to produce substituted pyrrolo[2,1-b]benzothiazole 2a was the prominent feature of this reaction. 
Compound $\mathbf{2 b}$ was also synthesized from $\mathbf{1}$ later on $^{3}$ by using trifluroacetic anhydride (TFAA), in hot toluene, as an activating reagent instead of Vilsmeier-Haack reagent. The applications of this methodology were limited due to added functionality in the product in the form of aldehydic or trifluroacetyl moiety in $\mathbf{2 a}$ and $\mathbf{2 b}$ respectively.
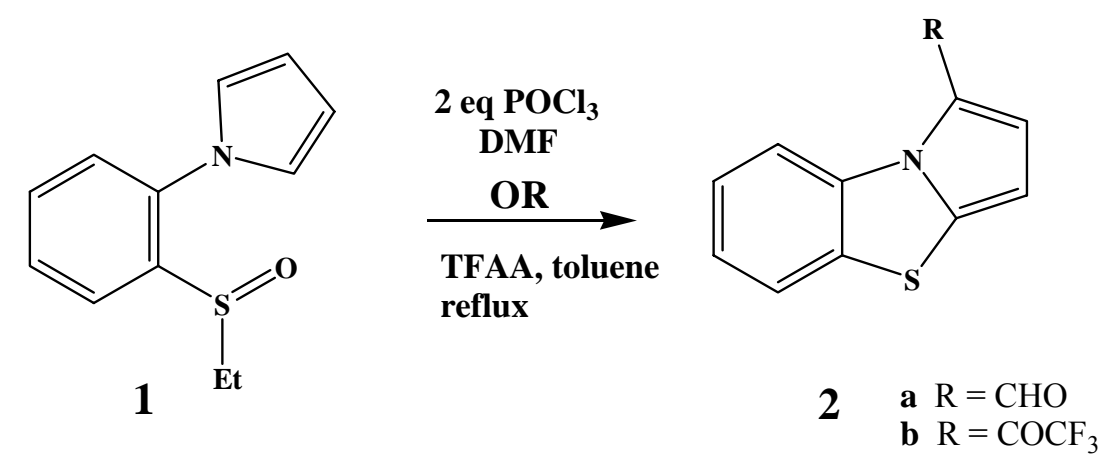

\section{Scheme 2}

Some 6-membered N,S-heterocycles were also synthesized from corresponding ethyl sulfoxides using the SES method (Scheme 3). ${ }^{4}$ Electrophilic activation (TFAA, large excess $3.7 \mathrm{eq}$ ) of 3 was carried out at room temperature (several hours) with pyridine (4 eq) as an acid scavenger to prevent the formation of $\mathbf{4 b}$. Unfortunately, in spite of this, $\mathbf{4 b}$ was isolated in $83 \%$ yield with the absence of $4 \mathbf{a}$. However, reducing the amount of TFAA to slight excess $(1.3 \mathrm{eq})$ in presence of pyridine $(1.5 \mathrm{eq})$ at $0{ }^{\circ} \mathrm{C}$ produced $4 \mathrm{a}$ in $55 \%$ yield along with $\mathbf{4 b}(10 \%)$ and unchanged starting material. In addition to electrophilic activation, thermal activation (heating sulfoxide in high boiling solvents such as benzene, toluene, $p$-xylene) of sulfoxide 3 (in $p$-xylene) produced $4 \mathbf{a}$ in $88 \%$ yield as well. 


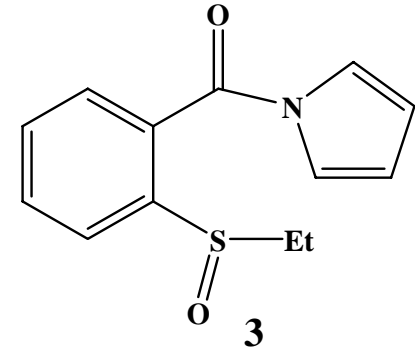

\section{Scheme 3}

The advantage of this mode of sulfoxide activation is that no other reagent is necessary to carry out this cyclization and the product obtained is therefore unsubstituted heterocycle. Furthermore, the by-products obtained from these reactions are alkyl alcohols depending on the sulfoxide side chain. For example, in case of $\mathbf{3}$, ethanol is produced as a byproduct along with $\mathbf{4 a}$. The by-product can easily be removed from the reaction mixture during reaction ( $p$-xylene, reflux, $140{ }^{\circ} \mathrm{C}$ ), after reaction (during concentration of the reaction mixture under vacuo) or during work-up procedures.

As research progressed, synthesis of some indole containing heterocycles was achieved using thermal activation of 5 to produce 6 in $66 \%$ yield (Scheme 4 ). ${ }^{5}$<smiles>CCS(=O)c1ccccc1C(=O)n1ccc2ccccc21</smiles>

5
Electrophilic Activation

Thermal Activation

Toluene, reflux

4 a $\mathrm{R}=\mathrm{H}$

b $\mathrm{R}=\mathrm{COCF}_{3}$<smiles>[R]c1ccc2sc3ccccc3c(=O)n12</smiles>

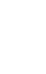


Other indole substrates produced rather unusual and mechanistically difficult to understand reaction products as shown in Scheme 5. Compound 7, upon heating in $p$ xylene, produced 8 and 9 in 15\% and 19\% yields respectively.

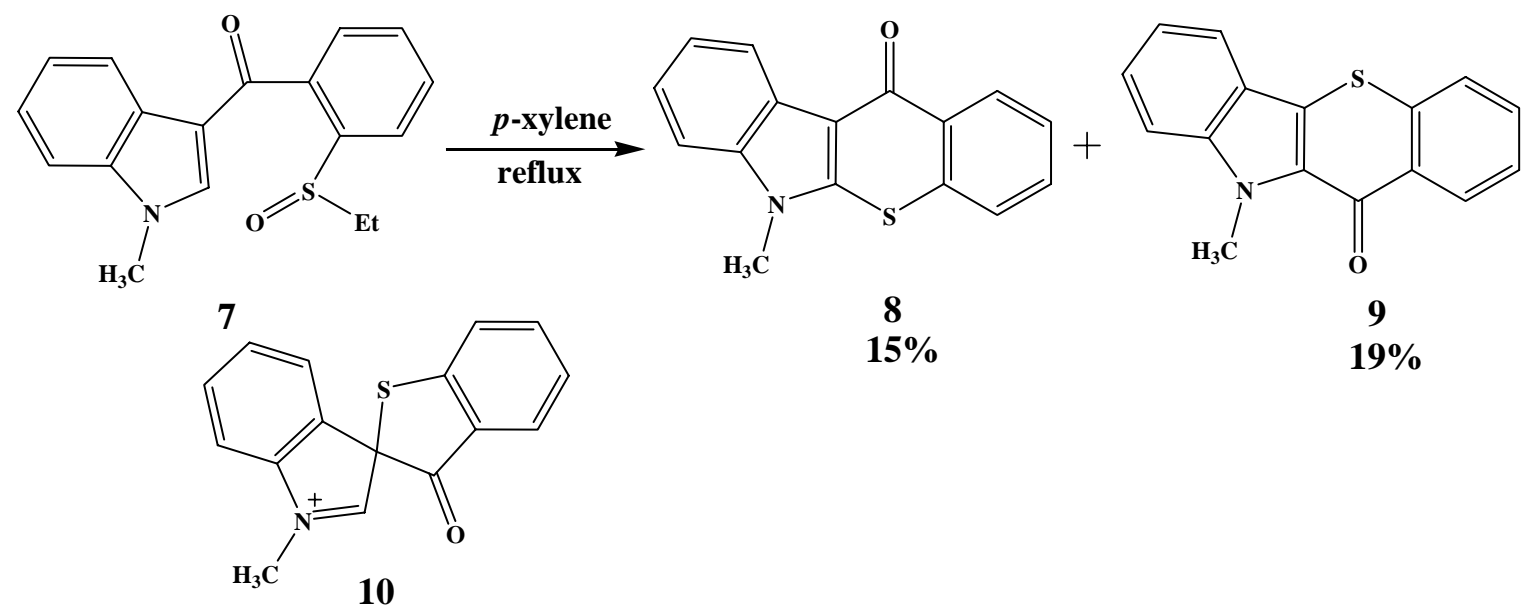

\section{Scheme 5}

While 8 was the expected product, formation of 9 was explained on the basis of the spirocyclic intermediate such as $\mathbf{1 0}$, followed by sulfur migration from the C-2 to C-3 position of the indole nucleus.

SES methodology was further extended towards synthesis of 7-membered ring thiazepinone compounds (Scheme 6). Ethyl sulfoxides tethered to indole-2-carboxamide derivatives (11-14) and indole-3-carboxamide derivatives (17-18) were synthesized and subjected to both electrophilic activation (TFAA, pyridine) and thermal activation ( $p$ xylene, reflux). 

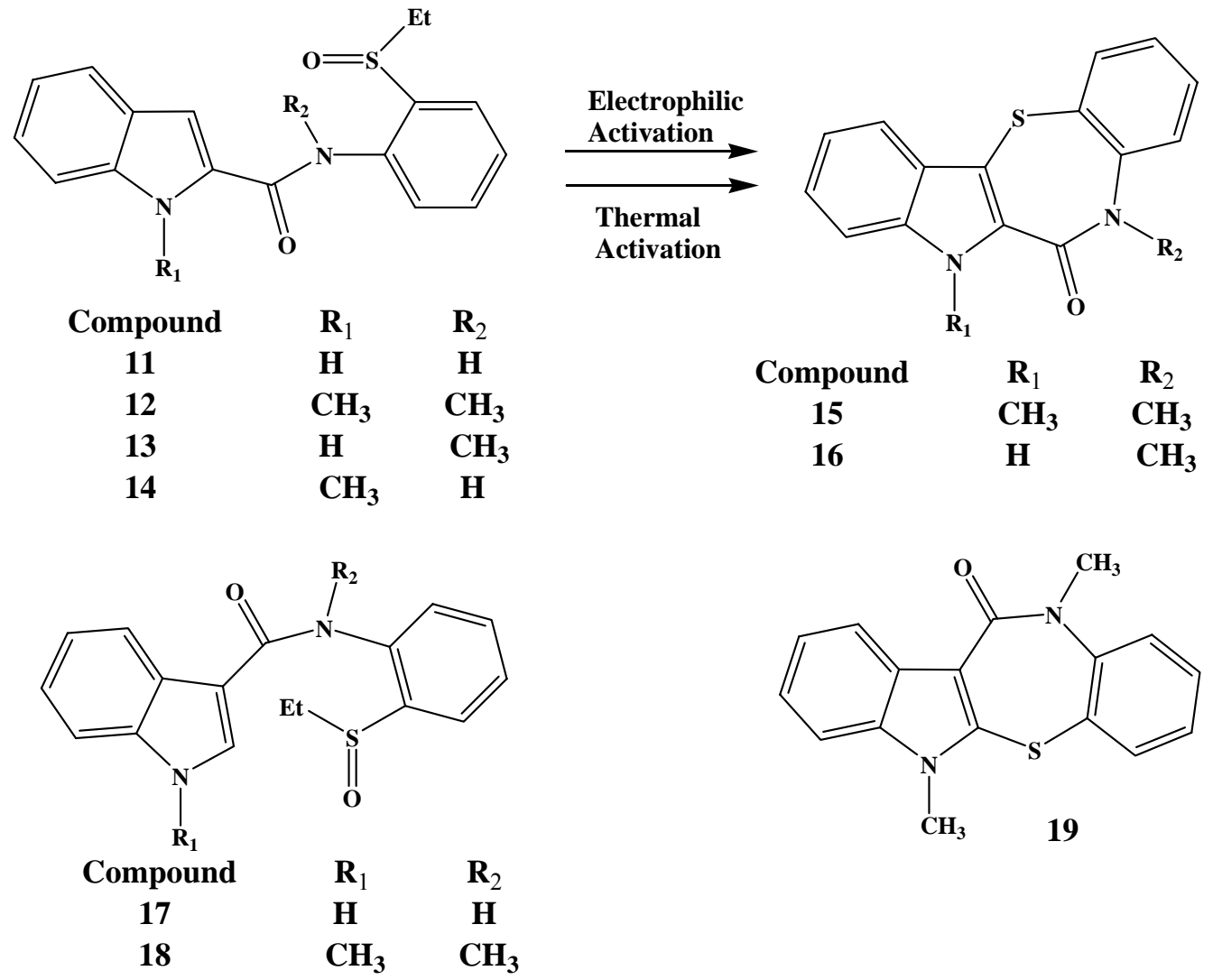

\section{Scheme 6}

Amidic substitution was essential in producing successful cyclization products, hence only 12 and 13 gave cyclized products 15 and 16 respectively, and no reaction was observed with $\mathbf{1 1}$ and $\mathbf{1 4}$. A striking result of this work was the formation of $\mathbf{1 5}$ instead of 19 from 3-substituted indole carboxamide 18. All of these results (contribution of Mary Mateo, former doctoral student of our laboratory) will be discussed in detail in Chapter 2. 


\section{Methods of Sulfoxide Activation:}

Common methods of sulfoxide activation involve electrophilic and thermal activation.

Electrophilic Activation:

As mentioned earlier, sulfoxides can be activated with the use of an electrophilic species. In all of the above-mentioned examples of SES reactions, we have used either TFAA or $\mathrm{POCl}_{3} / \mathrm{DMF}$ as an electrophilic species. Activation of sulfoxide mainly involves conversion of oxygen of sulfoxide into a better leaving group. This also involves formation of an electron deficient sulfonium ion as an electrophilic intermediate. An electron rich heterocycle then attacks this electrophilic intermediate similar to an aromatic electrophilic substitution reaction producing, in our case, a nucleophile-sulfur bond. Displacement of the alkyl side chain then produces a parent heterocycle. However, as observed in the above-mentioned examples, any excess of an activating reagent then reacts with this parent heterocycle and incorporates added functionality in the heterocycle.

Thermal Activation:

In the past, we have performed thermal activation of alkyl (ethyl) sulfoxides by heating the sulfoxide in electron rich, high-boiling solvents such as benzene, toluene, $p$-xylene, etc. to produce novel $N, S$-heterocycles. These reactions proceed well in either the presence or the absence of TFAA. The presence of TFAA yields a trifluroacetylated heterocycle because excess of TFAA reacts with the initially formed parent heterocycle. For example, compound 20, upon heating in refluxing toluene in the presence of TFAA, produced 24 in $76 \%$ yield (Scheme 7). ${ }^{3}$ 
<smiles>CCS(=O)c1ccccc1-n1cccc1</smiles>

20

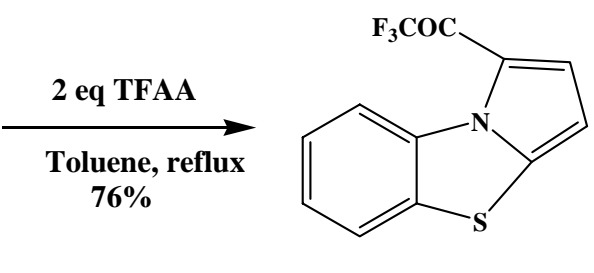

24

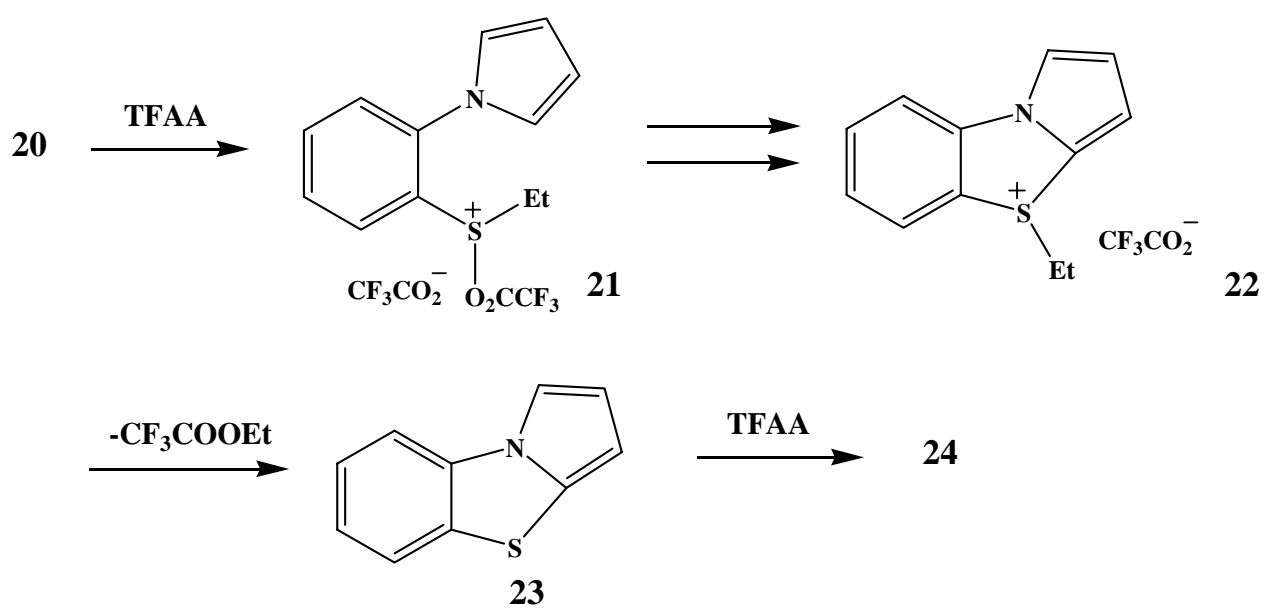

\section{Scheme 7}

We proposed that the reaction begins with the thermal activation of sulfoxide in the presence of TFAA to produce 21, followed by a nucleophilic attack of the electron rich pyrrole ring on sulfur to produce sulfonium salt 22. Sulfonium salt then undergoes thermal (toluene, reflux) displacement of the S-alkyl group to produce the parent heterocycle 23, which due to the added electrophilic activating influence of sulfur, undergoes further substitution to yield $\mathbf{2 4 .}$

As discussed earlier, we have also carried out the thermal activation of ethyl sulfoxide in the absence of TFAA simply by heating sulfoxide in $p$-xylene. We believe, in the absence of TFAA, sulfoxide activation still takes place in a very similar fashion as described in Scheme 7. In the absence of TFAA, it is probable that acidic protons of 
adjacent methylene carbons could intermolecularly protonate sulfoxide oxygen of another sulfoxide thereby activating it for the reaction.

There is literature precedence for another feasible mechanism of the thermal activation of sulfoxides in the case of penicillin to cephalosphorin conversions. ${ }^{6}$ It was proposed that this reaction proceeds via a species called "sulfenic acid" as the reactive intermediate as shown in Scheme 8.

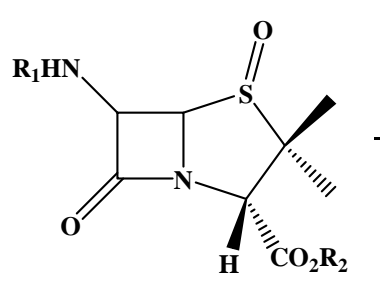

Penicillin Sulfoxide

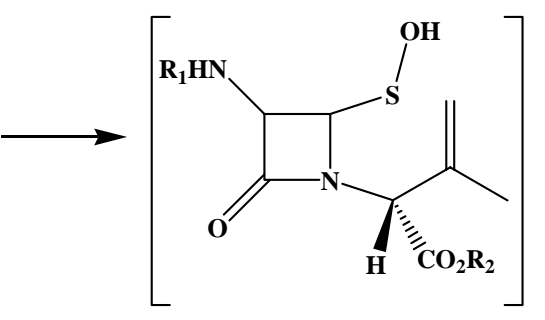

Sulfenic acid intermediate

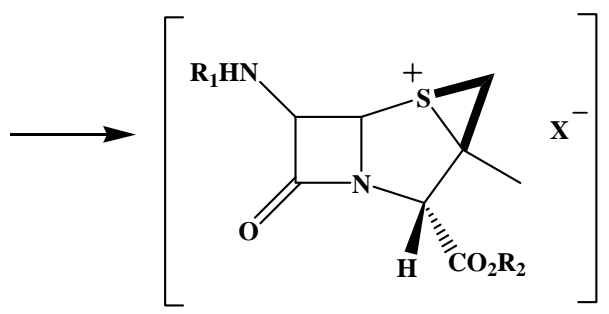

$\mathbf{Z}$

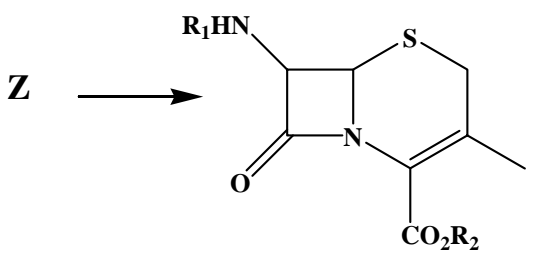

Cephalosphorin

\section{Scheme 8}

Understanding of sulfenic acid chemistry is essential to study the mechanistic aspects of thermal activation of sulfoxides. 


\section{Introduction to the sulfenic acid chemistry:}

Sulfenic acid is a highly reactive, difficult to isolate species, usually generated in situ by thermolysis of suitably substituted sulfoxide. ${ }^{7}$ Sulfoxides with one or more hydrogens on a carbon beta to a sulfur atom undergo decomposition at moderate temperatures to produce an alkene and sulfenic acid (R-S-OH). A commonly accepted mechanism of sulfenic acid formation is stereospecific cis-elimination ${ }^{8}$ as shown in Figure 1.

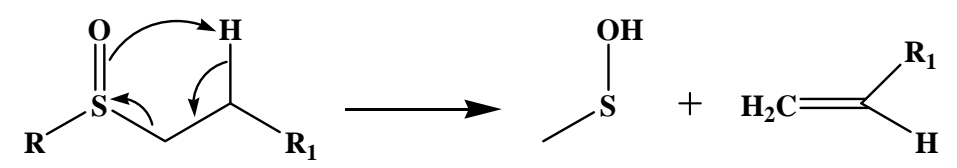

Figure 1

The temperature at which this thermolysis takes place varies with the $\mathrm{R}_{1}$ group (Figure 1). The commonly studied alkyl group is the t-butyl group and some other examples of the functional group include the propanenitrile ${ }^{9}$ group. Upon heating in high boiling solvents such as toluene, xylenes, etc., $t$-butyl sulfoxides undergo thermolysis to produce sulfenic acid with the elimination of isobutene. However, due to the steric bulk and the greater number of available beta-hydrogen atoms, $t$-butyl sulfoxides are capable of forming sulfenic acids even at low temperatures. ${ }^{10}$

As mentioned earlier, sulfenic acid is a very reactive species and hence undergoes a variety of reactions. Unfortunately, due to high reactivity, the principle reactions of sulfenic acids are not observed directly and most of the evidence for the existence of sulfenic acid has to be derived from the products obtained from a specific reaction. ${ }^{11}$ Some of the basic reactions of sulfenic acid involve formation of thiosulfinate $\mathbf{B}$ 
(equation 1). These thiosulfinate are unstable and thermally disproportionate in disulfides C (R-S-S-R) and thiosulfonates $\mathbf{D}\left(\mathrm{R}-\mathrm{SO}_{2}-\mathrm{S}-\mathrm{R}\right)$ as shown in equation 2. Thiosulfinate $\mathbf{B}$ and thiosulfonate $\mathbf{D}$ could also react with water (formed in equation 1) to produce sulfinic acid $\mathbf{E}$, thiol (F) and a molecule of sulfenic acid (equation 3, 4).

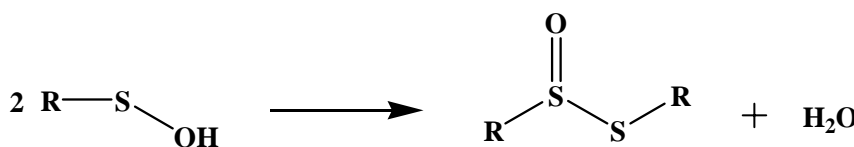

B

Eq 1<smiles>[R]SS([R])=O</smiles>

B

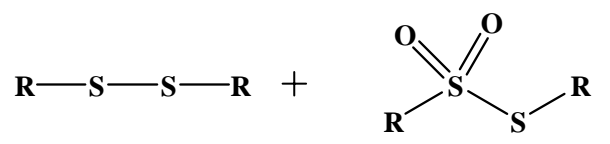

Eq 2

C

D<smiles>[R]S[SH]([R])=O</smiles><smiles>[R]S[I-][Na]</smiles><smiles>[R]SS([R])(=O)=O</smiles><smiles>CCCC</smiles><smiles>[R]S(=O)O[18OH]</smiles><smiles>[R]SO</smiles>

Eq 4

Synthetically useful reactions of sulfenic acids involve intramolecular and intermolecular additions of sulfenic acids to olefins and dienes. Examples of some of the known ${ }^{12}$ reactions of sulfenic acids are shown in Scheme 9. 

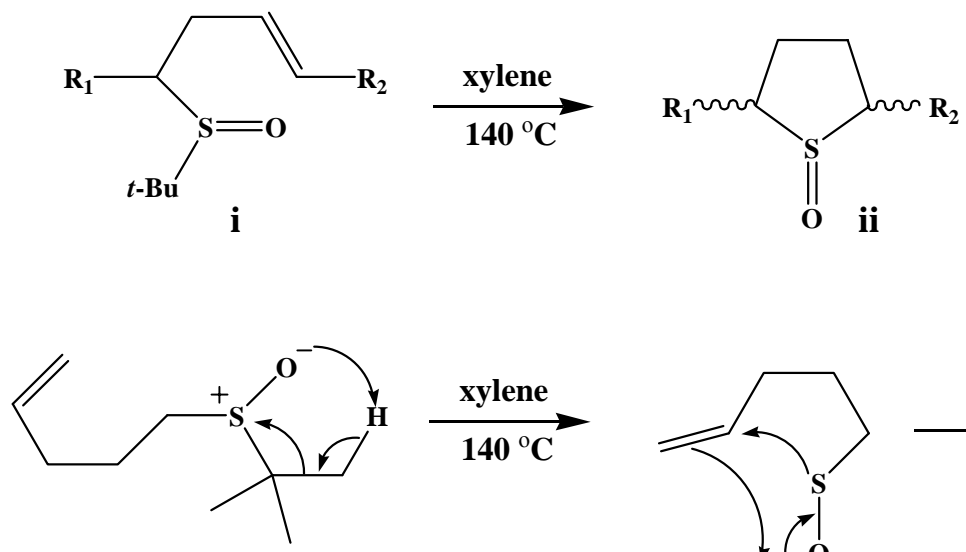

iii

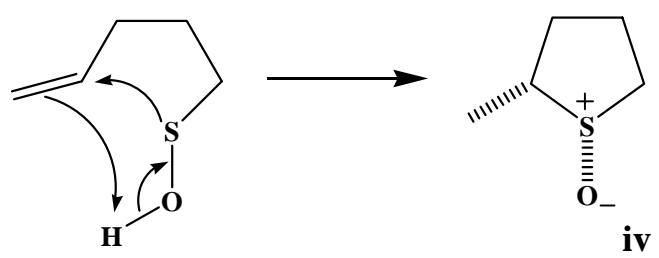

\section{Scheme 9}

Sulfenic acid mediated intramolecular cyclizations have also been reported in the synthesis of benzothiophenes by heating styrenyl sulfoxide in the presence of $p$-TSA (Scheme 10). ${ }^{13}$<smiles>COc1ccc(/C=C(/c2ccc(OC)cc2)S(=O)CC(C)C)cc1</smiles>

I

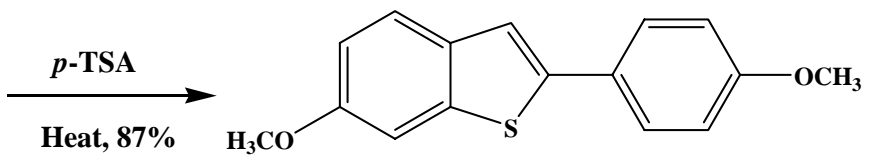

II

\section{Scheme 10}

The following reactions (Scheme 11) of sulfenic acids are of mechanistic importance. The presence of sulfenic acid in the reaction mixture can be confirmed by trapping it with alkyne or thiols. Methyl propiolate (25) is commonly used ${ }^{8,11,14}$ alkyne whereas 2- 
mercaptobenzothiazole (27) is an example ${ }^{6}$ of thiol used as a sulfenic acid trapping agent. Scheme 11 shows the typical products that are obtained by such trapping experiments.

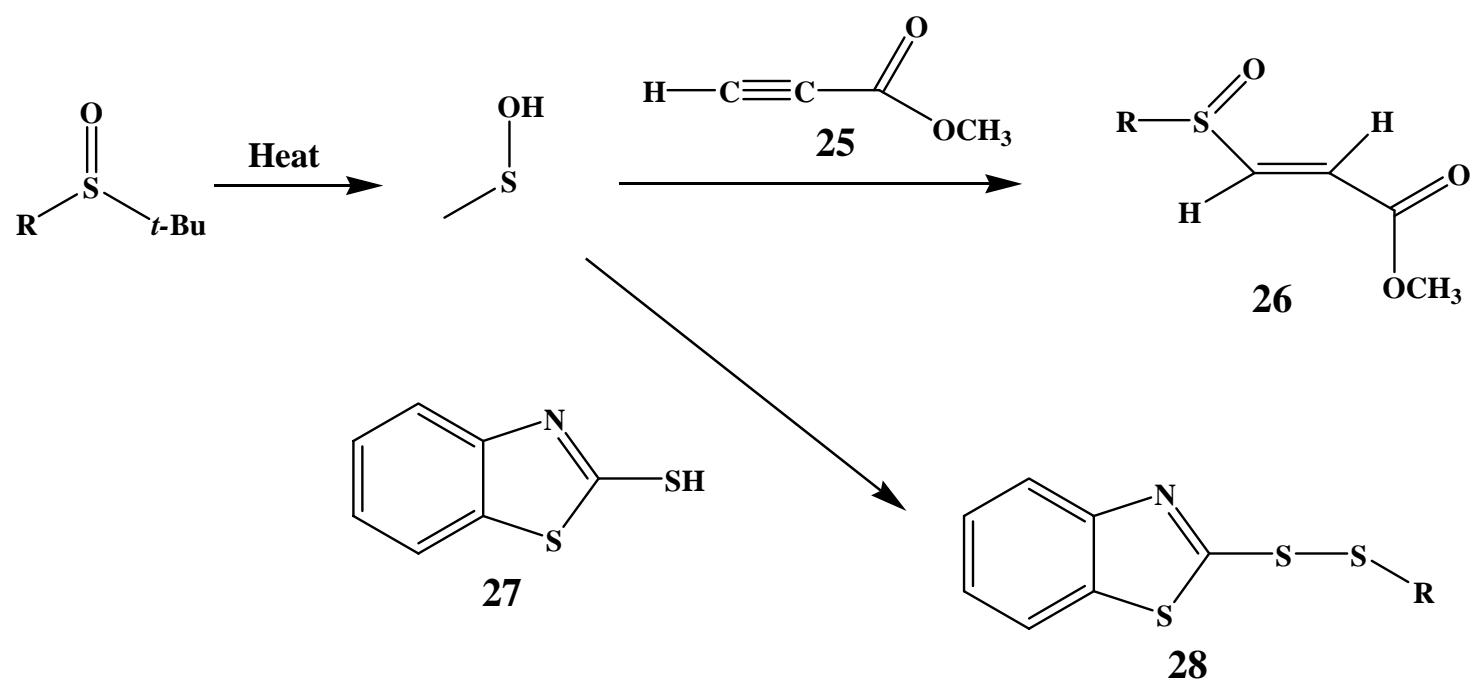

\section{Scheme 11}

Reaction of sulfenic acid with methyl propiolate can be seen as a reverse reaction of sulfenic acid formation and hence it is known ${ }^{11}$ that the formation of thiosulfinates (B) and 26 are competitive reactions. Reactions of sulfenic acids and $\mathbf{2 7}$ are generally clean producing corresponding disulfide (28) in high yields. ${ }^{6}$ These results indicate that 27 is a better sulfenic acid trap than methyl propiolate and avoids any complications in results. 


\section{Objective of this study:}

Because of the similarity of the sulfoxide substituent (ethyl group in our studies and $t$ butyl in sulfenic acid chemistry) and the temperature (toluene, reflux) at which we performed our thermal activation of sulfoxides, it was thought that our thermal activation process might involve sulfenic acid as a reactive intermediate.

Furthermore, Wright and co-workers ${ }^{15}$ synthesized specifically designed $t$-butyl sulfoxides and carried out thermal cyclization of these sulfoxides to produce benzisothiazolones (Scheme 12).

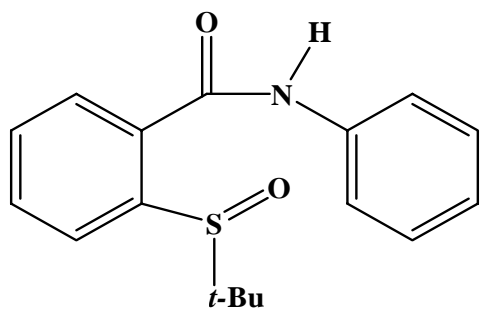

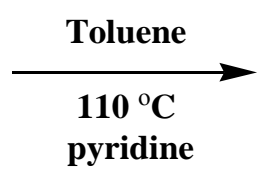

pyridine

\section{Scheme 12}

At the temperature of toluene reflux $100{ }^{\circ} \mathrm{C}$, they postulated that, the $t$-butyl sulfoxides will undergo thermal elimination to produce sulfenic acid, which would cyclize with the loss of water, to produce the desired benzisothiazolones. However, enough evidence for the formation of sulfenic acid in the reaction mixture was not provided (for example by chemical means such as trapping experiments or any other type of experiments). In addition, unlike other reported sulfenic acid based cyclizations, this cyclization was carried out in a basic medium (pyridine).

The above example lacks enough experimental evidence to support the existence of sulfenic acid in the reaction mixture. A thorough understanding of sulfenic acid 
chemistry along with experimental evidence is necessary to prove or disprove the presence of sulfenic acids as reactive intermediates in intramolecular cyclization reactions.

Our previous experience with intramolecular cyclizations using ethyl sulfoxides and the by-products obtained under those reaction conditions indicate that our thermal activation process follows the SES (formation of sulfonium salt) pathway. We obtained ethanol as a by-product in our cyclization reactions of ethyl sulfoxide. This is an indication of the absence of sulfenic acid (and corresponding alkene, ethene) being produced during the reaction. If sulfenic acid is to be produced in the reaction mixture, we would not have seen ethanol as a by-product of the reaction, as the ethene formed in the process of forming sulfenic acid would have been expelled (as a gas) from the reaction mixture.

Based on these observations, we persued the following study during which, we revisited our own system (discussed earlier, Scheme 6) and used t-butyl sulfoxides and propanenitrile sulfoxide (in one case) as intramolecular sulfenylating agents to carry out the synthesis of benzothiazepinones. The substituent effects and reactivity patterns were identical to the ones observed before for corresponding ethyl sulfoxides. Importantly, we performed a trapping experiment with 2-mercaptobenzothiazoles and were able to isolate thiazepinone 15 without any indication (by TLC) of the formation of a corresponding disulfide product 29.

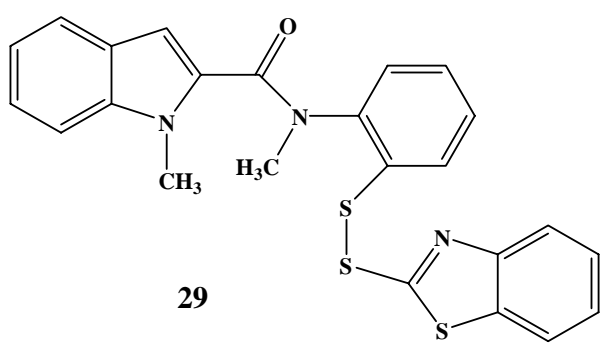


These results indicate that the products obtained in these cyclizations are SES reactions based on the attack of an electron rich heterocycle at the sulfoxide and do not involve a sulfenic acid mechanistic pathway. It is also probable that some results, which are reported in the literature (Wright and co-workers), ${ }^{15}$ to proceed via sulfenic acid, in fact follow the SES path.

In addition to this, we also synthesized compound 30, a t-butyl derivative of compound 18, in an attempt to understand the formation of $\mathbf{1 5}$ from 18. However, $\mathbf{3 0}$ did not

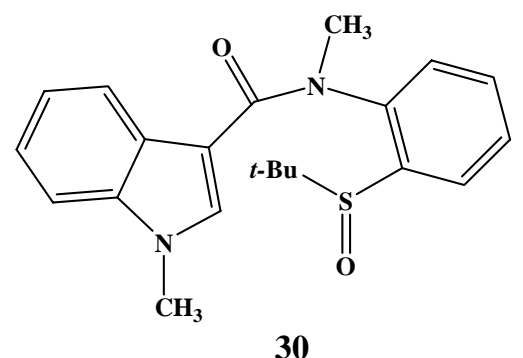

30 produce 18 under the reaction conditions used and we think that structural changes (by replacing the ethyl group) due to the bulky t-butyl group could be the reason behind this.

All of the above results of $t$-butyl, propanenitrile and ethyl sulfoxides along with experimental details and spectroscopic data are included in Chapter 2.

This research along with contribution from Mary Eggers (Mateo), former doctoral student in our research laboratory was presented at the ACS National Meeting, Jog, P. V.; Bates, D. K.; Eggers, M. E. Book of Abstracts, $228^{\text {th }}$ ACS National Meeting, Philadelphia, PA August 22-26, 2004. ORGN-675.

This research is also submitted for publication, Bates, D. K.; Eggers, M. E.; Jog, P. V. J. Org. Chem. Submitted for publication. 


\section{Part B: Sulfoxide as a protecting group}

This part covers the important aspects involved in the synthesis of thiazocine-2-acetic acid derivatives via Ring Closing Metathesis (RCM).

\section{Introduction:}

Application of organometallic chemistry in organic synthesis is ever increasing. Inorganic metal complexes act as catalysts in a variety of organic reactions. Alkene metathesis is one such well-known organic reaction, catalyzed by various types of tungsten-, molybdenum-, and ruthenium-based organometallic complexes. Initially, application of this reaction was limited in polymer chemistry with a Ring-Opening Metathesis Polymerization (ROMP) reaction. Soon after, an interesting reaction, namely, Ring Closing Metathesis (RCM) was discovered and within few years, it became the most powerful synthetic tool in carbon-carbon $(\mathrm{C}-\mathrm{C})$ bond forming reactions. Application of RCM is not only limited to the synthesis of novel compounds, but it has also proved to be the better alternative approach towards previously known systems. Subsequent chapter (Chapter 3) describes the new protocol, to synthesize thiazocine-2-acetic acid derivatives via RCM.

\section{Mechanism of RCM:}

The mechanism of RCM involves a series of alternating [2+2] cycloaddition and cycloreversion reactions between substrate and catalytic species (metal alkylidiene) (Figure 1). ${ }^{16}$ Also, there is some evidence of metalacyclobutanes as reactive intermediates. ${ }^{17}$ In alkene metathesis, all the individual steps of the catalytic cycle are 
reversible, however, ring-closing metathesis is a forward driven reaction due to an increase in entropy in going from one substrate to two products.

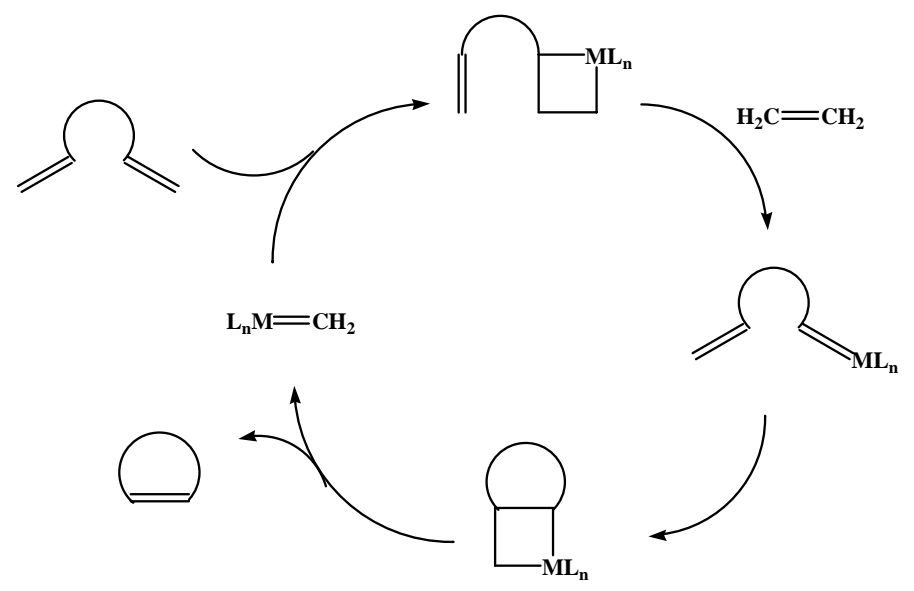

Figure 1: Mechanism of Ring Closing Metathesis.

\section{Catalysts used in RCM:}

RCM catalysts can be categorized as tungsten- (W), molybdenum- (Mo), or ruthenium$(\mathrm{Ru})$ based depending on the central transition metal atom in the complex. Tungstenbased catalysts $\mathbf{A}$ are less commonly used in RCM chemistry. ${ }^{16}$ Alkoxy imido Mo complexes $\mathbf{B}$ are the earlier developed catalysts ${ }^{17}$ followed, later on, by benzylidene $\mathrm{Ru}$ complexes $\mathbf{C}$ by Grubbs and co-workers ${ }^{18}$ (Figure 2). Due to their air and moisture sensitivity, short storage life and poor functional group tolerance, Mo complexes are limitedly used. However, they show pronounced metathesis activity towards a variety of substrates in varying steric and electronic environments. In contrast, Ru complexes show greater tolerance to varied functionalities from esters, ketones, acetals, ethers to even more polar alcohols, aldehydes and carboxylic acids. 

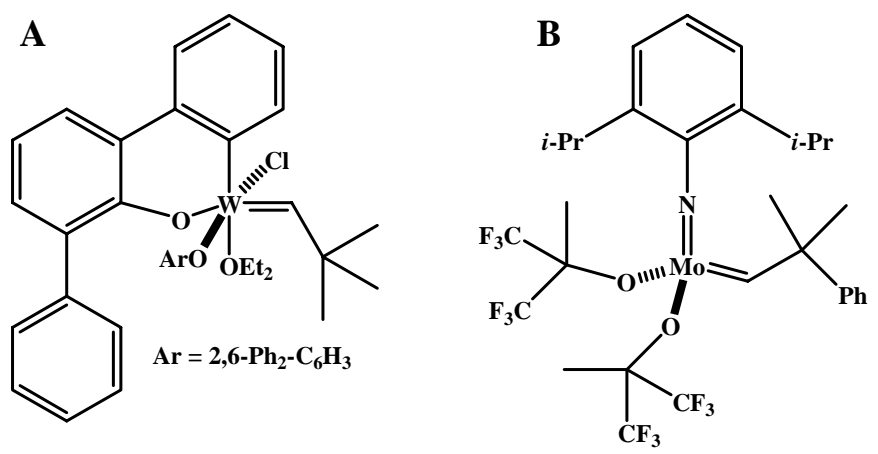

C

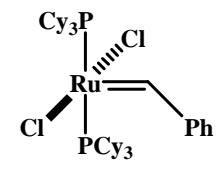

D

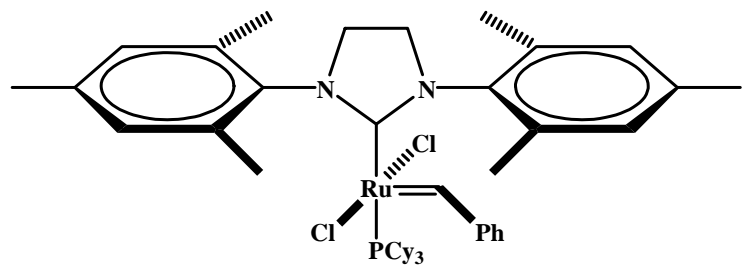

$\mathbf{E}$

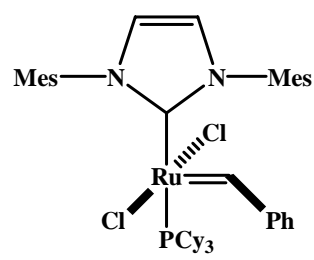

Figure 2: Various catalysts used in RCM.

This increased tolerance to functional groups, along with their ready availability and ease of handling, has made these Ru complexes commercially available over their Mo counterparts.

Later on, the focus of this research was shifted towards making more efficient, more functional group tolerant and air and moisture stable catalysts. Grubbs $2^{\text {nd }}$ generation catalysts $\mathbf{D}^{19}$ and $\mathbf{E}^{20}$ are the examples of such modern and efficient catalysts. The important feature of $\mathbf{D}$ and $\mathbf{E}$ is the presence of bulky electron donating imidazole and imidazoline ligand, which increases the ring-closing metathesis activity while retaining air and moisture stability. These complexes also show greater functional group and heteroatom compatibility compared to catalyst $\mathbf{C}$. 


\section{Factors affecting $\mathrm{RCM}$ reaction:}

Suppression of the competitive metathesis based polymerization of reactant under reaction conditions is important, in achieving successful RCM. This is achieved by performing the reaction under high dilution conditions. There is literature precedence that $\mathrm{RCM}$ is strongly favored over intermolecular polymerization by preexisting conformational constrains in the substrate. ${ }^{21}$ The choice of catalyst also plays an important role and often depends on the functional group in substrate and steric demands. $^{22}$ Ring size of the expected product, steric effect, effect of certain functional groups and the presence of heteroatom are some other important aspects governing the success of RCM chemistry. ${ }^{23}$ Discussion in this chapter is restricted to the application of RCM to sulfur containing substrates.

\section{RCM as applied to sulfur containing substrates:}

There is a lot of ongoing research towards synthesizing S-heterocycles using RCM methodology. This increasing interest is mainly due to well-known biologically active and synthetically useful (as intermediates) $S$-heterocycles. ${ }^{23}$ Our interest in $S$-heterocycles is centralized on the application of Sulfoxide Electrophilic Sulfenylation (SES) reaction in synthesizing novel $N, S$-heterocycles. SES is a novel sulfenylation method developed in our research laboratory, and uses sulfoxides as intramolecular sulfenylating agents. ${ }^{24}$ In order to synthesize a novel 12-membered ring $N, S$-heterocycle using SES methodology, we needed its immediate precursor, a thiazocine acetic acid derivative sulfoxide 3 (Scheme 1). Sulfoxide 3 is a novel 8 -membered ring $N, S$-heterocycle. Synthesis of higher membered heterocycles, specifically, 8-membered compounds still 


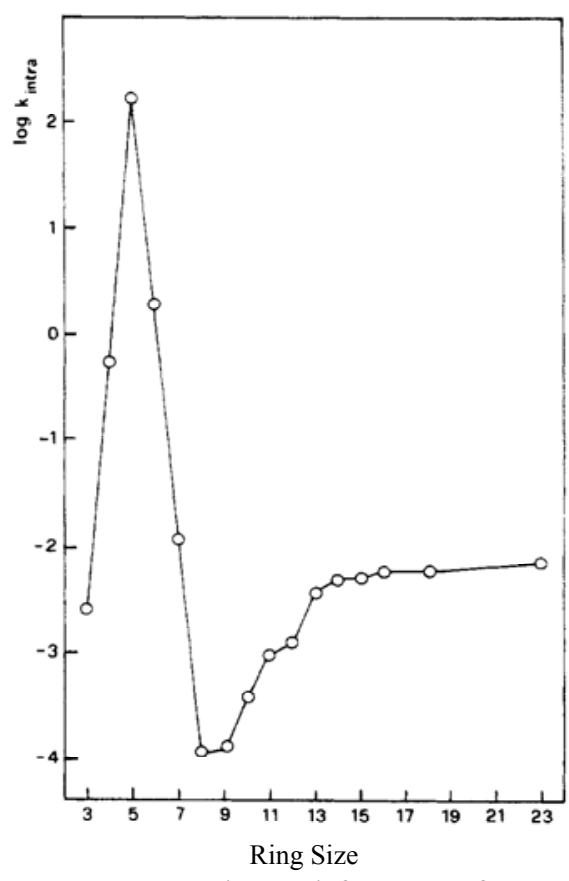

Figure 3: Adapted from Ref 25 remain as a formidable task. Figure 3 is an illustrative example of the difficulties involved in the synthesis of 8 -membered heterocycles. ${ }^{25}$ In this graph, the rate of cyclization (lactonization from $\omega$-bromoalkanoate) was plotted against the ring size (3 to 23 ) of the product obtained, which shows the decrease in the rate of lactonization for 8-membered ring heterocycles by a factor of $10^{6}$. In addition, the absence of examples with sulfur containing (as a nucleophile) systems displays the intensity of

the problems encountered specifically, in synthesizing 8-membered ring S-heterocycles.

However, there are examples in literature about 8-membered S-heterocycles. In one case, intramolecular cyclization of $\alpha, \omega$-dibromoalkanes in presence of $\mathrm{Na}_{2} \mathrm{~S} \cdot 9 \mathrm{H}_{2} \mathrm{O}$ was used to make 7-15 membered ring thiacycloalkanes (48\% yield of 8-membered thiacycloalkane). ${ }^{26}$ The other example used [2,3] the sigmatropic rearrangement of sulfur ylides to synthesize the 8-membered S-heterocycle. ${ }^{27}$ Additionally, examples of S-, N,S-, $N, O-O, O-, N, N$-hetrocycles, which are structurally analogous to $\mathbf{3}$ are also scarce. ${ }^{25,28}$ The need for 8 -membered $N, S$-heterocycles is increasing due to their occurrence in biologically active compounds as a substructure. This prompted us to design a suitable method for the synthesis of the 8-membered ring N,S-heterocycles.

RCM has proven its wide applicability in synthesizing medium-sized $N, O$-heterocyclic systems. ${ }^{28}$ We decided to apply RCM methodology to synthesize the 8-membered ring $N$ - 
S-heterocycle 2, with the hope that if this, otherwise improbable to achieve synthesis of the 8-membered heterocycle is successful, then RCM can be a very important synthetic tool in making other (9,10-membered and so on..) macrocyclic systems. We decided to employ RCM to synthesize rarely studied sulfur compounds of the type 2 with subsequent oxidation of sulfur to give sulfoxide 3 (Scheme 1). Retro-synthetic analysis gave us the structure of the RCM precursor as $\mathbf{1}$. However, our efforts to get the cyclized product 2, met with complete failure under varied RCM reaction conditions. Although, we were disappointed, the result was not unexpected looking at the volume of literature ${ }^{29}$ available about failures of sulfur containing compounds to produce successful RCM results.
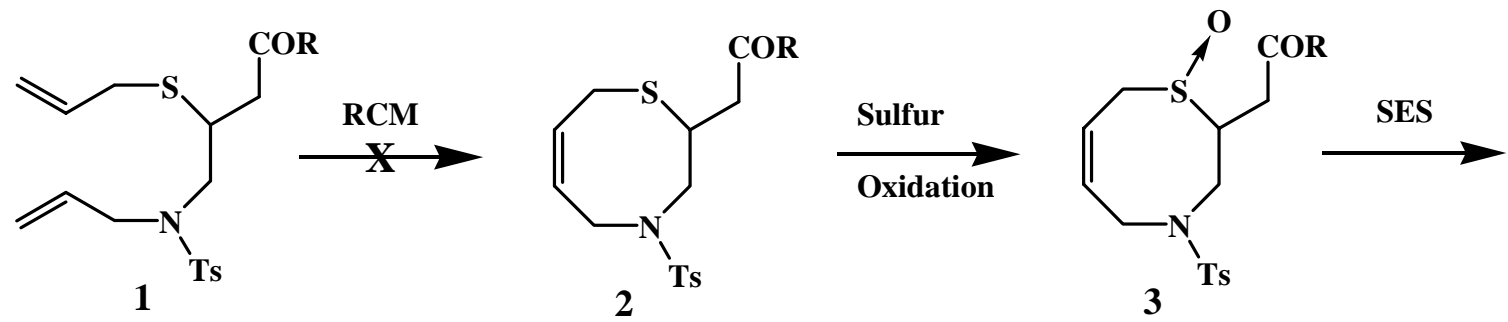

Scheme $1 \quad \mathrm{R}=\mathbf{O C H}_{3}$, Indole, Indoline

Basset and co-workers ${ }^{30}$ reported ring-closing metathesis on sulfur containing substrates as early as 1995 . Diallyl sulfides of the type $\mathbf{4}$ gave successful cyclized products with catalyst A. The same substrate, with catalyst $\mathbf{B}$ gave better yields than A, however, catalyst $\mathbf{C}$ did not produce a cyclized product.

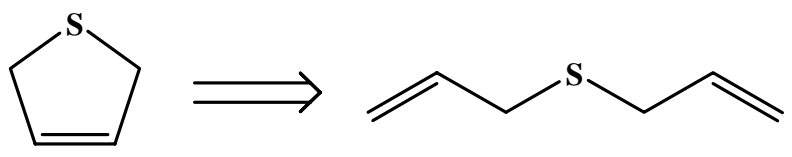


The main reason for the failure of catalyst $\mathbf{C}$ was $\mathrm{Ru}-\mathrm{S}$ (II) lone pair coordination, resulting in catalyst deactivation (poisoning). It was believed that, such Mo-S (II) coordination was avoided in case of catalyst $\mathbf{B}$ due to steric bulk around the central Mo atom. ${ }^{16}$ This example demonstrates the effect of changing the catalyst in order to get improved results. Unfortunately, due to financial constrains, instrumentation requirements, timeline of research, etc., it is not always possible for chemists to do such change readily.

As mentioned earlier, research in the development of a more efficient catalyst has answered these questions to some extent. For example, Grubb's $2^{\text {nd }}$ generation catalysts $\mathbf{D}$ and $\mathbf{E}$ showed improved heteroatom and functional group tolerance than generation one catalyst $\mathbf{C}$. The added air and moisture stability along with the commercial availability of $\mathbf{D}$ and $\mathbf{E}$ has increased their wide use over air senstitive and noncommercially available Mo-based catalysts.

Inspite of this, there is still no assurance of successful RCM chemistry on sulfur substrates as evident from unsuccessful cyclizations of some diallyl sulfides with catalyst $\mathbf{E}^{31}$ and our own example (Scheme 1), which uses catalyst $\mathbf{D}$. It is extremely discouraging for a chemist to see such unsuccessful results considering the time, labor and cost of chemicals involved in the synthesis of its precursors.

In parallel to the catalyst development studies, there are some reports where substrate modifications were helpful in getting successful results to synthesize $S$-heterocycles using RCM. For example, introduction of bulky t-butyl ester replacing hydrogen gave an almost quantitative yield (99\%) of the cyclized product $5 \mathbf{a}$ compared to a mere $19 \%$ of $\mathbf{5 b}$ when $\mathrm{R}_{1}=\mathrm{H}$. with catalyst $\mathbf{C}$ (Scheme 2$)^{32}$ 

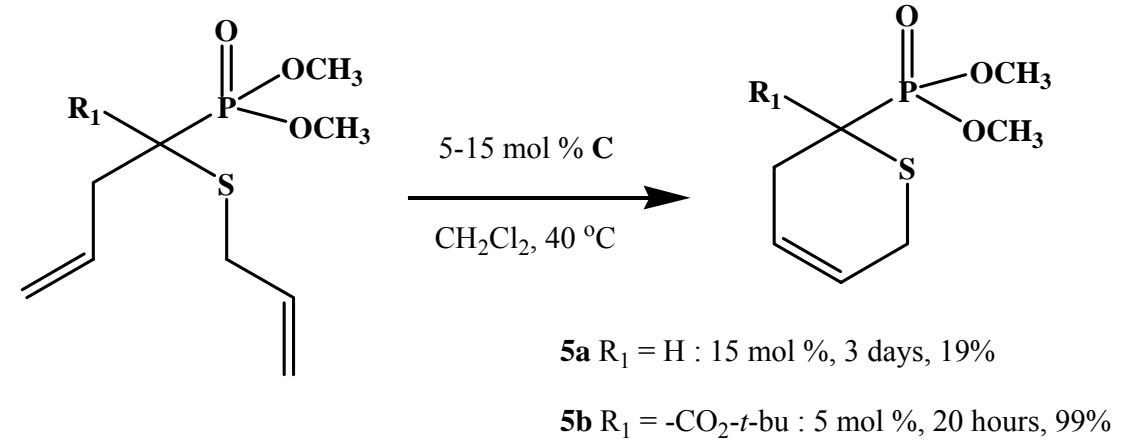

\section{Scheme 2}

Some other substrate modifications include complete oxidation of sulfides to corresponding sulfones. Sulfones tend to give much better results compared to their sulfide analogs. ${ }^{33}$ The success of these examples lies in avoiding the Ru-S(II) coordination by the steric bulk of $t$-butyl ester in one case (Scheme 2) and by the absence of lone pairs on sulfur, in case of sulfones.

An interesting point to note in both the above-mentioned examples is that success was achieved after making irreversible or permanent substrate modifications. This is important because, if the RCM reaction with S(II) containing substrates is to fail, then it may not always be possible for a chemist to make such irreversible substrate modifications. Furthermore, introduction of added functionality (for example, $t$-butyl ester in Scheme 2) could cause severe interference during the synthetic steps to be followed after RCM in any given reaction scheme depending on the chemical nature of functionality. Additionally, the need of sulfur (II) in the target compound is very high, as many of the biologically active $S$-heterocycles are in the form of sulfides. These issues, not only restrict the chemists ability in making their originally intended target compound but it also limits the scope of RCM as applied to sulfur chemistry. 
Change in the oxidation state of sulfur, is an attractive approach, but sulfone is not the only choice, specifically because the reduction of sulfone to produced desired sulfide is difficult to persue. Hence, we sought to carry out partial or incomplete (intermediate) oxidation of sulfide to get sulfoxide. Rather surprisingly, RCM studies on sulfoxides are rarely seen. To the best of our knowledge, prior to our work, there were only two reports of such studies with equal results of success ${ }^{34}$ and failure. ${ }^{35}$ It may be recalled that our earlier proposed reaction scheme involved reaction steps as $\mathrm{RCM} \rightarrow$ Sulfur oxidation $\rightarrow$ SES in that order (Scheme 1). After unsuccessful RCM results on sulfide 1, we exchanged the first two steps of the proposed scheme and carried out partial sulfur oxidation first, followed by RCM on sulfoxide $\mathbf{6}$. We were pleased to see that sulfoxide 6 sucessfully produced RCM cyclized sulfoxide 3 (Scheme 3). This success gave us one of our intended targets (compound 3), however, unsuccessful RCM on $\mathbf{1}$ precluded us in getting our other intended product, sulfide 2 . The idea of incomplete or partial oxidation of sulfide plays a crucial role here because, unlike previously reported substrate modifications, this modification is reversible. Individual oxidation and reduction steps are irreversible but the concept of performing these steps in a way shown below (Scheme 3) makes this modification reversible. For example, we achieved the reduction of sulfoxide 3 using Lawesson's Reagent (L.R.) and were able to successfully synthesize the intended sulfide 2.

The idea of using sulfoxide as an intermediate is advantageous, as there are plenty of reagents available to carry out sulfur oxidation and sulfoxide reduction. ${ }^{36}$ Such redox reactions are relatively cheap (in terms of cost of chemicals), less labor intensive and easy to perform. Importantly, by this approch, the change in catalyst is not required, 
which avoids the handling of the air sensitive catalyst and the need of a sophisticated instrumentation facility for such handling.

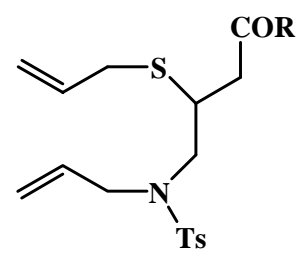

1
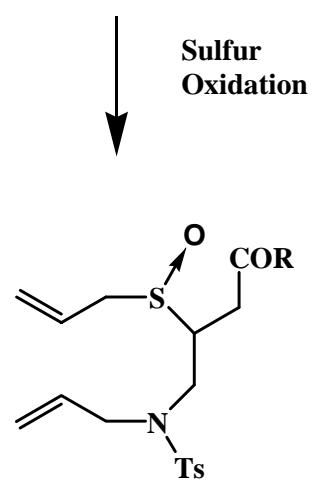

6

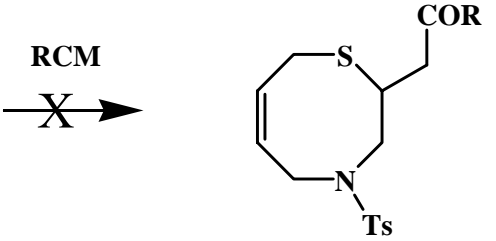

2
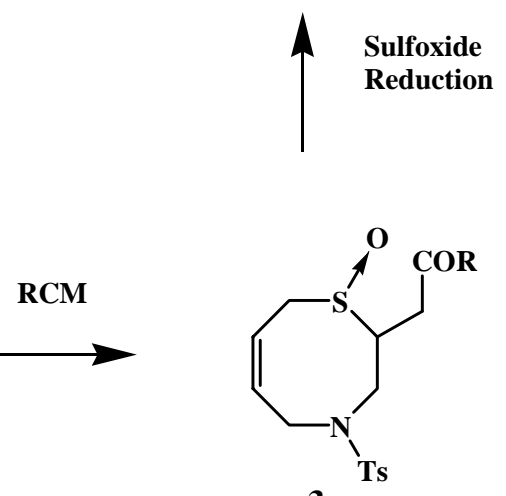

3

$$
\mathbf{R}=\text { Indole }
$$

\section{Scheme 3}

In other words, sulfoxide protects sulfur, presumably by preventing Ru-S(II) coordination during the RCM reaction thereby acting as a "protecting group" of sulfide. Based on all the advantages mentioned above, it is clear that using sulfoxide as a "protecting group" is the method of choice where RCM fails to produce the cyclized product with corresponding sulfide. It extends the scope and applicability of RCM on various sulfur containing substrates. It is even worth revisiting some of the earlier unsuccessful results with sulfides and applying this methodology. Importantly, with this 
powerful tool in hand, chemists will no longer have to make compromises in synthesizing their intended target compound, especially if it happens to be a sulfide [S(II)].

The demonstration of this concept with the most sensitive substrate (3) of this series, along with other synthetic efforts towards thiazocine-2-acetic acid derivatives was published in the following article:

Bates, D. K.; Li, X.; Jog, P. V. J. Org. Chem. 2004, 69, 2750.

This research was also presented at the ACS National Meeting, Bates, D. K.; Li, X.; Jog, P. V. Book of Abstracts, $227^{\text {th }}$ ACS National Meeting, Anaheim, CA March 28-April 1, 2004. ORGN-80.

My most important contribution was developing the redox protecting scheme for the indirect sulfide RCM methodology. This involved finding a suitable reagent, reaction conditions along with preparation of sulfide 2 (Scheme 3) and thorough spectroscopic characterization. I also synthesized various diene RCM precursors for exploratory work on scope and utility of this methodology. Due to space restrictions, thorough spectroscopic characterization such as COSY, HETCOR spectra of 2 along with the detailed chemical shift and the coupling constant information is included as an appendix 3 to this dissertation and is not included in the published article. 


\section{Part C: Sulfoxide as a "molecular brake"}

This part of the chapter demonstrates one of the unique applications of sulfide-sulfoxide redox chemistry.

\section{Introduction:}

Recently, there has been a lot of research interest in the area of molecular manufacturing ${ }^{37}$ to develop specific molecular systems with precisely positioned atoms and functional groups to achieve a desired behavior. The ultimate, rather far away, aim of this research is to synthesize a molecular analog of what we use in our everyday life, a machine. ${ }^{38}$ The idea of a molecular machine is based on the same principles as of any vehicle, appliances or even biological systems, muscles, etc. Molecular machines can achieve varied functions such as catalysis, nanoscale manipulations, information storage, and many more. ${ }^{39}$ In addition, an important factor, which adds a driving force to this research, is the nanoscale dimension of the molecular systems. In short, a lot can be achieved, if we were to make a nanoscale molecular machine to perform as many varied tasks as mentioned above.

The initial research in the designing of the molecular machine was challenging, mainly because there was nothing specifically known about any design. Equally important, as applicable to any machine, was to gain control over rather contineous molecular motions. Hence, the attention was shifted towards making smaller individual components first, with the ultimate assembling of each of these, to produce a complete molecular machine. This approach is commonly called as a bottom-up approach.

The integral component of the molecular machine is a "molecular brake". Kelley et al., were the early investigators of the molecular brake. ${ }^{40}$ Their design of the molecular brake 
involved a triptycene unit attached to a bipyridine unit. (Figure 1, adapted from reference 40).

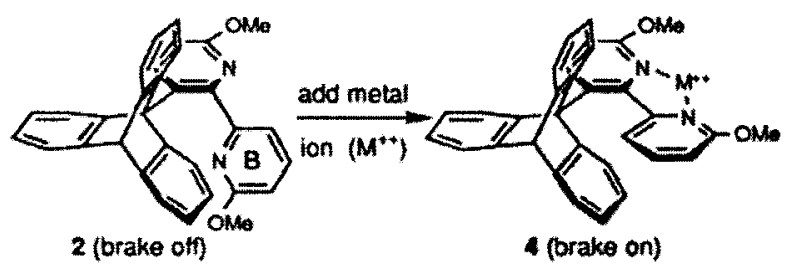

Brake "OFF" Brake "ON"

Figure 1: Example of the molecular brake

With the help of dynamic NMR spectroscopy (DNMR), they were able to show that the external addition of a metal ion solution $\left(\mathrm{Hg}^{+}\right.$ion) was capable of restricting the free rotation of the triptycene unit (brake "ON" mode). Disengagement of the brake was achieved with the addition of an EDTA solution, which selectively complexed the added metal ion resulting in the removal of the metal ion, from the system and free rotation of the triptycene unit (brake "OFF" mode).

Based on our knowledge of sulfur chemistry, we

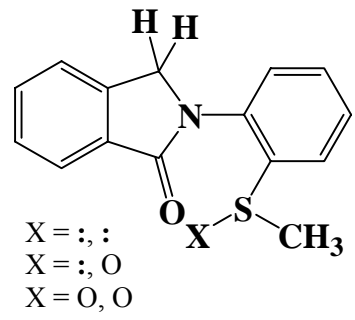

Figure 2: Isoindolines envisioned a molecular brake involving a sulfide-sulfoxide redox cycle. We designed a system Isoindoline-1-ones (Figure 2, detailed discussion about our design can be found in chapter 4), in which N-Aryl rotation was varied with the oxidation of the proximate sulfur atom. Dynamic spectroscopy studies on these compounds revealed that, sulfide [oxidation state of S(II)] shows free N-Aryl rotation while corresponding sulfoxide/sulfone resulted in hindered NAr rotation. 
This approach is novel for many reasons, first, it will add on to the current research of molecular brakes by giving a viable alternative to the scientists in this field, where the earlier example of the molecular brake (external addition of metal ion as rotation obstructive tool) could not be used. Secondly, since sulfur oxidation or reduction can be achieved easily by both chemical and electrochemical means, this type of molecular brake can be used to engage or disengage the brake externally (redox chemical reaction) or internally (redox electrochemical reaction). Importantly, our molecular brake satisfies the basic requirements of a molecular brake. Steric hindrance of sulfoxide oxygen provides the required hindrance to the free rotational process ( $\mathrm{N}$-Ar rotation) a brake "ON" mode and the ease of sulfide-sulfoxide redox chemistry gives the essential reversibility to the whole process.

The synthesis and characterization of isoindolines will be explained later (Chapter 4, supporting information), but before we go further it is important to understand some basic concepts and applications of dynamic NMR (DNMR) spectroscopy as applied to the research in the field of molecular machines.

\section{Principles and Applications of Dynamic NMR Spectroscopy ${ }^{41}$ :}

Dynamic NMR spectroscopy is a valuable tool for the chemists studying intramolecular motions. Intramolecular motions involve bond rotations, heteroatom inversions, ring inversions, etc. Although it is well known that the rotations about single bonds are rapid, there are examples of sterically or electronically hindered single bond rotations. Amides are probably the most widely studied example of functional groups by dynamic NMR 
spectroscopy ${ }^{42}$ and dimethylformamide (DMF, Figure 3 ) is the most common compound studied among other amides.

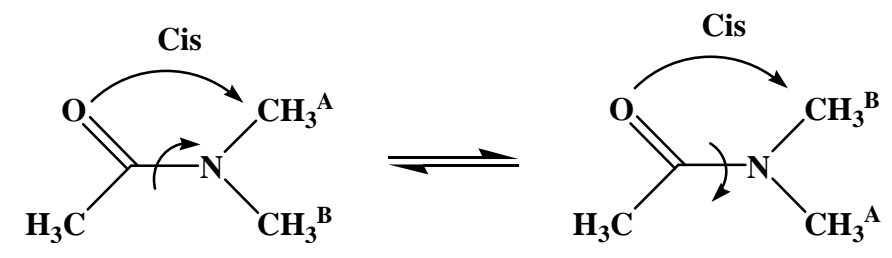

Figure 3: Dimethylformamide (DMF) with diastereotopic methyl grops.

The two methyl groups in DMF show separate signals in its ${ }^{1} \mathrm{H}$ NMR spectrum at room temperature. In this compound, due to amide resonance, the rotation about the amide bond is hindered. Methyl group cis to oxygen (Figure 3) is in a magnetically different environment than the methyl group trans to oxygen, which results in the two signals being separate. Chemically speaking, both methyl groups are identical. Such pairs of groups of atomic nuclei are called diastereotopic. If one of the groups is replaced by a different group then the compound forms a pair of diastereoisomers.

Another example of such class of compounds include the compound of the type $\mathbf{1}$ (Figure 4).

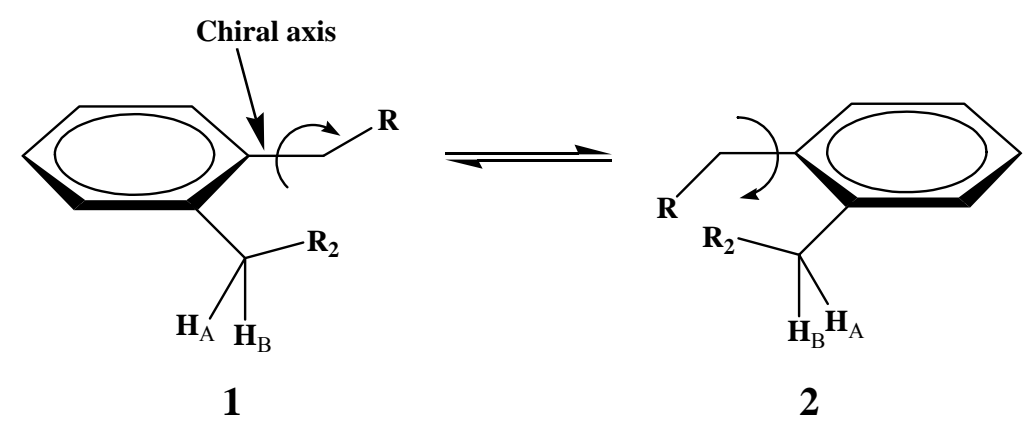

Figure 4: Schematic of enantiomeric rotational isomers. 
The molecule of the type $\mathbf{1}$ and $\mathbf{2}$ are called enantiomeric rotational isomers, because of the presence of the chiral axis. If a molecule in such a case is unsymmetrically substituted and adopts non-planar conformation, then it is probable for the two groups $\left(\mathrm{H}_{\mathrm{A}}\right.$ and $\mathrm{H}_{\mathrm{B}}$ in this case) to be magnetically nonequivalent (hence diastereotopic to each other) and give rise to two separate signals in the NMR spectra. The exact signal separation depends on the barrier to rotation about the chiral axis, a measure of ease by which these isomers interconvert. In addition, such magnetic nonequivalence is often temperature dependent (linear with temperature), in a specific range of temperatures, which is useful in quantification of the rotational barrier. This temperature dependence is non-linear for the molecules containing more than one stereogenic unit. For example, if the compounds similar to 1 contain additional stereocenter (eg. chiral center), in addition to the existing chiral axis, then such molecule shows conformational isomers when the rate of rotation is slow and configurational isomers when the rate of rotation is high (compound 3, Figure 5). Analysis of such systems with the dynamic NMR technique could be quite problematic and the NMR spectrum often displays unusual variations with changes in temperature.

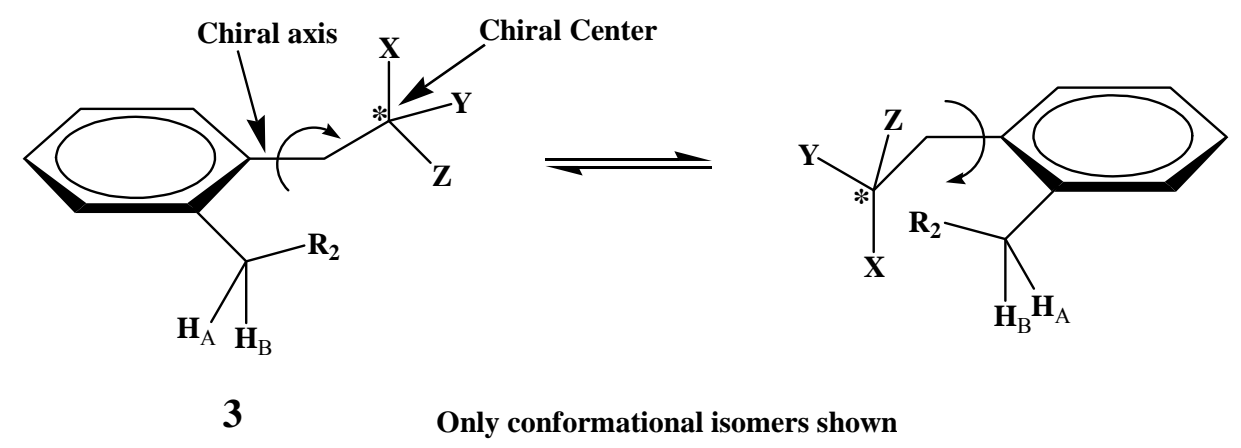

Figure 5 
The direct consequence of the hindered rotation is often encountered in ${ }^{1} \mathrm{H}$ NMR spectrum that shows some broad peaks in addition to normal sharp peaks. If the temperature is raised, the broad signal sharpens and if it is lowered, the original signal splits into one or more signals. Such behavior is an indication of an exchange in nuclei sites taking place at the temperature at which the spectrum is recorded. As temperature is raised, the rotation becomes faster due to an external supply of heat; similarly, a slow rotation is the result of cooling. A fast exchange limit is said to be reached when there is no further sharpening of the signal with a rise in temperature. Similarly, a stage at which the exchange of nuclei is negligibly small is called a slow exchange limit. It is in between these fast and slow exchange limits where dynamic NMR spectroscopy is valuable. The spectra recorded between these limits directly reflect the rate of exchange of nuclei. The most important temperature value for a dynamic NMR chemist is coalescence temperature. It is the temperature at which two signals coalsce (merge) to give one peak and it is denoted as Tc. Coalescence temperature gives information about the rate of exchange of nuclei (Eq 1). Equation 1 can only be used for the uncoupled nuclei. However, for the coupled systems (such as $\mathrm{H}_{\mathrm{A}}$ and $\mathrm{H}_{\mathrm{B}}$ ) equation 2 should be used to obtain the value of the rate of exchange. Using this value of the rate of exchange, the free energy of activation $\Delta G^{\mp}$ of exchange can be evaluated (Eq 3) along with other thermodynamic entities such as $\Delta H^{\mp}$ and $\Delta S^{\mp} .^{5}$

$$
\begin{aligned}
& k_{C}=2.22\left(\Delta v_{A B}\right) \\
& \left.k_{C}=2.22 \sqrt{\left(v_{A}\right.}-v_{B}\right)^{2}-6 J_{A B}{ }^{2}
\end{aligned}
$$




$$
\Delta G^{\mp}=4.575 \times 10^{-3} T_{C}\left[10.319+\log \left(\frac{T_{C}}{k}\right)\right]
$$

Free energy of activation $\Delta G^{\mp}$ is the most common way to express the intensity of hindered rotation. For example, the amide rotation in DMF has a free energy of activation of about $21.5 \mathrm{kcal} / \mathrm{mol}(89.9 \mathrm{~kJ} / \mathrm{mol}),{ }^{43}$ a value as high as that is an indication of highly hindered rotation. This is an example of a high rotational barrier value due to a preexisting constrain in the molecule. Extrapolating the same concept, it is reasonable to say that if for a specific molecule, the rotational barrier of a given rotation increases due to structural modification in the molecule or external stimuli, then that is the result of enforced restriction of the rotation about that bond.

It is well known that dynamic NMR spectroscopy is an invaluable technique to study intramolecular motions with activation energies of 5-25 kcal/mol (21-104 kJ/mol), which corresponds to about $10^{-4}$ to $10^{10} \mathrm{sec}^{-1}$ rate of exchange. Isomers interconverting at such rates are impossible to isolate as separate entities at room temperature.

It is extremely important to mention here that all the things said above, about fast and slow rotations are strictly based on what is being observed at the NMR time scale. For example, a slow rotation at the NMR time scale actually means that the rate of exchange is slower than the limit detectable by the dynamic NMR spectroscopy technique. Detection of slow or fast rotation often depends on the strength of the magnetic field under which the study is performed. 


\section{Line Shape Analysis ${ }^{41}$ :}

As mentioned earlier, the rate of exchanging nuclei can be calculated from the coalescence temperature (Tc), however it is always desirable to analyze data further to obtain the rate of exchange at various temperatures. Total line shape analysis is the method most commonly used to get the rate of exchange in the range of coalescence temperature. Line shape analysis is often performed with specifically designed computer

software. ${ }^{44}$ The computing program requires the values of chemical shifts, coupling constant and the experimentally recorded spectrum at any given temperature. It then simulates the signal shapes (line shape) and generates a "theoretical" spectrum at that temperature. This theoretical spectrum is a good visual match of the experimental spectrum, and more importantly, it provides the value of the rate of exchange at that temperature. Similar analysis near the range of coalescence temperature (usually $10{ }^{\circ} \mathrm{C}$ below and $2-3{ }^{\circ} \mathrm{C}$ above coalescence temperature) gives reliable data of the exchange rate. Plotting a graph (lnk vs $1 / \mathrm{T})$ of such data gives a value of the free energy of activation $\left(\Delta G^{\mp}\right)$, which eventually gives the estimate of other thermodynamic parameters such as $\Delta H^{\mp}$ and $\Delta S^{\mp}$.

\section{Computational Methods:}

A variety of computational methods such Molecular Mechanics (for example $\mathrm{MM}+$ ), Semi-empirical (for example, PM3), ab-initio (for example, STO3-G), etc. have been employed in evaluating the rotational barrier about sterically hindered rotations. A major advantage of computational methods is that it gives an exact nature of steric strain caused due to the precise positioning of the atoms during the complete rotational process 
(dihedral angles ranging from $0^{\circ}$ to $360^{\circ}$ ). This information is rarely available from the experimental methods. Furthermore, computational study helps to compare the data obtained from experimental techniques.

A chemist often has to make a choice of the computational method to be used for computation, based on available computational software sophistication and the time involved in doing the calculations. Molecular mechanics $(\mathrm{MM}+)$ is a popular method because of its high-speed ability to do calculations, however, data obtained from this method may not compare well with the experimental techniques and hence can be intriguing. In the MM+ method, molecules are treated as mechanical parts with atoms as spheres and bonds as springs. Such treatment, sometime results in false, unrealistic energy minima (local minima). A semi-empirical method (eg. PM3) is based on the experimentally derived parameters and although it takes more time than $\mathrm{MM}+$, it provides more accurate results than the $\mathrm{MM}+$ method. A highly sophisticated method is an ab-initio method of computation, which works best for highly complex molecules. This method provides accurate, intense (sometimes unnecessary) data but suffers from serious limitations in terms of the enormous time taken to do the calculations. Based on this information, it is easy to infer that within any given time limit, the semi-empirical (PM3) method is the best suitable for moderately complex molecules.

Among others, amides are the most commonly studied functional group by computational methods. There are reports of electronic and steric effects of nitrogen substitution on hindered amide rotations. ${ }^{45}$ There is a lot of interest in studying structure and geometries of strained amides. ${ }^{46}$ Particularly, in understanding how the rotation about such strained amide bonds takes place and if there is any change in hybridization of 
amidic nitrogen during the rotational process. While the study is still in progress, there are reports of otherwise $\mathrm{sp}^{2}$ hybridized amidic nitrogen undergoing change in hybridization from $\mathrm{sp}^{2}$ to $\mathrm{sp}^{3}$ as the substituent on nitrogen rotates. ${ }^{46}$

As noted by Streitwieser, Jr., A.; Heathcock, C. H.:

Ammonia itself and amines generally have a pyramidal structure (Section 23.1); the $\mathrm{H}-\mathrm{N}-\mathrm{H}$ bond angle in ammonia is $107.1^{\circ}$. The most effective conjugation of the nitrogen lone pair with the benzene ring would be obtained for a lone pair in a $p$-orbital parallel to the $p$-orbitals of the aromatic $\pi$-system. However, lone pairs are generally more stable in orbitals having some $s$-character. In the case of aniline, an energy compromise is reached in which the lone-pair orbital has more $p$-character than in ammonia but in which the orbital retains some s-character. As a result, the $\mathrm{NH}_{2}$ group in aniline is still pyramidal but with a larger $\mathrm{H}-$ $\mathrm{N}-\mathrm{H}$ angle $\left(113.9^{\circ}\right)$ than in ammonia. The $\mathrm{H}-\mathrm{N}-\mathrm{H}$ plane intersects the plane of the benzene ring at an angle of $39.4^{\circ}$.

--Streitwieser, Jr., A.; Heathcock, C. H. in Introduction to Organic Chemistry, $\left(3^{\text {rd }}\right.$ ed., Macmillan Publishing Company, New York, NY 1985.)

This effect applies, not only to anilines but also to amides. To avoid the concept of rehybridization $\left(\mathrm{sp}^{3}\right.$ in alkyl amines and $\mathrm{sp}^{2}$ in aryl amines, etc.) the concept of "nitrogen pyramidalization" was introduced. In this terminology, a pure $\mathrm{sp}^{3}$ hybridized nitrogen is said to have $100 \%$ pyramidalization. Any "flattening" of the nitrogen geometry decreases pyramidalization. Thus, planar nitrogen is said to have $0 \%$ of pyramidalization. Nitrogen pyramidalization is a conformational change that the molecule undergoes to minimize the steric interactions during rotation of the substituent. Nitrogen pyramidalization often results in reducing the rotational barrier of rotations. Rankin and Boyd ${ }^{47}$ developed a method to quantify nitrogen pyramidalization by measuring the virtual dihedral angle in 
case of prolyl amides. The arrows drawn (Figure 6), show how the virtual dihedral angle is defined. This value of this angle is $\pm 180^{\circ}$ for $\mathrm{sp}^{2}$ hybridized nitrogen and $\pm 120^{\circ}$ for the $\mathrm{sp}^{3}$ nitrogen.

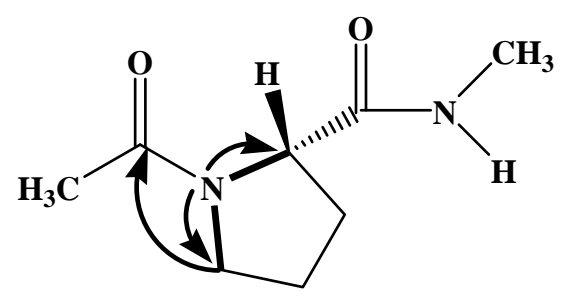

Figure 6: Definition of virtual dihedral angle as defined in prolyl amide.

The extent of nitrogen pyramidalization is a varying factor and it depends on the size of the substituent and its interaction with rest of the molecule during the rotational process. As the size of the substituent increases, its interaction with the rest of the molecule gets severe during its rotation. To reduce this sterically induced strain, nitrogen pyramidalization also increases and results in lowering the rotational barrier value of such process. However, if the steric strain is intentionally introduced in the molecule, then in that case, nitrogen pyramidalization partially nullifies the induced steric strain, giving less than the expected increase in the rotational barrier value.

It is reasonable to expect that nitrogen pyramidalization occurs only when there is a strong interaction between neighboring groups. Depending on the nature and size of the substituent, pyramidalization can occur only a few times during the complete rotational process (dihedral angles ranging from $0^{\circ}$ to $360^{\circ}$ ). In other words, rotation and pyramidalization are concerted or correlated events. Correlated rotations are known to show less than expected rotational barrier values. ${ }^{46 c}$ 
Lunazzi and co-workers ${ }^{48}$ developed a graphical method to show the presence of correlated rotations in molecules with the aid of computational methods. A 2D energy contour plot is plotted after driving the two dihedral angles under study (from $0^{\circ}$ to $360^{\circ}$ ) and obtaining the energy of the molecule at each fixed pair of dihedral angle. At each fixed pair of dihedral angle, the rest of the molecule is allowed to relax to its minimum energy conformation. A schematic of the 2D energy contour plot is shown in Figure 7. For example, let's assume that the contour plot obtained after driving dihedral angles A and B during such study looks like the one shown in Figure 7a. Then, since the line joining the energy minima is parallel to one axis (axis B in figure 7a) and perpendicular to another axis (axis $\mathrm{A}$ in Figure 7a), it can be deduced that the two rotations are noncorrelated to each other. In case of dihedral angles C and D however, (Figure 7b) such line is diagonal to one axis (axis D in figure $7 b$ ) and hence it can be said that the two rotations are correlated or geared.

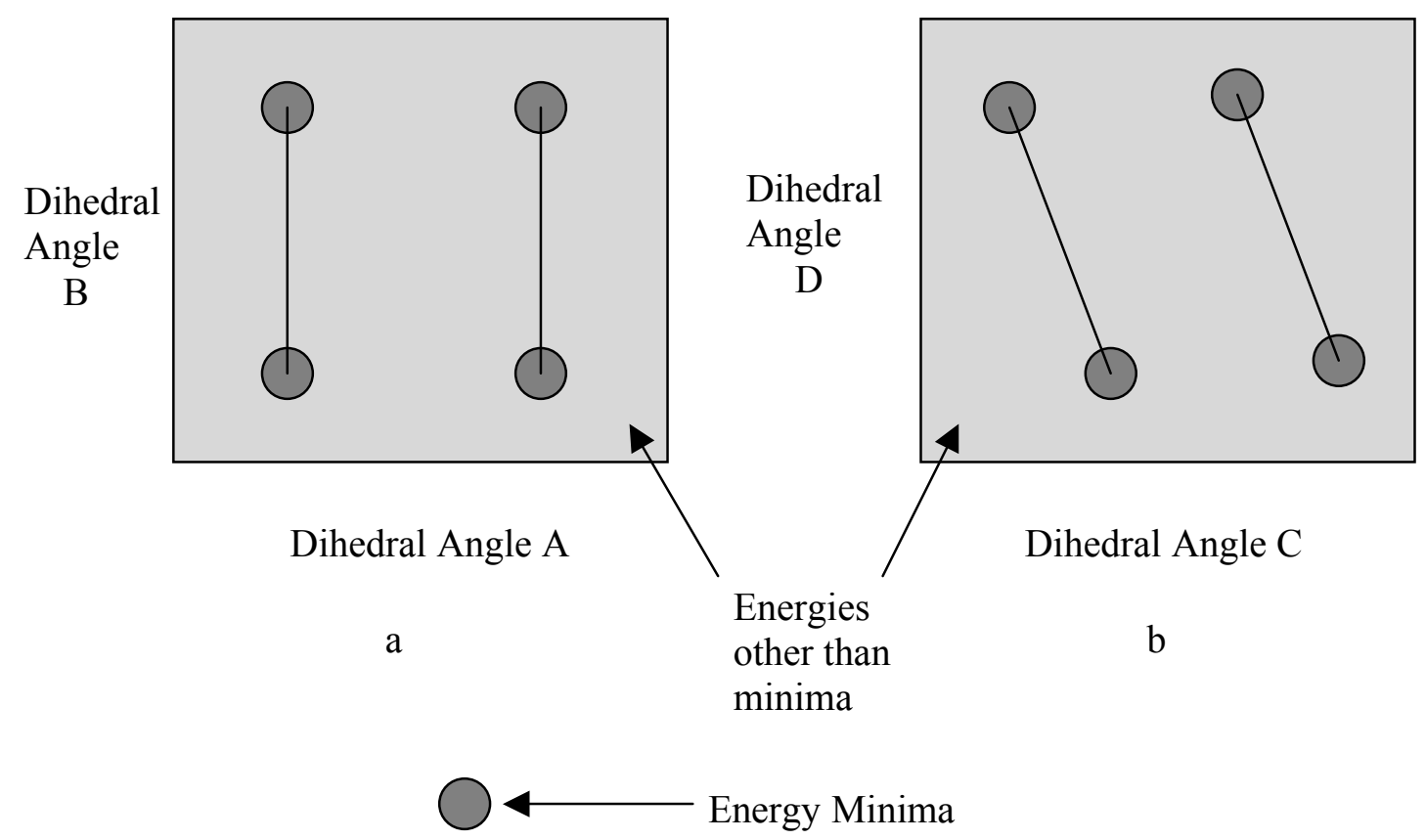

Figure 7: Schematic of 2D contour plot. 
Lunazzi and co-workers used ${ }^{48}$ this type of graphical notation to show the correlated or noncorrelated rotation between any given dihedral angles. We have designed a varient of this type of graph and plotted one dihedral angle involving complete rotation against another dihedral angle involved in nitrogen pyramidalization (virtual dihedral angle), while making sure (during the calculations) that both the dihedral angles cover the entire range of possible values. Detailed information of our results, along with the specific dihedral angles studied can be found in Chapter 4 .

All of the above discussion is helpful in understanding our sulfide-sulfoxide redox mediated molecular brake design which is published in the following article:

Jog, P. V.; Brown, R. E.; Bates, D. K. J. Org. Chem. 2003, 68, 8240.

This research was also presented at the ACS National Meeting, New Orleans, LA., Jog, P. V.; Brown, R. E.; Bates, D. K. Book of Abstracts, $225^{\text {th }}$ ACS National Meeting, New Orleans, LA March 23-27, 2003. ORGN-541. 


\section{References:}

1. Bates, D. K.; Tafel, K. A. J. Org. Chem. 1994, 59, 8076.

2. Bates, D. K.; Sell, B. A.; Picard, J. A. Tetrahedron Lett. 1987, 28, 3535.

3. Bates, D. K.; Winters, R. T.; Picard, J. A. J. Org. Chem. 1992, 57, 3094.

4. Tafel, K. A.; Bates, D. K. J. Org. Chem. 1992, 57, 3676.

5. Bates, D. K.; Habib, Q. A. J. Heterocyclic Chem. 1995, 32, 1477.

6. Kamiya, T.; Teraji, T.; Saito, Y.; Hashimoto, M.; Nakaguchi, O.; Oku, T. Tetrahedron Lett. 1973, 32, 3001.

7. Trost, B. M. Chem. Rev. 1978, 78, 363.

8. Shelton, J. P.; Davis, K. E. Int. J. Sulfur Chem. 1973, 3, 197.

9. (a) Aversa, M. C.; Barattucci, A.; Bonaccorsi, P.; Giannetto, P.; Jones, D. N. J. Org. Chem. 1997, 62, 4376. (b) Aucagne, V.; Aversa, M. C.; Barattucci, A.; Bonaccorsi, P.; Giannetto, P.; Rollin, P.; Tatibouet, A. J. Org. Chem. 2002, 67, 6925.

10. Emerson, D. W.; Kornski, T. J. J. Org. Chem. 1969, 34, 4115.

11. Davis, F. A.; Jenkins, R. H., Jr.; Rizvi, S. Q. A.; Yocklovich, S. G. J. Org. Chem. 1981, 46, 3467.

12. (a) Jones, D. N.; Hill, D. R.; Lewton, D. A.; Sheppard, C. J. Chem. Soc. Perkin Trans. 1 1977, 1574. (b) Grainger, R. S.; Tisselli, P.; Steed, J. W. Org. Biomol. Chem. 2004, 2, 151 and references therein.

13. Aikins, J. A.; Zhang, T. Y. Process for the synthesis of benzo(b)thiophenes. Patent Application WO 96-US9167 19960604, 1996; Chem. Abstr. 126:117861. 
14. Block, E.; O’Connor, J. J. Am. Chem. Soc. 1974, 96, 3921.

15. Wright, S. W.; Abelman, M. M.; Bostrom, L. L.; Corbett, R. L. Tetrahedron Lett. 1992, 33, 153.

16. Armstrong, S. K. J. Chem. Soc., Perkin Trans. 1, 1998, 371.

17. Schrock, R. R.; Murdzek, J. S.; Bazan, G. C.; Robbins, J.; DiMare, M.; O’Regan, M. J. Am. Chem. Soc. 1990, 112, 3875.

18. (a) Schwab, P.; France, M. B.; Ziller, J. W.; Grubbs, R. H. Angew. Chem., Int. Ed. Engl. 1995, 34, 2039. (b) Wu, Z.; Nguyen, S. T.; Grubbs, R. H.; Ziller, J. W. J. Am. Chem. Soc. 1995, 117, 5503. (c) Schwab, P.; Grubbs, R. H.; Ziller, J. W. J. Am. Chem. Soc. 1996, 118, 100.

19. Scholl, M.; Ding, S.; Lee, C. W.; Grubbs, R. H. Org. Lett. 1999, 1, 953.

20. Huang, J.; Stevens, E. D.; Nolan, S. P.; Peterson, J. L. J. Am. Chem. Soc. 1999, $121,2674$.

21. Furstner, A. Angew. Chem., Int. Ed. Engl. 2000, 39, 3012.

22. Schuster, M.; Blechert, S. Angew. Chem., Int. Ed. Engl. 1997, 36, 2036.

23. For detailed discussion along with leading references see: Li, X. Application of Ring Closing Metathesis to Synthesis of Sulfoxide Electrophilic Sulfenylation Substrates and Other Novel N,S-Heterocycles. M.S. Thesis, Michigan Technological University, Houghton, MI, 2001.

24. Bates, D. K.; Xia, M. J. Org. Chem. 1998, 63, 9190 and references therein.

25. Illuminati, G.; Mandolini, L. Acc. Chem. Res. 1981, 14, 95.

26. Mandolini, L.; Vontor, T. Synth. Commun. 1979, 9, 857.

27. Vedejs, E.; Hagen, J. P. J. Am. Chem. Soc. 1975, 97, 6878. 
28. Following the general trend for cyclane formation (Eliel, E. L.; Wilen, S. H.; Mander, L. N. Stereochemistry of Organic Compounds, Wiley Interscience, New York, New York, 1994, pp 678-684) cyclic thialkanes and thiazalkanes (MaGee, D. I.; Beck, E. J. J. Org. Chem. 2000, 65, 8367-8371) containing eight- or ninemembered rings form in very low yield by treatment of the corresponding alkyl dibromides with sodium sulfide nonahydrate. Some simple cyclic 1,4-thiazepines are available from tetrahydrothiopyran-4-one (Beckmann rearrangement of the derived oxime followed by amide reduction). (Doi, J. T.; Musker, W. K.; deLeeuw, D. L.; Hirschon, A. S. J. Org. Chem. 1981, 46, 1239-1243) and 8-, 9-, and 10- ring atom oxathiacycloalkenes are available by ring expansion of haloO,S-acetals (Coster, M. J.; DeVoss, J. J. Org. Lett. 2002, 4, 3047-3050.).

29. For examples of RCM failures with Grubbs' Gen 1 catalyst on sulfur-containing compounds, see: (a) Fuerstner, A.; Seidel, G.; Kindler, N. Tetrahedron 1999, 55, 8215-8230. (b) Armstrong, S. K. J. Chem. Soc., Perkin Trans. 1 1998, 371-388. (c) Bujard, M.; Gouverneur, V.; Mioskowski, C. J. Org. Chem. 1999, 64, 2119 2123. (d) Mascarenas, J. L.; Rumbo, A.; Castedo, L. J. Org. Chem. 1997, 62, $8620-8621$.

30. (a) Couturier, J. L.; Tanaka, K.; Leconte, M.; Basset, J. M.; Ollivier, J. Phosphorus, Sulfur Silicon Relat. Elem. 1993, 74, 383-384. (b) Leconte, M. P. S.; Mutch, A.; Lefebvre, F.; Basset, J. -M. Bull. Soc. Chim, Fr. 1995, 132, 1069.

31. Spagnol, G.; Heck, M. -P.; Nolan, S. P.; Mioskowski, C. Org. Lett. 2002, 4, 1767.

32. Moore, J. D.; Sprott, K. T.; Hanson, P. R. Synlett 2001, 605.

33. Yao, Q. Org. Lett. 2002, 4, 427. 
34. Cachoux, F.; Ibrahim-Ouali, M.; Santelli, M. Synlett 2002, 1987.

35. Michrowska, A.; Bieniek, M.; Kim, M.; Klajn, R.; Grela, K. Tetrahedron 2003, $59,4525$.

36. For leading references see Jog, P. V.; Brown, R. E.; Bates, D. K. J. Org. Chem. 2003, 68, 8240 .

37. Drexler, K. E. Proc. Natl. Acad. Sci. 1981, 78, 5275.

38. Kelly, T. R. Acc. Chem. Res. 2001, 34, 514.

39. Stevens, A. M.; Richards, C. J. Tetrahedron Lett. 1997, 38, 7805.

40. Kelly, T. R.; Bowyer, M. C.; Bhasker, K. V.; Bebbington, D.; Garcia, A.; Lang, F.; Kim, M. H.; Jette, M. P. J. Am. Chem. Soc. 1994, 116, 3657.

41. (a) Oki, M. Applications of Dynamic NMR Spectroscopy to Organic Chemistry; VCH Publishers, Deerfield Beach, Fla. 1985. (b) Sandstroem, J. Dynamic NMR Spectroscopy; Academic Press, London, UK. 1982.

42. Stewart, W. E.; Siddal (III), T. H. Chem. Rev. 1970, 70, 517.

43. Kessler, H. Angew. Chem. Int. Ed. Engl. 1970, 9, 219.

44. For example, we use gNMR (V4.1.0), Cherwell Scientific, Inc. to perform line shape analysis on spectra obtained with our system.

45. Yoder, C. H.; Sandberg, J. A.; Moore, W. S. J. Am. Chem. Soc. 1974, 96, 2260.

46. (a) Tsubrik, O.; Burk, P.; Pehk, T.; Maeorg, U. THEOCHEM, 2001, 546, 119. (b) Yamamoto, G.; Nakajo, F.; Mazaki, Y. Bull. Chem. Soc. Jpn. 2001, 74, 1973. (c) Kiefl, C. Eur. J. Org. Chem. 2000, 3279. (d) Glover, S. A.; Mo, G.; Tucker, D. J.; Turner, P. J. Chem. Soc., Perkin Trans. 2, 1999, 2053. (e) Ferretti, V.; Bertolasi, V.; Gilli, P.; Gilli, G. J. Phys. Chem. 1993, 97, 13568. (f) Bennet, A. J.; Somayaji, 
V.; Brown, R. S.; Santarsiero, B. D. J. Am. Chem. Soc. 1991, 113, 7563. (g) Gilli, G.; Bertolasi, V.; Bellucci, F.; Ferretti, V. J. Am. Chem. Soc. 1986, 108, 2420.

47. Rankin, K. N.; Boyd, R. J. J. Phys. Chem. A 2002, 106, 11168.

48. (a) Casarini, D.; Grilli, S.; Lunazzi, L.; Mazzanti, A. J. Org. Chem. 2001, 66, 2757. (b) Grilli, S.; Lunazzi, L.; Mazzanti, A. J. Org. Chem. 2001, 66, 4444. (c) Grilli, S.; Lunazzi, L.; Mazzanti, A. J. Org. Chem. 2001, 66, 5853. 


\section{Chapter 2}

\section{Sulfoxides as a sulfenylating agent}

\section{Intramolecular Sulfoxide Electrophilic Sulfenylation (SES) of 2- and 3- Indole Carbanilides: Formation of Indolo[3,2-b]-1,5-benzothiazepinones}

Bates, D. K.; Eggers, M. E.; Jog, P. V. J. Org. Chem. Submitted for publication.

The contribution of Parag Jog to this work included synthesis of all compounds containing $t$-butyl and 2-cyanoethyl groups as well as studies of thermal and TFAA activated reactions of the penultimate sulfoxide derivatives. He also did all of the work related to detection of sulfenic acid intermediates.

\section{Approved by co-author Dallas K. Bates}

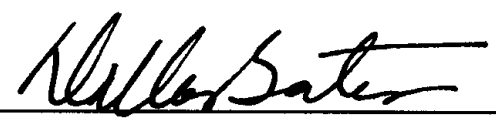




\title{
Intramolecular Sulfoxide Electrophilic
}

\author{
Sulfenylation (SES) of 2- and 3- Indole
}

\section{Carbanilides: Formation of Indolo[3,2-b]-1,5- benzothiazepinones}

Dallas K. Bates, * Mary E. Eggers, and Parag V. Jog

Department of Chemistry, Michigan Technological University, Houghton, MI 49931 


\begin{abstract}
A series of $\mathrm{N}$-[2-(alkylsulfinyl)phenyl]-1H-indole-2-carboxamides was prepared in which the indole and amidic nitrogens were either unsubstituted (2ab), partially substituted (indole N-H, amide N-Me, (4ab) and indole N-Me, amide N-H, (6ab), or fully substituted (indole $\mathrm{N}-\mathrm{Me}$, amide $\mathrm{N}-\mathrm{Me}$, (8abc). When these compounds were heated in an inert solvent (chloroform for $t$-butyl sulfoxides or $p$-xylene for ethyl sulfoxides) or treated with trifluoroacetic anhydride, all compounds $(\mathbf{4 a b}, \mathbf{8 a b c})$ in which the amidic nitrogen was methylated cyclize to indolo[3,2-b]-1,5-benzothiazepinones (9 or 10, depending on indolic substitution). Successful cyclization was attributed to an ability of the N-Me amides to readily adopt a conformation conducive to cyclization, which other derivatives were unable to achieve. The same pattern of reactivity was observed with $\mathrm{N}$-[2(alkylsulfinyl)phenyl]-1H-indole-3-carboxamides (15a, 17ab) under the same reaction conditions and the same product (10) was produced from (17a) upon heating in $p$-xylene. Rearrangement of a $3 \mathrm{H}$-indolinium spirocyclic intermediate is proposed to account for this result. Accumulating evidence, including cyclization in the presence of a sulfenic acid trapping agent, suggests these reactions take place via an sulfonium salt intermediate (electrophilic sulfur of sulfoxide attacked by a nucleophile) rather than via a sulfenic acid intermediate.
\end{abstract}




\section{Introduction}

Sulfoxides are widely used as electrophilic sulfenylating agents for alkenes and aromatic (and heteroaromatic) systems. There are two general mechanistic pathways by which sulfoxides are activated to become sulfenylating agents and both occur with reagents and conditions typical for the Pummerer Reaction. ${ }^{1}$ Many reported 'anomalous' reaction products from Pummerer Reactions are electrophilic sulfenylation reactions, with the 'anomalous' pathway favored by the presence of a proximate nucleophilic group $^{2}$ or a non-acidic hydrogen atom on the carbon $\alpha$ to the sulfoxide. ${ }^{3}$

One pathway to sulfenylation involves sulfenic acid intermediates. Treatment of a sulfoxide with an anhydride or trace of acid/heat in an inert solvent may form a mixed anhydride of a sulfenic acid ( $\mathrm{RSOCOCF}_{3}, \mathrm{RSOAc}$, etc.) or a sulfenic acid (RSOH, which presumably forms, in situ, $\mathrm{RSOH}_{2}{ }^{+}$as the active sulfenylating agent), ${ }^{4}$ which are reported to react with alkenes ${ }^{5,6}$ or arenes ${ }^{7}$ via electrophilic addition or electrophilic substitution, respectively. The other mechanistic pathway to sulfenylation with sulfoxides involves direct (nucleophilic) attack at (electrophilic) sulfur in an activated sulfoxide $\left(\mathrm{R}_{2} \mathrm{~S}\right.$ $\mathrm{O}_{2} \mathrm{CCF}_{3}^{+}$or $\mathrm{R}_{2} \mathrm{~S}-\mathrm{OH}^{+}$) forming an intermediate sulfonium salt which is dealkylated, either in situ or as a separate step, to form the sulfenylation product. These two pathways are shown in Scheme 1. There are numerous examples of electrophilic sulfenylations of $\operatorname{aromatics}^{8}$ and heteroaromatics ${ }^{9}$ utilizing this approach. 
Scheme 1. Alternative Pathways to Electrophilic Sulfenylation with Sulfoxides ${ }^{\text {a }}$

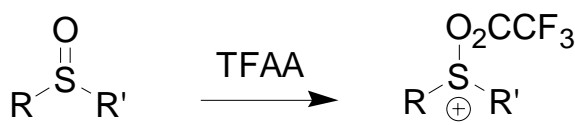

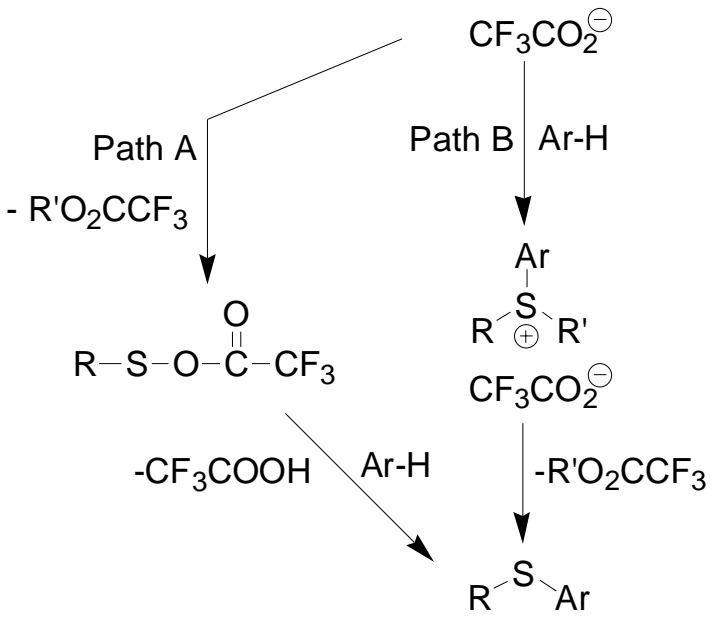

/a Illustrated with trifluoroacetic anhydride as the sulfoxide activator. Other reagents may be used (see text).

We have exploited this chemistry (sulfoxide electrophilic sulfenylation-SES) followed by variations in the mode of dealkylation to prepare new $N, S$-heterocyclic systems: SES/displacement ${ }^{10}$ (eq 1) and SES/ring enlargement ${ }^{11}$ (eq 2). We have also observed a third variant, SES/rearrangement ${ }^{12}$ in a pyrrole system (eq 3). In this process, a minor product under all conditions tried was the rearranged product shown. Its formation was rationalized as resulting from rearrangement of an initially formed spirocyclic intermediate. 


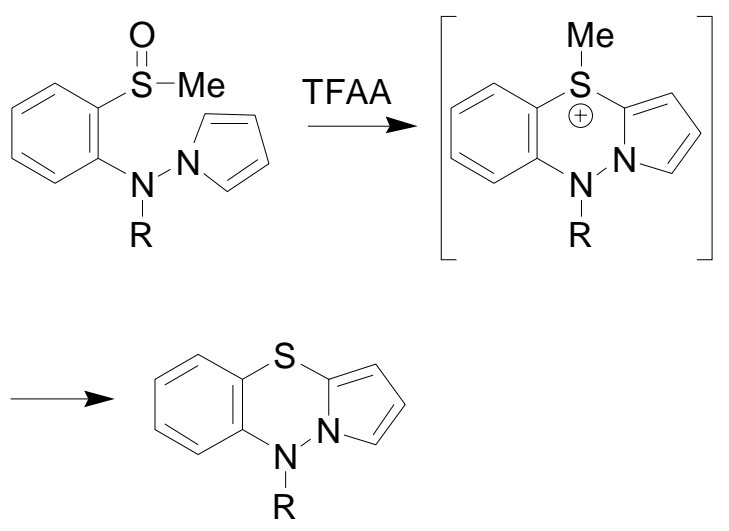

(1)
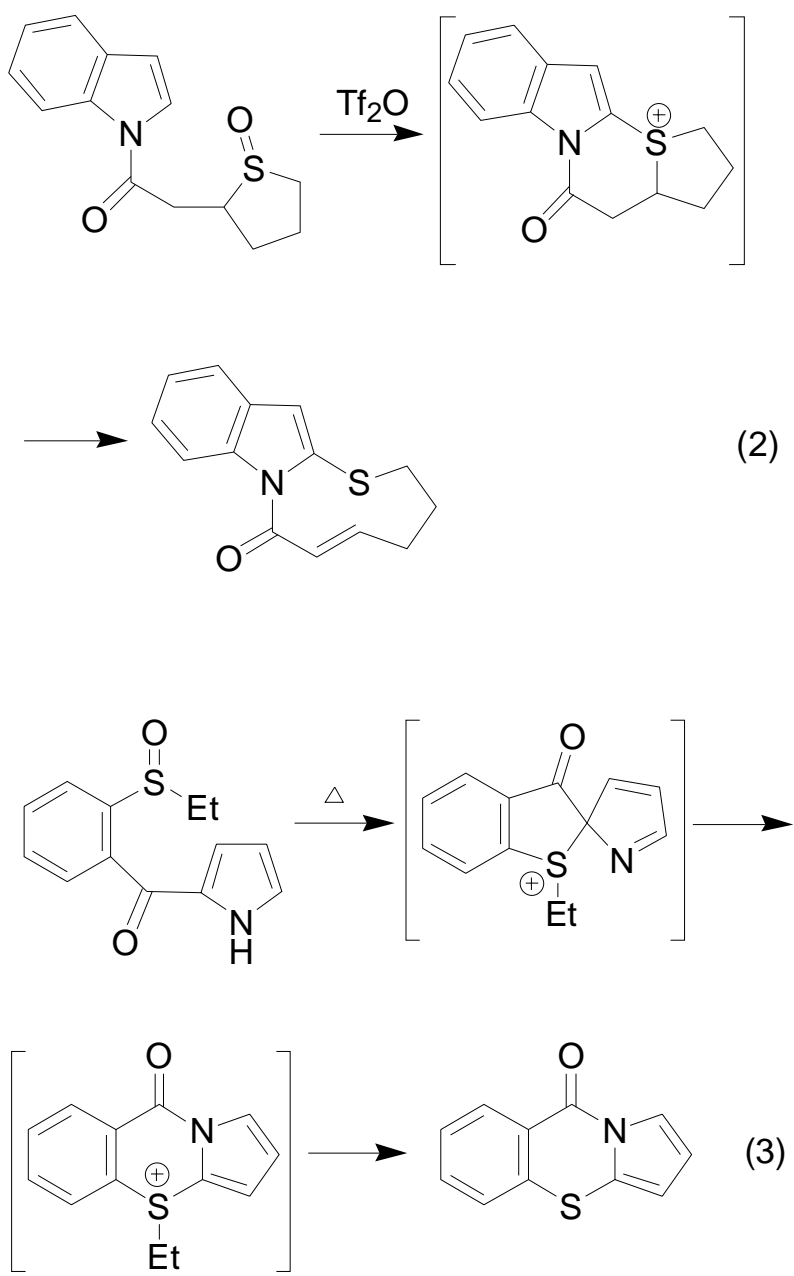

In this article we describe a study of SES/rearrangement cascade in a series of 2- and 3substituted indole compounds. Indoles exhibit a strong preference for electrophilic attack at C-3 even when that position is already substituted. The 3-substituted target compounds 
were selected to attempt to enhance rearrangement processes because 3,3-spirocyclic and other 3,3-disubstituted-3H-indolinium species resulting from indole/electrophile interactions are a common occurrence throughout indole chemistry. ${ }^{13}$ In each series, sulfoxides containing ethyl, t-butyl, and propanenitrile alkyl group were prepared. The latter two types of sulfoxides, having non-acidic alkyl groups, should undergo SES reactions (not Pummerer chemistry) but would be expected to dealkylate under milder conditions than ethyl substituted compounds. ${ }^{14}$ These compounds are of interest because t-butyl and propanenitrile sulfoxides also very easily thermally decompose to sulfenic acid derivatives ${ }^{15}$ providing a potential opportunity to study their relevance as intermediates in these reactions.

\section{Results and Discussion}

Several related (ethyl, t-butyl, and 2-propanenitrile) sulfoxides tethered to C-2 or C-3 of indole were prepared with indole and amidic nitrogen atoms in differing degrees of methylation. Preparation and reactions of the $\mathrm{C}-2$ and $\mathrm{C}-3$ substituted compounds are discussed separately below. 


\section{Scheme $2^{\text {a }}$}

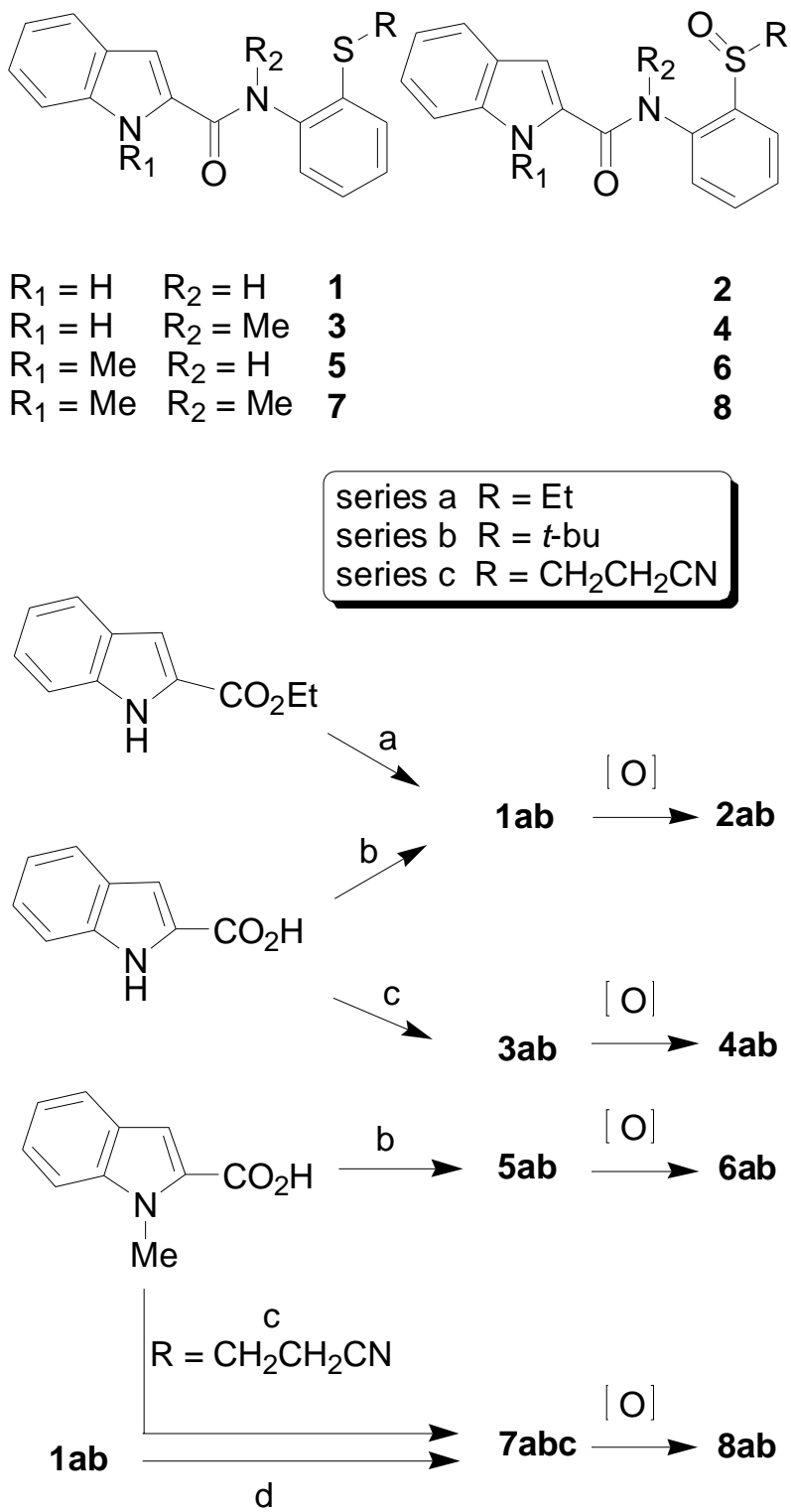

/a conditions: (a) 2-ethylthioaniline, $\mathrm{AlMe}_{3}$, toluene, reflux (b) $\mathrm{SOCl}_{2}, \mathrm{Et}_{2} \mathrm{O}, 2$ (alkylthio)aniline, rt (c) $\mathrm{SOCl}_{2}, \mathrm{Et}_{2} \mathrm{O}, \mathrm{N}$-methyl-2-(alkylthio)aniline, rt (d) $50 \% \mathrm{NaOH}$, $\mathrm{CH}_{3} \mathrm{I}, n-\mathrm{Bu}_{4} \mathrm{NHSO}_{4}$, toluene.

\section{Indoles substituted at C-2}

Indole-2-carboxylic acid chloride, formed in situ by reaction of indole-2-carboxylic acid with thionyl chloride at room temperature ${ }^{16}$, and a 2 -(alkylthio)aniline ${ }^{17}$ produced sulfides 1ac in modest yields. Problems have been reported in reaction of indole 
carboxylic acids with thionyl chloride ${ }^{18}$ and use of the unstable acid chloride (and consequent low yield of amide) could be avoided by trimethylaluminum-catalyzed condensation $^{19}$ of 2-(alkylthio)anilines and ethyl indole-2-carboxylate. For example, 1a was obtained in $92 \%$ yield by this approach. Oxidations were accomplished using standard methodologies: a slow, but selective, multi-phase reaction using $\mathrm{NaIO}_{4}$ in $\mathrm{CH}_{2} \mathrm{Cl}_{2} / \mathrm{MeOH} / \mathrm{H}_{2} \mathrm{O}^{3 a}$; potassium peroxy monosulfate (Oxone) in THF/MeOH/ $\mathrm{H}_{2} \mathrm{O}^{20}$; or $m$-chloroperbenzoic acid (m-CPBA) in dichloromethane. ${ }^{21}$

Monoalkylated compounds bearing the methyl substituent on the amide (4ab) were prepared similarly replacing the aniline component with an $N$-methyl-2(alkylthio)aniline, ${ }^{22}$ followed by sulfur oxidation.

Monoalkylated compounds bearing the methyl substituent on the indole (6ab) were prepared similarly using alkylthioanilines and $N$-methylindole carboxylic acid, followed by oxidation.

$N, N^{\prime}$-Dimethylated compounds (8ab) were prepared by dialkylation of amides (1ab) by catalytic phase transfer methylation, followed by oxidation. Sulfide (1c) decomposed under phase transfer conditions (retro-Michael) so compound 8c was prepared in low yield by reaction of 1-methyl- $1 H$-indole-2-carboxylic acid chloride with 2-propanenitrile2-(methylaminophenyl)sulfide).

When the sulfoxides were subjected to cyclization conditions [either activation by electrophilic species (TFAA) or thermally (refluxing in chloroform or $p$-xylene)] clear patterns of reactivity emerge. For unknown reasons, thermal conditions always produced lower yield of product than with TFAA activation. As expected, in successful reactions, $t$ butyl sulfoxide derivatives cyclize thermally in refluxing chloroform whereas ethyl 
sulfoxide derivatives require much higher temperatures (refluxing in $p$-xylene). In addition, as shown in Table 1, a substituent at the amidic nitrogen is essential for cyclization of all sulfoxides. Interestingly, in every case, the ${ }^{1} \mathrm{H}$ NMR spectrum of a sulfoxide that successfully cyclizes was very poorly resolved. This is indicative of conformational interconversion between two or more rotomers taking place on the millisecond time-scale at $25{ }^{\circ} \mathrm{C}$. Recording ${ }^{1} \mathrm{H}$ NMR spectra at $100{ }^{\circ} \mathrm{C}$ showed fewer, sharper peaks as expected for an increased rate of interconversion in a dynamic process. ${ }^{24}$ It seems logical that conformational flexibility could help the molecule readily obtain the geometry required for indole pi electron - sulfoxide sulfur atom interaction and it is well known that $N, N$-dialkyl amides have lower barriers to $\mathrm{C}(\mathrm{O})-\mathrm{N}$ rotation as well as higher energy, non-planar ground states (hence the lower rotational barrier) than primary or secondary amides. ${ }^{25}$ 
Table 1. Correlation of Cyclization and Spectral Data for Sulfoxides

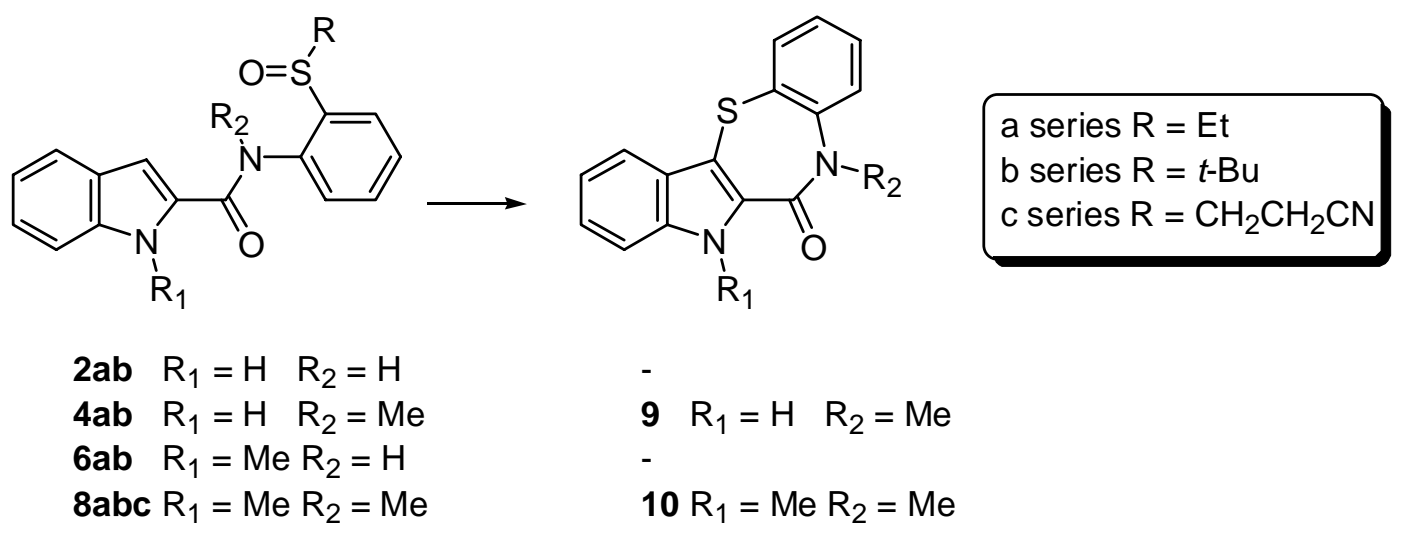

\begin{tabular}{|l|l|l|l|}
\hline Sulfoxide & $\begin{array}{l}\text { Cyclization product } \\
\text { \% yield (TFAA, thermal) }\end{array}$ & $\begin{array}{l}\text { H NMR } \\
(\mathrm{rt})\end{array}$ & $\begin{array}{l}\mathrm{U}_{\mathrm{S}=\mathrm{O}} \\
\left(\mathrm{cm}^{-1}\right)\end{array}$ \\
\hline $\mathbf{2 a}$ & none & sharp & 1009 \\
\hline $\mathbf{2 b}$ & none & sharp & 985 \\
\hline 4a & $\mathbf{9}(75,56)$ & broad & 1041 \\
\hline $\mathbf{4 b}$ & $\mathbf{9}(100,58)$ & broad & 1040 \\
\hline $\mathbf{6 a}$ & none & sharp & 1003 \\
\hline $\mathbf{6 b}$ & none & sharp & 999 \\
\hline $\mathbf{8 a}$ & $\mathbf{1 0}(90,22)$ & broad & 1025 \\
\hline $\mathbf{8 b}$ & $\mathbf{1 0}(100,66)$ & broad & 1039 \\
\hline $\mathbf{8 c}$ & $\mathbf{1 0}(50,41)$ & broad & 1039 \\
\hline
\end{tabular}

Sulfoxides which bear only a proton on the amidic nitrogen (2ab, 6ab) produced either decomposition products or were recovered unchanged. This may be due to factors preventing the sulfoxide sulfur atom and the indole pi-system from assuming the correct conformation for cyclization. There is evidence of $\mathrm{S}=\mathrm{O}---\mathrm{H}-\mathrm{N}$ intramolecular hydrogenbonding in ${ }^{1} \mathrm{H}$ NMR and IR spectra of these compounds. Sulfoxide peaks in the IR 
spectra of $2 \mathbf{a b}$ and $\mathbf{6 a b}$ appear at a lower frequency, which is to be expected if the oxygen of the sulfoxide is involved in hydrogen bonding ${ }^{26}$ (Table 1). Additionally, ${ }^{1} \mathrm{H}$ NMR spectra of 2ab and 6ab showed sharp and well-resolved peaks in contrast to ${ }^{1} \mathrm{H}$ NMR spectra of compounds in which the amidic nitrogen was methylated. Compounds in which the amidic site is methylated (but still contain an indole N-H) (4ab) cyclize both thermally and with TFAA activation to produce the same product, assigned the structure 10,11-dihydro-10-methyl-12H-indolo[3,2-b]-1,5-benzothiazepin-11-one (9). The failure of compounds containing amidic $\mathrm{N}-\mathrm{H}$ to cyclize is also attributable to the large energy difference in the cis- and trans- amide isomers, with the unfavorable (for cyclization) trans-isomer preferred. Not only are these compounds 'locked' into an arrangement wherein the sulfoxide oxygen is hydrogen bonded the amidic $\mathrm{NH}$, but the energy barrier to interconversion of the cis- and trans- amide forms is formidable. On the other hand, the amidic $\mathrm{N}(\mathrm{Me})$ compounds not only exhibit lower rotational barriers to cis-/transamide isomer interconversion, but the literature ${ }^{25 b}$ suggests that these compounds probably exist predominately in the cis-form (which in this case places the sulfoxide and indole entities near one another).

Compounds 8abc gave a compound assigned the structure 10,11-dihydro-10,12dimethylindolo[3,2-b]-1,5-benzothiazepin-11-one (10). Thermal conditions consistently produced lower yield of product than with TFAA activation with all sulfoxides. For reasons that are unclear, 2-propanenitrile compounds were inferior to both ethyl and $t$ butyl sulfoxides and were not pursued further. As with product $\mathbf{9}$, spectral data clearly shows cyclization was effected, however, additional proof of structure was sought due to the possibilities of scrambling of the acyl and sulfur locations either during the reaction 
or subsequent equilibration ${ }^{27,28}$ leading to compounds that would exhibit very similar spectral properties. Fortunately, the likely rearrangement product (13) is a known compound. The UV spectrum and melting point of our cyclization product differs considerably from that of $\mathbf{1 3}$ reported by Grandolini and co-workers ${ }^{29}$ ruling out this alternative isomer. In addition, $\mathbf{1 0}$ was synthesized via an unambiguous route (Scheme 3). Using the technique described by Atkinson, ${ }^{30}$ amino acid $\mathbf{1 1}$ was readily prepared. Refluxing 11 in toluene using $\mathrm{SiO}_{2}$ as catalyst ${ }^{31}$ gave 12 which on catalytic phase transfer methylation gave a product chromatographically and spectroscopically identical to the cyclization product (10), clearly establishing the sites of attachment of the sulfur and carbonyl groups on the indole ring. Catalytic phase transfer methylation of $\mathbf{9}$ also produced $10(76 \%)$ confirming the structure of that product as well. 
Scheme $3^{\text {a }}$

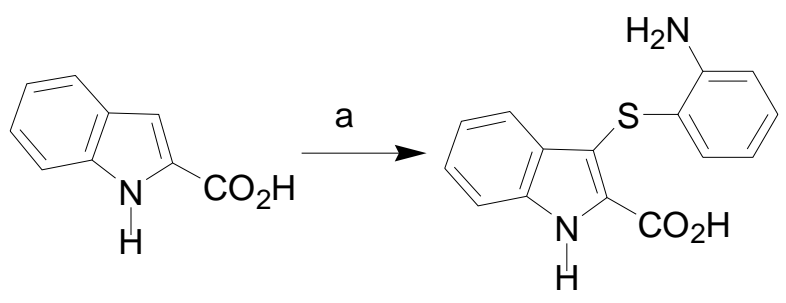

11

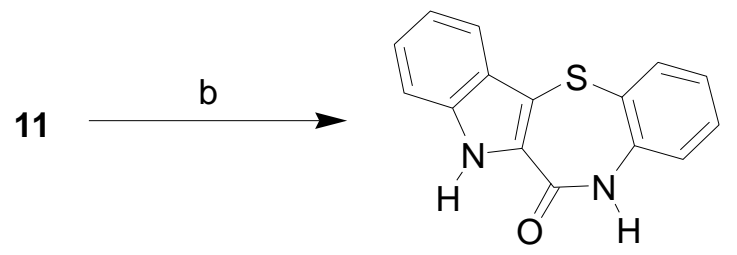

12

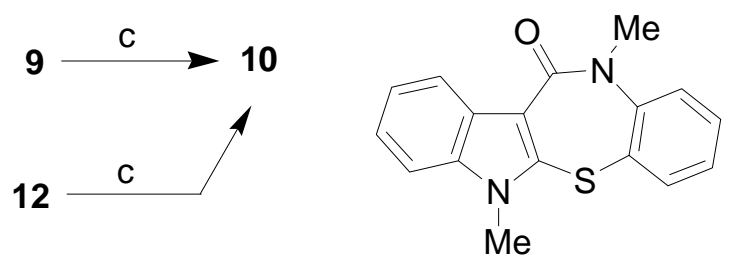

13

/a conditions: (a) 2,2'-diaminodiphenyldisulfide, $\mathrm{NaH}, \mathrm{DMF}$ (b) $\mathrm{SiO}_{2}$, toluene, reflux (c) $n-\mathrm{Bu}_{4} \mathrm{NHSO}_{4}, 50 \% \mathrm{NaOH}, \mathrm{CH}_{3} \mathrm{I}$, toluene, reflux.

\section{Indoles substituted at C-3}

3-Substituted indole sulfoxides were of great interest due to the propensity of indoles to react with electrophiles at C-3. The synthesis of sulfoxides (15a, 17ab) is outlined in Scheme 4. Indole-3-carboxylic acid ${ }^{32}$ was converted to the acid chloride in situ with the use of oxalyl chloride and then reacted with 2-(ethylthio)aniline to produce sulfide 14a which was oxidized to sulfoxides 15a. Catalytic phase transfer methylation of 14a to 16a followed by oxidation gave sulfoxide 17a. We encountered reproducibility problems with the reported preparation of 3-indole carboxylic acid so the t-butyl analogue was prepared by a different route. Indole and 2-(t-butylthio)aniline in the presence of triphosgene and 
pyridine directly produced (14b) in 33\% yield. Methylation and oxidation provided (17b).

Scheme $4^{\text {/a }}$
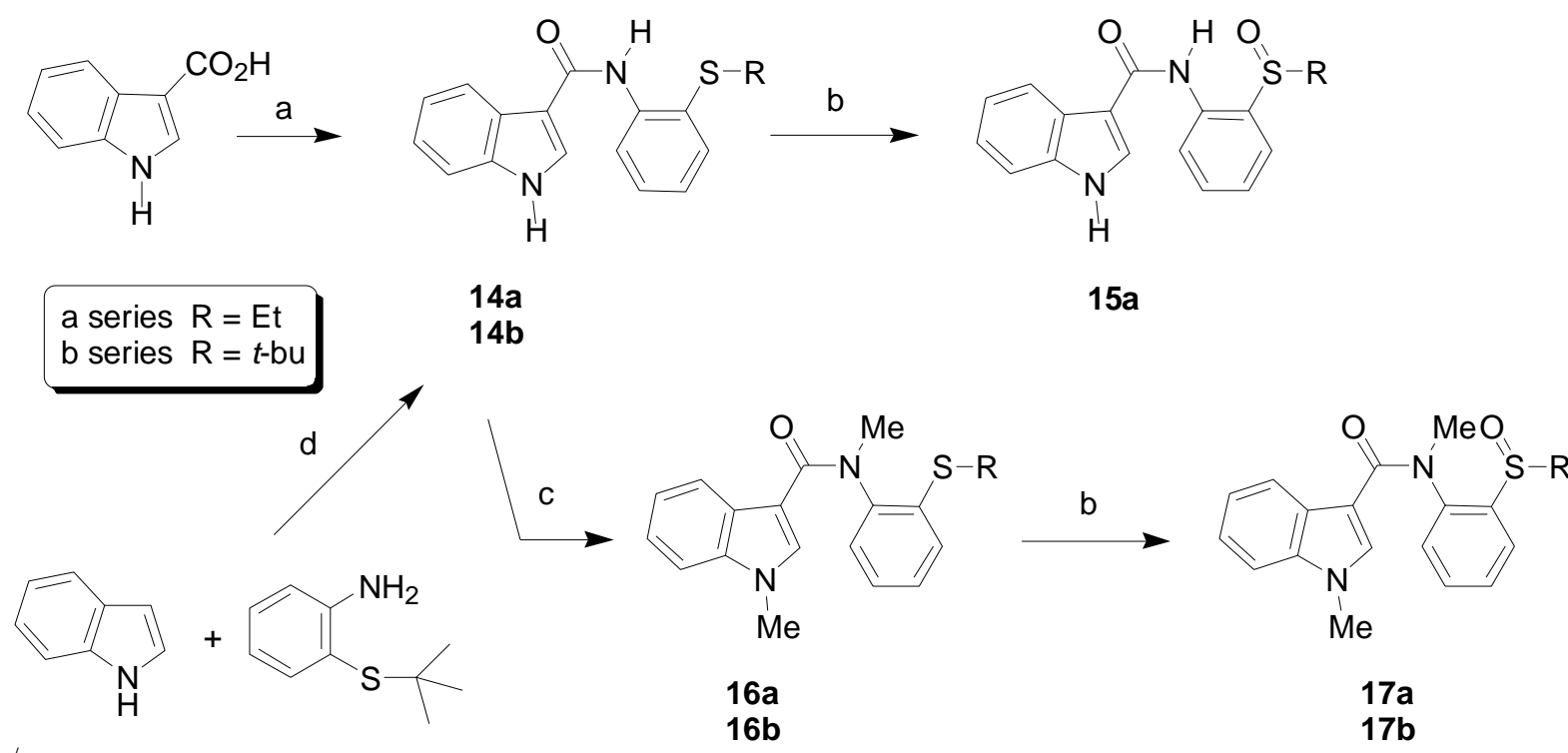

${ }^{/ a}$ Conditions: (a) 1. $(\mathrm{COCl})_{2}, \mathrm{THF}, 0{ }^{\circ} \mathrm{C} 2 . \mathrm{ArNH}_{2}, \mathrm{CH}_{2} \mathrm{Cl}_{2}$ (b) Oxone or m-CPBA (c) $50 \% \mathrm{NaOH}, \mathrm{CH}_{3} \mathrm{I}, n-\mathrm{Bu}_{4} \mathrm{NHSO}_{4}$, toluene (d) 1. triphosgene, toluene $/ \mathrm{CH}_{2} \mathrm{Cl}_{2} /$ pyr 2 . $\mathrm{ArNH}_{2}, \mathrm{CH}_{2} \mathrm{Cl}_{2}$.

Cyclizations of sulfoxides 15a, 17ab was attempted under both thermal and electrophilic activation. As shown in Table 2, results in this series were mixed. Like its 2substituted indole counterpart the compound with an amidic N-H (15a) did not cyclize under any conditions used. Compound 17a, which had a broad ${ }^{1} \mathrm{H}$ NMR spectrum, gave a cyclized product in good yield, but decomposed under TFAA activation. The t-butyl sulfoxide 17b, although it had the same ${ }^{1} \mathrm{H}$ NMR and IR patterns as successfully cyclized sulfoxides, decomposed under both thermal and TFAA activation. 
Table 2. Correlation of Cyclization and Spectral Data for Sulfoxides<smiles>[R]N(C(=O)c1cn([R1])c2ccccc12)c1ccccc1S([R])=O</smiles><smiles>[R]N1C(=O)c2c(c3ccccc3n2[R])Sc2ccccc21</smiles>

15a $\quad \mathrm{R}_{1}=\mathrm{H} \quad \mathrm{R}_{2}=\mathrm{H}$

$\begin{array}{ll}17 a b & R_{1}=M e R_{2}=M e\end{array}$

$10 \mathrm{R}_{1}=\mathrm{Me} \mathrm{R}_{2}=\mathrm{Me}$

\begin{tabular}{|l|l|l|l|}
\hline Sulfoxide & $\begin{array}{l}\text { Product\% yield } \\
(\text { TFAA, thermal) }\end{array}$ & $\begin{array}{l}{ }^{1} \mathrm{H} \text { NMR } \\
(\mathrm{rt})\end{array}$ & $\begin{array}{l}\mathrm{U}_{\mathrm{S}=\mathrm{O}} \\
\left(\mathrm{cm}^{-1}\right)\end{array}$ \\
\hline 15a & none & sharp & 1003 \\
\hline $\mathbf{1 7 a}$ & $\mathbf{1 0}($ none, 67\%) & broad & 1014 \\
\hline $\mathbf{1 7 b}$ & none & broad & 1030 \\
\hline
\end{tabular}

The product obtained from heating 17a under reflux for $15 \mathrm{~h}$ in $p$-xylene $(67 \%$ yield after chromatography) proved to be identical to 10, the cyclization product from the 2substituted indole sulfoxide. Mechanistically, there are several explanations for such a phenomenon. (a) migration of the acyl-amide group from $\mathrm{C}-3$ to $\mathrm{C}-2$ of indole prior to cyclization $(\mathbf{1 7 a} \rightarrow \mathbf{8 a} \rightarrow \mathbf{1 0})$. However, heating the sulfide 7a (which cannot cyclize via SES) for $12 \mathrm{~h}$ in $p$-xylene showed no evidence of acyl migration (95\% recovery of unchanged starting material). There is literature precedence for acyl migration from C-3 to $\mathrm{C}-2$ in indoles but typically much more strongly acidic conditions than used here are required. $^{33}$ (b) Initial formation of $\mathbf{1 3}$ by direct cyclization to $\mathrm{C}-2$, followed by rearrangement to $\mathbf{1 0}$ upon prolonged heating in $p$-xylene, as we had observed previosly in another indolic system. ${ }^{27}$ However, authentic 13 (synthesized as reported by Grandolini and co-workers ${ }^{29}$ ) showed no sign of rearrangement to $\mathbf{1 0}$ after $22 \mathrm{~h}$ in refluxing $p$ - 
xylene. Even after an additional $24 \mathrm{~h}$ at reflux in $0.1 \%$ TFA in $p$-xylene solution resulted in $92 \%$ recovery of unchanged starting material. (c) Initial formation of a $3 \mathrm{H}$-spirocyclic intermediate (such as 18) during SES at C-3, followed by acyl migration to C-2 and loss of a proton to give $\mathbf{1 0}$ (eq 4).

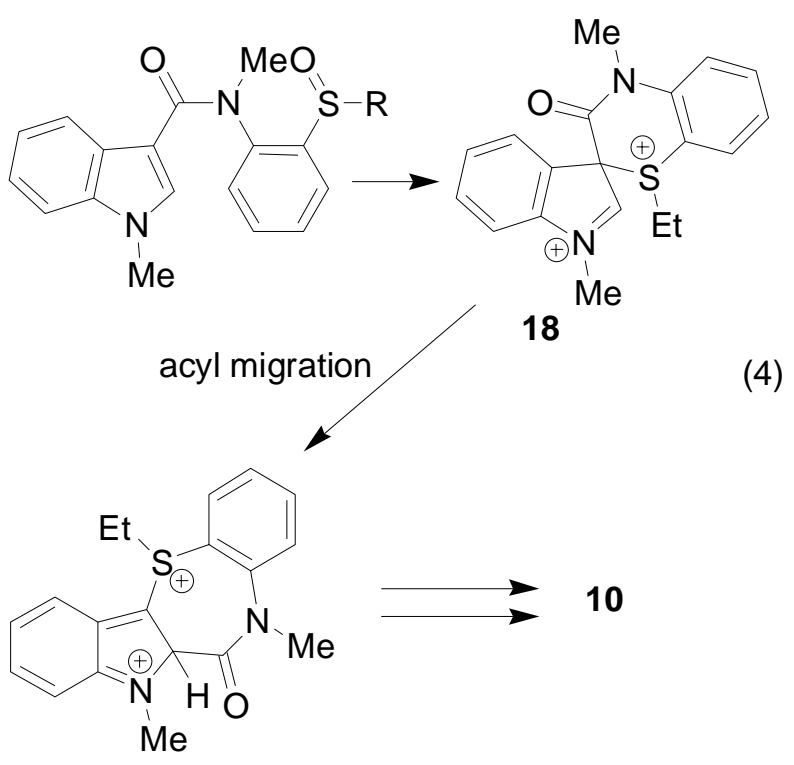

Formation of product $\mathbf{1 0}$ via a spirocyclic intermediate runs contrary to expectations based on the literature available. For example, formation of methyl 2-phenylthioindole-3carboxylate from sulfenyl chloride and methyl indole-3-carboxylate is proposed to occur by way of preferential sulfur migration in the $3 \mathrm{H}$-indolium species (19), although no evidence was provided to support this speculation. ${ }^{34}$ Additionally, Nagarajan ${ }^{35}$ proposed a spirocyclic intermediate (20) followed by preferential sulfur migration to C-2 to explain formation of 2,3,4,9-tetrahydrothiopyrano[2,3-b]indol-4-one from 3-(3indolylthio)propanoic acid. However, Hamel and co-workers ${ }^{36 a}$ have shown that 3-(3indolylthio)propanoic acid isomerizes to the 2-isomer prior to cyclization. This work also established a generalization about reaction conditions for cyclizations involving 3indolylthioalkanoic acids: when conducted in PPA C-3 to C-2 thioalkyl migration occurs 
prior to cyclization, but with PPE in $\mathrm{CH}_{2} \mathrm{Cl}_{2}$, migration is minimal. An apparent anomaly to this behavior, for which no formal explanation could be provided, involves 2-(3indolylthio)benzoic acid. ${ }^{28}$

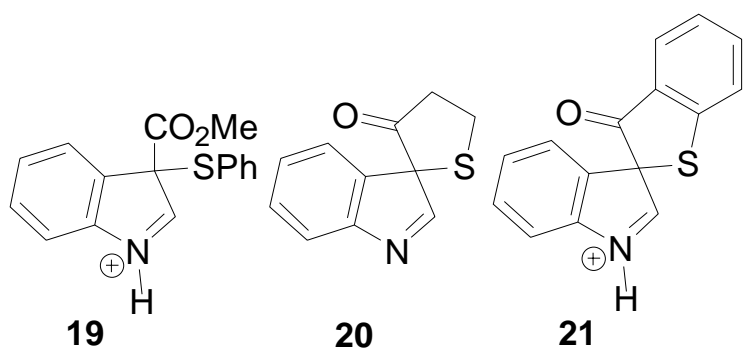

Formation of $\mathbf{1 0}$ via a spirocyclic intermediate requires migration of the acyl-amide group to occur in preference to an arylsulfonium group (or arylthio group) ${ }^{37}$ It also seems plausible that a similar intermediate from 21 could explain the apparently anamolous behavior reported by Hamel. ${ }^{28}$ Both 3-acylindoles ${ }^{33}$ and simple 3-alkylthioand 3-arylthioindoles ${ }^{36}$ undergo acid-catalyzed conversion, respectively, to their 2substituted counterpart in these thermodynamically-controlled processes. However, when both groups are present at indole $\mathrm{C}-2 / \mathrm{C}-3$, the thermodynamically more stable product can not be determined by inspection and in both Hamel's work and this work, neither the product nor the alternative isomer is interconverted under the conditions the reaction. Thus, the product that forms predominately could be a kinetic product derived by migration in a spirocyclic intermediate of the group having the greater migratory appitude: a resonance-stabilized acylium species [phenylacylium species (in the case of Hamel's cyclization) or of an amidic acyl (isocyanato) group in the present work in prefernce to a sulfur species]. Although the scant data available suggests sulfur substituents have a greater migratory aptitude than ester, aldehyde and alkyl ketone 
groups, no information is available concerning the potentially more stable migrating groups amide or phenyl ketone. ${ }^{38}$

\section{Eliminating the sulfenic acid pathway for SES chemistry}

Formation of sulfenic acids from sulfoxides and their sulfenylation of a nucleophilic nitrogen atom (to prepare a series of benzoisothiazolones) has been reported ${ }^{39}$ to occur under nearly the same conditions reported here (trichloroacetic anhydride/pyridine or refluxing toluene/pyridine). Glucosulfinylpropanenitriles ${ }^{40}$ as well as t-butyl alkyl sulfoxides, ${ }^{41}$ upon heating in an inert solvent, generate transient sulfenic acids which have been trapped using methyl propiolate or other alkynes.

To explore the possibility that $\mathbf{1 0}$ may also form via a transient sulfenic acid, we conducted thermal reactions of $\mathbf{8 b}$ in the presence of a sulfenic acid trapping agent. When (8b) was refluxed in chloroform in the presence of 2-mercaptobenzothiazole as a trapping agent $^{42}$ ( 1 molar equiv) cyclization product 10 was obtained in $31 \%$ yield (compared to $66 \%$ yield in the absence of the trapping agent) and no disulfide product is observed. In some cases when $t$-butyl sulfoxides were refluxed in $p$-xylene, minor amounts of 22 and 23 were isolated in addition to cyclized product. These compounds are indicative of sulfenic acid formation, ${ }^{43}$ but were never detected in reactions of ethyl sulfoxides. Yields of cyclized product from t-butyl sulfoxides were considerably lower when they were found in the crude reaction product. We believe sulfenic acids are not intermediates in these sulfenylation reactions and are instead formed as an undesired side reaction; it is important to conduct reactions under conditions below the threshold for sulfenic acid formation to obtain optimal yields of cyclized products. 

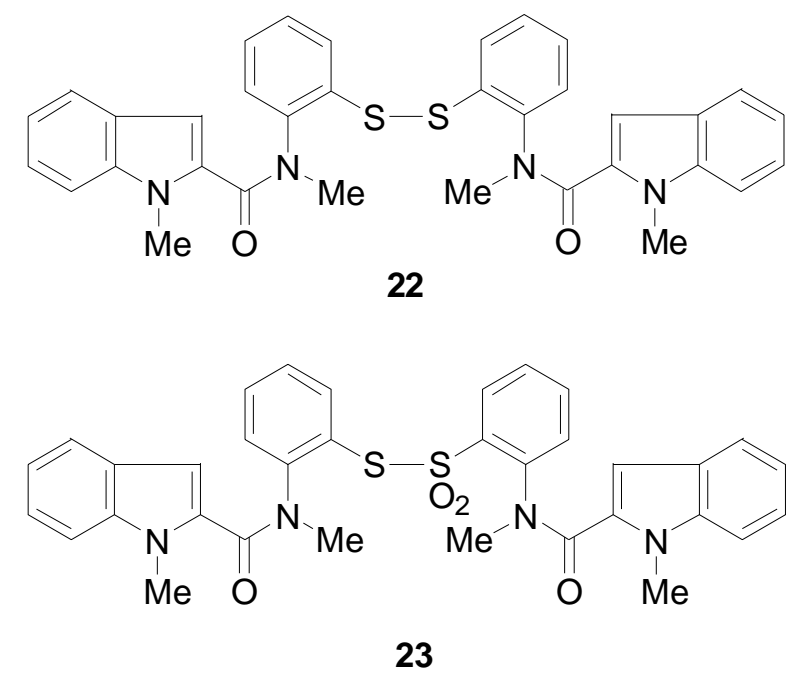

\section{Conclusions}

Contrary to expectations, both 2- and 3- indolecarbanilides (8abc and 17a) undergo cyclization to produce the same product - indolo[3,2-b]-1,5-benzothiazepin-11-one (10). For the 3-indolecarbanilides, the possibility of indole substituent migration before or after cyclization was eliminated and a $3 \mathrm{H}$-indolium spirocyclic intermediate, with preferential migration of the carbonyl-containing moiety from $\mathrm{C}-3$ to $\mathrm{C}-2$, is proposed to rationalize the results. We also discovered that successful cyclization in this series requires the absence of an amidic N-H in the compounds. The lack of reactivity of compounds containing an amidic $\mathrm{N}-\mathrm{H}$ is attributed to $\mathrm{N}-\mathrm{H}---\mathrm{O}=\mathrm{S}$ hydrogen bonding and a lower energy trans-amide conformation, both of which enforce a molecular geometry that prevents the sulfoxide sulfur atom from achieving an orientation conducive to interaction with indole pi-electrons. By extrapolation, if compounds containing an amidic N-H are required, one should consider introducing an easily removable amidic alkyl substituent (e.g., benzyl) into the SES substrate.

The reaction conditions used in this study have been reported to produce sulfenic acid intermediates and some authors have suggested a sulfenic acid pathway for some 
sulfenylation reactions of sulfoxides. Trapping experiments using benzothiazole and previous observations ${ }^{10 b, 11}$ suggest that both thermal and electrophile-activated sulfenylation reactions (including those with t-butyl sulfoxides) take place via a sulfonium salt pathway, not sulfenic acid intermediates.

\section{Experimental Section}

Melting points are uncorrected. ${ }^{1} \mathrm{H}$ and ${ }^{13} \mathrm{C}$ NMR spectra were determined in $\mathrm{CDCl}_{3}$ solutions unless otherwise indicated. IR spectra were recorded in $\mathrm{KBr}$ pellets for solid samples and neat on $\mathrm{NaCl}$ plates for liquid samples. Mass spectra were recorded at $70 \mathrm{eV}$ (EI) unless indicated otherwise.

$N$-[2-(ethylthio)phenyl]-1H-indole-2-carboxamide (1a). Method A. To a well-stirred solution of indole-2-carboxylic acid $(10.0 \mathrm{~g}, 60 \mathrm{mmol})$ in $\mathrm{Et}_{2} \mathrm{O}(194 \mathrm{~mL})$ at $0{ }^{\circ} \mathrm{C}$ under a drying tube was added dropwise, $\mathrm{SOCl}_{2}(9.6 \mathrm{~mL}, 140$ moles, 2.2 eq.) neat over $5 \mathrm{~min}$. The mixture was allowed to warm to room temperature. After stirring an additional $2 \mathrm{~h}$, volatiles were removed in vacuo leaving a yellow solid which was redissolved in $\mathrm{Et}_{2} \mathrm{O}$ $(96 \mathrm{~mL})$ and added to a well-stirred solution of 2-(ethylthio)aniline (2 eq. $18.4 \mathrm{~g}, 0.12$ moles) in $\mathrm{Et}_{2} \mathrm{O}(96 \mathrm{~mL})$ over 8 min at $0{ }^{\circ} \mathrm{C}$. The resulting yellow slurry was stirred for 50 min at $0{ }^{\circ} \mathrm{C}$ and at room temperature for $30 \mathrm{~min}$ at which time the mixture was diluted with EtOAc $(100 \mathrm{~mL})$. The combined organics were washed with 5\% aq $\mathrm{HCl}(3 \times 150$ $\mathrm{mL}), 5 \%$ aq. $\mathrm{NaHCO}_{3}(3 \times 150 \mathrm{~mL}), \mathrm{H}_{2} \mathrm{O}(2 \times 100 \mathrm{~mL})$, dried $\left(\mathrm{Na}_{2} \mathrm{SO}_{4}\right)$ and the solvent evaporated in vacuo to yield a wet orange solid. Column chromatography (1:1 $\mathrm{CHCl}_{3}$ :hexanes) gave a yellow solid, $1 \mathrm{a}(5.7 \mathrm{~g}, 32 \%)$. Analytically pure material could be obtained by recrystallization from acetone: $\mathrm{mp} 153-155^{\circ} \mathrm{C}$; IR 3350, 3298, $1654 \mathrm{~cm}^{-1} ;{ }^{1} \mathrm{H}$ NMR (200 MHz) $\delta 10.09(1 \mathrm{H}, \mathrm{d}, \mathrm{J}=0.6 \mathrm{~Hz}), 9.51(1 \mathrm{H}, \mathrm{s}), 8.60(1 \mathrm{H}, \mathrm{dd}, \mathrm{J}=1,6 \mathrm{~Hz})$, 
$7.65(1 \mathrm{H}, \mathrm{dd}, \mathrm{J}=0.6,8 \mathrm{~Hz}), 7.53(1 \mathrm{H}, \mathrm{dd}, \mathrm{J}=1,6 \mathrm{~Hz}), 7.44(1 \mathrm{H}, \mathrm{dd}, \mathrm{J}=0.6,8 \mathrm{~Hz}), 7.00-$

$7.40(5 \mathrm{H}, \mathrm{m}), 2.76(2 \mathrm{H}, \mathrm{q}, \mathrm{J}=7 \mathrm{~Hz}), 1.18(3 \mathrm{H}, \mathrm{t}, \mathrm{J}=7 \mathrm{~Hz},) ;{ }^{13} \mathrm{C}$ NMR $\delta 160.2,140.1$, $137.6,136.3,131.5,131.0,128.1,125.4,124.6,123.2,122.6,121.3,120.4,112.8,103.4$, 31.3, 15.3; MS [m/z (relative intensity] $296\left(\mathrm{M}^{+}, 54\right), 235$ (75), 153 (100); Anal. Calcd for $\mathrm{C}_{17} \mathrm{H}_{16} \mathrm{~N}_{2} \mathrm{OS}$ : C, 68.90; H, 5.44; N, 9.45. Found: C, 68.70; H, 5.55; N, 9.34.

Method B. To a $\mathrm{N}_{2}$-flushed flask containing toluene $(123 \mathrm{~mL})$ was added trimethylaluminum (2.0 $\mathrm{M}$ in hexane, $21 \mathrm{~mL}, 42 \mathrm{mmol}, 1.14$ eq.) via a syringe dropwise. CAUTION: pyrophoric. This solution was cooled to $5{ }^{\circ} \mathrm{C}$ and 2-(ethylthio)aniline (5.7 g, $37 \mathrm{mmol})$ in toluene $\left(18 \mathrm{~mL}\right.$ ) was added dropwise. After stirring $20 \mathrm{~min}$ at $5{ }^{\circ} \mathrm{C}$, the solution allowed to warm to room temperature over a 45 -min period. To the resulting solution was added dropwise ethyl indole-2-carboxylate $(7.0 \mathrm{~g}, 37 \mathrm{mmol})$ in toluene (62 $\mathrm{mL})$ and $\mathrm{CH}_{2} \mathrm{Cl}_{2}(18 \mathrm{~mL})$. When the addition was complete the reaction mixture was heated to reflux. After refluxing $16 \mathrm{~h}$, the cooled solution was hydrolyzed by slow addition of $2 \%$ aq. $\mathrm{HCl}(45 \mathrm{~mL})$. The layers were separated and the aqueous layer extracted with EtOAc $(3 \times 50 \mathrm{~mL})$. The combined organics were washed with saturated $\mathrm{NaCl}$ and then with $\mathrm{H}_{2} \mathrm{O}$, dried $\left(\mathrm{Na}_{2} \mathrm{SO}_{4}\right)$ and the solvent evaporated in vacuo to yield a yellow solid (9.8 $\mathrm{g}, 92 \%)$ as a single spot on TLC which was identical to the compound prepared above ( ${ }^{1} \mathrm{H}$ NMR and mixed melting point).

$N$-[2-(ethylsulfinyl)phenyl]-1H-indole-2-carboxamide (2a). To a well-stirred solution of $1 \mathrm{a}(0.5 \mathrm{~g}, 1.5 \mathrm{mmol})$ in $\mathrm{THF} / \mathrm{MeOH}(2.5 \mathrm{~mL} / 1 \mathrm{~mL})$ at $0{ }^{\circ} \mathrm{C}$ was added all at once a solution of Oxone in $\mathrm{H}_{2} \mathrm{O}(2.5 \mathrm{~mL})$. The resulting mixture was stirred at $0{ }^{\circ} \mathrm{C}$ for 5 min then at room temperature for $2.5 \mathrm{~h}$ then extracted with $\mathrm{CH}_{2} \mathrm{Cl}_{2}(2 \times 10 \mathrm{~mL})$. The combined organics were washed with $\mathrm{H}_{2} \mathrm{O}$, dried $\left(\mathrm{Na}_{2} \mathrm{SO}_{4}\right)$ and the solvent removed in 
vacuo to yield a light yellow solid which was recrystallized in $\mathrm{MeOH}$ to yield a very faint yellow solid, 2a (0.3 g, 58\%): mp 156-157 ${ }^{\circ} \mathrm{C}$; IR 3289, 3168, 1662, $1009 \mathrm{~cm}^{-1}$; ${ }^{1} \mathrm{H}$ NMR $(200 \mathrm{MHz}) \delta 11.84(1 \mathrm{H}, \mathrm{s}, \mathrm{br}), 10.29(1 \mathrm{H}, \mathrm{s}, \mathrm{br}), 8.80(1 \mathrm{H}, \mathrm{dd}, \mathrm{J}=1,8 \mathrm{~Hz}), 7.10-7.72$ $(8 \mathrm{H}, \mathrm{m}$, containing $1 \mathrm{H}$ at $\delta 7.45, \mathrm{dd}, \mathrm{J}=1,8 \mathrm{~Hz}), 3.07-3.19(2 \mathrm{H}, \mathrm{dm}), 1.26(3 \mathrm{H}, \mathrm{t}, \mathrm{J}=7$ $\mathrm{Hz}) ;{ }^{13} \mathrm{C}$ NMR $\delta 160.4,141.6,133.0,131.2,128.2,127.7,127.2,127.1,125.4,125.1$, 123.5, 122.9, 121.1, 112.4, 104.8, 48.9, 7.8; MS [m/z (relative intensity)] $312\left(\mathrm{M}^{+}, 63\right)$, 283 (20), 144 (100). Anal Calcd for $\mathrm{C}_{17} \mathrm{H}_{16} \mathrm{~N}_{2} \mathrm{O}_{2} \mathrm{~S}: \mathrm{C}, 65.36, \mathrm{H}, 5.16, \mathrm{~N}, 8.97$. Found: $\mathrm{C}$, 65.35, H, 5.33, N, 8.86.

$N$-[2-(tert-butylsulfinyl)phenyl]- $N$-methyl- $1 H$-indole-2-carboxamide (4b). To an ice-cooled solution of $\mathbf{3 b}(5.0 \mathrm{~g}, 14.8 \mathrm{mmol})$ in $\mathrm{CH}_{2} \mathrm{Cl}_{2}(150 \mathrm{~mL})$ was added slowly a solution of $m$-CPBA (77\%, 1.1 eq, $21.1 \mathrm{mmol}, 3.6 \mathrm{~g})$ in $\mathrm{CH}_{2} \mathrm{Cl}_{2}(50 \mathrm{~mL})$. The resulting mixture was stirred at $0{ }^{\circ} \mathrm{C}$ for $15 \mathrm{~min}$ and then put it in a freezer $\left(-8^{\circ} \mathrm{C}\right)$ overnight. The reaction mixture was then poured into $5 \% \mathrm{NaHCO}_{3}$ solution $(150 \mathrm{~mL})$ and extracted with $\mathrm{CH}_{2} \mathrm{Cl}_{2}(200 \mathrm{~mL})$. The combined organic layer was washed with distilled water, dried, and concentrated in vacuo. Column chromatography (EtOAc-hexane, 9:1) gave yellowish white solid which was recrystallized (hexane) to give a white solid (3.68 g, 70\%): mp 114-116 ${ }^{\circ} \mathrm{C}$; IR 3453, $1624 \mathrm{~cm}^{-1}$; ${ }^{1} \mathrm{H}$ NMR and ${ }^{13} \mathrm{C}$ NMR was not well-resolved due to presence of rotational isomers; MS [m/z (rel. intensity)] 144 (6), 56 (58), 41 (100).

$N$-[2-(ethylthio)phenyl]- $N$,1-dimethyl-1H-indole-2-carboxamide (7a). To a wellstirred suspension of $1 \mathrm{a}(5.2 \mathrm{~g} ; 17 \mathrm{mmol})$ and tetra-n-butylammonium sulfate $(0.6 \mathrm{~g} ; 2$ mmol; 0.1 eq.) in toluene $(22 \mathrm{~mL})$ was added $50 \%$ aq. $\mathrm{NaOH}$ solution $(22 \mathrm{~mL})$ in one portion. The resulting two-layer mixture was heated to reflux and a solution of $\mathrm{CH}_{3} \mathrm{I}(5.4$ $\mathrm{g} ; 38 \mathrm{mmol} ; 2.2 \mathrm{eq})$ in toluene $(5 \mathrm{~mL})$ added dropwise over $5 \mathrm{~min}$. This mixture was 
refluxed for $24 \mathrm{~h}$, cooled to room temperature and the layers separated. The organic layer was washed with $\mathrm{H}_{2} \mathrm{O}$ several times (until washings were neutral to litmus), dried $\left(\mathrm{Na}_{2} \mathrm{SO}_{4}\right)$ and the solvent evaporated in vacuo to yield a brown solid which was filtered through a silica gel column $\left(1: 1 \mathrm{CHCl}_{3}\right.$ :hexanes as eluent) to give $7 \mathbf{a}(4.7 \mathrm{~g}, 82 \%)$ as a white solid. Recrystallization (acetone) gave pure 7a: mp $127-128{ }^{\circ} \mathrm{C}$; IR $1635 \mathrm{~cm}^{-1} ;{ }^{1} \mathrm{H}$ NMR (200 MHz) $\delta$ 6.95-7.38 (8H, m), $6.07(1 \mathrm{H}, \mathrm{s}), 3.97(3 \mathrm{H}, \mathrm{s}), 3.41(3 \mathrm{H}, \mathrm{s}), 2.80(2 \mathrm{H}$, apparent qd, $\mathrm{J}=7.2,4.9,2.3 \mathrm{~Hz},), 1.20(2 \mathrm{H}, \mathrm{t}, \mathrm{J}=7.3 \mathrm{~Hz}) ;{ }^{13} \mathrm{C}$ NMR $\delta 164.6,143.1$, $138.4,136.5,132.3,129.0,128.8,128.6,127.9,126.5,123.7$ 122.2, 120.1, 110.1, 107.1, 37.2, 32.0, 26.4, 14.2; MS [m/z (relative intensity)] $324(\mathrm{M}+, 2), 158$ (100); Anal. Calcd for $\mathrm{C}_{19} \mathrm{H}_{20} \mathrm{~N}_{2} \mathrm{OS}$ : C, 70.34; H, 6.21; N, 8.63. Found: C, 70.27; H, 6.34; N, 8.58.

\section{0,11-Dihydro-10-methyl-12H-indolo[3,2-b][1,5]benzothiazepin-11-one (9) from}

4a.

Method A (Thermal Cyclization). A solution of 4a (1.7 g, $5 \mathrm{mmol})$ in $p$-xylenes (66 $\mathrm{mL}$ ) was heated under reflux for $12 \mathrm{~h}$. Upon cooling to room temperature, the solution was filtered to give a green solid, 9 (0.8 g, 56\%): $\mathrm{mp} 290{ }^{\circ} \mathrm{C}(\mathrm{dec})$; IR 3228, $1618 \mathrm{~cm}^{-1}$; ${ }^{1} \mathrm{H}$ NMR (200 MHz, DMSO-d 6 ) $\delta 12.13$ (1H, s, br), 7.15-7.67 (8H, m), 3.51 (3H, s); MS [m/z (relative intensity)] $280\left(\mathrm{M}^{+}, 100\right)$. Anal Calcd. for $\mathrm{C}_{16} \mathrm{H}_{12} \mathrm{~N}_{2} \mathrm{OS}: \mathrm{C}, 68.55, \mathrm{H}, 4.31$, N, 9.51. Found: C, 68.63, H, 4.65, N, 9.91.

Method B (excess of TFAA). The reaction was carried out as for $\mathbf{8}$ from TFAA $(0.2$ $\mathrm{mL}, 1.2 \mathrm{mmol}, 3.75$ eq) in $\mathrm{CH}_{2} \mathrm{Cl}_{2}(2 \mathrm{~mL})$, pyridine ( $0.1 \mathrm{~mL}, 1.2 \mathrm{mmol}, 4$ eq.), $4 \mathbf{a}(0.10$ g, $0.3 \mathrm{mmol})$ in $\mathrm{CH}_{2} \mathrm{Cl}_{2}(1 \mathrm{~mL})$. Stirring at $0{ }^{\circ} \mathrm{C}$ for $15 \mathrm{~min}$, then at room temperature for $0.5 \mathrm{~h}$, to yielded 9 as a yellow solid $(0.3 \mathrm{~g}, 75 \%)$. 
10,11-dihydro-10-methyl-12H-indolo[3,2-b]-1,5-benzothiazepin-11-one (9) from

$4 \mathbf{b}$.

Method A (Thermal Cyclization): A solution of $\mathbf{4 b}(0.73 \mathrm{~g}, 2.1 \mathrm{mmol})$ in chloroform (75 mL) was heated under reflux for 64 hours. The solution was allowed to cool to room temperature and the solid formed was filtered and air dried to give white solid $9(0.33 \mathrm{~g}$, 58\%): mp $295{ }^{\circ} \mathrm{C}(\mathrm{dec})$; IR $1619 \mathrm{~cm}^{-1} ;{ }^{1} \mathrm{H}$ NMR $\delta 10.61$ (broad, $\left.1 \mathrm{H}\right), 7.56(\mathrm{~d}, \mathrm{~J}=8.0 \mathrm{~Hz}$, 1H), $7.42(\mathrm{~d}, \mathrm{~J}=8.0 \mathrm{~Hz}, 1 \mathrm{H}), 7.28(\mathrm{~d}, \mathrm{~J}=8.28 \mathrm{~Hz}, 1 \mathrm{H}), 7.17-7.19(\mathrm{~m}, 2 \mathrm{H}), 7.11(\mathrm{dt}, \mathrm{J}=$ 6.99, $1.07 \mathrm{~Hz}, 1 \mathrm{H}), 6.95-7.03(\mathrm{~m}, 2 \mathrm{H}), 3.44(\mathrm{~s}, 3 \mathrm{H}) ;{ }^{13} \mathrm{C}$ NMR could not be taken due to insolubility of 9 in common solvents. MS [m/z (rel. intensity)] 280 [M+1, 100], 248(27).

Method B (Electrophilic Activation). To a well-stirred solution of TFAA (0.45 g, 2.1 mmol, 3.75 eq. $)$ in $\mathrm{CH}_{2} \mathrm{Cl}_{2}(20 \mathrm{~mL})$ at $0{ }^{\circ} \mathrm{C}$ was added pyridine (0.18 g, $2.3 \mathrm{mmol}, 4$ eq. $)$, neat via syringe. The reaction vessel was kept under a drying tube throughout the reaction. To the resulting yellow solution was added, a solution of $\mathbf{4 b}(0.20 \mathrm{~g}, 0.57 \mathrm{mmol})$ in $\mathrm{CH}_{2} \mathrm{Cl}_{2}(15 \mathrm{~mL})$ which was previously cooled to $0{ }^{\circ} \mathrm{C}$. This was stirred at $0{ }^{\circ} \mathrm{C}$ for 15 min then at room temperature for $30 \mathrm{~min}$. The solution was poured onto $10 \% \mathrm{Na}_{2} \mathrm{CO}_{3}(30$ $\mathrm{mL}$ ) and stirred for $5 \mathrm{~min}$. The layers were separated and the aqueous layer washed with $\mathrm{CH}_{2} \mathrm{Cl}_{2}(30 \mathrm{~mL})$. The combined organics were washed with $5 \%$ aqueous solution of $\mathrm{HCl}$ (3 X $40 \mathrm{~mL}$ ), 10\% aqueous solution of $\mathrm{Na}_{2} \mathrm{CO}_{3}(1 \mathrm{X} 40 \mathrm{~mL}), \mathrm{H}_{2} \mathrm{O}$ and dried over sodium sulfate. The solvent was removed in vacuo to yield pure yellow solid, $9(0.16 \mathrm{~g}, 100 \%)$.

10,11-Dihydro-10,12-dimethylindolo[3,2-b][1,5]benzothiazepin-11-one (10) from

8a.

Method A (Thermal Cyclization). A solution of 8a (1.0 g, $3 \mathrm{mmol})$ in p-xylenes (35 $\mathrm{mL}$ ) was heated under reflux for $30 \mathrm{~h}$. After solvent evaporation in vacuo, the brown 
solid was passed thru a silica gel column $\left(\mathrm{CHCl}_{3}\right)$ to give $\mathbf{1 0}(0.2 \mathrm{~g}, 22 \%)$ : mp 198-200

${ }^{\circ} \mathrm{C}$; IR $1627 \mathrm{~cm}^{-1}$; ${ }^{1} \mathrm{H}$ NMR $(200 \mathrm{MHz}) \delta 7.77(1 \mathrm{H}, \mathrm{d}, \mathrm{J}=0.9 \mathrm{~Hz}), 7.73(1 \mathrm{H}, \mathrm{d}, \mathrm{J}=0.9$ $\mathrm{Hz}), 7.08-7.58(6 \mathrm{H}, \mathrm{m}), 3.91(3 \mathrm{H}, \mathrm{s}), 3.62(3 \mathrm{H}, \mathrm{s}) ;{ }^{13} \mathrm{C} \mathrm{NMR} \delta 163.5,146.0,138.2$, $137.4,132.3,131.0,128.9,126.2,125.8,125.5,125.0,120.6,120.3,117.5,110.1,38.6$ 31.6; MS [m/z (relative intensity)] $294(\mathrm{M}+, 100)$. Anal. Calcd for $\mathrm{C}_{17} \mathrm{H}_{14} \mathrm{~N}_{2} \mathrm{OS}: \mathrm{C}$, 69.36; H, 4.79; N 9.52. Found: C, 69.35; H, 4.89; N, 9.28.

Method B (excess of TFAA). To a well-stirred solution of TFAA (1.4 mL, $11 \mathrm{mmol}$, 3.75 eq $)$ in $\mathrm{CH}_{2} \mathrm{Cl}_{2}(17 \mathrm{~mL})$ at $0{ }^{\circ} \mathrm{C}$ was added pyridine $(1 \mathrm{~mL}, 12 \mathrm{mmol}, 4$ eq. $)$, neat via a syringe. To this solution was added, $8 \mathrm{aa}(1 \mathrm{~g}, 3 \mathrm{mmol})$ in $\mathrm{CH}_{2} \mathrm{Cl}_{2}(9 \mathrm{~mL})$ which had been previously cooled to $0{ }^{\circ} \mathrm{C}$. After stirring at $0{ }^{\circ} \mathrm{C}$ for $15 \mathrm{~min}$ then at room temperature for 3 h., the solution was poured onto a $10 \%$ aqueous $\mathrm{Na}_{2} \mathrm{CO}_{3}(30 \mathrm{~mL})$ and stirred for $5 \mathrm{~min}$. The layers were separated and the aqueous layer washed with $\mathrm{CH}_{2} \mathrm{Cl}_{2}(1 \times 30 \mathrm{~mL})$. The combined organics were washed with $5 \% \mathrm{HCl}(3 \times 40 \mathrm{~mL}), 10 \% \mathrm{Na}_{2} \mathrm{CO}_{3}(1 \times 40 \mathrm{~mL})$, $\mathrm{H}_{2} \mathrm{O}$ and dried $\left(\mathrm{Na}_{2} \mathrm{SO}_{4}\right)$. The solvent was removed in vacuo to yield a relatively pure yellow solid $10(0.8 \mathrm{~g}, 90 \%)$. mp $196-198^{\circ} \mathrm{C}$.

Trapping experiment. To a solution of $\mathbf{8 b}(0.1 \mathrm{~g}, 0.27 \mathrm{mmol})$ in chloroform $(50 \mathrm{~mL})$ was added 2-mercaptobenzothiazole (1.0 eq., $0.27 \mathrm{mmol}, 45.0 \mathrm{mg})$ and the resulting mixture was heated under reflux for $72 \mathrm{~h}$ (until no starting material on TLC). The solution was allowed to cool to room temperature and the solvent evaporated in vacuo to yield a brown solid. Column chromatography $\left(\mathrm{CH}_{2} \mathrm{Cl}_{2}\right)$ gave white solid $\mathbf{1 0}(0.025 \mathrm{~g}$, $32 \%)$.

3-[(2-aminophenyl)thio]-1H-indole-2-carboxylic acid (11). To a well-stirred suspension of $\mathrm{NaH}(1.1 \mathrm{~g}, 45 \mathrm{mmol}, 3.0 \mathrm{eq})$ in $\mathrm{DMF}(30 \mathrm{~mL})$ under $\mathrm{N}_{2}$ at room 
temperature was added dropwise a solution of indole-2-carboxylic acid (2.4 g, $15 \mathrm{mmol})$ in DMF $(10 \mathrm{~mL})$. After the evolution of $\mathrm{H}_{2}$ had ceased, a solution of 2,2'diaminodiphenyldisulfide $(15 \mathrm{mmol})$ in DMF $(5 \mathrm{~mL})$ was added dropwise and the dark colored solution was heated at $50{ }^{\circ} \mathrm{C}$ for $24 \mathrm{~h}$, then poured to $\mathrm{H}_{2} \mathrm{O}(75 \mathrm{~mL})$ and extracted with $\mathrm{Et}_{2} \mathrm{O}(3 \times 50 \mathrm{~mL})$. The aqueous layer was acidified to $\mathrm{pH} \sim 5-6$ precipitating a light brown solid (11, $4.1 \mathrm{~g}, 93 \%)$. The solid was used in the next step without purification.

10,11-Dihydro-10,12H-indolo[3,2-b][1,5]benzothiazepin-11-one (12). A suspension of $11(5 \mathrm{~g}, 17.6 \mathrm{mmol})$ and $\mathrm{SiO}_{2}$ (column chromatographic grade, $20 \mathrm{~g}$ ) in toluene (250 $\mathrm{mL}$ ) was heated under reflux for $13 \mathrm{~h}$ under a Dean-Stark trap. The partially cooled reaction mixture was filtered through a sintered glass funnel $(10-15 \mu)$ and the $\mathrm{SiO}_{2}$ washed with 1:1 $\mathrm{CH}_{2} \mathrm{Cl}_{2}: \mathrm{MeOH}(50 \mathrm{~mL})$ and then with $\mathrm{MeOH}(2 \times 30 \mathrm{~mL})$. The combined organics were evaporated in vacuo and the light brown solid recrystallized to produce $12(2.95 \mathrm{~g}, 63 \%)$ as an off-white solid: $\mathrm{mp} 237-239{ }^{\circ} \mathrm{C}(50 \% \mathrm{EtOH})$; IR 3414 , 3197, $1654 \mathrm{~cm}^{-1} ;{ }^{1} \mathrm{H}$ NMR (200 MHz) $\delta 12.14(1 \mathrm{H}, \mathrm{s}, \mathrm{br}), 10.46(1 \mathrm{H}, \mathrm{s}, \mathrm{br}), 7.12-7.70$ $(8 \mathrm{H}, \mathrm{m}) ;{ }^{13} \mathrm{C}$ NMR $\delta 164.7,142.3,137.6,133.0,131.5,130.6,130,3,127.0,126.9,126.8$ 126.3, 125.2, 121.8, 120.8, 114.0; MS [m/z (relative intensity)] $266(\mathrm{M}+, 100)$. Anal. calcd for $\mathrm{C}_{15} \mathrm{H}_{10} \mathrm{~N}_{2} \mathrm{OS}$ : C, 67.65, H, 3.78, N, 10.52. Found: C, 67.48, H, 4.12, N, 10.42.

$N$-[2-(ethylthio)phenyl]-1H-indole-3-carboxamide (14a). To a magnetically stirred solution of indole-3-carboxylic acid $(1.5 \mathrm{~g}, 9 \mathrm{mmol})$ in $\mathrm{THF}(20 \mathrm{~mL})$ at $0{ }^{\circ} \mathrm{C}$ was added dropwise neat oxalyl chloride ( $2.3 \mathrm{~g}, 18 \mathrm{mmol}, 2 \mathrm{eq}$.$) . After 12 \mathrm{~h}$ at room temperature, the solvent was evaporated in vacuo and the yellow residue (in dichloroethane $(40 \mathrm{~mL})$ ) was added to a mechanically stirred solution of 2-(ethylthio)aniline ( $2.8 \mathrm{~g}, 18 \mathrm{mmol}, 2 \mathrm{eq})$ in dichloroethane $(30 \mathrm{~mL})$. After $5 \mathrm{~h}$ at room temperature, the mixture was washed with $5 \%$ 
$\mathrm{HCl}(3 \times 50 \mathrm{~mL}), 5 \% \mathrm{NaHCO}_{3}(3 \times 50 \mathrm{~mL}), \mathrm{H}_{2} \mathrm{O}$ and dried $\left(\mathrm{Na}_{2} \mathrm{SO}_{4}\right)$. The solvent was evaporated in vacuo to leave a dark brown oil. Column chromatography $\left(\mathrm{CHCl}_{3}\right)$ produced 14a as a faint brown solid, $(0.9 \mathrm{~g}, 35 \%)$ : $\mathrm{mp} 103-105^{\circ} \mathrm{C}$; IR 3352, 3216, 1638 $\mathrm{cm}^{-1} ;{ }^{1} \mathrm{H}$ NMR $(200 \mathrm{MHz}) \delta 9.31(1 \mathrm{H}, \mathrm{s}), 8.66(1 \mathrm{~h}, \mathrm{dd}, \mathrm{J}=1.3,7 \mathrm{~Hz}), 8.31-8.36(1 \mathrm{H}, \mathrm{m})$, $7.88(1 \mathrm{~h}, \mathrm{~d}, \mathrm{~J}=3 \mathrm{~Hz}), 7.58,(1 \mathrm{H}, \mathrm{dd}, \mathrm{J}=1.4,6.2 \mathrm{~Hz}), 7.25-7.48(4 \mathrm{H}, \mathrm{m}), 7.07(1 \mathrm{H}, \mathrm{td}, \mathrm{J}=$ 1.4, $6.2 \mathrm{~Hz}), 2.78(2 \mathrm{H}, \mathrm{q}, \mathrm{J}=7 \mathrm{~Hz}), 1.20(3 \mathrm{H}, \mathrm{t}, \mathrm{J}=7 \mathrm{~Hz}) ;{ }^{13} \mathrm{C}$ NMR $\delta 165.4,141.5$, $138.2,136.8,131.0,130.8,125.8,125.1,124.4,124.3,123.3,121.8,121.2,113.9,113.5$ 31.7, 16.1; MS [m/z (relative intensity)] $296\left(\mathrm{M}^{+}, 12\right), 153$ (100). Anal. Calcd. for $\mathrm{C}_{17} \mathrm{H}_{16} \mathrm{~N}_{2} \mathrm{OS}$ : C, 68.89; H, 5.44; N, 9.45. Found: C, 68.49; H, 5.62; N, 9.44.

$N$-[2-(tert-butylthio)phenyl]-1H-indole-3-carboxamide (14b). Triphosgene (1.1 g, $3.66 \mathrm{mmol}$ ) was dissolved in toluene $(5 \mathrm{~mL})$. This was added to a well-stirred solution of indole $(1.3 \mathrm{~g}, 11.0 \mathrm{mmol})$ and pyridine $(0.88 \mathrm{~g}, 11.0 \mathrm{mmol})$ in $\mathrm{CH}_{2} \mathrm{Cl}_{2}(40 \mathrm{~mL})$ dropwise over $30 \mathrm{~min}$ at $25^{\circ} \mathrm{C}$. The resulting dark red mixture was stirred for $3.5 \mathrm{~h}$ at $25^{\circ} \mathrm{C}$ under drying tube. At this point, the solvent was evaporated in vacuo to half of its volume and an additional $\mathrm{CH}_{2} \mathrm{Cl}_{2}(10 \mathrm{~mL})$ was added. To this, was added a solution of 2-(tertbutylthio)aniline $(4.0 \mathrm{~g}, 22.1 \mathrm{mmol})$ in $\mathrm{CH}_{2} \mathrm{Cl}_{2}(25 \mathrm{~mL})$ dropwise. The resulting dark green mixture was stirred at room temperature under a drying tube for $4 \mathrm{~h}$. The reaction mixture was washed with $5 \% \mathrm{HCl}(3 \times 50 \mathrm{~mL}), 5 \% \mathrm{Na}_{2} \mathrm{CO}_{3}(3 \times 50 \mathrm{~mL}), \mathrm{H}_{2} \mathrm{O}(1 \times 50$ $\mathrm{mL}$ ) and dried over sodium sulfate. The solvent was removed in vacuo to get a dark solid, which was purified by column chromatography $\left(\mathrm{CH}_{2} \mathrm{Cl}_{2}\right)$ to give white solid $(1.20 \mathrm{~g}$, 33\%): mp 185-187 ${ }^{\circ} \mathrm{C}$; IR 3335, $1637 \mathrm{~cm}^{-1}$; ${ }^{1} \mathrm{H}$ NMR $\delta 9.88$ (broad, 1H), 9.64 (broad, 1H), $8.76(\mathrm{~d}, \mathrm{~J}=7.88 \mathrm{~Hz}, 1 \mathrm{H}), 8.46(\mathrm{~d}, \mathrm{~J}=7.88 \mathrm{~Hz}, 1 \mathrm{H}), 7.89(\mathrm{~d}, \mathrm{~J}=2.64 \mathrm{~Hz}, 1 \mathrm{H}), 7.58$ (d, J = 7.74 Hz, 1H), $7.46(t, J=7.03 \mathrm{~Hz}, 2 \mathrm{H}), 7.32(\mathrm{t}, \mathrm{J}=7.03 \mathrm{~Hz}, 1 \mathrm{H}), 7.25(\mathrm{t}, \mathrm{J}=7.74$ 
$\mathrm{Hz}, 1 \mathrm{H}), 7.08(\mathrm{t}, \mathrm{J}=7.74 \mathrm{~Hz}, 1 \mathrm{H}), 1.27(\mathrm{~s}, 9 \mathrm{H}) ;{ }^{13} \mathrm{C}$ NMR $\delta 163.6,142.1,138.7,136.8$ $130.8,129.5,124.3,123.0,121.8,120.3,120.2,120.0,112.6,112.4,48.5,31.0,30.8 ; \mathrm{MS}$ [m/z (rel. intensity)] 324 [M+, 13], 268 (6), 144 (100), 125 (24), 57 (5).

$N$-[2-(ethylsulfinyl)phenyl]-1H-indole-3-carboxamide (15a). (96\%): $\mathrm{mp} \quad 60-61{ }^{\circ} \mathrm{C}$; IR 3217, 3168, 1652, $1003 \mathrm{~cm}^{-1}$; ${ }^{1} \mathrm{H}$ NMR $(200 \mathrm{MHz}) \delta 11.15(1 \mathrm{H}, \mathrm{s}) ; 9.72(1 \mathrm{H}, \mathrm{s}), 8.71$ $(1 \mathrm{H}, \mathrm{d}, \mathrm{J}=8 \mathrm{~Hz}), 8.40-8.45(1 \mathrm{H}, \mathrm{m}$, cont. $\mathrm{J}=3 \mathrm{~Hz}), 7.90(1 \mathrm{H}, \mathrm{d}, \mathrm{J}=3 \mathrm{~Hz}), 7.06-7.60$ $(6 \mathrm{H}, \mathrm{m}$, cont. $\mathrm{J}=1,8 \mathrm{~Hz}), 3.02-3.24(2 \mathrm{H}, \mathrm{dm}), 1.18(3 \mathrm{H}, \mathrm{t}, \mathrm{J}=7 \mathrm{~Hz}) ;{ }^{13} \mathrm{C}$ NMR $\delta 164.9$, $142.8,137.8,134.1,128.8,128.2,127.3,125.6,124.4,124.0,123.8,123.1,122.7,113.1$, 113.0, 49.3, 8.8; MS [m/z (relative intensity)] $312\left(\mathrm{M}^{+}, 15\right), 144$ (100). Anal. Calcd. for $\mathrm{C}_{17} \mathrm{H}_{16} \mathrm{~N}_{2} \mathrm{O}_{2} \mathrm{~S}: \mathrm{C}, 65.36 ; \mathrm{H}, 5.16 ; \mathrm{N}, 8.97$. Found: C, 65.48; H, 5.23; N, 8.99.

$N$-[2-(ethylthio)phenyl]- $N, 1$-dimethyl-1H-indole-3-carboxamide (16a). To a stirred suspension of 14a $(1.8 \mathrm{~g}, 6.1 \mathrm{mmol})$ and tetra-n-butylammonium sulfate in toluene (10 $\mathrm{mL}$ ) was added $50 \%$ aqueous $\mathrm{NaOH}(7 \mathrm{~mL})$ in one portion. After reflux for $2 \mathrm{~h}$, a solution of $\mathrm{CH}_{3} \mathrm{I}(1.9 \mathrm{~g}, 13.3 \mathrm{mmol}, 2.2$ eq. $)$ in toluene $(2 \mathrm{~mL})$ was added and the mixture heated under reflux for an additional $17 \mathrm{~h}$. The organics were collected, washed with $\mathrm{H}_{2} \mathrm{O}$ until neutral to litmus, dried $\left(\mathrm{Na}_{2} \mathrm{SO}_{4}\right)$, the volatiles removed in vacuo, and the brown solid chromatographed $\left(\mathrm{CHCl}_{3}\right)$ to produce $16 \mathrm{a}(1.8 \mathrm{~g}, 90 \%)$ as an off-white solid: $\mathrm{mp}$ 172-173 ${ }^{\circ} \mathrm{C}$ (acetone); IR $1621 \mathrm{~cm}^{-1} ;{ }^{1} \mathrm{H}$ NMR $(200 \mathrm{MHz}) \delta 8.41-8.46(1 \mathrm{H}, \mathrm{m}), 7.12-7.34$ $(7 \mathrm{H}, \mathrm{m}), 6.06(1 \mathrm{H}, \mathrm{d}, \mathrm{J}=0.6 \mathrm{~Hz}), 3.49(3 \mathrm{H}, \mathrm{s}), 3.38(3 \mathrm{H}, \mathrm{s}), 2.90(2 \mathrm{H}$, apparent qd, J = 7.1, $0.2 \mathrm{~Hz}), 1.27(3 \mathrm{H}, \mathrm{t}, \mathrm{J}=7.0 \mathrm{~Hz}) ;{ }^{13} \mathrm{C}$ NMR $\delta 166.7,143.6,138.4,136.8,132.6$, $130.3,124.4,124.2,127.5,126.7,123.5,123.2,122.0,110.0109 .837 .3,33.8,26.2,14.5$; MS [m/z (relative intensity)] $324\left(\mathrm{M}^{+}, 3\right), 158(100)$. Anal. Calcd. for $\mathrm{C}_{19} \mathrm{H}_{20} \mathrm{~N}_{2} \mathrm{OS}: \mathrm{C}$, 70.34; H, 6.21; N, 8.63. Found: C, 70,28; H, 6.41; N, 8.69. 


\section{$N$-[2-(tert-butylthio)phenyl]- $N, 1-d i m e t h y l-1 H$-indole-3-carboxamide (16b). To a}

well-stirred suspension of 14b (1.1 g, $3.4 \mathrm{mmol})$ and tetra-n-butylammonium hydrogen sulfate $(0.3 \mathrm{eq}, 1 \mathrm{mmol}, 0.35 \mathrm{~g})$ in toluene $(100 \mathrm{~mL})$ was added all at once $50 \% \mathrm{NaOH}$ (aq) solution $(100 \mathrm{~mL})$. The resulting two layer mixture was heated under reflux at which a solution of iodomethane $(2.5 \mathrm{eq}, 8.5 \mathrm{mmol}, 1.20 \mathrm{~g})$ in toluene $(10 \mathrm{~mL})$ was added dropwise over $5 \mathrm{~min}$. The resulting two phase mixture was maintained at reflux for $87 \mathrm{~h}$. It was cooled to room temperature and the layers separated. The organic layer was washed with water several times (until washings were neutral to litmus), dried over sodium sulfate and evaporated in vacuo to yield an off-white solid. Purification by column chromatography (chloroform:ether, 9:1) gave white compound (0.87 g, 73\%): mp 156-158 ${ }^{\circ} \mathrm{C}$; IR $1630 \mathrm{~cm}^{-1} ;{ }^{1} \mathrm{H}$ NMR $\delta 8.34-8.41(\mathrm{~m}, 1 \mathrm{H}), 7.59(\mathrm{~d}, \mathrm{~J}=7.72 \mathrm{~Hz}, 1 \mathrm{H}), 7.31-$ 7.39 (m, 2H), 7.28 (ddd, J = 7.71, 2.12 Hz, 1H), 7.17-7.22 (m, 2H), 7.10-7.16 (m, 1H), $5.81(\mathrm{~s}, 1 \mathrm{H}), 3.43(\mathrm{~s}, 6 \mathrm{H}), 1.29(\mathrm{~s}, 9 \mathrm{H}) ;{ }^{13} \mathrm{C} \mathrm{NMR} \delta 166.1,147.6,136.5,136.0,131.6$ $129.0,128.9,128.2,127.3,122.6,122.3,121.0,109.8,108.9,46.7,32.8,31.6 ; \mathrm{MS}[\mathrm{m} / \mathrm{z}$ (rel. intensity)] 295 (12), 263 (21), 158 (100).

\section{$N$-[2-(ethylsulfinyl)phenyl]- $N$,1-dimethyl-1H-indole-3-carboxamide (17a). (72\%).}

mp 188-189 ${ }^{\circ} \mathrm{C}$ (acetone); IR 1627, $1014 \mathrm{~cm}^{-1}$; ${ }^{1} \mathrm{H}$ NMR $\left(200 \mathrm{MHz}\right.$, taken at $100{ }^{\circ} \mathrm{C}$ in DMSO-d $\left._{6}\right) \delta 8.25-8.31(1 \mathrm{H}, \mathrm{m}), 7.96-8.01(1 \mathrm{H}, \mathrm{m}), 7.19-7.60(7 \mathrm{H}, \mathrm{m}), 6.25(1 \mathrm{H}, \mathrm{s}), 3.54$ $(3 \mathrm{H}, \mathrm{s}), 3.46(3 \mathrm{H}, \mathrm{s}), 2.56-2.80(2 \mathrm{H}, \mathrm{m}$, cont. $\mathrm{J}=6.6 \mathrm{~Hz}), 1.12(3 \mathrm{H}, \mathrm{t}, \mathrm{J}=6.6 \mathrm{~Hz}) ;{ }^{13} \mathrm{C}$ NMR $\delta 167.0,143.3,143.1,137.4,133.5,133.2,133.0,130.3,121.9,124.3,123.7$, 123.1, 123.0, 110.6, 110.5, 50.8, 49.5, 34.5, 7.3; MS [m/z (relative intensity)] $340(\mathrm{M}+$, 0.2), 158 (100). Anal. Calcd for $\mathrm{C}_{19} \mathrm{H}_{20} \mathrm{~N}_{2} \mathrm{O}_{2} \mathrm{~S}$ : C, 67.03; H, 5.92; N, 8.23. Found: C, $67.25 ; \mathrm{H}, 6.10 ; \mathrm{N}, 8.27$. 
$N$-[2-(tert-butylsulfinyl)phenyl]- $N$,1-dimethyl-1H-indole-3-carboxamide (17b). To an ice-cooled solution of $\mathbf{1 6 b}(0.82 \mathrm{~g}, 2.30 \mathrm{mmol})$ in $\mathrm{CH}_{2} \mathrm{Cl}_{2}(50 \mathrm{~mL})$ was added slowly a solution of $\mathrm{m}$-CPBA $(77 \%, 1.1 \mathrm{eq}, 3.32 \mathrm{mmol}, 0.57 \mathrm{~g})$ in $\mathrm{CH}_{2} \mathrm{Cl}_{2}(10 \mathrm{~mL})$. The resulting mixture was stirred at $0{ }^{\circ} \mathrm{C}$ for $15 \mathrm{~min}$ and then put it in a freezer $\left(-8{ }^{\circ} \mathrm{C}\right)$ overnight. The reaction mixture was then poured into $5 \% \mathrm{NaHCO}_{3}$ solution $(50 \mathrm{~mL})$ and extracted with $\mathrm{CH}_{2} \mathrm{Cl}_{2}(50 \mathrm{~mL})$. The combined organic layer was washed with distilled water, dried, and concentrated in vacuo. Column chromatography (acetone: $\mathrm{CH}_{2} \mathrm{Cl}_{2}, 1: 1$ ) gave white solid (0.42 g, 49\%): mp 159-160 ${ }^{\circ} \mathrm{C}$; IR 1631, $1030 \mathrm{~cm}^{-1} ;{ }^{1} \mathrm{H}$ NMR and ${ }^{13} \mathrm{C}$ NMR was not wellresolved due to presence of rotational isomers; MS [m/z (rel. intensity)] 294 (35), 158 (100).

\section{References and Footnotes}

1. For recent reviews see: (a) Lucchi, O. D.; Miotti, U.; Modena, G. Org. React., 1991, 40, 157-405. (b) Padwa, A.; Gunn, D. E., Jr.; Osterhout, M. H. Synthesis 1997, 13531377. (c) Padwa, A.; Waterson, A. G. Curr. Org. Chem. 2000, 4, 175-203. (d) Sano, T. Trends in Heterocyclic Chemistry, 2001, 7, 117-142. (e) Bur, S. K.; Padwa, A. Chem. Rev. 2004, 104, 2401-2432.

2. (a) Pyne, S. G.; Hajipour, A. R. Tetrahedron 1994, 50, 13501-13510. (b) Amat, M.; Hadida, S.; Pshenichnyi, G.; Bosch, J. J. Org. Chem. 1997, 62, 3158-3175.

3. (a) Bates, D. K.; Sell, B. A.; Picard, J. A. Tetrahedron Lett. 1987, 28, 3535-3538. (b) Bates, D. K.; Winters, R. T.; Picard, J. A. J. Org. Chem. 1992, 57, 3094-3097.

4. Chou, T. S.; Burgtorf, J. R.; Ellis, A. L.; Lammert, S. R.; Kukolja, S. P. J. Am. Chem. Soc. 1974, 96, 1609-1610. 
5. The most well studied example of this process is the Morin ring expansion ${ }^{5 a, b}$ used to convert penam sulfoxides into cepham antibiotics. Viehe ${ }^{5 c}$ has reported a related intermolecular counterpart which utilizes t-butyl sulfoxides as latent sulfenyl cations (via $\mathrm{RSOCOCF}_{3}$ ) for addition to alkenes. (a) Morin, R. B.; Jackson, B. G.; Mueller, R. A.; Lavagnino, E. R.; Scanlon, W. B.; Andrews, S. L. J. Am. Chem. Soc. 1963, 85, 18961897. (b) Morin, R. B.; Jackson, B. G.; Mueller, R. A.; Lavagnino, E. R.; Scanlon, W. B.; Andrews, S. L. J. Am. Chem. Soc. 1969, 91, 1401-1407. (c) Brichard, M. H.; Musick, M.; Janousek, Z.; Viehe, H. G. Syn. Commun. 1990, 20, 2379-2386.

6. Some optically active sulfoxides have been shown to racemize (even at room temperature) via a process of syn- beta elimination to an achiral sulfenic acid followed by recyclization to racemic sulfoxide: (a) Stoodley, R. J.; Wilkins, R. B. J. Chem Soc., Perkin Trans. 1, 1974, 1572-1579. (b) Berges, D. A.; Taggert, J. J. J. Org. Chem. 1985, $50,413-415$.

7. (a) Hoard, D. W.; Luke, W. D. U. S. Patent 5569772 A 19961029 1996; Chem. Abstr. 126:7983. (b) Hoard, D. W.; Luke, W. D. U. S. Patent 5514826 A 19960507 1996; Chem. Abstr. 125:86485. (c) Aikins, J. A.; Zhang, T. Y. Patent Application WO 96US9167 19960604, 1996; Chem. Abstr. 126:117861. (d) Hoard, D. W.; Luke, W. D.; Johnson, R. A. Book of Abstracts, 214 ${ }^{\text {th }}$ ACS National Meeting, Las Vegas, NV September 7-11, 1997. ORGN-326. (e) Zhang, T. Y.; O’Toole, J. C.; Aikins, J.; Sullivan, K. A. Book of Abstracts, $213^{\text {th }}$ ACS National Meeting, San Francisco, CA April 13-17, 1997. ORGN-175. 
8. (a) Wang, L. X.; Soczka-Guth, T.; Havinga, E.; Muellen, K. Angew. Chem. Int. Ed. Engl. 1996, 35, 1495-1497. (b) Becht, J. -M.; Wagner, A.; Mioskowski, C. J. Org. Chem. 2003, 68, 5758-5761.

9. (a) Hocker, J.; Ley, K.; Merten, R. Synthesis, 1975, 334-335. (b) Hartke, K.; Teuber, D.; Gerber, H. -D. Heterocycles 1986, 24, 2399-2402. (c) Hartke, K.; Teuber, D.; Gerber, H. -D. Tetrahedron, 1988, 44, 3261-3270. (d) Wendebourg, H. H.; Hartke, K. Synthesis 1989,329-331.

10. (a) Bates, D. K.; Tafel, K. A.; and Xu, J. Heterocycl. Commun. 1996, 2, 115-116. For an additional application see: (b) Picard, J. A.; Chen, S.; Bates, D. K. Heterocycles 1994, 38, 1775-1789.

11. Bates, D. K.; Xia, M. J. Org. Chem. 1998, 63, 9190-9196.

12. Bates, D. K.; Tafel, K. A. J. Org. Chem. 1994, 59, 8076-8080.

13. (a) Jackson, A. H.; Smith, A. E. Tetrahedron 1965, 21, 989-1000. (b) Jackson, A. H.; Naidoo, B.; Smith, P. Tetrahedron 1968, 24, 6119-6129. (c) Jackson, A. H.; Smith, A. E. Tetrahedron 1968, 24, 403-413. (d) Biswas, K. M.; Jackson, A. H. Tetrahedron 1969, 25, 227-241. (e) Jackson, A. H.; Naidoo, B. J. Chem. Soc., Perkin Trans. 2 1973, 548-551. (f) Hallett, D. J.; Gerhard, U.; Goodacre, S. C.; Hitzel, L.; Sparey, T. J.; Thomas, S.; Rowly, M.; Ball, R. G. J. Org. Chem. 2000, 65, 4984-4993. (h) Decker, M.; Faust, R.; Wedig, M.; Nieger, M.; Holzgrabe, U.; Lehmann, J. Heterocycles, 2001, 55, $1455-1466$.

14. Hughes, E. D.; Ingold, C. K.; Woolf, L. I. J. Chem. Soc., Abstr. 1948, 2084-2090. 
15. (a) Shelton, J. R.; Davis, K. E. Int. J. Sulfur Chem. 1973, 8, 197-204. (b) Shelton, J. R.; Davis, K. E. Int. J. Sulfur Chem. 1973, 3, 205-216.

16. Nippon Chemiphar Co. Ltd. Japan Patent JP 59,27,870; Chem. Abstr. 101, 110729f, 1982.

17. (a) alkyl = Et: Nieforth, K. A. J. Pharm. Sci. 1963, 52, 1136-1139. (b) alkyl $\mathrm{CH}_{2} \mathrm{CH}_{2} \mathrm{CN}$ : Lankin, D. C.; Petterson, R. C.; Velazquez, R. A. J. Org. Chem. 1974, 39, 2801-2803. (c) alkyl = t-Bu: Courtin, A.; von Tobel, H. - R.; Auerbach, G. Helv. Chem. Acta 1980, 63 1412-1419.

18. (a) Kermack, W. O.; Perkin, W. H., Jr.; Robinson, R. J. Chem. Soc. 1921, 119, 1602-1642 (Chem. Abstr. 16:367). (b) Szmuszkovicz, J. J. Org. Chem. 1964, 29, 178184. (c) Boatman, R. J.; Whitlock, H. W. J. Org. Chem. 1976, 41, 3050-3051. (d) Bergman J.; Carlsson, R.; Sjoberg, B. J. Heterocyclic Chem. 1977, 14, 1123-1134.

19. Augelli-Szafran, C. E.; Blankley, C. J.; Roth, B. D.; Trivedi, B. K.; Bousley, R. F.; Essenburg, A. D.; Hamelehle, K. L.; Krause, B. R.; Stanfield, R. L. J. Med. Chem. 1993, 36, 2943-2949.

20. Node, M.; Itoh, A.; Nishide, K.; Abe, H.; Kawabata, T.; Masaki, Y.; Fuji, K. Synthesis 1992, 1119-1124.

21. (a) Terney, A. L., Jr.; Chasar, D.W.; Sax, M. J. Org. Chem. 1967, 32, 2465-2470. (b)Terney, A. L., Jr.; Ens, L.; Herrmann, J.; Evans, S. J. Org. Chem. 1969, 34, 940-945.

22. (a) alkyl = Et: was prepared using the general procedure of Barluenga, J.; Bayon, A. M.; Asensio, G. J. Chem. Soc. Chem. Commun. 1984, 1334-1335. (b) see experimental 
section (c) Kuliev, A. M.; Agaeu, A. N.; Mamedov, F. A. Ser. Khim. Nauk. 1971, 32-36 (Chem. Abstr. 77:151594).

23. Gajda, T.; Zwierzak, A. Synthesis 1981, 1005-1008.

24. Jog, P. V.; Brown, R. E.; Bates, D. K. J. Org. Chem. 2003, 68, 8240-8243.

25. (a) Oki, M. Applications of Dynamic NMR Spectroscopy to Organic Chemistry; VCH Publishers, Deerfield Beach, Fla. 1985. (b) Saito, S.; Toriumi, Y.; Tomioka, N.; Itai, A. J. Org. Chem. 1995, 60, 4715-4720.

26. ${ }^{1} \mathrm{H}$ NMR data also suggest intramolecular N-H--O=S hydrogen bonding. Oxidation of $3 \mathbf{a}$ to $4 \mathbf{a}$ (and 1a to 2a) results in a chemical shift change of $\sim 0.2 \mathrm{ppm}$ (downfield) in the indolic N-H signal whereas oxidation of $5 \mathbf{a}$ to $6 \mathbf{a}$ (and 1a to 2a) results in a chemical shift change of $\sim 2.2 \mathrm{ppm}$ (downfield) in the amidic $\mathrm{N}-\mathrm{H}$ signal (in $\mathrm{CDCl}_{3}$ solution). Comparing ${ }^{1} \mathrm{H}$ NMR spectra recorded in $\mathrm{CDCl}_{3}$ to spectra recorded in DMSO- $\mathrm{d}_{6}$ for compounds 2a, 9, and $\mathbf{1 2}$ indicates causes a small ( $\sim 0.4 \mathrm{ppm})$ upfield shift in the amidic $\mathrm{N}-\mathrm{H}$ signal and a larger $(\sim 2.7 \mathrm{ppm})$ downfield shift in the indolic N-H. Additionally, the temperature dependence values for the amidic and indolic $\mathrm{N}-\mathrm{H}$ signals $\left(\Delta \delta \mathrm{NH} / \Delta \mathrm{T}, \mathrm{CDCl}_{3}\right.$ solution in the range $290-365 \mathrm{~K}$ ) are -1.0 and $-5.5 \mathrm{ppb} / \mathrm{K}$, respectively. ${ }^{26 \mathrm{~b}-\mathrm{d}}$ (a) Furukawa, N.; Fujihara, H. in The Chemistry of Sulfones and Sulfoxides (Patai, S.; Rappoport, Z.; Stirling, C. J. M., editors) 1988, John Wiley and Sons: New York, pp. 541-565. (b) Soth, M. J.; Nowick, J. S. J. Org. Chem. 1999, 64, 276-281. (c) Gung, B. W.; Zhu, Z.; Everingham, B. J. Org. Chem. 1997, 62, 3436-3437. (d) Kessler, H. Angew. Chem. Int. Ed. Engl. 1982, 21, 512-523.

27. Bates, D. K.; Habib, Q. A. J. Heterocycl. Chem. 1995, 32, 1477-1481. 
28. Hamel, P.; Girard, M.; Tsou, N. N. J. Heterocycl. Chem. 1999, 36, 643-652.

29. Ambrogi, V.; Furlani, A.; Grandolini, G.; Papaioannou, A.; Perioli, L.; Scarcia, V.; Tuttobello, L. Eur. J. Med. Chem. 1993, 28, 659-667.

30. Atkinson, J. G.; Hamel, P.; Girard, Y. Synthesis 1988, 480-481.

31. Blade-Font, A. Tetrahedron Lett. 1980, 21, 2443-2446.

32. Swain, C. J.; Baker, R.; Kneen, C.; Moseley, J.; Saunders, J.; Seward, E. M.; Stevenson, G.; Beer, M.; Stanton, J.; Waitling, K. J. Med. Chem. 1991, 34. 140-151.

33. Jackson, A. H.; Naidoo, B.; Smith, A. E.; Bailey, A. S.; Vandrevala, M. H. J. Chem. Soc. Chem. Commun. 1978, 779-781.

34. Engqvist, R.; Bergman, J. Tetrahedron 2003, 59, 9649-9653.

35. Nagarajan, K.; Arya, V. P.; Parthasarathy, T. N. Shenoy, S. J.; Shah, R. K.; Kulkarni, Y. S. Indian J. Chem. Soc. 1981, 20B, 672-679.

36. (a) Harris, R. L. N.; McFadden, H. G. Aust. J. Chem. 1986, 39, 887-892. (b) Plate, R.; Ottenheijm, H. C. J. Tetrahedron Lett. 1986, 42, 4511-4516. (c) Hamel, P.; Girard, Y.; Atkinson, J. G. Chem. Commun. 1989, 63-63. (d) Hamel, P.; Girard, Y.; Atkinson, J. G. J. Org. Chem. 1992, 57, 2694-2699.

37. The nature of the sulfur species may not be critical: sulfonium ions are observed to have relatively similar migratory aptitudes to sulfur as applied to the Stevenson and Sommelet rearrangements. ${ }^{37 a}$ The migratory aptitude of an alkylsulfonium species in pyrroles $^{9 \mathrm{a}}$ is roughly the same as an alkylthio group migrations in pyrroles ${ }^{37 \mathrm{~b}}$ under the 
same conditions (a) Barrett, G.C. In "Comprehensive Organic Chemistry", ed D.N. Jones, Pergamon Press, 1979, vol. 3, p.117. (b). Kakushima, M.; Frenette, R. J. Org. Chem. 1984, 49, 2025.

38. Gairns, R.S.; Moody, C.J.; Rees, C.W. J. Chem. Soc., Perkin Trans. I 1986, 501506.

39. Wright, S. W., Abelman, M. M.; Bostrom, L. L.; Corbett, R. L. Tetrahedron Lett. 1992, 33, 153- 156 .

40. Aucagne, V.; Aversa, M. C.; Batattucci, A.; Giannetto, P.; Rollin, P.; Tatibouet, A. J. Org. Chem. 2002, 67, 6925-6930.

41. Bell, R., Cottam, P. D.; Davies, J.; Jones, D. N. J. Chem. Soc., Perkin Trans. 1, 1981, 2106-2115.

42. (a) Kamiya, T.; Teraji, T.; Saito, Y.; Hashimoto, M.; Nakaguchi, O.; Oku, T. Tetrahedron Lett. 1973, 3001-3004. (b) see also Micetich, R. G.; Maiti, S. N.; Singh, M. P. Tanaka, M.; Yamazaki, T.; Ogawa, K. Tetrahedron Lett. 1985, 26, 5611-5614.

43. Block, E.; O’Connor, J. J. Amer. Chem. Soc. 1974, 96, 3929-3944.

Acknowledgment. The authors thank the NSF for an equipment grant (CHE-9512445). P. V. J. thanks the Chemistry Department of Michigan Technological University for financial support. The assistance of Mr. Jerry L. Lutz (NMR instrumental assistance) and Mr. Shane Crist (computer-related issues) are gratefully acknowledged.

Supporting Information Available: Experimental procedures for 2-(ethylthio)- $N$ methylaniline, 2-(tert-butylthio)- $N$-methylaniline, ethyl $N$-methylindole-2-carboxylate, 
1b, 2b, 3ab, 4a, 5ab, 6ab, 7bc, 8abc, and the thermal and TFAA-promoted cyclization of $\mathbf{8 b} ;{ }^{1} \mathrm{H}$ NMR and ${ }^{13} \mathrm{C}$ NMR spectra for $\mathbf{1 b}-\mathbf{3 b}, \mathbf{5 b - 8 b}, \mathbf{7 c}, \mathbf{1 0}, \mathbf{1 4 b}$, and $\mathbf{1 6 b} ;{ }^{1} \mathrm{H}$ NMR for 4b and 9. This material is available free of charge via the internet at http://pubs.acs.org. 


\section{Chapter 3}

\section{Sulfoxide as a "protecting group"}

\section{Simple Thiazocine-2-acetic acid Derivatives via Ring Closing Metathesis}

Bates, D. K.; Li, X.; Jog, P. V. J. Org. Chem. 2004, 69, 2750.

The contribution of Parag Jog to this work mainly involved design and execution of the use of sulfoxides as protecting groups in RCM chemistry. To my knowledge use of the sulfide $\leftarrow \rightarrow$ sulfoxide redox cycle has never been applied as a protecting group strategy. His work is very significant in this respect as the full potential of this approach to other areas where the S(II) oxidation state poses problems remains to be determined. He also conducted extensive NMR studies to assign all chemical shifts and coupling constants to the sulfide thiazocine derivative.

\section{Approved by co-author Dallas K. Bates}

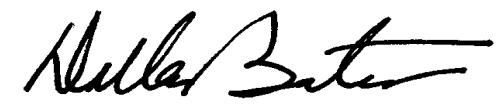




\title{
$\mathrm{JOC}_{\text {Article }}$
}

\section{Simple Thiazocine-2-acetic Acid Derivatives via Ring-Closing Metathesis}

\author{
Dallas K. Bates, ${ }^{*}$ Xiaofen Li, and Parag V. Jog \\ Department of Chemistry, Michigan Technological University, 1400 Townsend Drive \\ Houghton, Michigan 49931 \\ dbates@mtu.edu
}

Received November 17, 2003

\begin{abstract}
A new protocol for synthesis of 2-heterocylylacetic acid derivatives involving conjugate addition of allyl mercaptan to an acrylate containing a tethered olefinic site followed by RCM (ring-closing metathesis) is described. In this series, sulfanyl derivatives were unreactive, while sulfoxide and sulfone analogues provided the corresponding thiazocines in fair to excellent yields. Use of the sulfoxide oxidation state as a protecting group for sulfides inert to RCM is demonstrated also. Thus, oxidation of sulfide 9 [ $N$-allyl- $N$-[2-(allylthio)-4-(1H-indol-1-yl)-4-oxobutyl]-4-methylbenzenesulfonamide] followed by cyclization yielded the corresponding thiazocine sulfoxide 12. Deprotection (deoxygenation) of 12 was accomplished using Lawesson's reagent, producing 1-[[4-[4(methylphenyl)sulfonyl]-3,4,5,8-tetrahydro-2 $H$-1,4-thiazocin-2-yl]acetyl]-1H-indole (21) in 67\% unoptimized yield.
\end{abstract}

\section{Introduction}

Heterocylylacetic acid derivatives are a diverse and important group of compounds. In addition to examples of NSAIDS (Myalex/fenclozic acid) ${ }^{1}$ (including COX-2 specific inhibitors) ${ }^{2}$ and nonbenzodiazepine hypnotics (Ambien/zolpidem), ${ }^{3}$ compounds acting as blood platelet aggregation inhibitors, ${ }^{4}$ aldose reductase inhibitors, ${ }^{5}$ antimicrobial agents, ${ }^{6}$ TACE inhibitors, ${ }^{7}$ and medicinal chelating agents ${ }^{8}$ and many others exhibiting useful biological activity have been reported. Many synthetic efforts have concentrated on five-, six-, seven-, and eightmembered ring examples of $\mathbf{B}\left(\mathrm{Y}=\left(\mathrm{CH}_{2}\right)_{n}, \mathrm{X}=\mathrm{O}\right)$ because of their prominence as building blocks for

(1) Chalmers, T. M.; Pohl, J. E. F.; Platt, D. S. Ann. Rheum. Dis. $1969,28,590-594$

(2) (a) Caron, S.; Vazquez, E.; Stevens, R. W.; Nakao, K.; Koike, H.; Murata, Y. J. Org. Chem. 2003, 68, 4104-4107. (b) Kreft, A. F. Caufield, C. E.; Failli, A. A.; Caggiano, T. J.; Greenfield, A. A.; Kubrak D. M. U. S. Patent 5776967, 1998; Chem. Abstr. 129, 122569. (c) Jin F.; Confalone, P. N. U. S. Patent 5668159, 1997; Chem. Abstr. 127 293226.

(3) George, C. F. P. Lancet 2001, 358, 1623-1626.

(4) (a) Sanderson, P. E. J.; Cutrona, K. J.; Savage, K. L.; NaylorOlsen, A. M.; Bickel, D. J.; Bohn, D. L.; Clayton, F. C.; Krueger, J. A.; Lewis, S. D.; Lucas, B. J.; Lyle, E. A.; Wallace, A. A.; Welsh, D. C.; Yan, Y. Bioorg. Med. Chem. Lett. 2003, 13, 1441-1444. (b) Kanter, J.; Marlowe, C.: Mullins, J. J. G.: Pandey, A.: Scarborough. R.: Butke, G.; Jacobsen, B.; Walker, D. PCT Int. Appl. WO 2001092249, 2001; G.; Jacobsen, B,; Walker, it

(5) (a) Reddy, R. R.; Reddy, T. M.; Reddy, V. M. Indian J. Heterocycl. Chem. 1998, 7, 185-188. (b) Mylari, B. L.; Zembrowski, W. J.; Beyer, T. A.; Aldinger, C. E.; Siegel, T. W. J. Med. Chem. 1992, 35, 21552162.

(6) Tatsuta, K.; Kurita, Y. Jpn. Kokai Tokkyo Koho 05074047, 1993 Chem. Abstr. 119, 138980).

(7) Duan J. J.-W.; Lu, Z.; Xue, C.-B.; He, X.; Seng, J. L.; Roderick J. J.; Wasserman, Z. R.; Liu, R.-Q.; Covington, M. B.; Magolda, R. L. Newton, R. C.; Trzaskos, J. M.; Decicco, C. P. Bioorg. Med. Chem Lett 2003, 13, 2035-2040.

(8) Campbell, M. M. U. S. Patent 4582907, 1986; Chem. Abstr. 105, 78924 . synthesis of fused polyether marine toxins (e.g., ciguatoxin) ${ }^{9}$ and other natural products. ${ }^{10}$ Compounds $\mathbf{B}(\mathrm{X}=$ S) have also been prepared, but examples are scarce. Of the synthetic approaches developed, most target a specific member and are not general. Common general protocols (typically applicable only to five-, six-, and sevenmembered ring compounds) involve conjugate addition to an $\alpha, \beta$-unsaturated ester. Specifically, Michael addition of $\mathrm{N}$-, O-, or S-centered nucleophiles to tethered acrylates $^{11}$ (A, eq 1, path a) or Michael addition of a protected nucleophile to an acrylate (for example, C) followed by deprotection and cyclization ${ }^{12}$ (eq 1, path b) ${ }^{13}$ have been used extensively.
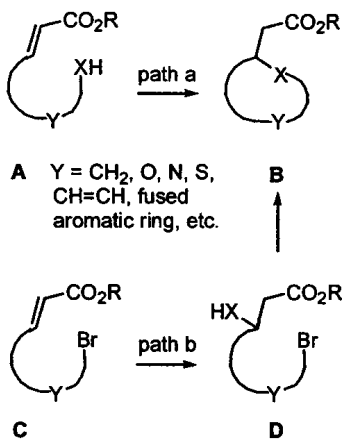

Here we describe a new protocol for assembling compounds of type $\mathbf{B}$ making use of conjugate addition followed by ring-closing metathesis (RCM) (eq 2). This approach is illustrated by preparation of 2-thiazocinyl-

(9) Oishi, T.; Nagumo, Y.; Shoji, M.: LeBrazidec, J.-Y.; Uehara, H.; Hirama, M. Chem. Commun. 1999, 2035-2036.

10.1021/jo035692z CCC: $\$ 27.50 \bigcirc 2004$ American Chemical Society Published on Web 03/12/2004 
acetic acid derivatives and should be readily adaptable to other analogues as well. The specific target compounds were chosen because RCM has been shown to be an excellent methodology for $\mathrm{N}$ - and $\mathrm{O}$-containing mediumsized ring synthesis ${ }^{14}$ and eight-membered rings are the smallest simple $S$ - and $N, S$-cycloalkanes for which conventional synthetic approaches fail. ${ }^{15}$

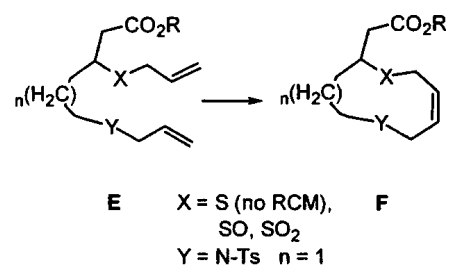

(2)

Although olefin metathesis has experienced explosive growth, reports of applications of RCM to sulfur-contain ing compounds are comparatively rare. Initial work found

(10) (a) Crimmins, M. T.; Emmitte, K. A. Synthesis 2000, 899-903. (b) Evans, P. A.; Roseman, J. D. J. Org. Chem. 1996, 61, 2252-2253. (c) Holmes, C. P.; Bartlett, P. A. J. Org. Chem. 1989, 54, 98-108. Nitrogen systems (B) appear in natural products: Bardou, A.; Celerier. Nitrogen systems (B) appear in natural products: Bardou, A.;

(11) (a) Shimizu, K. Takimoto, M. Mori, M. Org. Lett. 2003, 5 (11) (a) Shimizu, K.; Takimoto, M.; Mori, M. Org. Lett. 2003,
2323-2325. (b) Vares, L.; Rein, T. J. Org. Chem. 2002, 67, 7226-7237

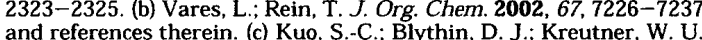
and references therein. (c) Kuo, S.-C.; Blythin, D. J.; Kreutner, W. U. S. Patent 5929236, 1999; Chem. Abstr. 131, 116239. (d) Banwell, M. Trans. 1 1996, 967-969. (e) Srinivasan, A.; Lyle, L. R.; Rajagopalan R. WO Patent 9603429, 1996; Chem. Abstr. 125, 59147. (f) Kuo, S.-C. Blythin, D. J.; Kreutner, W. WO Patent 9422843 , 1994; Chem. Abstr 122, 81384. (g) Bunce, R. A.; Bennett, M. J. Synth. Commun. 1993 23, 1009-1020. (h) Desmaele, D.: Pain, G.; D'Angelo, J. Tetrahedron: Asymmetry. 1992, 3, 863-866. (i) Bunce, R. A.; Peeples, C. J.; Jones, B. J. Org. Chem 1992 57, 1727-1733. (j) Tsutomu, T. Aust J Chem 1986, 39, 503-510. For a somewhat related radical based approach. 1986. Navarro-Vazquez, A.; Garcia, A.; Dominguez, D. J. Org. Chem. 2002, 67, 3213-3220

(12) (a) O'Brien, P.; Porter, D. W.; Smith. N. M. Synlett 2000, 13361338. (b) Enders, D. Wiedemann, J. Liebigs Ann./Recueil 1997, 699706.

(13) Another general approach, never applied to S-based compounds (i.e., to sulfenium ion intermediates) involves reactive oxenium or iminium species (generated in situ) reacting with various synthons for ${ }^{4-} \mathrm{CH}_{2} \mathrm{COOH}^{\prime:}$ (a) Takeuchi, Y.; Azuma, K.; Oshige, M.; Abe, H.; Nishioka, H.; Sasaki, K.; Harayama, T. Tetrahedron 2003, 59, 16391646. (b) Okitsu, O.; Suzuki, R.; Kobayashi, S. J. Org. Chem. 2001, 66, 809-823 and references therein. (c) Dixon, D. J.; Ley, S. V.; Tate, E. W. J. Chem Soc Perkin Trans. I 2000, 1829-1836. (d) Hayashi, M.: Sugiyama, M.; Toba, T.; Oguni, N. Chem. Commun. 1990, 767768. (e) Homma, K. Takenoshita, $\mathrm{H}$; Mukaiyama, T. Bull. Chem. Soc. Jpn 1990 63, 1898-1915 (f) Nagasaka T. Koseki Y. Hayashi, $\mathrm{H}$. Yasuda, Y.; Hamaguchi, F. Yakugaku Zasshi 1989, 109, 823-826; Yhem. Abstr. 112, 216668 .

(14) For a review, see: Maier, M. E. Angew. Chem., Int. Ed. 2000, 39, 2073-2077. See also (a) Papaioannou N.; Blank, J. T.; Miller, S. J. J. Org. Chem. 2003, 68, 2728-2734. (b) Hoffmann, T.; Waibel, R.; Gmeiner, P. J. Org. Chem. 2003, 68, 62-69. (c) Clark, J. S.; Middleton. M. D. Org. Lett. 2002, 4, 765-768. (d) Boyer, F.-D.; Hanna, I.; Nolan, S. P. J. Org. Chem. 2001, 66, 4094-4096. (e) Creighton, C. J.; Reitz, A. B. Org. Lett. 2001, 3, 893-895

(15) For a review of the difficulties of medium size ring synthesis via standard cyclization methodologies, see: Illuminati, G. Acc. Chem. Res. 1981, 14, 95. Following the general trend for cyclane formation (Eliel, E. L.; Wilen, S. H.; Mander, L. N. Stereochemistry of Organic Compounds. Wiley-Interscience: New York NY, 1994; pp 678-684) cyclic thialkan and thiazalkanes (MaCoe, DY, 1994; pp 678-684) cyclic thialkanes and thiazalkanes (MaGee, D. I., Beck, E. J. J. Org. Chem. 2000, 65, 8367-8371) containing eight- or nine-membered rings form in very low yield by treatment of the corresponding alkyl dibromides with sodium sulfide nonahydrate. Some simple cyclic 1,4thiazepines are avallable from tetrahydrothiopyran-4-one (Beckmann rearrangement of the derived axime followed by amide reduction) (Doi, J. T.; Musker, W. K.; deleeuw, D. L.: Hirschon, A. S. J. Org. Chem. 1981, 46, 1239-1243), and eight-, nine-, and ten-ring atom oxathiacycloalkenes are available by ring expansion of halo- $O, S$-acetals (Coster, M. J.; DeVoss, J. J. Org. Lett. 2002, 4, 3047-3050.).
SCHEME 1 a<smiles>C=CCN(C)CC=CC(C)=CC=CC</smiles>

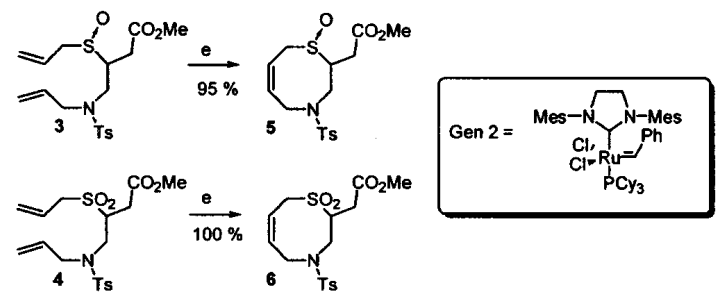

a Reagents: (a) $\mathrm{N}$-tosylallylamine, $\mathrm{NaOH}, \mathrm{TBAH}, \mathrm{DCM}, \mathrm{rt}$, $100 \%$; (b) allyl mercaptan, $\mathrm{NaOMe}, \mathrm{MeOH}$, reflux, 68\%; (c) $\mathrm{NaIO}_{4}$, acetone/water, $22 \mathrm{~h}$, rt, 35\%; (d) Oxone ( 3 equiv), $\mathrm{MeOH} /$ water, 3 d, rt, 58\%; (e) $5 \mathrm{~mol} \%$ Gen 2, DCM, reflux ( $6 \mathrm{~h}$ for $5,8 \mathrm{~h}$ for 6 ).

the Grubbs first generation catalyst inferior to Mo- and W-based catalysts with respect to sulfur(II) tolerance. ${ }^{16,17}$ Although Mo-based catalysts are quite tolerant of sulfur(II) sites in the substrate, the highly active $\mathrm{N}$-heterocyclic carbene catalysts such as Grubbs' second generation catalyst (Gen 2; see Scheme 1) are more widely used as a result of their improved tolerance for sulfur(II) and their air and moisture stability. Numerous examples of additional successful RCM reactions of sulfides, disulfides, and dithioacetals using Ru/carbene complexes have been reported. ${ }^{18}$ The literature suggests the sulfonyl oxidation state (sulfones, ${ }^{19}$ sulfonamides, ${ }^{20}$ etc. ${ }^{21}$ ) is better tolerated in RCM substrates than lower oxidation states of sulfur. Deactivation via Ru-sulfonyl oxygen coordination does not occur (even though Ru-sulfonyl ligation to assist ring closure has been suggested). ${ }^{22}$ In contrast to the body of work available for sulfides and sulfones,

(16) For examples of RCM failures with Grubbs' Gen 1 catalyst on sulfur-containing compounds, see: (a) Fuerstner, A.; Seidel, G.; Kindler, N. Tetrahedron 1999, 55, 8215-8230. (b) Armstrong, S. K. J Chem. Soc., Perkin Trans. 1 1998, 371-388. (c) Bujard, M.: Gouverneur, V.; Mioskowski, C. J. Org. Chem. 1999, 64, 2119-2123. (d) Mascarenas, J. L.; Rumbo, A.; Castedo, L. J. Org. Chem. 1997, 62 8620-8621.

(17) For successful application of Mo-based catalysts to sulfur(II) compounds, see: (a) Armstrong, S. K.; Christie, B. A. Tetrahedron Lett. 1996, 37, 9373-9376. (b) Barrett, A. G. M.; Baugh, S. P. D.; Gibson, V. C.; Giles, M. R.; Marshall, E. L.; Procopiou, P. A. Chem. Commun. 1997, 155-156. (c) Shon, Y.-S.; Lee, T. R. Tetrahedron Lett. 1997, 38 1997, 155-156. (c) Shon, Y.-S.; Lee, T. R. Tetrahedron Lett. 1997, 38, Couturier, J. L.; Tanaka, K.; Leconte, M.; Basset. J. M.; Ollivier, J. Couturier, J. L.; Tanaka, K.; Leconte, M.; Basset, J. M.; OI
Phosphorus, Sulfur Silicon Relat. Elem. 1993, 74, 383-384.

(18) (a) Spagnol, G.; Heck, M.-P.; Nolan, S. P.; Mioskowski, C. Org
. Lett. 2002, 4, 1767-1770. (b) Smulik, J. A.; Giessert, A. J.; Diver, S. T. Tetrahedron Lett. 2002, 43, 209-211. (c) Moore, J. D.; Sprott, K T.; Hanson, P. R. Synlett 2001, 605-608. (d) Garbaccio, R. M.; Danishefsky, S. J. Org. Lett. 2000, 2, 3127-3129.

(19) (a) Michrowska, A.; Bieniek, M.; Kim, M.; Klajn, R.; Grela, K. Tetrahedron 2003, 59, 4525-4531. (b) Yao, Q. Org. Lett. 2002, 4, 427430. (c) Basu, K.; Cabral, J. A.; Paquette, L. A. Tetrahedron Lett. 2002 43. 5453-5456. (d) Grela, K.; Bieniek, M. Tetrahedron Lett. 2001, 42 , 6425-6428. (e) Randl, S.; Connon, S. J.; Blechert, S. Chem. Commun. 2001, 1796-1797. (f) Blackwell, H. E.; O'Leary, D. J.; Chatterjee, A K.; Wasenfelder, R. A.; Busmann, D. A.; Grubbs, R. H. J. Am. Chem K.; Wasenfelder, R. A.; Busmann, D. A.; Grubbs, R. H. J. Am. Chem.
Soc. 2000, 122, 58-71. (g) Paquette, L. A.; Fabris, F.; Tae, J.; Gallucci Soc. 2000, 122, 58-71. (g) Paquette, L. A.; Fabris, F.; Tae, J.; Gallucci,
J. C.; Hofferberth, J. E. J. Am. Chem. Soc. 2000, 122, 3391-3398. (h) Fuerstner, A.; Ackermann, L. Chem. Commun. 1999, 95-96. (i) Fuerstner, A.; Ackermann, L. Chem. Commun. 1999, 95-96. (i) Chatterjee, A. K.; Grubbs, R. H. Org. Lett. 1999, 1, 1751-1753. (j)
Fuerstner, A.; Gastner, T.; Weintritt, H. J. Org. Chem. 1999, 64, 23612366. (k) Miller, J. F.; Termin, A.; Koch, K.; Piscopio, A. D. J. Org. Chem. 1998, 63, 3158-3159.

J. Org. Chem, Vol. 69, No. 8, 20042751 


\section{JOCArticle}

sulfoxides have been studied very little. DMSO treatment has been proposed ${ }^{23}$ as a means of removing Ru-containing contaminants from RCM reaction mixtures, presumably due to coordination Ru. Only two reports of RCM chemistry applied to sulfoxides have been reported. One was successful ${ }^{24}$ and the other was not. ${ }^{19 a}$ Therefore, sulfur-containing substrates for this study were prepared not only in the sulfanyl and sulfonyl oxidation states but also in the sulfinyl state as well.

\section{Results and Discussion}

Reaction of methyl 4-bromocrotonate with $\mathrm{N}$-tosylallylamine ${ }^{25}$ under phase transfer conditions followed by conjugate addition of allyl mercaptan provided the key intermediate 2 in $68 \%$ overall yield (Scheme 1). Oxidation of 2 to sulfoxide 3 (obtained as a 1:1 mixture of diastereomers) and sulfone 4 was readily accomplished by conventional means. Although 2 failed to undergo $\mathrm{RCM}^{26}$ (Gen 2 catalyst), ${ }^{27}$ both sulfone 4 and sulfoxide 3 cyclized [to thiazocines $\mathbf{6}$ and 5 (obtained as a 2:3 mixture of diastereomers), respectively] in excellent yield.

(20) (a) Wanner, J.; Harned, A. M.; Probst, D. A.; Poon, K. W. C.; Klein, T. A.; Snelgrove, K. A.; Hanson, P. R. Tetrahedron Lett. 2002 43, 917-921. (b) Yao, Q. Angew. Chem., Int. Ed. 2000, 39, 3896-3898. (c) Long, D. D.: Termin, A. P. Tetrahedron Lett. 2000, 41, 6743-6747. (c) Long, D. D.; Termin, A. P. Tetrahedron Lett. 2000, 41, 6743-6747. (d) Lane, C.; Snieckus, V. Synlett 2000, 1294-1296. (e) Hanson, P. R.; Probst, D. A.; Robinson, R. E.; Yau, M. Tetrahedron Lett. 1999, 40, 4761-4764. (f) Paquette, L. A.; Leit, S. M. J. Am. Chem. Soc. 1999, 121, 8126-8927. (g) Fuerstner, A. Chem. Commun. 1998, 1315-1316.
(h) Cerezo, S.; Cortes, J.; Moreno-Manus, M.; Pleixats, R.; Roglans, A. (h) Cerezo, S.; Cortes. J.; Moreno-Manus, M.; Pleixats, R.; Roglans, A.
Tetrahedron 1998, 54, 14869-14884. (i) Visser, M. S.; Heron, N. M.; Didiuk, M. T.; Sagal, J. F.; Hoveyda, A. H. J. Am. Chem. Soc. 1996 $118,4291-4298$.

(21) Sulfonates: Karsch, S.; Schwab, P.; Metz, P. Synlett 2002 2019-2022.

(22) Paquette, L. A.; Fabris, F.; Tae, J.; Gallucci, J. C.; Hofferberth J. E. J. Am. Chem. Soc. 2000, 122, 3391-3398. Also see: MarcoContelles, J.; de Opazo, E. J. Org. Chem. 2000, 65, 5416-5419 and references therein.

(23) Ahn, Y. M.; Yang, K.; Georg, G. I. Org. Lett. 2001, 3, 14111413. (24) Cachoux, F.; Ibrahim-Ouali, M.; Santelli, M. Synlett 2002

(25) $N$-Tosyl compounds appear to be superior to alkyl-, benzoylor ethoxycarbonyl-protected amines in RCM processes: (a) Kinderman S. S.; van Maarseveen, J. H. Schoemaker, H. E.; Hiemstra, H.; Rutjes, F. P. J. T. Org. Lett. 2001, 3, 2045-2048. (b) van Otterlo, W. A. L. Pathak, R.; de Konig, C. B. Synlett 2003, 1859-1861.

(26) Starting material (2) was recovered unchanged quantitatively. The lack of reactivity is probably not due to $\mathrm{Ru}$ coordination with the ester [no reaction was observed when $\mathrm{Ti}(\mathrm{O} I \mathrm{Pr})_{4}$ was added to the $\mathrm{RCM}$ reaction according to Fuerstner's protocol (Fuerstner. A.; Langemann, K. J. Am. Chem. Soc. 1997, 119, 9130-9136) and both S-oxidize counterparts to the sulfide (3 and 4) readily and both S-oxidized counterparts to the sulfidy undergo RCM]. The problem is more likely due to inactivation of the catalyst by coordinamolecularly prior to interaction of the $\mathrm{Ru}$ with the olefinic sites or intramolecularly, perhaps as shown in structure $\mathbf{i}$.

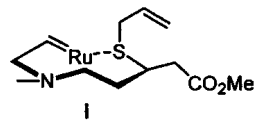
The latter process (involving coordination of ruthenium to hydroxyl
oxygen via a six-membered ring rather than the eight-membered ring oxygen via a six-membered ring rather than the eight-membered ring as in i) has been suggested previously (for example, Washburn, D. G.; Heidebrecht, R. W.: Martin, S. F. Org. Lett. $2003,5,3523-3525)$ to in the present context because of the size of the ring involved and the necessity to invoke selective reaction of the $N$-allylic olefinic site, even though the $S$-allylic olefinic site is sterically and electronically similar.

(27) (a) Chatterjee, A. K.; Morgan, J. P.; Scholl, M.; Grubbs, R. H J. Am. Chem. Soc. 2000, 122, 3783-3784. (b) Scholl, M.; Ding, S.; Lee C. W.; Grubbs, R. H. Org. Lett. 1999, 1, 953-956.
Bates et al.

SCHEME 2. Alternative Pathways to Thiazocine 12

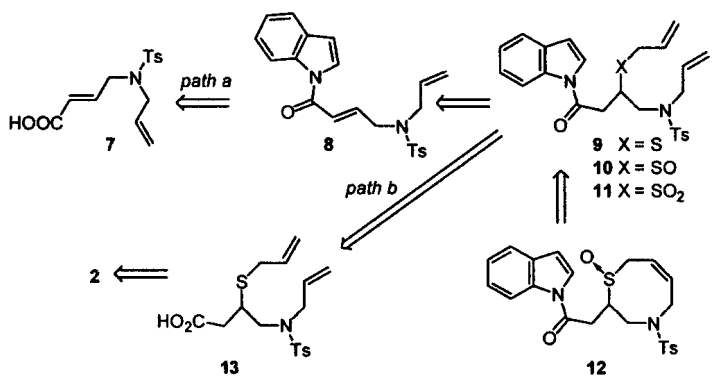

With this success in hand we applied the methodology to preparation of acyl indole 12 (Scheme 2), a system useful for SES/ring opening studies. ${ }^{28}$ Preparation of this target demonstrates some of the potential obstacles in syntheses of RCM substrates of the type shown in eq 2 . Thus, sulfide 9 was prepared in three steps from 1 in $19 \%$ overall yield, realizing path a in Scheme 2 . The low yield is due to poor conversion of the acid chloride of 7 to the acylindole. The problem stems from the lability of the initially formed acylindole to nucleophilic attack; no acylation procedure attempted involving a strong nucleophile $\left(\mathrm{OH}^{-}\right)$or nucleophilic solvent $\left(\mathrm{H}_{2} \mathrm{O}\right)$ gave satisfactory yields of 8 . Alternative acylation conditions were not thoroughly explored because an alternative process forming the labile acylindole later in the sequence was developed. Reversing the order of the reactions leading to 9 (forward synthesis version of path b, Scheme 2) did not improve overall yield because ester hydrolysis of 2 to $\mathbf{1 3}$ proceeded in poor yield as a result of unavoidable (in our hands) retro-Michael addition of allyl mercaptan.

These difficulties were overcome using the wellknown ${ }^{29}$ stability of indoline amides relative to their indole amide counterpart and the ease of conversion of the former to the latter. ${ }^{30}$ Execution of this strategy led to 9 in $44 \%$ yield (from 1, path a in Scheme 3). Reversing the sequence of Michael addition and indoline oxidation (path b, Scheme 3) was not effective. Although Michael addition of allyl mercaptan to 14 proceeded very cleanly, subsequent reaction of 15 with DDQ gave not only oxidation of the indoline but also retro-Michael addition providing mainly compound $\mathbf{8}$.

Oxidation $^{31}$ of indole 9 with 1 or 2 equiv of $m$-CPBA gave the corresponding sulfoxide (10, 72\%, 2:3 inseparable mixture of diastereomers) or sulfone $(11,99 \%)$. Treatment of these compounds with Gen $2(10 \mathrm{~mol} \%$ for the sulfoxide and $5 \mathrm{~mol} \%$ for the sulfone) produced the corresponding thiazocinyl acetamide derivatives 12 and 16 in $48 \%$ and $89 \%$, respectively (Scheme 4). Compound 12 was isolated as a 2:3 mixture of diastereomers.

(28) Bates, D. K.; Xia, M. J. Org. Chem. 1998, 63, 9190-9196.

(29) de Oliveira Baptista, M. J. V.; Barrett, A. G. M.; Barton, D. H R.; Girijavallabhan, M.; Jennings, R. C.; Kelly, J.; Papadimitriou, V. J.; Usher, N. A. J. Chem. Soc., Perkin Trans. 1 1977, 1477-1500.

(30) (a) Nicolaou, K. C.; Baran, P. S.; Zhong, Y.-L.; Fong, K. C.; He, Y.; Yoon, W. H.; Choi, H.-S. Angew. Chem., Int. Ed. 1999, 38, 16761678. (b) Bergman, J.; Carlsson, R.; Misztal, S. Acta Chem. Scand. B 1976, $30,853-862$.

(31) (a) Portoghese, P. S.; Telang, V. G. Tetrahedron 1971, 27, 18231829. (b) Johnson, C. R.; Diefenbach, H.; Keiser, J. E.; Sharp, J. C. Tetrahedron 1969, 25, 5649-5653.

2752 J. Org. Chem., Vol. 69, No. 8, 2004 
SCHEME 3. Acylindole 9 via Oxidation of Indoline Amide $8^{a}$

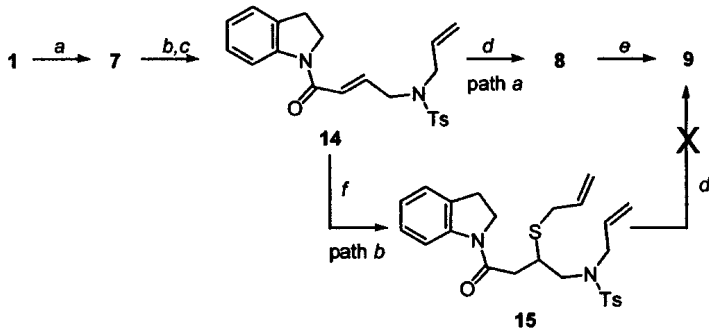

a Reagents: (a) $\mathrm{LiOH}, \mathrm{THF} / \mathrm{H}_{2} \mathrm{O}, 0^{\circ} \mathrm{C}, 95 \%$; (b) oxalyl chloride $\mathrm{THF}, 24 \mathrm{~h}, 0{ }^{\circ} \mathrm{C}$; (c) indoline, pyridine, DMAP, 64\%; (d) DDO dioxane, $70^{\circ} \mathrm{C}, 3 \mathrm{~d}, 89 \%$; (e) allyl mercaptan, $\mathrm{Et}_{3} \mathrm{~N}, \mathrm{CH}_{2} \mathrm{Cl}_{2}, 80 \%$; (f) allyl mercaptan, NaOMe, MeOH/THF, $15 \mathrm{~min}, 0{ }^{\circ} \mathrm{C}, 95 \%$.

SCHEME 4. RCM of Acylindoles 10 and 11

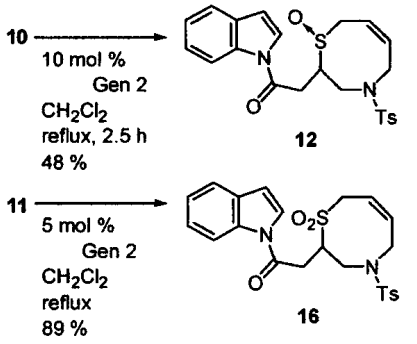

SCHEME 5. RCM of Indoline Amides 17 and 19

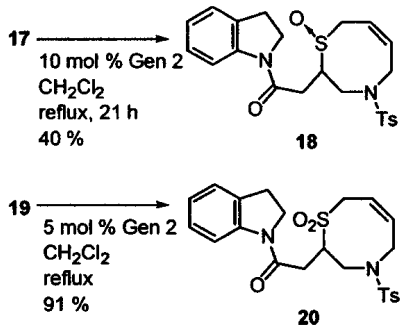

This pattern of reactivity was duplicated in the corresponding indoline analogues. Thus, treatment of 15 with either 1 or 2 equiv of $m$-CPBA gave the corresponding sulfoxide 17 (as an inseparable 2:3 ratio of diastereomers) or sulfone (19). Treatment of these compounds with Gen $2(10 \mathrm{~mol} \%$ for the sulfoxide and $5 \mathrm{~mol} \%$ for the sulfone $)$ produced the corresponding thiazocines 18 and 20 in $40 \%$ and $91 \%$, respectively (Scheme 5 ). Compound 18 was isolated as a 3:4 mixture of diastereomers.

In view of the mixed history of success with S(II) substrates in RCM processes, our current results on successful RCM on sulfoxides where the corresponding sulfides fail, the abundance of simple oxidation procedures for the sulfide to sulfoxide transformation, ${ }^{32}$ and the ease of the reverse deoxygenation process ${ }^{32}$ suggest sulfoxides may be useful protecting groups for thioethers undergoing RCM. ${ }^{33}$ As a demonstration of the concept, the most labile sulfoxide in the series (compound 12) was

(32) For leading references see Jog, P. V.; Brown, R. E.; Bates, D. K. J. Org. Chem. 2003, 68, 8240-8243. treated with Lawesson's reagent ${ }^{34}$ to produce sulfide 21 in $67 \%$ yield.

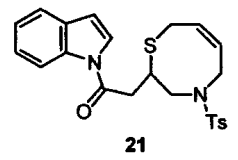

In conclusion, sulfone and sulfoxide derivatives of compound $\mathbf{E}(\mathrm{Y}=N$-Ts, eq 2) undergo $\mathrm{RCM}$ in good to excellent yield with Grubbs second generation catalyst, providing a new route to (1,4-thiazocinyl)-2-acetic acid derivatives. Sulfide derivatives in the series do not undergo RCM with Grubbs' second generation catalyst, but such compounds are available through a protection/ deprotection sequence involving the corresponding sulfoxide. Application of the methodology to other 2-heterocyclyl acetic acid derivatives is underway.

\section{Experimental Section}

Methyl (2E)-4-[Allyl[(4-methylphenyl)sulfonyl]amino]but-2-enoate (1). To a well-stirred mixture of $N$-allyl-4methylbenzensulfonamide $(0.42 \mathrm{~g}, 2 \mathrm{mmol})$, powdered $\mathrm{NaOH}$ (2.0 equiv, $0.16 \mathrm{~g}, 4 \mathrm{mmol}$ ), and tetrabutylammonium hydrogen sulfate ( 0.06 equiv, $0.04 \mathrm{~g}$ ) in $\mathrm{CH}_{2} \mathrm{Cl}_{2}(15 \mathrm{~mL})$ was added dropwise a solution of methyl $\gamma$-bromocrotonate (1.5 eq, 0.537 $\mathrm{g}, 3 \mathrm{mmol})$ in $\mathrm{CH}_{2} \mathrm{Cl}_{2}(5 \mathrm{~mL})$ in an ice-water bath. The resulting mixture was stirred at $0{ }^{\circ} \mathrm{C}$ for $5 \mathrm{~min}$ and at room temperature for $2 \mathrm{~h}$. After filtering, the filtrate was washed with distilled water, dried over anhydrous $\mathrm{Na}_{2} \mathrm{SO}_{4}$, and concentrated in vacuo. Column chromatography on silica ge (EtOAc/hexane, 1:4) gave $0.62 \mathrm{~g}(100 \%)$ of 1 as a white solid: $\mathrm{mp} 58-60^{\circ} \mathrm{C}$; IR $1724 \mathrm{~cm}^{-1}$; ' ${ }^{\mathrm{H}} \mathrm{NMR} \delta 7.65$ (d, $2 \mathrm{H}, J=8.0$ $\mathrm{Hz}), 7.27(\mathrm{~d}, 2 \mathrm{H}, J=8.0 \mathrm{~Hz}), 6.68(\mathrm{dt}, 1 \mathrm{H}, J=15.6,6.0 \mathrm{~Hz})$, $5.87(\mathrm{~d}, 1 \mathrm{H}, J=15.6 \mathrm{~Hz}), 5.55(\mathrm{~m}, 1 \mathrm{H}), 5.13-5.07(\mathrm{~m}, 2 \mathrm{H})$ $3.87(\mathrm{~d}, 2 \mathrm{H}, J=6.0 \mathrm{~Hz}$ ), $3.75(\mathrm{~d}, 2 \mathrm{H}, J=6.4 \mathrm{~Hz}), 3.68(\mathrm{~s}, 3 \mathrm{H})$, $2.38(\mathrm{~s}, 3 \mathrm{H}) ;{ }^{13} \mathrm{C}$ NMR $\delta 166.0,143.6,142.6,136.7,132.1,129.8$, $127.1,123.4,119.7,51.6,50.4,47.2,21.4 ; \mathrm{MS}[\mathrm{m} / z] 278\left(\mathrm{M}^{+}\right)$. Anal. Calcd for $\mathrm{C}_{15} \mathrm{H}_{19} \mathrm{O}_{4} \mathrm{NS}$ : C, $58.23 ; \mathrm{H}, 6.19 ; \mathrm{N}, 4.53$. Found: C, 58.36; H, 6.32; N, 4.53 .

Methyl 4-[Allyl[(4-methylphenyl)sulfonyl]amino]-3(allylthio)butanoate (2). To a well-stirred solution of allyl mercaptan (5.0 equiv, $0.20 \mathrm{~mL}, 2.5 \mathrm{mmol}$ ) and $\mathrm{MeONa}(1.1$ equiv, $0.03 \mathrm{~g}, 0.6 \mathrm{mmol})$ in methanol $(7 \mathrm{~mL})$ was added a solution of $1(0.16 \mathrm{~g}, 0.5 \mathrm{mmol})$ in $\mathrm{MeOH}(4 \mathrm{~mL})$ at room temperature. The resulting mixture was refluxed for $3 \mathrm{~h}$, cooled, poured into ice water, and extracted with EtOAc. The combined organic layers were washed with distilled water, dried over anhydrous $\mathrm{Na}_{2} \mathrm{SO}_{4}$, and concentrated in vacuo. Column chromatography on silica gel (EtOAc/hexane, 1:4) gave $0.13 \mathrm{~g}(68 \%)$ of 2 as an oily liquid: IR $1739 \mathrm{~cm}^{-1} ;{ }^{1} \mathrm{H}$ NMR $\delta$ 7.65 (d, $2 \mathrm{H}, J=8.0 \mathrm{~Hz}), 7.27(\mathrm{~d}, 2 \mathrm{H}, J=8.0 \mathrm{~Hz}), 5.74(\mathrm{~m}$, $1 \mathrm{H}), 5.48(\mathrm{~m}, 1 \mathrm{H}), 5.16-5.03(\mathrm{~m}, 4 \mathrm{H}), 3.80-3.76(\mathrm{~m}, 2 \mathrm{H}), 3.67$ $(\mathrm{s}, 3 \mathrm{H}), 3.37$ (dd, $1 \mathrm{H}, J=14.0,10.0 \mathrm{~Hz}), 3.22(\mathrm{~m}, 1 \mathrm{H}), 3.14(\mathrm{~d}$, $2 \mathrm{H}, J=6.8 \mathrm{~Hz}$ ), 3.09 (dd, $1 \mathrm{H}, J=14.0,4.8 \mathrm{~Hz}), 2.87$ (dd, $1 \mathrm{H}$, $J=16.4,4.8 \mathrm{~Hz}), 2.49$ (dd, $1 \mathrm{H}, J=16.4,8.4 \mathrm{~Hz}), 2.39(\mathrm{~s}, 3 \mathrm{H})$; ${ }^{13} \mathrm{C}$ NMR $\delta 171.9,143.5,136.4,134.4,132.4,129.7,127.2$, $119.7,117.4,51.7,51.6,51.5,39.1,37.4,34.8,21.4 ; \mathrm{MS}[\mathrm{m} / \mathrm{z}$ (rel intensity)] 228 (78), 224 (79), $155(100)$

Methyl 4-[Allyl[(4-methylphenyl)sulfonyl]amino]-3(allylsulfinyl)butanoate (3). To an ice-cooled solution of 2

(33) Gladysz and co-workers have reported use of a cationic rhenium compound that serves both as a protecting group for S(II) and RCM catalyst for thioethers: Martin-Alvarez, J. M.; Hampel, F.; Arif, A. M. Gladysz, J. A. Organometallics 1999, 18, 955-957.

(34) (a) Bartsch, H.; Erker, T. Tetrahedron Lett. 1992, 33, 199-200 (b) Tewari, N.; Kumar, Y.; Thaper, R. K.; Khanna, J. M. Synth. Commun. 1996, 26, 1169-1173. 
$(0.81 \mathrm{~g}, 2.12 \mathrm{mmol})$ in acetone $(12 \mathrm{~mL})$ was added a solution of sodium periodate ( 1.05 equiv, $0.48 \mathrm{~g}, 2.23 \mathrm{mmol}$ ) in $\mathrm{H}_{2} \mathrm{O}(6$ $\mathrm{mL}$ ) at $0{ }^{\circ} \mathrm{C}$. The resulting mixture was stirred at room temperature for $22 \mathrm{~h}$ and concentrated in vacuo. The residue was diluted with $\mathrm{H}_{2} \mathrm{O}(40 \mathrm{~mL})$ and extracted with $\mathrm{CHCl}_{3}$. The combined organic layers were washed with distilled water, dried over anhydrous $\mathrm{Na}_{2} \mathrm{SO}_{4}$, and concentrated in vacuo. Column chromatography on silica gel (EtOAc) gave $0.30 \mathrm{~g}$ (35\%) of 3 as a colorless sticky liquid (mixture of two diastereomers in a 1:1 ratio): IR $1725 \mathrm{~cm}^{-1}$; ${ }^{1} \mathrm{H}$ NMR $\delta 7.66$ (d, $2 \mathrm{H}$ $J=7.6 \mathrm{~Hz}), 7.29(\mathrm{~d}, 2 \mathrm{H}, J=7.6 \mathrm{~Hz}), 5.85(\mathrm{~m}, 1 \mathrm{H}), 5.55-5.39$ $(\mathrm{m}, 3 \mathrm{H}), 5.19-5.11(\mathrm{~m}, 2 \mathrm{H}), 3.82-3.75(\mathrm{~m}, 2 \mathrm{H}), 3.70(\mathrm{~s}, 3 \mathrm{H})$ 3.69 (s, 3H), 3.60 (dd, $1 \mathrm{H}, J=12.0,6.8 \mathrm{~Hz}$ ), $3.51(\mathrm{~m}, 1 \mathrm{H}), 3.41$ (dd, $2 \mathrm{H}, J=13.6,7.6 \mathrm{~Hz}$ ), 3.27 (dd, $1 \mathrm{H}, J=14.8,7.6 \mathrm{~Hz}$ ), 2.96 (dd, $1 \mathrm{H}, J=18.0,5.2 \mathrm{~Hz}$ ), 2.89 (dd, $1 \mathrm{H}, J=18.0,5.6$ $\mathrm{Hz}$ ), 2.79 (dd, $1 \mathrm{H}, J=18.0,8.0 \mathrm{~Hz}$ ), 2.52 (dd, $1 \mathrm{H}, J=18.0$ $6.4 \mathrm{~Hz}), 2.40(\mathrm{~s}, 3 \mathrm{H}) ;{ }^{13} \mathrm{C}$ NMR $\delta 171.9,171.4,143.9,135.6$, $135.5,132.1,132.0,129.9,129.8,127.4,127.3,125.9,125.8$, $123.9,123.8,120.5,120.4,54.6,53.9,53.4,52.4,52.3,52.2,52.1$, $52.0,47.4,44.6,32.0,28.3,21.5 ; \mathrm{MS}[\mathrm{m} / \mathrm{z}$ (rel intensity)] 224 (47), $155(66)$

Methyl 4-[Allyl[(4-methylphenyl)sulfonyl]amino]-3(allylsulfonyl)butanoate (4). To an ice-cooled solution of 2 $(0.73 \mathrm{~g}, 1.91 \mathrm{mmol})$ in $\mathrm{MeOH}(15 \mathrm{~mL})$ was added a solution of Oxone ( 3.0 equiv, $3.52 \mathrm{~g}, 5.73 \mathrm{mmol})$ in $\mathrm{H}_{2} \mathrm{O}(15 \mathrm{~mL})$ at $0{ }^{\circ} \mathrm{C}$ The resulting cloudy slurry was stirred at room temperature for $3 \mathrm{~d}$. After filtering, the filtrate was extracted with EtOAc. The combined organic layers were dried over anhydrous $\mathrm{Na}_{2}$ $\mathrm{SO}_{4}$ and concentrated in vacuo. Column chromatography on silica gel (EtOAc/hexane, 2:3) gave $0.46 \mathrm{~g}(58 \%)$ of 4 as a colorless sticky liquid: IR $1739 \mathrm{~cm}^{-1}$; ${ }^{1} \mathrm{H}$ NMR $\delta 7.67(\mathrm{~d}, 2 \mathrm{H}$ $J=8.0 \mathrm{~Hz}), 7.31(\mathrm{~d}, 2 \mathrm{H}, J=8.0 \mathrm{~Hz}), 5.89(\mathrm{~m}, 1 \mathrm{H}), 5.54-5.40$ $(\mathrm{m}, 3 \mathrm{H}), 5.19-5.12(\mathrm{~m}, 2 \mathrm{H}), 4.07(\mathrm{~m}, 1 \mathrm{H}), 3.85-3.76(\mathrm{~m}, 4 \mathrm{H})$ $3.73(\mathrm{~s}, 3 \mathrm{H}), 3.45-3.42(\mathrm{~m}, 2 \mathrm{H}), 2.99(\mathrm{dd}, 1 \mathrm{H}, J=18.0,6.4$ $\mathrm{Hz}$ ), 2.84 (dd, $1 \mathrm{H}, J=18.0,5.6 \mathrm{~Hz}), 2.42(\mathrm{~s}, 3 \mathrm{H}) ;{ }^{13} \mathrm{C}$ NMR $\delta$ $171.0,144.2,135.3,131.7,131.0,127.5,125.5,123.9,120.8$ $58.3,56.1,52.5,52.4,46.1,30.9,21.5 ; \mathrm{MS}[\mathrm{m} / \mathrm{z}$ (rel intensity)] 260 (5), 224 (1), 155 (8).

Methyl [4-(4-Methylphenylsulfonyl]-1-oxido-3,4,5,8-tetrahydro-2H-1,4-thiazocin-2-yl]acetate (5). To a solution of $3(0.26 \mathrm{~g}, 0.65 \mathrm{mmol})$ in $\mathrm{CH}_{2} \mathrm{Cl}_{2}(10 \mathrm{~mL})$ was added a solution of commercial Gen 2 catalyst $\left(5 \% \mathrm{~mol}, 0.028 \mathrm{~g}\right.$ ) in $\mathrm{CH}_{2} \mathrm{Cl}_{2}(4$ $\mathrm{mL}$ ) under a nitrogen atmosphere. The resulting mixture was refluxed for $6 \mathrm{~h}$. The mixture was cooled to room temperature and concentrated in vacuo. Column chromatography of the residue on silica gel (EtOAc) gave $0.23 \mathrm{~g}(95 \%)$ of 5 separable mixture of two diastereomers in a 2:3 ratio both of which were viscous, tacky semiliquids. Minor diastereomer (higher $R$ ): IR $1733 \mathrm{~cm}^{-1} ;{ }_{1} \mathrm{H}$ NMR $\delta 7.65(\mathrm{~d}, 2 \mathrm{H}, J=8.0 \mathrm{~Hz}), 7.31(\mathrm{~d}, 2 \mathrm{H}$, $=8.0 \mathrm{~Hz}$ ), $5.84(\mathrm{dd}, 1 \mathrm{H}, J=11.2,4.0 \mathrm{~Hz}), 5.57(\mathrm{~m}, 1 \mathrm{H}), 4.14$ $(\mathrm{d}, 2 \mathrm{H}, J=8.8 \mathrm{~Hz}), 4.00-3.76(\mathrm{~m}, 3 \mathrm{H}), 3.70(\mathrm{~s}, 3 \mathrm{H}), 3.48(\mathrm{~m}$ $1 \mathrm{H}), 3.04$ (d, $1 \mathrm{H}, J=14.8 \mathrm{~Hz}$ ), 2.83 (dd, $1 \mathrm{H}, J=16.4,6.4 \mathrm{~Hz}$ ), 2.44 (dd, $1 \mathrm{H}, J=16.4,8.0 \mathrm{~Hz}), 2.40$ (s, 3H); ${ }^{13} \mathrm{C}$ NMR $\delta 170.6$ $144.2,133.5,133.1,130.0,127.4,119.0,53.1,52.2,49.2,45.5$ $44.2,33.3,21.5 ; \mathrm{MS}\left[\mathrm{m} / z\right.$ (rel intensity)] $371\left(\mathrm{M}^{+}, 9\right), 216(8)$ 155 (68). Major diastereomer (lower $R_{f}$ ): IR $1736 \mathrm{~cm}^{-1}$; ${ }^{1} \mathrm{H}$ NMR $\delta 7.59(\mathrm{~d}, 2 \mathrm{H}, J=8.4 \mathrm{~Hz}), 7.30(\mathrm{~d}, 2 \mathrm{H}, J=7.6 \mathrm{~Hz}), 5.87-5.74$ $(\mathrm{m}, 2 \mathrm{H}), 4.30$ (dd, $1 \mathrm{H}, J=14.0,8.0 \mathrm{~Hz}), 4.21$ (d, $1 \mathrm{H}, J=18.0$ $\mathrm{Hz}$ ), 3.92 (dd, $1 \mathrm{H}, J=14.0,8.0 \mathrm{~Hz}$ ), 3.65 (s, $3 \mathrm{H}), 3.60-3.50$ $(\mathrm{m}, 3 \mathrm{H}), 3.14-3.07(\mathrm{~m}, 2 \mathrm{H}), 2.60(\mathrm{dd}, 1 \mathrm{H}, J=17.2,9.2 \mathrm{~Hz})$ $2.40(\mathrm{~s}, 3 \mathrm{H}) ;{ }^{13} \mathrm{C}$ NMR $\delta 171.0,144.2,134.1,132.2,130.0,127.1$, $118.8,55.6,52.2,49.748 .6,47.9,31.8,21.5 ; \mathrm{MS}[\mathrm{m} / z(\mathrm{re}$ intensity)] $371\left(\mathrm{M}^{+}, 15\right), 216(11), 155(56)$

Methyl [4-(4-Methylphenylsulfonyl]-1,1-dioxido-3,4,5,8tetrahydro-2H-1,4-thiazocin-2-yl]acetate (6). To a solution of $4(0.40 \mathrm{~g}, 0.96 \mathrm{mmol})$ in $\mathrm{CH}_{2} \mathrm{Cl}_{2}(10 \mathrm{~mL})$ was added a solution of commercial Gen 2 catalyst $(5 \% \mathrm{~mol}, 0.041 \mathrm{~g})$ in $\mathrm{CH}_{2} \mathrm{Cl}_{2}(5$ $\mathrm{mL}$ ) under a nitrogen atmosphere. The resulting mixture was refluxed for $8 \mathrm{~h}$. The mixture was cooled to room temperature and concentrated in vacuo. Column chromatography of the residue on silica gel (EtOAc/hexane, 2:3) gave $0.38 \mathrm{~g}(100 \%)$ of 6 as a white solid: $\mathrm{mp} 155-7{ }^{\circ} \mathrm{C}$; IR $1740 \mathrm{~cm}^{-1}$; ${ }^{1} \mathrm{H}$ NMR $\delta$ $7.63(\mathrm{~d}, 2 \mathrm{H}, J=8.0 \mathrm{~Hz}), 7.29(\mathrm{~d}, 2 \mathrm{H}, J=8.0 \mathrm{~Hz}), 5.77-5.66$ $(\mathrm{m}, 2 \mathrm{H}), 4.39(\mathrm{~m}, 1 \mathrm{H}), 4.12-3.94(\mathrm{~m}, 3 \mathrm{H}), 3.77-3.69(\mathrm{~m}, 2 \mathrm{H})$ $3.66(\mathrm{~s}, 3 \mathrm{H}), 3.22$ (dd, $1 \mathrm{H}, J=14.4,2.8 \mathrm{~Hz}$ ), 3.03 (dd, $1 \mathrm{H}, J=$ $16.8,3.6 \mathrm{~Hz}), 2.41-2.35(\mathrm{~m}, 4 \mathrm{H}) ;{ }^{13} \mathrm{C}$ NMR $\delta 170.2,144.3$ $134.4,132.7,130.0,127.3,118.7,55.6,52.8,52.1,49.4,49.0$ 29.0, 21.3; MS $[\mathrm{m} / \mathrm{z}$ (rel intensity)] 232 (14), 155 (32). Anal Calcd for $\mathrm{C}_{16} \mathrm{H}_{21} \mathrm{O}_{6} \mathrm{NS}_{2}: \mathrm{C}, 49.60 ; \mathrm{H}, 5.46 ; \mathrm{N}, 3.62$. Found: $\mathrm{C}$, $49.54 ; \mathrm{H}, 5.56 ; \mathrm{N}, 3.64$.

1-[I4-[4-(Methylphenyl)sulfonyl]-3,4,5,8-tetrahydro-2 $H$ 1,4-thiazocin-2-yl]acetyl]-1 $\mathrm{H}$-indole (21). To a solution of 12 (see Supporting Information for preparation; $30.8 \mathrm{mg}$, $0.0675 \mathrm{mmol})$ in $\mathrm{CH}_{2} \mathrm{Cl}_{2}(5 \mathrm{~mL})$ was added portionwise a freshly solution of Lawesson's reagent [2,4-bis (4-methoxyphenyl)-1,3,2,4-dithiadiphosphetane-2,4-disulfide, $27.3 \mathrm{mg}, 0.0654$ mmol] in $\mathrm{CH}_{2} \mathrm{Cl}_{2}(3 \mathrm{~mL})$. The mixture was stirred at room temperature until TLC indicated the reaction was complete (15 min), at which time solvent was evaporated in vacuo. One milliliter of methanol was added, and the mixture was stirred $(1 \mathrm{~min})$ during which time the product was obtained as a white solid $(20.0 \mathrm{mg}, 67 \%)$ : $\mathrm{mp} 177-178.5^{\circ} \mathrm{C}$; ${ }^{1} \mathrm{H}$ NMR $\delta 8.39$ (d, $1 \mathrm{H}, J=7.9 \mathrm{~Hz}), 7.61(\mathrm{~d}, 2 \mathrm{H}, J=8.2 \mathrm{~Hz}), 7.55(\mathrm{~d}, 1 \mathrm{H}, J=7.3$ $\mathrm{Hz}), 7.40(\mathrm{~d}, 1 \mathrm{H}, J=3.6 \mathrm{~Hz}), 7.34-7.26(\mathrm{~m}, 4 \mathrm{H}), 6.65(\mathrm{~d}, 1 \mathrm{H}$, $J=3.6 \mathrm{~Hz}$ ), 5.79 (ddd, $1 \mathrm{H}, J=11.3,7.6,2.7 \mathrm{~Hz}$ ), 5.66 (ddd, $11.3,4.0,3.7 \mathrm{~Hz}), 4.37$ (dd, $1 \mathrm{H}, J=13.1,8.6 \mathrm{~Hz}), 4.25$ (dd, $1 \mathrm{H}, J=17.7,3.7 \mathrm{~Hz}), 3.99-3.94(\mathrm{~m}, 1 \mathrm{H}), 3.77(\mathrm{dd}, 1 \mathrm{H}, J=$ $13.1,9.2 \mathrm{~Hz}$ ), 3.55 (dd, $1 \mathrm{H}, J=17.7,4.0 \mathrm{~Hz}$ ), 3.36 (dd, $1 \mathrm{H}, J$ $=13.1,2.7 \mathrm{~Hz}), 3.23-3.17(\mathrm{~m}, 2 \mathrm{H}), 3.04(\mathrm{dd}, 1 \mathrm{H}, J=16.4,8.2$ $\mathrm{Hz}), 2.38(\mathrm{~s}, 3 \mathrm{H}) ;{ }^{13} \mathrm{C}$ NMR $\delta 168.3,143.9,135.8,135.0,130.6$, $130.1,128.1,127.3,125.6,125.5,124.5,124.2,121.2,116.7$, $110.1,58.1,49.4,38.3,37.0,27.2,21.7 ;$ MS $[\mathrm{m} / z$, rel int) $]: 440$ (6), $117(100)$.

Acknowledgment. The authors thank the NSF for an equipment grant (CHE-9512445). X.L. thanks the Chemistry Department of Michigan Technological University for financial support and $\mathrm{K}$. Li for helpful discussions. The assistance of Mr. Jerry L. Lutz (NMR instrumental assistance) and Mr. Shane Crist (computer system administration) are gratefully acknowledged.

Supporting Information Available: Full experimental details for preparation of compounds 7-12 and 14-20 and selective spectroscopic and analytical data for these compounds and copies of ${ }^{1} \mathrm{H}$ and ${ }^{13} \mathrm{C}$ NMR spectra for compounds $1-12$ and 14-21. This material is available free of charge via the Internet at http://pubs.acs.org.

JO035692Z 


\section{Chapter 4}

Sulfoxide as a "molecular brake”

\section{A Redox-Mediated Molecular Brake: Dynamic NMR Study of 2-[2-(Methylthio)phenyl]isoindolin-1-one and S-Oxidized Counterparts}

Jog, P. V.; Brown, R. E.; Bates, D. K. J. Org. Chem. 2003, 68, 8240. 


\section{$\mathrm{JOC}_{\text {Note }}$}

\section{A Redox-Mediated Molecular Brake: Dynamic NMR Study of 2-[2-(Methylthio)phenyl]isoindolin-1-one and $S$-Oxidized Counterparts}

Parag V. Jog, Richard E. Brown, and Dallas K. Bates* Department of Chemistry, Michigan Technological University, 1400 Townsend Drive, Houghton, Michigan 49931

dbates@mtu.edu

Recelved May 9, 2003

Abstract: A redox-mediated molecular brake based on the sulfide-sulfoxide redox cycle is illustrated by modulation of the rotation rate of an N-Ar "shaft" by varying the oxidation state of sulfur in 2-[2-(sulfur-substituted)phenyl]isoindolin-1-ones. $\mathbf{N}$-Ar rotational barriers in methylsulfinyl (2) and methylsulfonyl (3) derivatives (13.6 kcal mol ${ }^{-1}$ ) are $\sim 5 \mathrm{kcal} \mathrm{mol}^{-1}$ higher than sulfide 1 . Rate reduction for $\mathrm{N}-\mathrm{Ar}$ rotation is $\sim 10^{4} \mathrm{~s}^{-1}(280 \mathrm{~K})$ upon oxidation. Correlated $N$-pyramidalization $/ \mathrm{N}$-Ar rotation reduces the effectiveness of the brake by decreasing the energy barrier to $\mathrm{N}-\mathrm{Ar}$ bond rotation.

Rotational motion is an integral behavior of molecules. Controlling these motions in a reproducible fashion is a key design element for molecular machines. For a molecular brake, ${ }^{1}$ an integral component of a molecular machine, reversibility and hindrance to motion are the two most important aspects. Recently, organic molecules designed specifically to achieve certain desired motions leading to a molecular motor have been reported. ${ }^{2}$ For example, a ratcheted "drive shaft" containing intramolecular features which allow rotation of the shaft in only one direction has been reported, ${ }^{3}$ and approaches to intramolecular brakes based upon changes of $\mathrm{pH},{ }^{4}$ metal ion concentration, ${ }^{1,5}$ or coordination number (or oxidation state of the metal) ${ }^{6}$ have been disclosed.

We now wish to introduce the concept of an organicbased redox-mediated molecular brake. The system is based on the sulfide-sulfoxide redox cycle and is illustrated in a minimalist system by modulation of the

* To whom correspondence should be addressed. Fax: (906) 487 2061. Phone: (906) 4872059.

(1) Kelly, T. R.; Bowyer, M. C.; Bhaskar, K. V.; Bebbington. D. Garcia, A.; Lang, F.; Kim, M. H.; Jette, M. P. J. Am. Chem. Soc. 1994 116,3657 .

(2) Kelly, T. R.; Silva, R. A.; Silva, H. D.; Jasmin, S.; Zhao, Y. J. Am. Chem. Soc. $2000,122,6935$

(3) Kelly, T. R. Acc. Chem. Res. 2001, 34, 514.

(4) (a) Amendola, V.; Fabbrizzi, L.; Mangano, C.; Pallavicini, P. Struct. Bonding 2001, 99, 79 and references therein. (b) Lee, J. W. Kim, K.; Kim, K. Chem. Commun. 2001, 1042. (c) Bergamini. J.-F Belabbas, M.; Jouini, M.; Aeiyach, S.; Lacroix, J.-C.; Chane-Ching, K. I.; Lacaze, P.-C. J. Electroanal. Chem. 2000, 482, 156.

(5) (a) Stevens, A. M.; Richards, C. J. Tetrahedron Lett. 1997, 38, 7805. (b) Tomohiro, Y.; Stake, A.; Kobuke, Y. J. Org. Chem. 2001, 66 8442 .

(6) (a) Cardenas, D. J.; Livoreil, A.; Sauvage, J.-P. J. Am. Chem. Soc. 1996, 118, 11980 . (b) Collin, J.-P.; Dietrich-Buchecker, C.; Gavina, P.; Jimenez-Molero, M. C.; Sauvage, J.P. Acc. Chem. Res. 2001, 34, 477. (c) Kalny, D.; Elhabiri, M.; Moav, T.; Vaskevich, A.; Rubinstein, I.; Shanzer, A.; Albrecht-Gary, A. M. Chem. Commun. 2002, 1426. (d) Switches, Wiley-VCH: New York, 2001; p 249. rate of rotation of an $\mathrm{N}-\mathrm{Ar}$ "shaft" by varying the oxidation state of a proximate sulfur atom in 2-[2-(sulfursubstituted)phenyllisoindolin-1-ones 1-3. Oxidized sulfur acts as the braking mode (rotation hindered) while the reduced [S(II)] counterpart shows "free" rotation. This approach is attractive because there are numerous highyield chemical and electrochemical processes for both sulfur oxidation to sulfoxide $\mathrm{e}^{7.8}$ and sulfoxide deoxygenation to sulfide. ${ }^{9,10}$ Thus, the processes of applying and removing the brake are readily reversible, easily controllable actions.

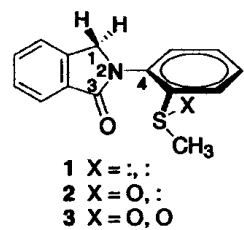

Simple isoindolin-1-ones exist as enantiomeric rotational isomers with slow rotation about the aryl $\mathrm{C}-\mathrm{N}$ bond giving rise to diastereotopic methylene protons. ${ }^{11}$ Ortho-substitution of the aryl group of 2-phenylisoindolin-1-one with sulfur-containing groups (compounds 1-3) had a pronounced line broadening effect on methylene proton signals in the ${ }^{1} \mathrm{H}$ NMR spectra $\left(25^{\circ} \mathrm{C}\right.$ ) (Figure 1) indicating the sulfur oxidation level dramatically affects the rate of rotation about the $\mathrm{N}-\mathrm{Ar}$ bond in these compounds.

On cooling below $-20^{\circ} \mathrm{C}$, the methylene "bump" in sulfone (3) splits into an $A B$ quartet $(\Delta v=191.5 \mathrm{~Hz}$ and $J_{\mathrm{AB}}=16.8 \mathrm{~Hz}$ at $400 \mathrm{MHz}$ in the absence of exchange)

(7) Chemical methods: (a) Madesclaire, M. Tetrahedron 1986, 42 5459. (b) Hajipour, A. R.; Mallakpour, S. E.; Adibi, H. J. Org. Chem 2002, 67, 8666. (c) Bethell, D.; Page, P. C. B.; Vahedi, H. J. Org. Chem. 2000, 65,6756 (d) Tohma, H. Takizawa, S. Watanabe, H. Fukuoka, Y.: Maegawa, T.: Kita, Y. J. Org. Chem. 1999, 64, 3519. (e) Yamanoi, Y.: Imamoto, T. J. Org Chem 1997, 62 8560 (f) Ochiai, M. Nakanishi, Y.: Imamoto, T. J. Org. Chem 1997, 62, 8560. (f) Ochia, M.: Nakanishi, A.; Ito, T. J. Org. Chem. 1997, 62, 4253. (g) Kakarla, R.; Dulina, R. G.; Hatzenbuhler, N. T.; Hui, Y. W.; Sofia, M. J. J. Org. Chem. 1996, 61 8347. (h) Arterburn, J. B.; Nelson, S. L. J. Org. Chem. 1996, 61, 2260. (i) Aldea, R.; Alper, H. J. Org. Chem. 1995, 60, 8365. (j) Brunel, J.-M.; Diter, P.; Duetsch, M; Kagan, H. B. J. Org. Chem. 1995, 60, 8086. (k) DesMarteau, D. D.; Petrov, V. A.; Montanari, V.; Pregnolato, M.; Resnati, G. J. Org. Chem. 1994, 59, 2762. (1) Komatsu, N.; Hashizume, M.; Sugita, T.; Uemura, S. J. Org. Chem. 1993, 58, 4529. (m) Davis F. A.; Reddy, R. T.; Han, W.; Carroll, P. J. J. Am. Chem. Soc. 1992, 114, 1428. (n) Davis, F. A.; Thimma R. R.; Weismiller, M. C. J. Am Chem. Soc. 1989, 111, 5964. (o) Gasparrini, F.; Giovannoli, M.; Misiti. D.; Natile, G.; Palmieri, G. J. Org. Chem. 1990, 55, 1323. (p) Davis, F. A.; Lal, S. G.; Durst, H. D. J. Org. Chem 1988, 53, 5004. (q) Massa, A.; Siniscalchi, F. R.; Bugatti, V.; Lattanzi, A.; Scettri, A. Tetrahe dror. Asymmetry 2002, 13, 1277. (r) Kim, S. S.; Nehru, K.; Kim, S. S.; Kim, D. W.; Jung, H. C. Synthesis 2002, 17, 2484. (s) Batigalhia, F.: Zaldini-Hernandes, M.; Ferreira, A. G.; Malvestiti, I.; Cass, Q. B. Tetrahedron 2001, 57, 9669. (t) Martin, S. E.; Rossi, L. I. Tetrahedron Lett. 2001, 42, 7147. (u) Chen, Y.-J.; Huang, Y.-P. Tetrahedron Lett. 2000 41, 5233 (v) Skarzewski J. Ostrychar . T. Siedleck Let 2000, 41, 5233. (v) Skarzewski, J.; Ostrycharz, E.; Siedlecka, R Tetrahedron. Asymmetry 1999, 10, 3457. (x) Bolm, C.; Dabard, O. A G. Synlett 1999, 360, (y) Iwahama, T.; Sakaguchi, S.; Ishii, Y. Tetrahedron Lett. 1998, 39, 9059. (x) Hashmat A. M.; Bohnert, G. J. Synthesis 1998, 1238. (z) Hirano, M.; Yak

(8) Electrochemical methods: (a) Chiba, K.; Yamaguchi, Y.; Tada M. Tetrahedron Lett. 1998, 39, 9035 . (b) Le Guillanton, G.; Martynov A.; Do, Q. T.; Elothmani, D. Electrochim. Acta 2002, 48, 191. (c) Yamagishi, A.; Aramata, A. J. Electroanal. Chem. 1985, 191, 449. 


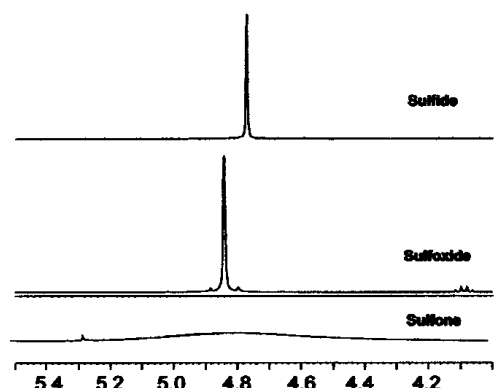

FIGURE 1. ' ${ }^{H} \mathrm{H}$ NMR spectra of isoindolin-1-one sulfide (1), sulfoxide (2), and sulfone (3) $\left(400 \mathrm{MHz}, \mathrm{CDCl}_{3}, 25^{\circ} \mathrm{C}\right)$.

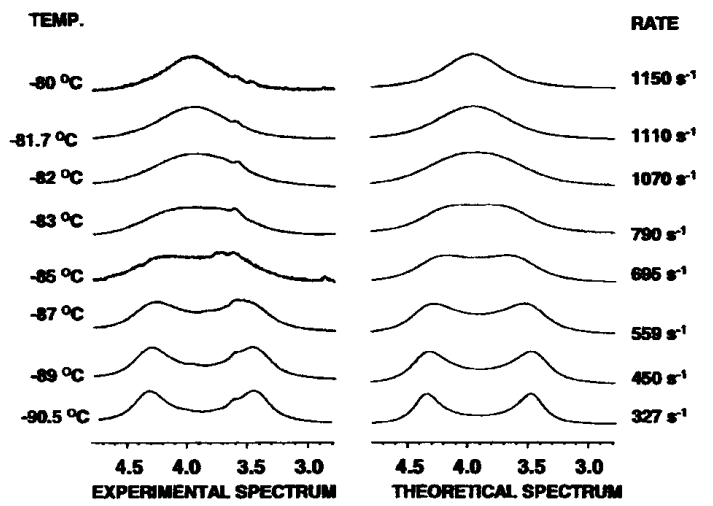

FIGURE 2. Line-shape analysis of 1.

TABLE 1. NMR Parameters and Thermodynamic Data for Isoindolin-1-ones (1-3)

\begin{tabular}{|c|c|c|c|c|c|}
\hline compd & $\begin{array}{l}T_{\mathcal{C}} \\
(\mathrm{K})\end{array}$ & $\begin{array}{c}\Delta H^{\mp} \\
\left(\mathrm{kcal} \mathrm{mol}^{-1}\right)\end{array}$ & $\begin{array}{c}\Delta S^{+} \\
\left(\mathrm{cal} \mathrm{mol}^{-1}\right)\end{array}$ & $\begin{array}{c}\Delta G^{4} c \\
\left(\mathrm{kcal} \mathrm{mol}^{-1}\right)\end{array}$ & $\begin{array}{c}\Delta G^{+} d \\
\left.\text { (kcal mol }{ }^{-1}\right) \\
\text { (computed) }\end{array}$ \\
\hline & $\begin{array}{r}<188 \\
280\end{array}$ & & -1.5 & & \\
\hline & & 12.2 & 4.9 & $13.6(13.7)$ & $17.2,13.5$ \\
\hline
\end{tabular}

a NMR in toluene- $d_{8 .}{ }^{b} \mathrm{NMR}$ in $\mathrm{CDCl}_{3}{ }^{c}$ Experimental value from line-shape analysis or (coalescence measurement). ${ }^{d} \mathrm{Calcu}-$ lated from minima on either side of the transition state.

with a coalescence temperature $\left(T_{\mathrm{c}}\right)$ of $22^{\circ} \mathrm{C}$. These data provide a value of $13.7 \mathrm{kcal} / \mathrm{mol}(57.2 \mathrm{~kJ} / \mathrm{mol})$ for the free energy of activation $\left(\Delta G^{*}\right)$ of 3. Line-shape analysis of spectra obtained at various temperatures confirms this value $\left(\Delta G^{*}=13.6,56.9 \mathrm{~kJ} / \mathrm{mol}\right)$ and allows calculation of $\Delta H^{*}$ and $\Delta S^{*}$ (Table 1). Similarly, sulfide (1) gives $T_{\mathrm{c}}$ $=-85^{\circ} \mathrm{C}(188 \mathrm{~K})$ and $\Delta G^{*}=8.56 \mathrm{kcal} / \mathrm{mol}(35.8 \mathrm{~kJ} / \mathrm{mol})$ $\left[\Delta G^{*}=8.40 \mathrm{kcal} / \mathrm{mol}(35.1 \mathrm{~kJ} / \mathrm{mol})\right.$ by line-shape analysis]. The rate data obtained from line-shape analysis of ${ }^{1} \mathrm{H}$ NMR spectra of 1 are shown in Figure 2.

Analyses of the variable-temperature (VT) ${ }^{1} \mathrm{H}$ NMR spectra of sulfoxide 2 were problematic. The methylene signals appeared as an $\mathrm{AB}$ quartet in the absence of exchange $\left(\mathrm{H}_{\mathrm{a}}=4.95, \mathrm{H}_{\mathrm{b}}=4.85, J=16.78 \mathrm{~Hz}, \mathrm{CDCl}_{3}\right.$, $-60^{\circ} \mathrm{C}$ ). As the sample was warmed these peaks gradually collapsed to a single broad peak and, upon further heating, decoalesced into another $\mathrm{AB}$ quartet $\left(\mathrm{H}_{\mathrm{a}}=4.86\right.$, $\mathrm{H}_{\mathrm{b}}=4.80, J=16.78 \mathrm{~Hz}, \mathrm{CDCl}_{3}, 50^{\circ} \mathrm{C}$ ). Throughout this process, the centerline of the absorptions migrated linearly upfield, becoming constant in appearance and chemical shift at about $120^{\circ} \mathrm{C}\left(\mathrm{CDBr}_{3}\right)$. This behavior is unique to the sulfoxide; both the sulfide and the sulfone showed classical decoalescence upon warming from the absence of exchange regime to give a singlet having a chemical shift equal to the average values for $\mathrm{H}_{\mathrm{a}}$ and $\mathrm{H}_{\mathrm{b}}$ in the low-temperature $A B$ quartet. In analogy with sulfide (1) and sulfone (3), coalescence of the lowtemperature $A B$ quartet in sulfoxide (2) is a physical manifestation of increased $\mathrm{N}-\mathrm{Ar}$ rotation. However, in 2 , even as $H_{a}$ and $H_{b}$ rapidly exchange, they are diastereotopic due to the presence of the chiral sulfoxide moiety, hence the reappearance of separate signals for $\mathrm{H}_{\mathrm{a}}$ and $\mathrm{H}_{b}$ at higher temperatures. In other words, as noted by Lunazzi, ${ }^{12}$ sulfoxides which contain a chiral center and also a stereogenic axis (in our case the $\mathrm{N}-\mathrm{Ar}$ bond) will display a set of conformational enantiomers (or atropisomers) at low temperature (under conditions of slow exchange) and a pair of configurational enantiomers when exchange is rapid.

For sulfoxide $2, \Delta G^{*}$ and $k$ were determined at the coalescence temperature $\left(280 \mathrm{~K}, 13.6 \mathrm{kcal} \mathrm{mol}^{-1}\right.$ and 1.3 $\times 10^{2} \mathrm{~s}^{-1}$, respectively). By comparison, $k_{280}$ for 1 and 3 are $1.6 \times 10^{6}$ and $1.4 \times 10^{2} \mathrm{~s}^{-1}$, respectively. As expected from the scant literature available, ${ }^{13}$ the sulfoxide and sulfone barriers to rotation are very similar. Thus, at 280 $\mathrm{K}$, oxidation of sulfide 1 to either the sulfoxide 2 or sulfone 3 slows this rate of rotation by about $10^{4} \mathrm{~s}^{-1} .^{14}$

Based on a "rigid shaft" model, we had expected an increase in rotational barrier of more than $5 \mathrm{kcal} / \mathrm{mol}$ upon sulfur oxidation. Examination in models of the $\mathrm{N}-$ Ar rotation reveals a severe steric interaction during

(9) Chemical methods: (a) Madesclaire, M. Tetrahedron 1988, 44, 6537. (b) Iranpoor, N.; Firouzabadi, H.; Shaterian, H. R. J. Ong. Chem. 2002, 67, 2826. (c) Yoo, B. W.; Choi, K. H.; Lee, S. J.; Yoon, C. M.; Collier, T. R.; Wu, W. Tetrahedron Lett. 2000, 41, 3781. (e) Kobayashi, K.: Kubota, Y.: Furukawa, N. Chem. Lett. 2000, 400. (f) Wang Y. Koreeda, M. Synlett 1996, 885 (g) Ruano. J. L. G.; Castro, A. M. M.; Ramos, J.H. R Tetrahedron Lett 1996, 374569 (h) Wan. J. M.; Ramos, Y. H. R. Tetrahedron Lett. 1996, 37 45. (h) Wang, J. Q.; Zhang, Y. M. Synth. Commun. 1995, 25, 3545. (1) Zhang. Y.; Yu, Y.; Bao, W. Synth. Com B.; Malajczyk, M. Synlett 1952, 252. (k) Bartsch, H.; Erker, T. Tetrahedron Lett. 1992, 33, 199. (1) Balicki, R. Synthesis 1991, 155. (m) Bernard, A. M.; Caredda, M. G.; Piras, P. P.; Serra, E. Synthesis 1990, 329. (n) Nicolaou, K. C.; Koumbis, A. E.; Snyder, S. A.; Simonsen, K. B. Angew. Chem., Int. Ed. 2000, 39, 2529. (o) Yadav, J. S.; Reddy, B. V. S.; Srinivas, C.; Srihari, P. Synlett 2001, 854. (p) Firouzabadi, H.; Karimi, B. Synthesis 1999, 500. (q) Khurana, J.; Ray, A.; Singh, S. Tetrahedron Lett. 1998, 39, 3829. (r) Wang. X.; Woo, L. K. J. Org. Chem. 1998, 63, 356. (s) Mohanazadeh, F.; Momeni, A. R.; Ranjbar, Y. Tetrahedron Lett. 1994, 35, 6127. (t) Lee, G. H.; Choi, E. B.; Lee, E.; Pak, C. S. Tetrahedron Lett. 1994, 35, 2195. (u) Shiao, M. J.; Lai, L. L.; Ku, W. S.; Lin, P. Y.; Hwu, J. R. J. Ong. Chem. 1993, 58, 4742. (v) Miller, R. D.; Renaldo, A. F.; Ito, H. J. Org. Chem. 1988, 53, 5571. (x) Narayana, C.; Padmanabhan, S.; Kabalka, G. W. Synlett 1991, 125. (10) Electrochemical methods: (a) Abo, M.; Dejima, M.: Asano, F.; Okubo, A.; Yamazaki, S. Tetrahedrort Asymmetry 2000, 11, 823. (b) (11) (a), V. Y. Coord. Chem. Rev. 1995, 139, 375.

(11) (a) Khadim, M. A.; Colebrook, L. D.; Hall, L. D. J. Heterocycl. Chem. 1980, 17,651. (b) Khadim, M. A.; Colebrook, L. D. Magn. Reson. Chem. 1985, 23, 259

(12) Casarini, D.; Foresti, E.; Gasparini, F.: Lunazzi, L.; Macciantelli, D.; Misiti, D.; Villani, C. J. Org. Chem. 1993, 58, 5674.

(13) (a) Braverman, S.; Zafrani, Y.; Gottlieb, H. E. J. Ong. Chem. 2002, 67, 3277. (b) Sakamoto, K.: Okj, M. Chem. Lett. 1974, 1173. Note, however, that the conformational free energy difference in cyclohexanes between the methylsulfonyl group and the methylsulfinyl group is large: Eliel, E. L.: Kandasamy, D. J. Org. Chem. 1976, 41, 3899. Juaristi, E.; Labastida, V.; Antunez, S. J. Org. Chem. 2000, 65, 969. Parts $a$ and $b$ of ref 13 are more representative of the molecular motions in $1-3$.

J. Org. Chem, Vol. 68, No. 21, 20038241 


\section{JOCNote}

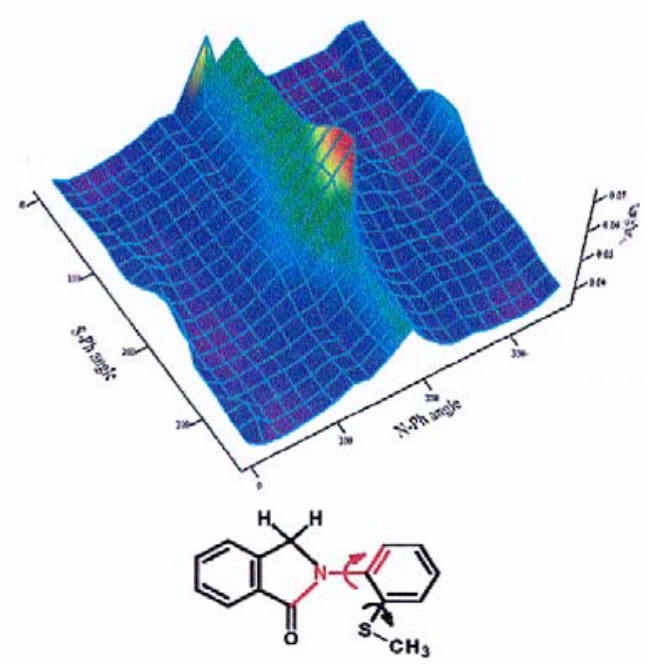

FIGURE 3. Potential energy surface plot for $\mathbf{1}$ (Gaussian 98)

the passage of the ortho substituents over the carbonyl group during rotational interconversion of enantiomers. Transition-state nitrogen pyramidalization to avoid steric interactions during rotational motion has been reported. ${ }^{1 .}$ To get a better picture of rotational processes and to determine theoretical rotational barriers, semiempirical molecular orbital calculations were performed on compounds $1-3 .^{16}$ Figure 3 shows the potential energy surface diagram for sulfide 1 for concurrent $\mathrm{N}-\mathrm{Ar}$ and $\mathrm{S}-\mathrm{Ar}$ rotations, with central maxima and minima on either side indicating noncorrelated $\mathrm{N}-\mathrm{Ar}$ and $\mathrm{S}-\mathrm{Ar}$ rotations. ${ }^{17}$ The calculated barrier is $\sim 9 \mathrm{kcal} / \mathrm{mol}$ for passage of $o$-SMeAr over the carbonyl group, which matches well with the values obtained from experimental

(14) As a practical matter, oxidation to the sulfone state offers little or no increase in the barrier to rotation, while methods for converting sulfones to sulfides are not nearly as diverse nor simply executed as for sulfoxide deoxygenation. This is why our focus is on the $S \leftrightarrow S=0$ redox cycle even though DNMR studies are, in general, easier to (15)

(15) (a) Tsubrik, O.; Burk, P.; Pehk, T.; Maeorg, U. THEOCHEM 2001. 546, 119. (b) Yamamoto, G.; Nakajo, F.; Mazaki, Y. Bull. Chem Soc. Jpn. 2001, 74, 1973. (c) Kief, C. Eur. J. Org. Chem. 2000, 3279 (d) Glover, S. A.: Mo. G.; Tucker, D. J.; Turner, P. J. Chem. Soc, Perkin Trans. 21999,2053 . (e) Ferretti, V.: Bertolasi, V.: Gilli, P.; Gilli, G. J Phys. Chem. 1993. 97, 13568. (f) Bennet, A. J.; Somayaji, V.: Brown R. S.; Santarsiero, B. D. J. Am. Chem. Soc. 1991, 113, 7563. (g) Gilli, G.: Bertolasi, V.: Bellucci, F.; Ferretti, V. J. Am. Chem. Soc. 1986, 108, 2420 .

(16) PM3 method as implemented in Gaussian 98: Frisch, M. J. Trucks, G. W.; Schlegel, H. B.; Scuseria, G. E.; Robb, M. A.; Cheeseman J. R.; Zakrzewski, V. G.; Montgomery, J. A., Jr.; Stratmann. R. E. Burant, J. C.; Dapprich, S.; Millam, J. M.; Daniels, A. D.; Kudin. K. N.; Strain, M. C.; Farkas, O.; Tomasi, J.; Barone, V.: Cossi, M.; Cammi. R.; Mennucci, B.; Pomelli, C.; Adamo, C.; Clifford, S.; Ochterski, J.; Petersson, G. A.; Ayala, P. Y.; Cui. Q.: Morokuma. K.: Malick., D. K.; Rabuck, A. D.: Raghavachari, K.. Foresman, J. B., Cioslowski, J.: Ortiz J. ... Stefanov, B. B., Liu, G.. Liashenko, A., Piskorz, P.. Komaromi I.; Gomperts, R.; Martin, R. L.: Fox, D. J.; Keith, T.; Al-laham, M. A. Peng, C. Y.; Nanayakkara, A.; Gonzalez, C.; Challacombe, M.; Gill, P M.: Johnson, B. G.; Chen, W.; Wong, M. W.; Andres, J. L.; HeadGordon, M.; Replogle, E. S.: Pople. J. A. Gaussian 98, revision A.7 Gaussian, Inc.: Pittsburgh, PA, 1998.

(17) (a) Casarini, D.: Grilli. S.: Lunazzi, L.: Mazzanti, A. J. Org. Chem. 2001, 66, 2757. (b) Grilli, S.; Lunazzi, L.; Mazzanti, A. J. Org Chem. 2001, 66. 4444. (c) Grilli. S.; Lunazzi, L.: Mazzanti, A. J. Org. Chem. 2001, 66, 5853.

8242 J. Org. Chem., Vol. 68, No. 21, 2003

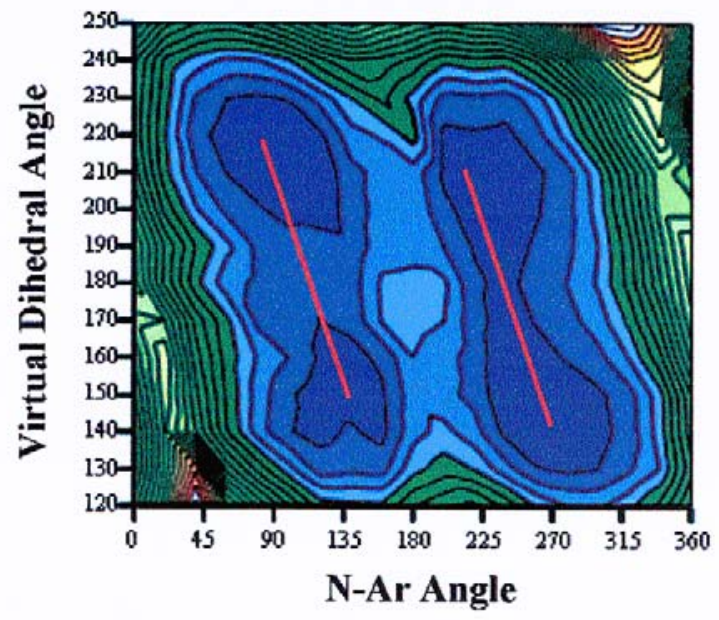

FIGURE 4. Surface plot of $\mathrm{N}-\mathrm{Ar}$ angle and virtual dihedral angle for 1 .

work $\left(\sim 8.5 \mathrm{kcal} \mathrm{mol}^{-1}\right)$. Similar calculations were performed on $\mathbf{2}$ and $\mathbf{3}$ (Table 1). These values also agree quite well with experimental results but are not as close as for 1. Theoretical values may deviate slightly from the experimental values due to chirality considerations or parametrizations in the PM3 program. The barrier to passage of $a-S M e A r$ over the methylene portion of the isoindolinone is calculated to be much lower than for passage over the carbonyl $\left(3.3 \mathrm{kcal} \mathrm{mol}^{-1}\right)$.

A major finding of the calculations is that pyramidalization of the amidic nitrogen is predicted in all three 2-(o-substituted phenyl)isoindolinone derivatives. This process cannot be observed by NMR in our model systems. Calculations show the degree of nitrogen pyramidalization ${ }^{18}$ in the highest energy conformations greatly increases with the initial oxidation from $-\mathrm{S}-$ to $\mathrm{S}=\mathrm{O}$ $\left(9.6^{\circ}\right.$ to $\left.13.3^{\circ}\right)$, but changes little upon further oxidation $\left(13.3^{\circ}\right.$ to $13.9^{\circ}$ in the sulfone). One can visualize nitrogen pyramidalization during $\mathrm{N}-\mathrm{Ar}$ bond rotation in our model system as a "wobbling" rotating shaft rather than a rigid rotating shaft. At torsion angles where steric repulsion of the indolinone ring and $\mathrm{o}-\mathrm{Ar}$ substituent is minimal, N-pyramidalization is also minimal. However as the steric interaction increases so does $\mathrm{N}$-pyramidalization.

The calculations suggest (and it is reasonable to expect) $\mathrm{N}-\mathrm{Ar}$ rotation and $\mathrm{N}$-pyramidalization to be coupled or correlated motions. The coordinated movement of two proximate groups in order to minimize steric interactions during rotation of these groups is termed correlated or cogwheel rotation and is a well-studied phenomenon. ${ }^{17,19-21}$ Motions in which rotation is coupled to nitrogen inversion

(18) Different measures have been used to describe nitrogen pyramidalization: (a) Degree of pyramidalization equals $360-\Sigma$ bone angles to the nitrogen atom (Ganguly, B.; Freed, D. A.; Kozlowski, M. C. J. Org. Chem. 2001, 66, 1103). (b) Measurement of a virtual dihedral angle (Rankin, K. N.: Boyd, R. J. J. Phys. Chem. A 2002, 106, 11168) and (c) measurement of the distance from the nitrogen atom to the plane containing by the three atoms bonded to nitrogen (Schweizer. W. B.: Procter, G.: Kaftory. M.: Dunitz, J. D. Helv. Chim. Acta 1978. $61,2783)$.

(19) Mislow, K. Acc. Chem. Res. 1976, 9, 26. 
have been studied extensively by both experimental and computational methods ${ }^{22}$ and correlated rotation-nitrogen pyramidalization has been studied computationally. ${ }^{23}$ Lunazzi ${ }^{17}$ has developed a graphical method to demonstrate correlated bond rotations by generating a contour map of the potential energy surface described by incremental rotations of the dihedral angles about the bonds suspected of correlated rotation. Correlated rotation is present when the rotation pathways (indicated by lines joining energy minima) run diagonal to the $x-y$ axes and parallel to each other. To demonstrate computationally the correlated nature of $\mathrm{N}-\mathrm{Ar}$ rotation and $\mathrm{N}$-pyramidalization in 1-3, we have developed a variant of the Lunazzi graphical approach: a "virtual dihedral angle" is plotted against the $\mathrm{N}-\mathrm{Ar}$ torsion angle. Rankin and Boyd ${ }^{18 b}$ introduced the virtual dihedral angle as a means of quantitating nitrogen pyramidalization. The virtual dihedral angle given by $\mathrm{C} 1-\mathrm{N} 2-\mathrm{C} 3-\mathrm{C} 4$ (see structure 1) in compounds $1-3$ having a value of $180^{\circ}$ corresponds to planar nitrogen (flat, $\mathrm{sp}^{2}$, degree of pyramidalization ${ }^{18}$ $=0^{\circ}$ ) and a value of $120^{\circ}$ corresponds to tetrahedral nitrogen $\left(\mathrm{sp}^{3}\right.$, degree of pyramidalization $\left.=31.5^{\circ}\right)$. In the calculations (PM3), the virtual dihedral angle and the $\mathrm{N}-$ Ar torsion angle were locked, and then the other angles and bond distances were allowed to relax to their minima for each of the locked values. The $\mathrm{N}-\mathrm{Ar}$ torsion angle values were fixed in $20^{\circ}$ increments from 0 to $360^{\circ}$ and virtual dihedral angle values were fixed in $10^{\circ}$ increments from $-90^{\circ}$ to $+90^{\circ}$. The resulting plot (Figure 4) has the appearance of a Lunazzi diagram, but relates $\mathrm{N}-\mathrm{Ar}$ rotation to $\mathrm{N}$-pyramidalization. Thus, diagonal lines joining the energy minima in Figure 4 are indicative of correlated motions.

Redox disengagement/engagement of the molecular brake can be observed in situ in an NMR tube. Addition of a solution of Lawesson's reagent ${ }^{\mathrm{9k}}$ (in $\mathrm{CDCl}_{3}$ ) to an NMR sample of compound 2 equilibrated to $-40{ }^{\circ} \mathrm{C}$ in the NMR probe leads to a rapid replacement of the $A B$ quartet $(\delta$ 4.95) observed for the methylene hydrogen atoms in the "stopped" sulfoxide with a broad singlet ( $\delta$ 4.78) corresponding to the methylene hydrogen atoms in sulfide 1 as rotation about the $\mathrm{N}-\mathrm{Ar}$ bond takes place.

(20) Kuttenberger, M.; Frieser, M.; Hofweber, M.; Mannschreck, A Tetrahedron: Asymmetry 1998, 9, 3629.

(21) (a) Clayden, J.; Pink, J. H. Angew. Chem., Int. Ed. 1998, 37 1937. (b) Bragg, R. A.; Clayden, J. Org. Lett. $2000,2331$.

(22) (a) Bushweller, C. H.; Anderson. W. G. Tetrahedron Lett. 1972 129. (b) Bushweller, C. H.; O'Neil, J. W.; Bilofsky, H. S. J. Am. Chem 129. (b) Soc. 1971, 93, 542. (c) Anderson, J. E.; Tocher, D. A.; Casarini, D. Lunazzi, L. J. Org. Chem. 1991, 56, 1731. (d) Casarini, D.; Lunazzi L.; Anderson, J. E. J. Org. Chem. 1993, 58, 714. (e) Garcia, M. B.; Grill S.; Lunazzi, L.; Mazzanti, A.; Orelli, L. R. J. Org. Chem. 2001, 66 (23) (a) Jennings, W. B.; Worley, S. D. Tetrahedron Lett. 1977, 1435. (b) Birkett, H. E.: Cherryman, J. C.; Chippendale, A. M.; Hazendonk P.: Harris, R. K. THEOCHEM 2002, 602/603, 59.
Conversely engagement of the brake may be observed by addition of a solution of $m$-CPBA (in $\mathrm{CDCl}_{3}$ ) to an NMR sample of compound 1 equilibrated to $-40^{\circ} \mathrm{C}$ in the NMR probe. This leads to a rapid replacement of the broad singlet $(\delta 4.78)$ corresponding to the methylene hydrogen atoms in the slowly rotating sulfide with an $A B$ quartet $(\delta 4.95)$ corresponding to the methylene hydrogen atoms in the absence of $\mathrm{N}-\mathrm{Ar}$ rotation in sulfoxide 2 . These reagents are nearly ideal for these experiments because the reactions are rapid (even at $-40^{\circ} \mathrm{C}$ ), quantitative, and exhibit no interfering peaks in the region $\delta 4-5$. A sample spectrum from titration of 1 with $m$-CPBA to approximately $80 \%$ conversion at $-40^{\circ} \mathrm{C}$ in an NMR tube is shown in Figure 5 (Supporting Information). The redox conversions may also be monitored by changes in intensity of the methyl signals in $1(\delta 2.41)$ and $2(\delta 3.05)$.

In conclusion, we have shown that the rate of $\mathrm{N}-\mathrm{Ar}$ rotation in isoindolin-1-ones 1-3 is controlled by the oxidation state of sulfur in a proximate ortho substituent. The rate difference between sulfane and the sulfinyl/ sulfonyl forms is $\sim 10^{4} \mathrm{~s}^{-1}$. The model compounds are shown computationally to undergo correlated N-pyramidalization and $\mathrm{C}-\mathrm{N}$ bond rotation. As the $\sigma-\mathrm{Ar}$ substituent passes the plane of the isoindolinone, $\mathrm{N}$-pyramidalization reaches maximum, but the nitrogen becomes planar when the isoindolinone and Ar groups are nearly orthogonal. Pyramidalization of nitrogen causes the redox brake to be less effective than expected. Design of a system in which shaft element flexing (in the form of atom pyramidalization) is avoided should provide more dramatic rate reductions using this molecular brake concept.

Acknowledgment. We thank the NSF for an equipment grant (CHE-9512445). P.V.J. thanks the Chemistry Department of Michigan Technological University for financial support. The assistance of Mr. Jerry L. Lutz (NMR instrumental assistance) and Mr. Shane Crist (computer system administration) is gratefully acknowledged.

Supporting Information Available: Experimental details for preparation and characterization of compounds $1-3$. ${ }^{1} \mathrm{H}$ and ${ }^{13} \mathrm{C}$ NMR spectra for compounds 1-3, 3D potential energy surface plots for 2 and 3. PM3-computed $Z$-matrices for the lowest energy conformation of compounds 1-3 (when $\mathrm{N}-\mathrm{Ar}$ and $\mathrm{S}-\mathrm{Ar}$ torsion angles are locked and incremented), 2D surface plots of $\mathrm{N}-\mathrm{Ar}$ torsion angle vs virtual dihedral angle for 2 and 3, rate data for 3 from line-shape analysis, and PM3-computed Z-matrices for the lowest energy conformation of compounds 1-3 (when $\mathrm{N}-\mathrm{Ar}$ and the virtual dihedral angle torsion angle are locked and incremented). This material is available free of charge via the Internet at http://pubs.acs.org.

JO034613G 


\section{Future Directions}

The use of sulfoxide as a source of intramolecular sulfenylating agents was well-known. Different modes (electrophilic and thermal) of sulfoxide activation were also well documented. However, the following observation warrants further study in understanding the actual process of sulfoxide activation. Mass spectrometric analysis of $t$-butyl, propane nitrile, and ethyl sulfoxides, which successfully produced a cyclized product in reaction conditions, was eventful. These sulfoxides, which are proven to have several rotomeric isomers in solution, ambiguously yet cleanly produce the intended cyclized product (based on $\mathrm{M}+$ values) directly from solid samples during the mass spectrometric data collection. Further work is necessary to understand this phenomenon, especially because this phenomenon was observed for only those sulfoxides, which successfully produced the cyclized product under both electrophilic and thermal reaction conditions in the laboratory.

In chapter 3, sulfoxide was introduced as a "protecting group" for thioether which was unreactive under RCM reaction conditions. A well-known Ru-S(II) coordination was presumably avoided in the sulfoxide oxidation state. The next step in this research is to define the scope and utility of this approach. It is important to note that in an example described in chapter 3, one of the unsaturated components in the RCM diene precursor was directly attached to a sulfur atom. It would be interesting to apply this methodology of using sulfoxide as a protecting group to a sulfur containing system in which either of the alkene components is not directly attached to the sulfur atom. The results obtained from such an experiment would provide more insight in understanding the exact nature of 
the interaction between the Ru-based catalyst and the sulfur containing substrate. Precisely, the validity of using sulfoxide as a protecting group of thioether needs to be tested with respect to the precise positioning of both the alkenes undergoing RCM reaction and the sulfur atom in substrate.

As discussed in chapter 4, nitrogen pyramidalization lowers the rotational barrier in sulfoxide, which results in a lower than expected increase in the rotational barrier. A system where nitrogen pyramidalization can be avoided should provide a more effective molecular brake based on the sulfide-sulfoxide redox cycle. If nitrogen pyramidalization is unavoidable, then the sulfide [S(II)] oxidation state of such system should have even lower barrier than Isoindoline $\mathbf{1}$ in chapter 4 to be a more efficient molecular brake than the one described in chapter 4 based on this redox cycle. 


\section{Appendix 1}

Intramolecular Sulfoxide Electrophilic Sulfenylation (SES) of 2- and 3- Indole Carbanilides: Formation of Indolo[3,2-b]-1,5-benzothiazepinones

Dallas K. Bates, * Mary E. Eggers and Parag V. Jog

Supplemental Information

(Compound numbers correspond to the numbers in the article:

Bates, D. K.;Eggers, M. E.; Jog, P. V. J. Org. Chem. Submitted for publication)

Table of Contents

Experimental procedures for 2-(ethylthio)- $N$-methylaniline, 2-(tert-butylthio)- $N$-methylaniline, ethyl $N$-methylindole

-2-carboxylate, 1b, 2b, 3ab, 4a, 5ab, 6ab, 7bc, 8abc, and

the thermal and TFAA-promoted cyclization of $8 b$

104-116

${ }^{1} \mathrm{H}$ NMR Spectrum of $N$-[2-(tert-butylthio)phenyl]acetamide

117

${ }^{13} \mathrm{C}$ NMR Spectrum of $N$-[2-(tert-butylthio)phenyl]acetamide

${ }^{1} \mathrm{H}$ NMR Spectrum of $N$-[2-(tert-butylthio)phenyl]- $N$-methylacetamide

${ }^{13}$ C NMR Spectrum of $N$-[2-(tert-butylthio)phenyl]- $N$-methylacetamide

${ }^{1} \mathrm{H}$ NMR Spectrum of 2-(tert-butylthio)- $N$-methylaniline

${ }^{13}$ C NMR Spectrum of 2-(tert-butylthio)- $N$-methylaniline

${ }^{1} \mathrm{H}$ NMR Spectrum of $\mathbf{1 b}$

${ }^{13} \mathrm{C}$ NMR Spectrum of $1 \mathrm{~b}$

${ }^{1} H$ NMR Spectrum of $\mathbf{2 b}$

${ }^{13} \mathrm{C}$ NMR Spectrum of $\mathbf{2 b}$ 
${ }^{1}$ H NMR Spectrum of $3 b$

${ }^{13} \mathrm{C}$ NMR Spectrum of $3 b$

128

${ }^{1} \mathbf{H}$ NMR Spectrum of $\mathbf{4 b}$

129

${ }^{1} \mathbf{H}$ NMR Spectrum of $5 b$

130

${ }^{13} \mathrm{C}$ NMR Spectrum of $5 b$

${ }^{1}$ H NMR Spectrum of $6 b$

${ }^{13} \mathrm{C}$ NMR Spectrum of $6 \mathrm{~b}$

${ }^{1}$ H NMR Spectrum of $7 b$

${ }^{13} \mathrm{C}$ NMR Spectrum of $7 \mathrm{~b}$

135

${ }^{1}$ H NMR Spectrum of 7c

136

${ }^{13}$ C NMR Spectrum of 7c

${ }^{1}$ H NMR Spectrum of $8 b$

${ }^{1}$ H NMR Spectrum of 9

${ }^{1} \mathrm{H}$ NMR Spectrum of 10

${ }^{13}$ C NMR Spectrum of 10

${ }^{1} \mathrm{H}$ NMR Spectrum of $14 \mathrm{~b}$

${ }^{13}$ C NMR Spectrum of 14b 143

${ }^{1} \mathrm{H}$ NMR Spectrum of $\mathbf{1 6 b}$ 144

${ }^{13} \mathrm{C}$ NMR Spectrum of $\mathbf{1 6 b}$

145 
2-(ethylthio)- $N$-methylaniline. Sodium metal (29.9 g, $260 \mathrm{mmol}$ ) was added to $\mathrm{MeOH}$ (390 mL) over 30 min with vigorous stirring. After $\mathrm{H}_{2}$ evolution was complete (10 min), neat 2-(ethylthio)aniline (40.3 g, $260 \mathrm{mmol}$ ) was added dropwise then this solution was added dropwise to a well-stirred suspension of paraformaldehyde $(10.9 \mathrm{~g}, 360 \mathrm{mmol})$ in $\mathrm{MeOH}(260 \mathrm{~mL})$. After stirring $7 \mathrm{~h}$ at room temperature, neat $\mathrm{NaBH}_{4}(9.1 \mathrm{~g}, 0.26 \mathrm{~mol})$ was added to the reddish brown mixture. After refluxing $45 \mathrm{~min}, 1 \mathrm{M} \mathrm{KOH}(400 \mathrm{~mL})$ was added to the cooled orange solution, which was then stirred for $30 \mathrm{~min}$. This mixture was reduced to $1 / 2$ its original volume in vacuo and poured into a saturated $\mathrm{NaCl}$ solution (200 mL). Extraction with $\mathrm{CH}_{2} \mathrm{Cl}_{2}(3 \times 200 \mathrm{~mL})$ followed by washing the combined organic layers with saturated aqueous $\mathrm{NaCl}$, drying $\left(\mathrm{Na}_{2} \mathrm{SO}_{4}\right)$ and solvent evaporation in vacuo produced a brown liquid. Flash chromatography (1:1 $\mathrm{CHCl}_{3}$ :hexanes) gave pure 2(ethylthio)- $N$-methylaniline (18.2 g, 42\%): IR $3390 \mathrm{~cm}^{-1} ;{ }^{1} \mathrm{H}$ NMR (200 MHz) $\delta 7.18$ $7.41(2 \mathrm{H}, \mathrm{m}), 6.57-6.67(2 \mathrm{H}, \mathrm{m}), 5.10(1 \mathrm{H}, \mathrm{s}, \mathrm{br}), 2.86(3 \mathrm{H}, \mathrm{s}), 2.70(2 \mathrm{H}, \mathrm{q}, \mathrm{J}=7.3 \mathrm{~Hz})$, $1.20(3 \mathrm{H}, \mathrm{t}, \mathrm{J}=7.3 \mathrm{~Hz}) ;{ }^{13} \mathrm{C}$ NMR $\delta 150.8,136.6,130.6,117.9,117.1,110.1,31.2,29.5$, 15.5; MS [m/z (relative intensity)] $167(\mathrm{M}+, 100)$.

2-(tert-butylthio)- $\mathrm{N}$-methylaniline. A mixture of 2-(tert-butylthio)aniline (3.5 g, 19 mmol) and $100 \mathrm{~mL}$ of glacial acetic acid was heated under reflux for $14 \mathrm{~h}$. The reaction mixture was cooled to room temperature and poured onto an ice-water mixture. The organic layer was extracted with chloroform $(2 \times 250 \mathrm{~mL})$, washed with water $(2 \times 250$ $\mathrm{mL}$ ) and dried over sodium sulfate. Evaporation of solvent in vacuo gave $\mathrm{N}$-[2-(tertbutylthio)phenyl]acetamide as a brown liquid. (4.2 g, 97\%): IR 3343, $1693 \mathrm{~cm}^{-1} ;{ }^{1} \mathrm{H}$ NMR $\delta 8.79$ (broad, 1H), $8.44(\mathrm{~d}, \mathrm{~J}=8.16,1 \mathrm{H}), 7.46(\mathrm{ddd}, \mathrm{J}=7.72,1.67,0.40 \mathrm{~Hz}, 1 \mathrm{H})$, 
$7.34(\mathrm{dt}, \mathrm{J}=7.52,1.64 \mathrm{~Hz}, 1 \mathrm{H}), 6.99(\mathrm{dt}, \mathrm{J}=7.61,1.33 \mathrm{~Hz}, 1 \mathrm{H}), 2.16(\mathrm{~s}, 3 \mathrm{H}), 1.25(\mathrm{~d}, \mathrm{~J}=$ $0.67 \mathrm{~Hz}, 9 \mathrm{H}) ;{ }^{13} \mathrm{C}$ NMR $\delta 167.9,141.4,138.5,130.7,123.1,119.7,119.5,48.2,30.9$, 30.8, 24.8; MS [m/z (rel. intensity)] $223\left[\mathrm{M}^{+}, 16\right], 125$ (100), 57 (36).

To a well-stirred suspension of $N$-[2-(tert-butylthio)phenyl]acetamide (12.8 g, $57 \mathrm{mmol})$ and tetra- $n$-butylammonium hydrogen sulfate $(0.2 \mathrm{eq}, 11.5 \mathrm{mmol}, 3.89 \mathrm{~g})$ in toluene $(250$ $\mathrm{mL}$ ) was added all at once $50 \% \mathrm{NaOH}$ aqueous solution $(500 \mathrm{~mL})$. The resulting two layer mixture was heated under reflux at which a solution of iodomethane (excess, (2.0 eq), $114 \mathrm{mmol}, 16.3 \mathrm{~g})$ in toluene $(40 \mathrm{~mL})$ was added dropwise over $15 \mathrm{~min}$. The resulting two phase mixture was maintained at reflux for $24 \mathrm{~h}$. It was cooled to room temperature and the layers separated. The organic layer was washed with water several times (until washings were neutral to litmus), dried over sodium sulfate and evaporated in vacuo to yield $N$-[2-(tert-butylthio)phenyl]- $N$-methylacetamide as an off-white solid (8.5 g, 63\%): mp 56-58 ${ }^{\circ} \mathrm{C}$; IR $1651 \mathrm{~cm}^{-1} ;{ }^{1} \mathrm{H}$ NMR $\delta 7.63(\mathrm{dd}, \mathrm{J}=7.23,1.37 \mathrm{~Hz}, 1 \mathrm{H})$, $7.31(\mathrm{ddd}, \mathrm{J}=7.45,1.85 \mathrm{~Hz}, 1.18 \mathrm{~Hz}, 1 \mathrm{H}), 7.27$ (ddd, J = 7.45, 1.89, $1.18 \mathrm{~Hz}, 1 \mathrm{H}), 7.18$ (ddd, J = 6.86, 1.81, $0.94 \mathrm{~Hz}, 1 \mathrm{H}), 3.18(\mathrm{dd}, \mathrm{J}=1.37,0.3 \mathrm{~Hz}, 3 \mathrm{H}), 1.75$ (dd, J = 1.45, $0.35 \mathrm{~Hz}, 3 \mathrm{H}), 1.34(\mathrm{~d}, \mathrm{~J}=1.35 \mathrm{~Hz}, 9 \mathrm{H}) ;{ }^{13} \mathrm{C}$ NMR $\delta$ 170.8, 146.6, 136.6, 133.5, 129.0, 128.5, 127.8, 47.2, 37.1, 31.7, 31.6, 22.7; MS [m/z (rel. intensity)] 181 (34), 57 (17). To a solution of $N$-[2-(tert-butylthio)phenyl]- $N$-methylacetamide $(8.3 \mathrm{~g}, 35 \mathrm{mmol})$ in $95 \%$ ethanol $(400 \mathrm{~mL})$ was added a solution of $30 \%$ aqueous $\mathrm{HCl}(70 \mathrm{~mL})$. The resulting reaction mixture was refluxed for $6 \mathrm{~d}$. The cooled reaction mixture was neutralized with $30 \%$ aqueous $\mathrm{NaOH}$ solution untill $\mathrm{pH}=8$. Organic layer was extracted with chloroform $(2 \times 250 \mathrm{~mL})$, washed with water $(2 \times 400 \mathrm{~mL})$ and dried over sodium sulfate.

Evaporation of solvent in vacuo gave 2-(tert-butylthio)- $N$-methylaniline as brown liquid 
(5.7 g, 83\%): IR $3400 \mathrm{~cm}^{-1} ;{ }^{1} \mathrm{H}$ NMR $\delta 7.41(\mathrm{dd}, \mathrm{J}=7.44,1.63 \mathrm{~Hz}, 1 \mathrm{H}), 7.29(\mathrm{dt}, \mathrm{J}=$ 7.38, $1.67 \mathrm{~Hz}, 1 \mathrm{H}), 6.60-6.67(\mathrm{~m}, 2 \mathrm{H}), 5.37$ (broad, $1 \mathrm{H}), 2.87(\mathrm{~s}, 3 \mathrm{H}), 1.33(\mathrm{~s}, 9 \mathrm{H}) ;{ }^{13} \mathrm{C}$ NMR $\delta 151.9,139.1,130.7,115.7,115.2,109.3,47.3,30.8,30.2 ;$ MS [m/z (rel. intensity)] 195 (16), 139 (100), 57 (13).

Ethyl $N$-methylindole-2-carboxylate. To a well-stirred solution of ethyl indole-2carboxylate (5.0 g, $26 \mathrm{mmol}), \mathrm{CH}_{3} \mathrm{I}(11.2 \mathrm{~g}, 79 \mathrm{mmol}, 3 \mathrm{eq})$ and tetra- $n$-butylammonium sulfate $(9.0 \mathrm{~g}, 26 \mathrm{mmol}, 1 \mathrm{eq})$ in toluene $(50 \mathrm{~mL})$ at $0{ }^{\circ} \mathrm{C}$ was added $50 \%$ aqueous $\mathrm{NaOH}$ $(25 \mathrm{~mL})$ in one portion. The resulting two-phase mixture was heated to reflux for $1 \mathrm{~h}$ and, after cooling, the layers separated. The organic layer was washed with $5 \% \mathrm{HCl}(2 \times 50$ $\mathrm{mL}), \mathrm{H}_{2} \mathrm{O}(1 \times 50 \mathrm{~mL})$, dried $\left(\mathrm{Na}_{2} \mathrm{SO}_{4}\right)$ and the solvent evaporated in vacuo to yield a wet yellow solid. Column chromatography $\left(\mathrm{CHCl}_{3}\right)$ and collection of the higher $\mathrm{R}_{\mathrm{f}}$ component gave ethyl $\mathrm{N}$-methylindole-2-carboxylate $(4.2 \mathrm{~g}, 80 \%)$ as an off-white solid: mp 59-60 ${ }^{\circ} \mathrm{C}$; IR $1704 \mathrm{~cm}^{-1} ;{ }^{1} \mathrm{H}$ NMR $\delta 7.70(1 \mathrm{H}, \mathrm{dd}, \mathrm{J}=0.8,7 \mathrm{~Hz}), 7.17-7.39$ (4H, m), $4.40(2 \mathrm{H}, \mathrm{q}, \mathrm{J}=7 \mathrm{~Hz}), 4.08(3 \mathrm{H}, \mathrm{s}), 1.43(3 \mathrm{H}, \mathrm{t}, \mathrm{J}=7 \mathrm{~Hz}) ; \mathrm{MS}$ [m/z (relative intensity)] $203\left(\mathrm{M}^{+}, 46\right), 89$ (100). Anal. Calcd for $\mathrm{C}_{12} \mathrm{H}_{13} \mathrm{NO}_{2}$ : C, 70.82; H, 6.45; N, 6.89. Found: C, 70.76; H, 6.47; N, 6.98 .

$N$-[2-(tert-butylthio)phenyl]-1H-indole-2-carboxamide (1b). To a well-stirred solution of indole-2-carboxylic acid $(4.25 \mathrm{~g}, 26.4 \mathrm{mmol})$ in ether $(100 \mathrm{~mL})$ at $0{ }^{\circ} \mathrm{C}$ under a drying tube was added dropwise thionyl chloride neat ( $3 \times 3.66$ g portionwise over $12 \mathrm{~h}, 3.5$ eq.). The reaction mixture was allowed to stir at room temperature during $12 \mathrm{~h}$. The resulting clear yellow solution was evaporated in vacuo to produce yellow solid (4.50 g, 
95\%). This solid was redissolved in ether $(100 \mathrm{~mL})$ and added to a well-stirred solution of 2-(tert-butylthio)aniline (1.2 eq, $5.64 \mathrm{~g}, 31 \mathrm{mmol})$ in dioxane-water-ether (40-5-50 $\mathrm{mL}$ ) mixture over $10 \mathrm{~min}$ at room temperature. The reaction mixture was stirred at room temperature for $3 \mathrm{~h}$ and then diluted with ethyl acetate $(150 \mathrm{~mL})$. The combined organics were washed with $5 \% \mathrm{HCl}\left(\mathrm{aq}, 3 \times 150 \mathrm{~mL}\right.$ ), $5 \% \mathrm{NaHCO}_{3}(\mathrm{aq}, 3 \times 150 \mathrm{~mL}$ ), water ( 2 x $150 \mathrm{~mL}$ ) dried over sodium sulfate and solvent evaporated in vacuo to yield a off-white solid. Recrystallization with hexane gave white compound (6.1 g, 71\%): mp 191-193 ${ }^{\circ} \mathrm{C}$; IR 3453, $1660 \mathrm{~cm}^{-1}$; ${ }^{1} \mathrm{H}$ NMR $\delta 10.4$ (broad, 1H), 9.9 (broad, $\left.1 \mathrm{H}\right), 8.80(\mathrm{~d}, \mathrm{~J}=8.16 \mathrm{~Hz}$, 1H), $7.76(\mathrm{~d}, \mathrm{~J}=7.91 \mathrm{~Hz}, 1 \mathrm{H}), 7.61(\mathrm{~d}, \mathrm{~J}=7.91 \mathrm{~Hz}, 1 \mathrm{H}), 7.54(\mathrm{~d}, \mathrm{~J}=7.91 \mathrm{~Hz}, 1 \mathrm{H}), 7.49$ $(\mathrm{d}, \mathrm{J}=7.91 \mathrm{~Hz}, 1 \mathrm{H}), 7.32(\mathrm{t}, \mathrm{J}=7.35 \mathrm{~Hz}, 1 \mathrm{H}), 7.09-7.22(\mathrm{~m}, 3 \mathrm{H}), 1.36(\mathrm{~s}, 9 \mathrm{H}),{ }^{13} \mathrm{C} \mathrm{NMR}$ $\delta 159.5,141.2,138.7,137.0,131.1,130.9,127.7,124.8,123.5,122.1,120.8,120.5$, 119.6, 112.2, 102.9, 48.5, 30.9; MS [m/z (rel. intensity)] $324\left[\mathrm{M}^{+}, 9\right], 144$ (100), 89 (20), 57 (14). Anal. Calc. for $\mathrm{C}_{19} \mathrm{H}_{20} \mathrm{~N}_{2} \mathrm{OS}$ : C, 70.34; H, 6.21; N, 8.63. Found: C, 70.21; H, $6.43 ; \mathrm{N}, 8.56$.

$N$-[2-(tert-butylsulfinyl)phenyl]-1H-indole-2-carboxamide (2b). To an ice-cooled solution of $\mathbf{1 b}(5.10 \mathrm{~g}, 15.7 \mathrm{mmol})$ in $\mathrm{CH}_{2} \mathrm{Cl}_{2}(150 \mathrm{~mL})$ was added slowly a solution of $m$-CPBA ( 77\%, 1.1 eq, $22.4 \mathrm{mmol}, 3.9 \mathrm{~g})$ in $\mathrm{CH}_{2} \mathrm{Cl}_{2}(50 \mathrm{~mL})$. The resulting mixture was stirred at $0{ }^{\circ} \mathrm{C}$ for $15 \mathrm{~min}$ and then put it in a freezer $\left(-8^{\circ} \mathrm{C}\right)$ overnight. The reaction mixture was then poured into $5 \% \mathrm{NaHCO}_{3}$ solution $(150 \mathrm{~mL})$ and extracted with $\mathrm{CH}_{2} \mathrm{Cl}_{2}$ (200 mL). The combined organic layer was washed with distilled water, dried, and concentrated in vacuo. Column chromatography (EtOAc-hexane, 3:7) gave yellowish white solid which was recrystallized (hexane) to give a white solid (3.51 g, 66\%): $\mathrm{mp}$ 
228-230 ${ }^{\circ} \mathrm{C}(\mathrm{dec})$; IR $1664 \mathrm{~cm}^{-1}$; ${ }^{1} \mathrm{H}$ NMR $\delta 12.1$ (broad, 1H), 9.46 (broad, $\left.1 \mathrm{H}\right), 8.74(\mathrm{dd}$, $\mathrm{J}=8.34,0.62 \mathrm{~Hz}, 1 \mathrm{H}), 7.72(\mathrm{~d}, \mathrm{~J}=8.15 \mathrm{~Hz}, 1 \mathrm{H}), 7.53(\mathrm{dt}, \mathrm{J}=8.49,1.70 \mathrm{~Hz}, 1 \mathrm{H}), 7.44$ $(\mathrm{d}, \mathrm{J}=8.25,0.83 \mathrm{~Hz}, 1 \mathrm{H}), 7.26-7.31(\mathrm{~m}, 2 \mathrm{H}), 7.09-7.19(\mathrm{~m}, 3 \mathrm{H}), 1.28(\mathrm{~s}, 9 \mathrm{H}) ;{ }^{13} \mathrm{C}$ NMR $\delta 159.6,142.5,136.7,132.4,131.1,128.8,127.9,124.8,122.6,122.4,122.3,120.8$ 120.7, 111.8, 104.2, 59.1, 23.4; MS [m/z (rel. intensity)] $340\left[\mathrm{M}^{+}, 1\right], 144$ (100), 89 (26), 57 (18). Anal. Calc. for $\mathrm{C}_{19} \mathrm{H}_{20} \mathrm{~N}_{2} \mathrm{O}_{2} \mathrm{~S}: \mathrm{C}, 67.03 ; \mathrm{H}, 5.92 ; \mathrm{N}, 8.23$. Found: C, 67.04; H, $6.07 ; \mathrm{N}, 8.18$.

$N$-[2-(ethylthio)phenyl]- $N$-methyl-1H-indole-2-carboxamide (3a). This compound was prepared analogously to compound 1a. The crude orange solid was chromatographed $\left(\mathrm{CHCl}_{3}\right)$ to give 3a as a yellow solid (38\%): $\mathrm{mp} 188-190{ }^{\circ} \mathrm{C}$ (acetone); IR 3316, $1611 \mathrm{~cm}^{-}$

'; ${ }^{1} \mathrm{H}$ NMR (200 MHz) $\delta 9.73$ (s, 1H, br), 6.95-7.49 (8H, m), $5.25(1 \mathrm{H}, \mathrm{s}), 3.45(3 \mathrm{H}, \mathrm{s})$, $2.88(2 \mathrm{H}$, apparent qd, J=7.3, $0.1 \mathrm{~Hz}), 1.24(3 \mathrm{H}, \mathrm{t}, \mathrm{J}=7 \mathrm{~Hz}) ;{ }^{13} \mathrm{C}$ NMR $\delta 163.3,142.3$, $138.7,136.5,130.5,130.3,130.2,128.8,128.3,127.2,125.2,123.0,120.9,112.6,107.2$ 38.1, 26.7, 14.7; MS [m/z (relative intensity)] $310\left(\mathrm{M}^{+}, 15\right), 249$ (100). Anal. Calcd for $\mathrm{C}_{18} \mathrm{H}_{18} \mathrm{~N}_{2} \mathrm{OS}: \mathrm{C}, 69.65, \mathrm{H}, 5.84, \mathrm{~N}, 9.02$. Found: C, 69.53, H, 5.95, N, 8.86.

$N$-[2-(tert-butylthio)phenyl]- $N$-methyl-1H-indole-2-carboxamide (3b). To a wellstirred solution of indole-2-carboxylic acid $(3.75 \mathrm{~g}, 23 \mathrm{mmol})$ in ether $(100 \mathrm{~mL})$ at $0{ }^{\circ} \mathrm{C}$ under a drying tube was added dropwise thionyl chloride neat $(3 \times 3.23 \mathrm{~g}$ portionwise over $12 \mathrm{~h}, 82 \mathrm{mmol}, 3.5 \mathrm{eq}$.). The reaction mixture was allowed to stir at room temperature during $12 \mathrm{~h}$. The resulting clear yellow solution was evaporated in vacuo to produce yellow solid $(4.0 \mathrm{~g}, 96 \%)$. This solid was redissolved in ether $(100 \mathrm{~mL})$ and 
added to a well-stirred solution of $N$-[2-(tert-butylthio)phenyl]- $N$-methylamine (1.1 eq, $5.0 \mathrm{~g}, 26 \mathrm{mmol})$ in dioxane-water-ether $(40-5-50 \mathrm{~mL})$ mixture over $10 \mathrm{~min}$ at room temperature. The reaction mixture was stirred at room temperature for $3 \mathrm{~h}$ and then diluted with ethyl acetate $(150 \mathrm{~mL})$. The combined organics were washed with $5 \% \mathrm{HCl}$ (aq, $3 \times 150 \mathrm{~mL}$ ), $5 \% \mathrm{NaHCO}_{3}$ (aq, $3 \times 150 \mathrm{~mL}$ ), water $(2 \times 150 \mathrm{~mL}$ ) dried over sodium sulfate and solvent evaporated in vacuo to give yellowish white solid, which was

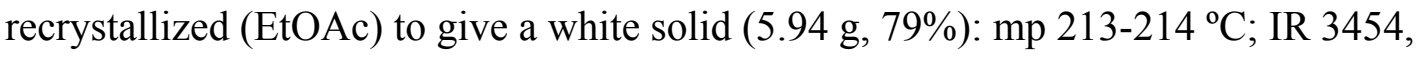
$1622 \mathrm{~cm}^{-1} ;{ }^{1} \mathrm{H}$ NMR $\delta 9.37$ (broad, $\left.1 \mathrm{H}\right), 7.69(\mathrm{dd}, \mathrm{J}=7.24,1.79 \mathrm{~Hz}, 1 \mathrm{H}), 7.37-7.47(\mathrm{~m}$, $3 \mathrm{H}), 7.35(\mathrm{~d}, \mathrm{~J}=8.35 \mathrm{~Hz}, 1 \mathrm{H}), 7.30(\mathrm{~d}, \mathrm{~J}=8.35 \mathrm{~Hz}, 1 \mathrm{H}), 7.17(\mathrm{t}, \mathrm{J}=7.43 \mathrm{~Hz}, 1 \mathrm{H}), 6.96$ $(\mathrm{t}, \mathrm{J}=7.40 \mathrm{~Hz}, 1 \mathrm{H}), 5.11(\mathrm{~s}, 1 \mathrm{H}), 3.45(\mathrm{~s}, 3 \mathrm{H}), 1.29(\mathrm{~s}, 9 \mathrm{H}) ;{ }^{13} \mathrm{C}$ NMR $\delta$ 162.6, 146.3, $137.2,135.2,134.4,129.9,129.4,128.9,128.4,127.7,124.3,122.1,120.0,111.5,106.3$, 47.1, 38.7, 31.8; MS [m/z (rel. intensity)] 338 [M+1, 6], 249 (41), 144 (100), 89 (25). Anal. Calc. for $\mathrm{C}_{20} \mathrm{H}_{22} \mathrm{~N}_{2} \mathrm{OS}: \mathrm{C}, 70.97 ; \mathrm{H}, 6.55 ; \mathrm{N}$, 8.28. Found: C, 70.82; H, 6.54; N, 8.17.

$N$-[2-(ethylsulfinyl)phenyl]- $N$-methyl-1H-indole-2-carboxamide (4a). (59\%): mp 177$179{ }^{\circ} \mathrm{C}$ (acetone); IR 3282, 1628, $1041 \mathrm{~cm}^{-1} ;{ }^{1} \mathrm{H}$ NMR $\left(200 \mathrm{MHz}\right.$ taken at $100{ }^{\circ} \mathrm{C}$ in DMSO-d $\left._{6}\right) \delta 9.53(1 \mathrm{H}, \mathrm{s}, \mathrm{br}), 8.04-8.09(1 \mathrm{H}, \mathrm{m}), 6.95-7.73(7 \mathrm{H}, \mathrm{m}), 5.39(1 \mathrm{H}, \mathrm{s}), 3.48$ $(3 \mathrm{H}, \mathrm{s}), 2.5-2.8(2 \mathrm{H}, \mathrm{m}$, cont. $\mathrm{J}=6.5 \mathrm{~Hz}), 1.13(3 \mathrm{H}, \mathrm{t}, \mathrm{J}=6.4 \mathrm{~Hz}) ;{ }^{13} \mathrm{C}$ NMR $\delta 164.2$, 143.4, 142.3, 137.7, 134.4, 132.0, 131.7, 130.5 129.2, 129.1, 126.9, 124.1, 122.4, 113.8, 108.8, 50.1, 40.6, 7.6; MS [m/z (relative intensity)] $326\left(\mathrm{M}^{+}, 8\right), 249$ (100). Anal. Calcd for $\mathrm{C}_{18} \mathrm{H}_{18} \mathrm{~N}_{2} \mathrm{O}_{2} \mathrm{~S}$ : C, 66.23, H, 5.56, N, 8.58. Found: C, 65.98, H, 5.70, N, 8.26. 
$N$-[2-(ethylthio)phenyl]-1-methyl-1H-indole-2-carboxamide (5a). This compound was prepared analogously to compound 9 from toluene $(15 \mathrm{~mL})$, trimethylaluminum $(2.0 \mathrm{M}$ in hexane, $2.5 \mathrm{~mL}, 4.96 \mathrm{mmol}, 1.14$ eq.), 2-(ethylthio)aniline (0.7 g, $4.4 \mathrm{mmol})$ in toluene (2 $\mathrm{mL})$, ethyl $N$-methylindole-2-carboxylate $(0.9 \mathrm{~g}, 4.4 \mathrm{mmol})$ in toluene $(7 \mathrm{~mL})$ and $\mathrm{CH}_{2} \mathrm{Cl}_{2}$ (5 drops). The orange oil $5 a(1.1 \mathrm{~g}, 84 \%)$ solidified on standing: mp 86-87.5 ${ }^{\circ} \mathrm{C}$ (light yellow solid, acetone/hexane); IR 3321, $1660 \mathrm{~cm}^{-1}$; ${ }^{1} \mathrm{H}$ NMR (200 MHz) $\delta 9.49$ $(1 \mathrm{H}, \mathrm{d}, \mathrm{J}=1 \mathrm{~Hz}), 8.56(1 \mathrm{H}, \mathrm{dd}, \mathrm{J}=1,7 \mathrm{~Hz}), 7.72(1 \mathrm{H}, \mathrm{dd}, \mathrm{J}=1,7 \mathrm{~Hz}), 7.59(1 \mathrm{H}, \mathrm{dd}, \mathrm{J}=$ 2, $6 \mathrm{~Hz}), 7.05-7.42(8 \mathrm{H}, \mathrm{m}$, containing $1 \mathrm{H}, \mathrm{d}, \mathrm{dd}, \mathrm{J}=2,6 \mathrm{~Hz}), 4.14(3 \mathrm{H}, \mathrm{s}), 2.83(2 \mathrm{H}, \mathrm{q}$, $\mathrm{J}=7 \mathrm{~Hz}), 1.25(3 \mathrm{H}, \mathrm{t}, \mathrm{J}=7 \mathrm{~Hz}) ;{ }^{13} \mathrm{C} \mathrm{NMR} \delta 161.5,141.2,140.3,136.8,133.2,131.1$, 127.3, 125.2, 123.3, $123.0120 .9,111.5,105.9,33.0,31.9,16.2 ;$ MS [m/z (relative intensity)] $310\left(\mathrm{M}^{+}, 7\right), 158$ (100). Anal. Calcd for $\mathrm{C}_{18} \mathrm{H}_{18} \mathrm{~N}_{2} \mathrm{OS}$ : C, 69.57; H, 5.84; N, 9.02. Found: C, 69.23; H, 5.95; N, 9.06.

$N$-[2-(tert-butylthio)phenyl]-1-methyl-1H-indole-2-carboxamide (5b). To a wellstirred solution of 1-methyl-1H-indole-2-carboxylic acid (3.25 g, $18.6 \mathrm{mmol})$ in ether $(100 \mathrm{~mL})$ at $0{ }^{\circ} \mathrm{C}$ under a drying tube was added dropwise thionyl chloride neat $(3 \times 2.6$ g portionwise over $12 \mathrm{~h}, 3.5$ eq.). The reaction mixture was allowed to stir at room temperature during $12 \mathrm{~h}$. The resulting clear yellow solution was evaporated in vacuo to produce yellow solid (3.0 g, 84\%). This solid was redissolved in ether (100 mL) and added to a well-stirred solution of 2-(tert-butylthio)aniline (1.1 eq, $3.69 \mathrm{~g}, 20.4 \mathrm{mmol})$ in dioxane-water-ether (40-5-50 $\mathrm{mL})$ mixture over $10 \mathrm{~min}$ at room temperature. The reaction mixture was stirred at room temperature for $3 \mathrm{~h}$ and then diluted with ethyl acetate $(150 \mathrm{~mL})$. The combined organics were washed with $5 \% \mathrm{HCl}(\mathrm{aq}, 3 \times 150 \mathrm{~mL})$, 
$5 \% \mathrm{NaHCO}_{3}$ (aq, $\left.3 \times 150 \mathrm{~mL}\right)$, water $(2 \times 150 \mathrm{~mL})$ dried over sodium sulfate and solvent evaporated in vacuo to yield a greenish solid which was purified by column chromatography (chloroform: hexane, 8:2) to give pale yellow solid (3.71 g, 59\%): mp 86-88 ${ }^{\circ} \mathrm{C}$; IR 3326, $1670 \mathrm{~cm}^{-1}$; ${ }^{1} \mathrm{H}$ NMR $\delta 9.78$ (broad, $\left.1 \mathrm{H}\right), 8.65(\mathrm{dd}, \mathrm{J}=8.34,1.19 \mathrm{~Hz}$, 1H), $7.74(\mathrm{dt}, \mathrm{J}=8.07,0.88 \mathrm{~Hz}, 1 \mathrm{H}), 7.58(\mathrm{dd}, \mathrm{J}=7.74,1.58 \mathrm{~Hz}, 1 \mathrm{H}), 7.42-7.49(\mathrm{~m}, 2 \mathrm{H})$, 7.37 (ddd, $\mathrm{J}=6.79,1.12 \mathrm{~Hz}, 1 \mathrm{H}), 7.20(\mathrm{ddd}, \mathrm{J}=6.88,1.15 \mathrm{~Hz}, 1 \mathrm{H}), 7.13(\mathrm{~s}, 1 \mathrm{H}), 7.10$ (dt, J = 7.53, $1.40 \mathrm{~Hz}, 1 \mathrm{H}), 4.14$ (s, 3H), 1.34 (s, 9H); ${ }^{13} \mathrm{C}$ NMR $\delta$ 160.0, 141.6, 139.4, $138.6,131.9,130.8,126.0,124.5,123.3,122.1,120.7,120.4,119.4,110.2,104.6,48.4$ 31.7, 30.9; MS [m/z (rel. intensity)] 338 [M+, 7], 158 (100), 89 (29), 57 (12). Anal. Calc. for $\mathrm{C}_{20} \mathrm{H}_{22} \mathrm{~N}_{2} \mathrm{OS}$ : C, 70.97; H, 6.55; N, 8.28. Found: C, 70.88; H, 6.79; N, 8.22.

$N$-[2-(ethylsulfinyl)phenyl]-1-methyl-1H-indole-2-carboxamide (6a). (73\%): mp 96$99{ }^{\circ} \mathrm{C}$ (acetone); IR (KBr) 3219, 1667, $1003 \mathrm{~cm}^{-1} ;{ }^{1} \mathrm{H}$ NMR (200 MHz) $\delta 11.60(1 \mathrm{H}, \mathrm{s}$, br), $8.67(1 \mathrm{H}, \mathrm{d}, \mathrm{J}=8 \mathrm{~Hz}), 7.08-7.71(8 \mathrm{H}, \mathrm{m}), 4.15(3 \mathrm{H}, \mathrm{s}), 3.02-3.22(2 \mathrm{H}, \mathrm{dm}$, cont. $\mathrm{J}=$ $7 \mathrm{~Hz}), 1.24(3 \mathrm{H}, \mathrm{t}, \mathrm{J}=7 \mathrm{~Hz}) ;{ }^{13} \mathrm{C} \mathrm{NMR} \delta 162.0,142.4,140.9,133.8,132.3,128.0,127.3$, 126.2, 125.9, 124.2, 123.8, 123.7, 121.7, 111.4, 107.0, 49.6, 33.1, 8.7; MS [m/z/(relative intensity) $326\left(\mathrm{M}^{+}, 10\right), 158(100)$. Anal. Calcd for $\mathrm{C}_{18} \mathrm{H}_{18} \mathrm{~N}_{2} \mathrm{O}_{2} \mathrm{~S}: \mathrm{C}, 66.23 ; \mathrm{H}, 5.56 ; \mathrm{N}$ 8.58. Found: C, $65.88, \mathrm{H}, 5.63, \mathrm{~N}, 8.77$.

$N$-[2-(tert-butylsulfinyl)phenyl]-1-methyl-1H-indole-2-carboxamide (6b). To an icecooled solution of $5 \mathbf{b}(4.0 \mathrm{~g}, 11.8 \mathrm{mmol})$ in $\mathrm{CH}_{2} \mathrm{Cl}_{2}(150 \mathrm{~mL})$ was added slowly a solution of $m$-CPBA (77\%, 1.1 eq, $16.8 \mathrm{mmol}, 2.90 \mathrm{~g})$ in $\mathrm{CH}_{2} \mathrm{Cl}_{2}(50 \mathrm{~mL})$. The resulting mixture was stirred at $0{ }^{\circ} \mathrm{C}$ for $15 \mathrm{~min}$ and then put it in a freezer $\left(-8{ }^{\circ} \mathrm{C}\right)$ overnight. The 
reaction mixture was then poured into $5 \% \mathrm{NaHCO}_{3}$ solution $(150 \mathrm{~mL})$ and extracted with $\mathrm{CH}_{2} \mathrm{Cl}_{2}(200 \mathrm{~mL})$. The combined organic layer was washed with distilled water, dried, and concentrated in vacuo. Column chromatography (chloroform-ether, 9:1) gave white solid (2.6 g, 62\%): mp 135-137 ${ }^{\circ} \mathrm{C}$; IR $1669 \mathrm{~cm}^{-1} ;{ }^{1} \mathrm{H}$ NMR $\delta 11.87$ (broad, $\left.1 \mathrm{H}\right), 8.69$ (dd, $\mathrm{J}=8.49,0.99 \mathrm{~Hz}, 1 \mathrm{H}), 7.71(\mathrm{dt}, \mathrm{J}=8.05,0.96 \mathrm{~Hz}, 1 \mathrm{H}), 7.51(\mathrm{dt}, \mathrm{J}=7.21,1.74 \mathrm{~Hz}, 1 \mathrm{H})$, 7.35-7.41 (m, 2H), 7.33 (dt, J = 6.80, 1.07 Hz, 1H), 7.12-7.18 (m, 2H), $7.10(\mathrm{dt}, \mathrm{J}=7.26$, $1.03 \mathrm{~Hz}, 1 \mathrm{H}), 4.14$ (s, 3H), 1.28 (s, 9H); ${ }^{13} \mathrm{C}$ NMR $\delta 160.1,142.5,139.6,132.3,131.3$, $128.7,126.1,124.5,122.6,122.4,122.1,120.9,120.5,110.1,106.0,59.1,31.9,23.4 ; \mathrm{MS}$ [m/z (rel. intensity)] $354\left[\mathrm{M}^{+}, 2\right], 158$ (100), 89 (16). Anal. Calc. for $\mathrm{C}_{20} \mathrm{H}_{22} \mathrm{~N}_{2} \mathrm{O}_{2} \mathrm{~S}$ : C, 67.77; H, 6.26; N, 7.90. Found: C, 68.09; H, 6.22; N, 7.88.

$N$-[2-(tert-butylthio)phenyl]- $N, 1-d i m e t h y l-1 H$-indole-2-carboxamide (7b). To a wellstirred suspension of $\mathbf{1 b}(6.5 \mathrm{~g}, 20 \mathrm{mmol})$ and tetra- $n$-butylammonium hydrogen sulfate $(0.3 \mathrm{eq}, 6 \mathrm{mmol}, 2.0 \mathrm{~g})$ in toluene $(250 \mathrm{~mL})$ was added all at once $50 \% \mathrm{NaOH}(\mathrm{aq})$ solution $(200 \mathrm{~mL})$. The resulting two layer mixture was heated under reflux at which a solution of iodomethane $(2.5 \mathrm{eq}, 50 \mathrm{mmol}, 7.11 \mathrm{~g})$ in toluene $(10 \mathrm{~mL})$ was added dropwise over 5 min The resulting two phase mixture was maintained at reflux for $24 \mathrm{~h}$. It was cooled to room temperature and the layers separated. The organic layer was washed with water several times (until washings were neutral to litmus), dried over sodium sulfate and evaporated in vacuo to yield an off-white solid. Recrystallization with hexane gave white compound (5.6 g, 79\%): mp 105-106 ${ }^{\circ} \mathrm{C}$; IR $3007,1636 \mathrm{~cm}^{-1} ;{ }^{1} \mathrm{H}$ NMR $\delta 7.48(\mathrm{~d}, \mathrm{~J}=7.99 \mathrm{~Hz}, 1 \mathrm{H}), 7.42(\mathrm{dt}, \mathrm{J}=7.33,1.42 \mathrm{~Hz}, 1 \mathrm{H}), 7.37$ (d, J = 7.9 Hz, 1H), $7.29(\mathrm{~d}, \mathrm{~J}=8.24 \mathrm{~Hz}, 2 \mathrm{H}), 7.17(\mathrm{q}, \mathrm{J}=7.72 \mathrm{~Hz}, 2 \mathrm{H}), 6.95(\mathrm{t}, \mathrm{J}=7.43 \mathrm{~Hz}, 1 \mathrm{H}), 5.72$ 
(s, 1H), $4.02(\mathrm{~s}, 3 \mathrm{H}), 3.49$ (s, 3H), 1.24 (s, 9H); ${ }^{13} \mathrm{C}$ NMR $\delta$ 164.4, 148.4, 138.3, 137.9, $132.4,131.5,129.6,126.9,126.8,126.0,123.3,121.7,119.5,109.7,106.8,46.5,38.4$, 31.6, 31.4; MS [m/z (rel. intensity)] 263 (100), 158 (80), 89 (33). Anal. Calc. for $\mathrm{C}_{21} \mathrm{H}_{24} \mathrm{~N}_{2} \mathrm{OS}$ : C, 71.55; H, 6.86; N, 7.95. Found: C 71.79; H 7.06; N 7.91.

$N$-\{2-[(2-cyanoethyl)thio]phenyl\}- $N$,1-dimethyl-1H-indole-2-carboxamide (7c). To a well-stirred solution of 1-methyl-1H-indole-2-carboxylic acid $(0.65 \mathrm{~g}, 3.71 \mathrm{mmol})$ in ether $(50 \mathrm{~mL})$ at $0{ }^{\circ} \mathrm{C}$ under a drying tube was added dropwise thionyl chloride neat $(3 \mathrm{x}$ $0.5 \mathrm{~g}$ portionwise over $12 \mathrm{~h}, 3.5 \mathrm{eq}$.$) . The reaction mixture was allowed to stir at room$ temperature during $12 \mathrm{~h}$. The resulting clear yellow solution was evaporated in vacuo to produce yellow solid $(0.6 \mathrm{~g}, 84 \%)$. This solid was redissolved in ether $(50 \mathrm{~mL})$ and added to a well-stirred solution of 2-propanenitrile-2-(methylaminophenyl)sulfide (1.1 eq, 0.65 $\mathrm{g}, 3.38 \mathrm{mmol})$ in dioxane-water-ether $(20-3-25 \mathrm{~mL})$ mixture over $10 \mathrm{~min}$ at room temperature. The reaction mixture was stirred at room temperature for $3 \mathrm{~h}$ and then diluted with ethyl acetate $(150 \mathrm{~mL})$. The combined organics were washed with $5 \% \mathrm{HCl}$ (aq, $3 \times 150 \mathrm{~mL}$ ), $5 \% \mathrm{NaHCO}_{3}$ (aq, $3 \times 150 \mathrm{~mL}$ ), water $(2 \times 150 \mathrm{~mL})$ dried over sodium sulfate and solvent evaporated in vacuo to yield a sticky solid which was purified by column chromatography (chloroform: Ether, 9:1) to give white solid (0.35 g, 27\%): $\mathrm{mp}$ 124-125 ${ }^{\circ} \mathrm{C}$; IR 2240, $1635 \mathrm{~cm}^{-1}$; ${ }^{1} \mathrm{H}$ NMR $\delta$ 6.92-7.80 (m, 8H), 5.95 (s, 1H), $3.98(\mathrm{~s}$, 3H), $3.44(\mathrm{~s}, 3 \mathrm{H}), 2.41(\mathrm{~s}, 2 \mathrm{H}), 2.30(\mathrm{~s}, 2 \mathrm{H}) ;{ }^{13} \mathrm{C}$ NMR was not well-resolved due to presence of rotational isomers; MS [m/z (rel. intensity)] 349 [M+1 , 3], 263 (72), 158 (100), 89 (24). Anal. Calc. for $\mathrm{C}_{20} \mathrm{H}_{19} \mathrm{~N}_{3} \mathrm{OS}: \mathrm{C}, 68.74 ; \mathrm{H}, 5.48 ; \mathrm{N}, 12.02$. Found: $\mathrm{C} 68.80 ; \mathrm{H}$ $5.58 ; \mathrm{N} 11.87$. 
$N$-[2-(ethylsulfinyl)phenyl]- $N$,1-dimethyl-1H-indole-2-carboxamide (8a). (95\%); mp $165-167^{\circ} \mathrm{C}$ (acetone); IR 1633, 1052, $1025 \mathrm{~cm}^{-1} ;{ }^{1} \mathrm{H}$ NMR $\left(200 \mathrm{MHz}\right.$, taken at $\left.100{ }^{\circ} \mathrm{C}\right)$

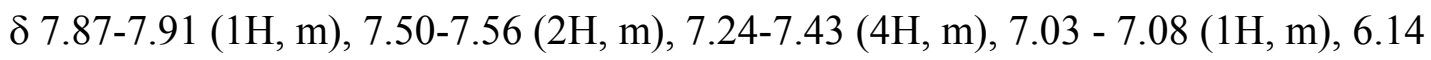
$(1 \mathrm{H}, \mathrm{s}), 3.97(3 \mathrm{H}, \mathrm{s}), 3.40(3 \mathrm{H}, \mathrm{s}), 2.65(2 \mathrm{H}, \mathrm{m}$, containing $\mathrm{J}=7 \mathrm{~Hz}), 1.19(3 \mathrm{H}, \mathrm{t}, \mathrm{J}=7$ Hz); ${ }^{13}$ C NMR $\delta 163.0,141.8,138.8,133.1,132.0,129.5,129.3,129.0,126.9,126.8$, 124.9, 122.6, 121.0, 110.6, 108.8, 49.2, 39.0, 32.3, 7.0; MS [m/z (relative intensity)] 340 $\left(\mathrm{M}^{+}, 1\right), 158$ (100). Anal. Calcd for $\mathrm{C}_{19} \mathrm{H}_{20} \mathrm{~N}_{2} \mathrm{O}_{2} \mathrm{~S}: \mathrm{C}, 67.02 ; \mathrm{H}, 6.11 ; \mathrm{N}, 8.00$. Found: $\mathrm{C}$, $67.03 ; \mathrm{H}, 5.92 ; \mathrm{N}, 8.23$.

$N$-[2-(tert-butylsulfinyl)phenyl]- $N$,1-dimethyl-1H-indole-2-carboxamide (8b). To an ice-cooled solution of $\mathbf{7 b}(5.38 \mathrm{~g}, 15.2 \mathrm{mmol})$ in $\mathrm{CH}_{2} \mathrm{Cl}_{2}(150 \mathrm{~mL})$ was added slowly a solution of $m$-CPBA (77\%, $1.1 \mathrm{eq}, 21.7 \mathrm{mmol}, 3.74 \mathrm{~g})$ in $\mathrm{CH}_{2} \mathrm{Cl}_{2}(50 \mathrm{~mL})$. The resulting mixture was stirred at $0{ }^{\circ} \mathrm{C}$ for $15 \mathrm{~min}$ and then put it in a freezer $\left(-8{ }^{\circ} \mathrm{C}\right)$ overnight. The reaction mixture was then poured into $5 \% \mathrm{NaHCO}_{3}$ solution $(150 \mathrm{~mL})$ and extracted with $\mathrm{CH}_{2} \mathrm{Cl}_{2}(200 \mathrm{~mL})$. The combined organic layer was washed with distilled water, dried, and concentrated in vacuo. Column chromatography (EtOAc-hexane, 6:4) gave yellowish white solid which was recrystallized (hexane) to give a white solid (3.42 g, 61\%): mp 125-127 ${ }^{\circ} \mathrm{C}$; IR 3003, $1639 \mathrm{~cm}^{-1}$; ${ }^{1} \mathrm{H}$ NMR and ${ }^{13} \mathrm{C}$ NMR were not well-resolved due to presence of rotational isomers; MS [m/z (rel. intensity)] 158 (100), 89 (26). Anal. Calc. for $\mathrm{C}_{21} \mathrm{H}_{24} \mathrm{~N}_{2} \mathrm{O}_{2} \mathrm{~S}: \mathrm{C}, 68.45 ; \mathrm{H}, 6.56 ; \mathrm{N}, 7.60$. Found: $\mathrm{C} 68.30 ; \mathrm{H} 6.64 ; \mathrm{N}$ 7.51.

$N$-\{2-[(2-cyanoethyl)sulfinyl]phenyl\}- $N$,1-dimethyl-1 $H$-indole-2-carboxamide (8c). To an ice-cooled solution of $7 \mathbf{c}(0.2 \mathrm{~g}, 0.57 \mathrm{mmol})$ in $\mathrm{CH}_{2} \mathrm{Cl}_{2}(50 \mathrm{~mL})$ was added slowly 
a solution of $m$-CPBA $(77 \%, 1.1 \mathrm{eq}, 0.81 \mathrm{mmol}, 0.141 \mathrm{~g})$ in $\mathrm{CH}_{2} \mathrm{Cl}_{2}(50 \mathrm{~mL})$. The resulting mixture was stirred at $0{ }^{\circ} \mathrm{C}$ for $15 \mathrm{~min}$ and then put it in a freezer $\left(-8^{\circ} \mathrm{C}\right)$ overnight. The reaction mixture was then poured into $5 \% \mathrm{NaHCO}_{3}$ solution $(50 \mathrm{~mL})$ and extracted with $\mathrm{CH}_{2} \mathrm{Cl}_{2}(75 \mathrm{~mL})$. The combined organic layer was washed with distilled water, dried, and concentrated in vacuo. Column chromatography (EtOAc:hexane, 9:1) gave sticky liquid (0.15 g, 72\%): IR 1637, $1039 \mathrm{~cm}^{-1} ;{ }^{1} \mathrm{H}$ NMR and ${ }^{13} \mathrm{C}$ NMR were not well-resolved due to presence of rotational isomers; MS [m/z (rel. intensity)] 312 (15), 294 (38), 158 (100), 53 (99).

\section{0,11-dihydro-10,12-dimethylindolo[3,2-b]-1,5-benzothiazepin-11-one (10) from 8b.} Method A (Thermal Cyclization): A solution of $\mathbf{8 b}(0.3 \mathrm{~g}, 0.82 \mathrm{mmol})$ in chloroform $(50 \mathrm{~mL})$ was heated under reflux for $6 \mathrm{~d}$. The solution was allowed to cool to room temperature and the solvent evaporated in vacuo to yield a brown solid. Column chromatography $\left(\mathrm{CHCl}_{3}\right.$ : ether, 9:1) gave white solid (0.16 g, 67\%): mp 196-198 ${ }^{\circ} \mathrm{C}$; IR $1624 \mathrm{~cm}^{-1} ;{ }^{1} \mathrm{H}$ NMR $\delta 7.73(\mathrm{dt}, \mathrm{J}=8.06,0.93 \mathrm{~Hz}, 1 \mathrm{H}), 7.54(\mathrm{dd}, \mathrm{J}=7.75,0.8 \mathrm{~Hz}), 7.26-$ $7.34(\mathrm{~m}, 4 \mathrm{H}), 7.17(\mathrm{ddd}, \mathrm{J}=8.11,4.54,1.12 \mathrm{~Hz}, 2 \mathrm{H}), 3.91(\mathrm{~s}, 3 \mathrm{H}), 3.61(\mathrm{~s}, 3 \mathrm{H}) ;{ }^{13} \mathrm{C}$ NMR $\delta 163.4,146.0,138.2,137.4,132.3,131.0,128.9,126.2,125.8,125.5,125.0$, 120.6, 120.2, 117.5, 110.1, 38.6, 31.6; MS [m/z (rel. intensity)] $294\left[\mathrm{M}^{+}, 100\right]$. Method B (Electrophilic Activation): To a well-stirred solution of TFAA (0.64 g, 3.1 mmol, 3.75 eq. $)$ in $\mathrm{CH}_{2} \mathrm{Cl}_{2}(20 \mathrm{~mL})$ at $0{ }^{\circ} \mathrm{C}$ was added pyridine $(0.25 \mathrm{~g}, 3.2 \mathrm{mmol}, 4$ eq. $)$, neat via syringe. The reaction vessel was kept under a drying tube throughout the reaction. To the resulting yellow solution was added, a solution of $\mathbf{8 b}(0.3 \mathrm{~g}, 0.82 \mathrm{mmol})$ in $\mathrm{CH}_{2} \mathrm{Cl}_{2}(15 \mathrm{~mL})$ which was previously cooled to $0{ }^{\circ} \mathrm{C}$. This was stirred at $0{ }^{\circ} \mathrm{C}$ for 15 
min then at room temperature for $3 \mathrm{~h}$. The solution was poured onto $10 \% \mathrm{Na}_{2} \mathrm{CO}_{3}(25$ $\mathrm{mL}$ ) and stirred for $5 \mathrm{~min}$. The layers were separated and the aqueous layer washed with $\mathrm{CH}_{2} \mathrm{Cl}_{2}(30 \mathrm{~mL})$. The combined organics were washed with $5 \%$ aqueous solution of $\mathrm{HCl}$ (3 X $40 \mathrm{~mL}), 10 \%$ aqueous solution of $\mathrm{Na}_{2} \mathrm{CO}_{3}(1 \mathrm{X} 40 \mathrm{~mL}), \mathrm{H}_{2} \mathrm{O}$ and dried over sodium sulfate. The solvent was removed in vacuo to yield pure yellow solid, $10(0.23 \mathrm{~g}, 100 \%)$. 
${ }^{1} \mathrm{H}$ NMR Spectrum of $\boldsymbol{N}$-[2-(tert-butylthio)phenyl]acetamide

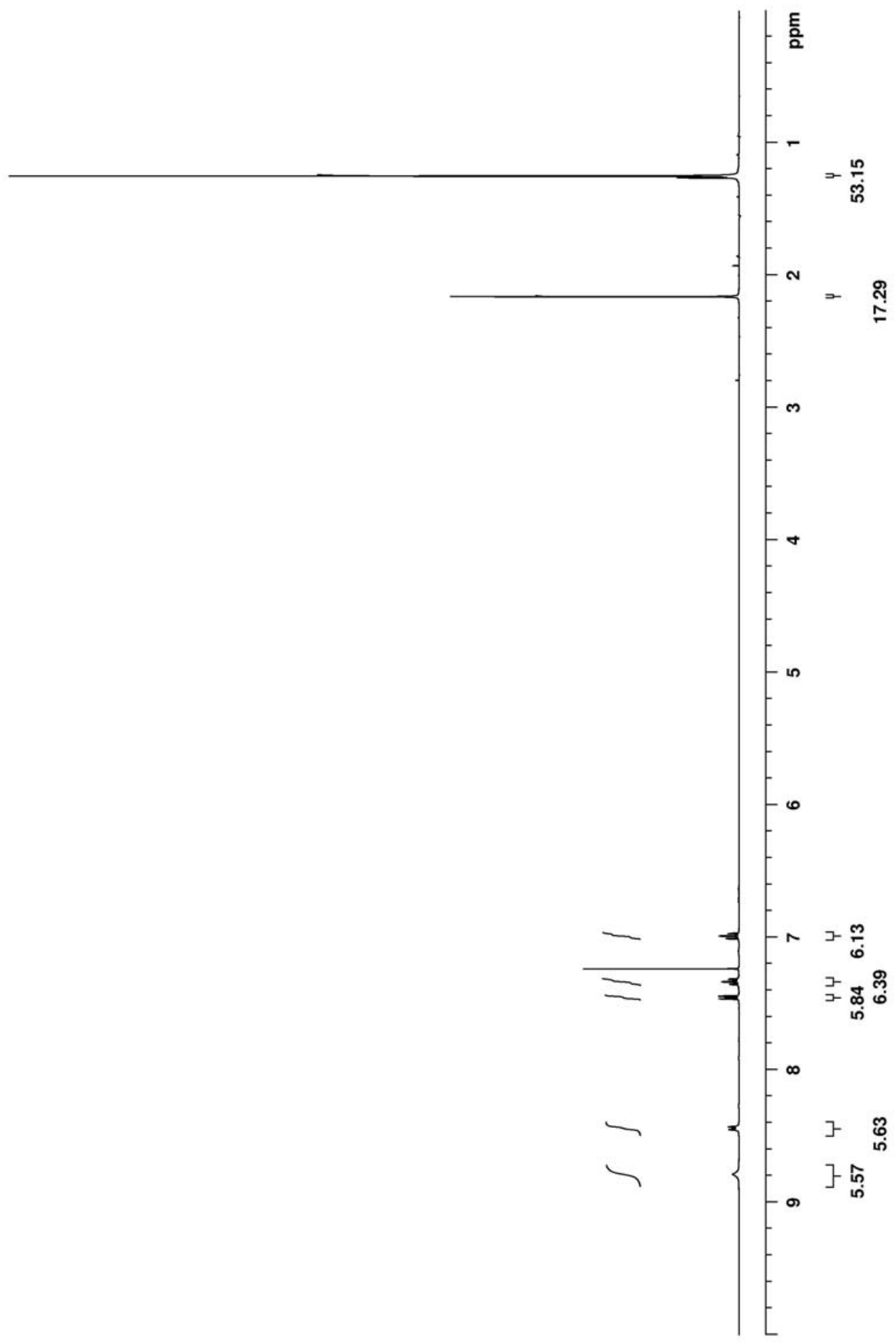


${ }^{13} \mathrm{C}$ NMR Spectrum of $N$-[2-(tert-butylthio)phenyl]acetamide

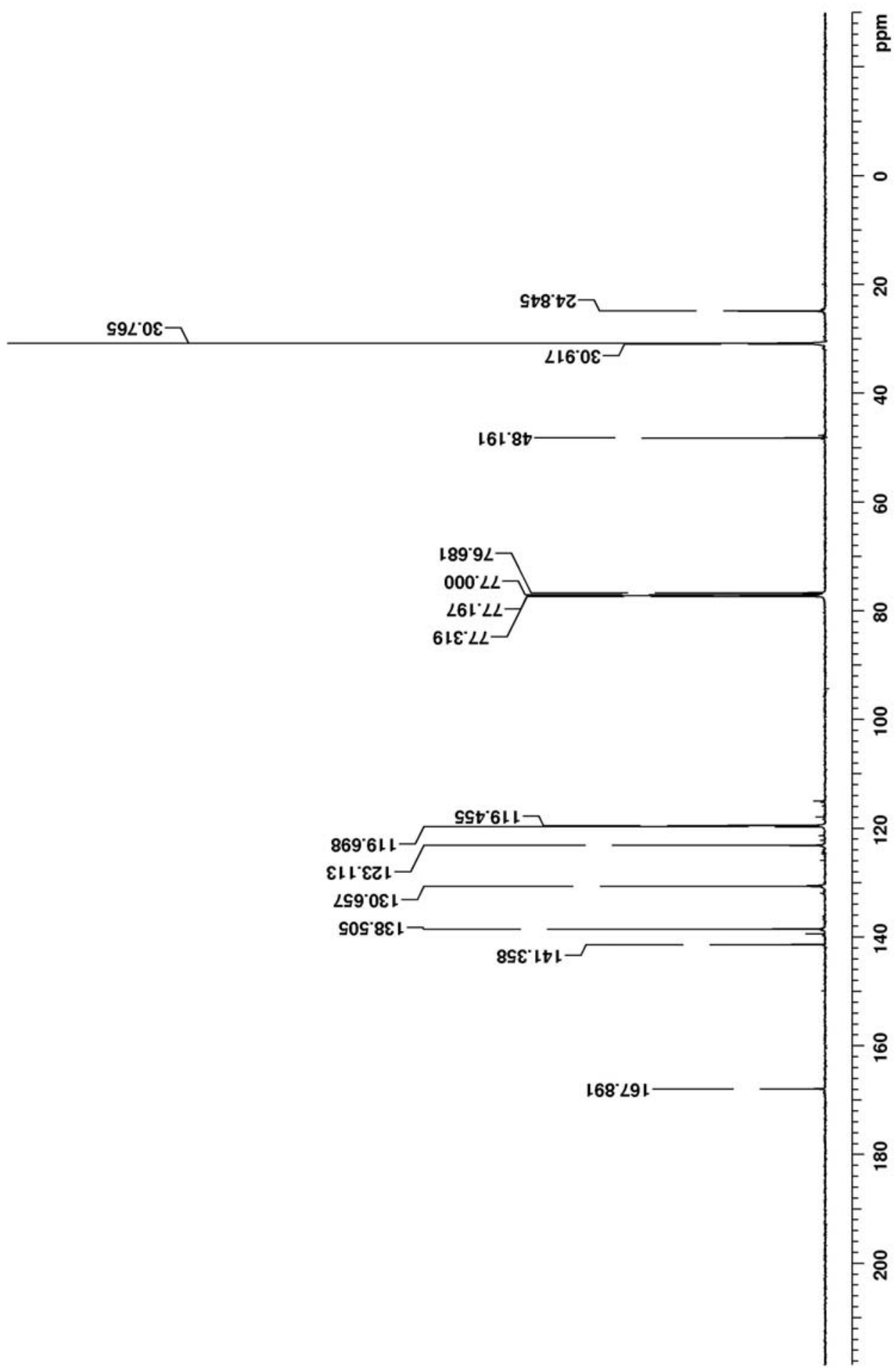




\section{${ }^{1} \mathrm{H}$ NMR Spectrum of $N$-[2-(tert-butylthio)phenyl]- $N$-methylamide}

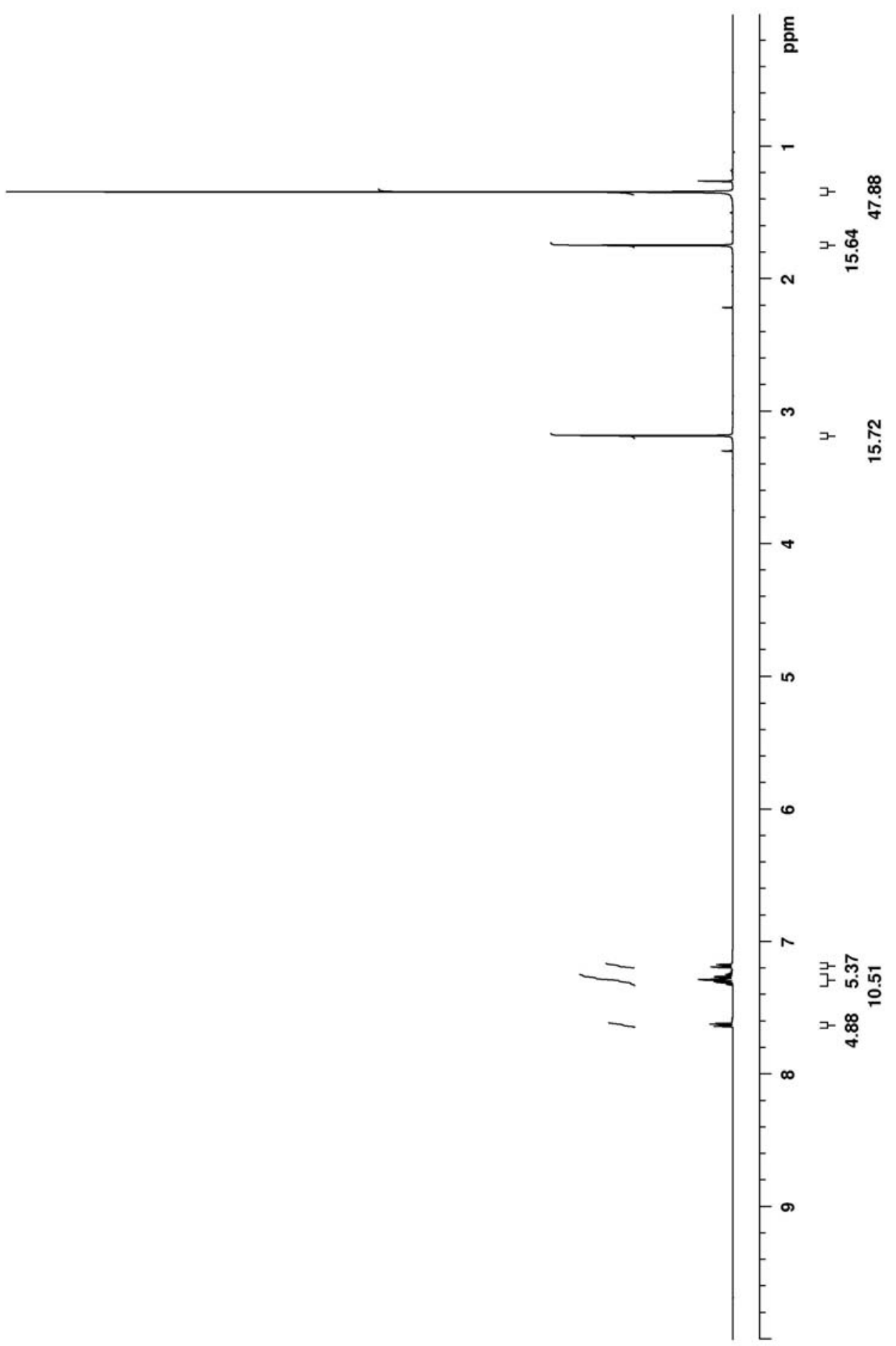


${ }^{13} \mathrm{C}$ NMR Spectrum of $N$-[2-(tert-butylthio)phenyl]- $N$-methylamide

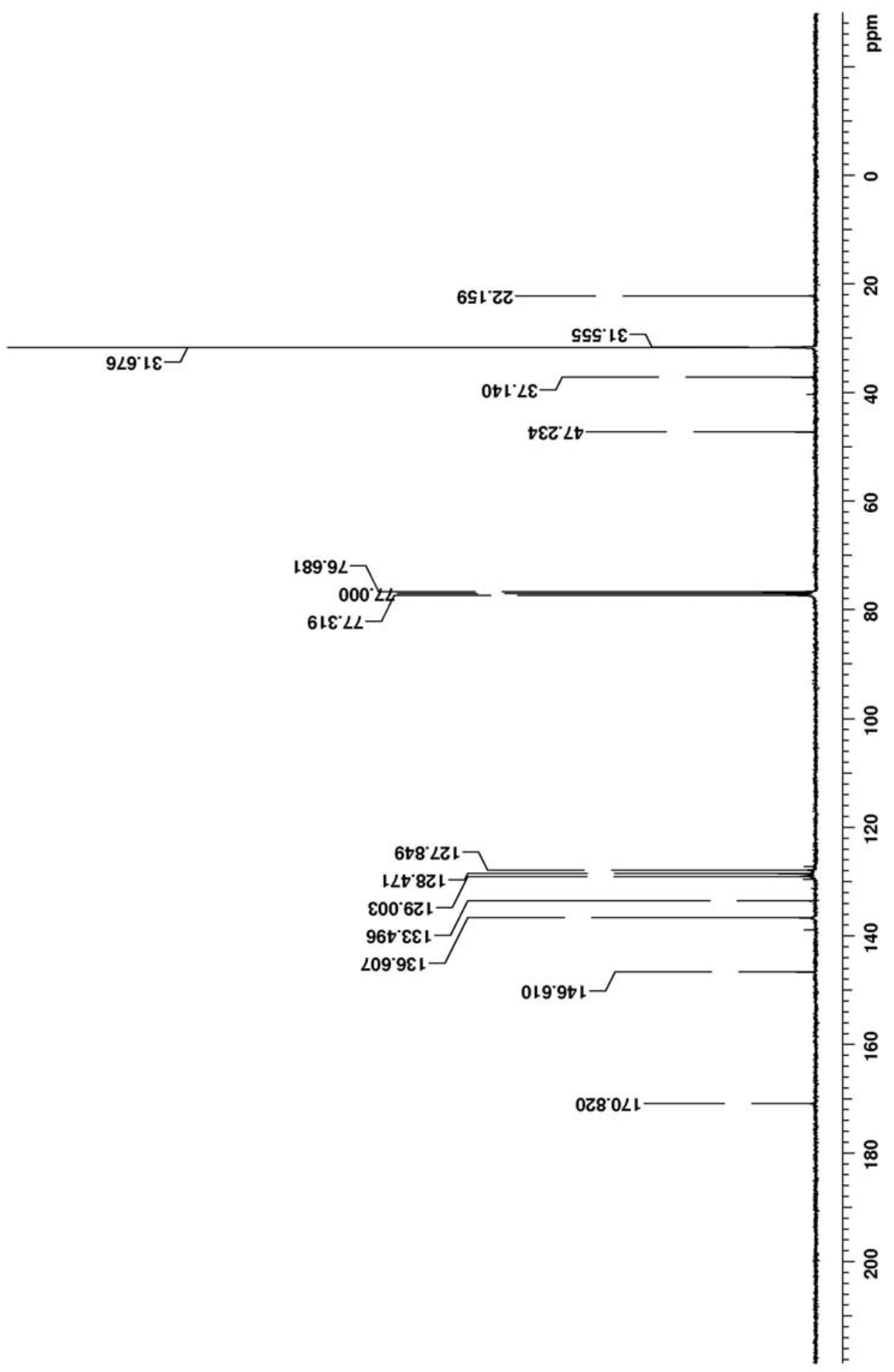


${ }^{1} \mathrm{H}$ NMR Spectrum of $N$-[2-(tert-butylthio)phenyl]- $N$-methylamine

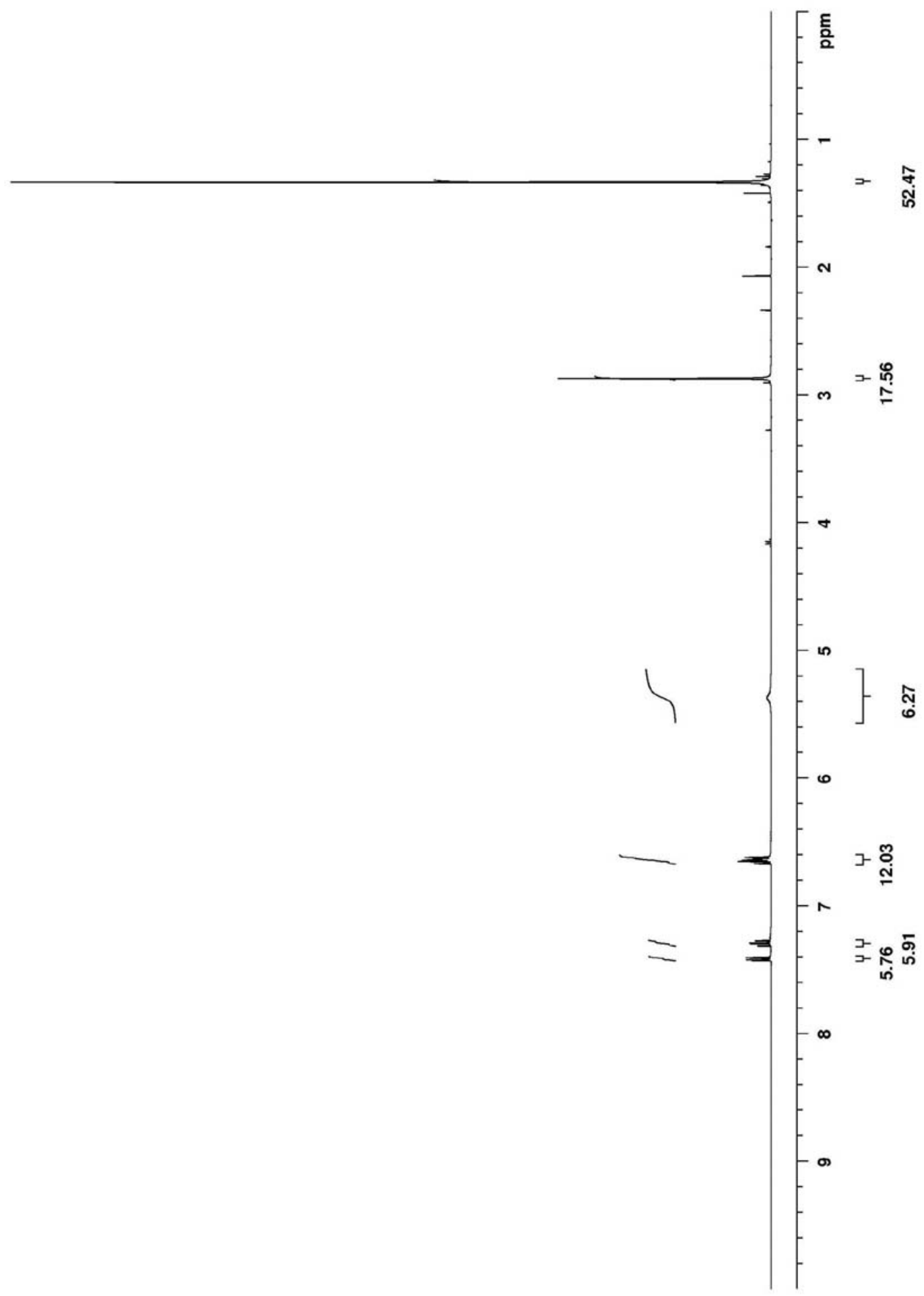


${ }^{13} \mathrm{C}$ NMR Spectrum of $\boldsymbol{N}$-[2-(tert-butylthio)phenyl]- $\boldsymbol{N}$-methylamine

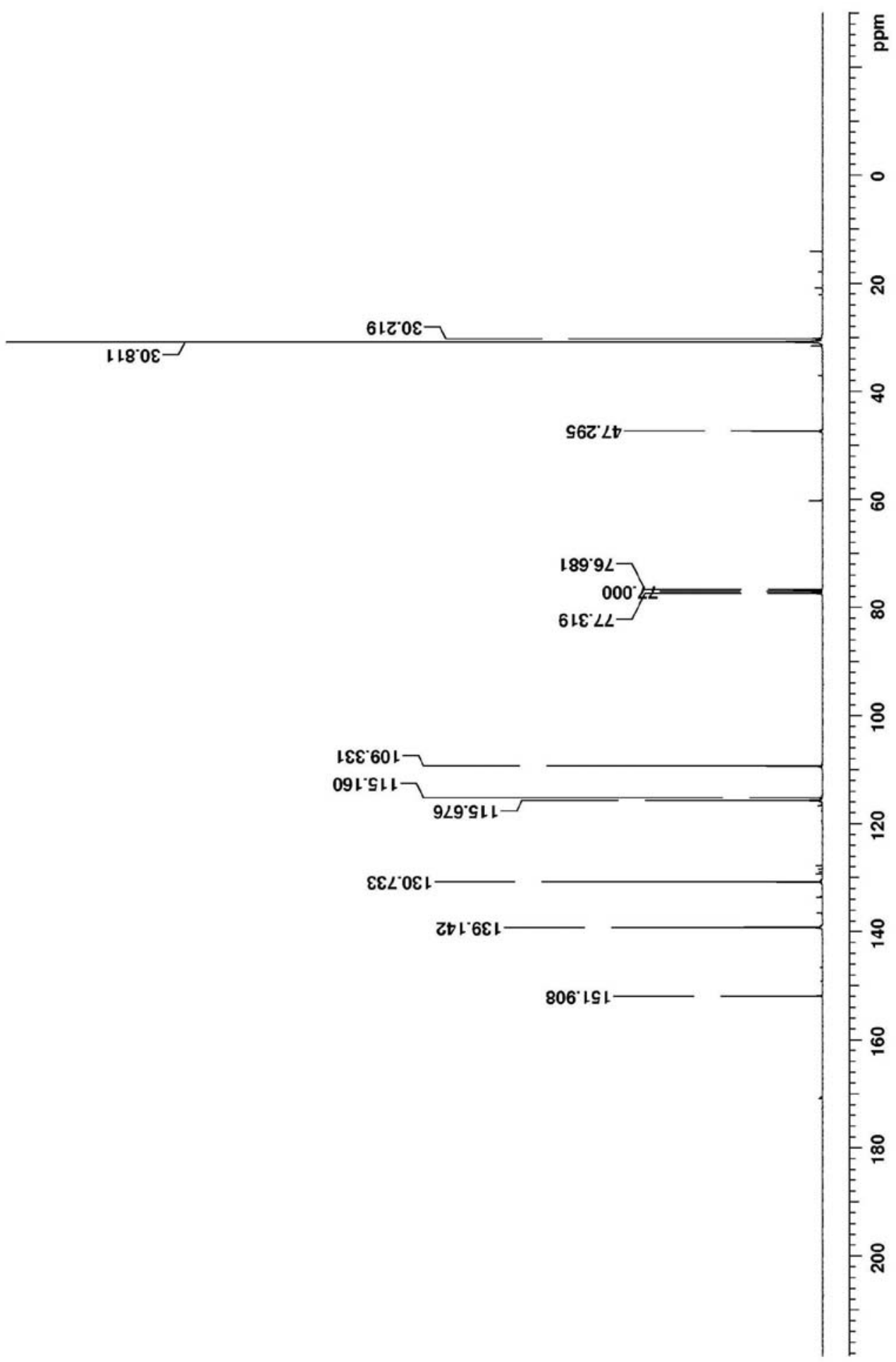


${ }^{1} \mathrm{H}$ NMR Spectrum of $\mathbf{1 b}$

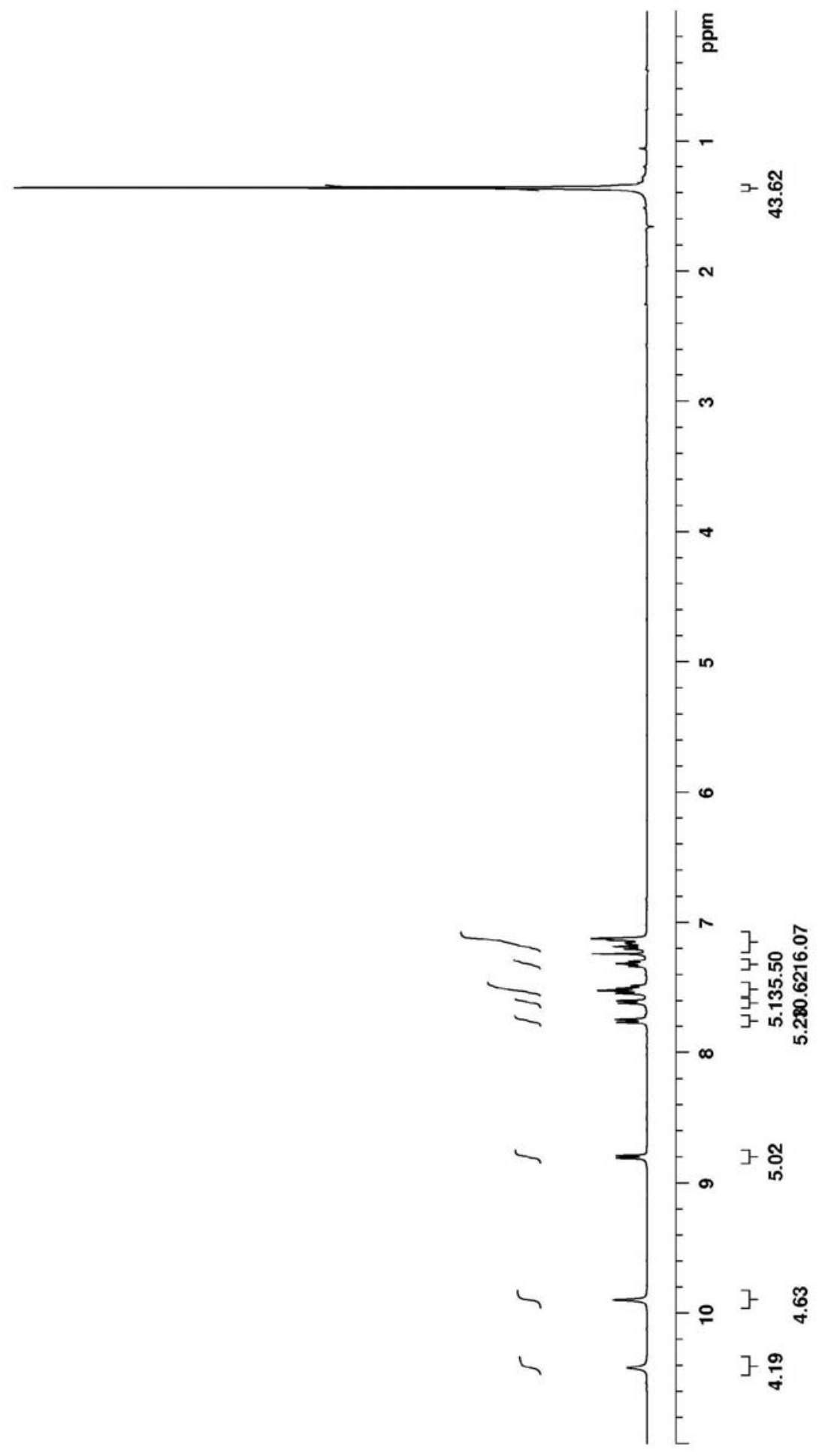


${ }^{13} \mathrm{C}$ NMR Spectrum of $\mathbf{1 b}$

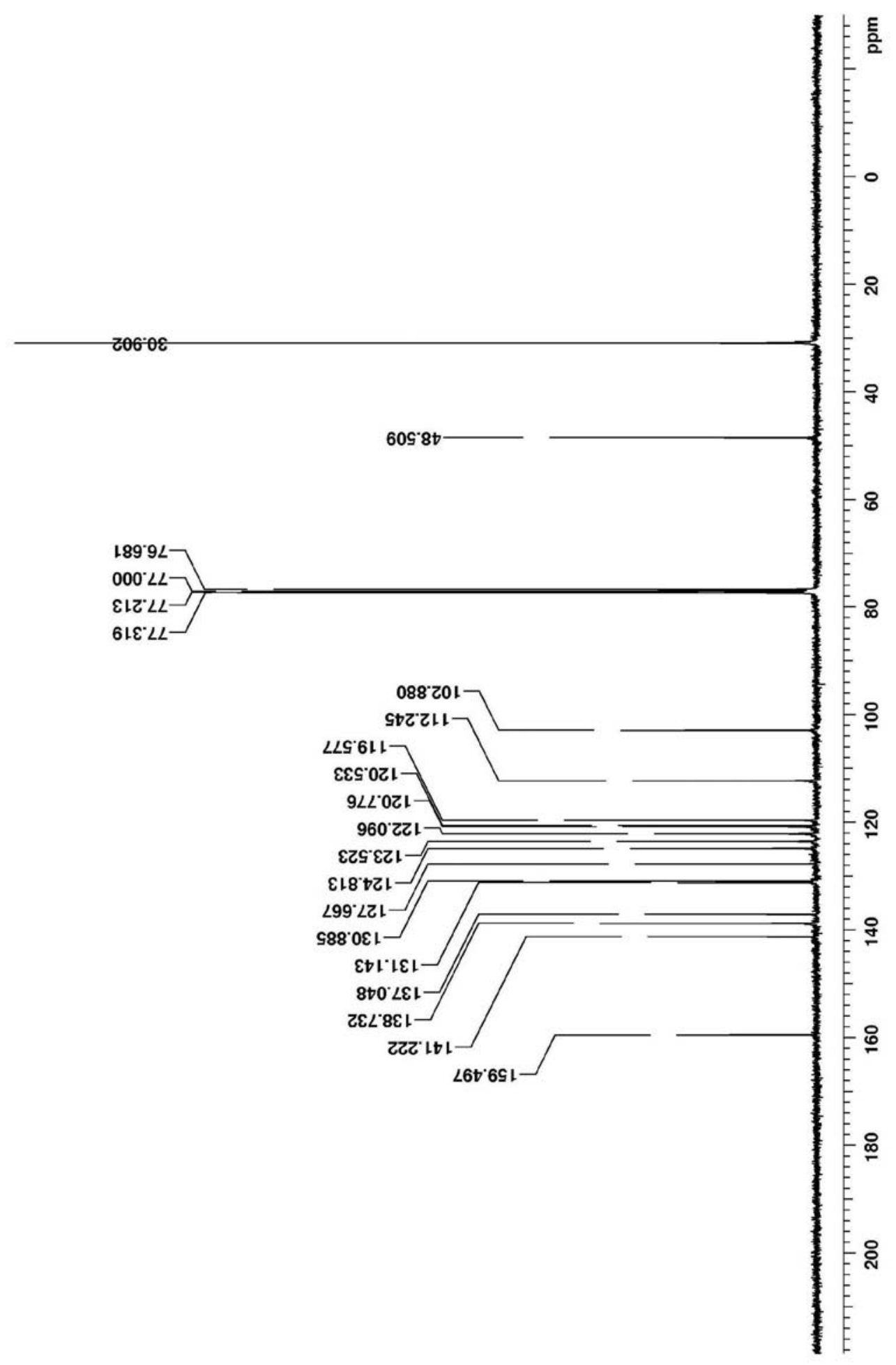


${ }^{1} \mathrm{H}$ NMR Spectrum of $\mathbf{2} \mathbf{b}$

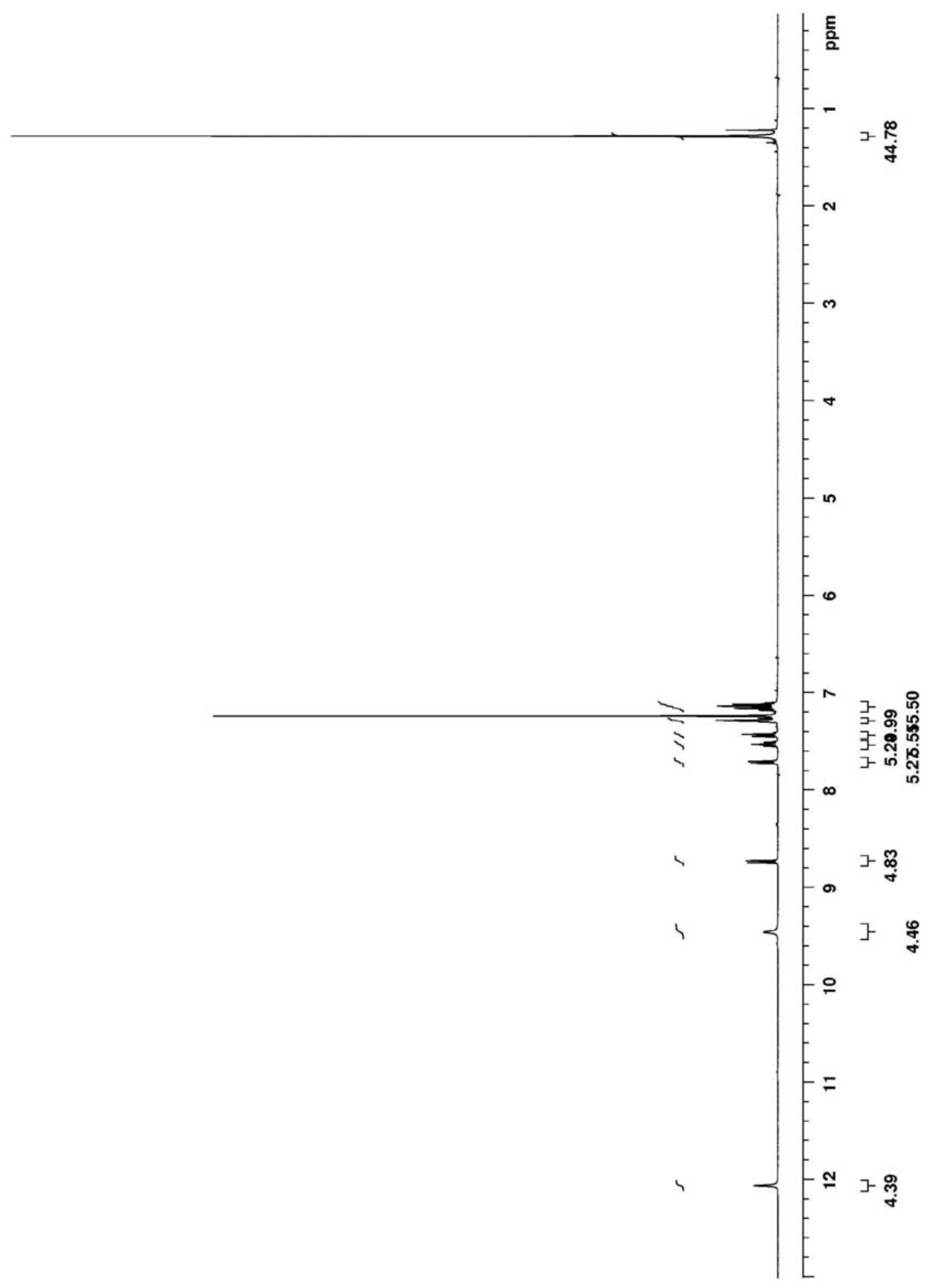


${ }^{13} \mathrm{C}$ NMR Spectrum of $\mathbf{2 b}$

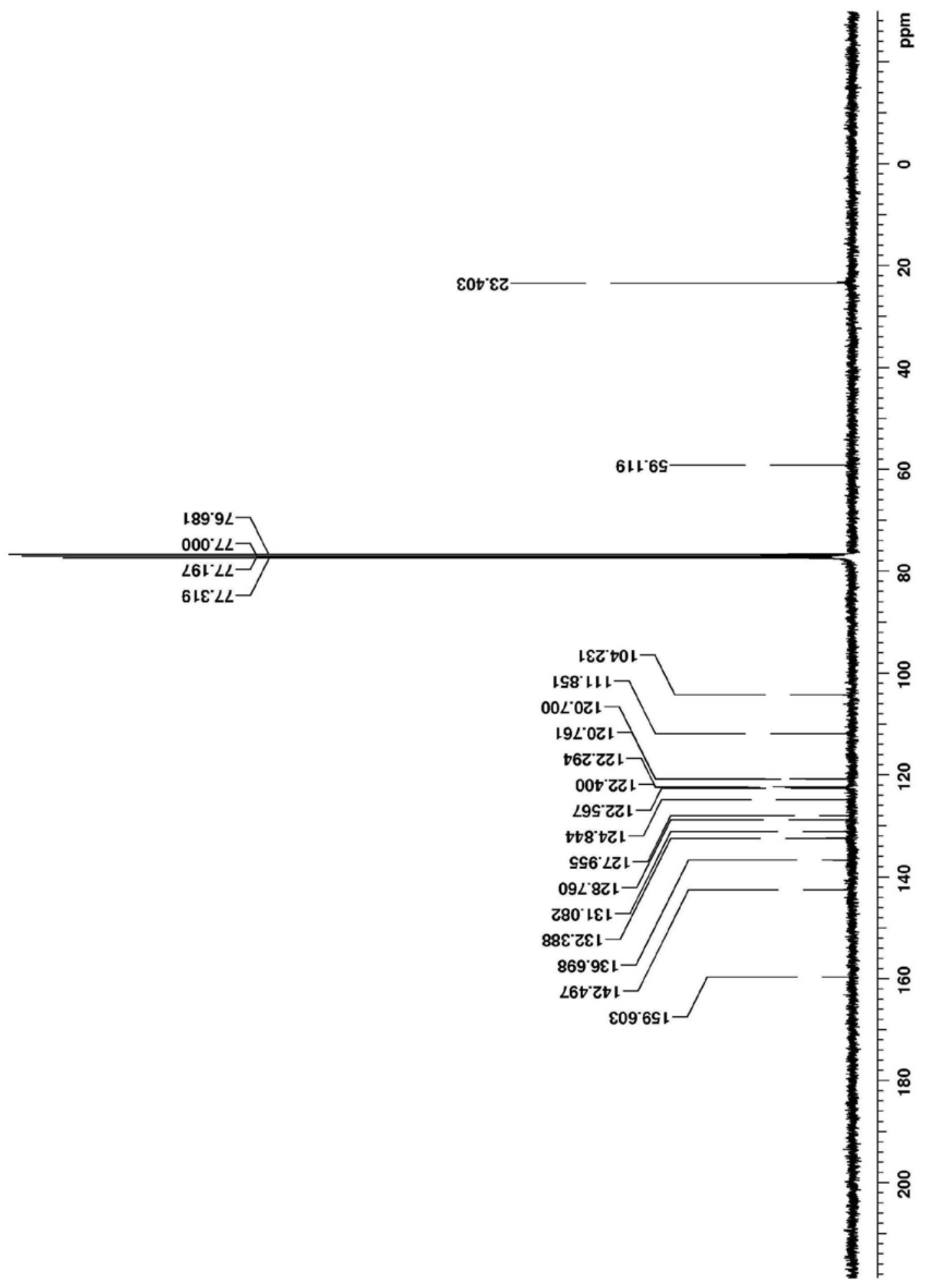




\section{${ }^{1} \mathrm{H}$ NMR Spectrum of $\mathbf{3 b}$}

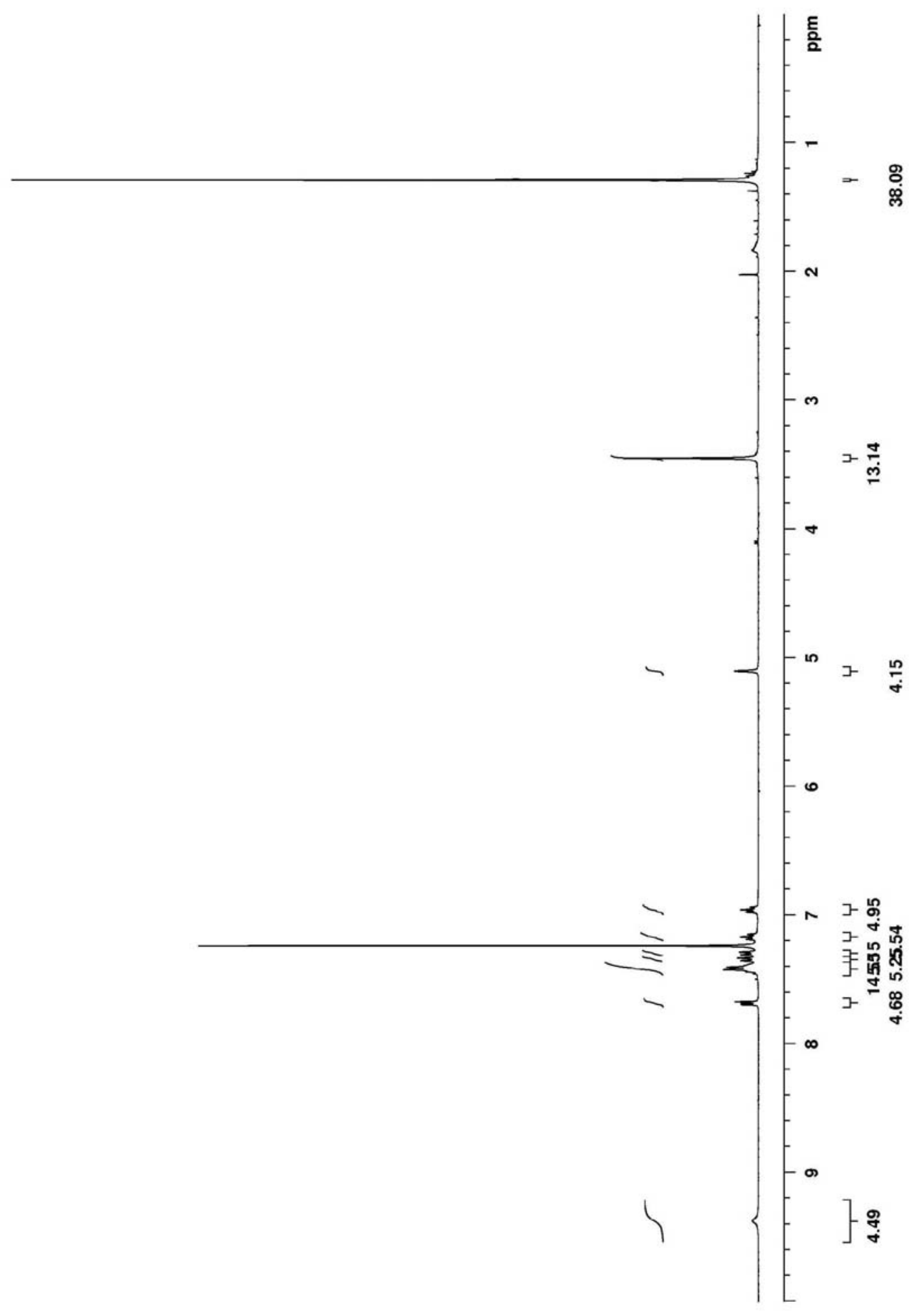


${ }^{13}$ C NMR Spectrum of $\mathbf{3 b}$

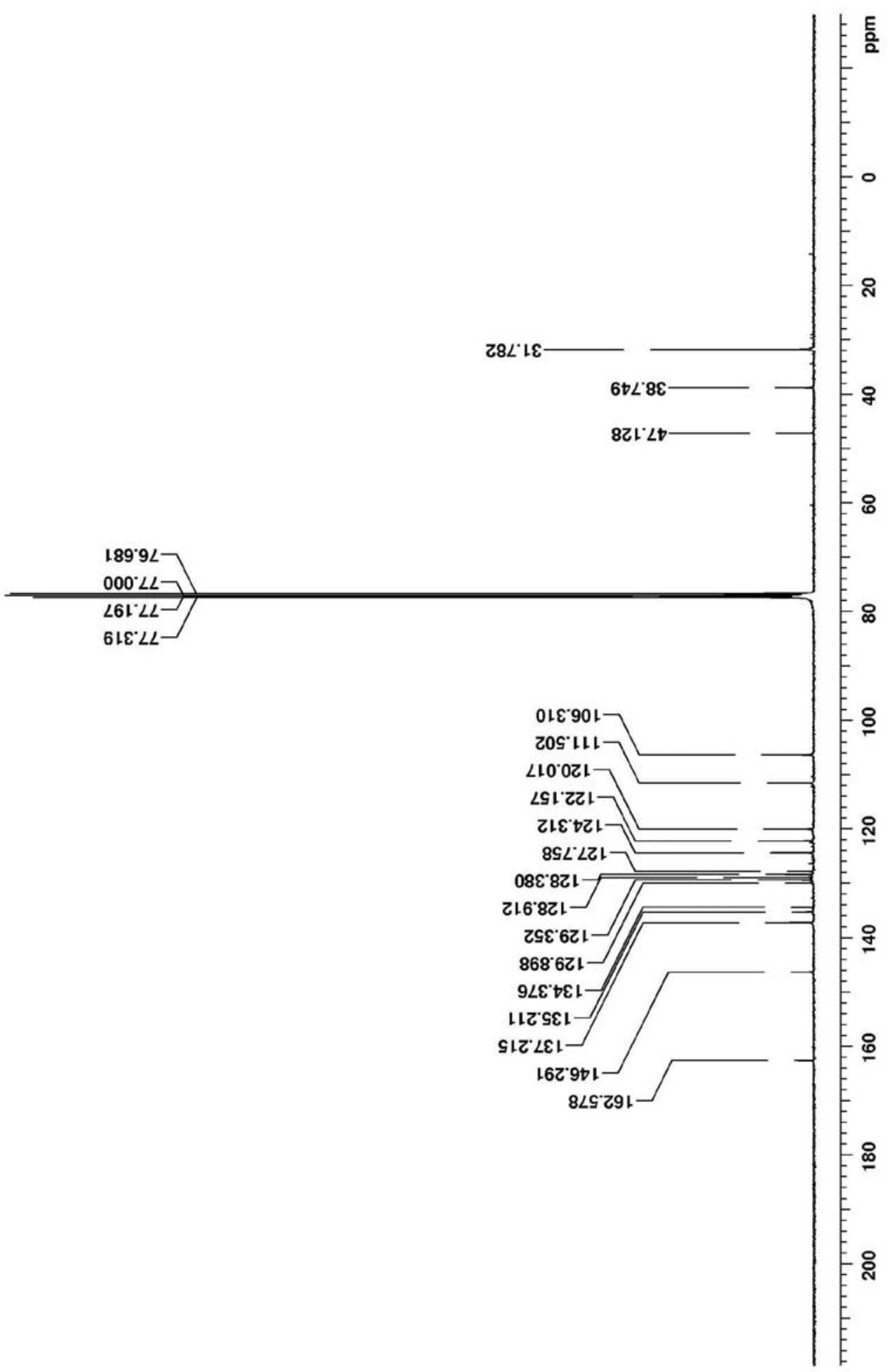




\section{${ }^{1} \mathrm{H}$ NMR Spectrum of $\mathbf{4 b}$}

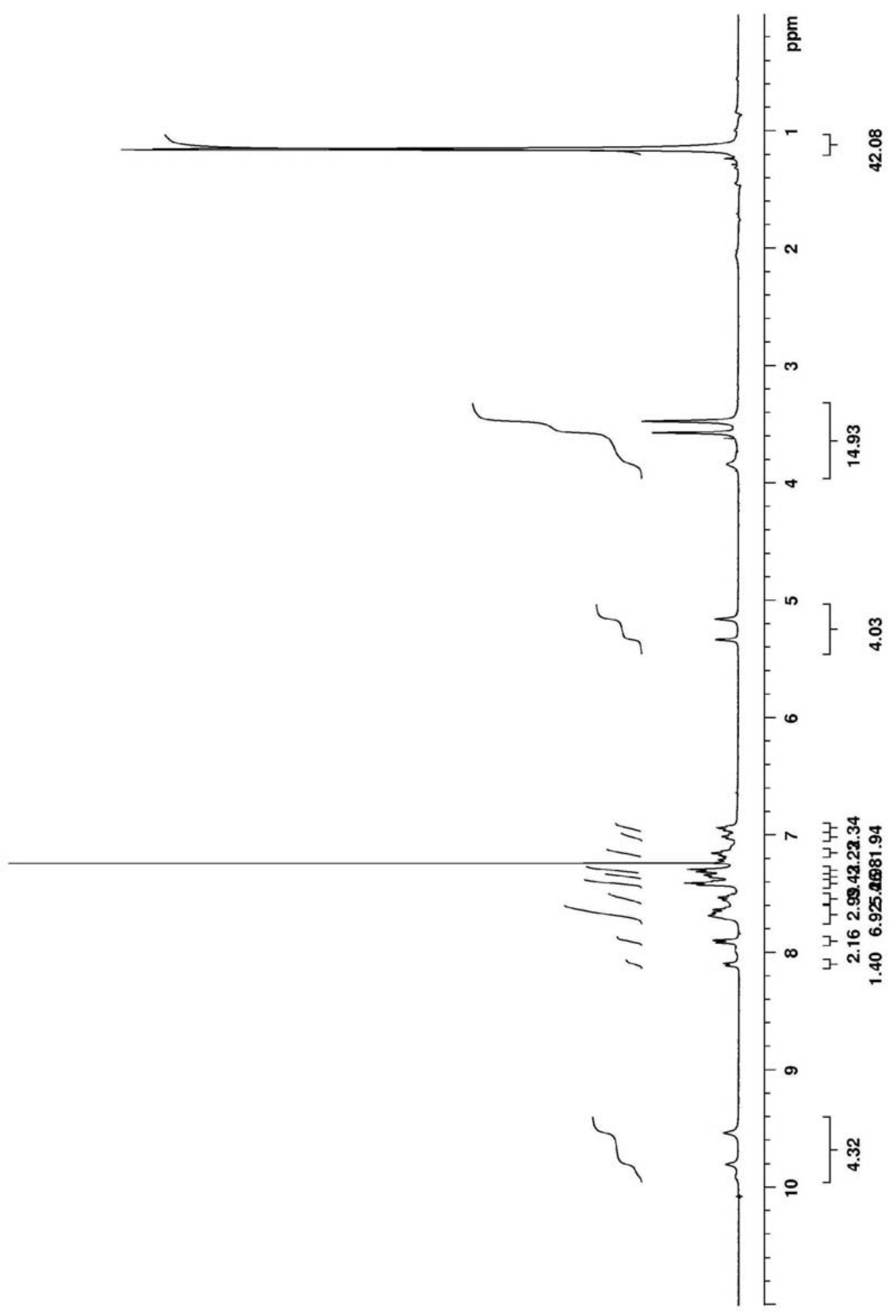




\section{${ }^{1} \mathrm{H}$ NMR Spectrum of $\mathbf{5 b}$}

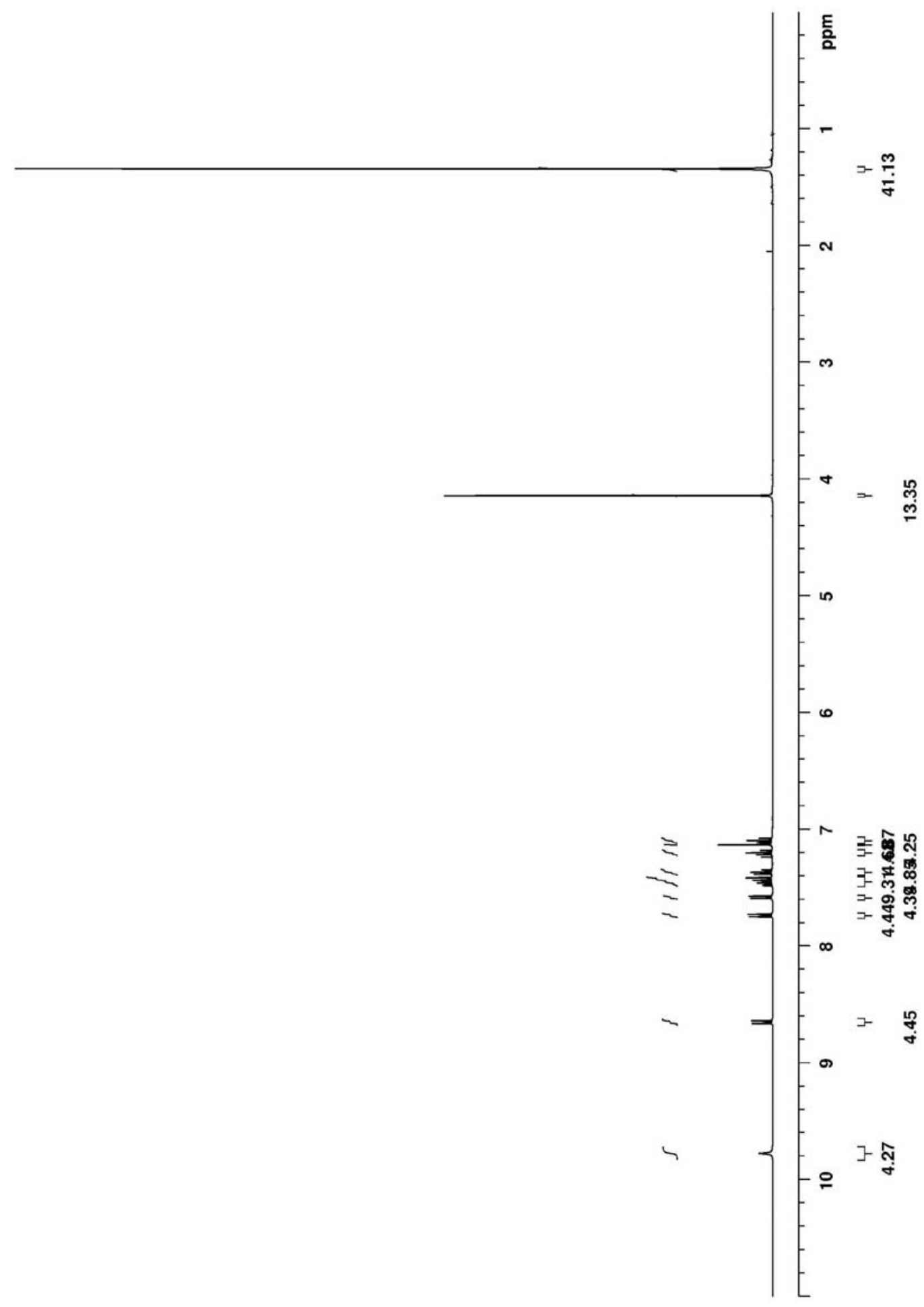




\section{${ }^{13}$ C NMR Spectrum of $\mathbf{5 b}$}

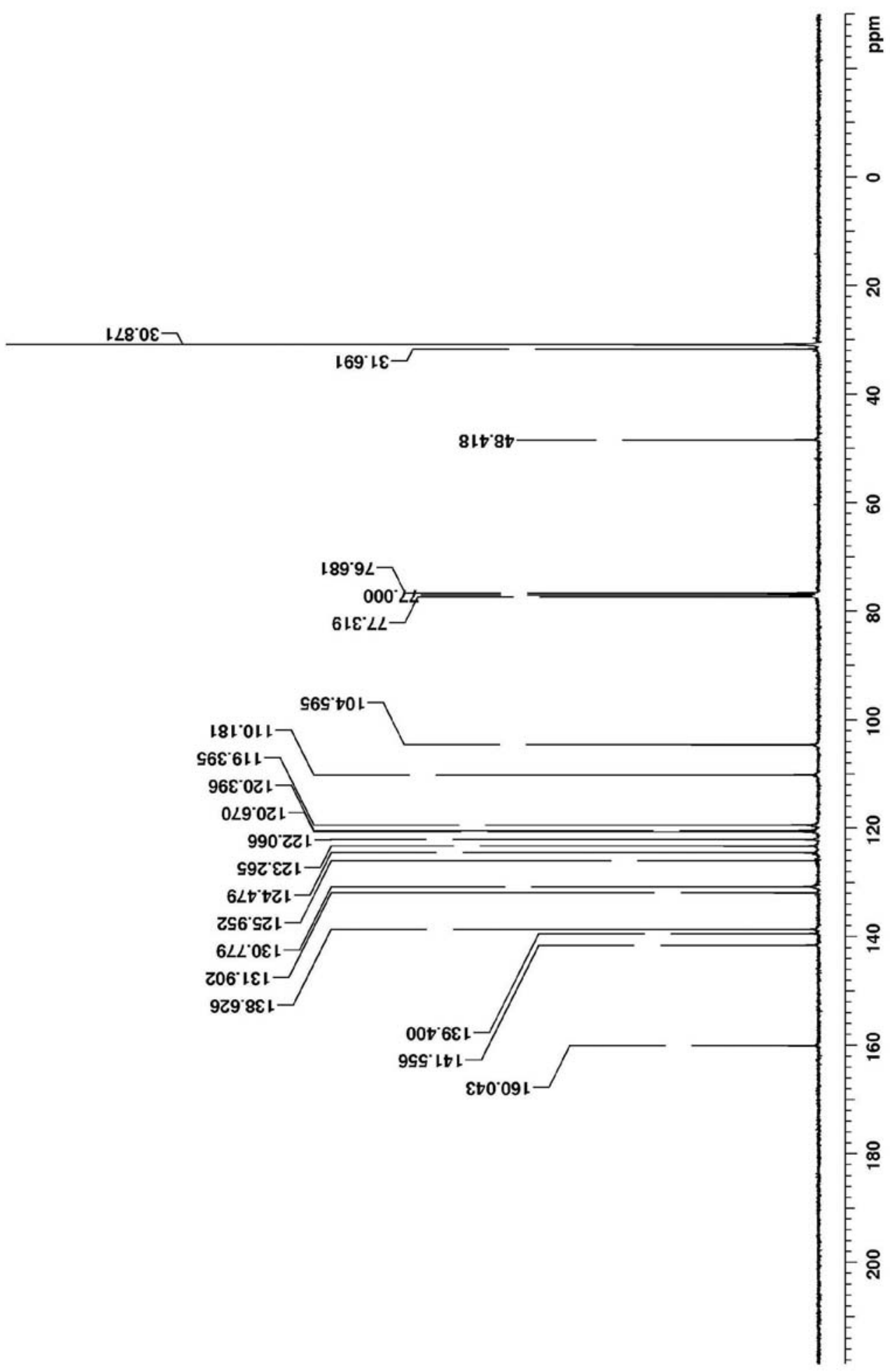




\section{${ }^{1} \mathrm{H}$ NMR Spectrum of $\mathbf{6 b}$}

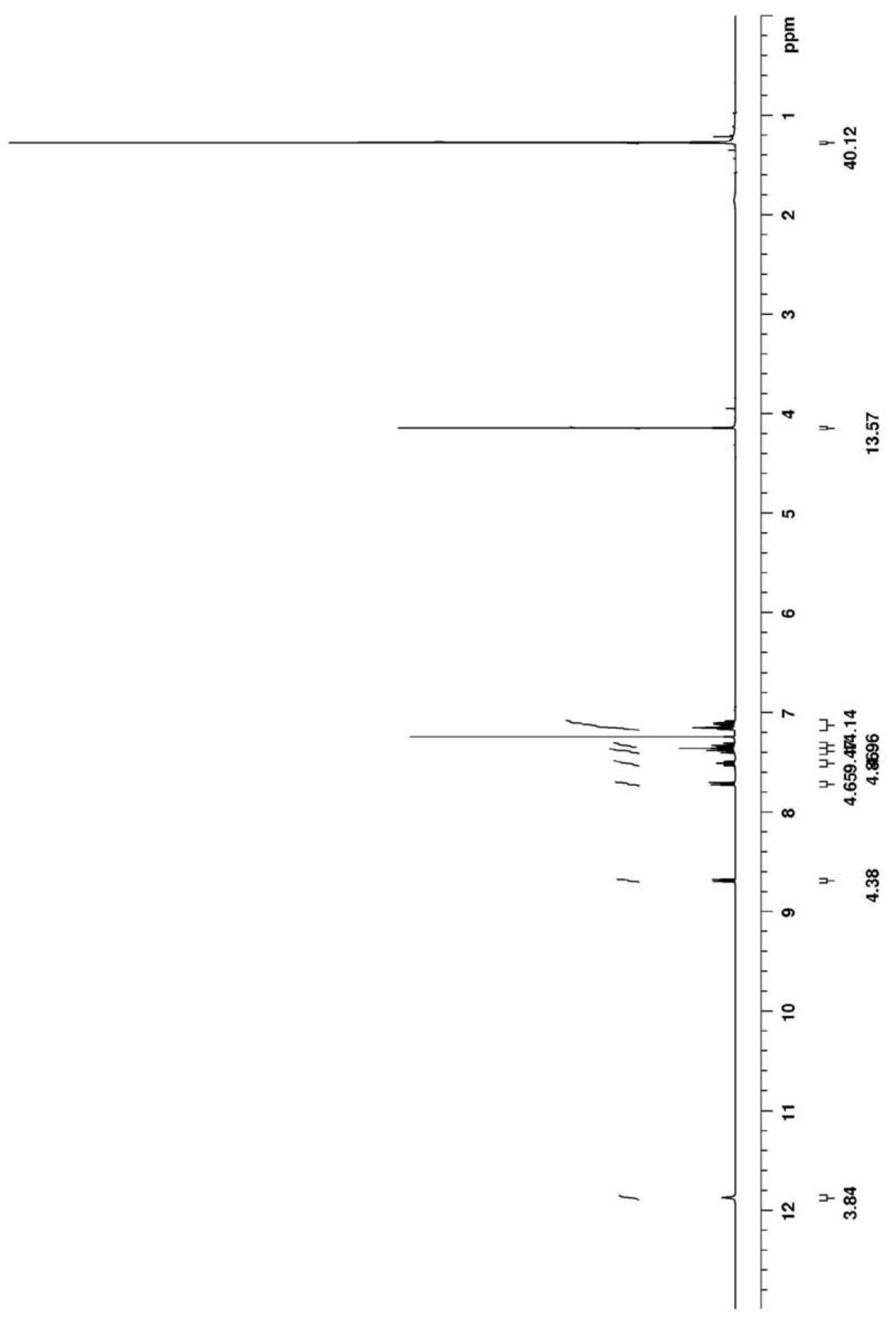


${ }^{13} \mathrm{C}$ NMR Spectrum of $\mathbf{6 b}$

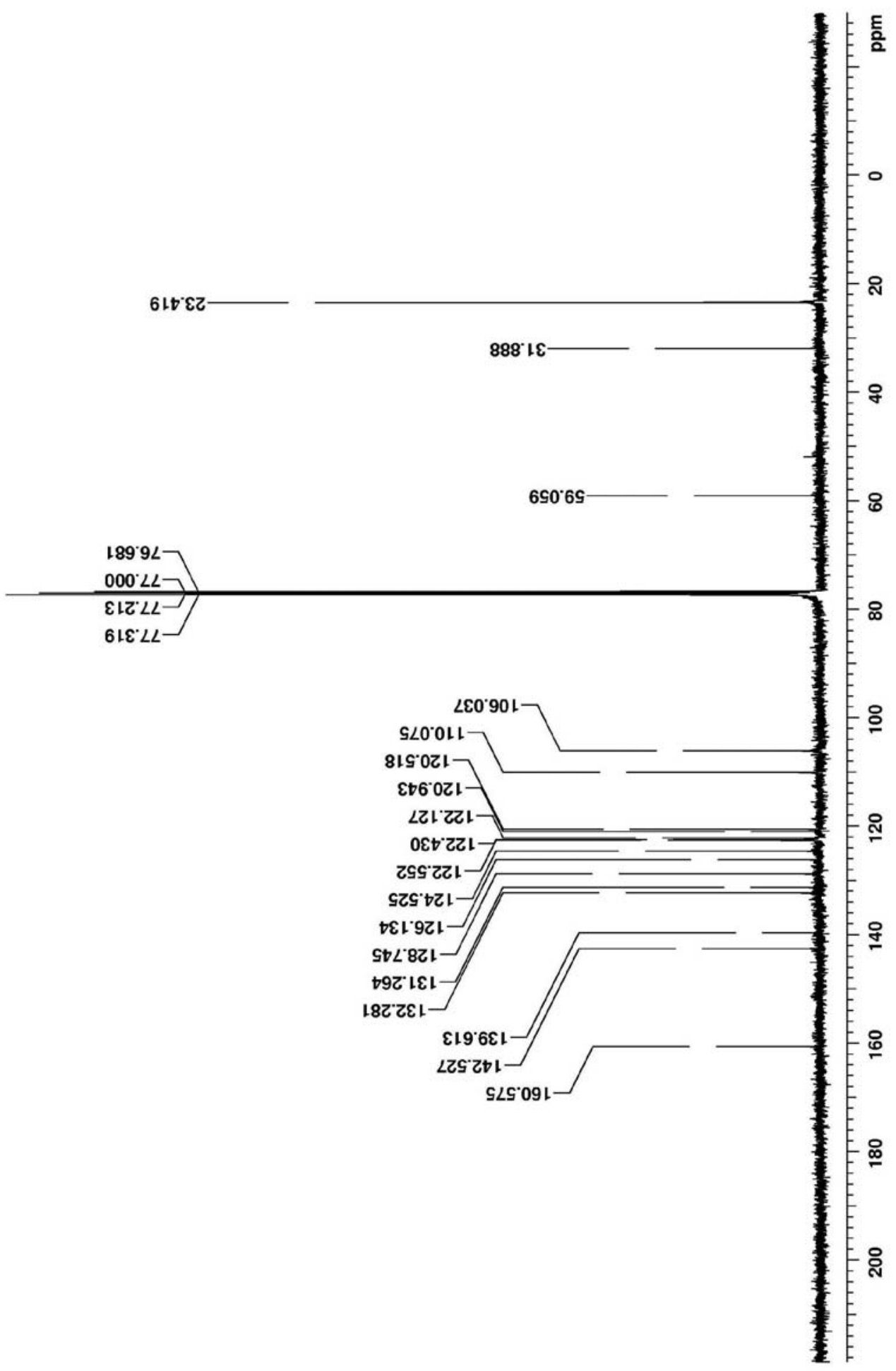


${ }^{1} \mathrm{H}$ NMR Spectrum of $\mathbf{7 b}$

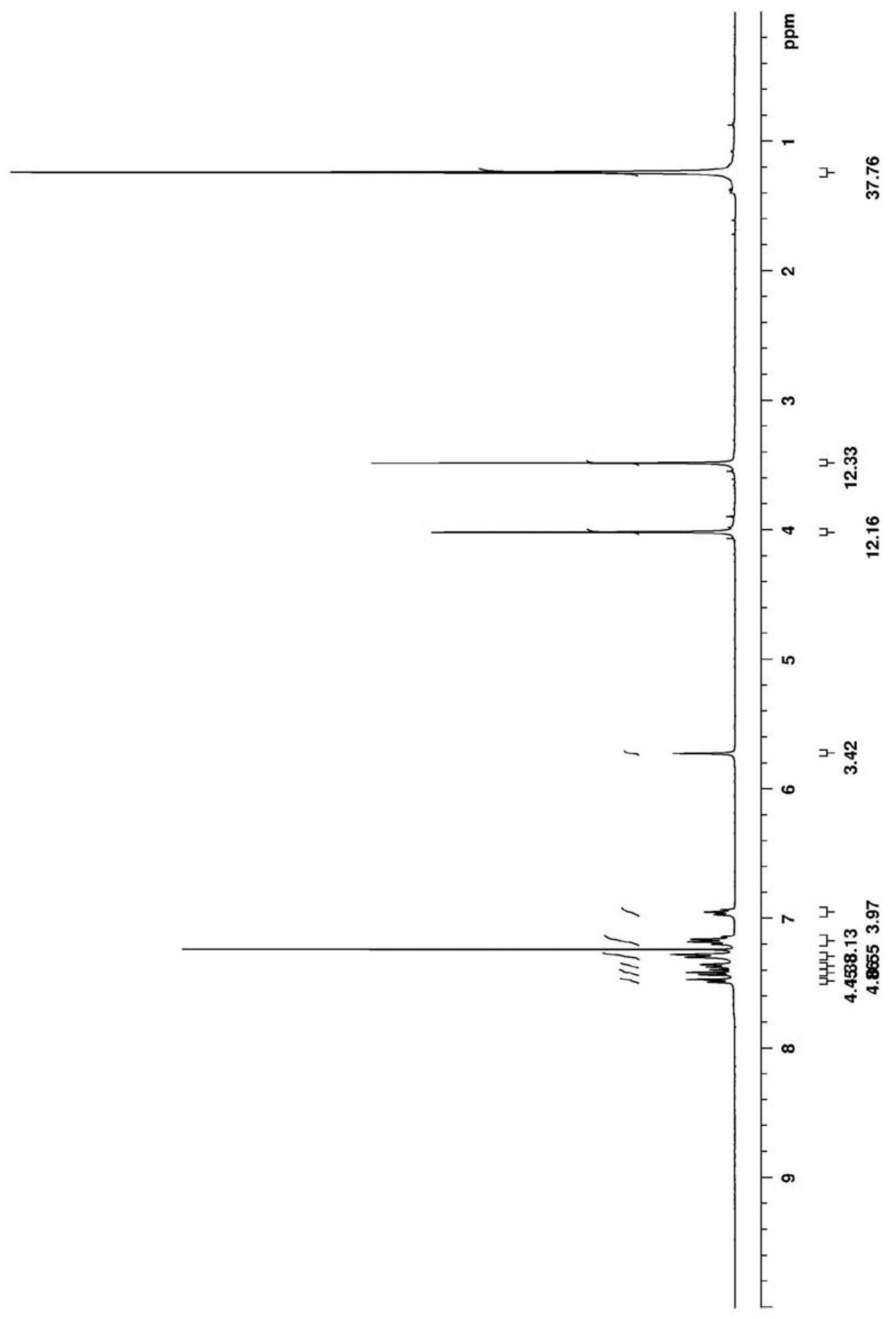


${ }^{13}$ C NMR Spectrum of $7 \mathbf{b}$

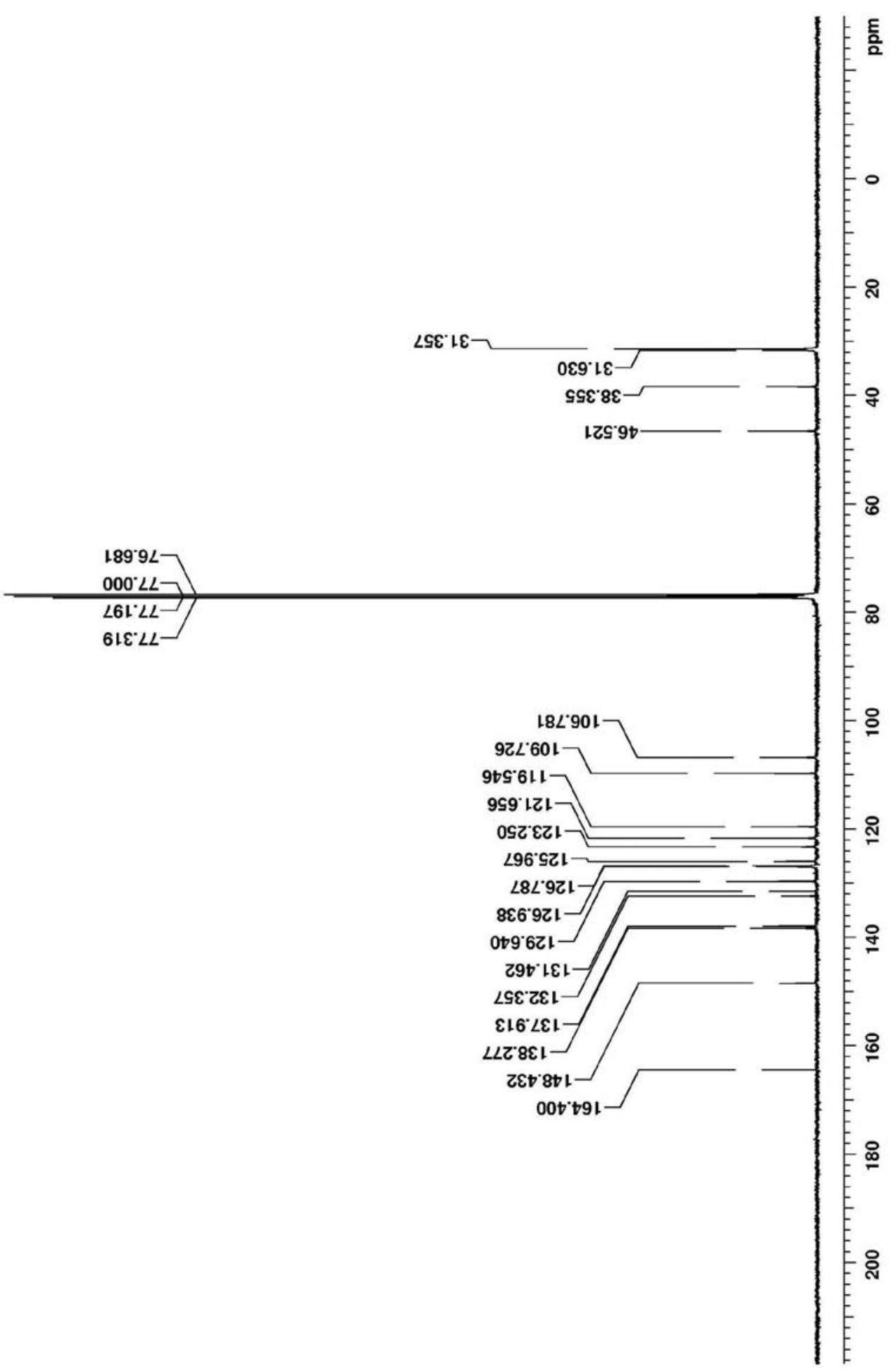




\section{${ }^{1} \mathrm{H}$ NMR Spectrum of $\mathbf{7 c}$}

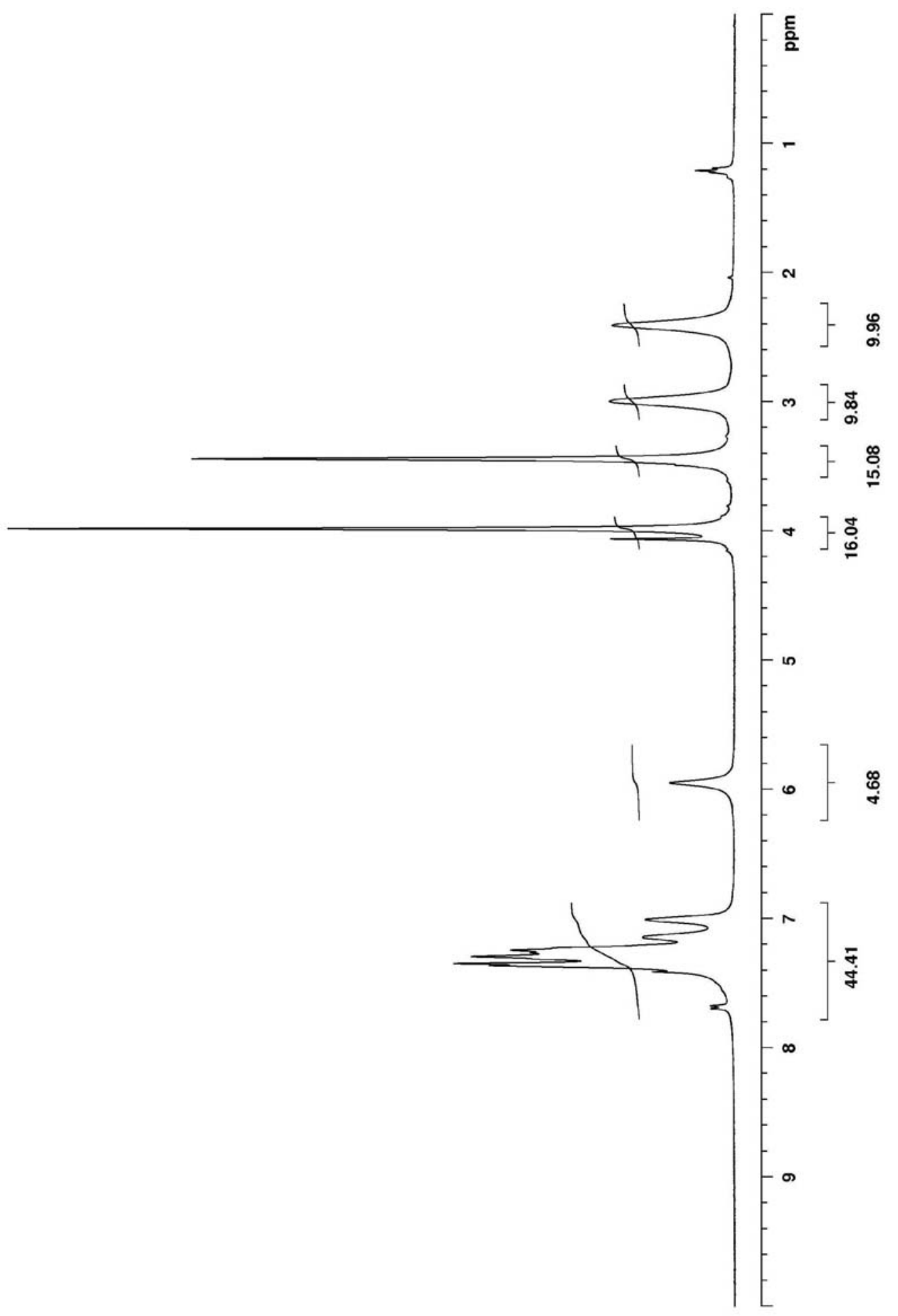


${ }^{13}$ C NMR Spectrum of 7c

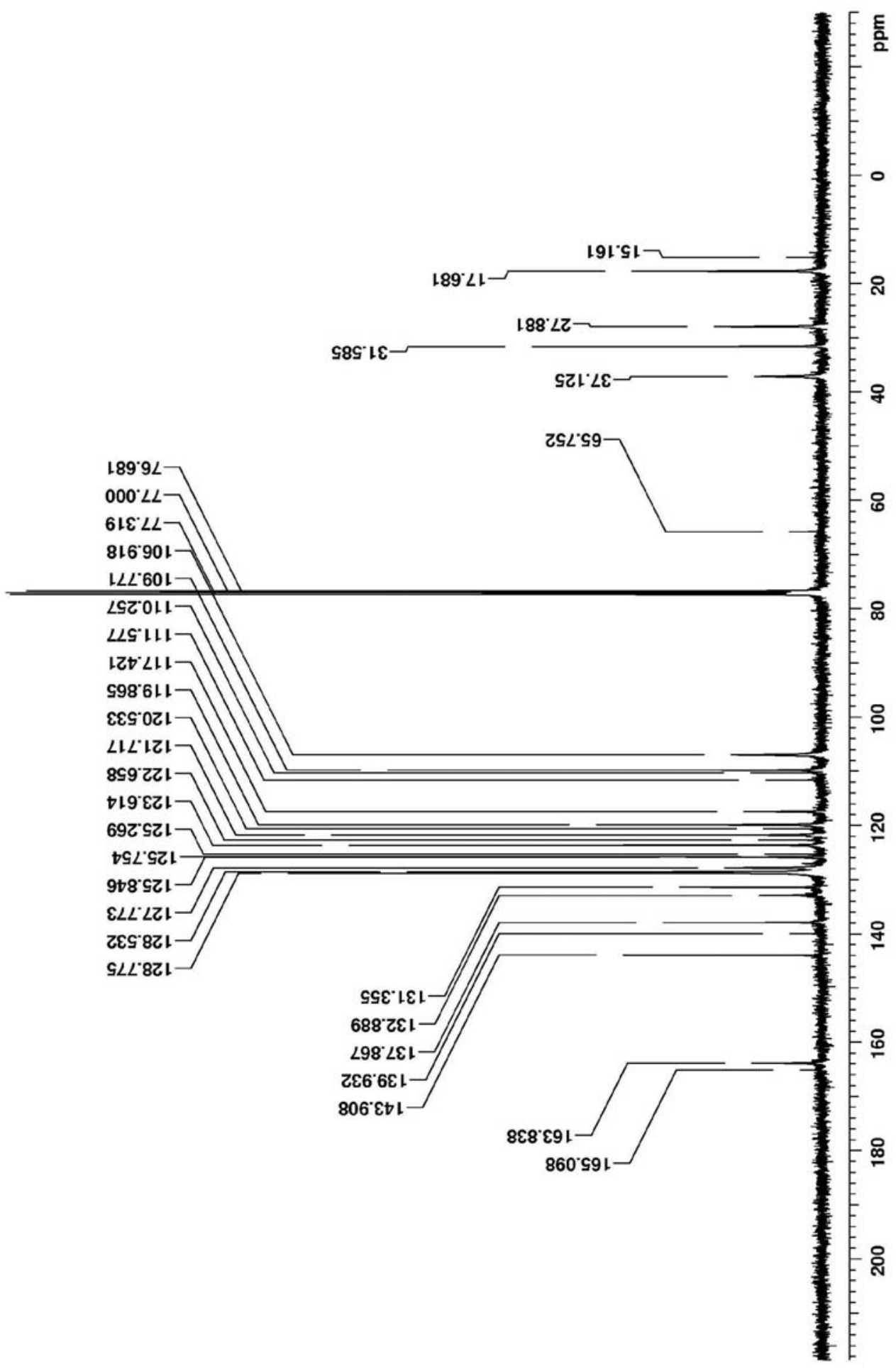




\section{${ }^{1} \mathrm{H}$ NMR Spectrum of $\mathbf{8 b}$}

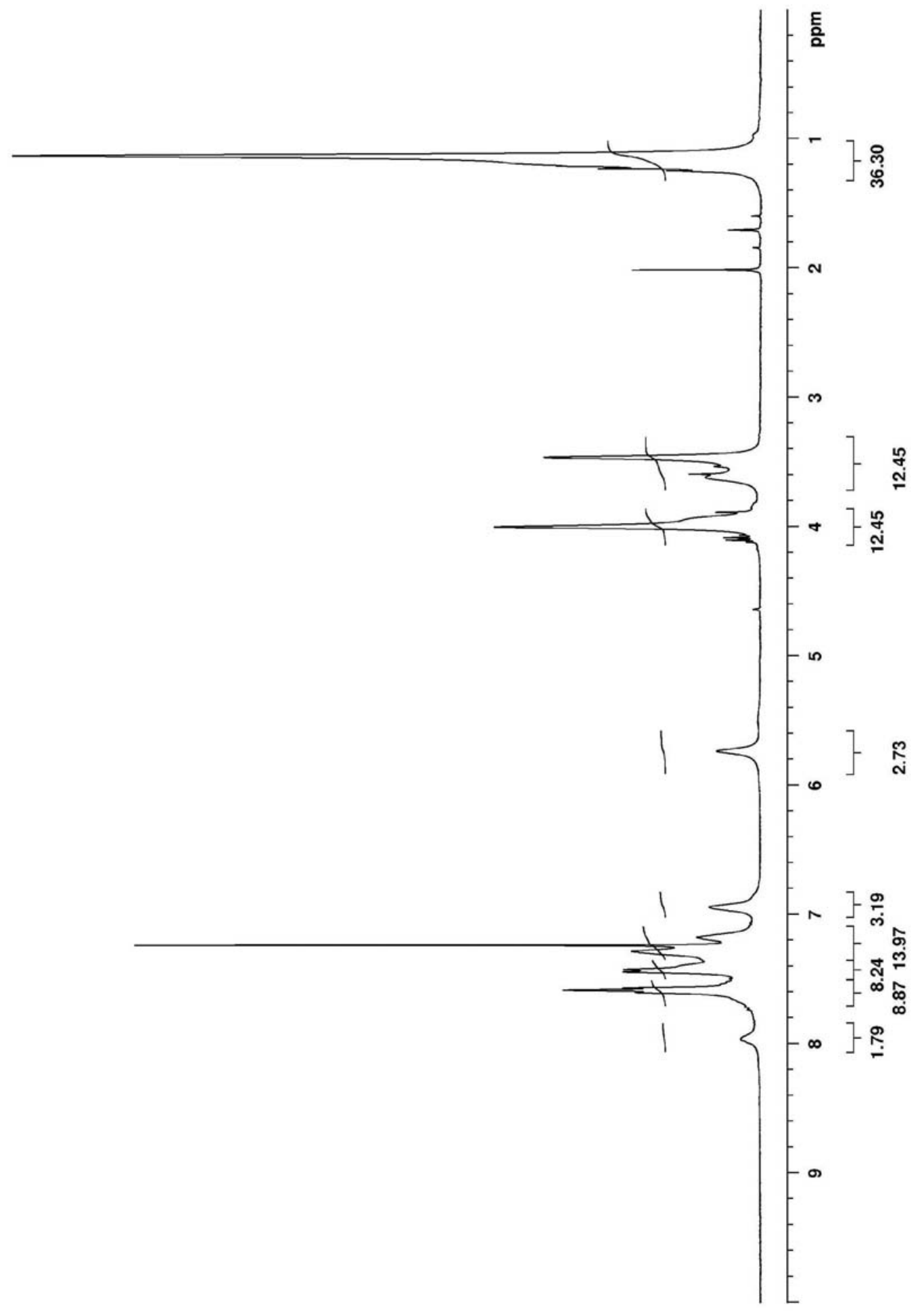


${ }^{1} \mathrm{H}$ NMR Spectrum of 9

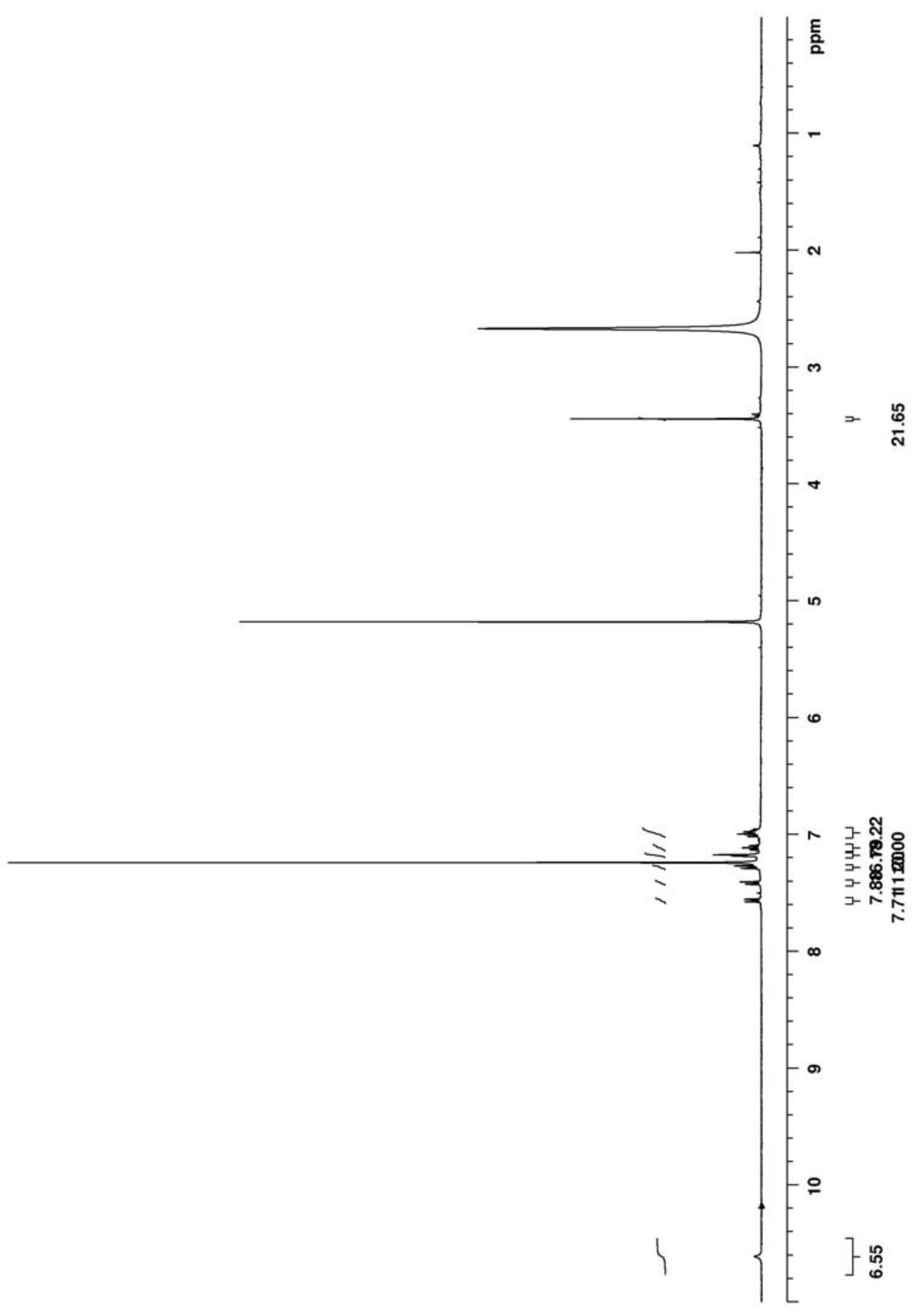


${ }^{1}$ H NMR Spectrum of $\mathbf{1 0}$

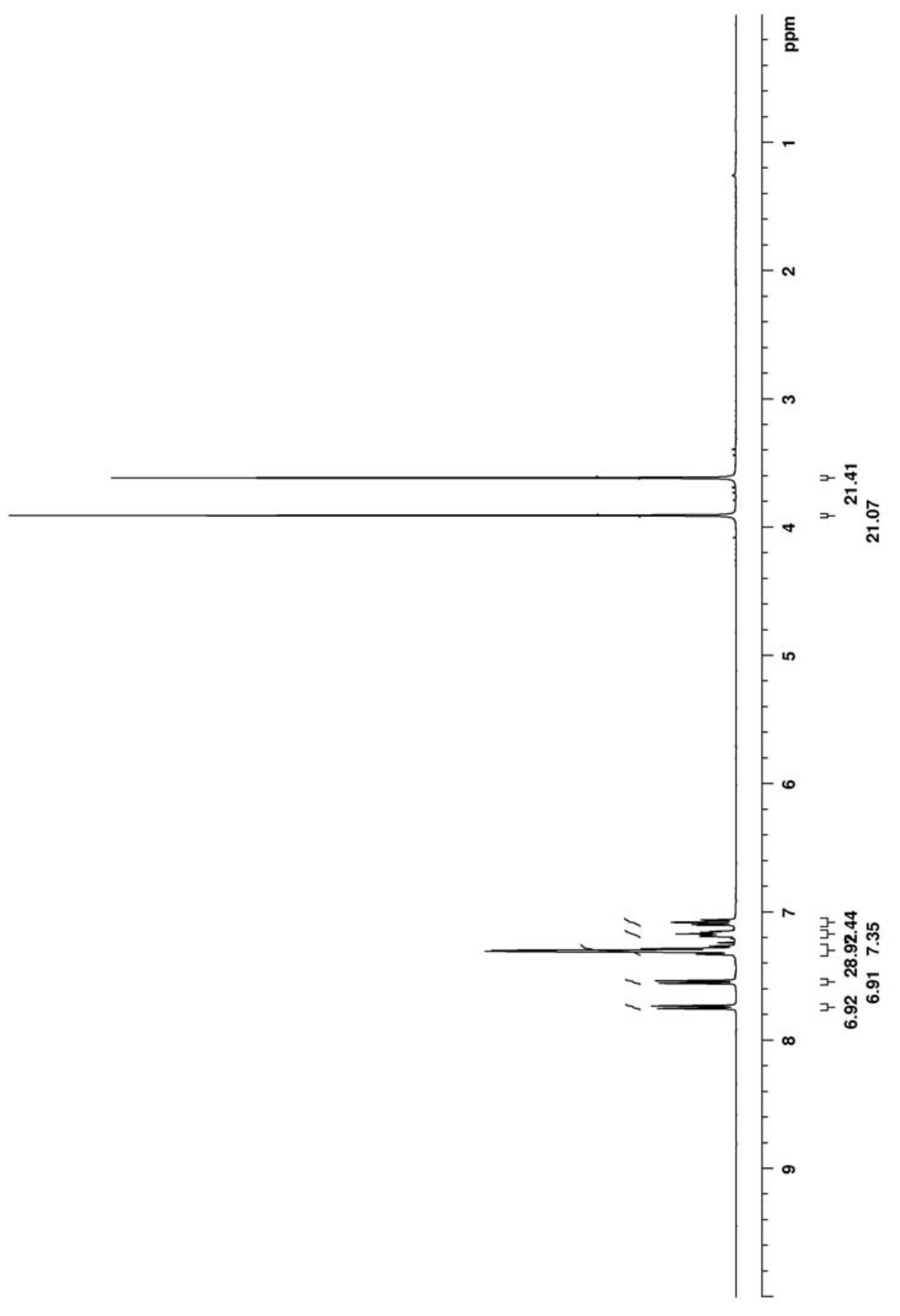


${ }^{13} \mathrm{C}$ NMR Spectrum of $\mathbf{1 0}$

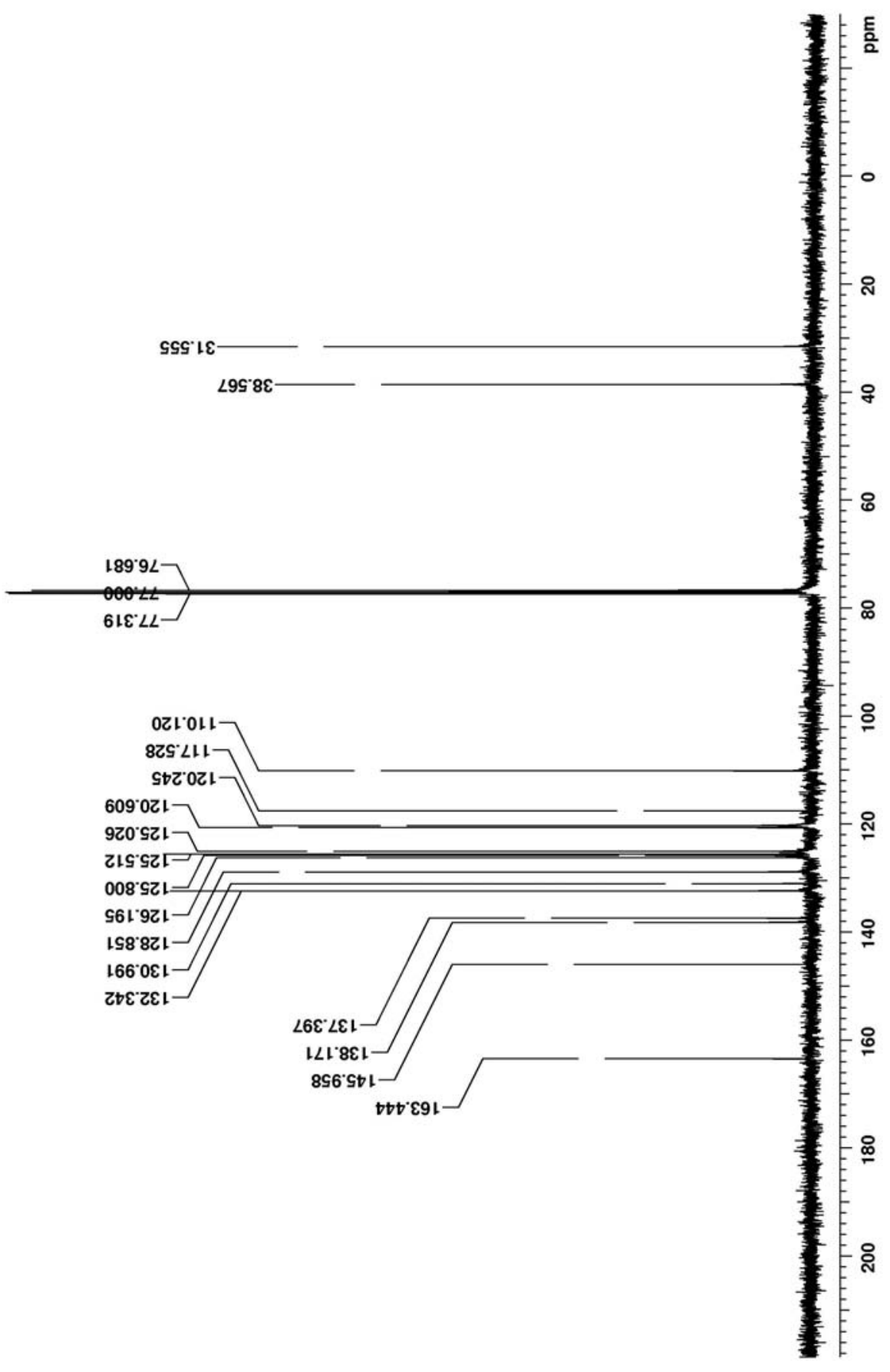


${ }^{1} \mathrm{H}$ NMR Spectrum of $\mathbf{1 4 b}$

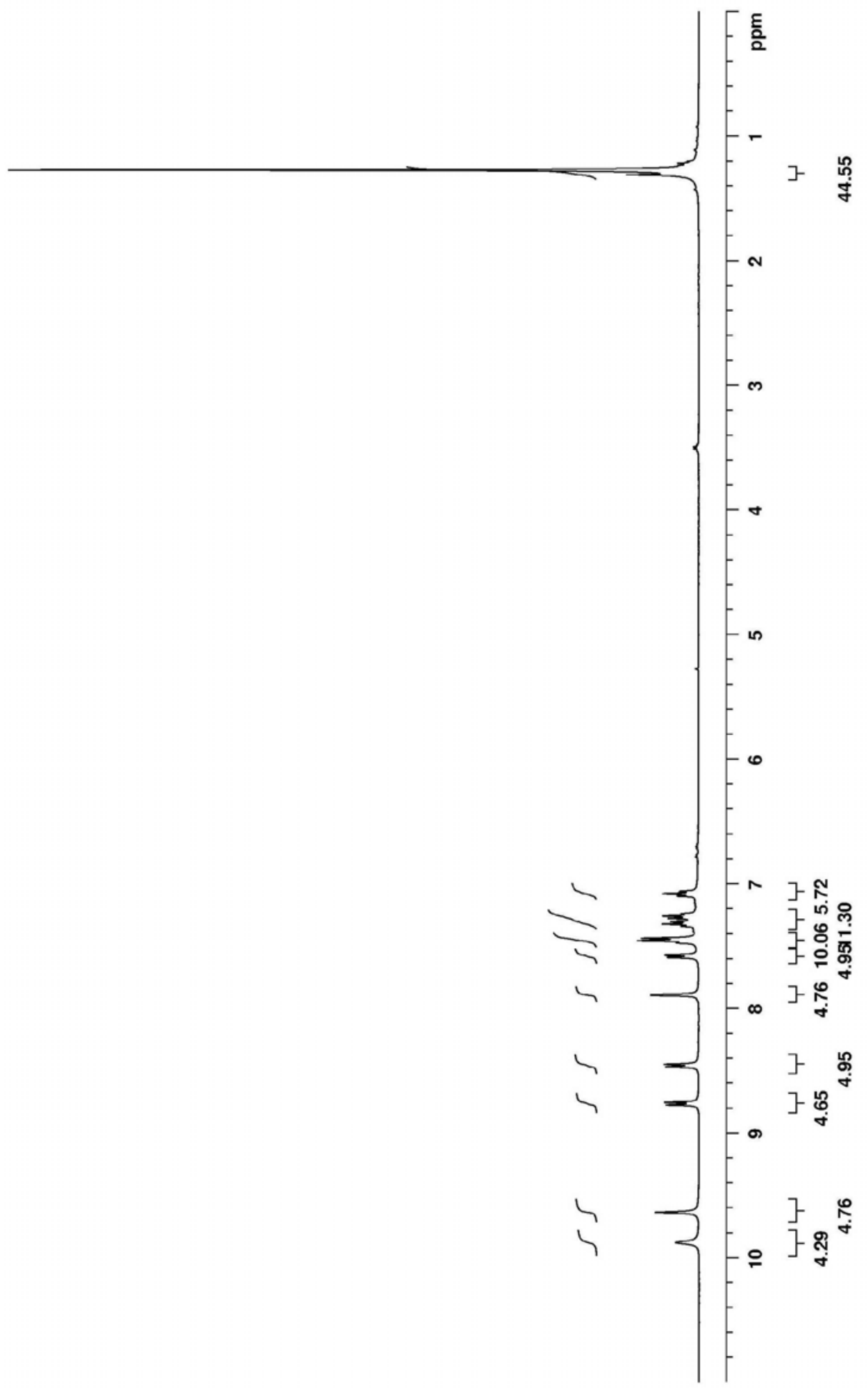


${ }^{13} \mathrm{C}$ NMR Spectrum of $\mathbf{1 4 b}$

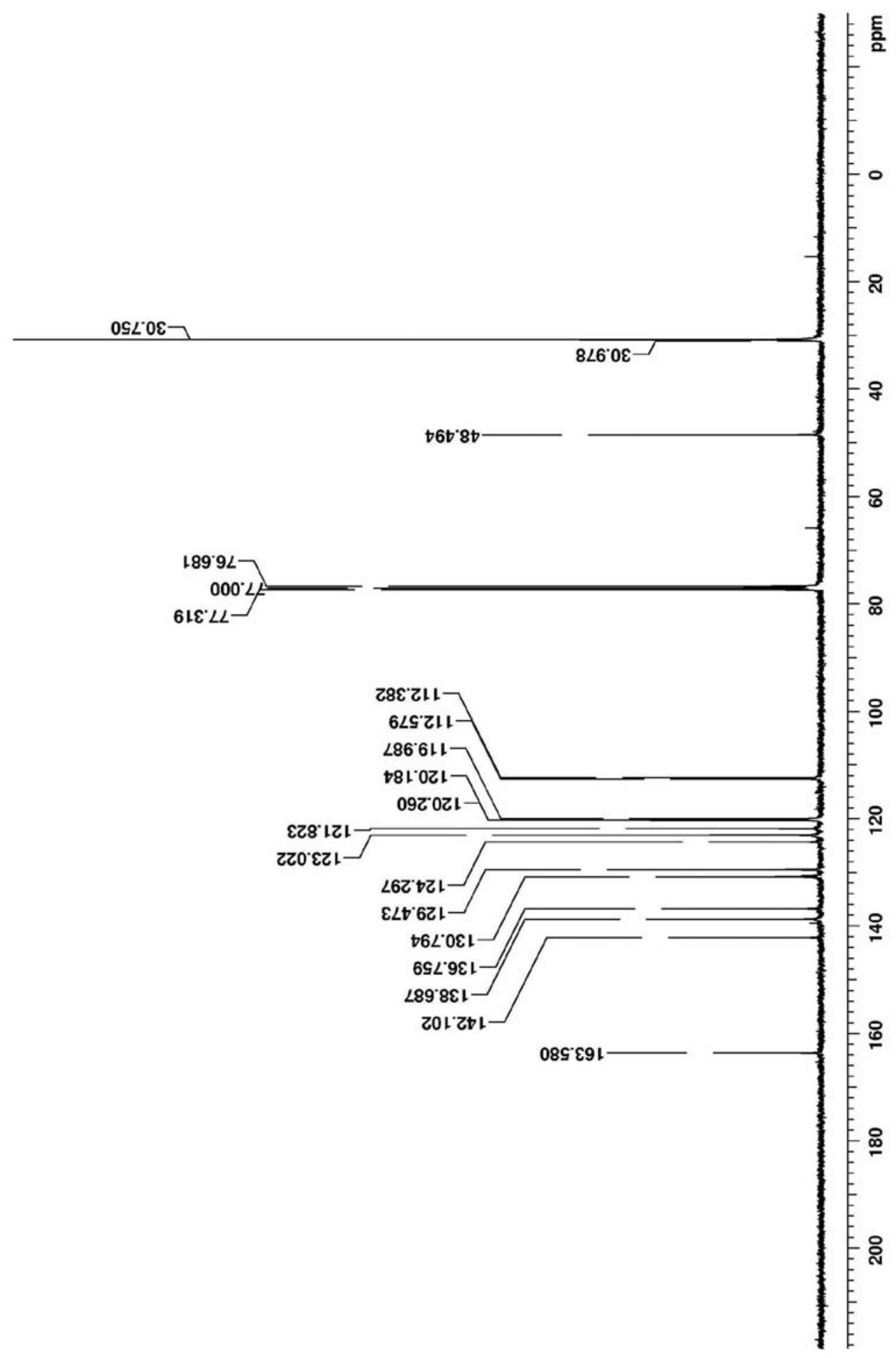


${ }^{1} \mathrm{H}$ NMR Spectrum of $\mathbf{1 6 b}$

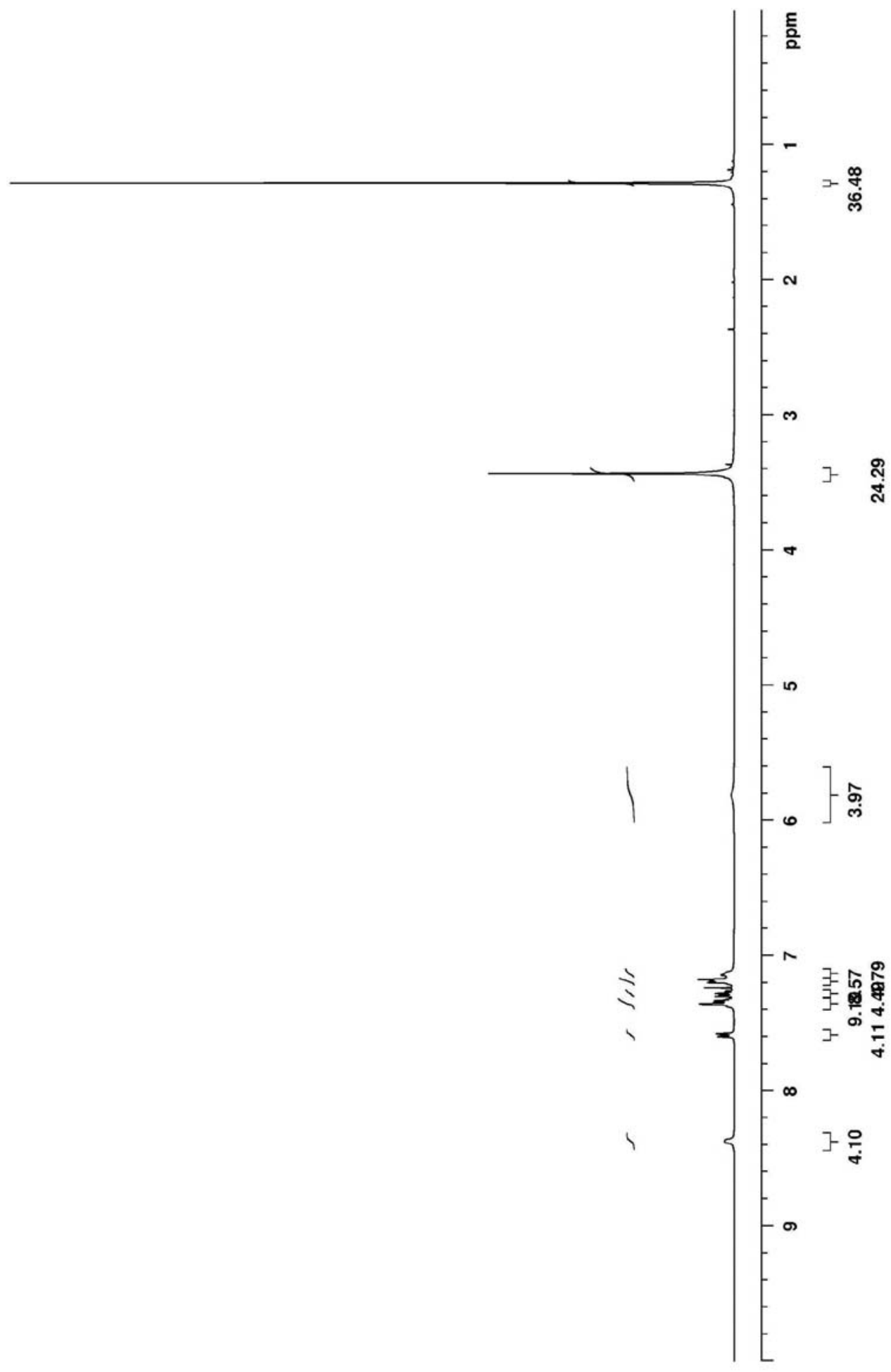


${ }^{13} \mathrm{C}$ NMR Spectrum of $\mathbf{1 6 b}$

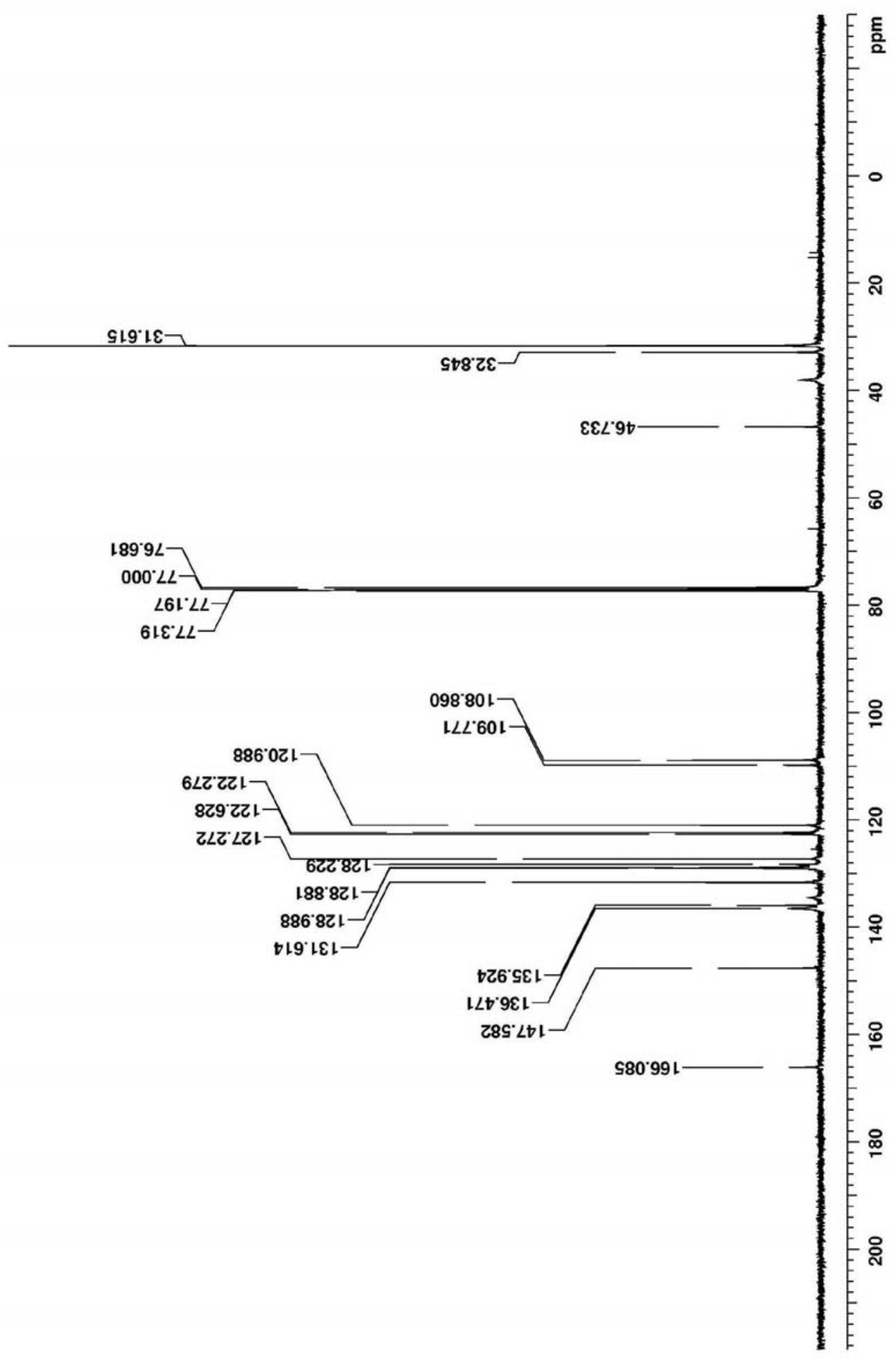




\section{Appendix 2}

Simple Thiazocine-2-acetic Acid Derivatives via Ring Closing Metathesis

Dallas K. Bates, * Xiaofen Li and Parag V. Jog

\section{Supplemental Information}

(Compound numbers correspond to the numbers in the article:

Bates, D. K.; Li, X.; Jog, P. V. J. Org. Chem. 2004, 69, 2750)

Table of Contents

Experimental section

$149-160$

${ }^{1}$ H NMR Spectrum of 1

${ }^{13} \mathrm{C}$ NMR Spectrum of 1

${ }^{1} \mathrm{H}$ NMR Spectrum of 2

163

${ }^{13}$ C NMR Spectrum of 2

${ }^{1} \mathrm{H}$ NMR Spectrum of 3

165

${ }^{13}$ C NMR Spectrum of 3

$\begin{array}{ll}{ }^{1} \text { H NMR Spectrum of } 4 & 167\end{array}$

${ }^{13} \mathrm{C}$ NMR Spectrum of $4 \quad 168$

${ }^{1} H$ NMR Spectrum of 5 (high $R_{f}$ diastereomer) 169

${ }^{13} C$ NMR Spectrum of 5 (high $R_{f}$ diastereomer) 170

${ }^{1} H$ NMR Spectrum of 5(low $R_{f}$ diastereomer) 171

${ }^{13}$ C NMR Spectrum of 5(low $R_{\mathrm{f}}$ diastereomer) 172

${ }^{1}$ H NMR Spectrum of $6 \quad 173$

${ }^{13}$ C NMR Spectrum of $6 \quad 174$ 
${ }^{1}$ H NMR Spectrum of 7

${ }^{13}$ C NMR Spectrum of 7

${ }^{1}$ H NMR Spectrum of 8

${ }^{13} \mathrm{C}$ NMR Spectrum of 8

178

${ }^{1} \mathrm{H}$ NMR Spectrum of 9

${ }^{13} \mathrm{C}$ NMR Spectrum of 9

${ }^{13} \mathrm{C}{ }^{1} \mathrm{H}$ NMR Spectrum of 10

${ }^{13} \mathrm{C}$ NMR Spectrum of 10

${ }^{1}$ H NMR Spectrum of 11

183

${ }^{13}$ C NMR Spectrum of 11

${ }^{1}$ H NMR Spectrum of 12

${ }^{13}$ C NMR Spectrum of 12 186

${ }^{1}$ H NMR Spectrum of 14

${ }^{13}$ C NMR Spectrum of 14 188

${ }^{1}$ H NMR Spectrum of 15

${ }^{13} \mathrm{C}$ NMR Spectrum of 15 190

${ }^{1}$ H NMR Spectrum of 16

${ }^{13} \mathrm{C}$ NMR Spectrum of 16

${ }^{1}$ H NMR Spectrum of 17

${ }^{13} \mathrm{C}$ NMR Spectrum of 17

${ }^{1}$ H NMR Spectrum of 18

195

${ }^{13} \mathrm{C}$ NMR Spectrum of 18 196

${ }^{1}$ H NMR Spectrum of 19 
${ }^{13}$ C NMR Spectrum of $19 \quad 198$

\begin{tabular}{l}
${ }^{1}$ H NMR Spectrum of 20 \\
\hline
\end{tabular}

${ }^{13}$ C NMR Spectrum of $20 \quad 200$

${ }^{1}$ H NMR Spectrum of $21 \quad 201$

${ }^{13}$ C NMR Spectrum of $21 \quad 202$ 


\section{Experimental Section (Introduction and compounds 7-12 and 13-20)}

All reagents were used without purification unless otherwise noted. Silica gel (70-230 mesh, $60 \AA$ ) was used for column chromatography. Melting points were taken on a Fisher-Johns melting point apparatus and are uncorrected. IR spectra were recorded in chloroform solution. NMR spectra were recorded in $\mathrm{CDCl}_{3}$ solution unless otherwise noted $\left({ }^{1} \mathrm{H}\right.$ NMR at $400 \mathrm{MHz}$ and ${ }^{13} \mathrm{C} \mathrm{NMR}$ at $\left.100 \mathrm{MHz}\right)$. Mass spectra were obtained under electron impact at $70 \mathrm{eV}$. Gen2 refers to Grubbs' second Generation catalyst [CAS registry number 246047-72-3, 1,3-bis(2,4,6-trimethylphenyl)-2imidazolidinylidene)dichloro(phenylmethylene)(tricycohexylphosphine)ruthenium].

Methyl $\gamma$-bromocrotonate was prepared according to the literature: ${ }^{35}{ }^{1} \mathrm{H}$ NMR $\delta 6.95(\mathrm{~m}$, 1H), $5.99(\mathrm{dt}, 1 \mathrm{H}, J=15.6,1.2 \mathrm{~Hz}), 3.97(\mathrm{dd}, 2 \mathrm{H}, J=7.6,1.2 \mathrm{~Hz}), 3.71(\mathrm{~s}, 3 \mathrm{H}) ;{ }^{13} \mathrm{C}$ NMR $\delta 165.9,141.9,124.1,51.8,29.0 . \quad N$-Allyl-4-methylbenzensulfonamide was prepared according to the literature: mp $62-63{ }^{\circ} \mathrm{C}\left(\right.$ lit. $\left.{ }^{36} 63-65{ }^{\circ} \mathrm{C}\right) ;{ }^{1} \mathrm{H}$ NMR $\delta 7.73(\mathrm{~d}$, $2 \mathrm{H}, J=8.0 \mathrm{~Hz}), 7.29(\mathrm{~d}, 2 \mathrm{H}, J=8.0 \mathrm{~Hz}), 5.70(\mathrm{~m}, 1 \mathrm{H}), 5.14(\mathrm{dd}, 1 \mathrm{H}, J=17.2,1.2 \mathrm{~Hz})$, $5.08(\mathrm{dd}, 1 \mathrm{H}, J=9.2,1.2 \mathrm{~Hz}), 4.50(\mathrm{br} \mathrm{s}, 1 \mathrm{H}), 3.56(\mathrm{t}, 2 \mathrm{H}, J=6.0 \mathrm{~Hz}), 2.41(\mathrm{~s}, 3 \mathrm{H}) ;{ }^{13} \mathrm{C}$ NMR $\delta 143.5,137.0,133.0,129.7,127.1,117.7,45.8,21.5$.

(2E)-4-[allyl[(4-methylphenyl)sulfonyl]amino]but-2-enoic acid (7). To an ice-cooled solution of $\mathrm{LiOH}^{\cdot} \mathrm{H}_{2} \mathrm{O}(1.5 \mathrm{eq}, 0.95 \mathrm{~g}, 22.5 \mathrm{mmol}) \mathrm{in}_{2} \mathrm{O}(40 \mathrm{~mL})$ and THF $(40 \mathrm{~mL})$ was added at $0{ }^{\circ} \mathrm{C}$ a solution of $\mathbf{1}(4.64 \mathrm{~g}, 15 \mathrm{mmol})$ in THF $(40 \mathrm{~mL})$. This mixture was stirred at $0{ }^{\circ} \mathrm{C}$ for $3 \mathrm{~h}$ under a nitrogen atmosphere, then extracted with EtOAc. The aqueous layer was acidified with $5 \% \mathrm{HCl}$ solution followed by extraction with EtOAc. The combined organic layers were dried over anhydrous $\mathrm{Na}_{2} \mathrm{SO}_{4}$ and concentrated in vacuo to 
give $4.22 \mathrm{~g}(95 \%)$ of $\mathbf{7}$ as a white solid, which was used directly for preparation of $\mathbf{8}$ or 14. ${ }^{1} \mathrm{H}$ NMR $\delta 7.68(\mathrm{~d}, 2 \mathrm{H}, J=8.0 \mathrm{~Hz}), 7.30(\mathrm{~d}, 2 \mathrm{H}, J=8.0 \mathrm{~Hz}), 6.81(\mathrm{dt}, 1 \mathrm{H}, J=15.6$, $5.6 \mathrm{~Hz}), 5.85(\mathrm{dt}, 1 \mathrm{H}, J=15.6,1.6 \mathrm{~Hz}), 5.59(\mathrm{~m}, 1 \mathrm{H}), 5.17-5.10(\mathrm{~m}, 2 \mathrm{H}), 3.93(\mathrm{dd}, 2 \mathrm{H}, J$ $=5.6,1.6 \mathrm{H} \mathrm{z}), 3.79(\mathrm{~d}, 2 \mathrm{H}, J=6.4 \mathrm{~Hz}), 2.42(\mathrm{~s}, 3 \mathrm{H}) ;{ }^{13} \mathrm{C} \mathrm{NMR} \delta 174.6,145.3,143.8$, $136.7,132.2,129.8,127.2,119.9,117.6,50.6,47.3,21.6$.

\section{$N$-allyl- $N$-[(2E)-4-(1H-indol-1-yl)-4-oxobut-2-enyl]-4-methylbenzenesulfonamide}

(8). Method 1: To an ice-cooled solution of $7(0.71 \mathrm{~g}, 2.4 \mathrm{mmol})$ in THF $(25 \mathrm{~mL})$ was added dropwise oxalyl chloride $(12.0 \mathrm{eq}, 2.52 \mathrm{~mL}, 28.9 \mathrm{mmol})$ at $0{ }^{\circ} \mathrm{C}$. The resulting mixture was stirred at room temperature for $24 \mathrm{~h}$ and concentrated in vacuo. The residue was used directly for the following reaction. To a well-stirred mixture of indole (1.1 eq, $0.31 \mathrm{~g}, 2.7 \mathrm{mmol}$ ), powdered $\mathrm{NaOH}(1.5 \mathrm{eq}, 0.14 \mathrm{~g}, 3.6 \mathrm{mmol})$ and tetrabutylammonium hydrogen sulfate ( $0.01 \mathrm{eq}, 0.01 \mathrm{~g}$ ) in $\mathrm{CH}_{2} \mathrm{Cl}_{2}(15 \mathrm{~mL})$ was added dropwise a solution of above residue in $\mathrm{CH}_{2} \mathrm{Cl}_{2}(10 \mathrm{~mL})$ in an ice-water bath. The resulting mixture was stirred at $0{ }^{\circ} \mathrm{C}$ for $15 \mathrm{~min}$ and at room temperature for $3 \mathrm{~h}$, poured into ice-water, and extracted with $\mathrm{CH}_{2} \mathrm{Cl}_{2}$. The combined organic layers were washed with distilled water, dried over anhydrous $\mathrm{Na}_{2} \mathrm{SO}_{4}$, and concentrated in vacuo. Column chromatography on silica gel (EtOAc/hexane, 1:9) gave $0.24 \mathrm{~g}(25 \%)$ of 8 as a sticky liquid.

Method 2: To a solution of $\mathbf{1 4}(1.10 \mathrm{~g}, 2.78 \mathrm{mmol})$ in dioxane $(50 \mathrm{~mL})$ was added a solution of DDQ (6.0 eq, $3.78 \mathrm{~g}, 16.68 \mathrm{mmol})$ in dioxane $(30 \mathrm{~mL})$ at $55{ }^{\circ} \mathrm{C}$ through a syringe. The resulting mixture was then heated to $70{ }^{\circ} \mathrm{C}$ and stirred for 3 days. The mixture was vacuum filtered and the filtrate was concentrated in vacuo. The residue was then stirred in $\mathrm{MeOH}(60 \mathrm{~mL})$ at room temperature for $1 \mathrm{~h}$. After the evaporation of 
$\mathrm{MeOH}$, the residue was diluted with $\mathrm{CH}_{2} \mathrm{Cl}_{2}(100 \mathrm{~mL})$, washed with $5 \% \mathrm{NaHCO}_{3}$ solution followed by distilled water and concentrated in vacuo. Column chromatography on silica gel (EtOAc/hexane, 1:4) gave $0.98 \mathrm{~g}(89 \%)$ of 8 as a sticky liquid: IR $1690 \mathrm{~cm}^{-1}$; ${ }^{1} \mathrm{H}$ NMR $\delta 8.45(\mathrm{~d}, 1 \mathrm{H}, J=7.6 \mathrm{~Hz}), 7.72(\mathrm{~d}, 2 \mathrm{H}, J=8.4 \mathrm{~Hz}), 7.55(\mathrm{~d}, 1 \mathrm{H}, J=7.6 \mathrm{~Hz})$, $7.39(\mathrm{~d}, 1 \mathrm{H}, J=4.0 \mathrm{~Hz}), 7.36-7.25(\mathrm{~m}, 4 \mathrm{H}), 6.98(\mathrm{dt}, 1 \mathrm{H}, J=15.2,5.2 \mathrm{~Hz}), 6.76(\mathrm{dt}, 1 \mathrm{H}$, $J=15.2,1.6 \mathrm{~Hz}), 6.64(\mathrm{dd}, 1 \mathrm{H}, J=4.0,0.8 \mathrm{~Hz}), 5.64(\mathrm{~m}, 1 \mathrm{H}), 5.19-5.14(\mathrm{~m}, 2 \mathrm{H}), 4.05$

(dd, $2 \mathrm{H}, J=5.2,1.6 \mathrm{~Hz}), 3.85(\mathrm{~d}, 2 \mathrm{H}, J=6.4 \mathrm{~Hz}), 2.39(\mathrm{~s}, 3 \mathrm{H}) ;{ }^{13} \mathrm{C}$ NMR $\delta 163.3,144.1$, $143.8,136.7,135.7,132.3,130.6,130.0,127.2,125.1,124.6,123.9,123.0,120.9,119.9$, 116.7, 109.4, 50.9, 47.7, 21.5; MS [m/z (rel intensity)] $394\left(\mathrm{M}^{+}, 27\right), 278$ (8), 239 (13), 155 (69), 123 (6), 117 (60).

\section{$N$-allyl- $N$-[2-(allylthio)-4-(1H-indol-1-yl)-4-oxobutyl]-4-}

methylbenzenesulfonamide (9). To an ice-cooled solution of allyl mercaptan (1.2 eq, $0.07 \mathrm{~mL}, 0.9 \mathrm{mmol})$ and $\mathrm{Et}_{3} \mathrm{~N}(1.2 \mathrm{eq}, 0.125 \mathrm{~mL}, 0.9 \mathrm{mmol})$ in $\mathrm{CH}_{2} \mathrm{Cl}_{2}(10 \mathrm{~mL})$ was added a solution of $8(0.30 \mathrm{~g}, 0.75 \mathrm{mmol})$ in $\mathrm{CH}_{2} \mathrm{Cl}_{2}(6 \mathrm{~mL})$ at $0{ }^{\circ} \mathrm{C}$. The resulting mixture was stirred at $0{ }^{\circ} \mathrm{C}$ for $15 \mathrm{~min}$ and at room temperature for $6 \mathrm{~h}$, then diluted with $\mathrm{CH}_{2} \mathrm{Cl}_{2}$, washed with $5 \% \mathrm{HCl}$ solution followed by distilled water. The organic layer was dried over anhydrous $\mathrm{Na}_{2} \mathrm{SO}_{4}$, and concentrated in vacuo. Column chromatography on silica gel (EtOAc/hexane, 1:4) gave $0.28 \mathrm{~g}(80 \%)$ of 9 as a sticky liquid: IR $1705 \mathrm{~cm}^{-1}$; ${ }^{1} \mathrm{H}$ NMR $\delta 8.47(\mathrm{~d}, 1 \mathrm{H}, J=8.0 \mathrm{~Hz}), 7.67(\mathrm{~d}, 2 \mathrm{H}, J=8.0 \mathrm{~Hz}), 7.55(\mathrm{~d}, 1 \mathrm{H}, J=7.6 \mathrm{~Hz})$, $7.47(\mathrm{~d}, 1 \mathrm{H}, J=4.0 \mathrm{~Hz}), 7.36-7.26(\mathrm{~m}, 4 \mathrm{H}), 6.64(\mathrm{~d}, 1 \mathrm{H}, J=3.6 \mathrm{~Hz}), 5.76(\mathrm{~m}, 1 \mathrm{H}), 5.56$ $(\mathrm{m}, 1 \mathrm{H}), 5.23-5.02(\mathrm{~m}, 4 \mathrm{H}), 3.89-3.78(\mathrm{~m}, 2 \mathrm{H}), 3.57-3.46(\mathrm{~m}, 3 \mathrm{H}), 3.29-3.06(\mathrm{~m}, 4 \mathrm{H})$, $2.40(\mathrm{~s}, 3 \mathrm{H}) ;{ }^{13} \mathrm{C}$ NMR $\delta 169.3,143.7,136.3,135.7,134.5,132.5,130.4,129.8,127.3$, 
$125.0,124.7,123.7,120.8,120.0,117.6,116.7,109.3,52.0,51.8,39.1,38.6,35.5,21.5$

MS [m/z (rel intensity)] 313 (65), 224 (72), 155 (89), 117 (68).

\section{$N$-allyl- $N$-[2-(allylsulfinyl)-4-(1H-indol-1-yl)-4-oxobutyl]-4-}

methylbenzenesulfonamide (10). To an ice-cooled solution of $9(1.5 \mathrm{~g}, 3.21 \mathrm{mmol})$ in $\mathrm{CH}_{2} \mathrm{Cl}_{2}(25 \mathrm{~mL})$ was added slowly a solution of $m$-CPBA $(1.0 \mathrm{eq})$ in $\mathrm{CH}_{2} \mathrm{Cl}_{2}(20 \mathrm{~mL})$. The resulting mixture was stirred at $0{ }^{\circ} \mathrm{C}$ for $15 \mathrm{~min}$ and put in a freezer $\left(-20{ }^{\circ} \mathrm{C}\right)$ overnight. The reaction mixture was then poured into $5 \% \mathrm{NaHCO}_{3}$ solution. After separating and saving the organic layer, the aqueous layer was extracted with $\mathrm{CH}_{2} \mathrm{Cl}_{2}$. The combined organic layers were washed with distilled water, dried over anhydrous $\mathrm{Na}_{2} \mathrm{SO}_{4}$, and concentrated in vacuo. Column chromatography on silica gel (EtOAc/hexane, 3:2) gave $1.12 \mathrm{~g}(72 \%)$ of $\mathbf{1 0}$ as a sticky liquid (as a mixture of two diastereomers in a 2:3 ratio): IR $1698 \mathrm{~cm}^{-1},{ }^{1} \mathrm{H}$ NMR $\delta 8.38(\mathrm{~d}, 1 \mathrm{H}, J=7.6 \mathrm{~Hz}), 7.69$ (d, $2 \mathrm{H}, J=8.0 \mathrm{~Hz}), 7.55(\mathrm{~d}, 1 \mathrm{H}, J=7.6 \mathrm{~Hz}), 7.47(\mathrm{~d}, 1 \mathrm{H}, J=3.6 \mathrm{~Hz}), 7.36-7.25(\mathrm{~m}, 4 \mathrm{H})$, $6.67(\mathrm{~d}, 1 \mathrm{H}, J=4.0 \mathrm{~Hz}), 5.97(\mathrm{~m}, 1 \mathrm{H}), 5.59(\mathrm{~m}, 1 \mathrm{H}), 5.48-5.43(\mathrm{~m}, 2 \mathrm{H}), 5.22(\mathrm{dd}, 1 \mathrm{H}, J=$ 17.2, $1.2 \mathrm{~Hz}), 5.16(\mathrm{dd}, 1 \mathrm{H}, J=10.0,1.2 \mathrm{~Hz}), 3.86(\mathrm{~d}, 2 \mathrm{H}, J=6.4 \mathrm{~Hz}), 3.79(\mathrm{~m}, 1 \mathrm{H}), 3.69$ (m, 1H), 3.64-3.59 (m, 2H), $3.57(\mathrm{~m}, 1 \mathrm{H}), 3.48(\mathrm{~m}, 1 \mathrm{H}), 3.43(\mathrm{dd}, 1 \mathrm{H}, J=7.2,2.0 \mathrm{~Hz})$ $2.39(\mathrm{~s}, 3 \mathrm{H}) ;{ }^{13} \mathrm{C}$ NMR $\delta 168.6,144.0,135.6,132.4,130.4,130.0,127.4,127.2,126.0$, 125.3, 124.4, 124.0, 123.9, 121.1, 120.5, 116.5, 110.2, 54.2, 52.5, 51.0, 44.7, 34.1, 21.5.

\section{$N$-allyl- $N$-[2-(allylsulfonyl)-4-(1H-indol-1-yl)-4-oxobutyl]-4-}

methylbenzenesulfonamide (11). To an ice-cooled solution of $9(0.28 \mathrm{~g}, 0.60 \mathrm{mmol})$ in $\mathrm{CH}_{2} \mathrm{Cl}_{2}(8 \mathrm{~mL})$ was added a solution of $m$-CPBA $(2.0$ eq $)$ in $\mathrm{CH}_{2} \mathrm{Cl}_{2}(6 \mathrm{~mL})$. The 
resulting mixture was stirred at room temperature for $12 \mathrm{~h}$ and poured into $5 \% \mathrm{NaHCO}_{3}$ solution. After separating and saving the organic layer, the aqueous layer was extracted with $\mathrm{CH}_{2} \mathrm{Cl}_{2}$. The combined organic layers were washed with distilled water, dried over anhydrous $\mathrm{Na}_{2} \mathrm{SO}_{4}$, and concentrated in vacuo. Column chromatography on silica gel (EtOAc/hexane, 3:7) gave $0.17 \mathrm{~g}(57 \%)$ of 11 as a sticky liquid: IR $1703 \mathrm{~cm}^{-1} ;{ }^{1} \mathrm{H}$ NMR $\delta$ $8.42(\mathrm{~d}, 1 \mathrm{H}, J=8.0 \mathrm{~Hz}), 7.68(\mathrm{~d}, 2 \mathrm{H}, J=8.0 \mathrm{~Hz}), 7.56(\mathrm{~d}, 1 \mathrm{H}, J=7.6 \mathrm{~Hz}), 7.48(\mathrm{~d}, 1 \mathrm{H}, J$ $=4.0 \mathrm{~Hz}), 7.35-7.33(\mathrm{~m}, 1 \mathrm{H}), 7.30-7.26(\mathrm{~m}, 3 \mathrm{H}), 6.67(\mathrm{dd}, 1 \mathrm{H}, J=4.0,0.4 \mathrm{~Hz}), 5.93(\mathrm{~m}$, 1H), 5.59-5.48 (m, 3H), $5.23(\mathrm{dd}, 1 \mathrm{H}, J=17.2,1.2 \mathrm{~Hz}), 5.16(\mathrm{dd}, 1 \mathrm{H}, J=10.0,1.2 \mathrm{~Hz})$, $4.37(\mathrm{~m}, 1 \mathrm{H}), 3.99-3.78(\mathrm{~m}, 4 \mathrm{H}), 3.70-3.59(\mathrm{~m}, 2 \mathrm{H}), 3.53-3.46(\mathrm{~m}, 2 \mathrm{H}), 2.39(\mathrm{~s}, 3 \mathrm{H}) ;{ }^{13} \mathrm{C}$ NMR $\delta 168.0,144.2,135.6,135.2,131.8,130.5,130.0,127.5,125.7,125.3,124.4$ $124.1,124.0,121.1,121.0,116.5,110.2,58.8,54.9,52.4,46.2,32.6,21.5$.

\section{1-[[4-[4-(methylphenyl)sulfonyl]-1-oxido-3,4,5,8-tetrahydro-2H-1,4-thiazocin-2-}

yl]acetyl]-1H-indole (12). To a solution of $\mathbf{1 0}(0.79 \mathrm{~g}, 1.63 \mathrm{mmol}, 1: 1 \mathrm{ratio})$ in $\mathrm{CH}_{2} \mathrm{Cl}_{2}$ $(10 \mathrm{~mL})$ was added a solution of Gen 2 catalyst $(10 \% \mathrm{~mol}, 0.14 \mathrm{~g})$ in $\mathrm{CH}_{2} \mathrm{Cl}_{2}(8 \mathrm{~mL})$ under a nitrogen atmosphere. The resulting mixture was refluxed for $2.5 \mathrm{~h}$, cooled to room temperature and concentrated in vacuo, Column chromatography of the residue on silica gel (EtOAc) gave $0.36 \mathrm{~g}(48 \%)$ of 12 as a white solid (a mixture of two diastereomers in a $2: 3$ ratio): $\mathrm{mp} 149-51{ }^{\circ} \mathrm{C}$.

minor diastereomer: ${ }^{1} \mathrm{H}$ NMR $\delta 8.39(\mathrm{~d}, 1 \mathrm{H}, J=8.0 \mathrm{~Hz}), 7.66(\mathrm{~d}, 2 \mathrm{H}, J=8.4 \mathrm{~Hz})$, $7.54(\mathrm{~d}, 1 \mathrm{H}, J=7.6 \mathrm{~Hz}), 7.41(\mathrm{~d}, 1 \mathrm{H}, J=4.0 \mathrm{~Hz}), 7.35-7.25(\mathrm{~m}, 4 \mathrm{H}), 6.64(\mathrm{~d}, 1 \mathrm{H}, J=4.0$ Hz), $5.90(\mathrm{~m}, 1 \mathrm{H}), 5.64(\mathrm{~m}, 1 \mathrm{H}), 4.42(\mathrm{~m}, 1 \mathrm{H}), 4.17-3.79(\mathrm{~m}, 5 \mathrm{H}), 3.47(\mathrm{dd}, 1 \mathrm{H}, J=17.2$, $6.0 \mathrm{~Hz}), 3.09-3.03(\mathrm{~m}, 2 \mathrm{H}), 2.36(\mathrm{~s}, 3 \mathrm{H})$. 
major diastereomer: IR $1702 \mathrm{~cm}^{-1} ;{ }^{1} \mathrm{H}$ NMR $\delta 8.32(\mathrm{~d}, 1 \mathrm{H}, J=8.4 \mathrm{~Hz}), 7.59(\mathrm{~d}, 2 \mathrm{H}, J$ $=8.4 \mathrm{~Hz}), 7.54(\mathrm{~d}, 1 \mathrm{H}, J=7.2 \mathrm{~Hz}), 7.47(\mathrm{~d}, 1 \mathrm{H}, J=4.0 \mathrm{~Hz}), 7.34-7.26(\mathrm{~m}, 4 \mathrm{H}), 6.66(\mathrm{~d}$, $1 \mathrm{H}, J=4.0 \mathrm{~Hz}), 5.95(\mathrm{dt}, 1 \mathrm{H}, J=11.6,3.2 \mathrm{~Hz}), 5.84(\mathrm{~m}, 1 \mathrm{H}), 4.44(\mathrm{~m}, 1 \mathrm{H}), 4.30(\mathrm{~d}, 1 \mathrm{H}$, $J=18.4 \mathrm{~Hz}), 3.99(\mathrm{dd}, 1 \mathrm{H}, J=14.0,8.8 \mathrm{~Hz}), 3.91(\mathrm{~m}, 1 \mathrm{H}), 3.83(\mathrm{dd}, 1 \mathrm{H}, J=17.2,2.4$ $\mathrm{Hz}), 3.74-3.64(\mathrm{~m}, 2 \mathrm{H}), 3.26(\mathrm{dd}, 1 \mathrm{H}, J=14.0,2.4 \mathrm{~Hz}), 3.16(\mathrm{dd}, 1 \mathrm{H}, J=17.2,10.0 \mathrm{~Hz})$, 2.39 (s, 3H); ${ }^{13} \mathrm{C}$ NMR $\delta 168.0,167.5,144.2,144.1,135.6,135.5,134.0,133.7,132.7$, $130.4,130.1,130.0,127.4,127.1,125.4,125.3,124.3,124.1,124.0,121.1,118.8,116.4$, 116.3, 110.3, 110.2, 55.4, 51.2, 49.9, 49.2, 48.8, 48.1, 46.2, 44.6, 34.7, 33.6, 21.46. Anal. Calcd for $\mathrm{C}_{23} \mathrm{H}_{24} \mathrm{O}_{5} \mathrm{~N}_{2} \mathrm{~S}_{2}: \mathrm{C}, 60.51 ; \mathrm{H}, 5.30 ; \mathrm{N}, 6.14$. Found: $\mathrm{C}, 60.18 ; \mathrm{H}, 5.43 ; \mathrm{N}, 6.00$ (on diastereomer mixture).

\section{$N$-Allyl- $N$-[(2E)-4-(2,3-dihydro-1H-indol-1-yl)-4-oxobut-2-enyl]-4-}

methylbenzenesulfonamide (14). To an ice-cooled solution of 7 (0.68 g, $2.3 \mathrm{mmol})$ in THF $(25 \mathrm{~mL})$ was added dropwise oxalyl chloride $(12.0 \mathrm{eq}, 2.41 \mathrm{~mL}, 27.66 \mathrm{mmol})$ at 0 ${ }^{\circ} \mathrm{C}$. The resulting mixture was stirred at room temperature for $24 \mathrm{~h}$ then concentrated in vacuo and the residue was used directly in the following reaction. To an ice-cooled solution of indoline (1.2 eq, $0.33 \mathrm{~g}, 2.8 \mathrm{mmol})$, pyridine (10.0 eq, $1.88 \mathrm{~mL}, 23.1 \mathrm{mmol})$ and DMAP $(0.05 \mathrm{~g})$ in $\mathrm{CH}_{2} \mathrm{Cl}_{2}(15 \mathrm{~mL})$ was added slowly a solution of the above residue in $\mathrm{CH}_{2} \mathrm{Cl}_{2}(15 \mathrm{~mL})$ at $0{ }^{\circ} \mathrm{C}$. The resulting mixture was stirred at $0{ }^{\circ} \mathrm{C}$ for $15 \mathrm{~min}$ and at room temperature overnight. The solution was poured into $5 \%$ aqueous $\mathrm{HCl}$ and extracted with $\mathrm{CH}_{2} \mathrm{Cl}_{2}$. The combined organic layers were washed with $5 \% \mathrm{HCl}$ solution followed by distilled water, dried over anhydrous $\mathrm{Na}_{2} \mathrm{SO}_{4}$ and concentrated in vacuo. Column chromatography on silica gel (EtOAc/hexane, 2:3) gave $0.59 \mathrm{~g}(64 \%)$ of 14 as a 
white solid: $\mathrm{mp} 123-5{ }^{\circ} \mathrm{C}$; IR $1666 \mathrm{~cm}^{-1}$; ${ }^{1} \mathrm{H}$ NMR $\delta 8.22(\mathrm{~d}, 1 \mathrm{H}, J=7.2 \mathrm{~Hz}), 7.69(\mathrm{~d}, 2 \mathrm{H}$, $J=8.4 \mathrm{~Hz}), 7.28(\mathrm{~d}, 2 \mathrm{H}, J=8.0 \mathrm{~Hz}), 7.17(\mathrm{t}, 2 \mathrm{H}, J=7.6 \mathrm{~Hz}), 7.00(\mathrm{t}, 1 \mathrm{H}, J=7.6 \mathrm{~Hz})$, $6.77(\mathrm{dt}, 1 \mathrm{H}, J=14.8,5.2 \mathrm{~Hz}), 6.37(\mathrm{~d}, 1 \mathrm{H}, J=14.8 \mathrm{~Hz}), 5.61(\mathrm{~m}, 1 \mathrm{H}), 5.15-5.11(\mathrm{~m}$, 2H), 4.10-4.05 (m, 2H), $3.98(\mathrm{~d}, 2 \mathrm{H}, J=4.8 \mathrm{~Hz}), 3.81(\mathrm{~d}, 2 \mathrm{H}, J=6.4 \mathrm{~Hz}), 3.16(\mathrm{t}, 2 \mathrm{H}, J$ $=7.6 \mathrm{~Hz}), 2.39(\mathrm{~s}, 3 \mathrm{H}) ;{ }^{13} \mathrm{C}$ NMR $\delta 163.2,143.6,142.7,140.2,136.9,132.3,131.5$, $129.8,127.5,127.1,124.5,123.9,119.6,117.4,50.4,48.0,47.6,27.9,21.4 ;$ MS [m/z (rel intensity)] $396\left(\mathrm{M}^{+}, 10\right), 241$ (4), 155 (29), 119 (83). Anal. Calcd for $\mathrm{C}_{22} \mathrm{H}_{24} \mathrm{O}_{3} \mathrm{~N}_{2} \mathrm{~S}: \mathrm{C}$, 66.64; H, 6.10; N, 7.07. Found: C, 66.56; H, 6.16; N, 7.07.

\section{$N$-allyl- $N$-[2-(allylthio)-4-(2,3-dihydro-1H-indol-1-yl)-4-oxobutyl]-4-}

methylbenzenesulfonamide (15). To an ice-cooled solution of allyl mercaptan (3.0 eq, $0.36 \mathrm{~mL}, 4.5 \mathrm{mmol})$ and sodium methoxide $(1.12 \mathrm{eq}, 0.09 \mathrm{~g}, 1.7 \mathrm{mmol})$ in $\mathrm{MeOH}(15$ $\mathrm{mL})$ and THF $(5 \mathrm{~mL})$ was added a solution of $14(0.59 \mathrm{~g}, 1.5 \mathrm{mmol})$ in THF $(10 \mathrm{~mL})$ at 0 ${ }^{\circ} \mathrm{C}$. The resulting mixture was stirred at $0{ }^{\circ} \mathrm{C}$ for $15 \mathrm{~min}$. The solution was then poured into ice water and extracted with $\mathrm{CH}_{2} \mathrm{Cl}_{2}$. The combined organic layers were washed with distilled water, dried over anhydrous $\mathrm{Na}_{2} \mathrm{SO}_{4}$, and concentrated in vacuo. Column chromatography on silica gel (EtOAc/hexane, 3:7) gave $0.67 \mathrm{~g}(95 \%)$ of 15 as a sticky liquid: IR $1659 \mathrm{~cm}^{-1} ;{ }^{1} \mathrm{H}$ NMR $\delta 8.22(\mathrm{~d}, 1 \mathrm{H}, J=8.8 \mathrm{~Hz}), 7.65(\mathrm{~d}, 2 \mathrm{H}, J=8.4 \mathrm{~Hz}), 7.27$ (d, 2H, $J=8.4 \mathrm{~Hz}), 7.16(\mathrm{t}, 2 \mathrm{H}, J=7.2 \mathrm{~Hz}), 6.98(\mathrm{t}, 1 \mathrm{H}, J=7.2 \mathrm{~Hz}), 5.78(\mathrm{~m}, 1 \mathrm{H}), 5.49$ (m, 1H), 5.19-5.04 (m, 4H), 4.11-4.05 (m, 2H), $3.84(\mathrm{~d}, 2 \mathrm{H}, J=6.4 \mathrm{~Hz}), 3.55-3.42(\mathrm{~m}$, 2H), 3.28-3.13 (m, 5H), $3.00(\mathrm{dd}, 1 \mathrm{H}, J=16.8,4.4 \mathrm{~Hz}), 2.65(\mathrm{dd}, 1 \mathrm{H}, J=16.8,7.6 \mathrm{~Hz})$, 2.39 (s, 3H); ${ }^{13} \mathrm{C}$ NMR $\delta 168.7,143.4,143.0,136.7,134.6,132.3,131.3,129.7,127.4$, 
$127.2,124.5,123.6,119.8,117.4,117.0,51.5,51.4,48.1,39.0,38.7,35.4,27.9,21.5 ; \mathrm{MS}$ [m/z (rel intensity)] 315 (25), 224 (21), 155 (52), 119 (65).

\section{1-[[4-[4-(methylphenyl)sulfonyl]-1,1-dioxido-3,4,5,8-tetrahydro-2H-1,4-thiazocin-} 2-yl]acetyl]-1H-indole (16). To a solution of $\mathbf{1 1}(0.07 \mathrm{~g}, 0.14 \mathrm{mmol})$ in $\mathrm{CH}_{2} \mathrm{Cl}_{2}(4 \mathrm{~mL})$ was added a solution of commercial Gen 2 catalyst $(5 \% \mathrm{~mol}, 0.006 \mathrm{~g})$ in $\mathrm{CH}_{2} \mathrm{Cl}_{2}(3 \mathrm{~mL})$ under a nitrogen atmosphere. The mixture was refluxed until TLC showed the reaction to be complete, cooled to room temperature and concentrated in vacuo. Column chromatography of the residue on silica gel (EtOAc/hexane, 3:2) gave $0.06 \mathrm{~g}(91 \%)$ of $\mathbf{1 6}$ as a white solid: $\mathrm{mp} 237-9{ }^{\circ} \mathrm{C}$; IR $1711 \mathrm{~cm}^{-1}$; ${ }^{1} \mathrm{H}$ NMR $\delta 8.32(\mathrm{~d}, 1 \mathrm{H}, J=8.0 \mathrm{~Hz}), 7.65(\mathrm{~d}$, $2 \mathrm{H}, J=8.4 \mathrm{~Hz}), 7.55(\mathrm{~d}, 1 \mathrm{H}, J=7.2 \mathrm{~Hz}), 7.45(\mathrm{~d}, 1 \mathrm{H}, J=4.0 \mathrm{~Hz}), 7.34-7.25(\mathrm{~m}, 4 \mathrm{H})$, $6.67(\mathrm{~d}, 1 \mathrm{H}, J=4.0 \mathrm{~Hz}), 5.88(\mathrm{~d}, 1 \mathrm{H}, J=12.4 \mathrm{~Hz}), 5.79(\mathrm{~m}, 1 \mathrm{H}), 4.80(\mathrm{~m}, 1 \mathrm{H}), 4.42-4.35$ (m, 2H), 4.02-3.92 (m, 2H), 3.79-3.73 (m, 2H), $3.22(\mathrm{dd}, 1 \mathrm{H}, J=13.2,3.2 \mathrm{~Hz}), 3.03(\mathrm{dd}$, $1 \mathrm{H}, J=17.6,10.8 \mathrm{~Hz}), 2.39$ (s, 3H); ${ }^{13} \mathrm{C}$ NMR $\delta 167.1,144.4,135.5,133.8,130.4,130.2$, $127.3,125.4,124.3,124.1,121.2,117.7,116.3,110.6,60.4,53.1,49.8,49.1,30.2,21.5$. Anal. Calcd for $\mathrm{C}_{23} \mathrm{H}_{24} \mathrm{O}_{5} \mathrm{~N}_{2} \mathrm{~S}_{2}$ : C, 58.46; H, 5.12; N, 5.93. Found: C, 58.18; H, 5.28; N, 5.76 .

\section{$N$-allyl- $N$-[2-(allylsulfinyl)-4-(2,3-dihydro-1H-indol-1-yl)-4-oxobutyl]-4-}

methylbenzenesulfonamide (17). To an ice-cooled solution of $\mathbf{1 5}(0.85 \mathrm{~g}, 1.81 \mathrm{mmol})$ in $\mathrm{CH}_{2} \mathrm{Cl}_{2}(20 \mathrm{~mL})$ was added slowly a solution of $m$-CPBA $\left(1.0\right.$ eq) in $\mathrm{CH}_{2} \mathrm{Cl}_{2}(10 \mathrm{~mL})$. The resulting mixture was stirred at $0{ }^{\circ} \mathrm{C}$ for $15 \mathrm{~min}$ and put in a freezer $\left(-20{ }^{\circ} \mathrm{C}\right)$ overnight. The reaction mixture was then poured into $5 \% \mathrm{NaHCO}_{3}$ solution. After 
separating and saving the organic layer, the aqueous layer was extracted with $\mathrm{CH}_{2} \mathrm{Cl}_{2}$. The combined organic layers were washed with distilled water, dried over anhydrous $\mathrm{Na}_{2} \mathrm{SO}_{4}$, and concentrated in vacuo. Column chromatography on silica gel (EtOAc) gave $0.87 \mathrm{~g}(99 \%)$ of $\mathbf{1 7}$ as a sticky liquid (a mixture of two diastereomers present in a ratio of 2:3).

minor diastereomer: IR $1656 \mathrm{~cm}^{-1} ;{ }^{1} \mathrm{H}$ NMR $\delta 8.14(\mathrm{~d}, 1 \mathrm{H}, J=7.6 \mathrm{~Hz}), 7.67(\mathrm{~d}, 2 \mathrm{H}, J$ $=8.0 \mathrm{~Hz}), 7.28(\mathrm{~d}, 2 \mathrm{H}, J=8.0 \mathrm{~Hz}), 7.18-7.15(\mathrm{~m}, 2 \mathrm{H}), 7.00(\mathrm{~m}, 1 \mathrm{H}), 5.95(\mathrm{~m}, 1 \mathrm{H}), 5.53$ (m, 1H), 5.44-5.40 (m, 2H), $5.21(\mathrm{dd}, 1 \mathrm{H}, J=17.2,1.6 \mathrm{~Hz}), 5.14(\mathrm{dd}, 1 \mathrm{H}, J=10.0,0.8$ Hz), 4.12-4.06 (m, 2H), $3.84(\mathrm{~d}, 2 \mathrm{H}, J=6.8 \mathrm{~Hz}), 3.70-3.54(\mathrm{~m}, 4 \mathrm{H}), 3.38(\mathrm{dd}, 1 \mathrm{H}, J=$ $13.2,3.6 \mathrm{~Hz}), 3.20$ (t, $2 \mathrm{H}, J=8.4 \mathrm{~Hz}), 3.12(\mathrm{dd}, 1 \mathrm{H}, J=17.6,4.4 \mathrm{~Hz}), 2.91(\mathrm{dd}, 1 \mathrm{H}, J=$ 17.6, 8.0 Hz), 2.39 (s, 3H); ${ }^{13} \mathrm{C}$ NMR $\delta 167.8,143.8,142.6,135.9,132.3,131.2,129.9$, $127.4,127.3,126.3,124.7,124.0,123.6,120.3,116.9,54.3,52.1,51.3,48.0,44.7,34.2$, 27.9, 21.5.

major diastereomer: IR $1656 \mathrm{~cm}^{-1} ;{ }^{1} \mathrm{H}$ NMR $\delta 8.17(\mathrm{~d}, 1 \mathrm{H}, J=8.8 \mathrm{~Hz}), 7.68(\mathrm{~d}, 2 \mathrm{H}, J$ $=8.0 \mathrm{~Hz}), 7.28(\mathrm{~d}, 2 \mathrm{H}, J=8.0 \mathrm{~Hz}), 7.17(\mathrm{~m}, 2 \mathrm{H}), 7.01(\mathrm{td}, 1 \mathrm{H}, J=7.2,1.6 \mathrm{~Hz}), 5.87(\mathrm{~m}$, $1 \mathrm{H}), 5.53-5.39(\mathrm{~m}, 3 \mathrm{H}), 5.22-5.11(\mathrm{~m}, 2 \mathrm{H}), 4.17-4.06(\mathrm{~m}, 2 \mathrm{H}), 3.87-3.84(\mathrm{~m}, 3 \mathrm{H}), 3.59$ (dd, $1 \mathrm{H}, J=14.4,9.2 \mathrm{~Hz}), 3.54(\mathrm{dd}, 1 \mathrm{H}, J=13.6,8.0 \mathrm{~Hz}), 3.40$ (dd, $1 \mathrm{H}, J=12.8,7.2$ Hz), 3.30 (dd, 1H, $J=14.4,6.4 \mathrm{~Hz}), 3.22-3.13(\mathrm{~m}, 3 \mathrm{H}), 2.68$ (dd, 1H, $J=18.0,4.8 \mathrm{~Hz})$, $2.39(\mathrm{~s}, 3 \mathrm{H}) ;{ }^{13} \mathrm{C}$ NMR $\delta 168.1,143.8,142.8,135.9,132.4,132.0,131.3,129.9,127.3$, $127.2,126.0,124.7,123.9,120.4,116.9,54.9,52.1,51.7,48.0,29.0,28.0,21.5 ; \mathrm{MS}[\mathrm{m} / \mathrm{z}$ (rel intensity)] 396 (9), 241 (9), 155 (35), 119 (100) for diastereomer mixture. 


\section{1-[[4-[4-(methylphenyl)sulfonyl]-1-oxido-3,4,5,8-tetrahydro-2H-1,4-thiazocin-2-}

yl]acetyl]indoline (18). To a solution of $17(0.58 \mathrm{~g}, 1.193 \mathrm{mmol})$ in $\mathrm{CH}_{2} \mathrm{Cl}_{2}(12 \mathrm{~mL})$ was added a solution of commercial Gen 2 catalyst $(10 \% \mathrm{~mol}, 0.10 \mathrm{~g})$ in $\mathrm{CH}_{2} \mathrm{Cl}_{2}(10 \mathrm{~mL})$ under a nitrogen atmosphere. The resulting mixture was refluxed for $21 \mathrm{~h}$, cooled to room temperature and concentrated in vacuo. Column chromatography of the residue on silica gel (EtOAc) gave $0.22 \mathrm{~g}(40 \%)$ of $\mathbf{1 8}$ as a mixture of two diastereomers in a 3:4 ratio).

minor diastereomer: ${ }^{1} \mathrm{H}$ NMR $\delta 8.14(\mathrm{~d}, 1 \mathrm{H}, J=7.6 \mathrm{~Hz}), 7.67(\mathrm{~d}, 2 \mathrm{H}, J=8.0 \mathrm{~Hz})$, $7.28(\mathrm{~d}, 2 \mathrm{H}, J=8.0 \mathrm{~Hz}), 7.19-7.15(\mathrm{~m}, 2 \mathrm{H}), 7.02(\mathrm{~m}, 1 \mathrm{H}), 5.89(\mathrm{dt}, 1 \mathrm{H}, J=11.6,3.2 \mathrm{~Hz})$, $5.64(\mathrm{~m}, 1 \mathrm{H}), 4.47(\mathrm{~m}, 1 \mathrm{H}), 4.21-3.71(\mathrm{~m}, 7 \mathrm{H}), 3.19(\mathrm{t}, 2 \mathrm{H}, J=8.4 \mathrm{~Hz}), 3.04-2.95(\mathrm{~m}$, 2H), $2.57(\mathrm{dd}, 1 \mathrm{H}, J=17.2,4.0 \mathrm{~Hz}), 2.39(\mathrm{~s}, 3 \mathrm{H}) ;{ }^{13} \mathrm{C} \mathrm{NMR} \delta 166.7,144.0,142.5,134.2$, $134.0,131.2,130.0,127.5,127.4,124.7,124.2,118.9,116.9,49.1,48.0,46.6,44.6,37.5$ $34.9,28.0,21.5$.

major diastereomer: IR $1655 \mathrm{~cm}^{-1} ;{ }^{1} \mathrm{H}$ NMR $\delta 8.06(\mathrm{~d}, 1 \mathrm{H}, J=8.0 \mathrm{~Hz}), 7.59(\mathrm{~d}, 2 \mathrm{H}, J$ $=8.8 \mathrm{~Hz}), 7.27(\mathrm{~d}, 2 \mathrm{H}, J=8.0 \mathrm{~Hz}), 7.17-7.11(\mathrm{~m}, 2 \mathrm{H}), 7.00(\mathrm{~m}, 1 \mathrm{H}), 5.88(\mathrm{dt}, 1 \mathrm{H}, J=$ 11.6, $4.0 \mathrm{~Hz}), 5.80(\mathrm{~m}, 1 \mathrm{H}), 4.39(\mathrm{~m}, 1 \mathrm{H}), 4.28-3.61(\mathrm{~m}, 7 \mathrm{H}), 3.30(\mathrm{td}, 2 \mathrm{H}, J=14.0,2.8$ $\mathrm{Hz}), 3.18(\mathrm{t}, 2 \mathrm{H}, J=8.4 \mathrm{~Hz}), 2.66(\mathrm{dd}, 1 \mathrm{H}, J=16.8,10.0 \mathrm{~Hz}), 2.38(\mathrm{~s}, 3 \mathrm{H}) ;{ }^{13} \mathrm{C} \mathrm{NMR} \delta$ $167.3,144.1,142.4,134.2,134.0,132.7,130.0,127.4,127.1,124.7,124.1,118.5,116.7$, 55.6, 49.8, 48.6, 48.3, 48.0, 33.5, 28.0, 21.5; MS [m/z (rel intensity)] 155 (26), 119 (88) for diastereomer mixture.

\section{$N$-allyl- $N$-[2-(allylsulfonyl)-4-(2,3-dihydro-1H-indol-1-yl)-4-oxobutyl]-4-}

methylbenzenesulfonamide (19). To an ice-cooled solution of 15 (0.62 g, $1.32 \mathrm{mmol})$ in $\mathrm{CH}_{2} \mathrm{Cl}_{2}(20 \mathrm{~mL})$ was added a solution of $m$-CPBA $(2.0 \mathrm{eq})$ in $\mathrm{CH}_{2} \mathrm{Cl}_{2}(10 \mathrm{~mL})$. The 
resulting mixture was stirred at room temperature for $16 \mathrm{~h}$, poured into $5 \% \mathrm{NaHCO}_{3}$ solution. After separating and saving the organic layer, the aqueous layer was extracted with $\mathrm{CH}_{2} \mathrm{Cl}_{2}$. The combined organic layers were washed with distilled water, dried over anhydrous $\mathrm{Na}_{2} \mathrm{SO}_{4}$, and concentrated in vacuo. Column chromatography on silica gel (EtOAc/hexane, 3:7) gave $0.62 \mathrm{~g}(94 \%)$ of 19 as a sticky liquid: IR $1658 \mathrm{~cm}^{-1} ;{ }^{1} \mathrm{H}$ NMR $\delta$ $8.17(\mathrm{~d}, 1 \mathrm{H}, J=8.0 \mathrm{~Hz}), 7.68(\mathrm{~d}, 2 \mathrm{H}, J=8.4 \mathrm{~Hz}), 7.28(\mathrm{~d}, 2 \mathrm{H}, J=8.4 \mathrm{~Hz}), 7.19-7.16(\mathrm{~m}$, 2H), $7.01(\mathrm{td}, 1 \mathrm{H}, J=7.6,0.8 \mathrm{~Hz}), 5.91(\mathrm{~m}, 1 \mathrm{H}), 5.56-5.43(\mathrm{~m}, 3 \mathrm{H}), 5.21(\mathrm{dd}, 1 \mathrm{H}, J=$ 17.2, $1.2 \mathrm{~Hz}), 5.14(\mathrm{dd}, 1 \mathrm{H}, J=10.0,1.2 \mathrm{~Hz}), 4.28(\mathrm{~m}, 1 \mathrm{H}), 4.18-4.06(\mathrm{~m}, 2 \mathrm{H}), 3.96-3.77$ (m, 4H), $3.64(\mathrm{dd}, 1 \mathrm{H}, J=14.4,9.6 \mathrm{~Hz}), 3.45(\mathrm{dd}, 1 \mathrm{H}, J=14.4,5.2 \mathrm{~Hz}), 3.20(\mathrm{t}, 2 \mathrm{H}, J=$ $8.4 \mathrm{~Hz}), 3.13(\mathrm{dd}, 1 \mathrm{H}, J=18.0,6.4 \mathrm{~Hz}), 3.05(\mathrm{dd}, 1 \mathrm{H}, J=18.0,5.6 \mathrm{~Hz}), 2.40(\mathrm{~s}, 3 \mathrm{H}) ;{ }^{13} \mathrm{C}$ NMR $\delta 167.1,144.0,142.6,135.6,131.7,131.3,129.9,127.4,125.4,124.7,124.1$, $124.0,120.6,116.8,58.5,54.9,51.8,47.9,45.7,32.6,27.9,21.5 ; \mathrm{MS}[\mathrm{m} / \mathrm{z}$ (rel intensity)] 347 (16), 155 (43), 119 (100).

\section{1-[[4-[4-(methylphenyl)sulfonyl]-1,1-dioxido-3,4,5,8-tetrahydro-2H-1,4-thiazocin-}

2-yl]acetyl]indoline (20). To a solution of $19(0.31 \mathrm{~g}, 0.62 \mathrm{mmol})$ in $\mathrm{CH}_{2} \mathrm{Cl}_{2}(10 \mathrm{~mL})$ was added a solution of commercial Gen 2 catalyst $(5 \% \mathrm{~mol}, 0.026 \mathrm{~g})$ in $\mathrm{CH}_{2} \mathrm{Cl}_{2}(5 \mathrm{~mL})$ under a nitrogen atmosphere. The mixture was refluxed until TLC showed the reaction to be complete, then cooled to room temperature and concentrated in vacuo. Column chromatography of the residue on silica gel (EtOAc) gave $0.26 \mathrm{~g}(89 \%)$ of $\mathbf{2 0}$ as a white solid: mp 267-9 ${ }^{\circ} \mathrm{C}$; IR $1651 \mathrm{~cm}^{-1} ;{ }^{1} \mathrm{H}$ NMR $\delta 8.05$ (d, 1H, $\left.J=8.0 \mathrm{~Hz}\right), 7.65$ (d, 2H, $J=$ $8.4 \mathrm{~Hz}), 7.29$ (d, 2H, $J=8.0 \mathrm{~Hz}), 7.15(\mathrm{dd}, 2 \mathrm{H}, J=15.6,7.6 \mathrm{~Hz}), 7.01(\mathrm{td}, 1 \mathrm{H}, J=7.6$, $1.2 \mathrm{~Hz}), 5.86(\mathrm{~d}, 1 \mathrm{H}, J=11.6 \mathrm{~Hz}), 5.75(\mathrm{~m}, 1 \mathrm{H}), 4.85(\mathrm{dd}, 1 \mathrm{H}, J=13.6,7.2 \mathrm{~Hz}), 4.41(\mathrm{~d}$, 
1H, $J=19.6 \mathrm{~Hz}), 4.06-4.02(\mathrm{~m}, 2 \mathrm{H}), 3.97-3.85(\mathrm{~m}, 2 \mathrm{H}), 3.69$ (d, 2H, $J=19.2 \mathrm{~Hz}), 3.26-$ $3.17(\mathrm{~m}, 4 \mathrm{H}), 2.51(\mathrm{dd}, 1 \mathrm{H}, J=17.2,10.4 \mathrm{~Hz}), 2.39(\mathrm{~s}, 3 \mathrm{H}) ;{ }^{13} \mathrm{C}$ NMR $\delta 166.2,144.3$, $142.4,134.0,131.3,130.1,127.4,127.2,124.8,124.3,117.4,116.7,53.1,52.9,49.7$, 49.2, 47.9, 29.9, 27.9, 21.5; MS [m/z (rel intensity)] $474\left(\mathrm{M}^{+}, 1\right), 155$ (26), 119 (100). Anal. Calcd for $\mathrm{C}_{23} \mathrm{H}_{26} \mathrm{O}_{5} \mathrm{~N}_{2} \mathrm{~S}_{2}$ : C, 58.21; H, 5.52; N, 5.90. Found: C, 57.98; H, 5.69; N, 5.85 .

36. Ahmad, L.; Gedye, R. N.; Nechvatal, A. J. Chem. Soc. C 1968, 185-187.

37. Dang, H. S.; Roberts, B. P. J. Chem. Soc., Perkin Trans. 1 1996, 1493-1498. 
${ }^{1}$ H NMR Spectrum of 1

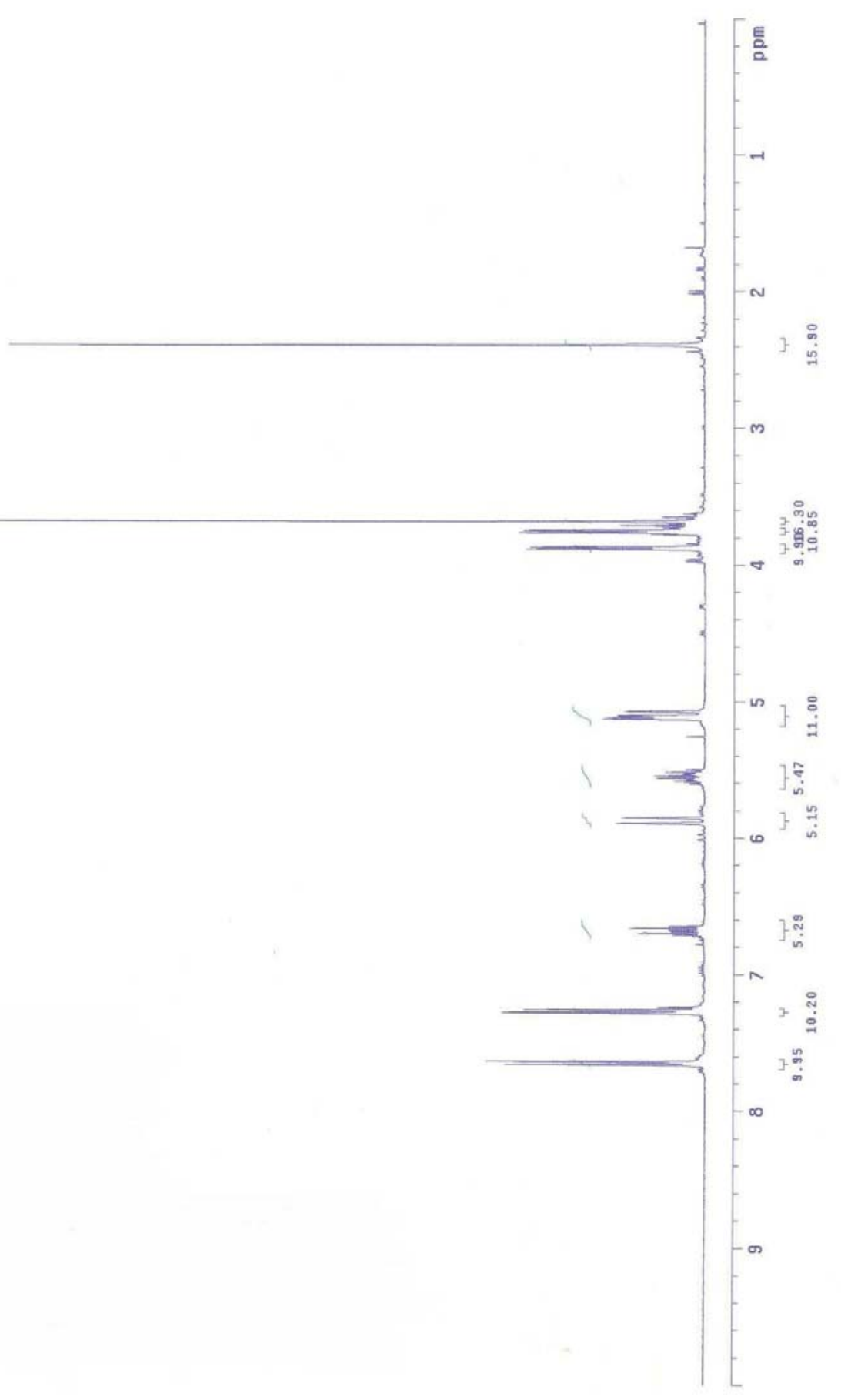




\section{${ }^{13} \mathrm{C}$ NMR Spectrum of 1}

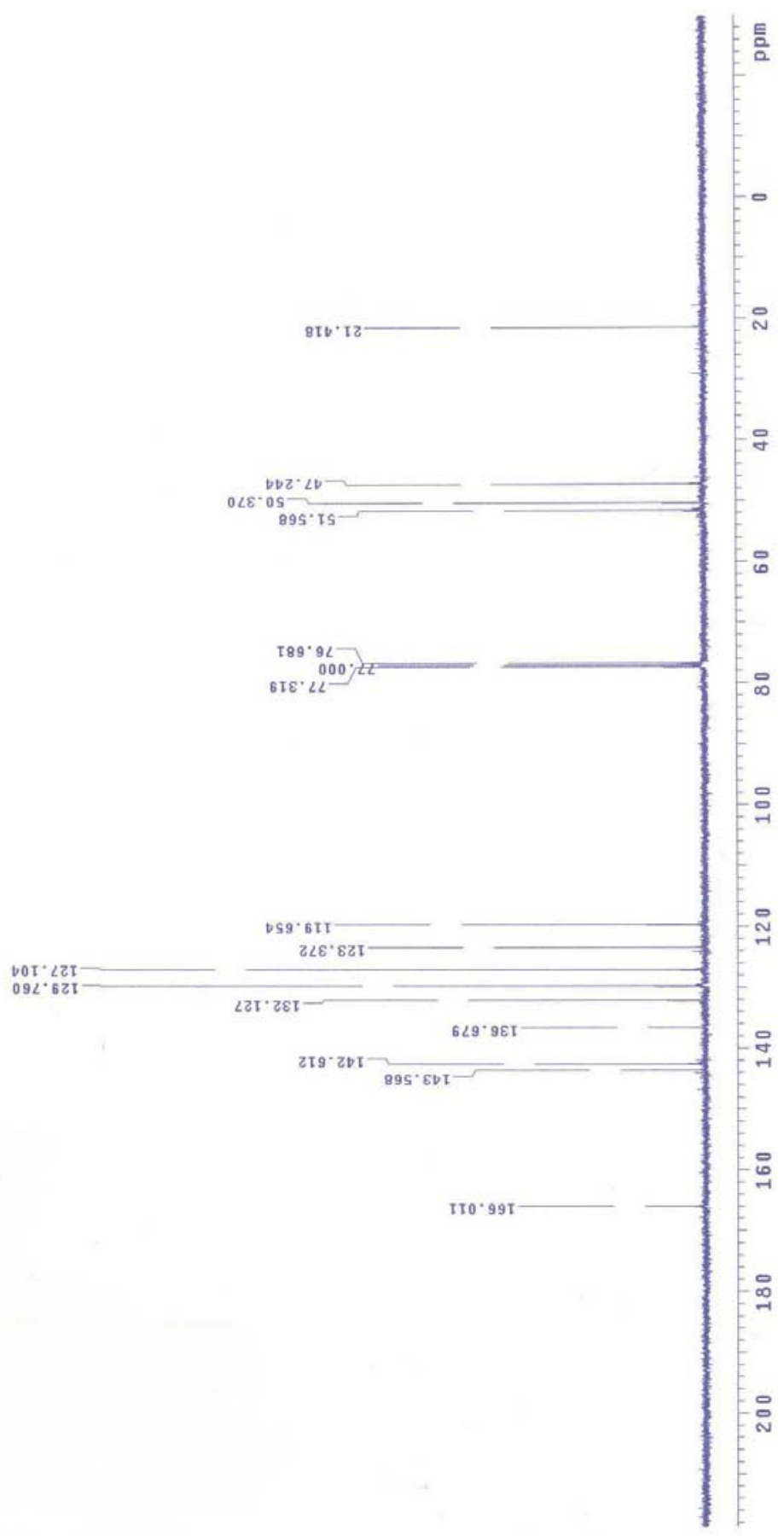




\section{${ }^{1} \mathrm{H}$ NMR Spectrum of 2}

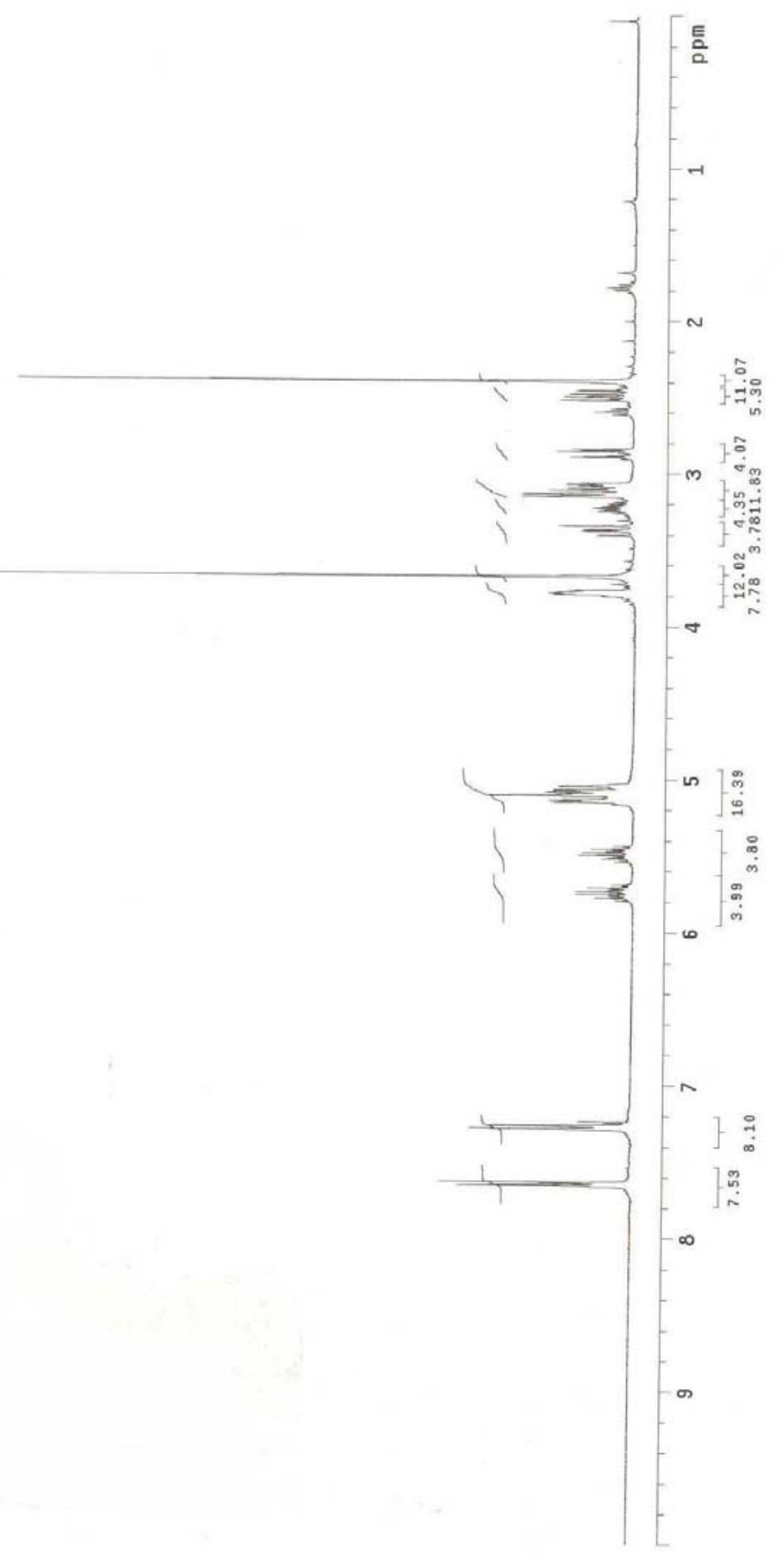




\section{${ }^{13}$ C NMR Spectrum of 2}

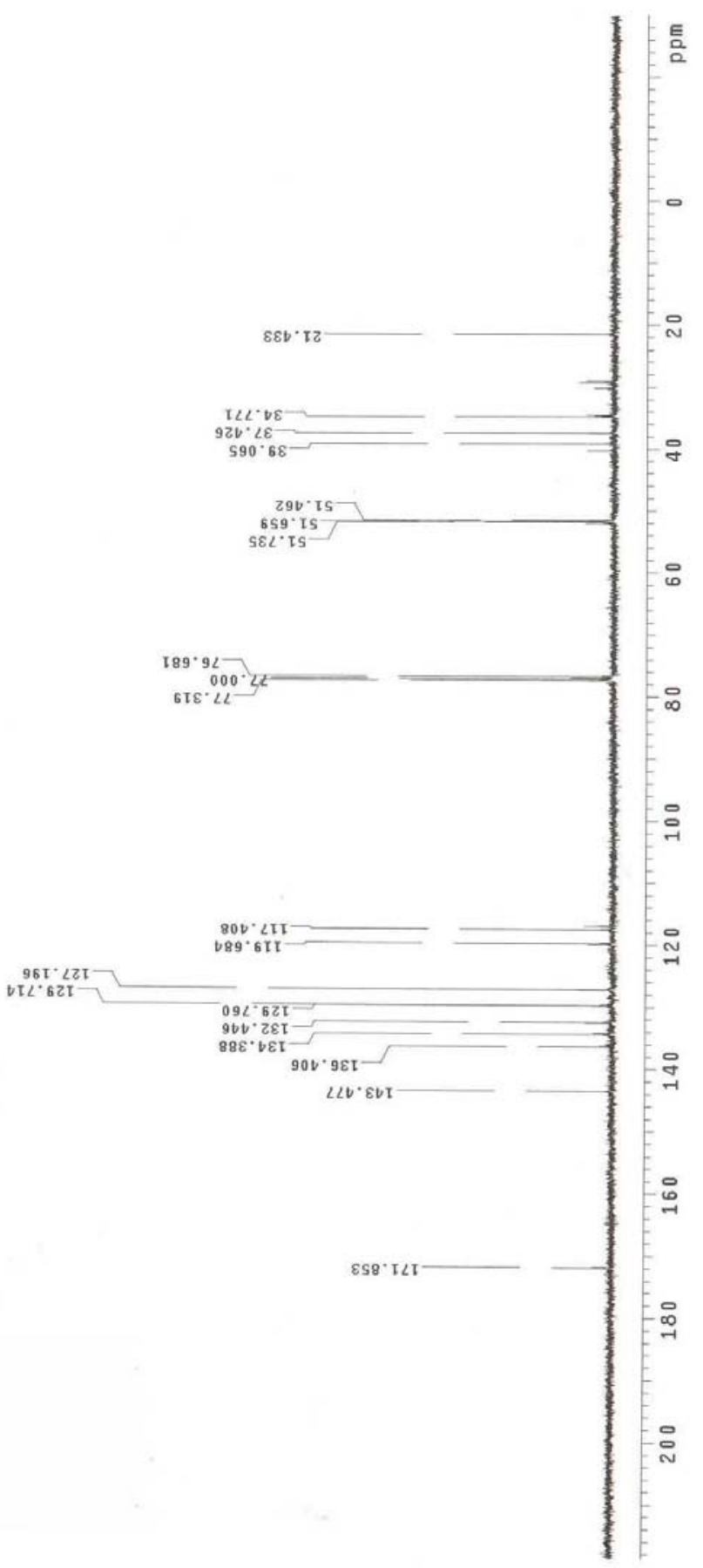




\section{${ }^{1} \mathrm{H}$ NMR Spectrum of 3}

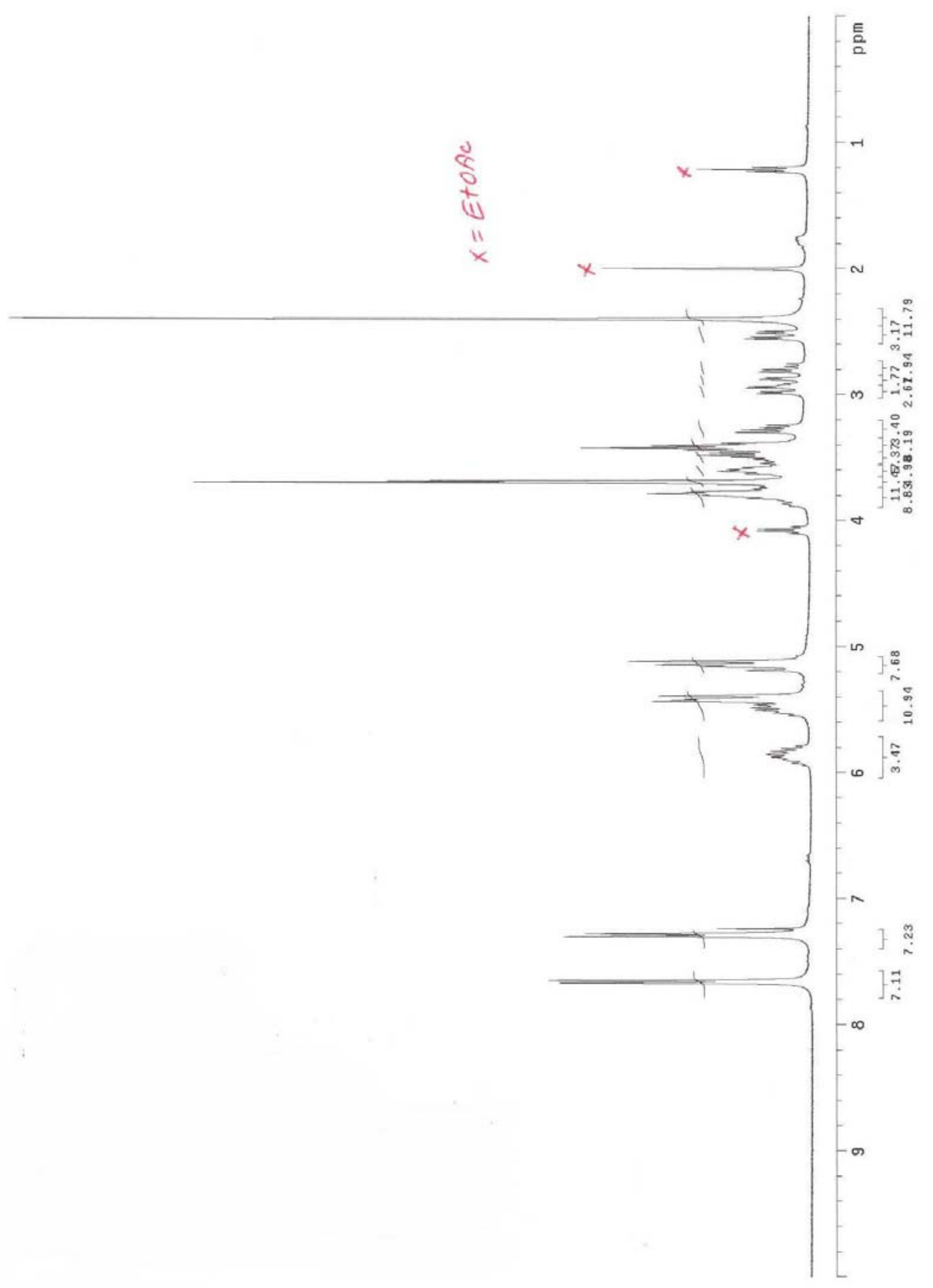




\section{${ }^{13} \mathrm{C}$ NMR Spectrum of 3}

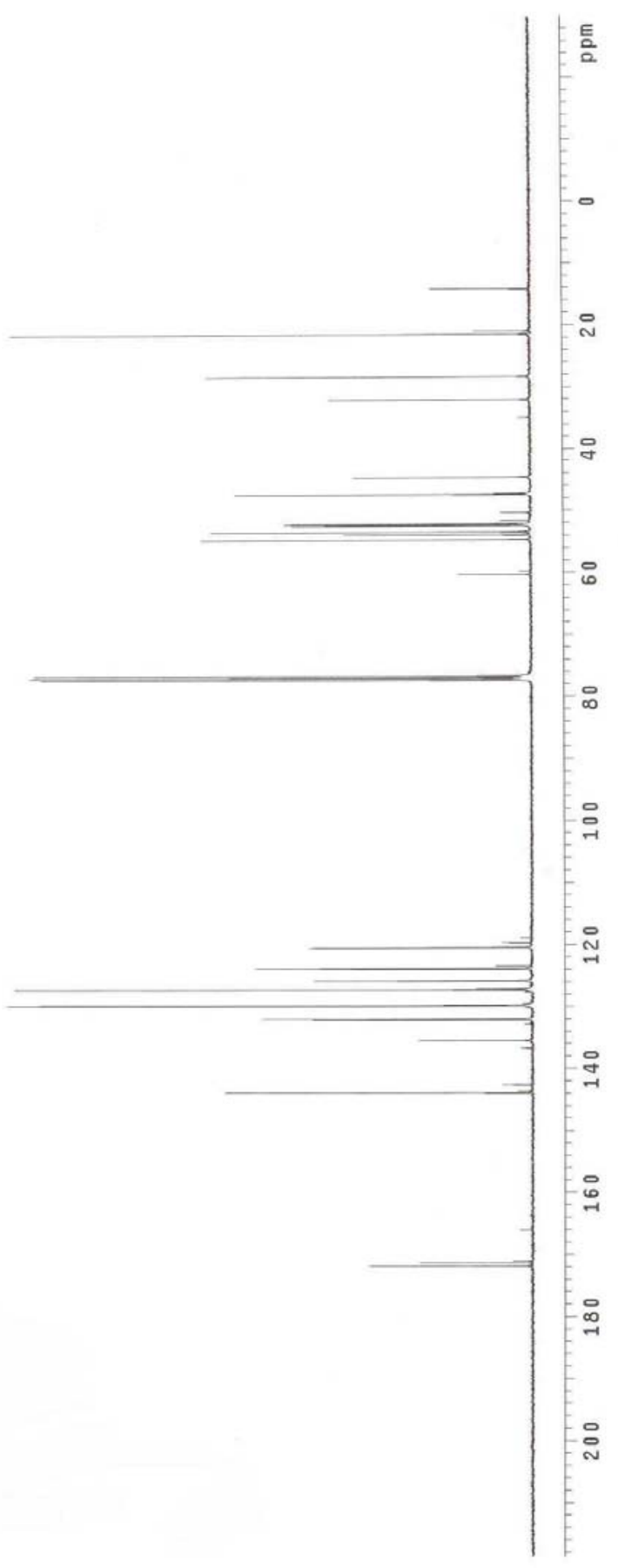




\section{${ }^{1} \mathrm{H}$ NMR Spectrum of 4}

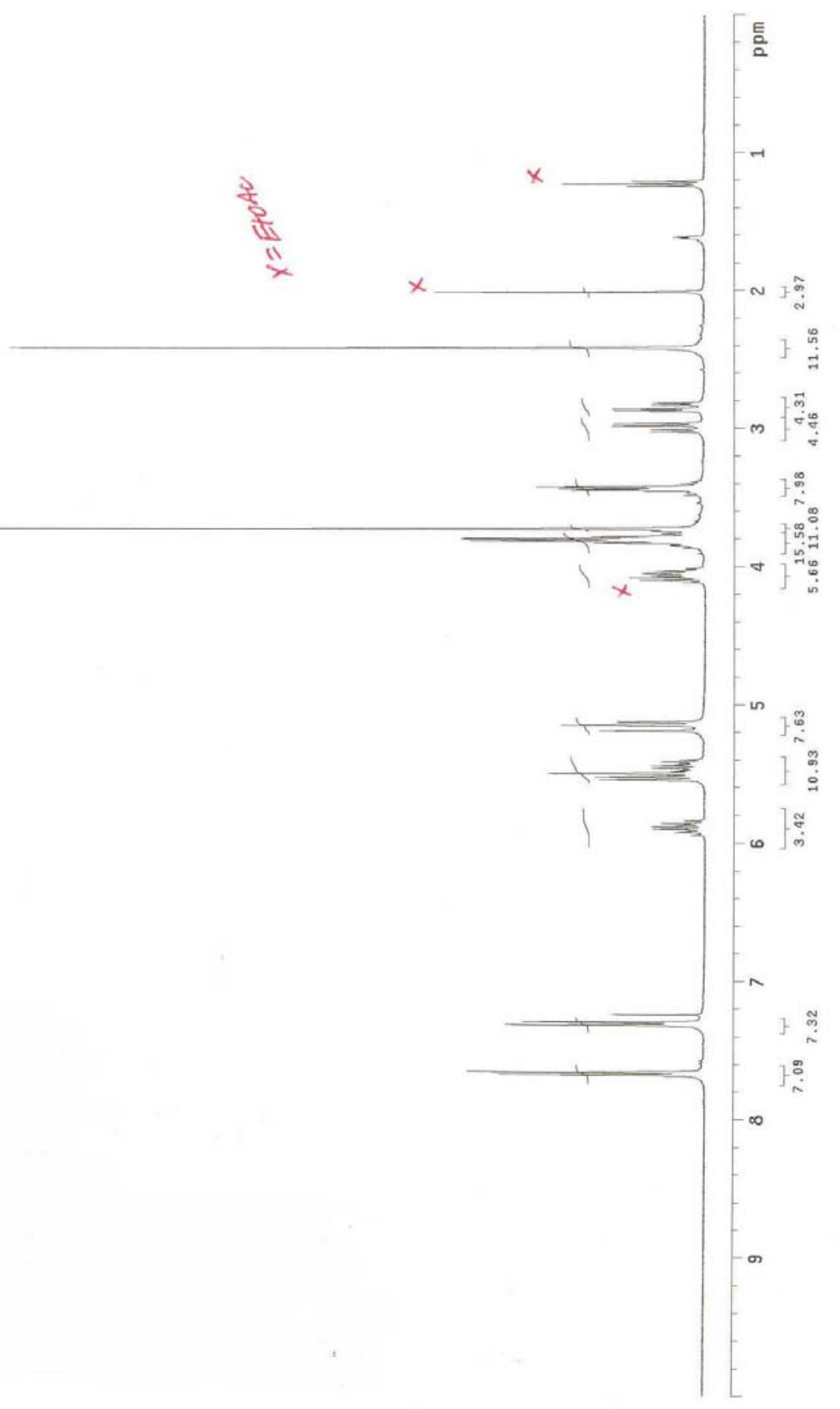


${ }^{13}$ C NMR Spectrum of 4

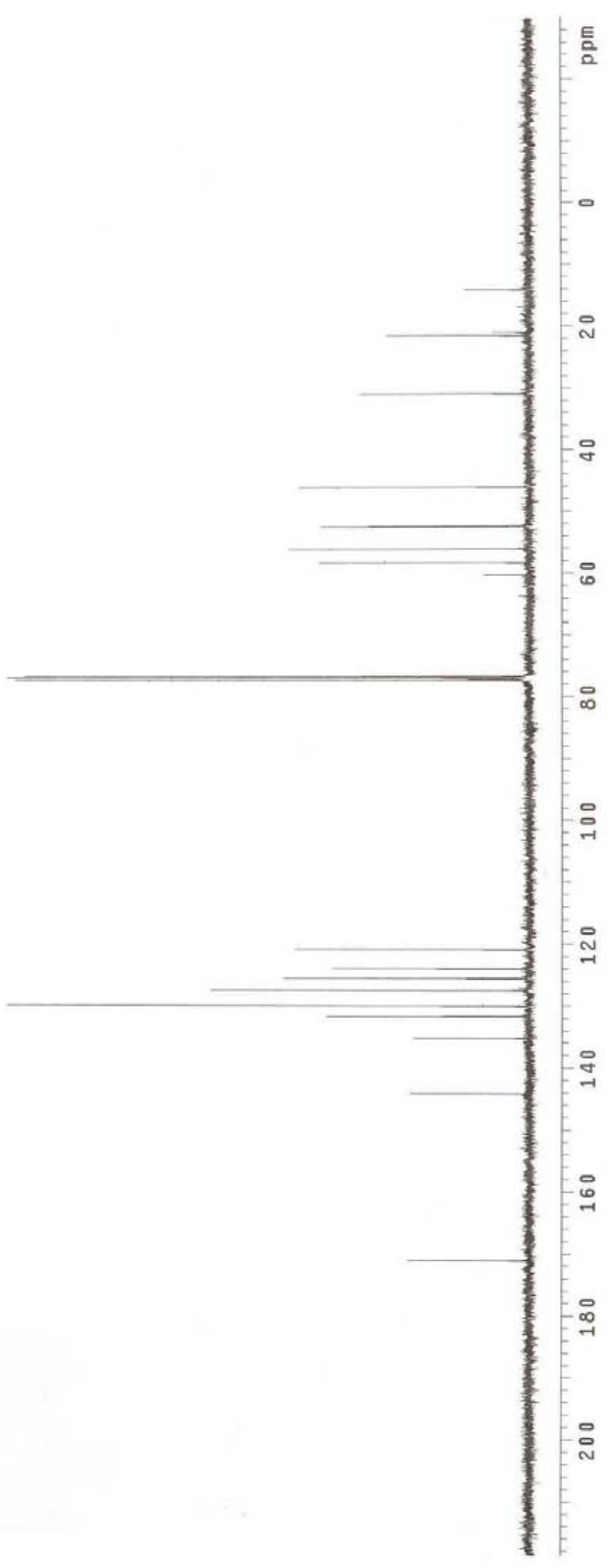


${ }^{1} \mathbf{H}$ NMR Spectrum of 5 (High $\mathbf{R}_{\mathbf{f}}$ diastereomer)

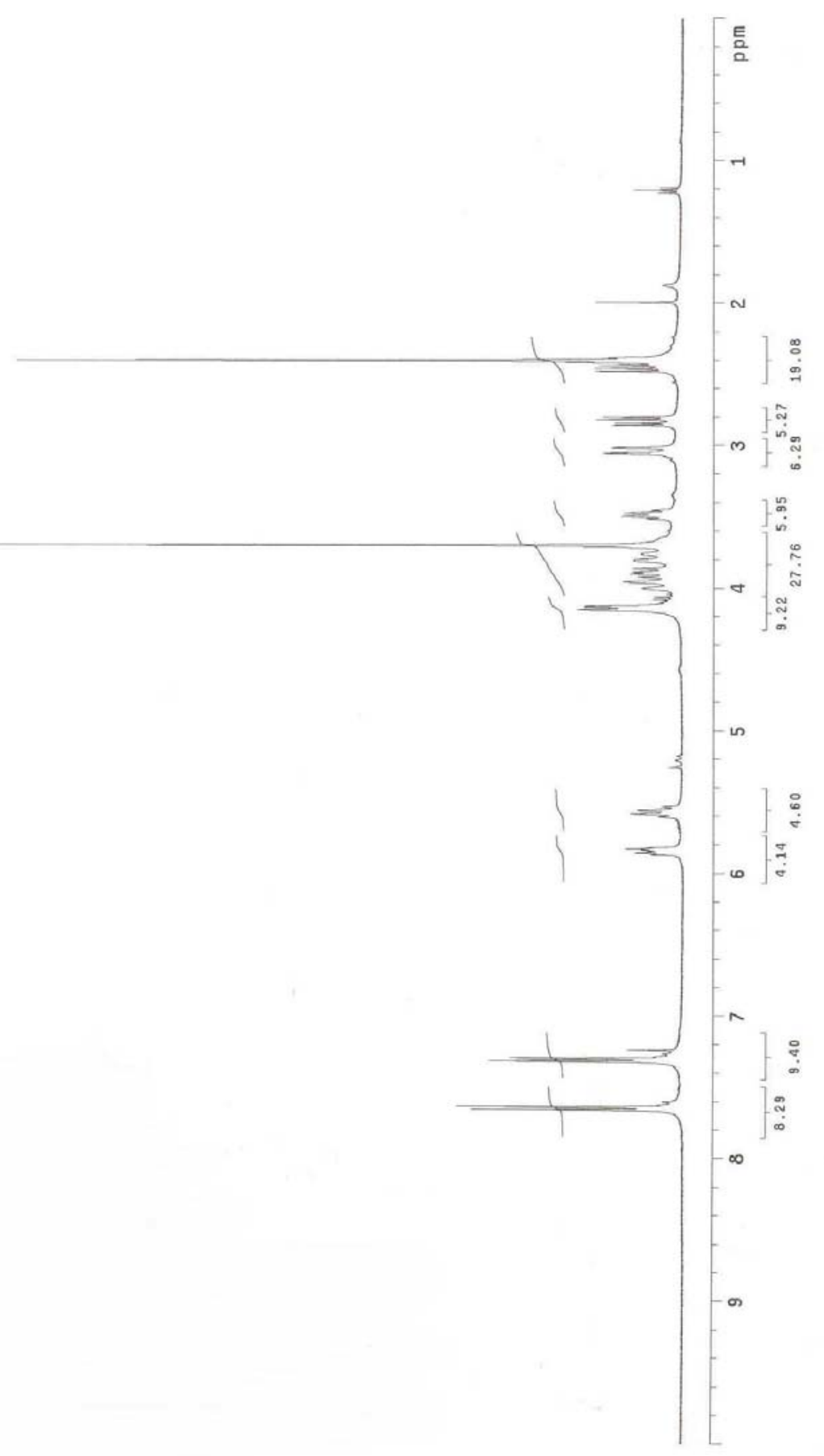


${ }^{13} \mathrm{C}$ NMR Spectrum of 5 (High $\mathbf{R}_{\mathrm{f}}$ diastereomer)

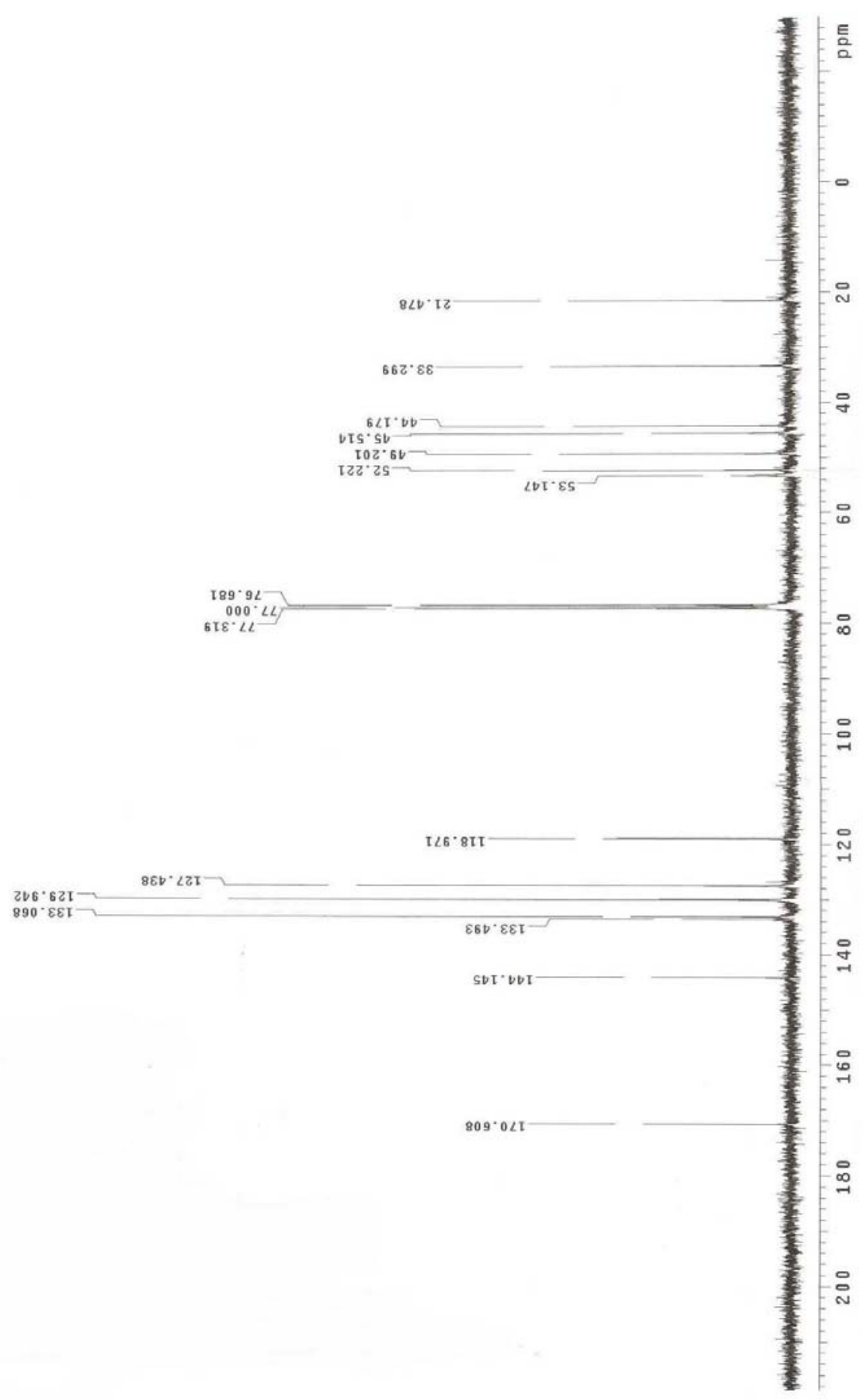


${ }^{1} \mathrm{H}$ NMR Spectrum of 5 (Low $\mathbf{R}_{\mathrm{f}}$ diastereomer)

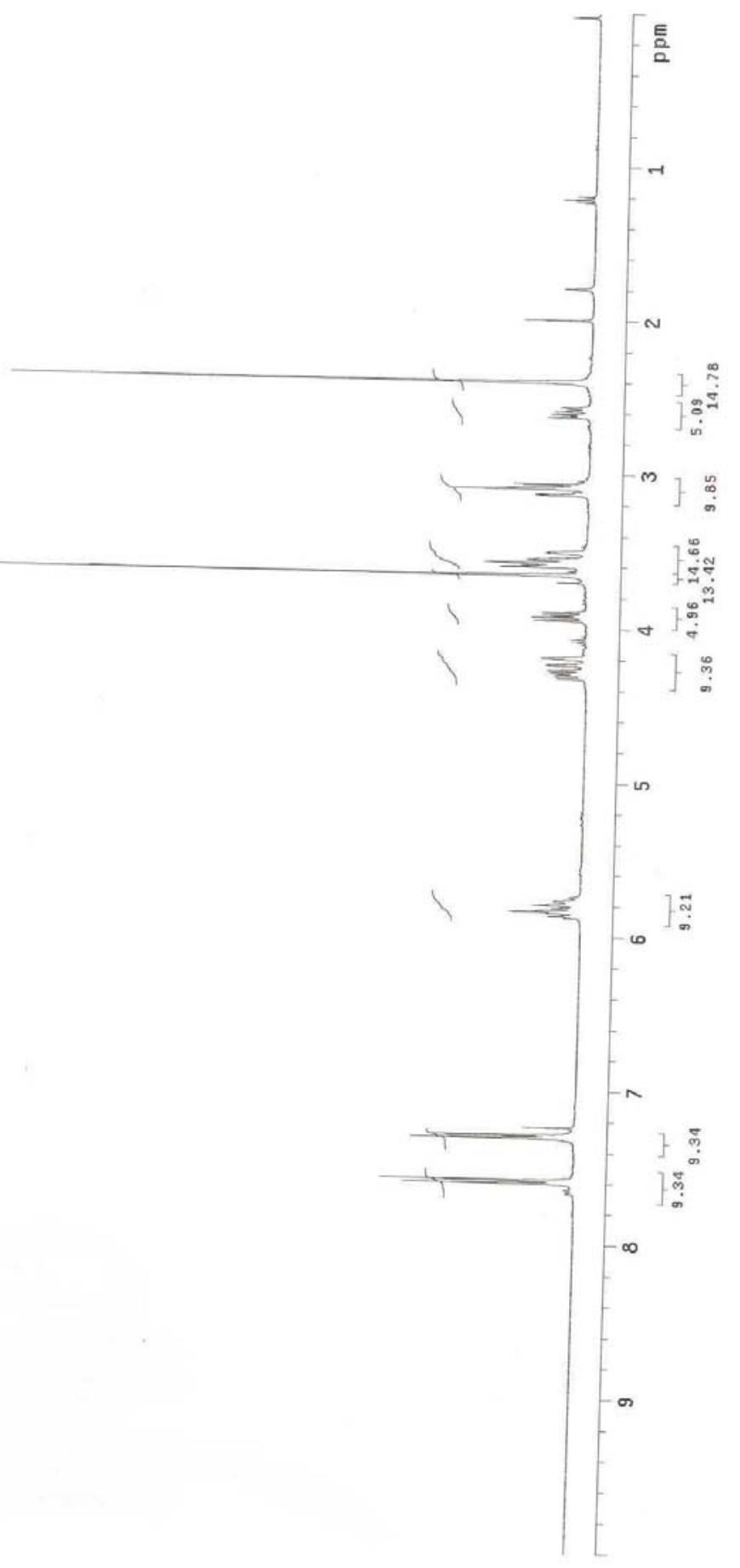


${ }^{13}$ C NMR Spectrum of 5 (Low $R_{f}$ diastereomer)

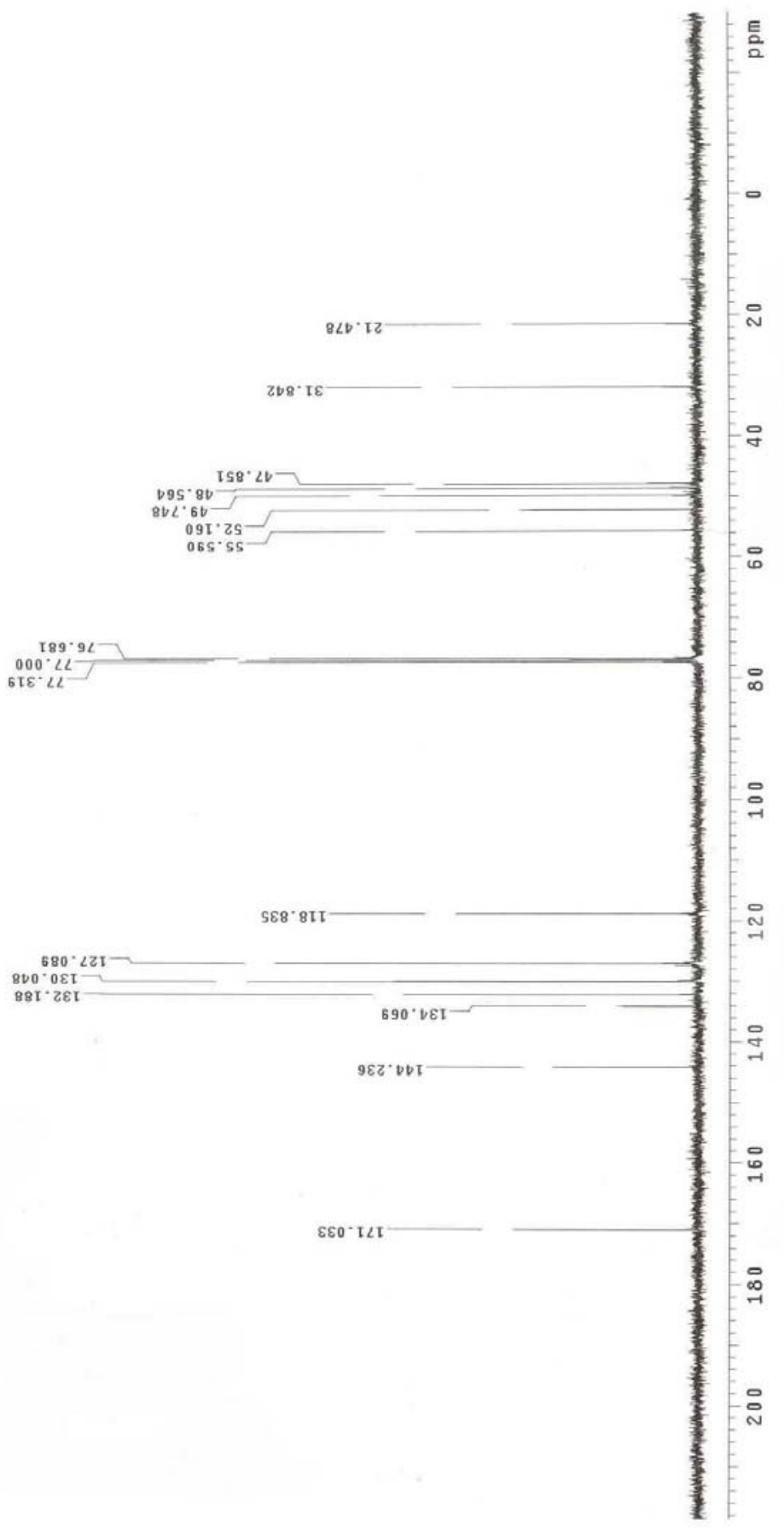




\section{${ }^{1}$ H NMR Spectrum of 6}

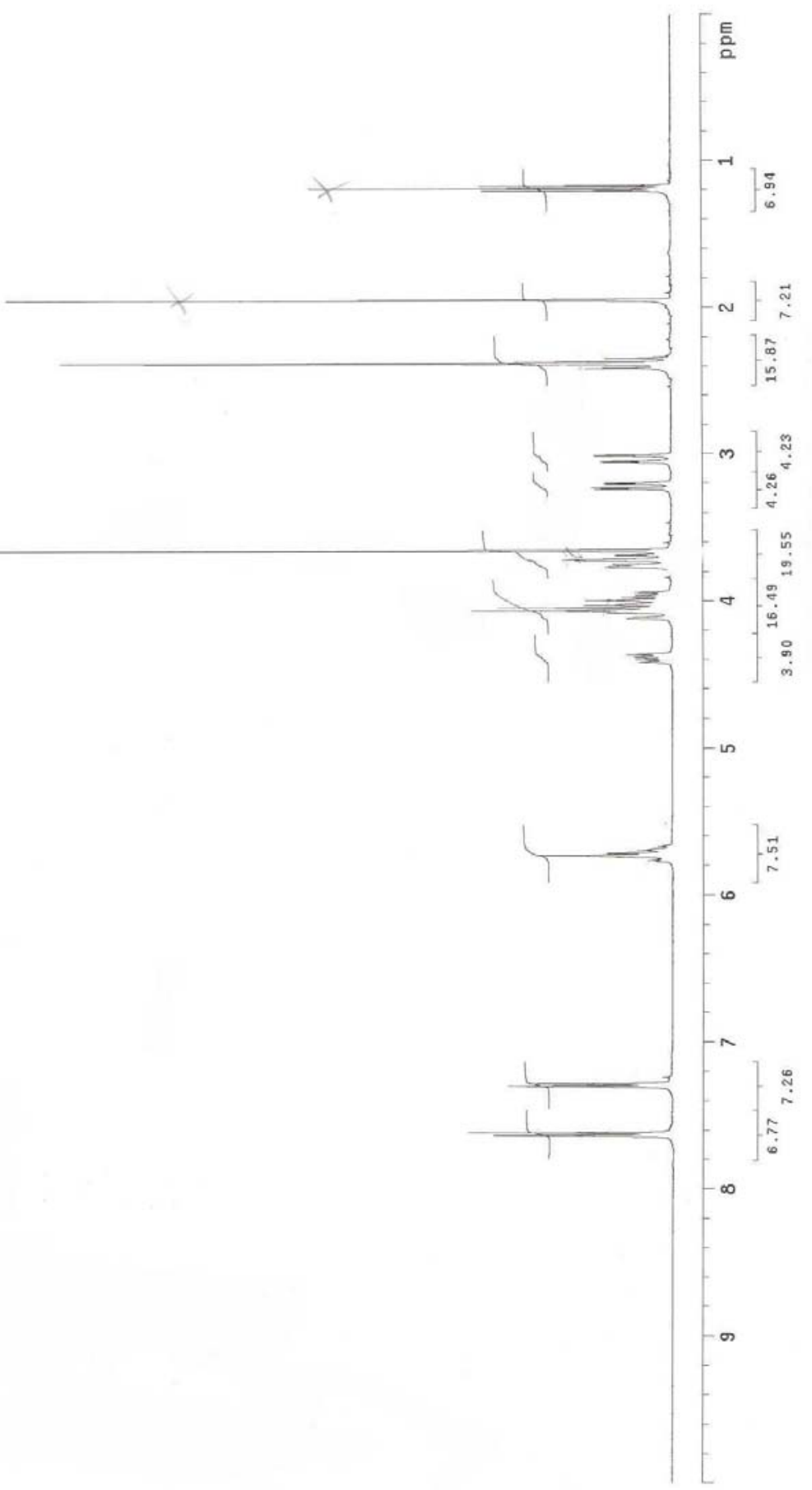


${ }^{13}$ C NMR Spectrum of 6

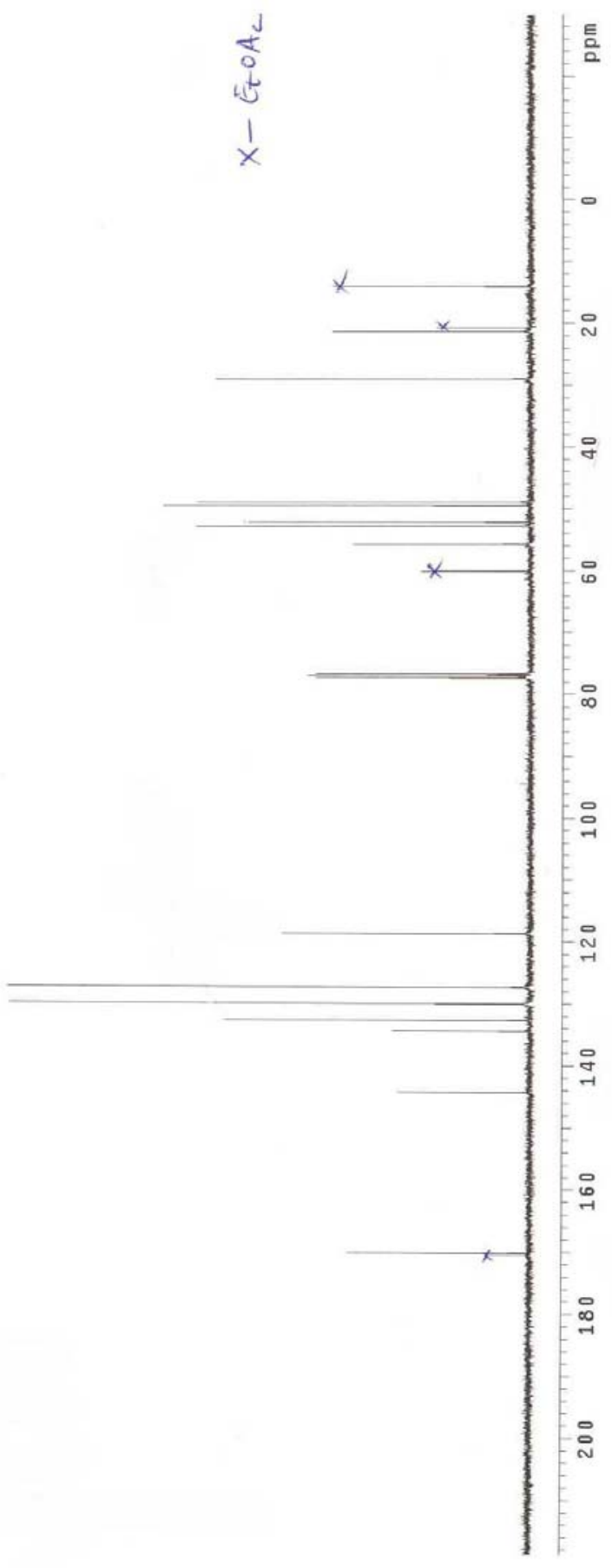




\section{${ }^{1} \mathrm{H}$ NMR Spectrum of 7}

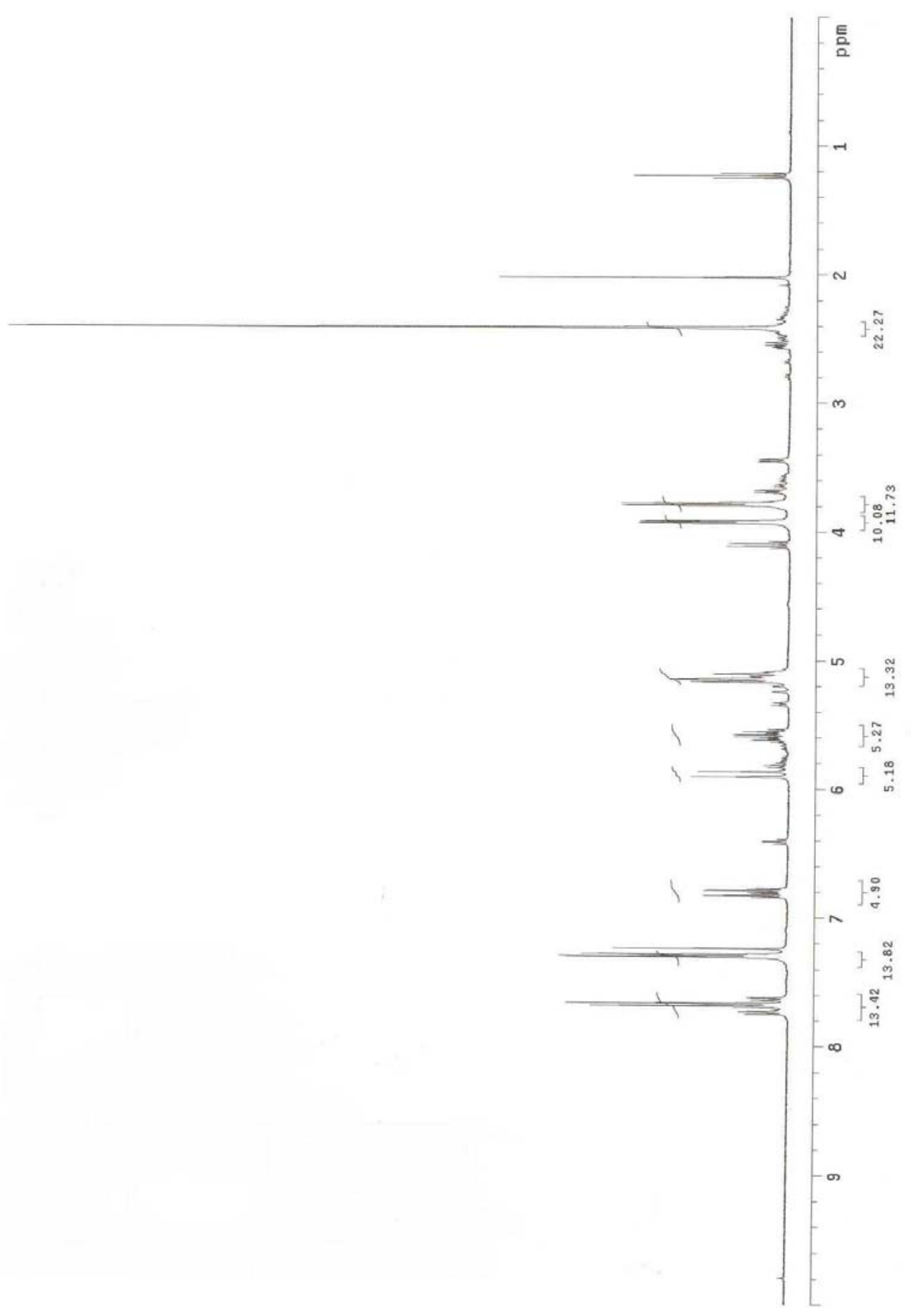


${ }^{13}$ C NMR Spectrum of 7

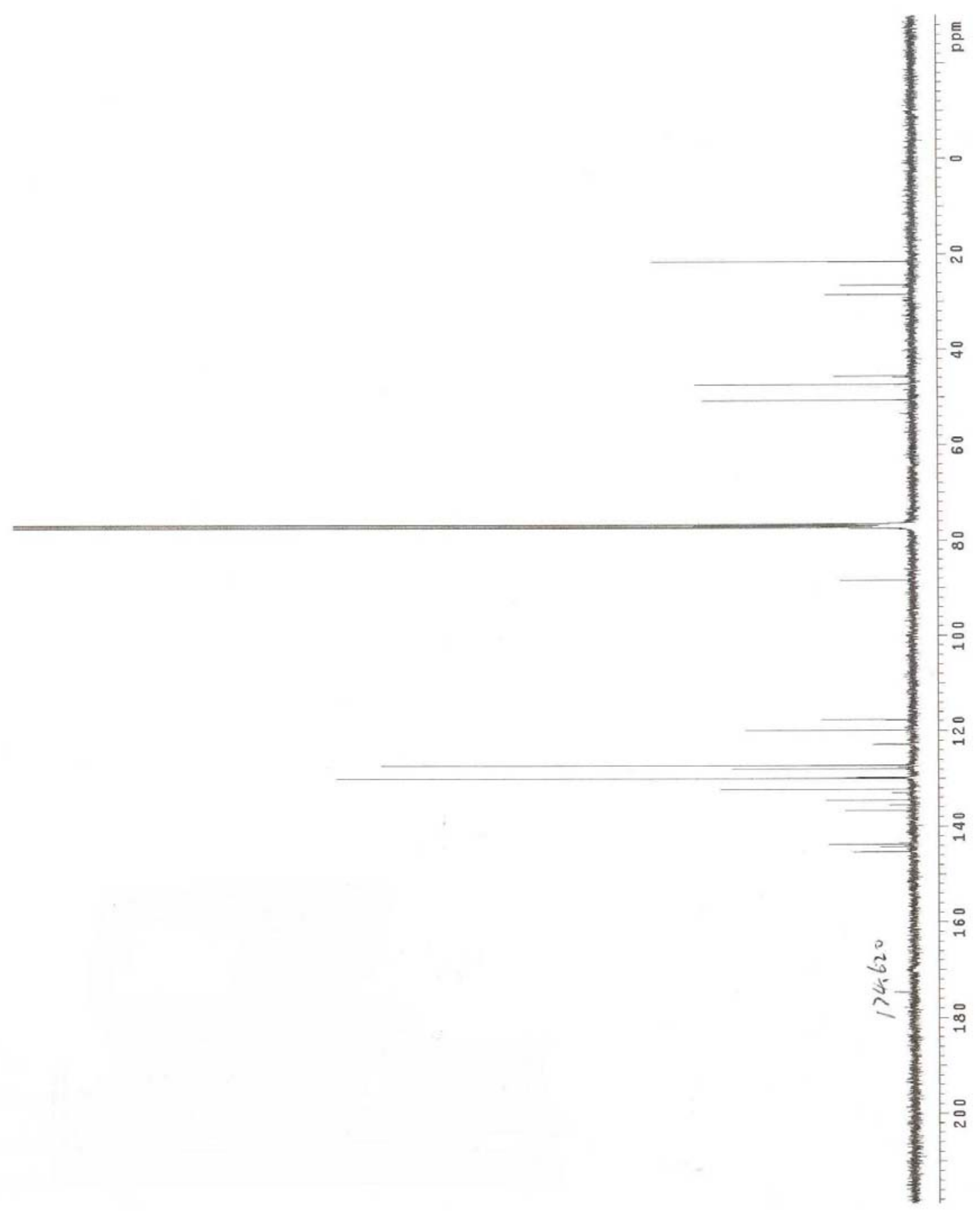




\section{${ }^{1} \mathrm{H}$ NMR Spectrum of 8}

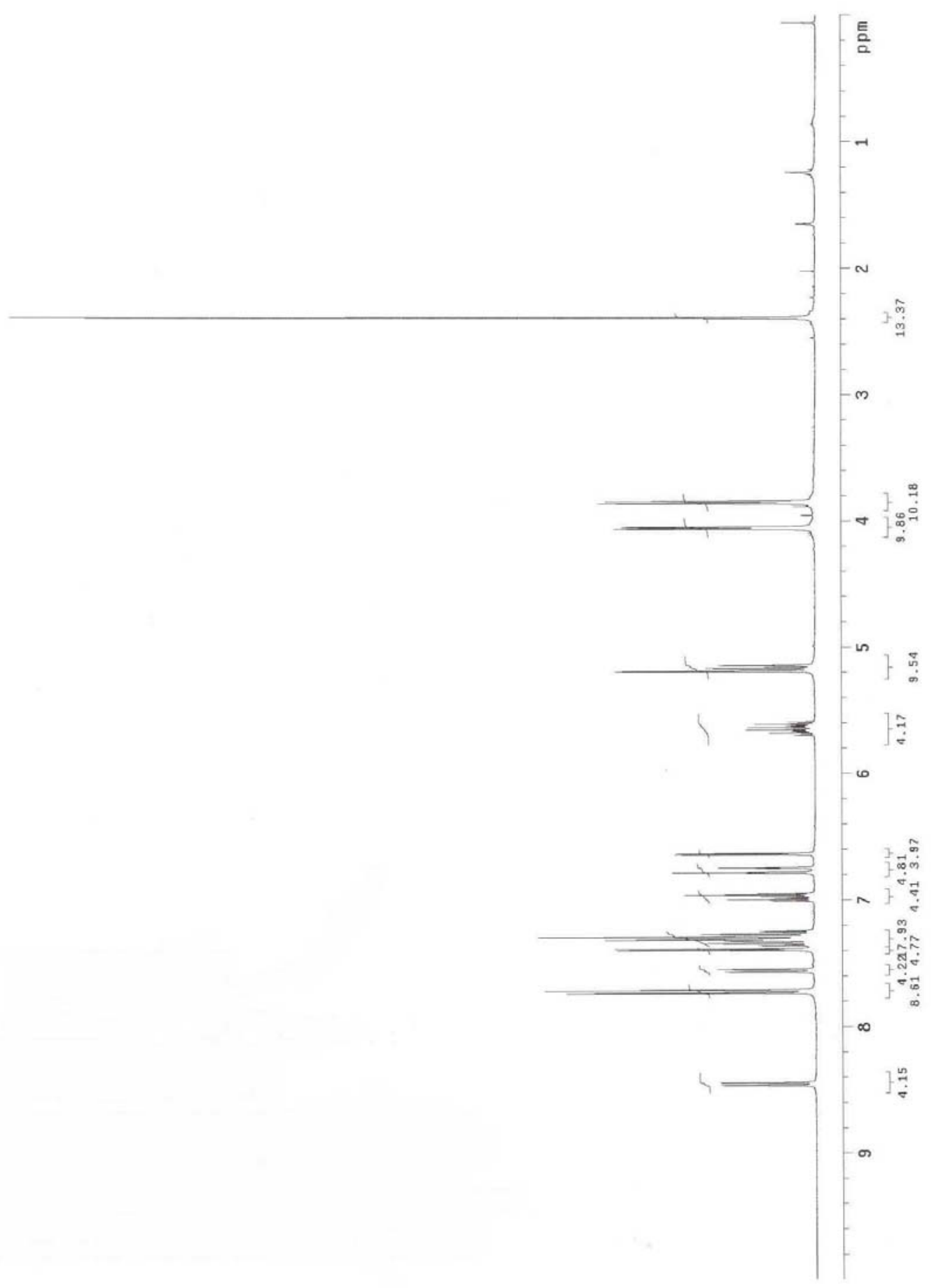




\section{${ }^{13} \mathrm{C}$ NMR Spectrum of 8}

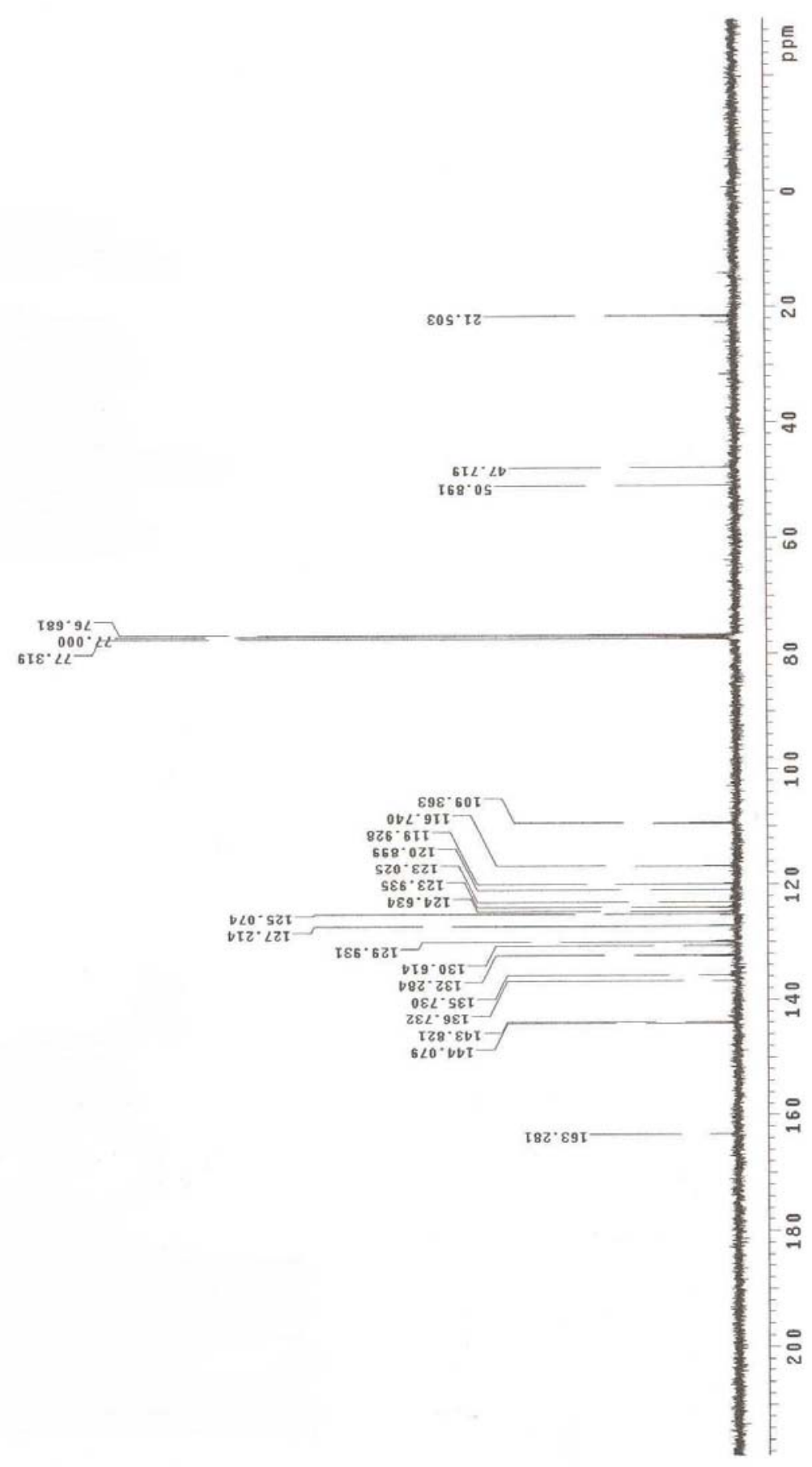




\section{${ }^{1} \mathrm{H}$ NMR Spectrum of 9}

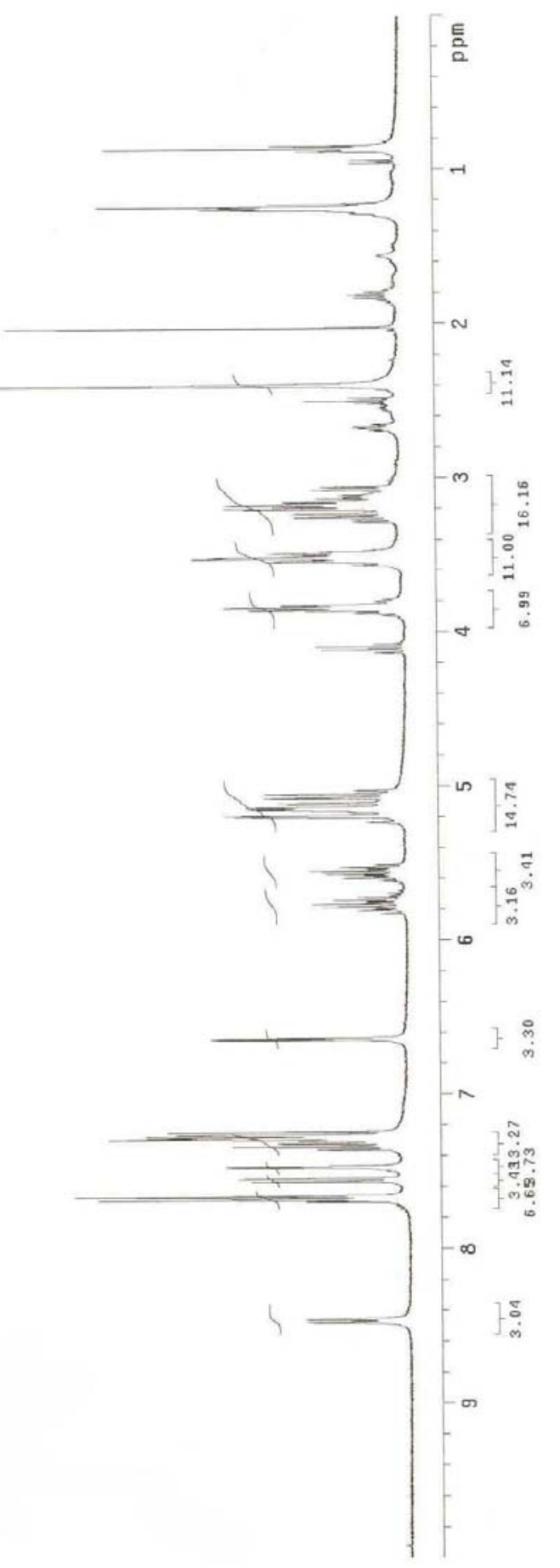




\section{${ }^{13}$ C NMR Spectrum of 9}

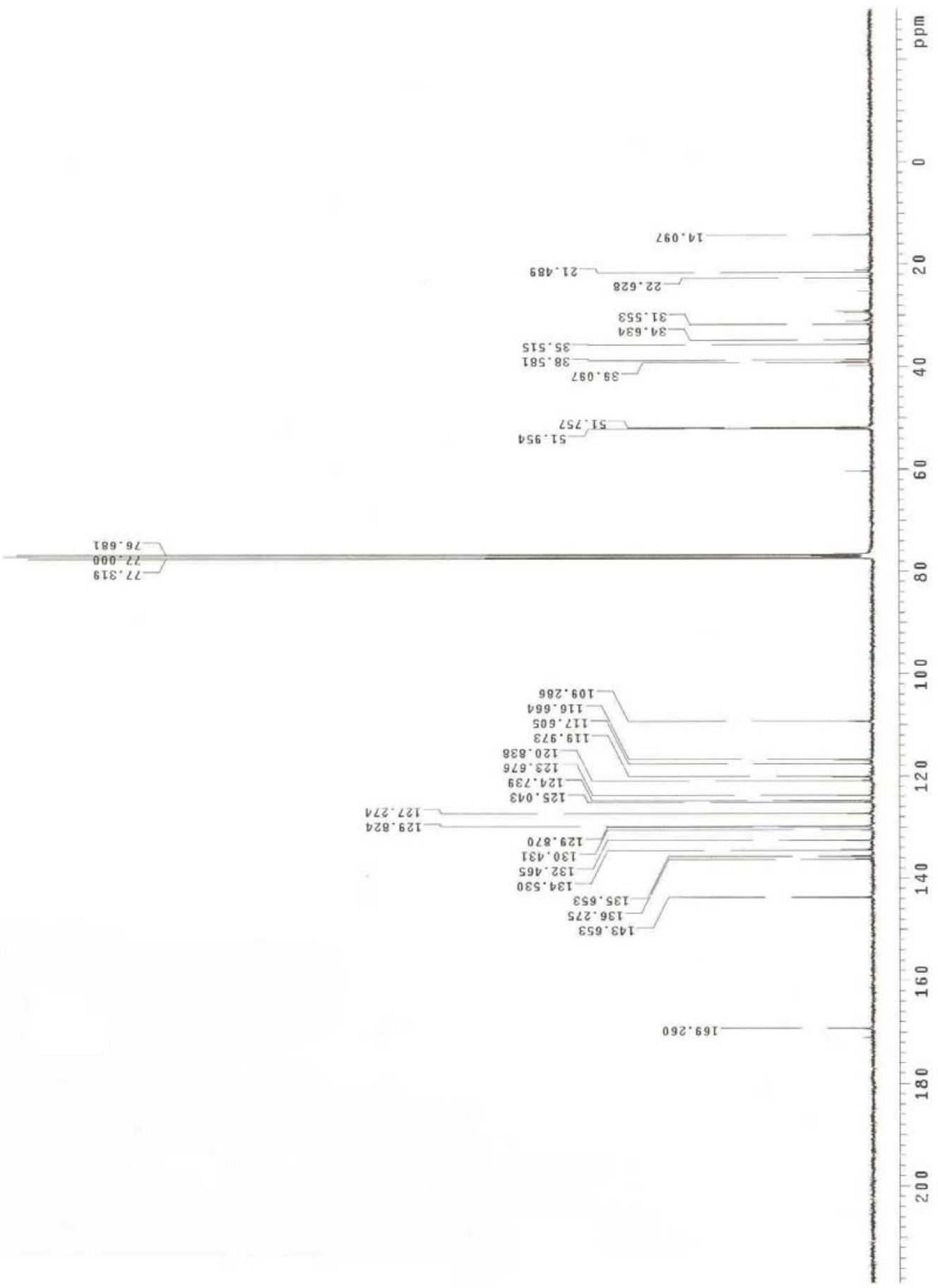


${ }^{1} \mathrm{H}$ NMR Spectrum of $\mathbf{1 0}$

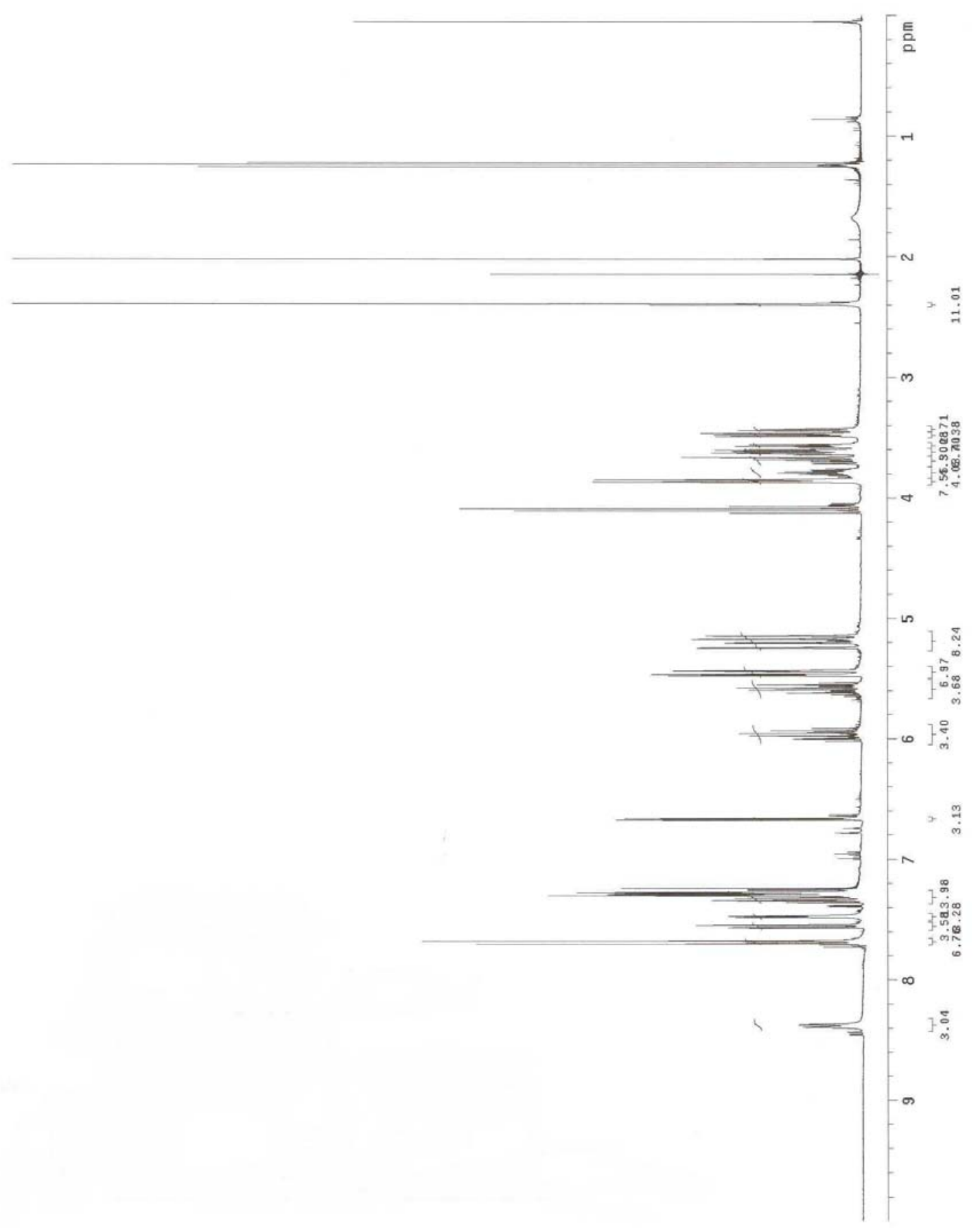


${ }^{13}$ C NMR Spectrum of 10

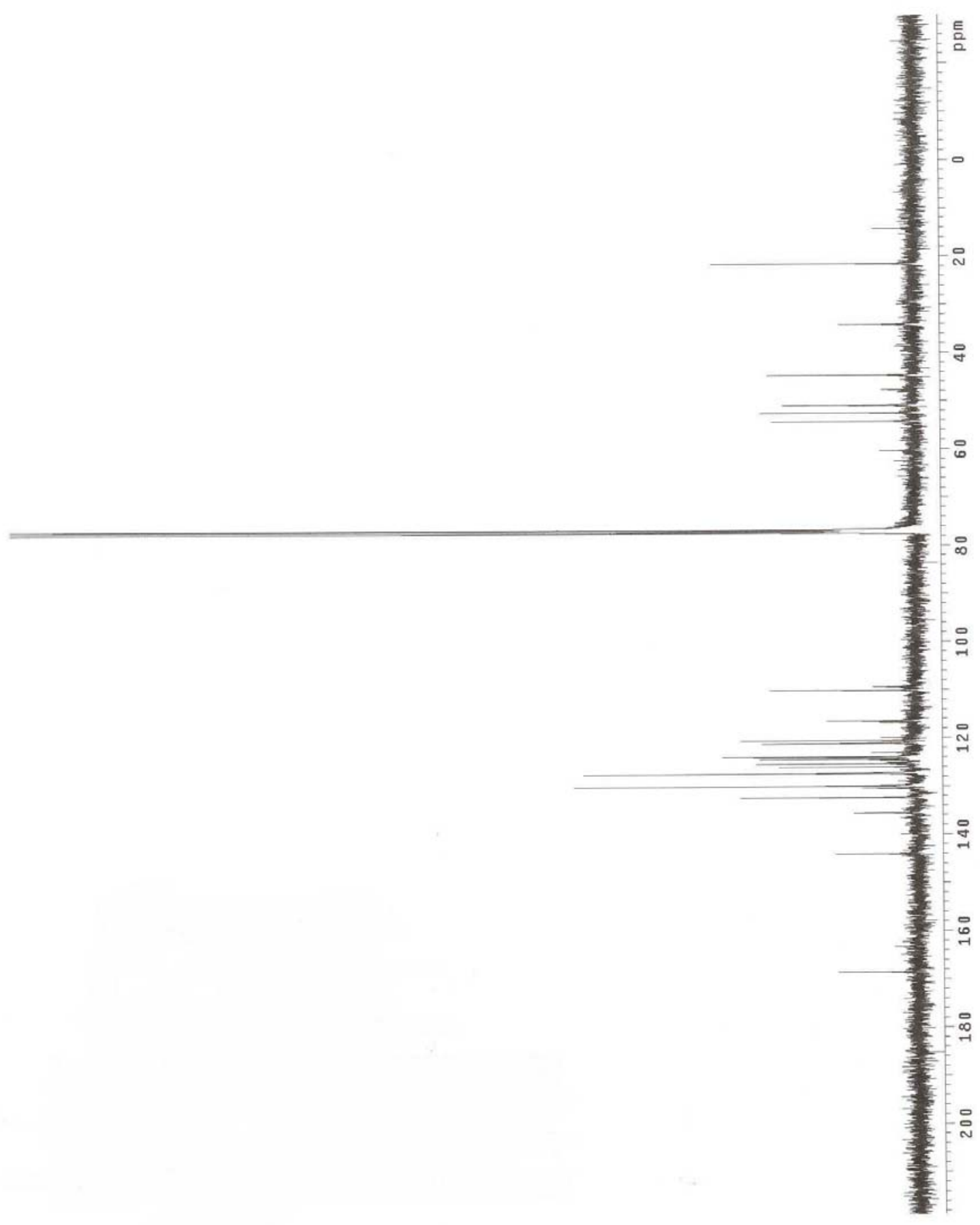




\section{${ }^{1} \mathrm{H}$ NMR Spectrum of 11}

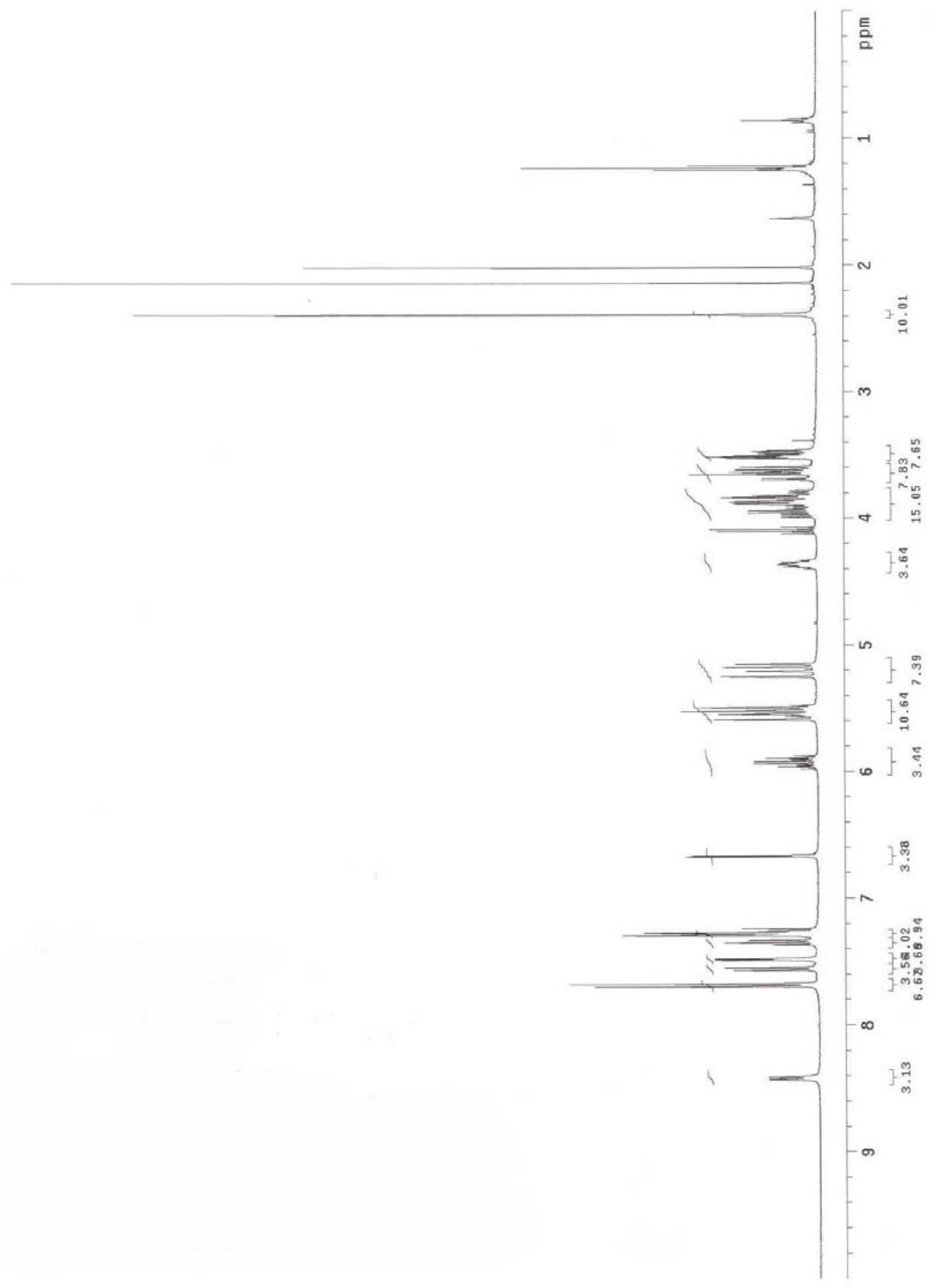


${ }^{13} \mathrm{C}$ NMR Spectrum of 11

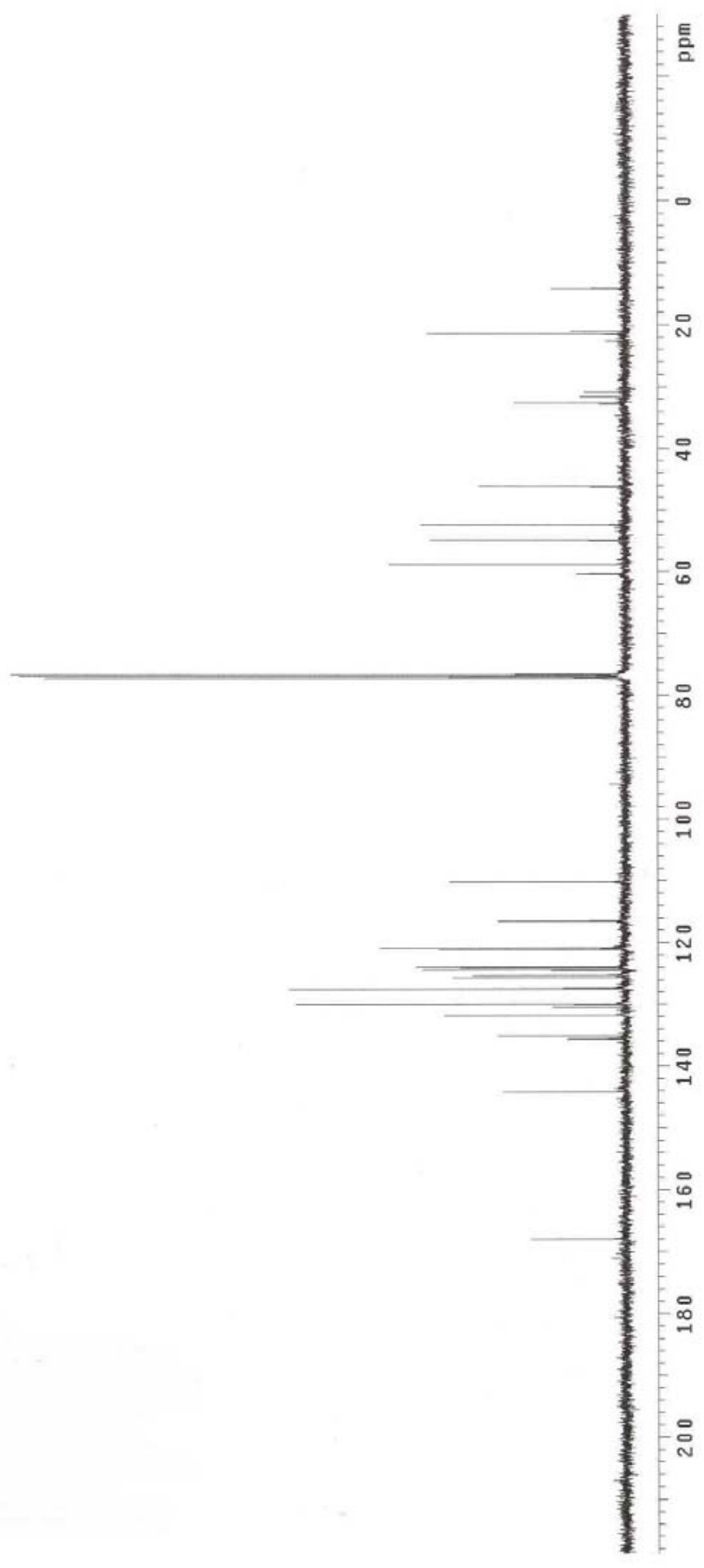




\section{${ }^{1}$ H NMR Spectrum of 12}

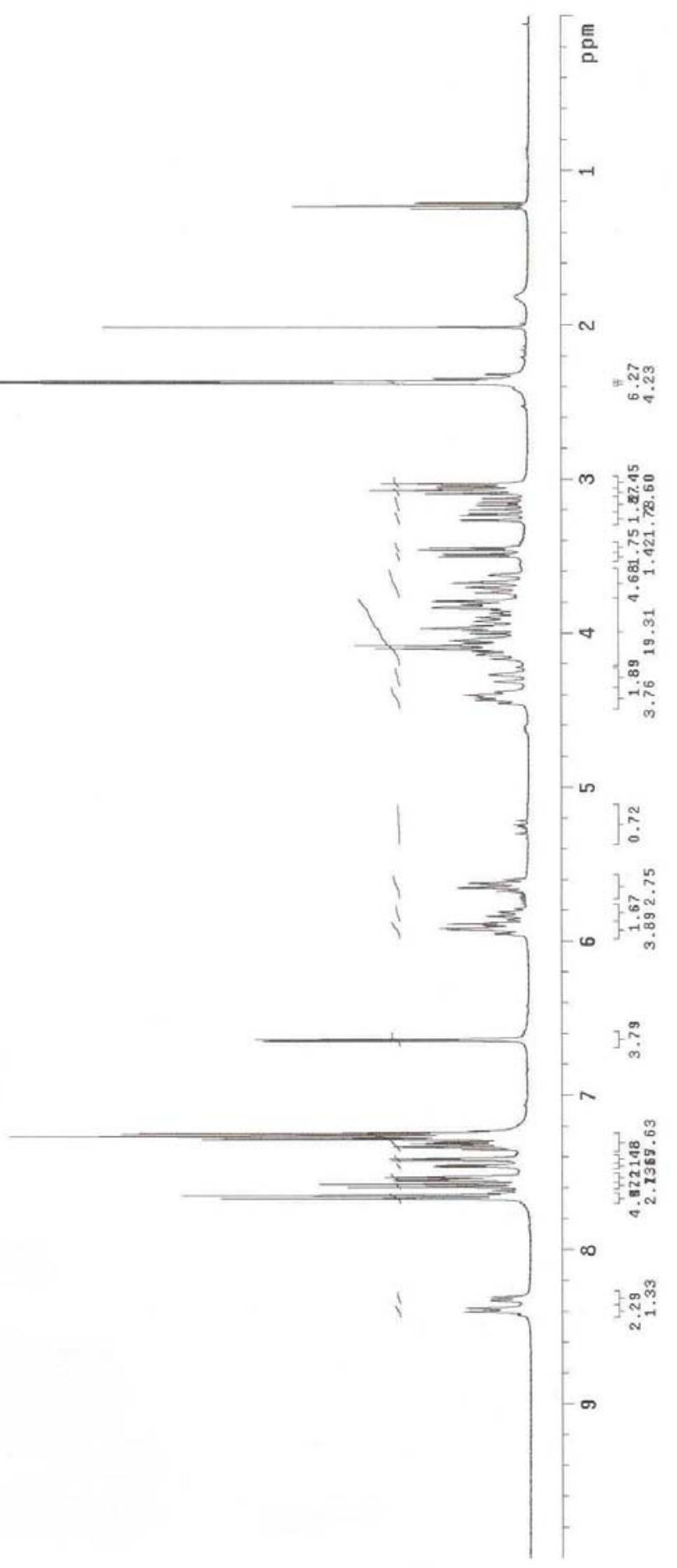


${ }^{13} \mathrm{C}$ NMR Spectrum of 12

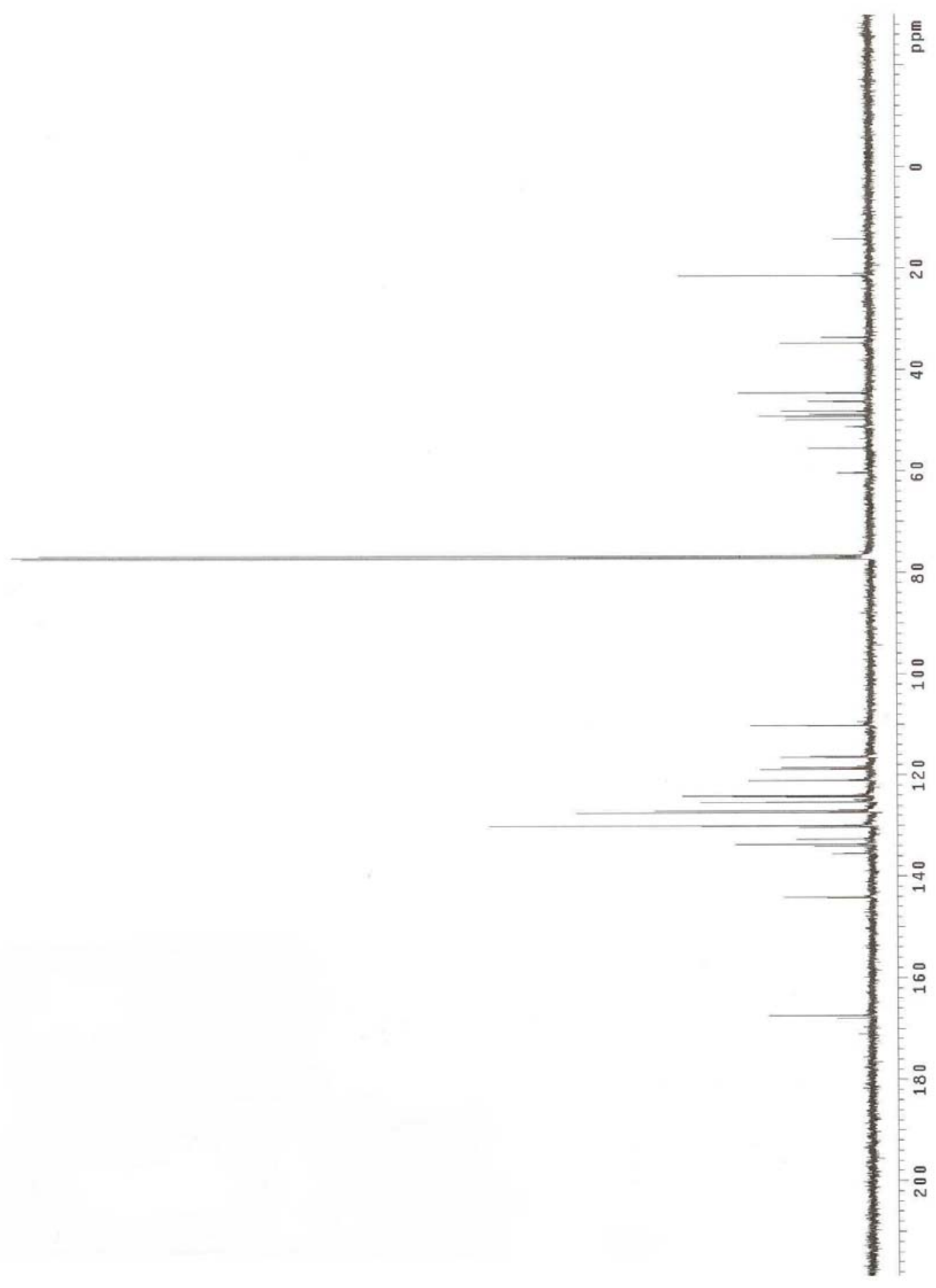




\section{${ }^{1} \mathrm{H}$ NMR Spectrum of 14}

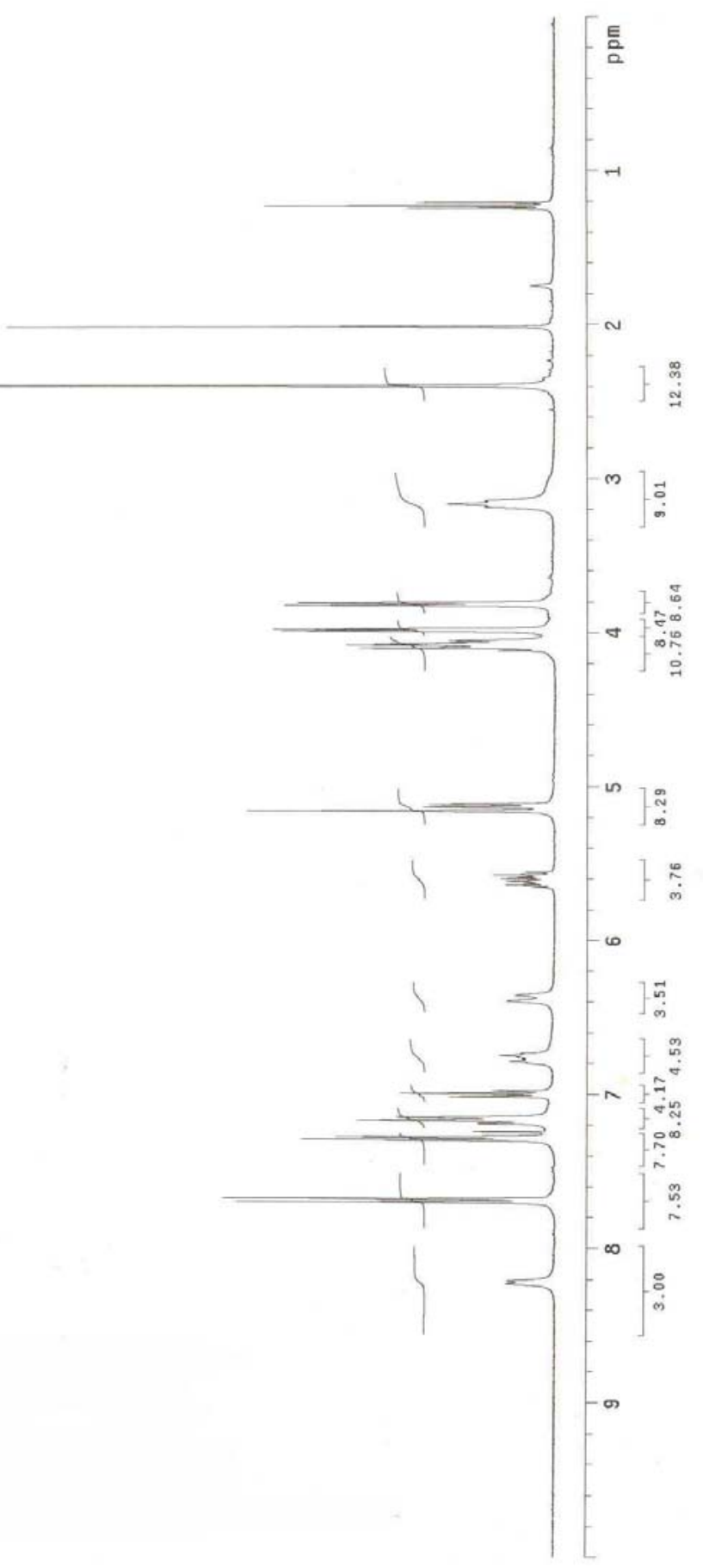


${ }^{13} \mathrm{C}$ NMR Spectrum of 14

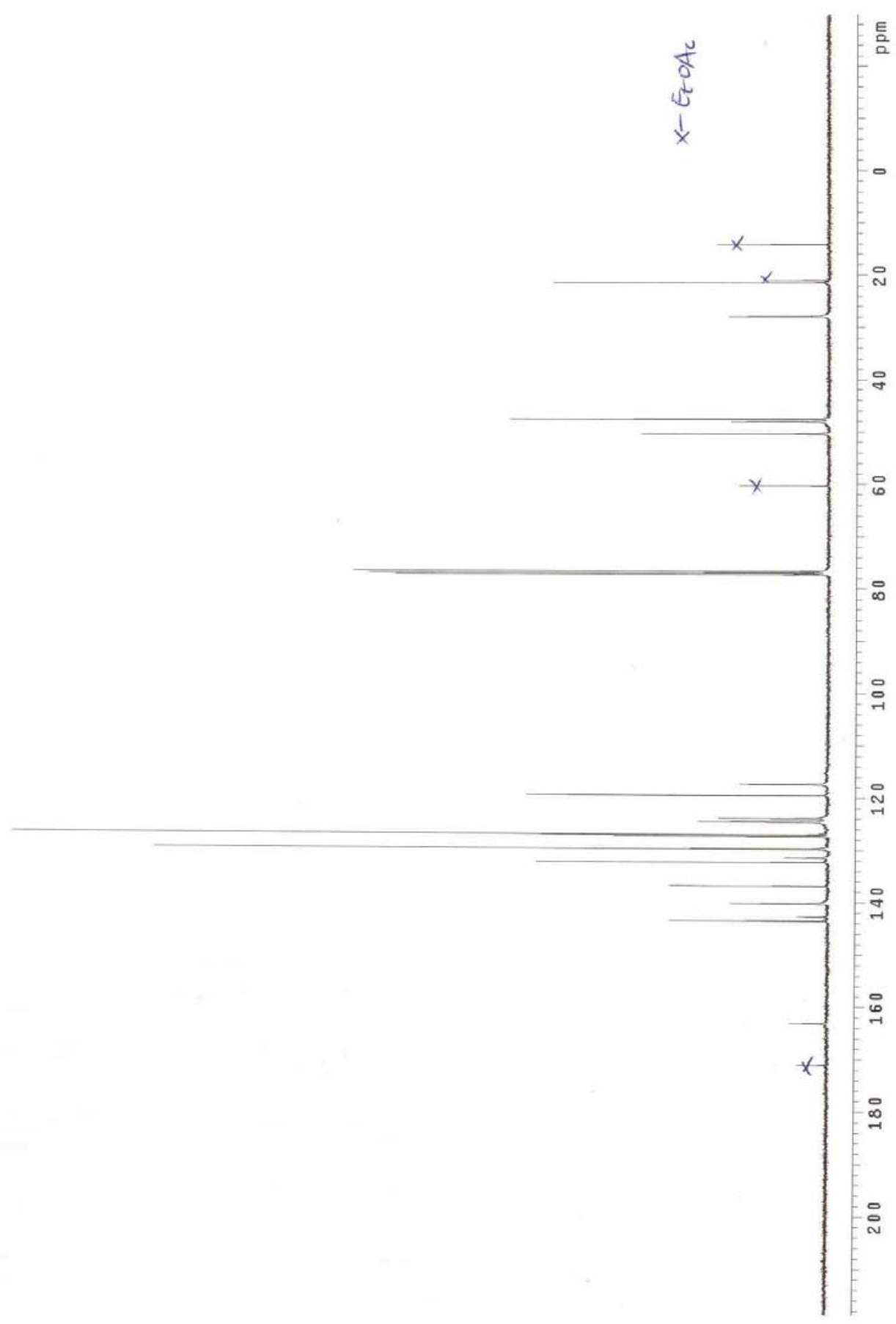




\section{${ }^{1} \mathrm{H}$ NMR Spectrum of 15}

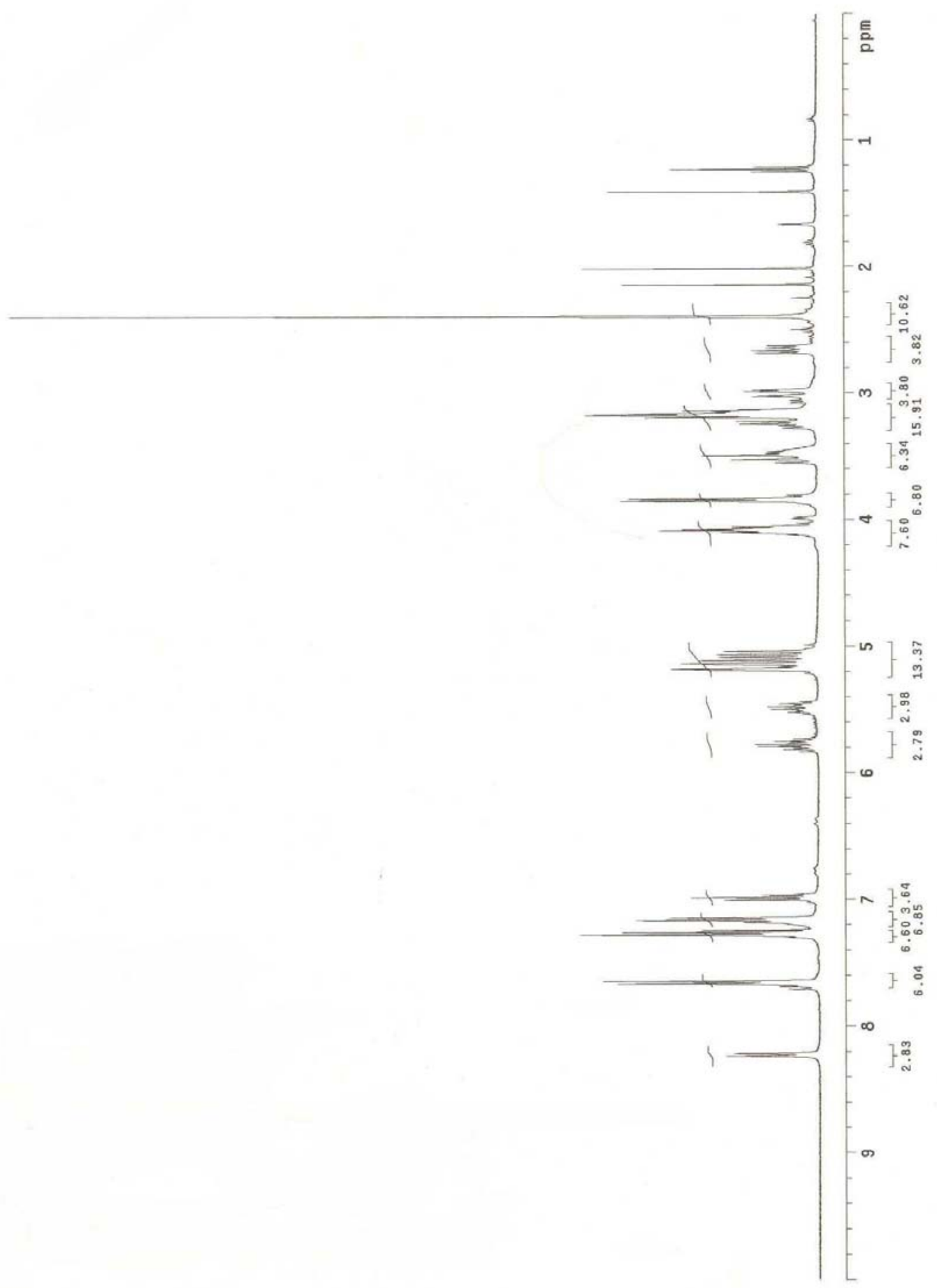


${ }^{13}$ C NMR Spectrum of 15

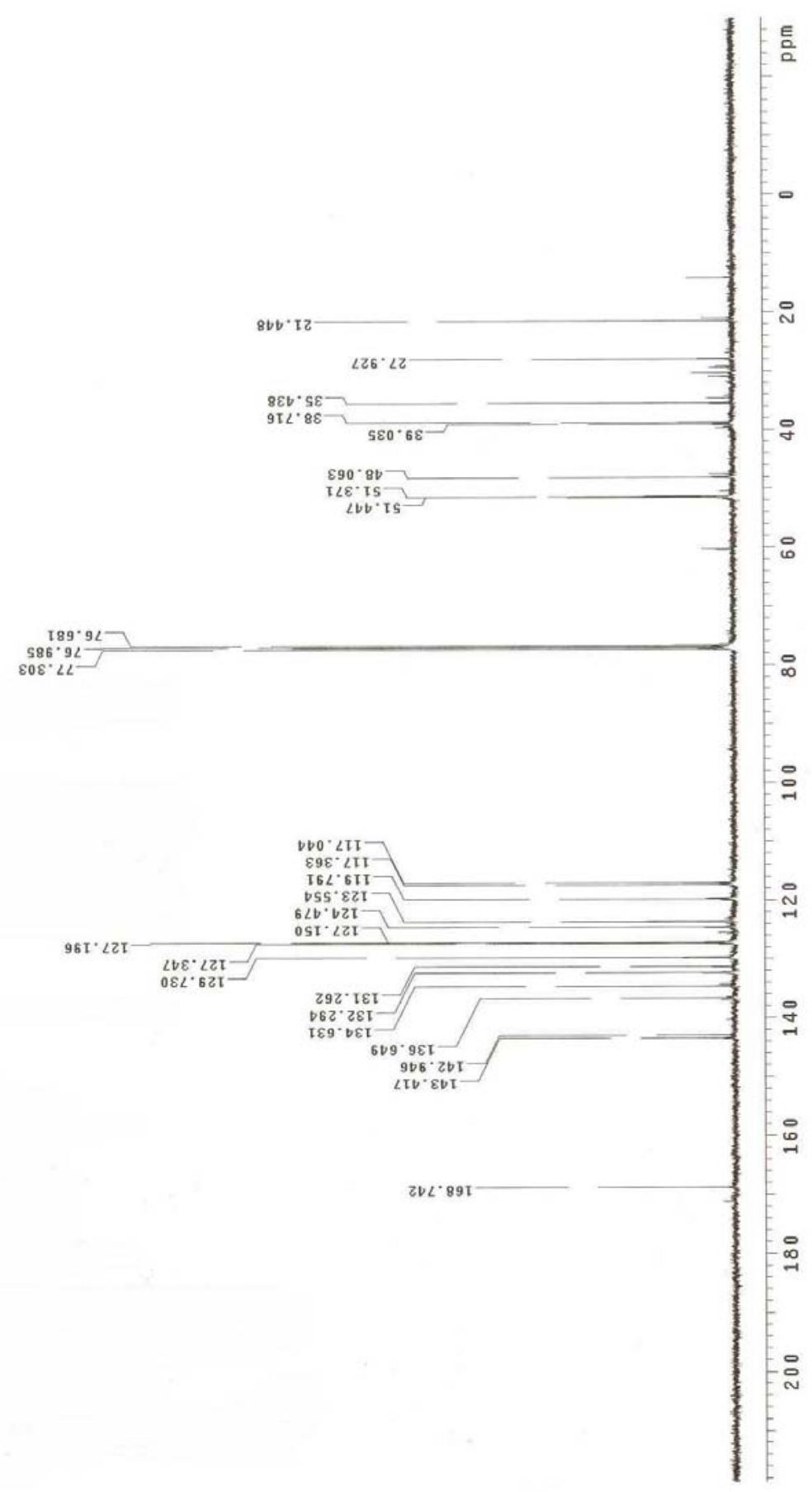




\section{${ }^{1} \mathrm{H}$ NMR Spectrum of 16}

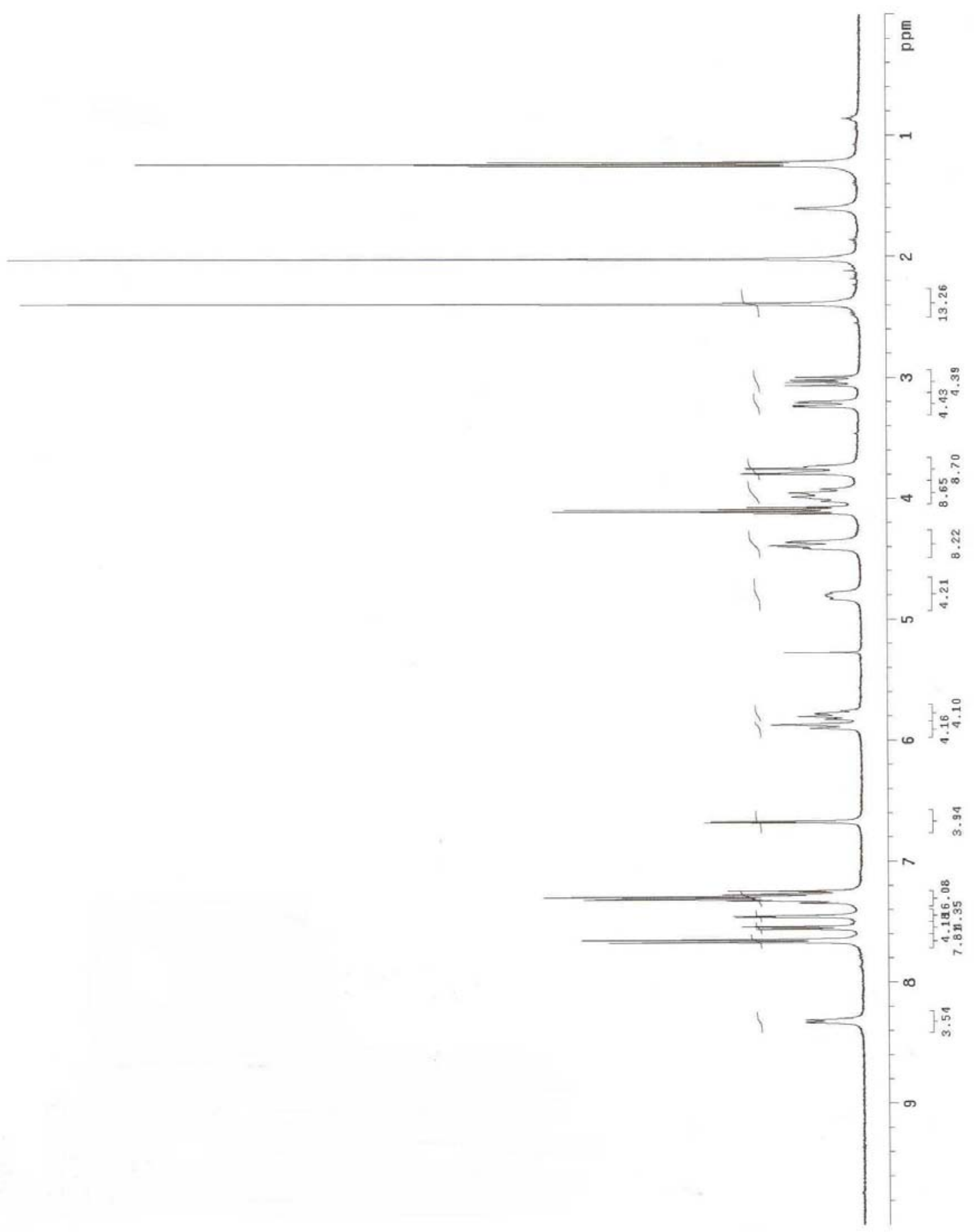


${ }^{13} \mathrm{C}$ NMR Spectrum of 16

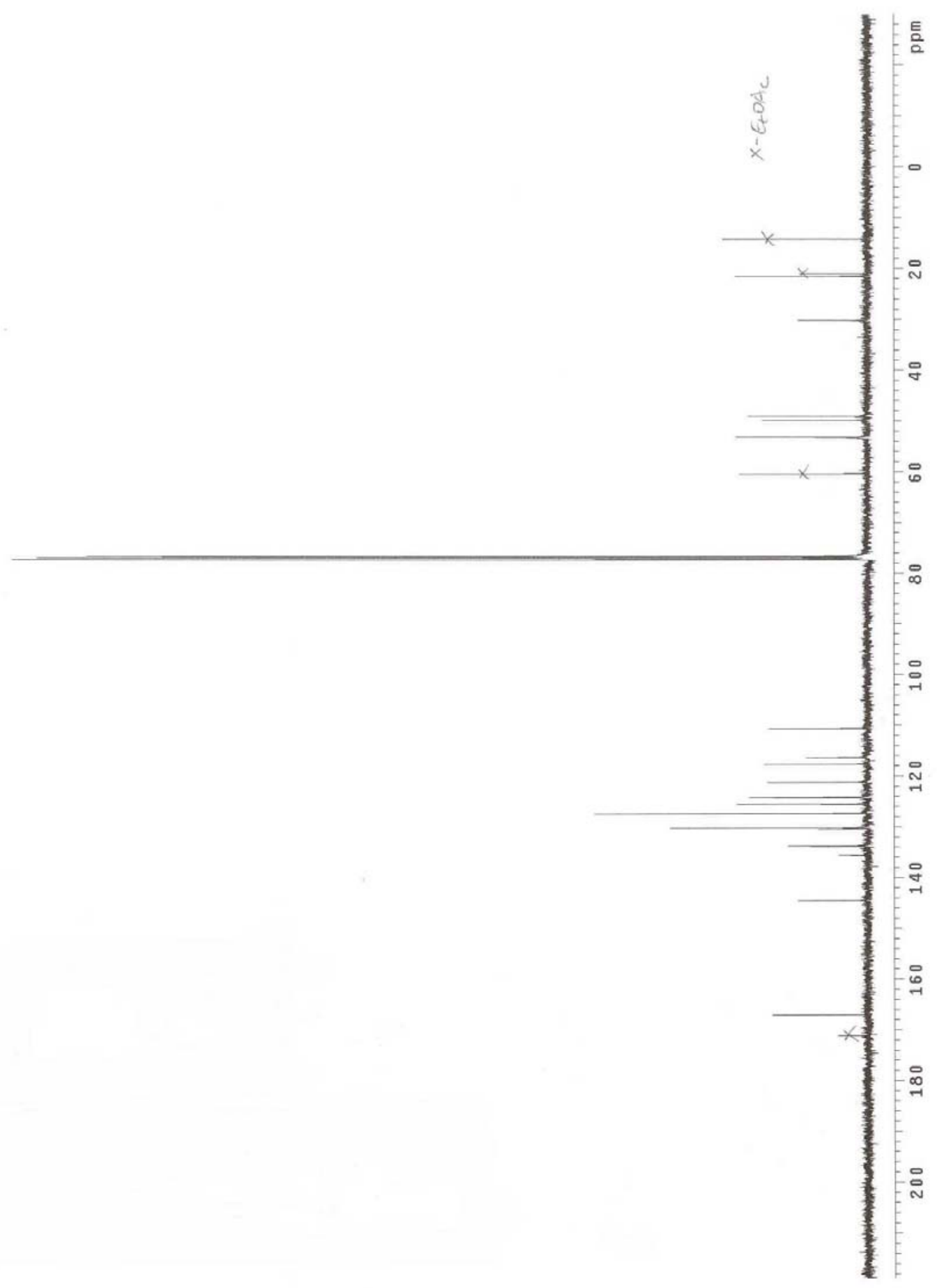




\section{${ }^{1} \mathrm{H}$ NMR Spectrum of 17}

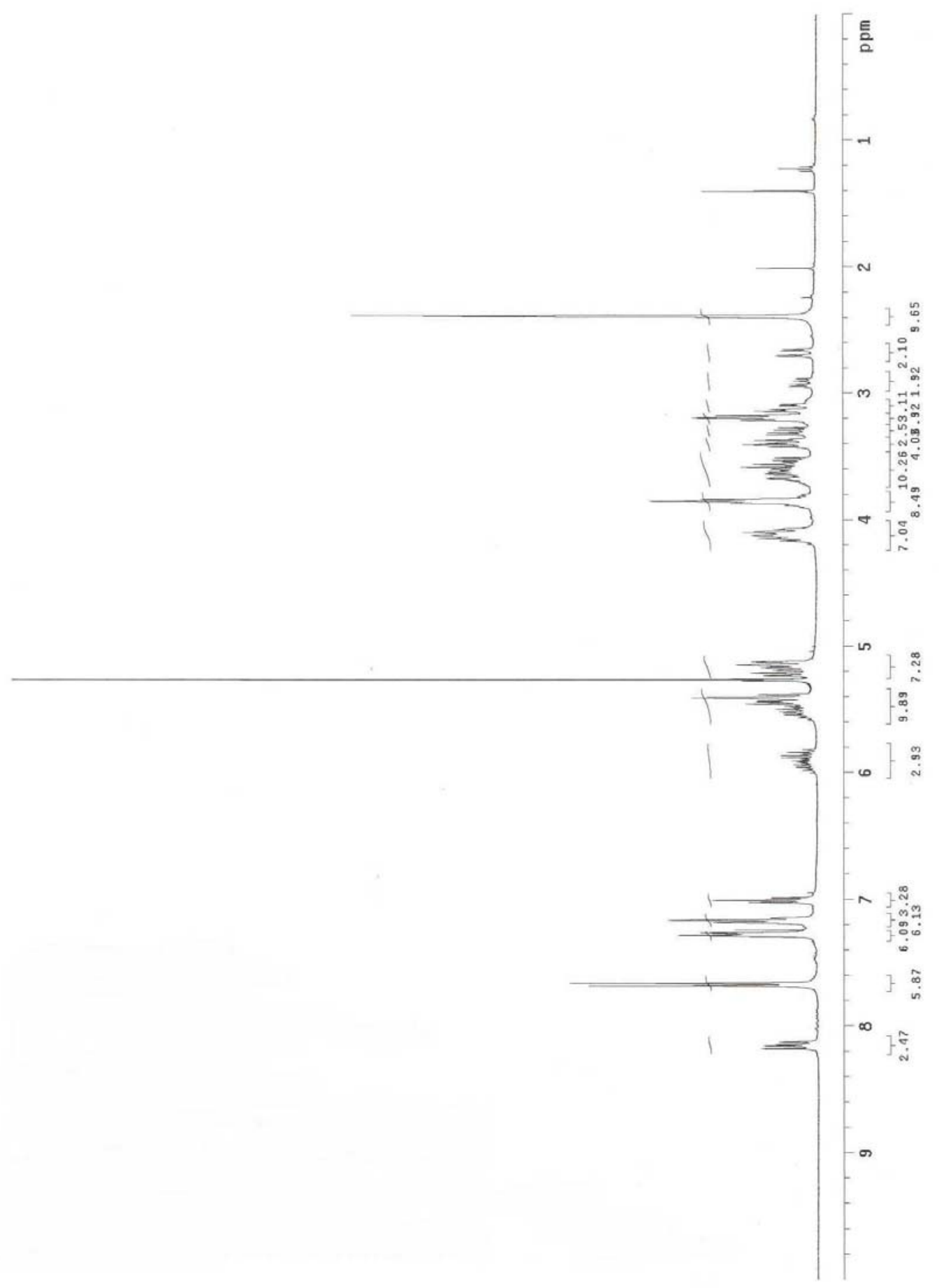


${ }^{13}$ C NMR Spectrum of 17

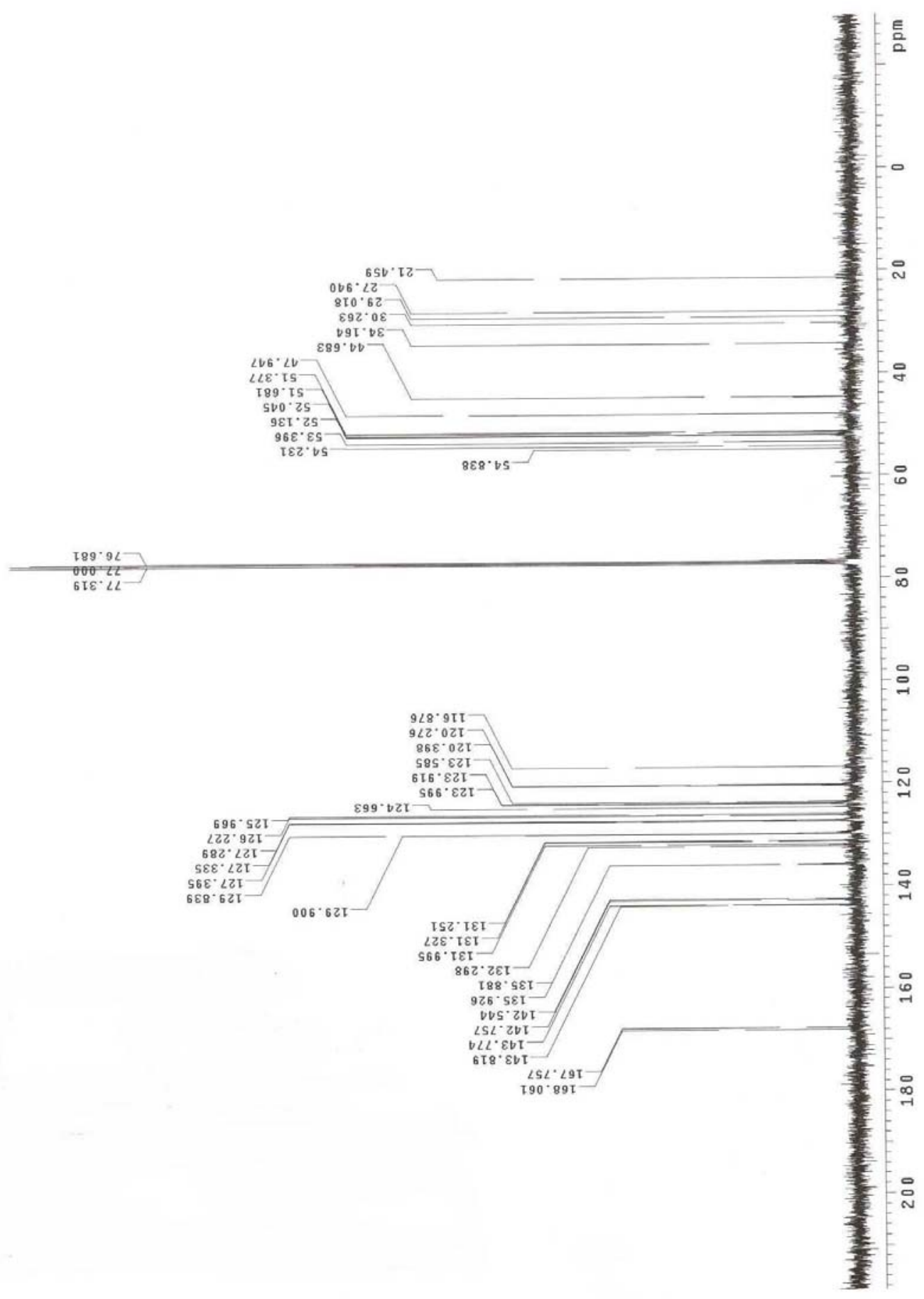




\section{${ }^{1} \mathrm{H}$ NMR Spectrum of 18}

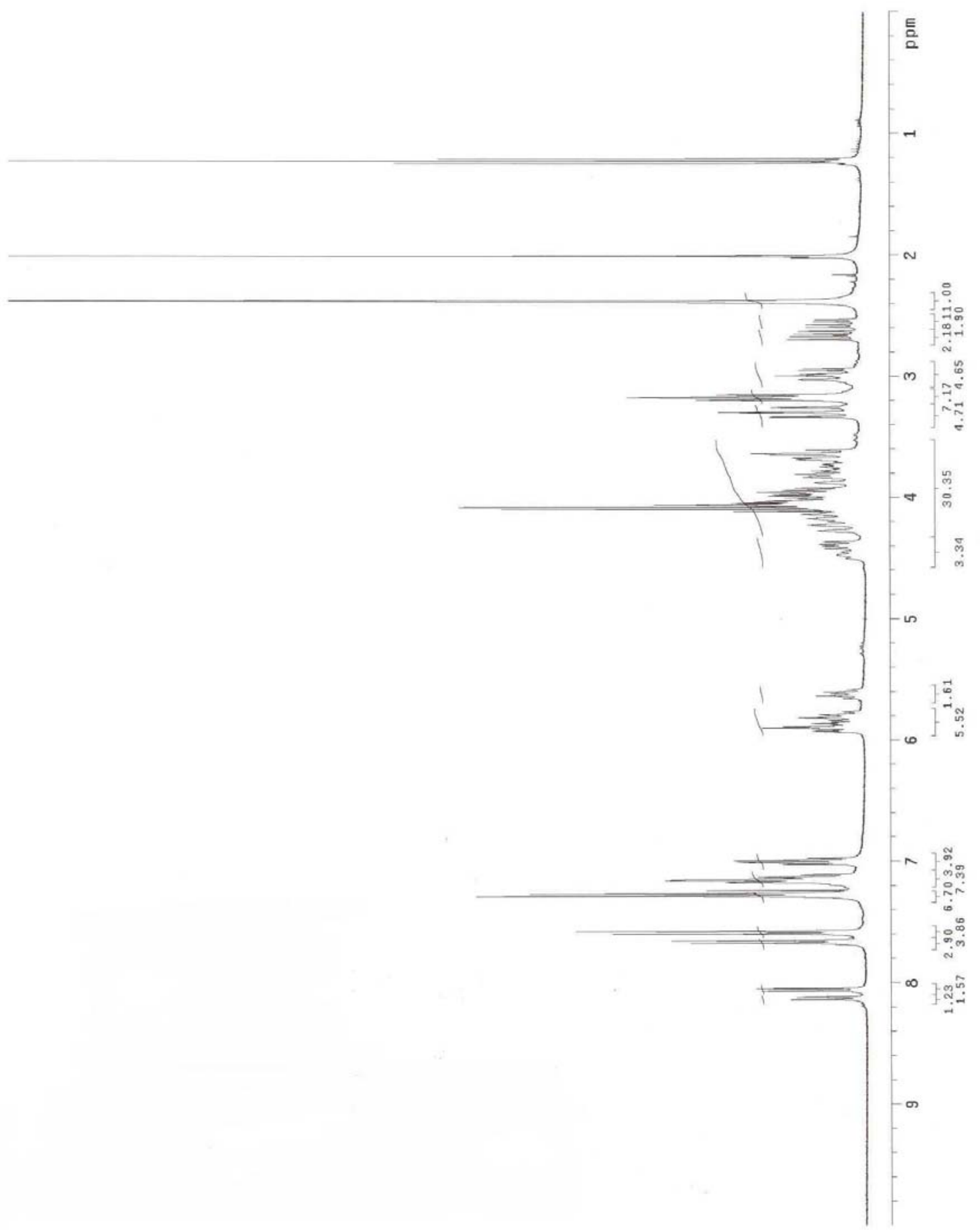




\section{${ }^{13}$ C NMR Spectrum of 18}

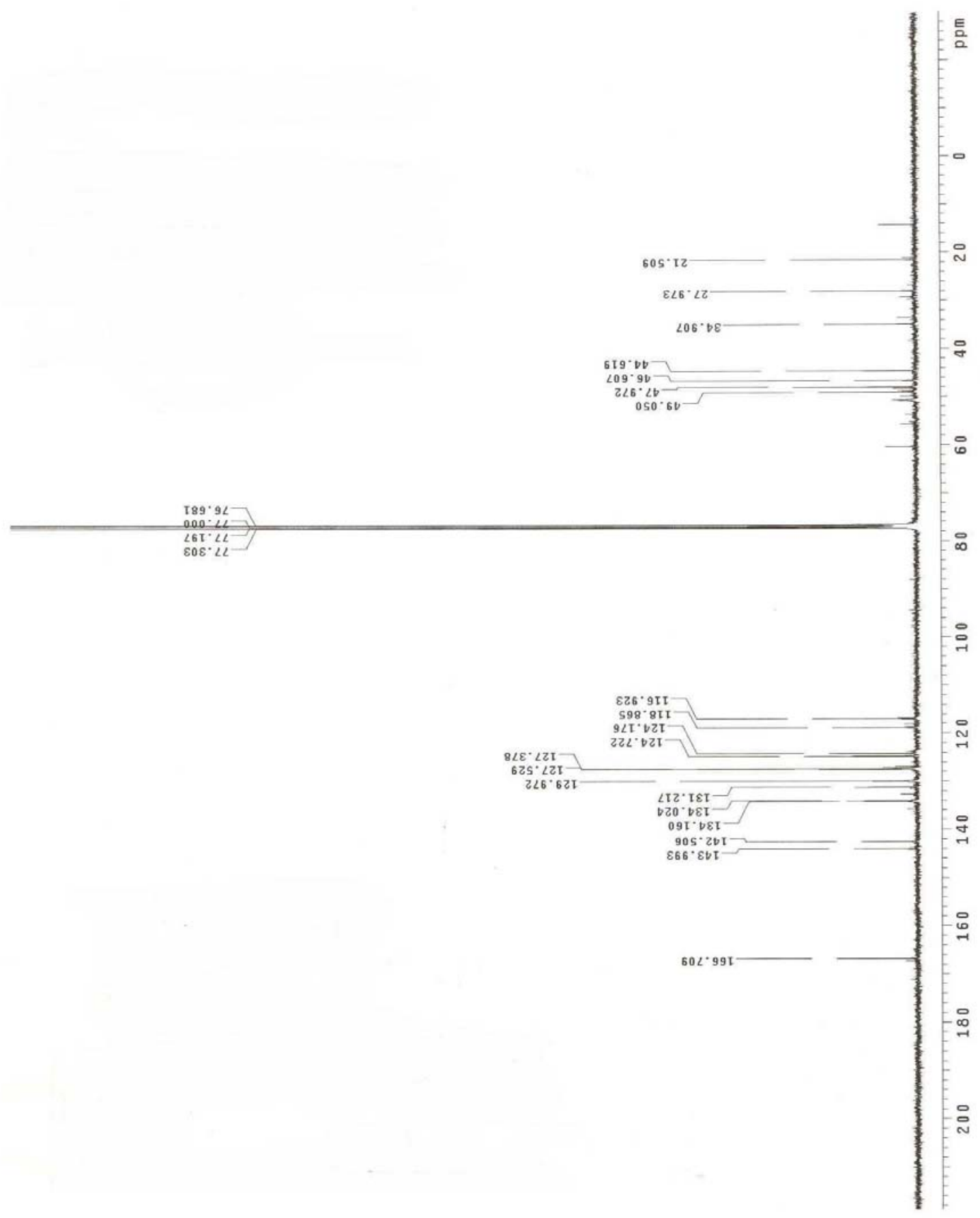




\section{${ }^{1} \mathrm{H}$ NMR Spectrum of 19}

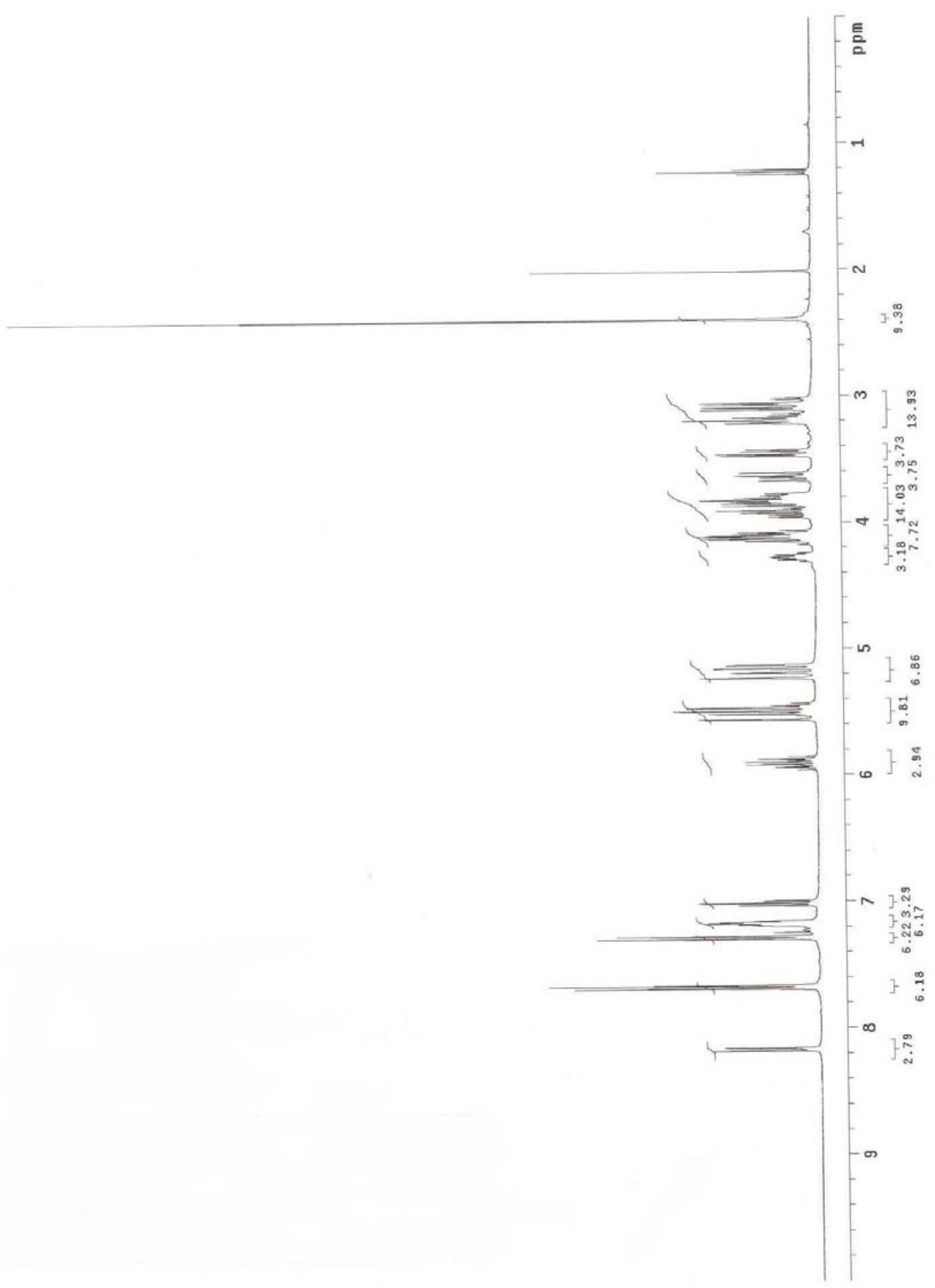




\section{${ }^{13} \mathrm{C}$ NMR Spectrum of 19}

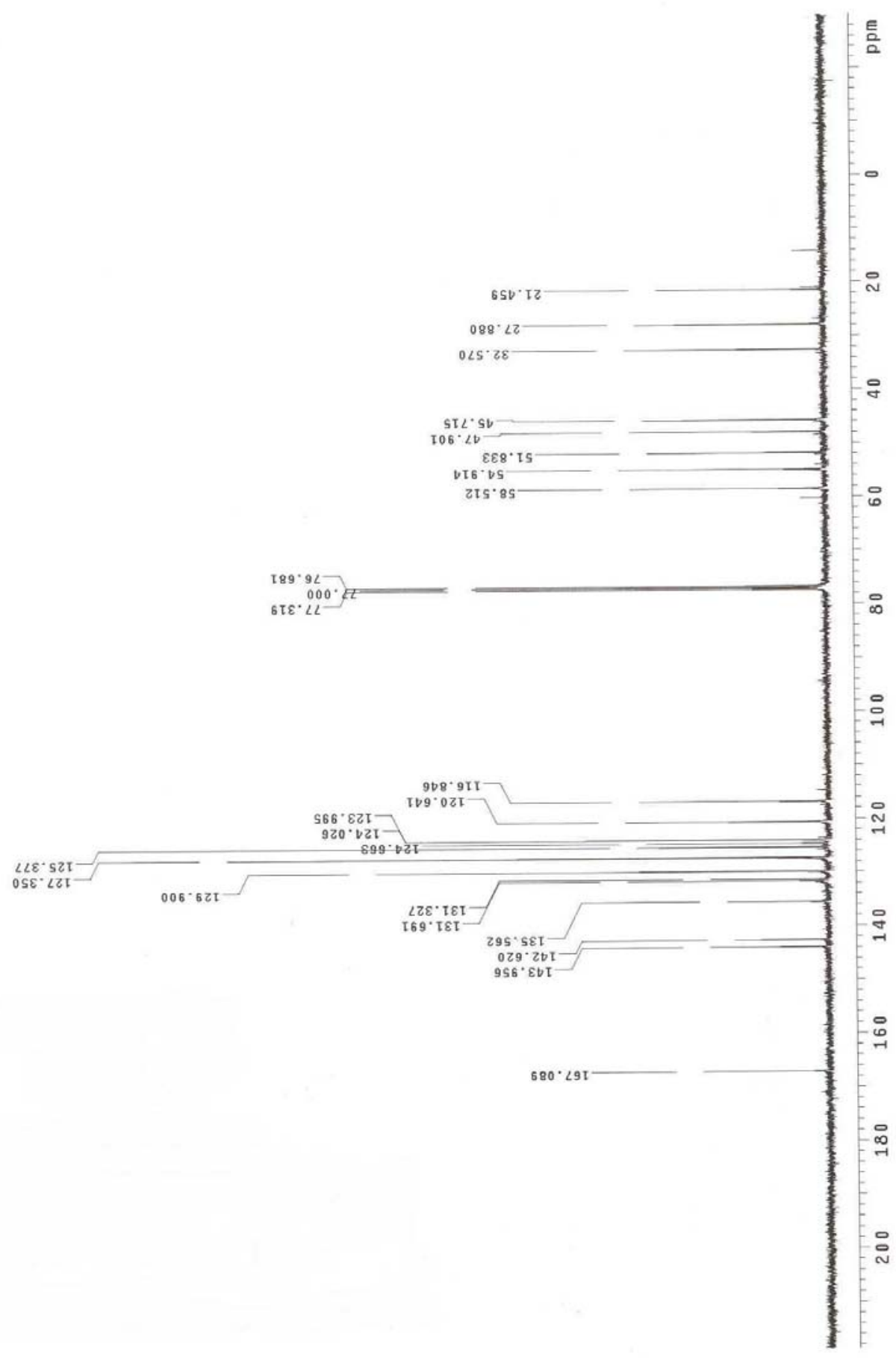


${ }^{1} \mathrm{H}$ NMR Spectrum of 20

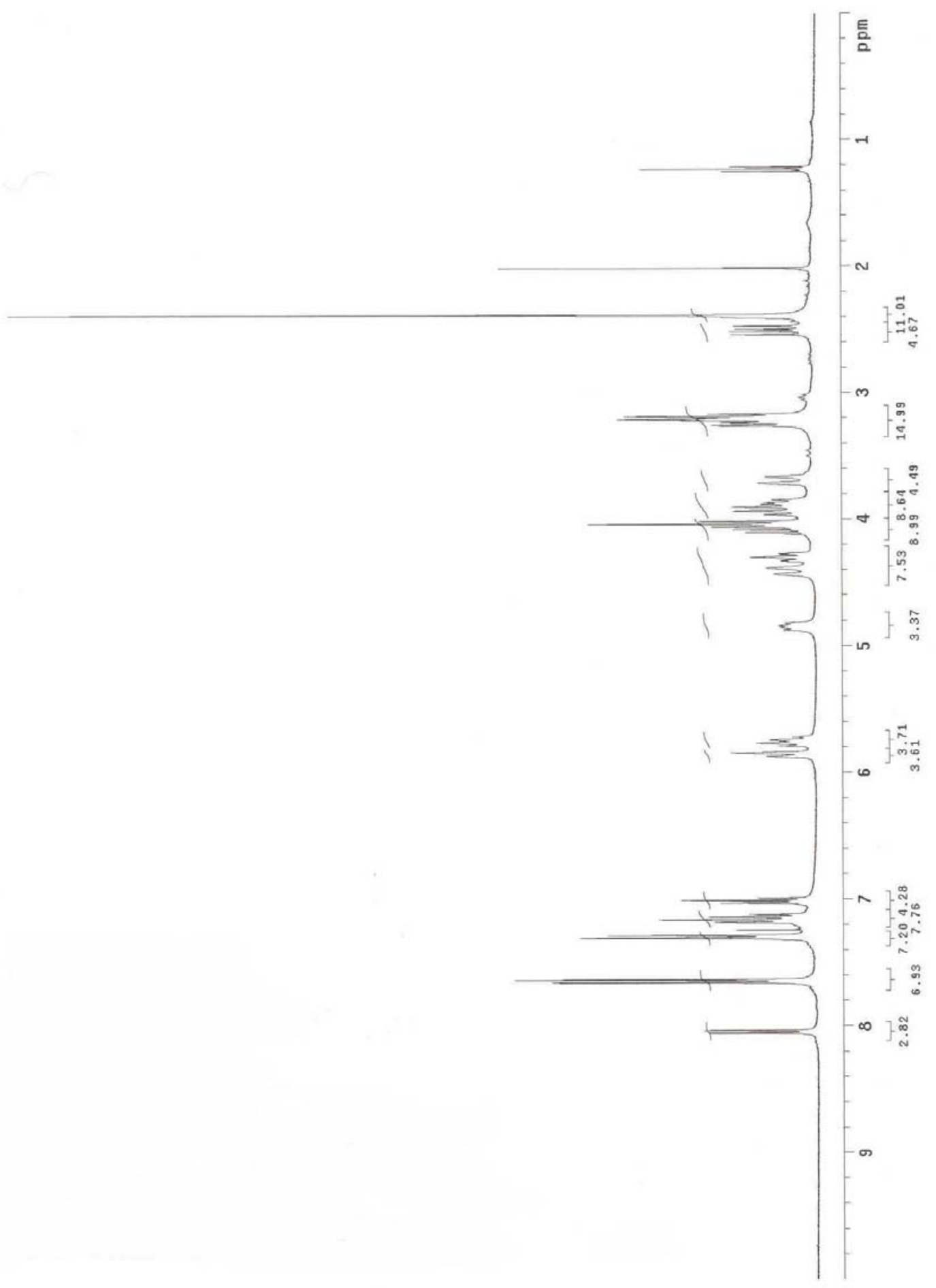


${ }^{13}$ C NMR Spectrum of 20

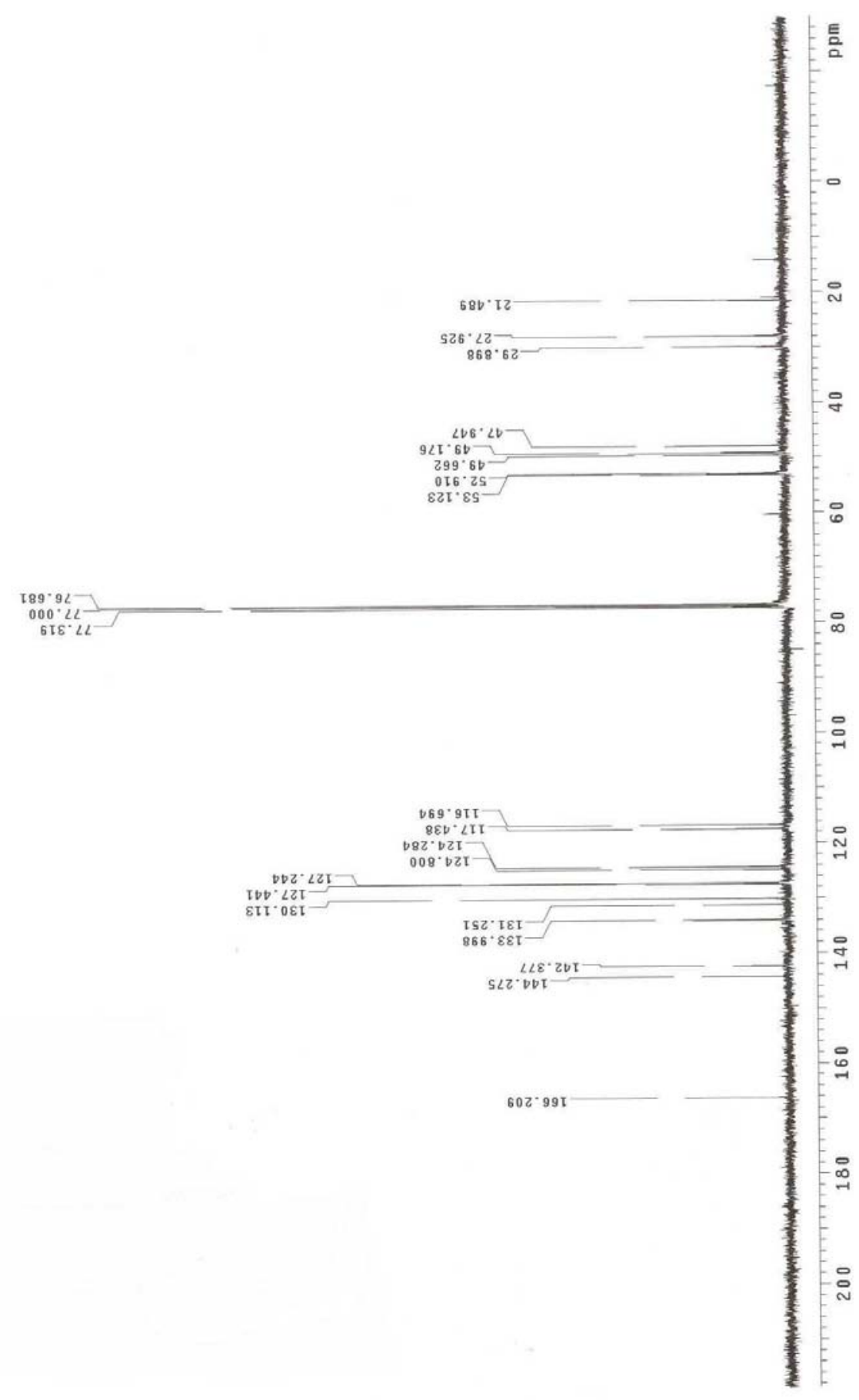




\section{${ }^{1}$ H NMR Spectrum of 21}

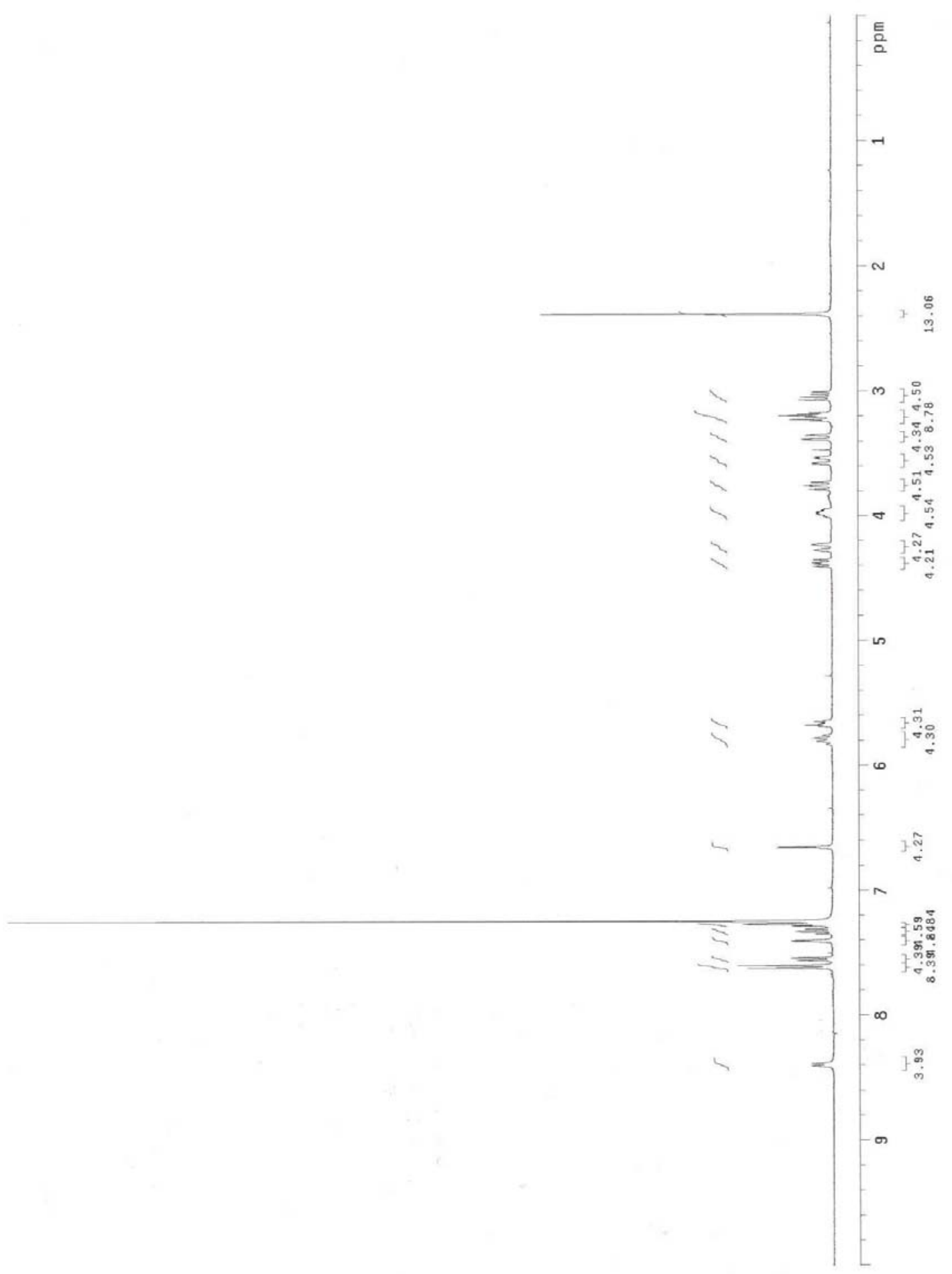


${ }^{13}$ C NMR Spectrum of 21

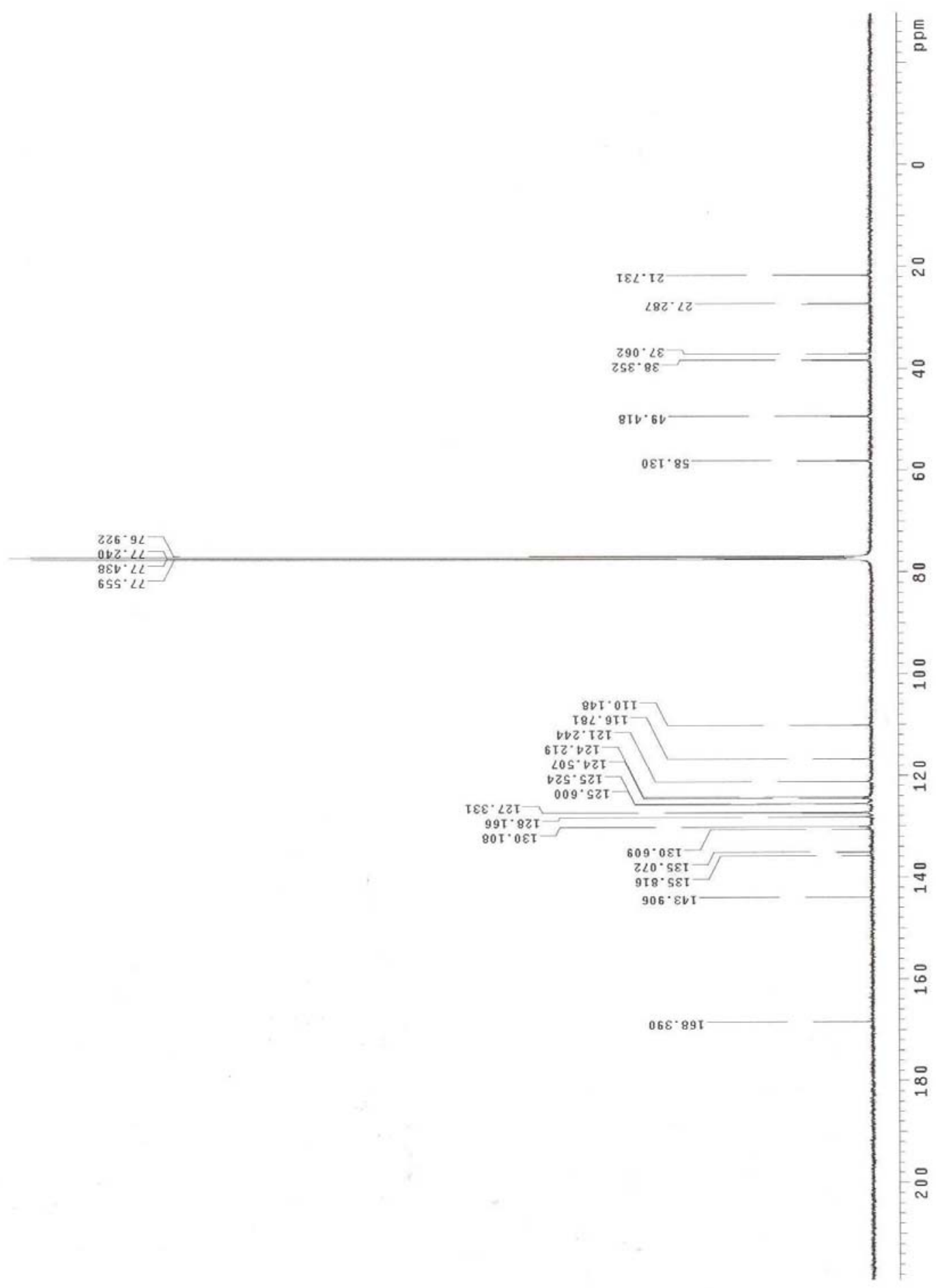




\section{Appendix 3 \\ Simple Thiazocine-2-acetic Acid Derivatives via Ring Closing Metathesis \\ Dallas K. Bates, * Xiaofen Li and Parag V. Jog \\ Additional Supplemental Information}

(Compound numbers correspond to the numbers in the article:

Bates, D. K.; Li, X.; Jog, P. V. J. Org. Chem. 2004, 69, 2750)

${ }^{1} \mathrm{H}-{ }^{1} \mathrm{H}$ COSY NMR Sprctrum of 21

${ }^{1} \mathrm{H}-{ }^{1} \mathrm{H}$ COSY NMR Sprctrum of 21 (Expansion 1) 205

${ }^{1} \mathrm{H}^{1} \mathrm{H}$ COSY NMR Sprctrum of 21 (Expansion 2) 206

${ }^{1} \mathrm{H}-{ }^{13} \mathrm{C}$ HETCOR NMR Sprctrum of 21

${ }^{1} \mathrm{H}-{ }^{13} \mathrm{C}$ HETCOR NMR Sprctrum of 21 (Expansion 1) 208

${ }^{1} \mathrm{H}-{ }^{13} \mathrm{C}$ HETCOR NMR Sprctrum of 21 (Expansion 2) 209

$\begin{array}{lr}\text { Peak Assignments in Compound } 21 & 210\end{array}$ 
${ }^{1} \mathrm{H}-{ }^{1} \mathrm{H}$ COSY NMR Spectrum of 21

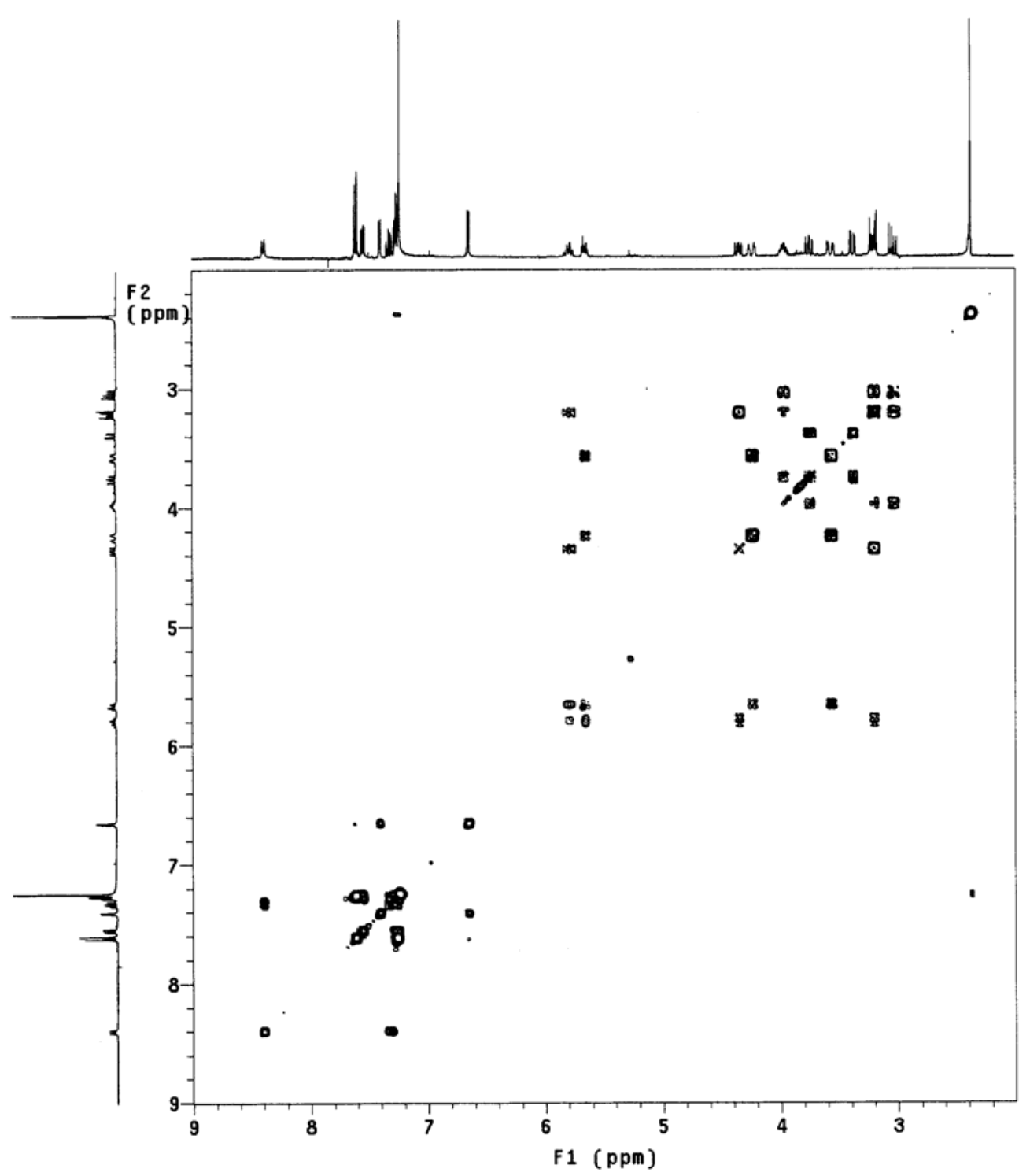




\section{${ }^{1} \mathrm{H}-{ }^{1} \mathrm{H}$ COSY NMR Spectrum of 21 (Expansion 1)}

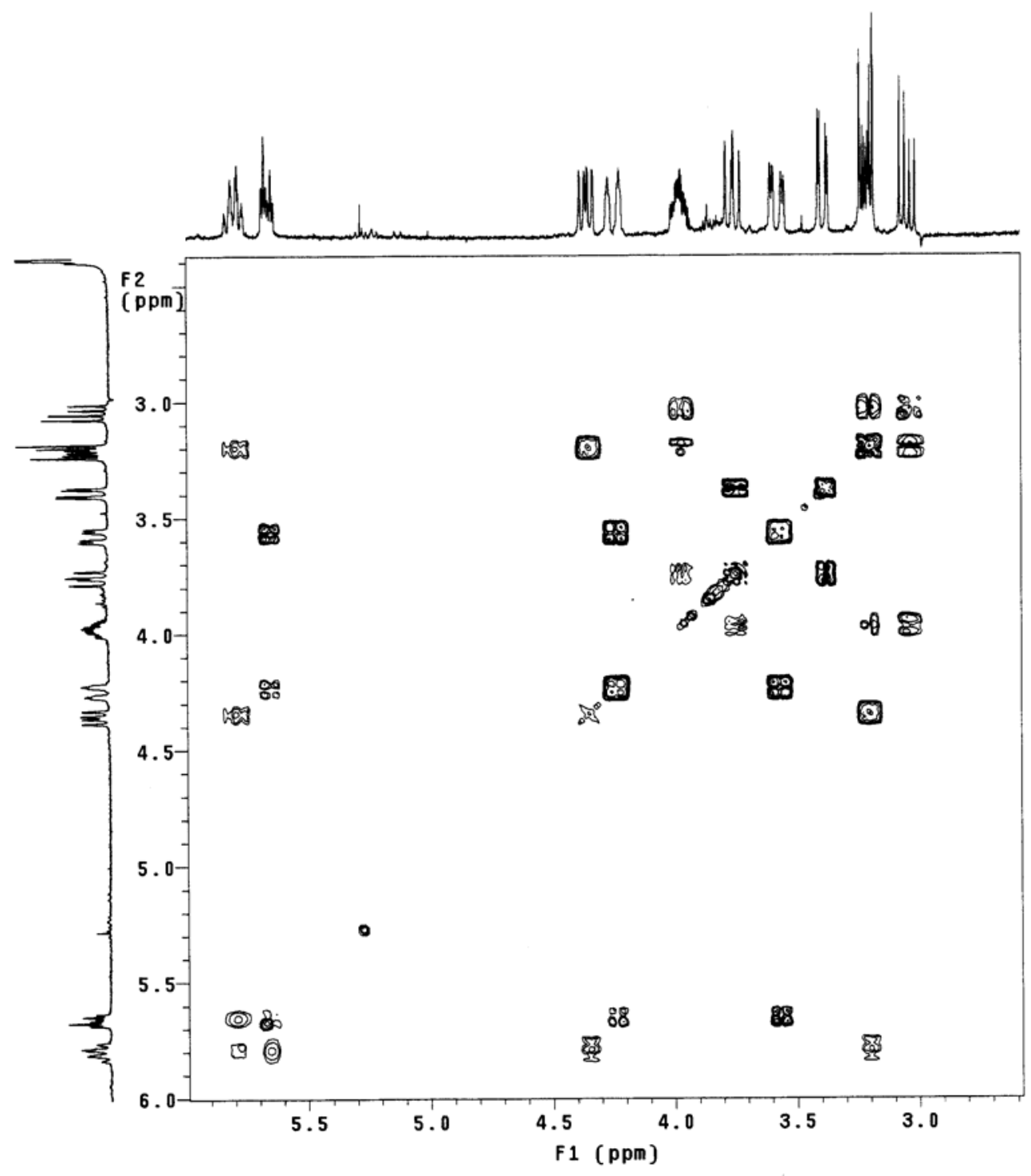




\section{${ }^{1} \mathrm{H}-{ }^{1} \mathrm{H}$ COSY NMR Spectrum of 21 (Expansion 2)}

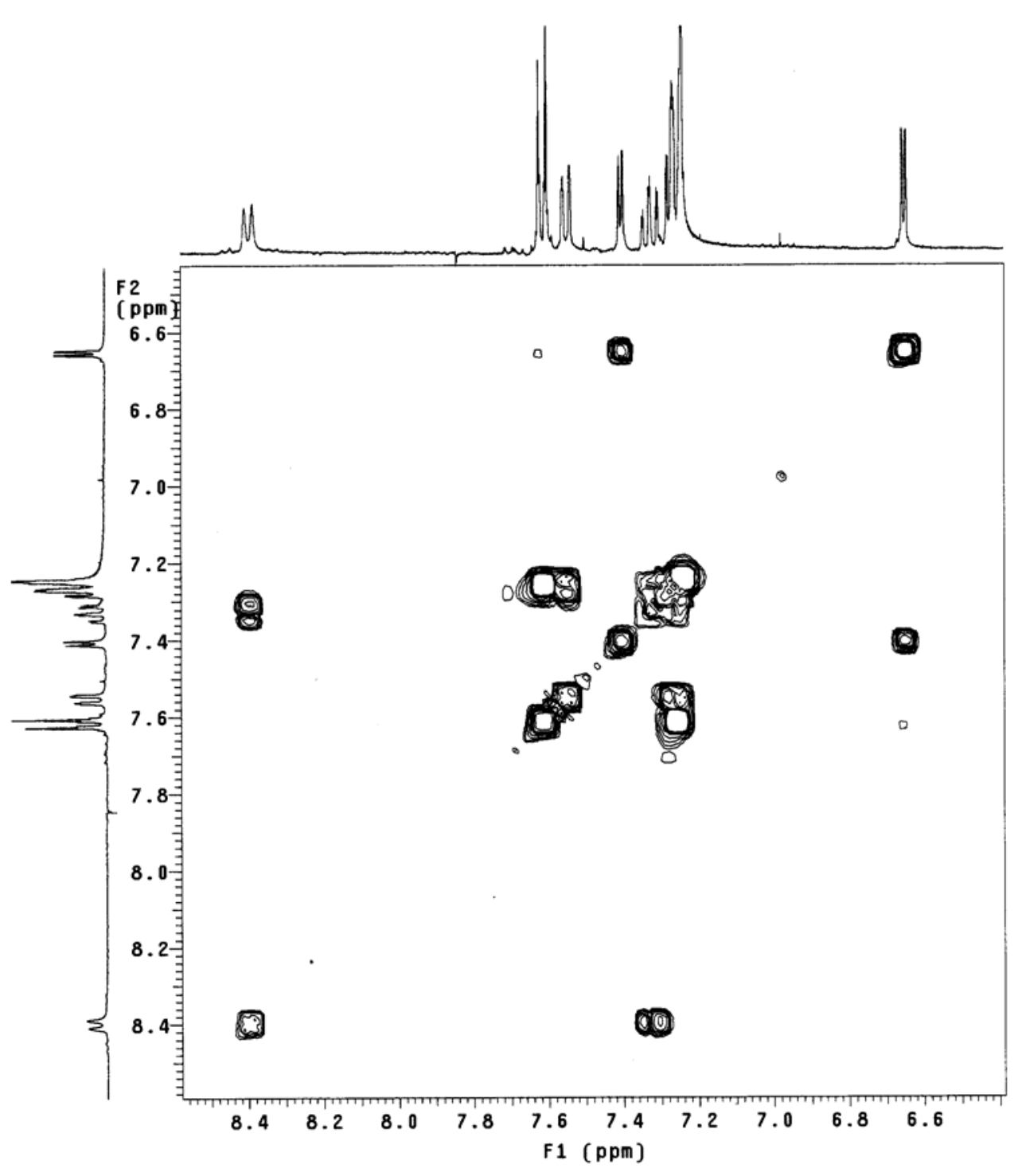


${ }^{1} \mathrm{H}-{ }^{13} \mathrm{C}$ HETCOR NMR Spectrum of 21

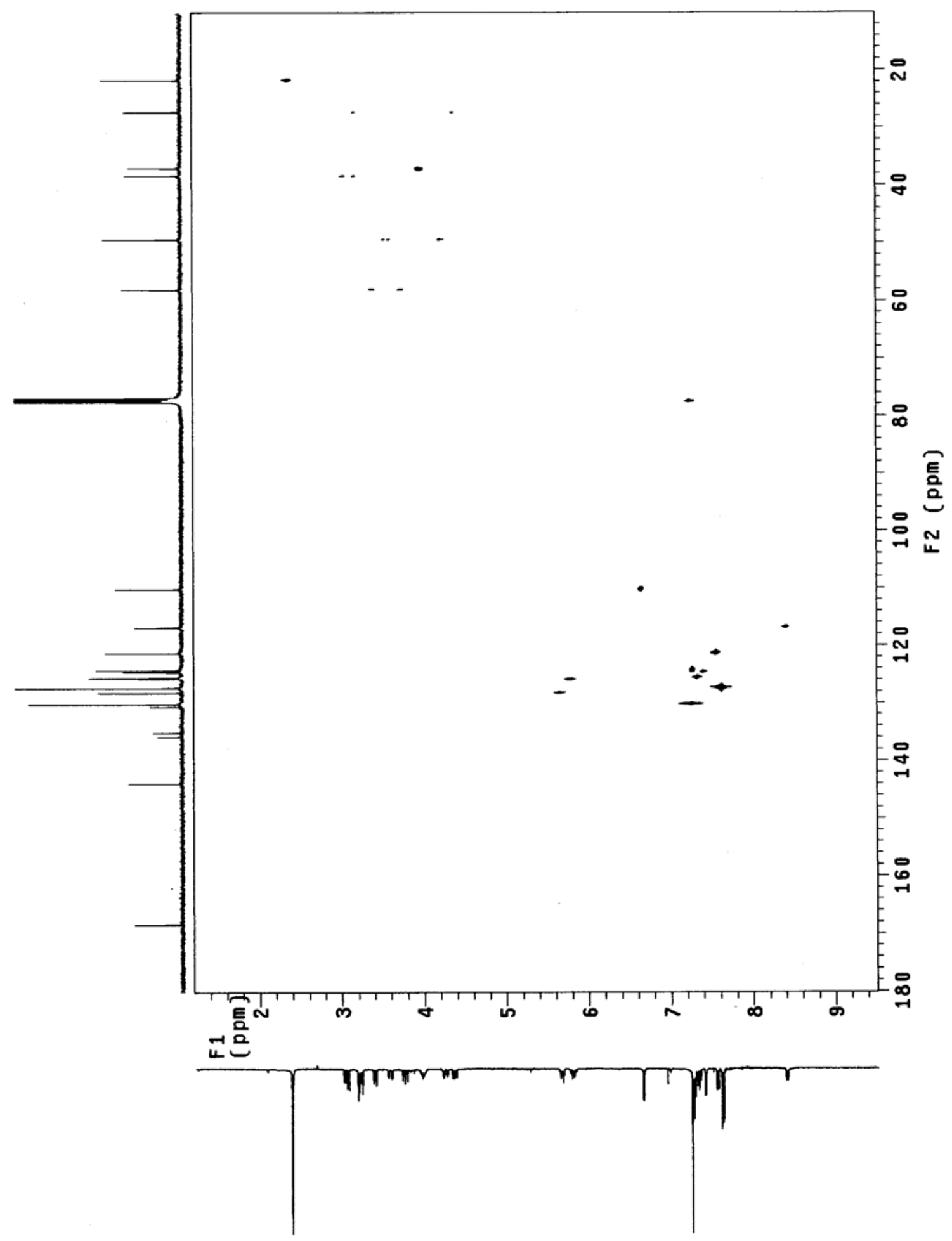


${ }^{1} \mathrm{H}-{ }^{13} \mathrm{C}$ HETCOR NMR Spectrum of 21 (Expansion 1)

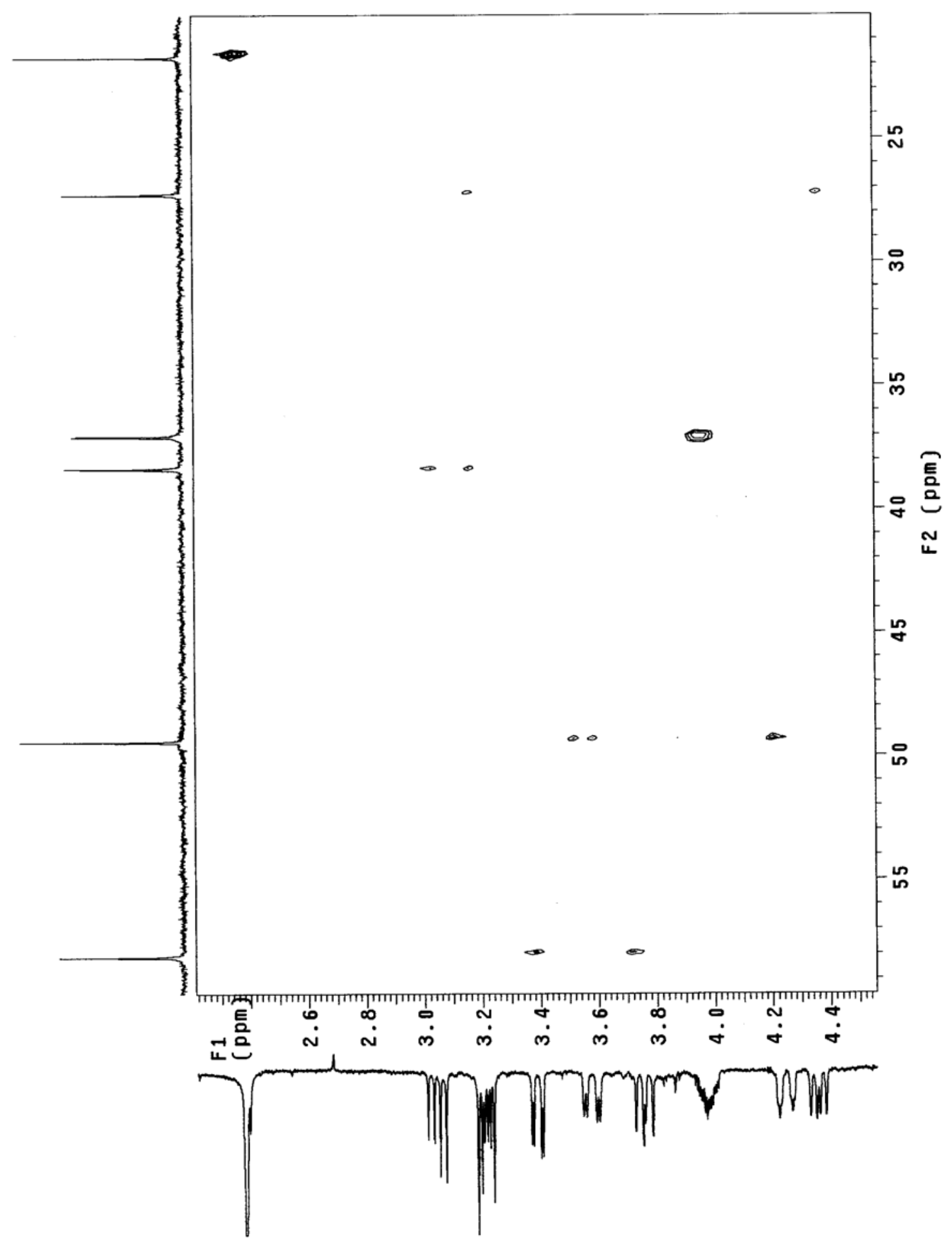


${ }^{1} \mathrm{H}-{ }^{13} \mathrm{C}$ HETCOR NMR Spectrum of 21 (Expansion 2)

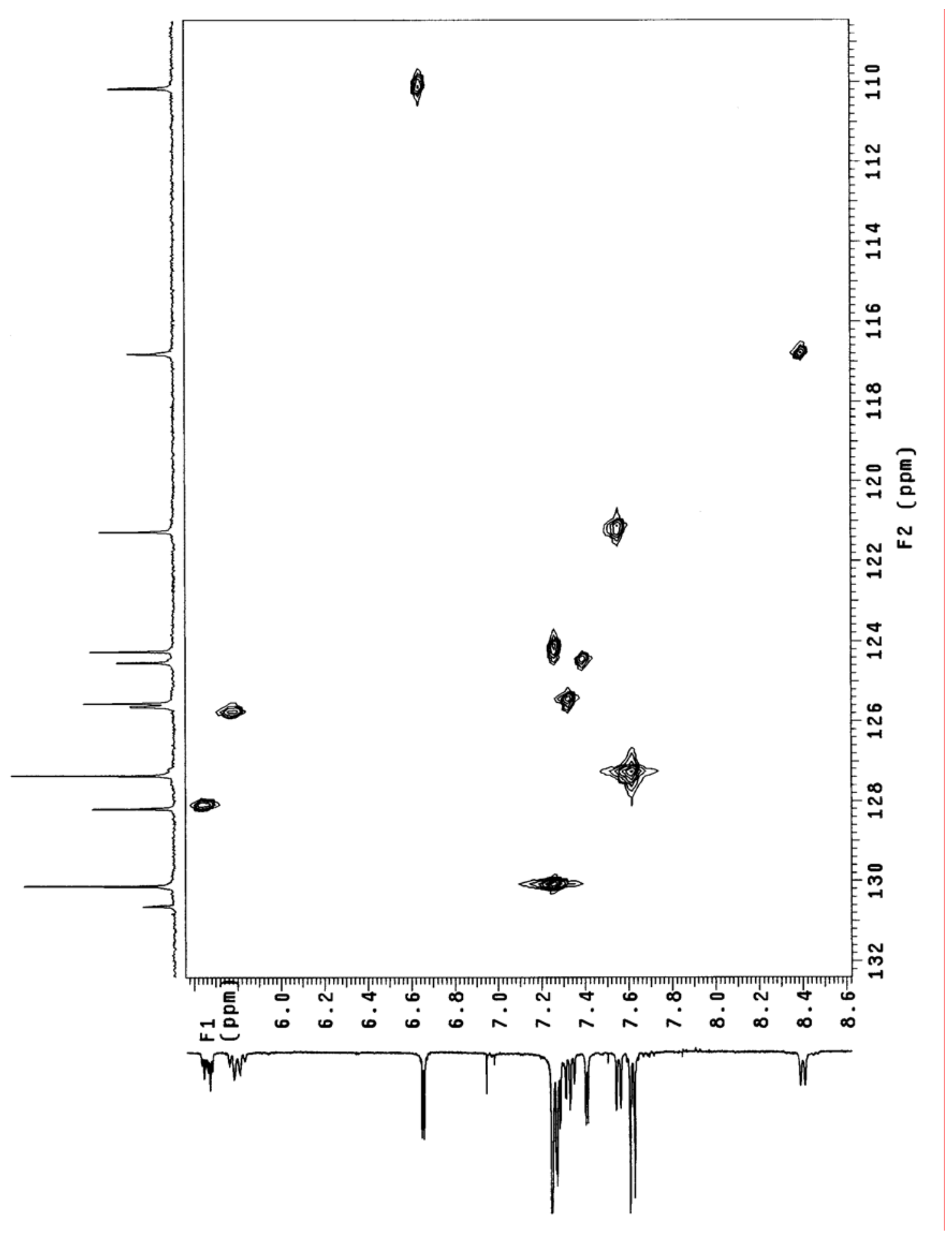


Peak Assignments in Compound 21

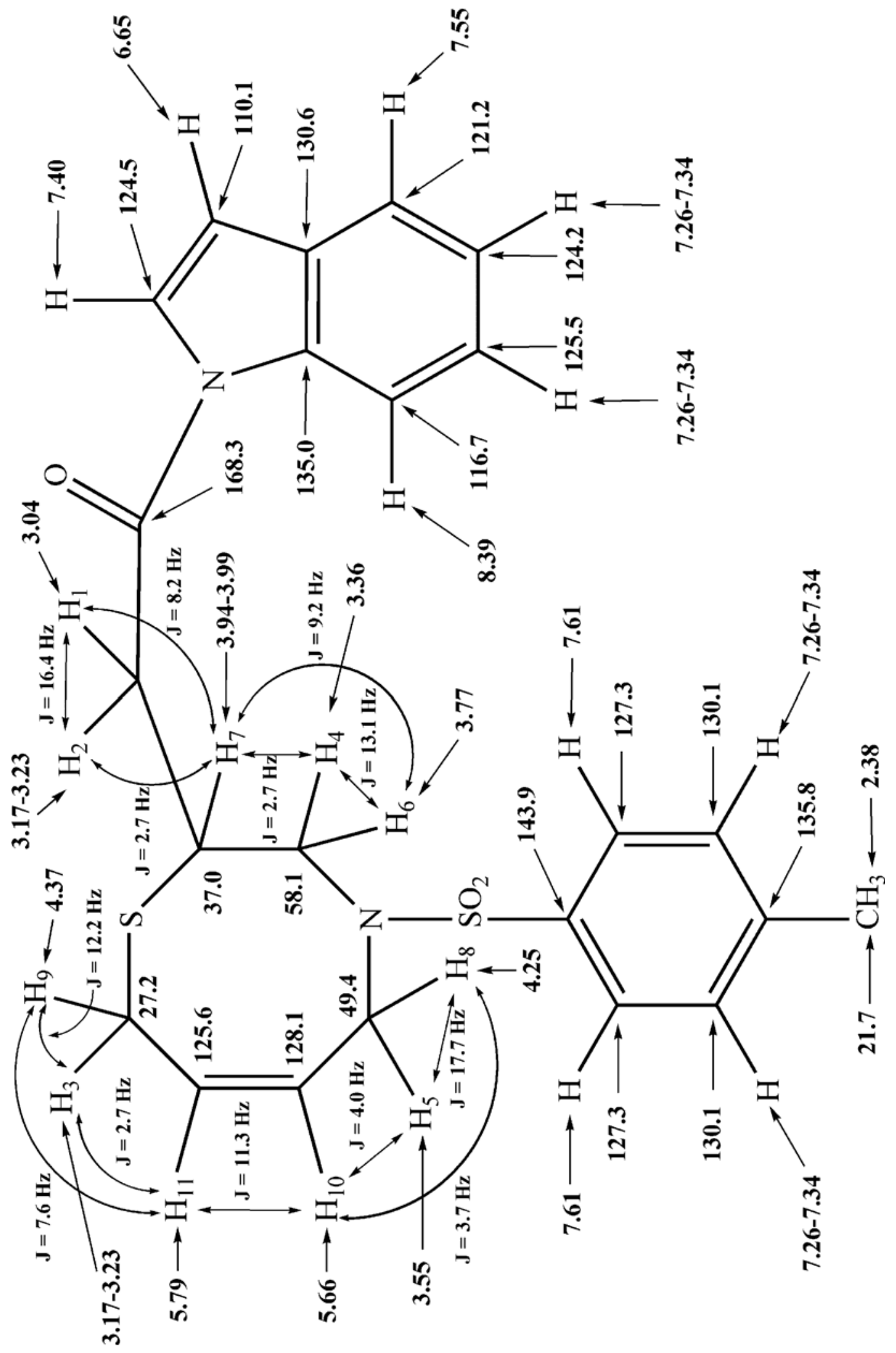




\section{Appendix 4}

\section{A Redox-Mediated Molecular Brake: Dynamic NMR Study of 2-[2- (Methylthio)phenyl]isoindolin-1-one and $S$-Oxidized Counterparts}

Parag V. Jog, Richard E. Brown and Dallas K. Bates*

(Compound numbers correspond to the numbers in the article:

Jog, P. V.; Brown, R. E.; Bates, D. K. J. Org. Chem. 2003, 68, 8240)

\section{Supplemental Material Table of Contents}

Experimental section

213-215

${ }^{1}$ H NMR Spectrum of 1

${ }^{13}$ C NMR Spectrum of 1

${ }^{1}$ H NMR Spectrum of 2

${ }^{13}$ C NMR Spectrum of 2

${ }^{1}$ H NMR Spectrum of 3

${ }^{13} \mathrm{C}$ NMR Spectrum of 3

PE Surface Plot for 2

PE Surface Plot for 3

Z- matrix for 1 [N-Ar \& S-Ar locked] (Lowest A)

Z- matrix for 1 [N-Ar \& S-Ar locked] (Lowest B)

Z-matrix for 1 [N-Ar \& S-Ar locked] (TS)

Z- matrix for 2 [N-Ar \& S-Ar locked] (Lowest A) 
Z- matrix for 2 [N-Ar \& S-Ar locked] (Lowest B)

Z-matrix for 2 [N-Ar \& S-Ar locked] (TS)

Z- matrix for 3 [N-Ar \& S-Ar locked] (Lowest A)

Z- matrix for 3[N-Ar \& S-Ar locked] (Lowest B)

Z-matrix for 3 [N-Ar \& S-Ar locked] (TS)

Figure 6. Partial ${ }^{1} \mathrm{H}$ NMR of 1 (and 2) [-40 $\left.{ }^{\circ} \mathrm{C}, \mathrm{CDCl}_{3}\right]$

in the presence of $m$-CPBA as a limiting reagent.

2D plot of $\mathrm{N}$-Ar torsion angle vs virtual dihedral angle (VDA) for 2

2D plot of $\mathrm{N}$-Ar torsion angle vs virtual dihedral angle (VDA) for 3

Line-shape analysis for 3

Z-matrix for 1 [N-Ar \& VDA locked] (Lowest Energy)

Z-matrix for 2 [N-Ar \& VDA locked] (Lowest Energy)

Z-matrix for 3 [N-Ar \& VDA locked] (Lowest Energy)

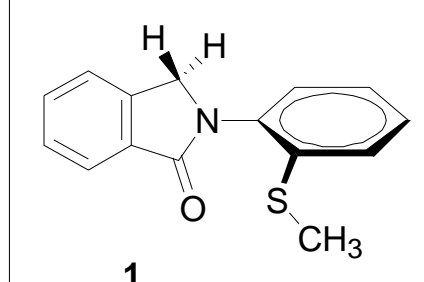

1

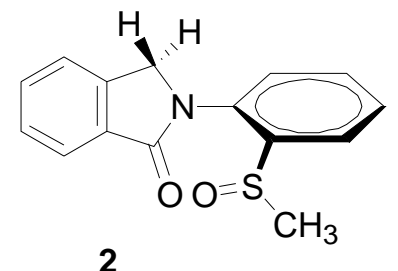

2

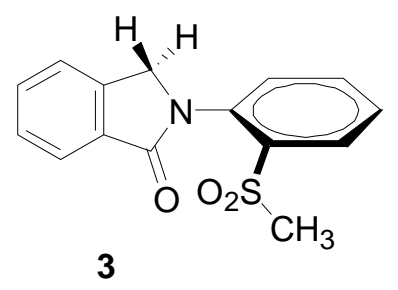




\section{Experimental Section}

${ }^{1} \mathrm{H}$ and ${ }^{13} \mathrm{C}$ NMR spectra were recorded in $\mathrm{CDCl}_{3}$ (unless otherwise noted) and were recorded at $400 \mathrm{MHz}$ or $100 \mathrm{MHz}$, respectively. Mass spectra were obtained by electron impact $(70 \mathrm{eV})$, relative intensities are given in parenthesis. IR spectra were recorded in chloroform solution. Column chromatography was carried out on silica gel (0.06-0.20 $\mathrm{mm}$, pore diameter $\sim 6 \mathrm{~nm}$ ). Organic phases were dried over anhydrous $\mathrm{Na}_{2} \mathrm{SO}_{4}$. Phthalaldehyde, 2-aminothiophenol, m-chloroperbenzoic acid were commercial samples and used without further purification.

2-[2-(methylthio)phenyl]isoindolin-1-one (1). o-Phthalaldehyde (4 mmol, $0.5 \mathrm{~g})$ in AcOH $(20 \mathrm{~mL})$ was heated to reflux. To this was added dropwise 2-(methylthio)aniline $(1.4 \mathrm{~g}, 10 \mathrm{mmol})$ in $2 \mathrm{~mL}$ of $\mathrm{AcOH}$. After $20 \mathrm{~min}$ at reflux the mixture was poured into water and extracted with EtOAc. The organic layer was washed sequentially with $2 \mathrm{~N}$ $\mathrm{HCl}$, saturated $\mathrm{NaHCO}_{3}$ and brine and then dried. Solvent was evaporated and the crude orange-red solid was purified by column chromatography (benzene/EtOAc, 80/20) and recrystallization (EtOAc) to give a white solid (0.32 g, $30 \%$ : mp 153-154 ${ }^{\circ} \mathrm{C}$; IR v 1692

$\mathrm{cm}^{-1} ;{ }^{1} \mathrm{H}$ NMR $\delta 2.40(\mathrm{~s}, 3 \mathrm{H}), 4.77(\mathrm{~s}, 2 \mathrm{H}), 7.21-7.38(\mathrm{~m}, 4 \mathrm{H}), 7.49(\mathrm{t}, \mathrm{J}=7.94 \mathrm{~Hz}, 2 \mathrm{H})$, $7.58(\mathrm{dt}, \mathrm{J}=7.62,0.89 \mathrm{~Hz}, 1 \mathrm{H}), 7.94(\mathrm{~d}, \mathrm{~J}=7.56 \mathrm{~Hz}, 1 \mathrm{H}) ;{ }^{13} \mathrm{C} \mathrm{NMR} \delta 15.5,52.2,123.0$ $124.6,126.1,126.9,128.3,129.1,129.1,132.0,132.3,136.1,138.3,142.0,168.3$; MS (EI) $\left[\mathrm{m} / \mathrm{z}\right.$ (rel. intensity)] 255 [M+ $\left.\mathrm{M}^{+}, 43\right], 208$ (100), 180 (25). Anal. Calc. for $\mathrm{C}_{15} \mathrm{H}_{13} \mathrm{NOS}$ : C, 70.56; H, 5.13; N, 5.49. Found: C, 70.80; H, 5.32; N, 5.59.

2-[2-(methylsulfinyl)phenyl]isoindolin-1-one (2). To an ice-cooled solution of 1 (0.1 $\mathrm{g}, 0.39 \mathrm{mmol})$ in $\mathrm{CH}_{2} \mathrm{Cl}_{2}(20 \mathrm{~mL})$ was added slowly a solution of $m$-CPBA (1.1eq, 0.43 mmol, $0.074 \mathrm{~g})$ in $\mathrm{CH}_{2} \mathrm{Cl}_{2}(5 \mathrm{~mL})$. The resulting mixture was stirred at $0{ }^{\circ} \mathrm{C}$ for $15 \mathrm{~min}$ 
and then put it in a freezer $\left(-8{ }^{\circ} \mathrm{C}\right)$ overnight. The reaction mixture was then poured into $5 \% \mathrm{NaHCO}_{3}$ solution $(40 \mathrm{~mL})$ and extracted with $\mathrm{CH}_{2} \mathrm{Cl}_{2}(40 \mathrm{~mL})$. The combined organic layer was washed with distilled water, dried, and concentrated in vacuo. Column chromatography (EtOAc) gave yellowish white solid (0.082 g, $77 \%$ ), which was recrystallized (EtOAc-hexane, 8:2) to give a white solid: $\mathrm{mp} 161-162^{\circ} \mathrm{C}$; IR $\vee 1698 \mathrm{~cm}^{-1}$; ${ }^{1} \mathrm{H}$ NMR $\delta 2.88(\mathrm{~s}, 3 \mathrm{H}), 4.84(\mathrm{~s}, 2 \mathrm{H}), 7.29(\mathrm{dd}, \mathrm{J}=$ 7.67, $1.44 \mathrm{~Hz}, 1 \mathrm{H}), 7.50-7.64(\mathrm{~m}$, $5 \mathrm{H}), 7.89(\mathrm{~d}, \mathrm{~J}=7.46 \mathrm{~Hz}, 1 \mathrm{H}), 8.11(\mathrm{dd}, \mathrm{J}=7.64,1.67 \mathrm{~Hz}, 1 \mathrm{H}) ;{ }^{13} \mathrm{C}$ NMR $\delta 43.3,53.4$, 123.2, 124.6, 125.5, 127.4, 128.8, 129.8, 131.3, 132.5, 132.7, 135.2, 141.7, 144.9, 169.1; MS (EI) [m/z (rel. intensity)] 271 [M+2, 23], 254 (100), 238 (77), 208 (53). Anal. Calc. for $\mathrm{C}_{15} \mathrm{H}_{13} \mathrm{NO}_{2} \mathrm{~S}: \mathrm{C}, 66.40 ; \mathrm{H}, 4.83 ; \mathrm{N}, 5.16$. Found: $\mathrm{C}, 66.18 ; \mathrm{H}, 4.94 ; \mathrm{N}, 4.97$.

2-[2-(methylsulfonyl)phenyl]isoindolin-1-one (3). To an ice-cooled solution of $\mathbf{1}$ ( $0.10 \mathrm{~g}, 0.39 \mathrm{mmol})$ in $\mathrm{CH}_{2} \mathrm{Cl}_{2}(20 \mathrm{~mL})$ was added a solution of $m$-CPBA (2.2 eq, 0.86 mmol, $0.15 \mathrm{~g})$ in $\mathrm{CH}_{2} \mathrm{Cl}_{2}(10 \mathrm{~mL})$. The resulting mixture was stirred at room temperature for $18 \mathrm{~h}$, then poured into $5 \% \mathrm{NaHCO}_{3}(40 \mathrm{~mL})$ solution. The aqueous layer was extracted with $\mathrm{CH}_{2} \mathrm{Cl}_{2}(30 \mathrm{~mL})$. The combined organic layer was washed with distilled water, dried, and concentrated in vacuo. Column chromatography (EtOAc: hexane, 6:4) gave yellowish white solid (0.091 g, 82\%), which was recrystallized (EtOAc-hexane, 7:3) to give a white solid: mp $186-187{ }^{\circ} \mathrm{C}$; IR $v 1694 \mathrm{~cm}^{-1}$; ${ }^{1} \mathrm{H}$ NMR $\delta 3.15(\mathrm{~s}, 3 \mathrm{H}), 4.84$ (br, 2H), $7.39(\mathrm{dd}, \mathrm{J}=7.83,1.2 \mathrm{~Hz}, 1 \mathrm{H}), 7.48-7.53(\mathrm{~m}, 2 \mathrm{H}), 7.59-7.64(\mathrm{~m}, 2 \mathrm{H}), 7.74(\mathrm{dt}, \mathrm{J}=$ 7.65, 4.6 Hz, 1H), $7.89(\mathrm{~d}, \mathrm{~J}=7.59 \mathrm{~Hz}, 1 \mathrm{H}), 8.17(\mathrm{~d}, \mathrm{~J}=7.94,1.48 \mathrm{~Hz}, 1 \mathrm{H}) ;{ }^{13} \mathrm{C}$ NMR $\delta$ $44.2,55.5,123.2,124.4,128.5,129.8,131.1,131.5,131.7,132.5,135.3,137.8,140.0$ 142.6, 169.8; MS (EI) [m/z (rel. intensity)] 287 [M+, 34], 208 (100), 179 (17), 105 (50). Anal. Calc. for $\mathrm{C}_{15} \mathrm{H}_{13} \mathrm{NO}_{3} \mathrm{~S}$ : C, 62.70; H, 4.56; N, 4.87. Found: C 62.69; H 4.59; N 4.76. 
Computational procedures. Rate constants at the coalescense temperature $\left(k_{\mathrm{c}}\right)$ were calculated using the standard equation for an $\mathrm{AB}$ coupled system (eq 1). $\Delta G^{\mp}$ (in Kcal $\mathrm{mol}^{-1}$ ) was calculated from rate constants obtained by line shape analysis of the methylene protons in ${ }^{1} \mathrm{H}$ NMR spectra taken at various temperatures using eq 2. Probe temperatures were measured using a neat methanol sample and automated calibration procedures. Plotting $\log \frac{k}{T} v s \frac{1}{1000 T}$ gives $\Delta H^{\mp}$ (in $\mathrm{Kcal} \mathrm{mol}^{-1}$ ) as -4.576 times the slope and $\Delta S^{\mp}$ (in cal $\mathrm{mol}^{-1}$ ) was calculated according to eq 3. For a detailed discussion of these procedures see: (a) Sandstrom, J. Dynamic NMR Spectroscopy; Academic Press: New York, 1982, pp. 84-100. (b) Nelson, J. H. Nuclear Magnetic Resonance Spectroscopy; Prentice Hall: Upper Saddle River, NJ, 2003, Chapter 11.

$$
\begin{aligned}
& \left.k_{C}=2.22 \sqrt{\left(v_{A}\right.}-v_{B}\right)^{2}-6 J_{A B}^{2} \\
& \Delta G^{\mp}=4.575 \times 10^{-3} T\left[10.319+\log \left(\frac{T}{k}\right)\right] \\
& \Delta S^{\mp}=4.576 \log \frac{k}{T}+\frac{\Delta H^{\mp}}{T}-47.218
\end{aligned}
$$

Structures 1, 2 and 3 were optimized in PM3 by driving the N-Ar and S-Ar torsion angles in $20^{\circ}$ steps from $0^{\circ}$ to $360^{\circ}$ and allowing the other angles and bond distances to relax to their minima for each fixed value of the torsion angles. The barriers $\left(\Delta G^{\mp}\right)$ were computed by locating the transition state (saddle point) by the QST3 method followed by frequency calculations of the minima on either side of the transition state and at the transition state (TS). Z-matrices for minima on both sides of the TS and for the TS are provided in the Supplemental Information (pp 224-232). 


\section{${ }^{1} \mathrm{H}$ NMR Spectrum of $\mathbf{1}$}

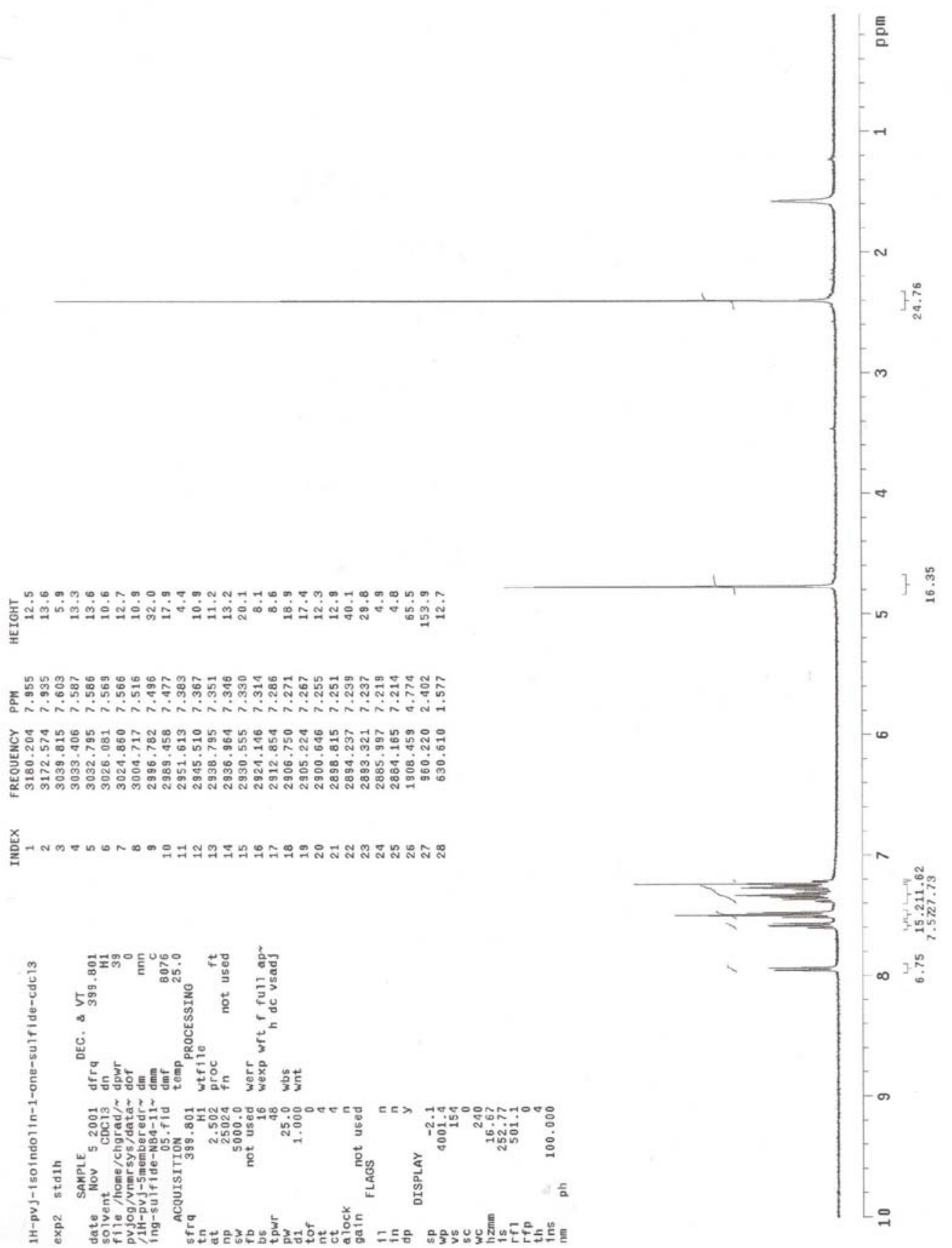




\section{${ }^{13} \mathrm{C}$ NMR Spectrum of $\mathbf{1}$}

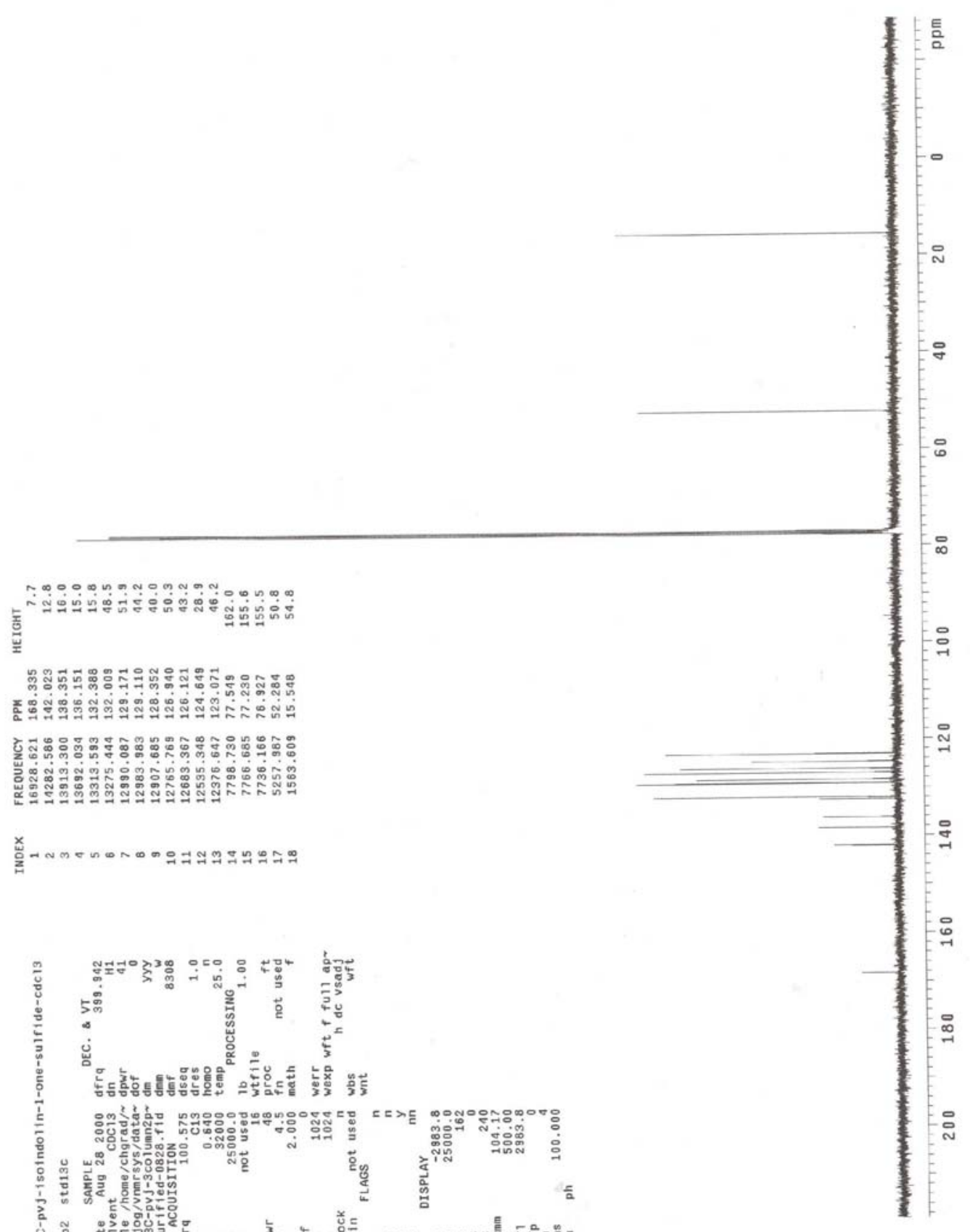




\section{${ }^{1} \mathrm{H}$ NMR Spectrum of $\mathbf{2}$}

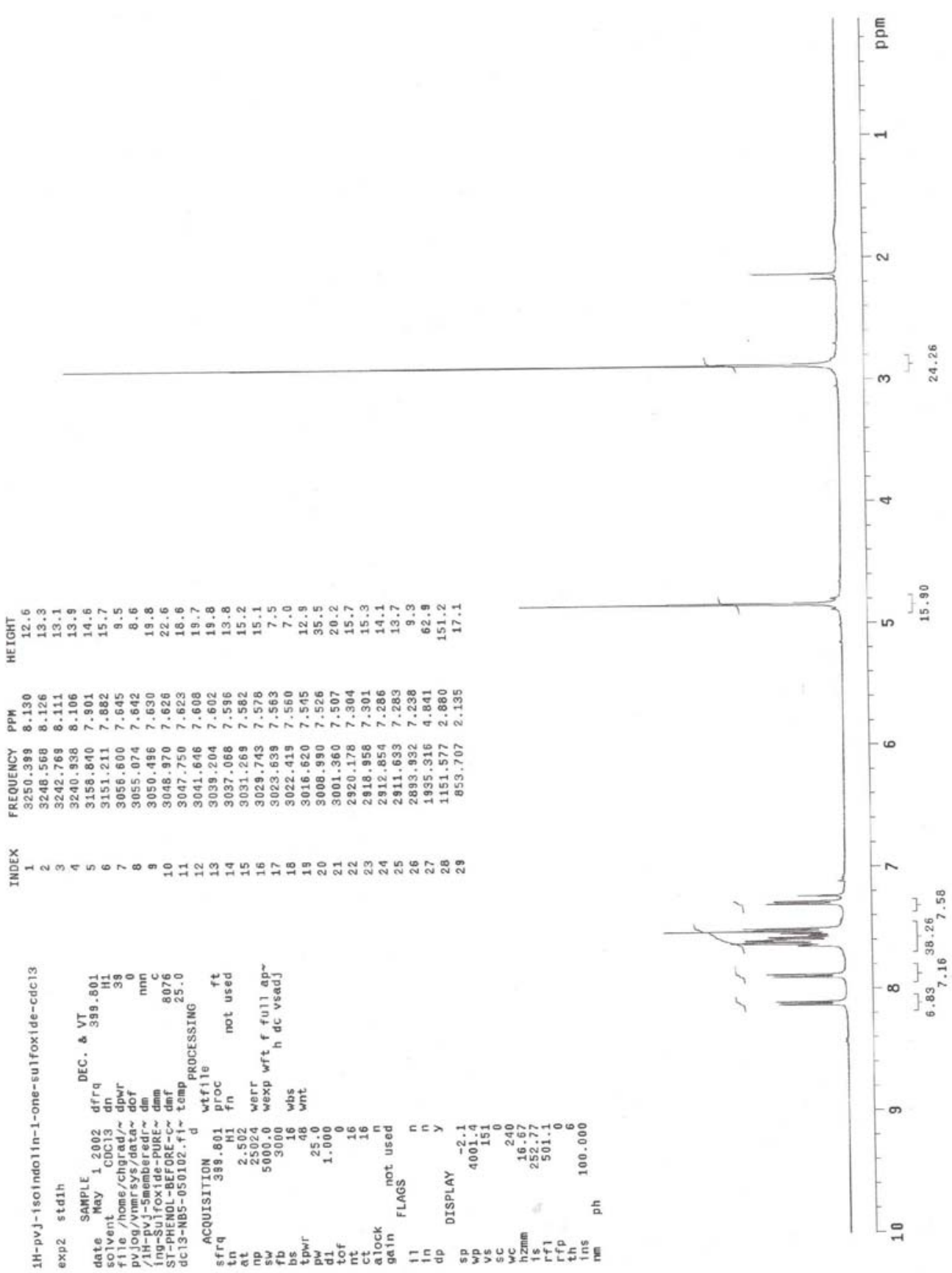


${ }^{13} \mathrm{C}$ NMR Spectrum of 2

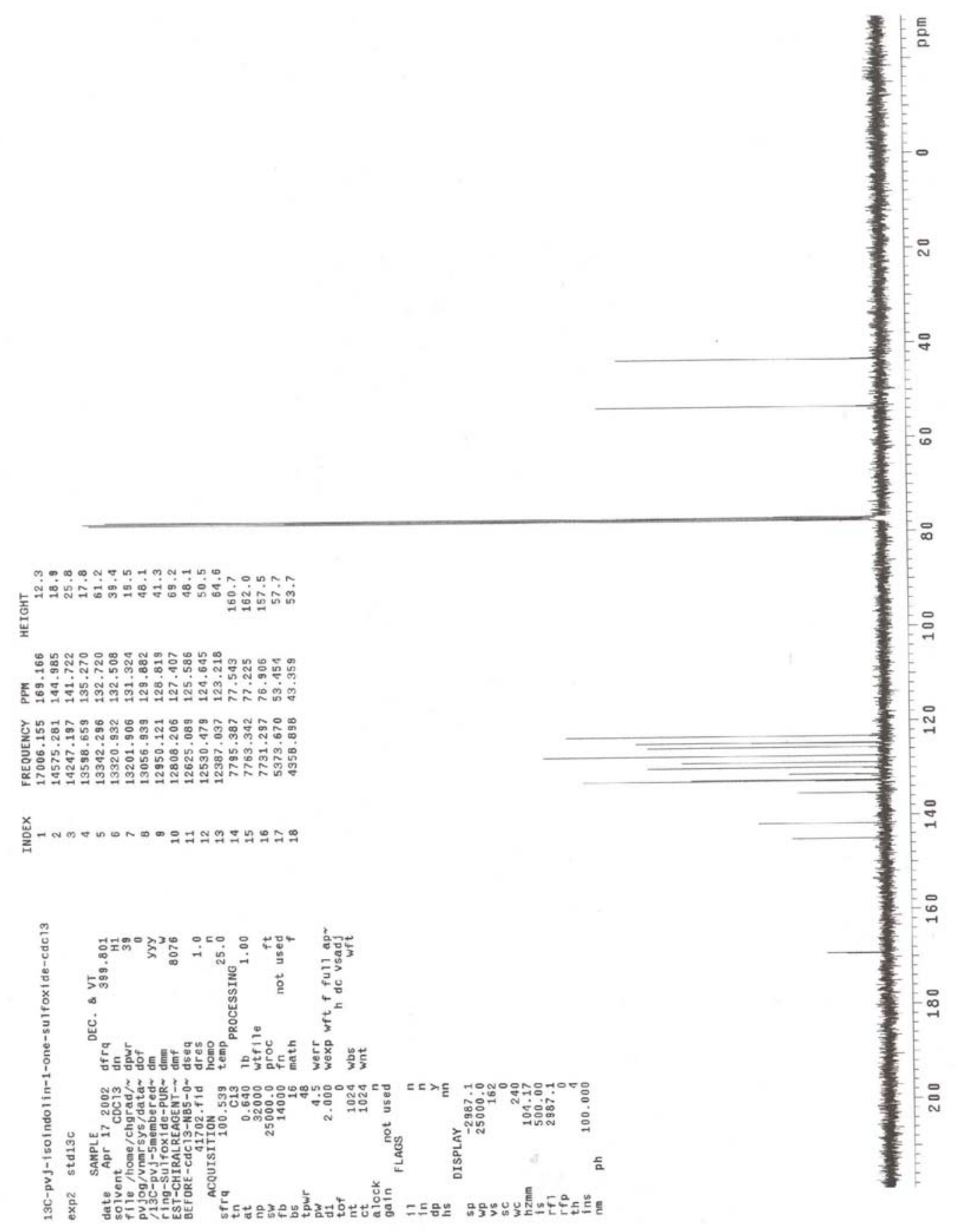




\section{${ }^{1} \mathrm{H}$ NMR Spectrum of $\mathbf{3}$}

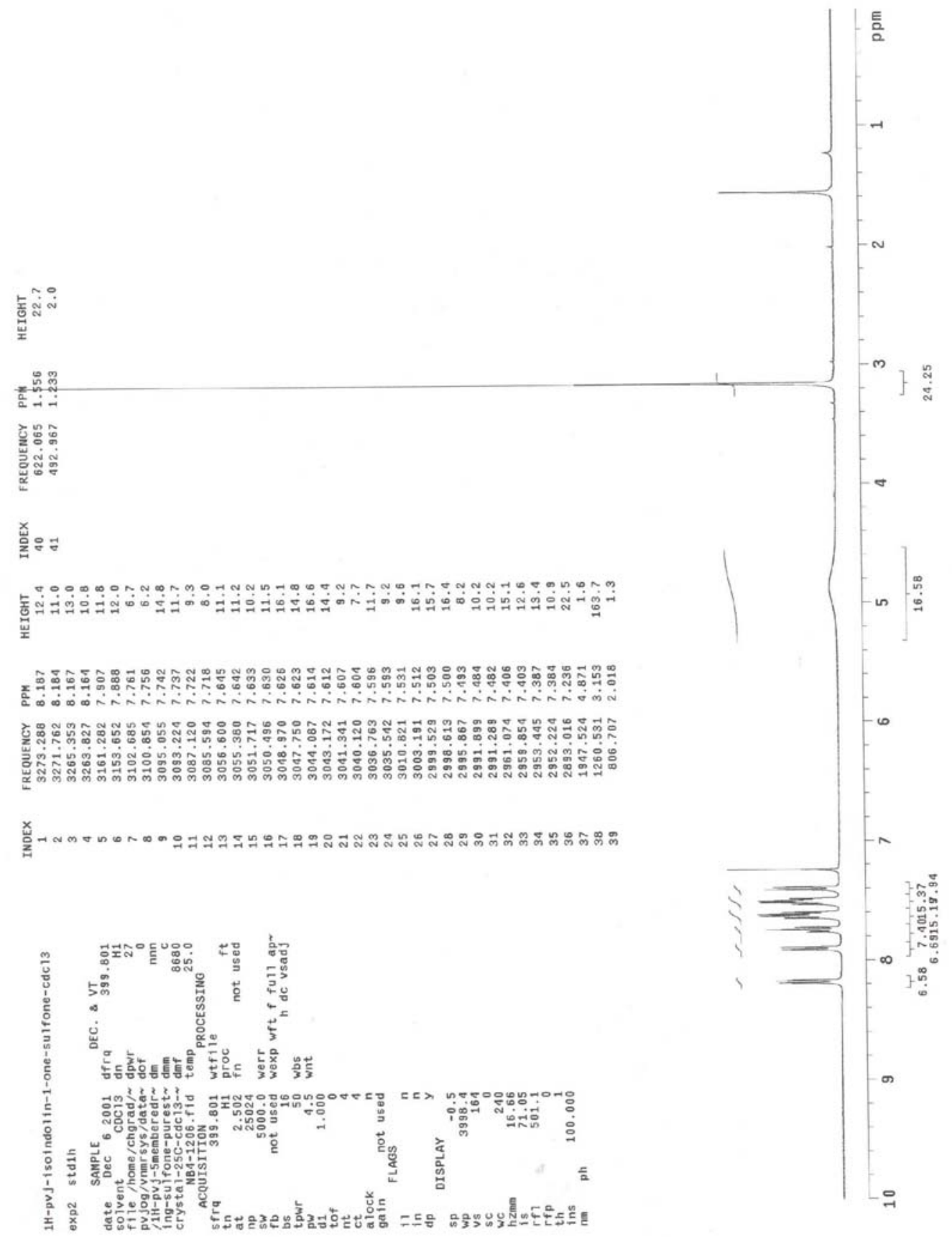




\section{${ }^{13} \mathrm{C}$ NMR Spectrum of 3}

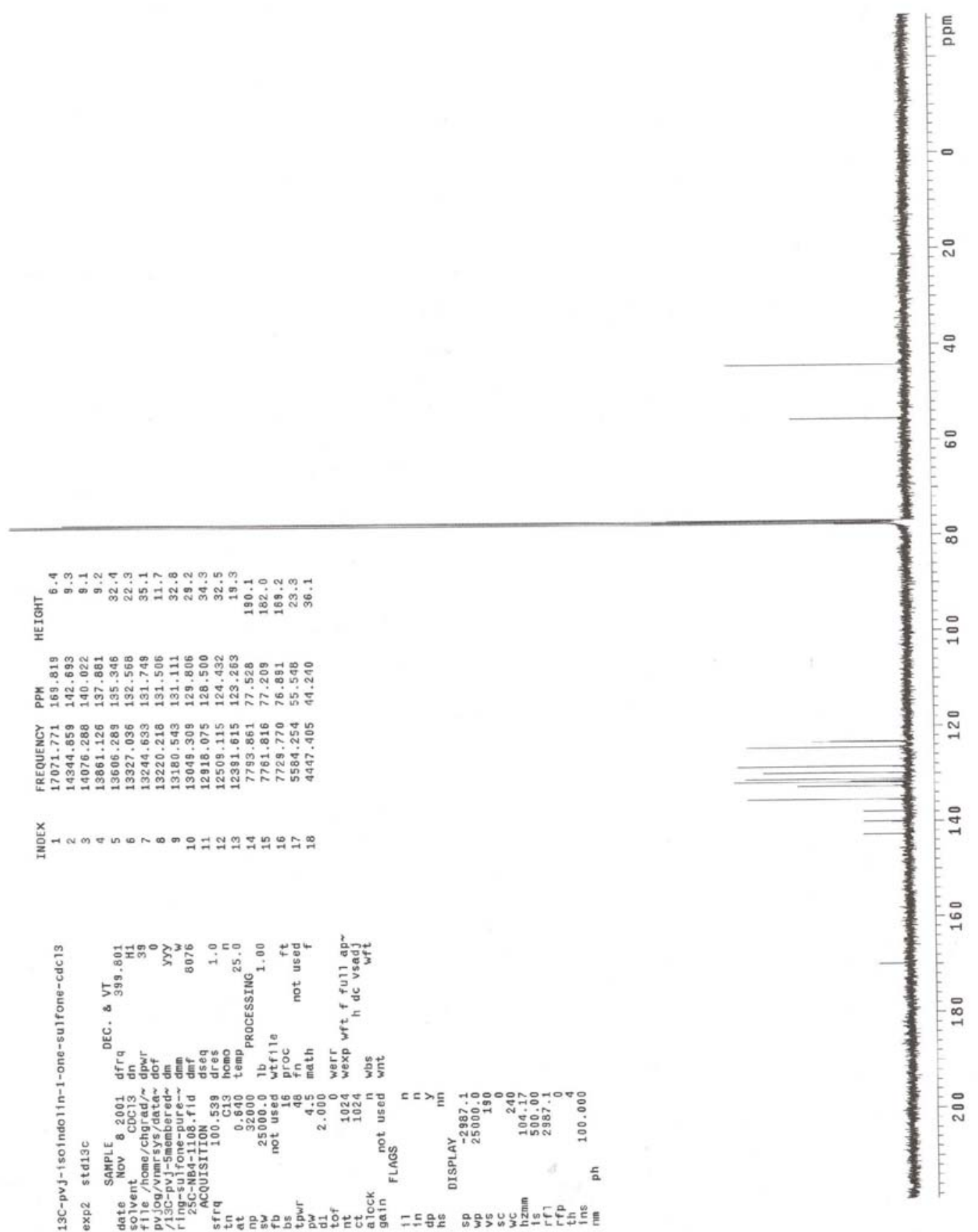


Potential Energy Surface Plot for Sulfoxide 2

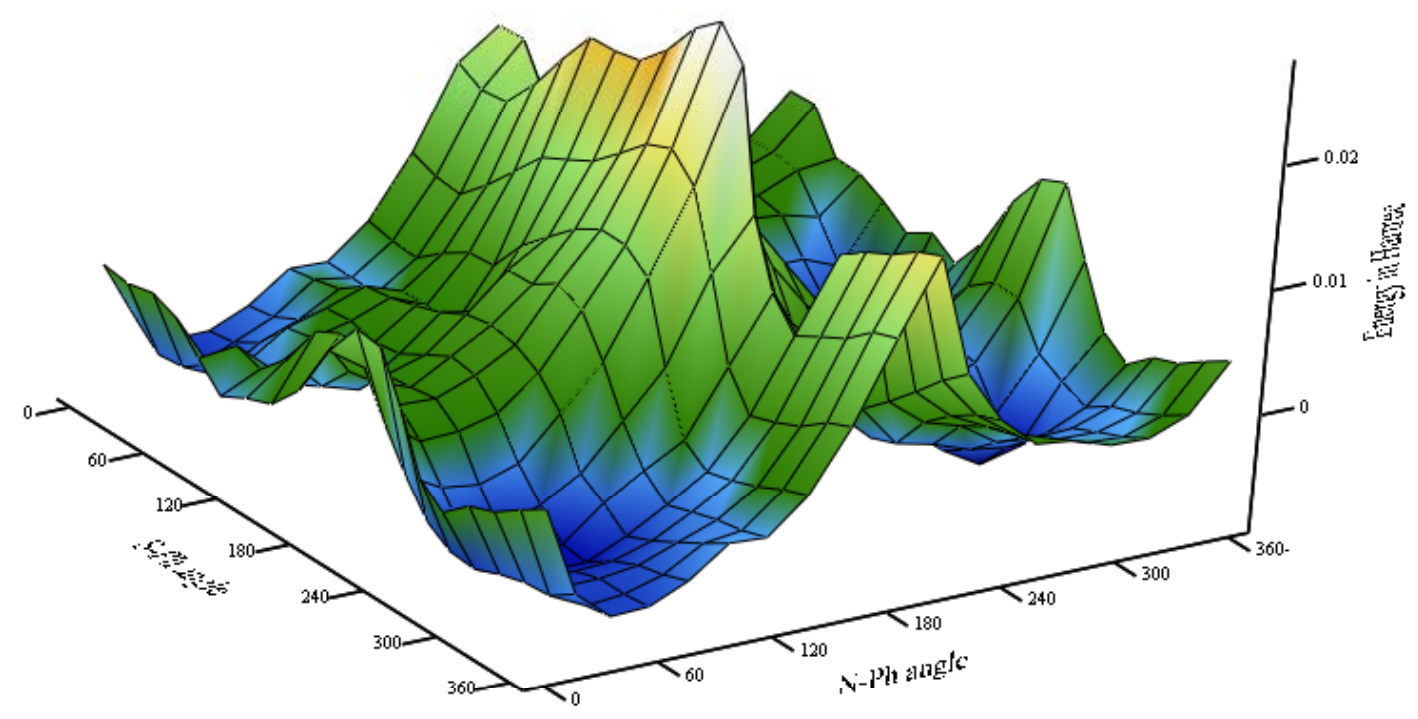

$(\mathrm{z}, \mathrm{s}, \mathrm{q})$ 
Potential Energy Surface Plot for Sulfone 3

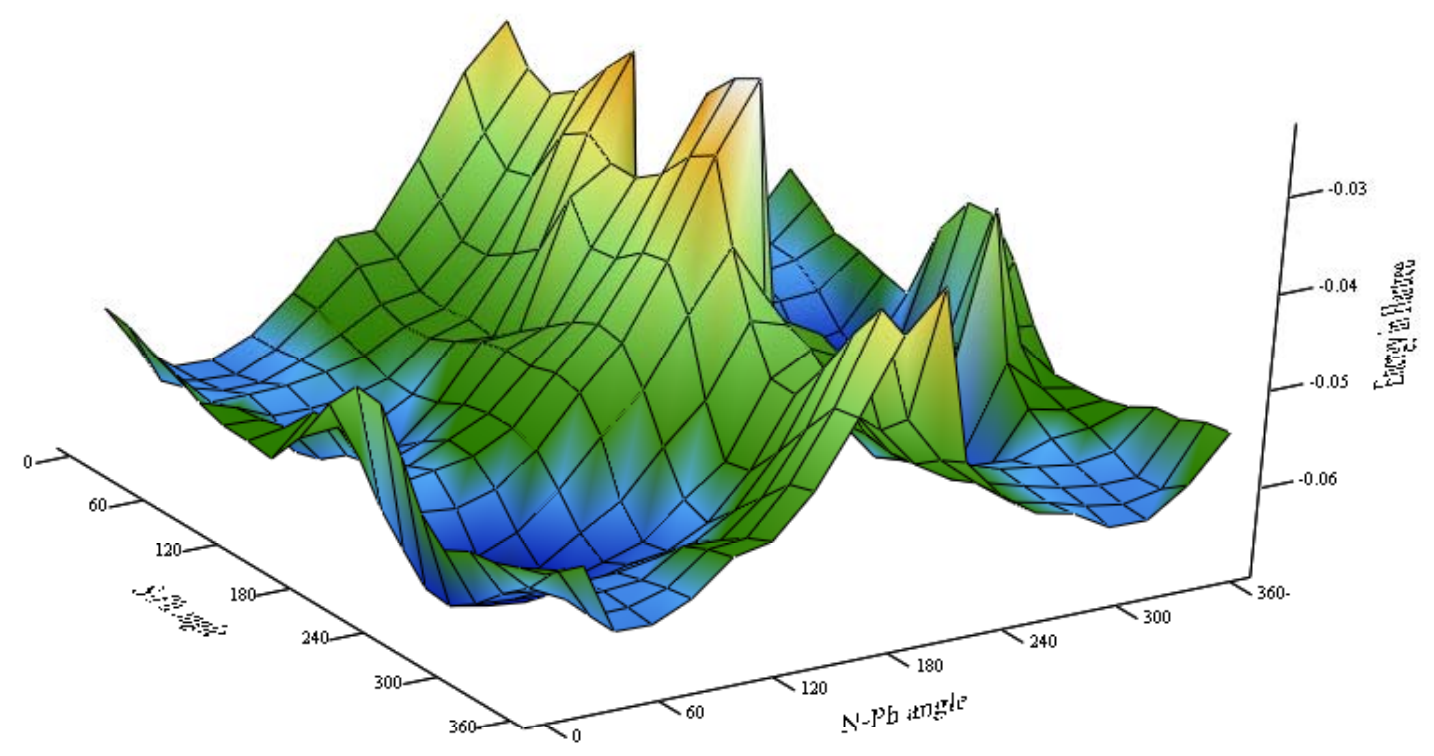

$(\mathrm{z}, \mathrm{v}, \mathrm{w})$ 


\section{Z-matrix for 1 [N-Ar \& S-Ar locked] (Lowest Energy Conformation)}

\begin{tabular}{|c|c|c|c|c|c|c|}
\hline 01 & & & $\operatorname{sch} 5$ & 107.46093 & $\operatorname{dih} 18$ & 0.04480 \\
\hline $\mathrm{h}$ & & & $\operatorname{dih} 5$ & -122.80381 & cc19 & 1.38323 \\
\hline c $1 \mathrm{ch} 2$ & & & $\operatorname{cs} 6$ & 1.77180 & $\operatorname{ccc} 19$ & 118.18847 \\
\hline h 2 hc 3 & 1 hch3 & & $\csc 6$ & 104.68279 & $\operatorname{dih} 19$ & 0.10850 \\
\hline h 2 hc 4 & 3 hch 4 & $1 \operatorname{dih} 4$ & $\operatorname{dih} 6$ & 273.94890 & $\operatorname{cc} 20$ & 1.49561 \\
\hline s $2 \operatorname{sc} 5$ & $3 \operatorname{sch} 5$ & $1 \operatorname{dih} 5$ & cc7 & 1.39784 & $\operatorname{ccc} 20$ & 128.80482 \\
\hline c $5 \operatorname{cs} 6$ & $2 \csc 6$ & $4 \operatorname{dih} 6$ & $\operatorname{ccs} 7$ & 121.14130 & $\operatorname{dih} 20$ & -179.71856 \\
\hline $6 \mathrm{cc} 7$ & $5 \operatorname{ccs} 7$ & $2 \operatorname{dih} 7$ & $\operatorname{dih} 7$ & 340.00000 & hc21 & 1.09441 \\
\hline c $7 \mathrm{cc} 8$ & $6 \operatorname{ccc} 8$ & $5 \operatorname{dih} 8$ & $\operatorname{cc} 8$ & 1.38719 & hec21 & 120.89667 \\
\hline c 8 cc 9 & $7 \operatorname{ccc} 9$ & 6 dih9 & $\operatorname{ccc} 8$ & 121.44158 & $\operatorname{dih} 21$ & -179.79813 \\
\hline c 9 cc10 & $8 \operatorname{ccc} 10$ & $7 \operatorname{dih} 10$ & $\operatorname{dih} 8$ & -178.14156 & hc22 & 1.10957 \\
\hline c $10 \mathrm{cc} 11$ & $9 \operatorname{ccc} 11$ & $8 \operatorname{dih} 11$ & cc9 & 1.39036 & hec22 & 112.67464 \\
\hline n $11 \mathrm{nc} 12$ & $10 \mathrm{ncc} 12$ & $9 \operatorname{dih} 12$ & $\operatorname{ccc} 9$ & 119.92977 & $\operatorname{dih} 22$ & 57.76910 \\
\hline c $12 \mathrm{cn} 13$ & $11 \mathrm{cnc} 13$ & $10 \operatorname{dih} 13$ & dih9 & 0.47929 & hc23 & 1.10793 \\
\hline c 13 cc14 & $12 \operatorname{con} 14$ & $11 \operatorname{dih} 14$ & $\operatorname{cc} 10$ & 1.38814 & hec23 & 111.99601 \\
\hline c $14 \mathrm{cc} 15$ & $13 \operatorname{ccc} 15$ & $12 \operatorname{dih} 15$ & $\operatorname{ccc} 10$ & 119.82708 & $\operatorname{dih} 23$ & -63.50499 \\
\hline c $15 \mathrm{cc} 16$ & $14 \operatorname{ccc} 16$ & $13 \operatorname{dih} 16$ & $\operatorname{dih} 10$ & -0.01575 & hc24 & 1.09540 \\
\hline c $16 \mathrm{cc} 17$ & $15 \operatorname{ccc} 17$ & $14 \operatorname{dih} 17$ & cc11 & 1.40013 & hec24 & 119.32075 \\
\hline c $17 \mathrm{cc} 18$ & $16 \operatorname{ccc} 18$ & $15 \operatorname{dih} 18$ & $\operatorname{ccc} 11$ & 120.43985 & $\operatorname{dih} 24$ & -179.96237 \\
\hline c $18 \mathrm{cc} 19$ & $17 \operatorname{ccc} 19$ & $16 \operatorname{dih} 19$ & $\operatorname{dih} 11$ & -0.57748 & hc25 & 1.09523 \\
\hline c $19 \operatorname{cc} 20$ & $18 \operatorname{ccc} 20$ & $17 \operatorname{dih} 20$ & nc12 & 1.44748 & hec25 & 119.69272 \\
\hline h $18 \mathrm{hc} 21$ & 17 hcc 21 & $16 \operatorname{dih} 21$ & ncc12 & 119.98005 & $\operatorname{dih} 25$ & 179.91169 \\
\hline h 20 hc 22 & 19 hcc 22 & $18 \operatorname{dih} 22$ & $\operatorname{dih} 12$ & -178.86414 & hc26 & 1.09533 \\
\hline h 20 hc 23 & 19 hcc23 & $18 \operatorname{dih} 23$ & cn13 & 1.44925 & hec26 & 120.72678 \\
\hline h 17 hc 24 & 18 hcc24 & $19 \operatorname{dih} 24$ & cnc13 & 123.18632 & $\operatorname{dih} 26$ & -179.87909 \\
\hline h 16 hc 25 & 17 hcc 25 & $18 \operatorname{dih} 25$ & $\operatorname{dih} 13$ & 40.00000 & oc27 & 1.21465 \\
\hline h 15 hc 26 & 14 hcc26 & $19 \operatorname{dih} 26$ & cc14 & 1.48613 & occ27 & 129.64647 \\
\hline o 13 oc 27 & 14 occ 27 & $19 \operatorname{dih} 27$ & $\operatorname{ccn} 14$ & 106.28642 & $\operatorname{dih} 27$ & -177.22970 \\
\hline h 10 hc 28 & 11 hcc 28 & $12 \operatorname{dih} 28$ & $\operatorname{dih} 14$ & -153.21541 & hc28 & 1.09724 \\
\hline h 9 hc29 & 10 hcc29 & $11 \operatorname{dih} 29$ & cc15 & 1.38357 & hec 28 & 120.23132 \\
\hline h 8 hc30 & 9 hcc30 & $10 \operatorname{dih} 30$ & $\operatorname{ccc} 15$ & 129.70692 & $\operatorname{dih} 28$ & -0.25801 \\
\hline h 7 hc31 & 8 hcc31 & $9 \operatorname{dih} 31$ & $\operatorname{dih} 15$ & -174.86795 & hc29 & 1.09497 \\
\hline & & & cc16 & 1.39713 & hec29 & 119.97327 \\
\hline $\operatorname{ch} 2$ & 1.1003 & & $\operatorname{ccc} 16$ & 118.05980 & $\operatorname{dih} 29$ & 179.33372 \\
\hline hc3 & 1.0962 & & $\operatorname{dih} 16$ & -179.83891 & hc30 & 1.09494 \\
\hline hch3 & 108.228 & & cc17 & 1.39069 & hec30 & 120.17359 \\
\hline hc4 & 1.0954 & & $\operatorname{ccc} 17$ & 120.86810 & $\operatorname{dih} 30$ & -179.97353 \\
\hline hch4 & 107.988 & & $\operatorname{dih} 17$ & -0.10851 & hc31 & 1.10200 \\
\hline $\operatorname{dih} 4$ & 116.050 & & cc18 & 1.39723 & hec31 & 119.28782 \\
\hline $\operatorname{sc} 5$ & 1.8045 & & $\operatorname{ccc} 18$ & 121.05078 & $\operatorname{dih} 31$ & -179.60645 \\
\hline
\end{tabular}




\section{Z-matrix for 1 [N-Ar \& S-Ar locked]-Lowest Energy Conformation (other side of the Transition State)}

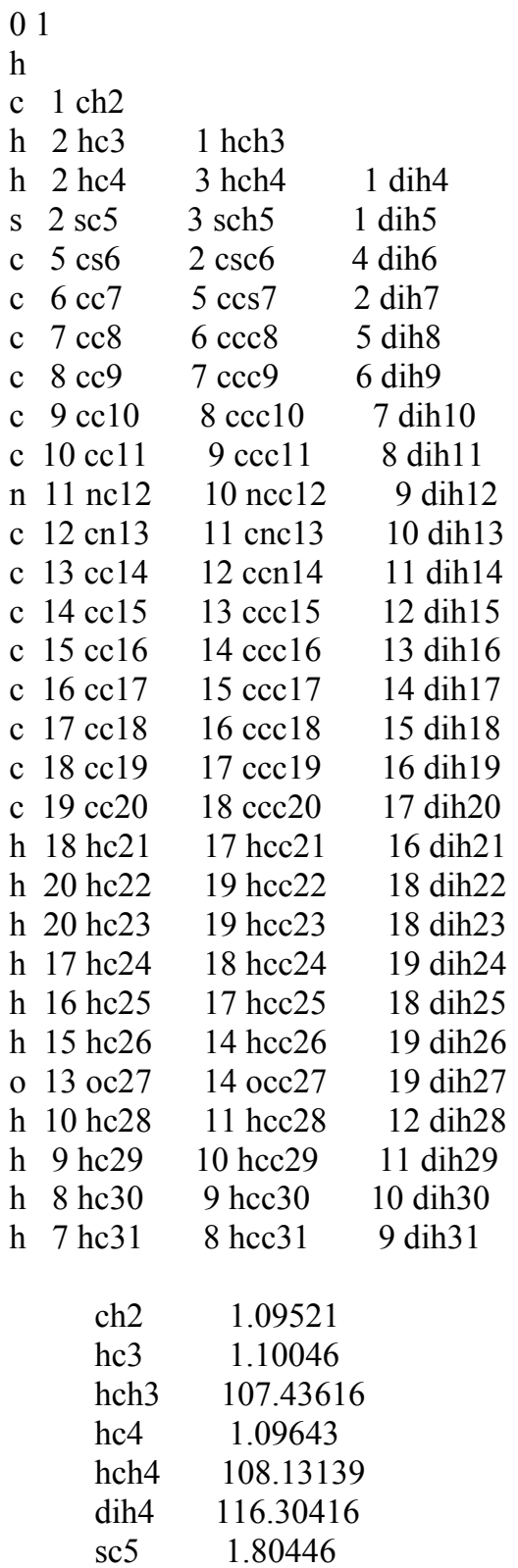

$\begin{array}{lc}\text { sch5 } & 113.36711 \\ \text { dih5 } & -124.71193 \\ \text { cs6 } & 1.77176 \\ \text { csc6 } & 104.47674 \\ \text { dih6 } & 156.37300 \\ \text { cc7 } & 1.39795 \\ \text { ccs7 } & 121.13414 \\ \text { dih7 } & 340.00000 \\ \text { cc8 } & 1.38714 \\ \text { ccc8 } & 121.48684 \\ \text { dih8 } & -178.59292 \\ \text { cc9 } & 1.39027 \\ \text { ccc9 } & 119.91684 \\ \text { dih9 } & 0.30620 \\ \text { cc10 } & 1.38809 \\ \text { ccc10 } & 119.80609 \\ \text { dih10 } & -0.07069 \\ \text { cc11 } & 1.40019 \\ \text { ccc11 } & 120.47830 \\ \text { dih11 } & 0.09825 \\ \text { nc12 } & 1.44817 \\ \text { ncc12 } & 119.89669 \\ \text { dih12 } & -180.84774 \\ \text { cn13 } & 1.45040 \\ \text { cnc13 } & 122.92543 \\ \text { dih13 } & 320.00000 \\ \text { cc14 } & 1.48601 \\ \text { ccn14 } & 106.32454 \\ \text { dih14 } & -207.53316 \\ \text { cc15 } & 1.38359 \\ \text { ccc15 } & 129.71296 \\ \text { dih15 } & -185.19813 \\ \text { cc16 } & 1.39712 \\ \text { ccc16 } & 118.05721 \\ \text { dih16 } & -180.15880 \\ \text { cc17 } & 1.39070 \\ \text { ccc17 } & 120.86550 \\ \text { dih17 } & 0.09091 \\ \text { cc18 } & 1.39721 \\ \text { ccc18 } & 121.05022 \\ & \\ & \end{array}$

$\begin{array}{lc}\text { dih18 } & -0.02993 \\ \text { cc19 } & 1.38326 \\ \text { ccc19 } & 118.19311 \\ \text { dih19 } & -0.09453 \\ \text { cc20 } & 1.49557 \\ \text { ccc20 } & 128.80335 \\ \text { dih20 } & -180.33293 \\ \text { hc21 } & 1.09438 \\ \text { hcc21 } & 120.89301 \\ \text { dih21 } & -180.21847 \\ \text { hc22 } & 1.10780 \\ \text { hcc22 } & 112.00740 \\ \text { dih22 } & 63.47436 \\ \text { hc23 } & 1.10916 \\ \text { hcc23 } & 112.70075 \\ \text { dih23 } & -57.88287 \\ \text { hc24 } & 1.09539 \\ \text { hcc24 } & 119.32389 \\ \text { dih24 } & -180.02871 \\ \text { hc25 } & 1.09522 \\ \text { hcc25 } & 119.69281 \\ \text { dih25 } & 180.09458 \\ \text { hc26 } & 1.09534 \\ \text { hcc26 } & 120.72589 \\ \text { dih26 } & -180.16468 \\ \text { oc27 } & 1.21447 \\ \text { occ27 } & 129.63091 \\ \text { dih27 } & -182.74933 \\ \text { hc28 } & 1.09727 \\ \text { hcc28 } & 120.22351 \\ \text { dih28 } & 0.46814 \\ \text { hc29 } & 1.09497 \\ \text { hcc29 } & 119.98013 \\ \text { dih29 } & 180.38169 \\ \text { hc30 } & 1.09494 \\ \text { hcc30 } & 120.18186 \\ \text { dih30 } & -179.70674 \\ \text { hc31 } & 1.10206 \\ \text { hcc31 } & 119.27032 \\ \text { dih31 } & \\ & \\ & \\ \end{array}$




\section{Z-matrix for 1 [N-Ar \& S-Ar locked] (Transition State)}

\begin{tabular}{|c|c|c|c|c|c|c|}
\hline & $\operatorname{ccs} 7$ & 113.860 & $\operatorname{dih} 22$ & 106.849 \\
\hline \multicolumn{3}{|l|}{1} & $\operatorname{dih} 7$ & -80.000 & hc23 & 1.108970 \\
\hline & $\operatorname{cc} 8$ & 1.383890 & hen 23 & 113.030 \\
\hline \multicolumn{3}{|l|}{$\begin{array}{l}\text { c } 1 \text { ch } 2 \\
\text { h } 2 \text { hc } 3\end{array}$} & $\operatorname{ccc} 8$ & 121.945 & $\operatorname{dih} 23$ & -11.940 \\
\hline $2 \mathrm{hc} 4$ & 1 hch 4 & $3 \operatorname{dih} 4$ & $\operatorname{dih} 8$ & -167.543 & hc24 & 1.095420 \\
\hline $\begin{array}{l}2 \operatorname{sc} 5 \\
5 \operatorname{se} 6\end{array}$ & $1 \operatorname{sch} 5$ & $3 \operatorname{dih} 5$ & cc9 & 1.391820 & hec24 & 119.626 \\
\hline c $5 \operatorname{cs} 6$ & $2 \csc 6$ & $4 \operatorname{dih} 6$ & $\operatorname{ccc} 9$ & 119.114 & $\operatorname{dih} 24$ & 179.891 \\
\hline c $6 \mathrm{cc} 7$ & $5 \operatorname{ccs} 7$ & $2 \operatorname{dih} 7$ & $\operatorname{dih} 9$ & 1.229 & hc25 & 1.095259 \\
\hline $7 \operatorname{cc} 8$ & $6 \operatorname{ccc} 8$ & $5 \operatorname{dih} 8$ & $\operatorname{cc} 10$ & 1.383731 & hec 25 & 119.441 \\
\hline $8 \mathrm{cc} 9$ & $7 \operatorname{ccc} 9$ & $6 \operatorname{dih} 9$ & $\operatorname{ccc} 10$ & 119.935 & $\operatorname{dih} 25$ & -179.847 \\
\hline $9 \operatorname{cc} 10$ & $8 \operatorname{ccc} 10$ & $7 \operatorname{dih} 10$ & $\operatorname{dih} 10$ & -3.221 & hc26 & 1.095240 \\
\hline $6 \mathrm{cc} 11$ & $5 \operatorname{ccs} 11$ & $2 \operatorname{dih} 11$ & cc11 & 1.406218 & hec 26 & 120.801 \\
\hline $11 \mathrm{nc} 12$ & 6 ncc12 & $5 \operatorname{dih} 12$ & $\operatorname{ccs} 11$ & 126.822 & $\operatorname{dih} 26$ & 1.136 \\
\hline \multirow{2}{*}{$\begin{array}{l}12 \mathrm{cn} 13 \\
13 \mathrm{cc} 14\end{array}$} & $11 \mathrm{cnc} 13$ & $6 \operatorname{dih} 13$ & $\operatorname{dih} 11$ & 108.433 & oc27 & 1.210421 \\
\hline & $12 \operatorname{ccn} 14$ & $11 \operatorname{dih} 14$ & nc12 & 1.448799 & ocn 27 & 122.107 \\
\hline c $14 \operatorname{cc} 15$ & $13 \operatorname{ccc} 15$ & $12 \operatorname{dih} 15$ & ncc12 & 127.185 & $\operatorname{dih} 27$ & -64.649 \\
\hline c $15 \mathrm{cc} 16$ & $14 \operatorname{ccc} 16$ & $13 \operatorname{dih} 16$ & $\operatorname{dih} 12$ & -13.679 & hc28 & 1.098219 \\
\hline c $16 \mathrm{cc} 17$ & $15 \operatorname{ccc} 17$ & $14 \operatorname{dih} 17$ & cn13 & 1.478770 & hec 28 & 117.944 \\
\hline c $17 \mathrm{cc} 18$ & $16 \operatorname{ccc} 18$ & $15 \operatorname{dih} 18$ & cnc13 & 125.317 & $\operatorname{dih} 28$ & -177.593 \\
\hline c $14 \operatorname{cc} 19$ & $13 \operatorname{ccc} 19$ & $12 \operatorname{dih} 19$ & $\operatorname{dih} 13$ & -3.546 & hc29 & 1.095250 \\
\hline $12 \mathrm{cn} 20$ & $11 \mathrm{cnc} 20$ & $6 \operatorname{dih} 20$ & cc14 & 1.483710 & hec29 & 120.096 \\
\hline h 18 hc 21 & 17 hcc 21 & $16 \operatorname{dih} 21$ & $\operatorname{ccn} 14$ & 108.296 & $\operatorname{dih} 29$ & 177.200 \\
\hline h 20 hc 22 & 12 hcn 22 & $11 \operatorname{dih} 22$ & $\operatorname{dih} 14$ & 126.389 & hc30 & 1.094580 \\
\hline h 20 hc23 & 12 hcn 23 & $11 \operatorname{dih} 23$ & cc15 & 1.383850 & hec30 & 120.432 \\
\hline h 17 hc2 24 & 16 hcc24 & $15 \operatorname{dih} 24$ & $\operatorname{ccc} 15$ & 129.947 & $\operatorname{dih} 30$ & 179.880 \\
\hline h 16 hc 25 & 15 hcc 25 & $14 \operatorname{dih} 25$ & $\operatorname{dih} 15$ & -175.077 & hc31 & 1.098531 \\
\hline h 15 hc 26 & 14 hcc 26 & $13 \operatorname{dih} 26$ & cc16 & 1.397120 & hcc31 & 118.543 \\
\hline о 13 oc 27 & $12 \operatorname{ocn} 27$ & $11 \operatorname{dih} 27$ & $\operatorname{ccc} 16$ & 117.940 & $\operatorname{dih} 31$ & 10.603 \\
\hline h 10 hc 28 & 9 hcc28 & $8 \operatorname{dih} 28$ & $\operatorname{dih} 16$ & -178.826 & & \\
\hline h 9 hc 29 & 8 hcc29 & $7 \operatorname{dih} 29$ & cc17 & 1.390980 & & \\
\hline h 8 hc 30 & 7 hec30 & $6 \operatorname{dih} 30$ & $\operatorname{ccc} 17$ & 120.875 & & \\
\hline h 7 hc31 & 6 hec31 & $5 \operatorname{dih} 31$ & $\operatorname{dih} 17$ & 0.237 & & \\
\hline & & & cc18 & 1.397160 & & \\
\hline $\operatorname{ch} 2$ & 1.096500 & & $\operatorname{ccc} 18$ & 121.050 & & \\
\hline hc3 & 1.095300 & & $\operatorname{dih} 18$ & -0.203 & & \\
\hline hch3 & 108.279 & & cc19 & 1.402776 & & \\
\hline hc4 & 1.095800 & & $\operatorname{ccc} 19$ & 108.787 & & \\
\hline hch4 & 108.038 & & $\operatorname{dih} 19$ & 5.953 & & \\
\hline $\operatorname{dih} 4$ & 116.613 & & cn20 & 1.523247 & & \\
\hline sc5 & 1.806980 & & cnc20 & 112.780 & & \\
\hline $\operatorname{sch} 5$ & 106.835 & & $\operatorname{dih} 20$ & 128.384 & & \\
\hline dih5 & -121.419 & & hc21 & 1.094360 & & \\
\hline cs6 & 1.774350 & & hcc21 & 120.923 & & \\
\hline $\csc 6$ & 103.566 & & $\operatorname{dih} 21$ & -179.905 & & \\
\hline dih6 & -65.629 & & hc22 & 1.107700 & & \\
\hline cc7 & 1.402980 & & $\mathrm{hcn} 22$ & 108.251 & & \\
\hline
\end{tabular}




\section{Z-matrix for 2 [N-Ar \& S-Ar locked] (Lowest Energy Conformation)}

$\begin{array}{lll}01 & \\ \text { h } & & \\ \text { c } 1 \text { ch2 } & & \\ \text { h } 2 \text { hc3 } & 1 \text { hch3 } & \\ \text { h } 2 \text { hc4 } & 3 \text { hch4 } & 1 \text { dih4 } \\ \text { s } 2 \text { sc5 } & 4 \text { sch5 } & 3 \text { dih5 } \\ \text { o } 5 \text { os6 } & 2 \text { osc6 } & 1 \text { dih6 } \\ \text { c } 5 \text { cs } 7 & 2 \text { csc7 } & 1 \text { dih7 } \\ \text { c } 7 \text { cc8 } & 5 \text { ccs } 8 & 2 \text { dih8 } \\ \text { c } 8 \text { cc9 } & 7 \text { ccc9 } & 5 \text { dih9 } \\ \text { c } 9 \text { cc10 } & 8 \text { ccc10 } & 7 \text { dih10 } \\ \text { c } 10 \text { cc11 } & 9 \text { ccc11 } & 8 \text { dih11 } \\ \text { c } 11 \text { cc12 } & 10 \text { ccc12 } & 9 \text { dih12 } \\ \text { n } 12 \text { nc13 } & 11 \text { ncc13 } & 10 \text { dih13 } \\ \text { c } 13 \text { cn14 } & 12 \text { cnc14 } & 11 \text { dih14 } \\ \text { c } 14 \text { cc15 } & 13 \text { ccn15 } & 12 \text { dih15 } \\ \text { c } 15 \text { cc16 } & 14 \text { ccc16 } & 13 \text { dih16 } \\ \text { c } 16 \text { cc17 } & 15 \text { ccc17 } & 14 \text { dih17 } \\ \text { c } 17 \text { cc18 } & 16 \text { ccc18 } & 15 \text { dih18 } \\ \text { c } 18 \text { cc19 } & 17 \text { ccc19 } & 16 \text { dih19 } \\ \text { c } 19 \text { cc20 } & 18 \text { ccc20 } & 17 \text { dih20 } \\ \text { c } 20 \text { cc21 } & 19 \text { ccc21 } & 18 \text { dih21 } \\ \text { h } 8 \text { hc22 } & 9 \text { hcc22 } & 10 \text { dih22 } \\ \text { h } 9 \text { hc23 } & 10 \text { hcc23 } & 11 \text { dih23 } \\ \text { h } 10 \text { hc24 } & 11 \text { hcc24 } & 12 \text { dih24 } \\ \text { h } 11 \text { hc25 } & 12 \text { hcc25 } & 13 \text { dih25 } \\ \text { h } 21 \text { hc26 } & 20 \text { hcc26 } & 19 \text { dih26 } \\ \text { h } 21 \text { hc27 } & 20 \text { hcc27 } & 19 \text { dih27 } \\ \text { h } 19 \text { hc28 } & 18 \text { hcc28 } & 17 \text { dih28 } \\ \text { h } 18 \text { hc29 } & 19 \text { hcc29 } & 20 \text { dih29 } \\ \text { h } 17 \text { hc30 } & 18 \text { hcc30 } & 19 \text { dih30 } \\ \text { h } 16 \text { hc31 } & 15 \text { hcc31 } & 20 \text { dih31 } \\ \text { o } 14 \text { oc32 } & 15 \text { occ32 } & 20 \text { dih32 }\end{array}$

$\begin{array}{lc}\text { dih5 } & -124.12739 \\ \text { os6 } & 1.55430 \\ \text { osc6 } & 103.54371 \\ \text { dih6 } & -170.36889 \\ \text { cs7 } & 1.81353 \\ \text { csc7 } & 99.20021 \\ \text { dih7 } & -62.34639 \\ \text { cc8 } & 1.39511 \\ \text { ccs8 } & 119.82700 \\ \text { dih8 } & 260.00000 \\ \text { cc9 } & 1.39024 \\ \text { ccc9 } & 121.18599 \\ \text { dih9 } & 182.70993 \\ \text { cc10 } & 1.38955 \\ \text { ccc10 } & 120.02758 \\ \text { dih10 } & -0.37211 \\ \text { cc11 } & 1.38963 \\ \text { ccc11 } & 119.87169 \\ \text { dih11 } & -0.40344 \\ \text { cc12 } & 1.39855 \\ \text { ccc12 } & 120.02754 \\ \text { dih12 } & 0.56045 \\ \text { nc13 } & 1.45486 \\ \text { ncc13 } & 120.03917 \\ \text { dih13 } & -178.40211 \\ \text { cn14 } & 1.45796 \\ \text { cnc14 } & 121.62636 \\ \text { dih14 } & 300.00000 \\ \text { cc15 } & 1.48572 \\ \text { ccn15 } & 106.24593 \\ \text { dih15 } & -210.52975 \\ \text { cc16 } & 1.38343 \\ \text { ccc16 } & 129.69823 \\ \text { dih16 } & -186.31504 \\ \text { cc17 } & 1.39752 \\ \text { ccc17 } & 118.04530 \\ \text { dih17 } & 179.98928 \\ \text { cc18 } & 1.39043 \\ \text { ccc18 } & 120.88130 \\ \text { dih18 } & 0.05553 \\ \text { cc19 } & 1.39767 \\ \text { ccc19 } & 121.04680 \\ & \\ & \end{array}$

$\begin{array}{lc}\text { dih19 } & -0.02548 \\ \text { cc20 } & 1.38282 \\ \text { ccc20 } & 118.17325 \\ \text { dih20 } & -0.13061 \\ \text { cc21 } & 1.49554 \\ \text { ccc21 } & 128.81170 \\ \text { dih21 } & 180.11303 \\ \text { hc22 } & 1.10096 \\ \text { hcc22 } & 119.41420 \\ \text { dih22 } & 180.50051 \\ \text { hc23 } & 1.09533 \\ \text { hcc23 } & 120.13650 \\ \text { dih23 } & 179.81577 \\ \text { hc24 } & 1.09529 \\ \text { hcc24 } & 119.90238 \\ \text { dih24 } & -179.45886 \\ \text { hc25 } & 1.09667 \\ \text { hcc25 } & 120.48748 \\ \text { dih25 } & 2.17593 \\ \text { hc26 } & 1.11309 \\ \text { hcc26 } & 113.13319 \\ \text { dih26 } & -56.53884 \\ \text { hc27 } & 1.10853 \\ \text { hcc27 } & 111.87746 \\ \text { dih27 } & 64.76130 \\ \text { hc28 } & 1.09444 \\ \text { hcc28 } & 120.84958 \\ \text { dih28 } & 179.87908 \\ \text { hc29 } & 1.09554 \\ \text { hcc29 } & 119.30489 \\ \text { dih29 } & -180.09249 \\ \text { hc30 } & 1.09541 \\ \text { hcc30 } & 119.69885 \\ \text { dih30 } & -179.92354 \\ \text { hc31 } & 1.09550 \\ \text { hcc31 } & 120.76047 \\ \text { dih31 } & 179.98403 \\ \text { oc32 } & 1.21189 \\ \text { occ32 } & 130.05433 \\ \text { dih32 } & -184.01239 \\ & \\ & \end{array}$

$\begin{array}{lc}\text { ch2 } & 1.10134 \\ \text { hc3 } & 1.09530 \\ \text { hch3 } & 107.87237 \\ \text { hc4 } & 1.09661 \\ \text { hch4 } & 106.98438 \\ \text { dih4 } & 115.61616 \\ \text { sc5 } & 1.82361 \\ \text { sch5 } & 109.12847\end{array}$

$\operatorname{ccc} 19-121.04680$ 


\section{Z-matrix for 2 [N-Ar \& S-Ar locked]-Lowest Energy Conformation}

(other side of the Transition State)

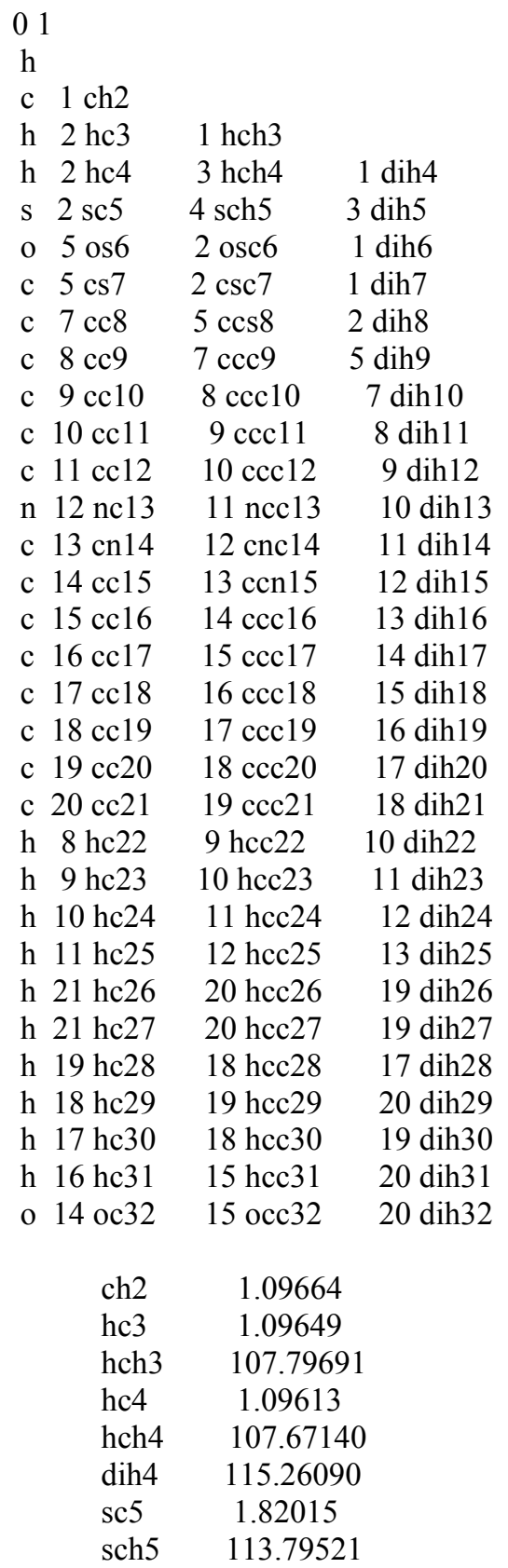

$\begin{array}{lc}\text { dih5 } & -124.04437 \\ \text { os6 } & 1.55766 \\ \text { osc6 } & 104.05640 \\ \text { dih6 } & 85.50938 \\ \text { cs7 } & 1.81315 \\ \text { csc7 } & 99.63681 \\ \text { dih7 } & 193.80876 \\ \text { cc8 } & 1.39509 \\ \text { ccs8 } & 119.82092 \\ \text { dih8 } & 260.00000 \\ \text { cc9 } & 1.39015 \\ \text { ccc9 } & 121.21552 \\ \text { dih9 } & 181.63503 \\ \text { cc10 } & 1.38946 \\ \text { ccc10 } & 119.99494 \\ \text { dih10 } & -0.15081 \\ \text { cc11 } & 1.38944 \\ \text { ccc11 } & 119.85499 \\ \text { dih11 } & 0.57367 \\ \text { cc12 } & 1.39890 \\ \text { ccc12 } & 120.08958 \\ \text { dih12 } & -0.25717 \\ \text { nc13 } & 1.45738 \\ \text { ncc13 } & 119.90203 \\ \text { dih13 } & -180.74428 \\ \text { cn14 } & 1.46098 \\ \text { cnc14 } & 119.92638 \\ \text { dih14 } & 60.00000 \\ \text { cc15 } & 1.48358 \\ \text { ccn15 } & 106.80321 \\ \text { dih15 } & -144.08230 \\ \text { cc16 } & 1.38379 \\ \text { ccc16 } & 129.78477 \\ \text { dih16 } & -175.00663 \\ \text { cc17 } & 1.39715 \\ \text { ccc17 } & 118.02809 \\ \text { dih17 } & 180.04681 \\ \text { cc18 } & 1.39075 \\ \text { ccc18 } & 120.86742 \\ \text { dih18 } & -0.08525 \\ \text { cc19 } & 1.39744 \\ \text { ccc19 } & 121.05986 \\ & \end{array}$

$\begin{array}{lc}\text { dih19 } & 0.07119 \\ \text { cc20 } & 1.38313 \\ \text { ccc20 } & 118.18491 \\ \text { dih20 } & 0.11656 \\ \text { cc21 } & 1.49611 \\ \text { ccc21 } & 128.76282 \\ \text { dih21 } & 180.20338 \\ \text { hc22 } & 1.10103 \\ \text { hcc22 } & 119.43878 \\ \text { dih22 } & 180.01546 \\ \text { hc23 } & 1.09531 \\ \text { hcc23 } & 120.16159 \\ \text { dih23 } & 180.32260 \\ \text { hc24 } & 1.09527 \\ \text { hcc24 } & 119.89841 \\ \text { dih24 } & -180.25913 \\ \text { hc25 } & 1.09674 \\ \text { hcc25 } & 120.48839 \\ \text { dih25 } & -1.06252 \\ \text { hc26 } & 1.10853 \\ \text { hcc26 } & 111.93514 \\ \text { dih26 } & -63.35717 \\ \text { hc27 } & 1.10678 \\ \text { hcc27 } & 112.26433 \\ \text { dih27 } & 57.60945 \\ \text { hc28 } & 1.09456 \\ \text { hcc28 } & 120.88112 \\ \text { dih28 } & 180.15011 \\ \text { hc29 } & 1.09557 \\ \text { hcc29 } & 119.31253 \\ \text { dih29 } & -179.91646 \\ \text { hc30 } & 1.09539 \\ \text { hcc30 } & 119.69713 \\ \text { dih30 } & -180.04044 \\ \text { hc31 } & 1.09542 \\ \text { hcc31 } & 120.77150 \\ \text { dih31 } & 179.97922 \\ \text { oc32 } & 1.21322 \\ \text { occ32 } & 130.00511 \\ \text { dih32 } & -178.09089 \\ & \\ & \end{array}$




\section{Z-matrix for 2 [N-Ar \& S-Ar locked] (Transition State)}

\begin{tabular}{|c|c|c|c|c|c|c|}
\hline 01 & & & cc9 & 1.381290 & hen 26 & 108.555 \\
\hline $\mathrm{h}$ & & & $\operatorname{ccc} 9$ & 122.665 & $\operatorname{dih} 26$ & -115.341 \\
\hline c $1 \operatorname{ch} 2$ & & & dih9 & -177.428 & hc27 & 1.109179 \\
\hline h 2 hc 3 & $1 \mathrm{hch} 3$ & & cc10 & 1.391639 & hen27 & 112.762 \\
\hline h 2 hc 4 & 3 hch 4 & $1 \operatorname{dih} 4$ & $\operatorname{ccc} 10$ & 118.609 & $\operatorname{dih} 27$ & 125.726 \\
\hline s $2 \operatorname{sc} 5$ & $3 \operatorname{sch} 5$ & $4 \operatorname{dih} 5$ & $\operatorname{dih} 10$ & 0.332 & hc28 & 1.094490 \\
\hline o 5 os 6 & 2 osc 6 & $1 \operatorname{dih} 6$ & cc11 & 1.384120 & hcc28 & 120.981 \\
\hline c $5 \operatorname{cs} 7$ & $2 \csc 7$ & $1 \operatorname{dih} 7$ & $\operatorname{ccc} 11$ & 119.891 & $\operatorname{dih} 28$ & -0.089 \\
\hline c $7 \mathrm{cc} 8$ & $5 \operatorname{ccs} 8$ & $6 \operatorname{dih} 8$ & $\operatorname{dih} 11$ & 1.584 & hc29 & 1.095581 \\
\hline c 8 cc 9 & $7 \operatorname{ccc} 9$ & 5 dih 9 & cc12 & 1.402553 & hec29 & 119.325 \\
\hline c $9 \operatorname{cc} 10$ & $8 \operatorname{ccc} 10$ & $7 \operatorname{dih} 10$ & $\operatorname{ccc} 12$ & 118.881 & $\operatorname{dih} 29$ & 179.808 \\
\hline c $10 \mathrm{cc} 11$ & $9 \operatorname{ccc} 11$ & 8 dih1 1 & $\operatorname{dih} 12$ & -2.477 & hc30 & 1.095410 \\
\hline c $7 \mathrm{cc} 12$ & $8 \operatorname{ccc} 12$ & $9 \operatorname{dih} 12$ & $\mathrm{nc} 13$ & 1.452680 & hec30 & 119.675 \\
\hline n $12 \mathrm{nc} 13$ & 7 ncc13 & $8 \operatorname{dih} 13$ & ncc13 & 128.169 & $\operatorname{dih} 30$ & -179.992 \\
\hline c $13 \mathrm{cn} 14$ & 12 cnc 14 & $7 \operatorname{dih} 14$ & $\operatorname{dih} 13$ & -173.352 & hc31 & 1.095360 \\
\hline c $14 \mathrm{cc} 15$ & $13 \operatorname{ccn} 15$ & $12 \operatorname{dih} 15$ & cn14 & 1.466101 & hec31 & 120.827 \\
\hline c $15 \mathrm{cc} 16$ & $14 \operatorname{ccc} 16$ & $13 \operatorname{dih} 16$ & cnc14 & 127.376 & $\operatorname{dih} 31$ & 0.677 \\
\hline c $16 \mathrm{cc} 17$ & $15 \operatorname{ccc} 17$ & $14 \operatorname{dih} 17$ & $\operatorname{dih} 14$ & -3.881 & oc32 & 1.212820 \\
\hline c $17 \mathrm{cc} 18$ & $16 \operatorname{ccc} 18$ & $15 \operatorname{dih} 18$ & cc15 & 1.482800 & ocn32 & 122.994 \\
\hline c $18 \mathrm{cc} 19$ & $17 \operatorname{ccc} 19$ & $16 \operatorname{dih} 19$ & $\operatorname{ccn} 15$ & 108.405 & $\operatorname{dih} 32$ & 166.499 \\
\hline c $15 \mathrm{cc} 20$ & $14 \operatorname{ccc} 20$ & $13 \operatorname{dih} 20$ & $\operatorname{dih} 15$ & 133.065 & & \\
\hline c $13 \mathrm{cn} 21$ & $14 \mathrm{cnc} 21$ & $15 \operatorname{dih} 21$ & cc16 & 1.384400 & & \\
\hline h 8 hc 22 & 7 hcc22 & $12 \operatorname{dih} 22$ & $\operatorname{ccc} 16$ & 129.834 & & \\
\hline h 9 hc23 & 8 hcc23 & $7 \operatorname{dih} 23$ & $\operatorname{dih} 16$ & -177.514 & & \\
\hline h 10 hc 24 & 9 hсc24 & $8 \operatorname{dih} 24$ & cc17 & 1.396809 & & \\
\hline h 11 hc 25 & 12 hcc 25 & $13 \operatorname{dih} 25$ & $\operatorname{ccc} 17$ & 117.925 & & \\
\hline h 21 hc 26 & $13 \mathrm{hcn} 26$ & $14 \operatorname{dih} 26$ & $\operatorname{dih} 17$ & -179.190 & & \\
\hline h 21 hc 27 & 13 hcn 27 & $14 \operatorname{dih} 27$ & $\operatorname{cc} 18$ & 1.391310 & & \\
\hline h 19 hc 28 & 20 hсc28 & $21 \operatorname{dih} 28$ & $\operatorname{ccc} 18$ & 120.883 & & \\
\hline h 18 hc 29 & 19 hcc29 & $20 \operatorname{dih} 29$ & $\operatorname{dih} 18$ & 0.226 & & \\
\hline h $17 \mathrm{hc} 30$ & 18 hcc 30 & $19 \operatorname{dih} 30$ & cc19 & 1.397120 & & \\
\hline h 16 hc 31 & 15 hcc31 & $14 \operatorname{dih} 31$ & $\operatorname{ccc} 19$ & 121.064 & & \\
\hline o 14 oc 32 & $13 \operatorname{ocn} 32$ & $21 \operatorname{dih} 32$ & $\operatorname{dih} 19$ & -0.096 & & \\
\hline & & & $\operatorname{cc} 20$ & 1.402509 & & \\
\hline $\operatorname{ch} 2$ & 6780 & & $\operatorname{ccc} 20$ & 108.913 & & \\
\hline hc3 & 6430 & & $\operatorname{dih} 20$ & 3.313 & & \\
\hline hch3 & 7.641 & & cn21 & 1.523925 & & \\
\hline hc4 & 5801 & & cnc21 & 106.686 & & \\
\hline hch4 & 7.807 & & $\operatorname{dih} 21$ & -4.485 & & \\
\hline $\operatorname{dih} 4$ & .637 & & hc22 & 1.120990 & & \\
\hline $\operatorname{sc} 5$ & 1640 & & hec22 & 114.589 & & \\
\hline $\operatorname{sch} 5$ & .034 & & $\operatorname{dih} 22$ & 175.797 & & \\
\hline $\operatorname{dih} 5$ & .649 & & hc23 & 1.094440 & & \\
\hline os6 & 1731 & & hec23 & 120.510 & & \\
\hline osc6 & .173 & & $\operatorname{dih} 23$ & -179.835 & & \\
\hline dih6 & .233 & & hc24 & 1.095301 & & \\
\hline $\operatorname{cs} 7$ & 8280 & & hec24 & 120.204 & & \\
\hline $\csc 7$ & .900 & & $\operatorname{dih} 24$ & -179.060 & & \\
\hline $\operatorname{dih} 7$ & .203 & & hc25 & 1.098290 & & \\
\hline $\operatorname{cc} 8$ & 0960 & & hec 25 & 120.549 & & \\
\hline $\operatorname{ccs} 8$ & .337 & & $\operatorname{dih} 25$ & -6.119 & & \\
\hline $\operatorname{dih} 8$ & & & hc26 & 1.108090 & & \\
\hline
\end{tabular}




\section{Z-matrix for 3 [N-Ar \& S-Ar locked] (Lowest Energy Conformation)}

\begin{tabular}{|c|c|c|c|c|c|c|}
\hline 01 & & & $\operatorname{dih} 5$ & -124.63797 & cc20 & 1.39722 \\
\hline $\mathrm{h}$ & & & os6 & 1.47390 & $\operatorname{ccc} 20$ & 121.07655 \\
\hline c 1 ch2 & & & osc6 & 108.64523 & $\operatorname{dih} 20$ & 0.07727 \\
\hline h 2 hc 3 & 1 hch3 & & $\operatorname{dih} 6$ & 131.88368 & cc21 & 1.38338 \\
\hline h 2 hc 4 & 1 hch4 & 3 dih4 & os7 & 1.47044 & $\operatorname{ccc} 21$ & 118.20547 \\
\hline s $2 \operatorname{sc} 5$ & $1 \operatorname{sch} 5$ & $4 \operatorname{dih} 5$ & osc7 & 110.11663 & $\operatorname{dih} 21$ & 0.14110 \\
\hline o 5 os 6 & 2 osc 6 & $3 \operatorname{dih} 6$ & $\operatorname{dih} 7$ & 3.20854 & $\operatorname{cc} 22$ & 1.49586 \\
\hline o 5 os 7 & 2 osc 7 & $3 \operatorname{dih} 7$ & $\operatorname{cs} 8$ & 1.80548 & $\operatorname{ccc} 22$ & 128.68415 \\
\hline c $5 \operatorname{cs} 8$ & $2 \csc 8$ & $3 \operatorname{dih} 8$ & $\csc 8$ & 102.43726 & $\operatorname{dih} 22$ & -179.68976 \\
\hline c $8 \operatorname{cc} 9$ & $5 \operatorname{ccs} 9$ & 2 dih9 & $\operatorname{dih} 8$ & -113.17858 & hc23 & 1.10086 \\
\hline c 9 cc10 & $8 \operatorname{ccc} 10$ & $5 \operatorname{dih} 10$ & $\operatorname{cc} 9$ & 1.39880 & hec23 & 118.78132 \\
\hline c $10 \mathrm{cc} 11$ & $9 \operatorname{ccc} 11$ & 8 dih1 1 & $\operatorname{ccs} 9$ & 117.65770 & $\operatorname{dih} 23$ & 180.46665 \\
\hline c $11 \mathrm{cc} 12$ & $10 \operatorname{ccc} 12$ & $9 \operatorname{dih} 12$ & $\operatorname{dih} 9$ & 240.00000 & hc24 & 1.09548 \\
\hline c $12 \mathrm{cc} 13$ & $11 \mathrm{ccc} 13$ & $10 \operatorname{dih} 13$ & cc10 & 1.38930 & hcc24 & 120.14084 \\
\hline n 13 nc14 & 12 ncc14 & $11 \operatorname{dih} 14$ & $\operatorname{ccc} 10$ & 121.73317 & $\operatorname{dih} 24$ & 180.06867 \\
\hline c 14 cn 15 & $13 \mathrm{cnc} 15$ & $12 \operatorname{dih} 15$ & $\operatorname{dih} 10$ & 182.60748 & hc25 & 1.09543 \\
\hline c $15 \mathrm{cc} 16$ & $14 \operatorname{ccn} 16$ & $13 \operatorname{dih} 16$ & cc11 & 1.38945 & hec25 & 120.02478 \\
\hline c $16 \mathrm{cc} 17$ & $15 \operatorname{ccc} 17$ & $14 \operatorname{dih} 17$ & $\operatorname{ccc} 11$ & 120.00626 & $\operatorname{dih} 25$ & 179.58515 \\
\hline c $17 \mathrm{cc} 18$ & $16 \operatorname{ccc} 18$ & $15 \operatorname{dih} 18$ & $\operatorname{dih} 11$ & 0.46026 & hc26 & 1.09695 \\
\hline c $18 \mathrm{cc} 19$ & $17 \operatorname{ccc} 19$ & $16 \operatorname{dih} 19$ & cc12 & 1.38878 & hec26 & 120.44742 \\
\hline c 19 cc 20 & $18 \operatorname{ccc} 20$ & $17 \operatorname{dih} 20$ & $\operatorname{ccc} 12$ & 119.66164 & $\operatorname{dih} 26$ & -0.88799 \\
\hline c 20 cc 21 & $19 \operatorname{ccc} 21$ & $18 \operatorname{dih} 21$ & $\operatorname{dih} 12$ & -0.10997 & hc27 & 1.10810 \\
\hline c $21 \mathrm{cc} 22$ & $20 \operatorname{ccc} 22$ & $19 \operatorname{dih} 22$ & cc13 & 1.39911 & hcc27 & 112.02833 \\
\hline h 9 hc23 & 10 hcc23 & $11 \operatorname{dih} 23$ & $\operatorname{ccc} 13$ & 120.10613 & $\operatorname{dih} 27$ & -63.47290 \\
\hline h 10 hc24 & 11 hec24 & $12 \operatorname{dih} 24$ & $\operatorname{dih} 13$ & -0.47293 & hc28 & 1.10898 \\
\hline h 11 hc25 & 12 hcc 25 & $13 \operatorname{dih} 25$ & nc14 & 1.45603 & hcc28 & 112.63312 \\
\hline h 12 hc26 & 13 hcc26 & $14 \operatorname{dih} 26$ & ncc14 & 119.47104 & $\operatorname{dih} 28$ & 58.05908 \\
\hline h 22 hc27 & 21 hcc27 & $20 \operatorname{dih} 27$ & $\operatorname{dih} 14$ & -180.31466 & hc29 & 1.09467 \\
\hline h 22 hc 28 & 21 hcc 28 & $20 \operatorname{dih} 28$ & cn15 & 1.45872 & hcc29 & 120.91787 \\
\hline h 20 hc29 & 19 hсc29 & $18 \operatorname{dih} 29$ & cnc15 & 120.43549 & $\operatorname{dih} 29$ & -179.68163 \\
\hline h 19 hc30 & 20 hcc30 & $21 \operatorname{dih} 30$ & $\operatorname{dih} 15$ & 60.00000 & hc30 & 1.09557 \\
\hline h 18 hc31 & 19 hcc31 & $20 \operatorname{dih} 31$ & cc16 & 1.48247 & hcc30 & 119.31294 \\
\hline h 17 hc32 & 16 hcc32 & $21 \operatorname{dih} 32$ & $\operatorname{ccn} 16$ & 106.60514 & $\operatorname{dih} 30$ & -179.95191 \\
\hline о 15 oc33 & 16 occ33 & $21 \operatorname{dih} 33$ & $\operatorname{dih} 16$ & -146.45113 & hc31 & 1.09533 \\
\hline & & & cc17 & 1.38404 & hcc31 & 119.69677 \\
\hline $\operatorname{ch} 2$ & 1.09971 & & $\operatorname{ccc} 17$ & 129.73281 & $\operatorname{dih} 31$ & 179.91773 \\
\hline hc3 & 1.09952 & & $\operatorname{dih} 17$ & -175.14111 & hc32 & 1.09532 \\
\hline hch3 & 106.21738 & & cc18 & 1.39682 & hcc32 & 120.77368 \\
\hline hc4 & 1.09959 & & $\operatorname{ccc} 18$ & 118.03437 & $\operatorname{dih} 32$ & -179.96664 \\
\hline hch4 & 105.9717 & & $\operatorname{dih} 18$ & -179.96996 & oc33 & 1.21398 \\
\hline $\operatorname{dih} 4$ & -112.40045 & & cc19 & 1.39096 & occ33 & 130.36602 \\
\hline sc5 & 1.78959 & & $\operatorname{ccc} 19$ & 120.85873 & $\operatorname{dih} 33$ & -178.22304 \\
\hline & & & & & & \\
\hline
\end{tabular}




\section{Z-matrix for 3 [N-Ar \& S-Ar locked]-Lowest Energy Conformation}

(other side of the Transition State)

\begin{tabular}{|c|c|c|}
\hline \multicolumn{3}{|l|}{01} \\
\hline & & \\
\hline h $2 \mathrm{hc} 3$ & 1 hch3 & \\
\hline h 2 hc4 & $1 \mathrm{hch} 4$ & $3 \operatorname{dih} 4$ \\
\hline $2 \operatorname{sc} 5$ & $1 \operatorname{sch} 5$ & $4 \operatorname{dih} 5$ \\
\hline 5 os6 & 2 osc 6 & $3 \operatorname{dih} 6$ \\
\hline 5 os 7 & $2 \operatorname{osc} 7$ & $3 \operatorname{dih} 7$ \\
\hline $5 \operatorname{cs} 8$ & $2 \csc 8$ & $3 \operatorname{dih} 8$ \\
\hline $8 \operatorname{cc} 9$ & $5 \operatorname{ccs} 9$ & $2 \operatorname{dih} 9$ \\
\hline $9 \operatorname{cc} 10$ & $8 \operatorname{ccc} 10$ & $5 \operatorname{dih} 10$ \\
\hline c $10 \mathrm{cc} 11$ & $9 \operatorname{ccc} 11$ & 8 dih11 \\
\hline c $11 \mathrm{cc} 12$ & $10 \operatorname{ccc} 12$ & $9 \operatorname{dih} 12$ \\
\hline c $12 \mathrm{cc} 13$ & $11 \operatorname{ccc} 13$ & $10 \operatorname{dih} 13$ \\
\hline n $13 \mathrm{nc} 14$ & 12 ncc14 & $11 \operatorname{dih} 14$ \\
\hline c $14 \mathrm{cn} 15$ & $13 \mathrm{cnc} 15$ & $12 \operatorname{dih} 15$ \\
\hline c $15 \mathrm{cc} 16$ & $14 \operatorname{con} 16$ & $13 \operatorname{dih} 16$ \\
\hline c $16 \mathrm{cc} 17$ & $15 \operatorname{ccc} 17$ & $14 \operatorname{dih} 17$ \\
\hline c $17 \mathrm{cc} 18$ & $16 \operatorname{ccc} 18$ & $15 \operatorname{dih} 18$ \\
\hline c 18 cc 19 & $17 \operatorname{ccc} 19$ & $16 \operatorname{dih} 19$ \\
\hline c $19 \operatorname{cc} 20$ & $18 \operatorname{ccc} 20$ & $17 \operatorname{dih} 20$ \\
\hline c $20 \mathrm{cc} 21$ & $19 \operatorname{ccc} 21$ & $18 \operatorname{dih} 21$ \\
\hline c $21 \mathrm{cc} 22$ & $20 \operatorname{ccc} 22$ & $19 \operatorname{dih} 22$ \\
\hline h 9 hc23 & 10 hcc 23 & $11 \operatorname{dih} 23$ \\
\hline h 10 hc 24 & 11 hcc24 & $12 \operatorname{dih} 24$ \\
\hline h 11 hc 25 & 12 hcc 25 & $13 \operatorname{dih} 25$ \\
\hline h 12 hc 26 & 13 hcc 26 & $14 \operatorname{dih} 26$ \\
\hline h 22 hc 27 & 21 hcc 27 & $20 \operatorname{dih} 27$ \\
\hline h 22 hc 28 & 21 hec28 & $20 \operatorname{dih} 28$ \\
\hline h 20 hc 29 & 19 hcc 29 & $18 \operatorname{dih} 29$ \\
\hline h $19 \mathrm{hc} 30$ & 20 hcc 30 & $21 \operatorname{dih} 30$ \\
\hline h 18 hc 31 & 19 hcc31 & $20 \operatorname{dih} 31$ \\
\hline h 17 hc32 & 16 hcc32 & $21 \operatorname{dih} 32$ \\
\hline o 15 oc 33 & 16 occ33 & $21 \operatorname{dih} 33$ \\
\hline
\end{tabular}

$\begin{array}{lc}\text { ch2 } & 1.09970 \\ \text { hc3 } & 1.10231 \\ \text { hch3 } & 105.74564 \\ \text { hc4 } & 1.09789 \\ \text { hch4 } & 105.95278 \\ \text { dih4 } & -112.75818 \\ \text { sc5 } & 1.79502\end{array}$

$\begin{array}{lc}\text { sch5 } & 111.21516 \\ \text { dih5 } & -122.87967 \\ \text { os6 } & 1.47375 \\ \text { osc6 } & 107.64784 \\ \text { dih6 } & 185.40933 \\ \text { os7 } & 1.45920 \\ \text { osc7 } & 109.80237 \\ \text { dih7 } & 57.35084 \\ \text { cs8 } & 1.80967 \\ \text { csc8 } & 101.71621 \\ \text { dih8 } & -60.86019 \\ \text { cc9 } & 1.39996 \\ \text { ccs9 } & 116.74231 \\ \text { dih9 } & 240.00000 \\ \text { cc10 } & 1.38894 \\ \text { ccc10 } & 121.96251 \\ \text { dih10 } & 181.94320 \\ \text { cc11 } & 1.38929 \\ \text { ccc11 } & 119.97856 \\ \text { dih11 } & 0.39892 \\ \text { cc12 } & 1.38823 \\ \text { ccc12 } & 119.53758 \\ \text { dih12 } & 0.48979 \\ \text { cc13 } & 1.39977 \\ \text { ccc13 } & 120.24649 \\ \text { dih13 } & -0.14237 \\ \text { nc14 } & 1.45672 \\ \text { ncc14 } & 19.11798 \\ \text { dih14 } & -182.08878 \\ \text { cn15 } & 1.46750 \\ \text { cnc15 } & 19.84711 \\ \text { dih15 } & 300.00000 \\ \text { cc16 } & 1.48528 \\ \text { ccn16 } & 06.28676 \\ \text { dih16 } & -212.02691 \\ \text { cc17 } & 1.38346 \\ \text { ccc17 } & 129.66633 \\ \text { dih17 } & -187.26582 \\ \text { cc18 } & 1.39762 \\ \text { ccc18 } & 118.02603 \\ \text { dih18 } & -180.32705 \\ \text { cc19 } & 1.39040 \\ \text { ccc19 } & 120.88550 \\ & \end{array}$

$\begin{array}{lc}\text { dih19 } & 0.06610 \\ \text { cc20 } & 1.39770 \\ \text { ccc20 } & 121.04128 \\ \text { dih20 } & 0.02836 \\ \text { cc21 } & 1.38284 \\ \text { ccc21 } & 118.15817 \\ \text { dih21 } & -0.11619 \\ \text { cc22 } & 1.49550 \\ \text { ccc22 } & 128.75587 \\ \text { dih22 } & -180.46183 \\ \text { hc23 } & 1.10117 \\ \text { hcc23 } & 118.50807 \\ \text { dih23 } & 180.27528 \\ \text { hc24 } & 1.09549 \\ \text { hcc24 } & 120.15787 \\ \text { dih24 } & 180.86882 \\ \text { hc25 } & 1.09538 \\ \text { hcc25 } & 120.08803 \\ \text { dih25 } & 180.43433 \\ \text { hc26 } & 1.09709 \\ \text { hcc26 } & 120.38783 \\ \text { dih26 } & -1.02120 \\ \text { hc27 } & 1.11203 \\ \text { hcc27 } & 112.37338 \\ \text { dih27 } & -56.47234 \\ \text { hc28 } & 1.10830 \\ \text { hcc28 } & 112.04060 \\ \text { dih28 } & 64.74615 \\ \text { hc29 } & 1.09439 \\ \text { hcc29 } & 120.84516 \\ \text { dih29 } & -180.22042 \\ \text { hc30 } & 1.09551 \\ \text { hcc30 } & 119.30561 \\ \text { dih30 } & -180.00989 \\ \text { hc31 } & 1.09541 \\ \text { hcc31 } & 119.69926 \\ \text { dih31 } & 180.18099 \\ \text { hc32 } & 1.09556 \\ \text { hcc32 } & 120.76542 \\ \text { dih32 } & -180.24083 \\ \text { oc33 } & 1.20973 \\ \text { occ33 } & 130.02882 \\ \text { dih33 } & -186.76067 \\ & \end{array}$


Z-matrix for 3 [N-Ar \& S-Ar locked] (Transition State)

\begin{tabular}{|c|c|c|c|c|c|c|}
\hline 01 & & & $\operatorname{dih} 5$ & -124.023 & $\operatorname{cc} 20$ & 1.397420 \\
\hline $\mathrm{h}$ & & & os6 & 1.470110 & $\operatorname{ccc} 20$ & 121.080 \\
\hline c $1 \mathrm{ch} 2$ & & & osc6 & 110.626 & $\operatorname{dih} 20$ & -0.262 \\
\hline h 2 hc 3 & $1 \mathrm{hch} 3$ & & dih6 & -134.467 & $\operatorname{cc} 21$ & 1.402138 \\
\hline h 2 hc4 & 3 hch 4 & $1 \operatorname{dih} 4$ & os 7 & 1.477129 & $\operatorname{ccc} 21$ & 108.789 \\
\hline s $2 \operatorname{sc} 5$ & $3 \operatorname{sch} 5$ & $1 \operatorname{dih} 5$ & osc7 & 108.060 & $\operatorname{dih} 21$ & 5.973 \\
\hline o 5 os6 & 2 osc 6 & $4 \operatorname{dih} 6$ & $\operatorname{dih} 7$ & -6.084 & cn22 & 1.529506 \\
\hline o 5 os 7 & $2 \operatorname{osc} 7$ & $4 \operatorname{dih} 7$ & $\operatorname{cs} 8$ & 1.812850 & cnc22 & 105.710 \\
\hline c $5 \operatorname{cs} 8$ & $2 \csc 8$ & 4 dih8 & $\csc 8$ & 103.415 & $\operatorname{dih} 22$ & -8.079 \\
\hline с 8 cc 9 & $5 \operatorname{ccs} 9$ & 6 dih9 & $\operatorname{dih} 8$ & 109.750 & hc23 & 1.103960 \\
\hline c $9 \operatorname{cc} 10$ & $8 \operatorname{ccc} 10$ & $5 \operatorname{dih} 10$ & $\operatorname{cc} 9$ & 1.408819 & hec23 & 119.015 \\
\hline c $10 \mathrm{cc} 11$ & $9 \operatorname{ccc} 11$ & 8 dih 11 & $\operatorname{ccs} 9$ & 111.067 & $\operatorname{dih} 23$ & 175.163 \\
\hline c $11 \mathrm{cc} 12$ & $10 \operatorname{ccc} 12$ & $9 \operatorname{dih} 12$ & $\operatorname{dih} 9$ & 122.586 & hc24 & 1.094950 \\
\hline c 8 cc 13 & $9 \operatorname{ccc} 13$ & $10 \operatorname{dih} 13$ & $\operatorname{cc} 10$ & 1.381291 & hcc24 & 120.472 \\
\hline n $13 \mathrm{nc} 14$ & 8 ncc 14 & $9 \operatorname{dih} 14$ & $\operatorname{ccc} 10$ & 123.568 & $\operatorname{dih} 24$ & -179.613 \\
\hline c 14 cn 15 & $13 \mathrm{cnc} 15$ & $8 \operatorname{dih} 15$ & $\operatorname{dih} 10$ & -178.699 & hc25 & 1.095320 \\
\hline c $15 \mathrm{cc} 16$ & $14 \operatorname{ccn} 16$ & $13 \operatorname{dih} 16$ & cc11 & 1.390710 & hec 25 & 120.493 \\
\hline c $16 \mathrm{cc} 17$ & $15 \operatorname{ccc} 17$ & $14 \operatorname{dih} 17$ & $\operatorname{ccc} 11$ & 118.887 & $\operatorname{dih} 25$ & -177.180 \\
\hline c 17 cc 18 & $16 \operatorname{ccc} 18$ & $15 \operatorname{dih} 18$ & $\operatorname{dih} 11$ & -0.068 & hc26 & 1.099151 \\
\hline c 18 cc19 & $17 \operatorname{ccc} 19$ & $16 \operatorname{dih} 19$ & $\mathrm{cc} 12$ & 1.380629 & hec26 & 120.428 \\
\hline c $19 \operatorname{cc} 20$ & $18 \operatorname{ccc} 20$ & $17 \operatorname{dih} 20$ & $\operatorname{ccc} 12$ & 119.163 & $\operatorname{dih} 26$ & -10.627 \\
\hline c 16 cc 21 & $15 \operatorname{ccc} 21$ & $14 \operatorname{dih} 21$ & $\operatorname{dih} 12$ & 3.842 & hc27 & 1.109131 \\
\hline c 14 cn 22 & 15 cnc 22 & $16 \operatorname{dih} 22$ & cc13 & 1.409000 & hen 27 & 112.960 \\
\hline h 9 hc23 & 8 hcc23 & $13 \operatorname{dih} 23$ & $\operatorname{ccc} 13$ & 116.943 & $\operatorname{dih} 27$ & 128.767 \\
\hline h 10 hc 24 & 9 hcc24 & $8 \operatorname{dih} 24$ & $\operatorname{dih} 13$ & -6.076 & hc28 & 1.108030 \\
\hline h 11 hc 25 & 10 hcc 25 & $9 \operatorname{dih} 25$ & nc14 & 1.454090 & hen 28 & 108.372 \\
\hline h 12 hc 26 & 13 hcc26 & $14 \operatorname{dih} 26$ & ncc14 & 129.566 & $\operatorname{dih} 28$ & -112.168 \\
\hline h 22 hc 27 & 14 hen 27 & $15 \operatorname{dih} 27$ & $\operatorname{dih} 14$ & -166.547 & hc29 & 1.094310 \\
\hline h 22 hc 28 & $14 \mathrm{hcn} 28$ & $15 \operatorname{dih} 28$ & cn15 & 1.474890 & hec29 & 121.022 \\
\hline h 20 hc 29 & 21 hcc 29 & $22 \operatorname{dih} 29$ & cnc 15 & 128.925 & $\operatorname{dih} 29$ & 0.413 \\
\hline h 19 hc30 & 20 hcc30 & $21 \operatorname{dih} 30$ & $\operatorname{dih} 15$ & -4.888 & hc30 & 1.095461 \\
\hline h 18 hc31 & 19 hcc31 & $20 \operatorname{dih} 31$ & cc16 & 1.480160 & hec30 & 119.297 \\
\hline h 17 hc32 & 16 hcc32 & $15 \operatorname{dih} 32$ & $\operatorname{ccn} 16$ & 108.764 & $\operatorname{dih} 30$ & 179.726 \\
\hline o 15 oc33 & $14 \operatorname{ocn} 33$ & $22 \operatorname{dih} 33$ & $\operatorname{dih} 16$ & 128.314 & hc31 & 1.095340 \\
\hline & & & cc17 & 1.384155 & hec31 & 119.673 \\
\hline $\operatorname{ch} 2$ & 0849 & & $\operatorname{ccc} 17$ & 129.875 & $\operatorname{dih} 31$ & 179.591 \\
\hline hc3 & 8890 & & $\operatorname{dih} 17$ & -174.318 & hc32 & 1.095350 \\
\hline hch3 & .006 & & cc18 & 1.397320 & hec32 & 120.843 \\
\hline hc4 & 0061 & & $\operatorname{ccc} 18$ & 117.828 & $\operatorname{dih} 32$ & 1.106 \\
\hline hch4 & .962 & & $\operatorname{dih} 18$ & -179.263 & oc33 & 1.210931 \\
\hline dih4 & 294 & & cc19 & 1.390990 & ocn33 & 121.728 \\
\hline $\operatorname{sc} 5$ & 6330 & & $\operatorname{ccc} 19$ & 120.914 & $\operatorname{dih} 33$ & 161.262 \\
\hline $\operatorname{sch} 5$ & .114 & & $\operatorname{dih} 19$ & 0.060 & & \\
\hline
\end{tabular}


Figure 6. Partial ${ }^{1} \mathrm{H}$ NMR of 1 (and 2) $\left[-40{ }^{\circ} \mathrm{C}, \mathrm{CDCl}_{3}\right]$ with $m$-CPBA as a limiting reagent ( 0.85 equiv) after $\sim 5$ min.

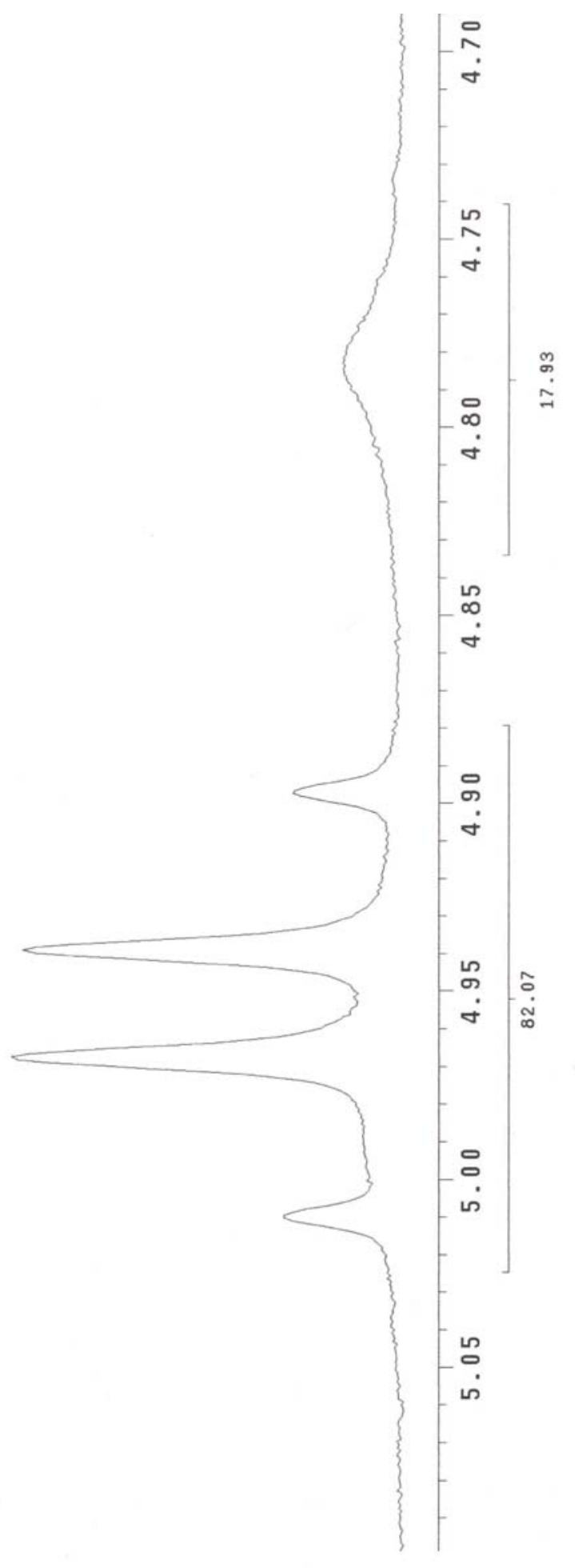


$\mathrm{N}$-Ar torsion angle $v s$ virtual dihedral angle for $\mathbf{2}$

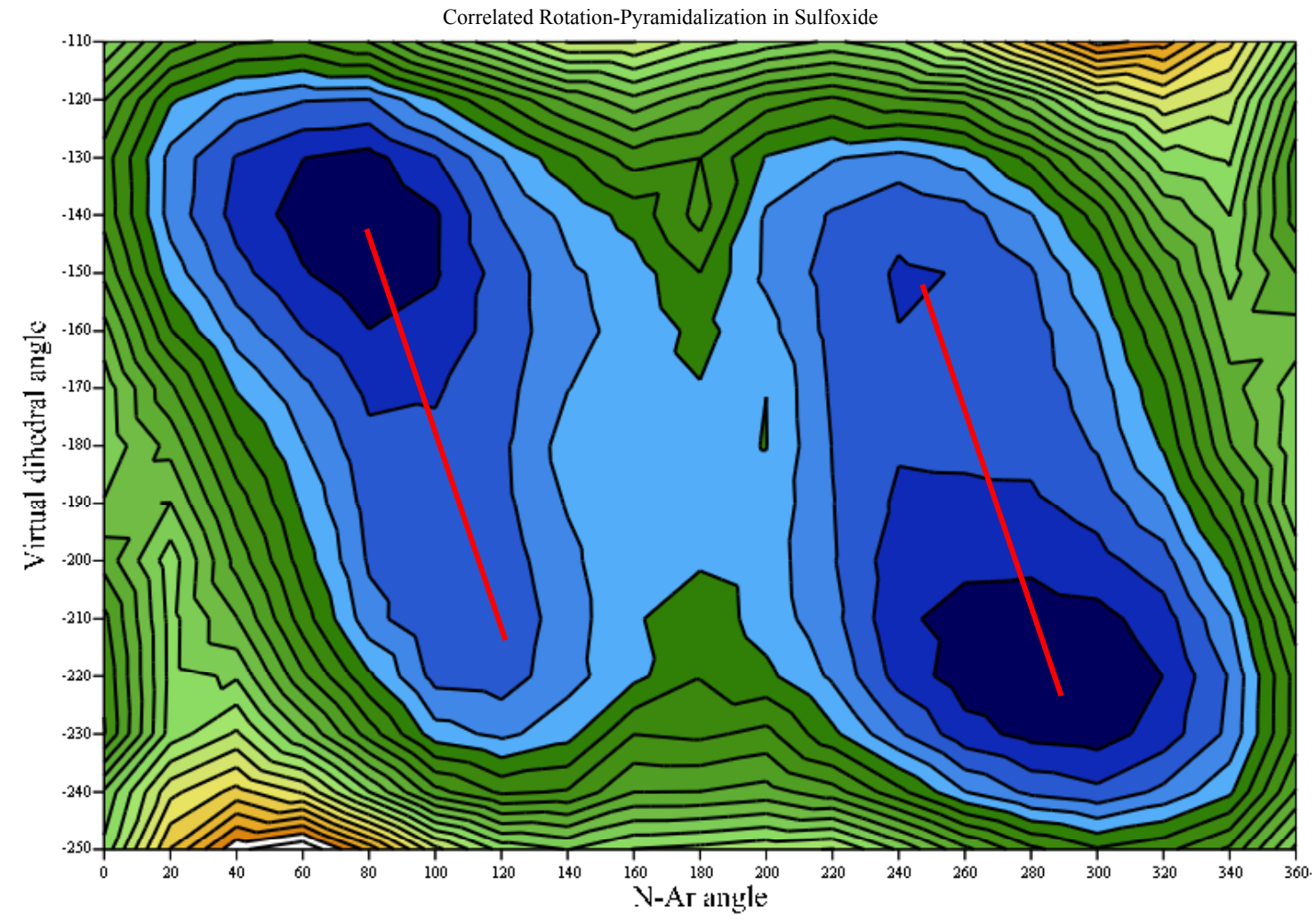

$(\mathrm{q}, \mathrm{w}, \mathrm{z})$ 
$\mathrm{N}$-Ar torsion angle $v s$ virtual dihedral angle for $\mathbf{3}$

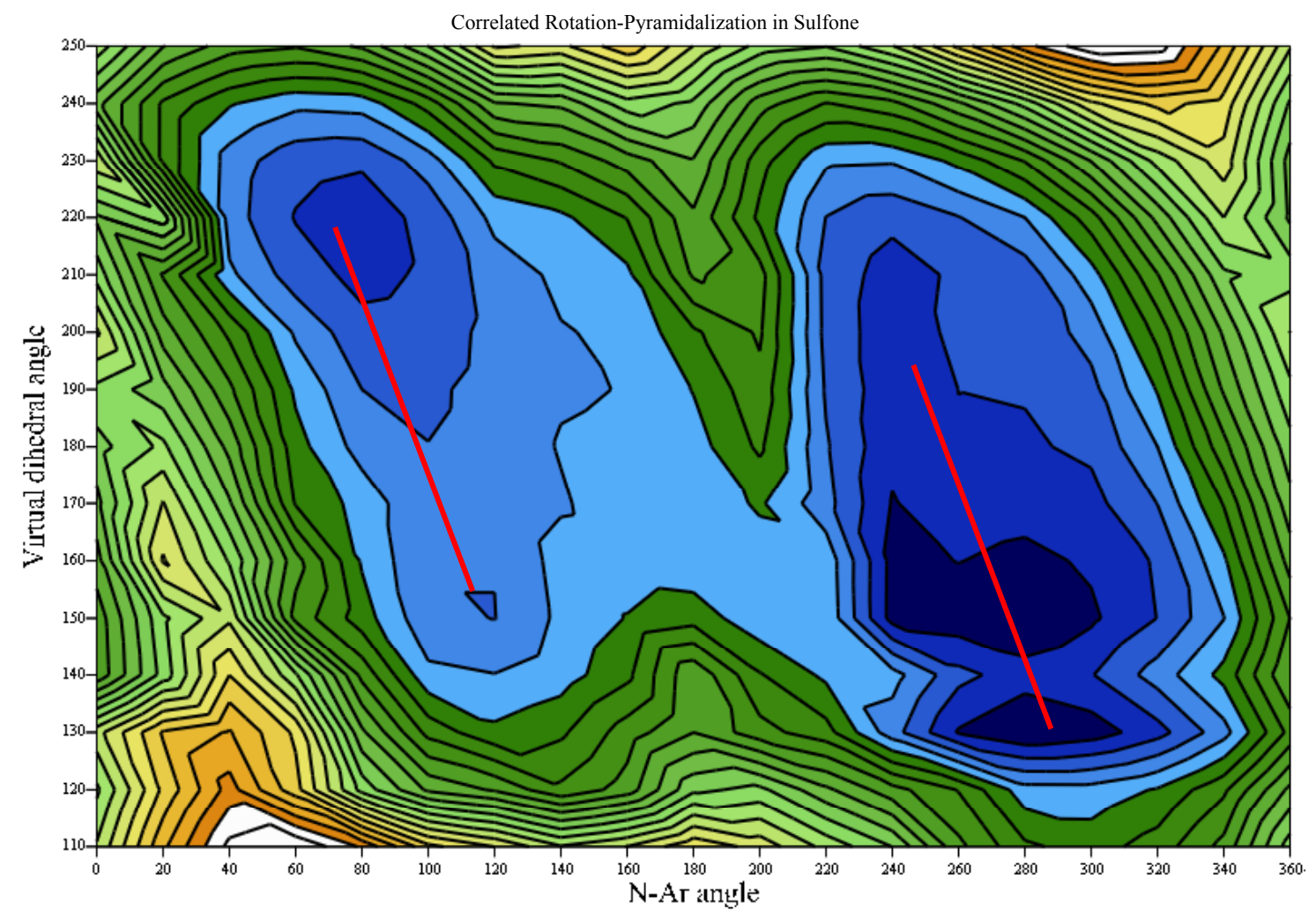

$(\mathrm{q}, \mathrm{w}, \mathrm{z})$ 
Line-shape analysis for 3

TEMP.

RATE
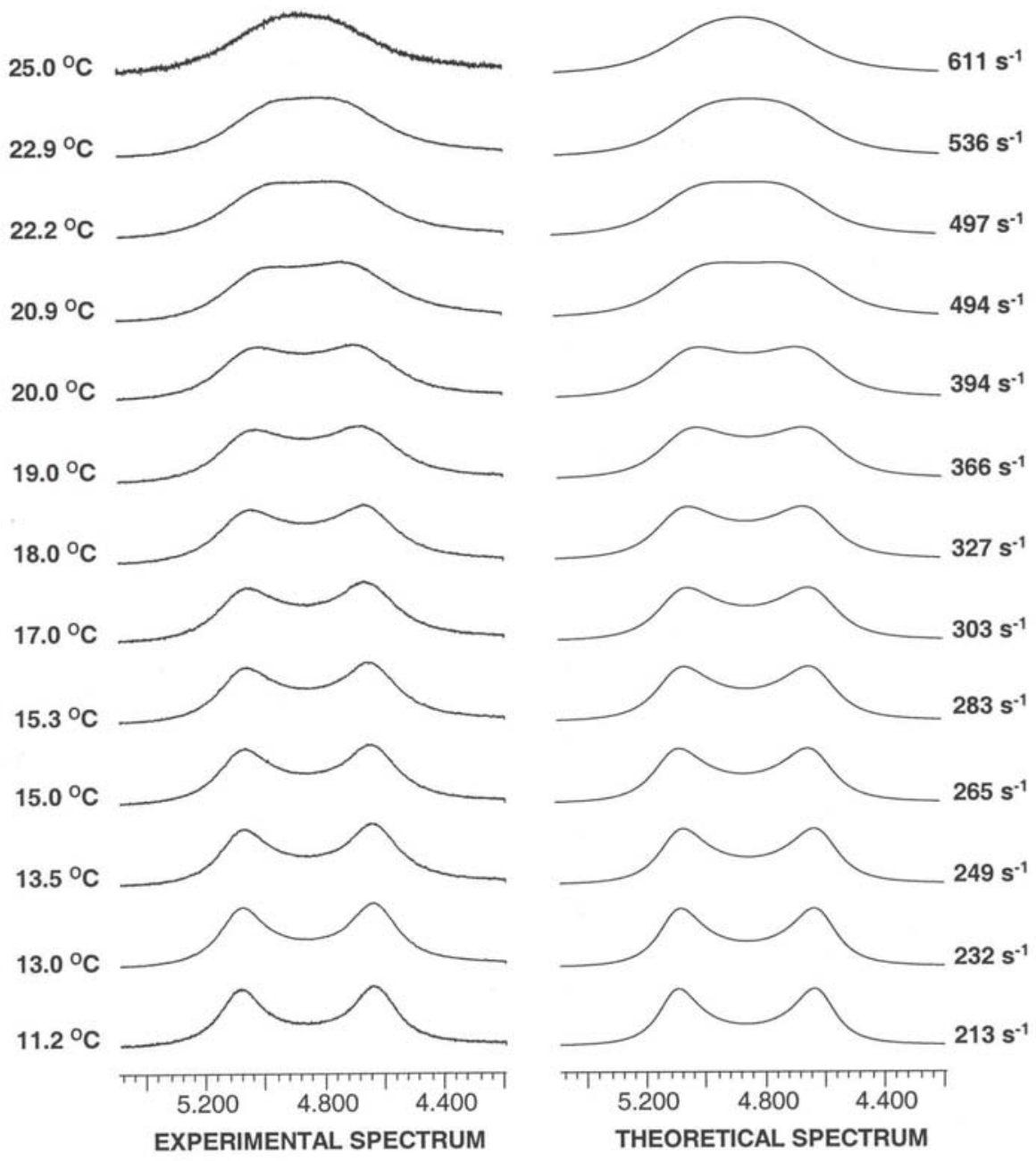


\section{Z-matrix for 1 (N-Ar \& Virtual Dihedral Angle locked)-Lowest Energy Conformation}

\begin{tabular}{|c|c|c|c|c|c|c|}
\hline 01 & & & $\operatorname{cc} 6$ & 1.38813 & $\operatorname{cc} 20$ & 1.49509 \\
\hline $\mathrm{c}$ & & & $\operatorname{ccc} 6$ & 120.32517 & $\operatorname{ccn} 20$ & 104.07437 \\
\hline n $1 \mathrm{nc} 2$ & & & dih6 & 180.30749 & $\operatorname{dih} 20$ & -7.77877 \\
\hline c $2 \mathrm{cn} 3$ & $1 \mathrm{cnc} 3$ & & cc7 & 1.39057 & hc21 & 1.09453 \\
\hline c $2 \mathrm{cn} 4$ & $1 \mathrm{cnc} 4$ & $3 \operatorname{dih} 4$ & $\operatorname{ccc} 7$ & 119.99521 & hec21 & 120.91192 \\
\hline c $4 \operatorname{cc} 5$ & $2 \operatorname{ccn} 5$ & $1 \operatorname{dih} 5$ & $\operatorname{dih} 7$ & 0.13847 & $\operatorname{dih} 21$ & 179.85103 \\
\hline c 5 cc 6 & $4 \operatorname{ccc} 6$ & 2 dih 6 & $\operatorname{cc} 8$ & 1.38826 & hc22 & 1.09548 \\
\hline c $6 \mathrm{cc} 7$ & $5 \operatorname{ccc} 7$ & $4 \operatorname{dih} 7$ & $\operatorname{ccc} 8$ & 119.88847 & hcc22 & 119.62039 \\
\hline c $7 \operatorname{cc} 8$ & $6 \operatorname{ccc} 8$ & $5 \operatorname{dih} 8$ & $\operatorname{dih} 8$ & -0.83974 & $\operatorname{dih} 22$ & 179.92606 \\
\hline c $8 \operatorname{cc} 9$ & $7 \operatorname{ccc} 9$ & $6 \operatorname{dih} 9$ & cc9 & 1.39864 & hc23 & 1.09528 \\
\hline s $9 \mathrm{sc} 10$ & $8 \operatorname{scc} 10$ & $7 \operatorname{dih} 10$ & $\operatorname{ccc} 9$ & 121.15526 & hcc 23 & 119.44568 \\
\hline c $10 \mathrm{cs} 11$ & $9 \csc 11$ & 8 dih11 & dih9 & 0.65545 & $\operatorname{dih} 23$ & -180.02428 \\
\hline h $11 \mathrm{hc} 12$ & $10 \mathrm{hcs} 12$ & $9 \operatorname{dih} 12$ & sc10 & 1.77391 & hc24 & 1.09535 \\
\hline h $11 \mathrm{hc} 13$ & $10 \mathrm{~h} \operatorname{cs} 13$ & 9 dih 13 & $\operatorname{scc} 10$ & 115.13803 & hcc24 & 120.74788 \\
\hline h $11 \mathrm{hc} 14$ & 10 hcs 14 & $9 \operatorname{dih} 14$ & $\operatorname{dih} 10$ & -182.92343 & $\operatorname{dih} 24$ & -0.14571 \\
\hline c $3 \mathrm{cc} 15$ & $2 \operatorname{ccn} 15$ & $1 \operatorname{dih} 15$ & $\operatorname{cs} 11$ & 1.80565 & oc 25 & 1.21366 \\
\hline c $15 \mathrm{cc} 16$ & $3 \operatorname{ccc} 16$ & $2 \operatorname{dih} 16$ & $\csc 11$ & 104.79719 & ocn 25 & 123.56947 \\
\hline c $16 \mathrm{cc} 17$ & $15 \operatorname{ccc} 17$ & $3 \operatorname{dih} 17$ & $\operatorname{dih} 11$ & 121.01170 & $\operatorname{dih} 25$ & 184.69508 \\
\hline c $17 \mathrm{cc} 18$ & $16 \operatorname{ccc} 18$ & $15 \operatorname{dih} 18$ & hc12 & 1.09502 & hc26 & 1.09723 \\
\hline c 18 cc19 & $17 \operatorname{ccc} 19$ & $16 \operatorname{dih} 19$ & hcs 12 & 112.92958 & hec26 & 120.38630 \\
\hline c $1 \operatorname{cc} 20$ & $2 \operatorname{ccn} 20$ & $3 \operatorname{dih} 20$ & $\operatorname{dih} 12$ & -53.82583 & $\operatorname{dih} 26$ & 1.02506 \\
\hline h 19 hc 21 & 18 hcc 21 & $17 \operatorname{dih} 21$ & hc13 & 1.09690 & hc27 & 1.09520 \\
\hline h 18 hc 22 & 17 hcc22 & $16 \operatorname{dih} 22$ & hcs 13 & 106.58306 & hcc27 & 119.85616 \\
\hline h $17 \mathrm{hc} 23$ & 16 hcc 23 & $15 \operatorname{dih} 23$ & $\operatorname{dih} 13$ & 187.62173 & $\operatorname{dih} 27$ & -179.86280 \\
\hline h 16 hc 24 & 15 hcc24 & $3 \operatorname{dih} 24$ & hc14 & 1.09534 & hc28 & 1.09501 \\
\hline о 3 oc 25 & 2 ocn 25 & $1 \operatorname{dih} 25$ & hcs 14 & 113.32572 & hcc 28 & 120.15262 \\
\hline h 5 hc 26 & 4 hcc26 & $2 \operatorname{dih} 26$ & $\operatorname{dih} 14$ & 68.90601 & $\operatorname{dih} 28$ & -180.29603 \\
\hline h 6 hc 27 & 5 hcc 27 & $4 \operatorname{dih} 27$ & cc15 & 1.48438 & hc29 & 1.09809 \\
\hline h 7 hc 28 & 6 hcc 28 & $5 \operatorname{dih} 28$ & $\operatorname{ccn} 15$ & 106.75227 & hcc29 & 119.88264 \\
\hline h 8 hc29 & 7 hcc29 & $6 \operatorname{dih} 29$ & $\operatorname{dih} 15$ & 8.05609 & $\operatorname{dih} 29$ & -179.21689 \\
\hline h 1 hc30 & $2 \mathrm{hcn} 30$ & $3 \operatorname{dih} 30$ & cc16 & 1.38385 & hc30 & 1.10803 \\
\hline h $1 \mathrm{hc} 31$ & 2 hen 31 & $3 \operatorname{dih} 31$ & $\operatorname{ccc} 16$ & 129.75096 & hen 30 & 112.44261 \\
\hline & & & $\operatorname{dih} 16$ & -185.05184 & $\operatorname{dih} 30$ & 113.49984 \\
\hline nc2 & 1.50975 & & cc17 & 1.39697 & hc31 & 1.10844 \\
\hline $\mathrm{cn} 3$ & 1.45978 & & $\operatorname{ccc} 17$ & 118.04217 & hen31 & 108.42942 \\
\hline cnc3 & 108.89349 & & $\operatorname{dih} 17$ & -180.06020 & $\operatorname{dih} 31$ & -127.97887 \\
\hline $\operatorname{cn} 4$ & 1.45190 & & cc18 & 1.39085 & & \\
\hline cnc4 & 115.49531 & & $\operatorname{ccc} 18$ & 120.85820 & & \\
\hline $\operatorname{dih} 4$ & 220.00000 & & $\operatorname{dih} 18$ & 0.07523 & & \\
\hline $\operatorname{cc5}$ & 1.40060 & & cc19 & 1.39719 & & \\
\hline $\operatorname{ccn} 5$ & 120.34846 & & $\operatorname{ccc} 19$ & 121.05840 & & \\
\hline $\operatorname{dih} 5$ & 100.00000 & & $\operatorname{dih} 19$ & -0.03954 & & \\
\hline
\end{tabular}




\section{Z-matrix for 2 (N-Ar \& Virtual Dihedral Angle locked)-Lowest Energy Conformation}

\begin{tabular}{|c|c|c|c|c|c|c|}
\hline 01 & & & $\operatorname{dih} 5$ & 80.00000 & $\operatorname{dih} 19$ & 0.05810 \\
\hline $\mathrm{c}$ & & & cc6 & 1.38930 & $\operatorname{cc} 20$ & 1.39760 \\
\hline $\mathrm{n} 1 \mathrm{nc} 2$ & & & $\operatorname{ccc} 6$ & 120.14908 & $\operatorname{ccc} 20$ & 121.04756 \\
\hline c $2 \operatorname{cn} 3$ & $1 \mathrm{cnc} 3$ & & $\operatorname{dih} 6$ & -179.95374 & $\operatorname{dih} 20$ & -0.02686 \\
\hline c $2 \mathrm{cn} 4$ & $1 \mathrm{cnc} 4$ & $3 \operatorname{dih} 4$ & cc7 & 1.38938 & cc21 & 1.49548 \\
\hline c $4 \operatorname{cc} 5$ & $2 \operatorname{ccn} 5$ & $1 \operatorname{dih} 5$ & $\operatorname{ccc} 7$ & 119.81206 & $\operatorname{ccn} 21$ & 103.94507 \\
\hline c $5 \operatorname{cc} 6$ & $4 \operatorname{ccc} 6$ & $2 \operatorname{dih} 6$ & $\operatorname{dih} 7$ & 0.21161 & $\operatorname{dih} 21$ & -9.72510 \\
\hline c $6 \mathrm{cc} 7$ & $5 \operatorname{ccc} 7$ & $4 \operatorname{dih} 7$ & $\operatorname{cc} 8$ & 1.38996 & hc22 & 1.09445 \\
\hline c $7 \operatorname{cc} 8$ & $6 \operatorname{ccc} 8$ & $5 \operatorname{dih} 8$ & $\operatorname{ccc} 8$ & 119.99130 & hec22 & 120.85500 \\
\hline c $8 \operatorname{cc} 9$ & $7 \operatorname{ccc} 9$ & $6 \operatorname{dih} 9$ & $\operatorname{dih} 8$ & -0.53188 & $\operatorname{dih} 22$ & 179.88917 \\
\hline s $9 \mathrm{sc} 10$ & $8 \operatorname{scc} 10$ & 7 dih10 & cc9 & 1.39567 & hc23 & 1.09554 \\
\hline o 10 os 11 & $9 \operatorname{osc} 11$ & $8 \operatorname{dih} 11$ & $\operatorname{ccc} 9$ & 121.31522 & hcc23 & 119.64446 \\
\hline c $10 \operatorname{cs} 12$ & $9 \csc 12$ & $8 \operatorname{dih} 12$ & $\operatorname{dih} 9$ & 0.13450 & $\operatorname{dih} 23$ & 179.93527 \\
\hline h 12 hc13 & 10 hcs 13 & $11 \operatorname{dih} 13$ & $\mathrm{sc} 10$ & 1.81380 & hc24 & 1.09541 \\
\hline h 12 hc14 & 10 hcs 14 & $11 \operatorname{dih} 14$ & $\operatorname{scc} 10$ & 119.27278 & hec24 & 119.42383 \\
\hline h 12 hc 15 & 10 hcs 15 & $11 \operatorname{dih} 15$ & $\operatorname{dih} 10$ & -174.97989 & $\operatorname{dih} 24$ & 179.95733 \\
\hline c $3 \mathrm{cc} 16$ & $2 \operatorname{ccn} 16$ & 1 dih16 & os11 & 1.55492 & hc25 & 1.09550 \\
\hline c $16 \mathrm{cc} 17$ & $3 \operatorname{ccc} 17$ & $2 \operatorname{dih} 17$ & osc 11 & 105.05411 & hcc25 & 120.76097 \\
\hline c $17 \mathrm{cc} 18$ & $16 \operatorname{ccc} 18$ & $3 \operatorname{dih} 18$ & $\operatorname{dih} 11$ & 2.37140 & $\operatorname{dih} 25$ & -0.16359 \\
\hline c $18 \mathrm{cc} 19$ & $17 \operatorname{ccc} 19$ & $16 \operatorname{dih} 19$ & cs12 & 1.82252 & oc26 & 1.21180 \\
\hline c 19 cc 20 & $18 \operatorname{ccc} 20$ & $17 \operatorname{dih} 20$ & $\csc 12$ & 100.13442 & ocn26 & 123.57681 \\
\hline c $1 \mathrm{cc} 21$ & $2 \operatorname{con} 21$ & $3 \operatorname{dih} 21$ & $\operatorname{dih} 12$ & -104.63445 & $\operatorname{dih} 26$ & -172.35769 \\
\hline h 20 hc 22 & 19 hcc 22 & $18 \operatorname{dih} 22$ & hc13 & 1.10017 & hc27 & 1.09677 \\
\hline h 19 hc 23 & 18 hcc 23 & $17 \operatorname{dih} 23$ & hes 13 & 111.02151 & hcc27 & 120.44826 \\
\hline h 18 hc24 & 17 hcc24 & $16 \operatorname{dih} 24$ & $\operatorname{dih} 13$ & 190.15458 & $\operatorname{dih} 27$ & 0.75426 \\
\hline h 17 hc 25 & 16 hec 25 & $3 \operatorname{dih} 25$ & hc14 & 1.09546 & hc28 & 1.09527 \\
\hline o 3 oc 26 & $2 \operatorname{ocn} 26$ & $1 \operatorname{dih} 26$ & hes 14 & 114.24206 & hec28 & 119.92624 \\
\hline h 5 hc 27 & 4 hcc27 & $2 \operatorname{dih} 27$ & $\operatorname{dih} 14$ & -47.66955 & $\operatorname{dih} 28$ & -179.59390 \\
\hline h 6 hc 28 & 5 hcc 28 & $4 \operatorname{dih} 28$ & hc15 & 1.09687 & hc29 & 1.09532 \\
\hline 7 hc29 & 6 hcc29 & $5 \operatorname{dih} 29$ & hcs 15 & 108.92310 & hec29 & 120.15927 \\
\hline h 8 hc 30 & 7 hcc30 & $6 \operatorname{dih} 30$ & $\operatorname{dih} 15$ & 71.80602 & $\operatorname{dih} 29$ & 179.89879 \\
\hline 1 hc31 & 2 hen31 & $3 \operatorname{dih} 31$ & cc16 & 1.48530 & hc30 & 1.10120 \\
\hline h 1 hc 32 & 2 hcn 32 & $3 \operatorname{dih} 32$ & $\operatorname{ccn} 16$ & 106.43772 & hec30 & 119.28023 \\
\hline & & & $\operatorname{dih} 16$ & 9.98057 & $\operatorname{dih} 30$ & -179.14006 \\
\hline nc2 & 1.5078 & & cc17 & 1.38353 & hc31 & 1.11234 \\
\hline $\operatorname{cn} 3$ & 1.4606 & & $\operatorname{ccc} 17$ & 129.72086 & hen31 & 108.53149 \\
\hline cnc3 & 108.992 & & $\operatorname{dih} 17$ & 173.83040 & $\operatorname{dih} 31$ & -129.97678 \\
\hline cn4 & 1.4559 & & cc18 & 1.39744 & hc32 & 1.10843 \\
\hline cnc4 & 115.575 & & $\operatorname{ccc} 18$ & 118.03958 & hen32 & 112.51186 \\
\hline dih4 & -140.000 & & $\operatorname{dih} 18$ & 179.92783 & $\operatorname{dih} 32$ & 111.49100 \\
\hline $\operatorname{cc} 5$ & 1.39903 & & cc19 & 1.39052 & & \\
\hline $\operatorname{ccn} 5$ & 119.733 & & $\operatorname{ccc} 19$ & 120.87865 & & \\
\hline
\end{tabular}




\section{Z-matrix for 3 (N-Ar \& Virtual Dihedral Angle locked)-Lowest Energy Conformation}

\begin{tabular}{|c|c|c|c|c|c|c|}
\hline 01 & & & $\operatorname{dih} 5$ & 280.00000 & $\operatorname{cc} 20$ & 1.39094 \\
\hline c & & & cc6 & 1.38865 & $\operatorname{ccc} 20$ & 120.86398 \\
\hline $\mathrm{n} 1 \mathrm{nc} 2$ & & & $\operatorname{ccc} 6$ & 120.17543 & $\operatorname{dih} 20$ & -0.12616 \\
\hline c $2 \mathrm{cn} 3$ & $1 \mathrm{cnc} 3$ & & $\operatorname{dih} 6$ & 180.43492 & $\operatorname{cc} 21$ & 1.39717 \\
\hline c $2 \mathrm{cn} 4$ & $1 \mathrm{cnc} 4$ & $3 \operatorname{dih} 4$ & cc7 & 1.38943 & $\operatorname{ccc} 21$ & 121.07761 \\
\hline $4 \operatorname{cc} 5$ & $2 \operatorname{ccn} 5$ & $1 \operatorname{dih} 5$ & $\operatorname{ccc} 7$ & 119.62737 & $\operatorname{dih} 21$ & 0.06926 \\
\hline c $5 \operatorname{cc} 6$ & $4 \operatorname{ccc} 6$ & $2 \operatorname{dih} 6$ & $\operatorname{dih} 7$ & -0.22696 & $\operatorname{cc} 22$ & 1.49620 \\
\hline c $6 \mathrm{cc} 7$ & $5 \operatorname{ccc} 7$ & $4 \operatorname{dih} 7$ & $\operatorname{cc} 8$ & 1.38907 & $\operatorname{ccn} 22$ & 103.28453 \\
\hline c $7 \operatorname{cc} 8$ & $6 \operatorname{ccc} 8$ & $5 \operatorname{dih} 8$ & $\operatorname{ccc} 8$ & 119.98758 & $\operatorname{dih} 22$ & 5.60369 \\
\hline c 8 cc 9 & $7 \operatorname{ccc} 9$ & $6 \operatorname{dih} 9$ & $\operatorname{dih} 8$ & -0.24592 & hc23 & 1.09462 \\
\hline s $9 \mathrm{sc} 10$ & $8 \operatorname{scc} 10$ & $7 \operatorname{dih} 10$ & $\operatorname{cc} 9$ & 1.39914 & hec23 & 120.91318 \\
\hline o 10 os 11 & 9 osc11 & $8 \operatorname{dih} 11$ & $\operatorname{ccc} 9$ & 121.81343 & $\operatorname{dih} 23$ & -179.63482 \\
\hline o 10 os 12 & 9 osc12 & $8 \operatorname{dih} 12$ & $\operatorname{dih} 9$ & 0.23831 & hc24 & 1.09553 \\
\hline c $10 \operatorname{cs} 13$ & $9 \csc 13$ & $8 \operatorname{dih} 13$ & $\mathrm{sc} 10$ & 1.80502 & hcc24 & 119.60882 \\
\hline h 13 hc14 & 10 hcs 14 & $12 \operatorname{dih} 14$ & $\operatorname{scc} 10$ & 117.45896 & $\operatorname{dih} 24$ & -179.81812 \\
\hline h 13 hc15 & 10 hcs 15 & $12 \operatorname{dih} 15$ & $\operatorname{dih} 10$ & -178.51055 & hc25 & 1.09530 \\
\hline h 13 hc16 & 10 hcs 16 & $12 \operatorname{dih} 16$ & os11 & 1.46922 & hcc25 & 119.44171 \\
\hline c $3 \operatorname{cc} 17$ & $2 \operatorname{con} 17$ & $1 \operatorname{dih} 17$ & osc11 & 110.10983 & $\operatorname{dih} 25$ & -179.96762 \\
\hline c $17 \mathrm{cc} 18$ & $3 \operatorname{ccc} 18$ & $2 \operatorname{dih} 18$ & $\operatorname{dih} 11$ & 130.58371 & hc26 & 1.09530 \\
\hline c $18 \mathrm{cc} 19$ & $17 \operatorname{ccc} 19$ & $3 \operatorname{dih} 19$ & os 12 & 1.47372 & hcc26 & 120.76568 \\
\hline c 19 cc 20 & $18 \operatorname{ccc} 20$ & $17 \operatorname{dih} 20$ & osc 12 & 108.75719 & $\operatorname{dih} 26$ & 0.19854 \\
\hline c $20 \mathrm{cc} 21$ & $19 \operatorname{ccc} 21$ & $18 \operatorname{dih} 21$ & $\operatorname{dih} 12$ & 2.07529 & oc27 & 1.21568 \\
\hline c 1 cc 22 & $2 \operatorname{con} 22$ & $3 \operatorname{dih} 22$ & $\operatorname{cs} 13$ & 1.78958 & ocn 27 & 123.06084 \\
\hline h 21 hc 23 & 20 hcc 23 & $19 \operatorname{dih} 23$ & $\csc 13$ & 101.70444 & $\operatorname{dih} 27$ & 176.48206 \\
\hline h 20 hc 24 & 19 hcc 24 & $18 \operatorname{dih} 24$ & $\operatorname{dih} 13$ & -112.38465 & hc28 & 1.09697 \\
\hline h 19 hc 25 & 18 hcc 25 & $17 \operatorname{dih} 25$ & hc14 & 1.09965 & hec 28 & 120.41018 \\
\hline h 18 hc 26 & 17 hcc 26 & $3 \operatorname{dih} 26$ & hcs 14 & 112.14765 & $\operatorname{dih} 28$ & -0.19398 \\
\hline o 3 oc 27 & $2 \operatorname{ocn} 27$ & $1 \operatorname{dih} 27$ & $\operatorname{dih} 14$ & 115.81963 & hc29 & 1.09543 \\
\hline h 5 hc 28 & 4 hcc 28 & $2 \operatorname{dih} 28$ & hc15 & 1.09956 & hec29 & 120.05234 \\
\hline h 6 hc29 & 5 hcc 29 & $4 \operatorname{dih} 29$ & hcs 15 & 112.17576 & $\operatorname{dih} 29$ & 179.69153 \\
\hline h 7 hc30 & 6 hcc30 & $5 \operatorname{dih} 30$ & $\operatorname{dih} 15$ & -3.55484 & hc30 & 1.09548 \\
\hline h 8 hc31 & 7 hcc31 & $6 \operatorname{dih} 31$ & hc16 & 1.10000 & hec30 & 120.15398 \\
\hline h 1 hc32 & $2 \mathrm{hcn} 32$ & $3 \operatorname{dih} 32$ & hcs 16 & 113.90818 & $\operatorname{dih} 30$ & -180.12789 \\
\hline h $1 \mathrm{hc} 33$ & 2 hcn 33 & $3 \operatorname{dih} 33$ & $\operatorname{dih} 16$ & -124.00706 & hc31 & 1.10091 \\
\hline & & & cc17 & 1.48301 & hcc31 & 118.70130 \\
\hline $\mathrm{nc} 2$ & 1.505 & & $\operatorname{ccn} 17$ & 106.29511 & $\operatorname{dih} 31$ & -179.46263 \\
\hline $\mathrm{cn} 3$ & 1.446 & & $\operatorname{dih} 17$ & -6.02150 & hc32 & 1.10926 \\
\hline cnc3 & $110.25 c$ & & cc18 & 1.38395 & hen32 & 109.08437 \\
\hline $\mathrm{cn} 4$ & 1.4519 & & $\operatorname{ccc} 18$ & 129.66198 & $\operatorname{dih} 32$ & 125.42292 \\
\hline cnc4 & 118.260 & & $\operatorname{dih} 18$ & -176.11360 & hc33 & 1.10783 \\
\hline $\operatorname{dih} 4$ & 150.000 & & cc19 & 1.39682 & hen33 & 112.04193 \\
\hline $\operatorname{cc} 5$ & 1.3995 & & $\operatorname{ccc} 19$ & 118.03828 & $\operatorname{dih} 33$ & -115.34331 \\
\hline $\operatorname{ccn} 5$ & 118.76 & & $\operatorname{dih} 19$ & -179.87980 & & \\
\hline
\end{tabular}




\section{Appendix 5}

\section{Step by step guide to convert and analyze NMR spectra from VNMR to gNMR}

There are 3 different programs, which you have to use,

1. gCVT: This program is used to import spectrum from VNMR (which is our NMR processing software.

2. gSPG: This is the program in which imported spectra from gCVT opens up. In this program you can process your spectrum. For example, you can adjust your spectra for Line width, Phasing and other things. Be sure to SAVE your spectrum after you think it's perfect for processing in gNMR.

3. gNMR: This is the program which gives your theoretical and experimental spectrum

Now, following are the instructions for using gCVT program:

(Before doing this you might want to make a NEW sub-folder (as gSPG) in your main folder where you have your all VNMR spectrums for that particular compound. But you can do this later.)

1. Open gCVT program.

2. Along with gCVT program a window opens up which says Convert File. There are 4 different options in this window.

i) VNMR Parameter file: procpar file

ii) VNMR spectrum file: NONE

iii) VNMR FID file: fid file 
iv) VNMR text file: text file

Now, next to each of those there is a browse option. Click on that and go to your own spectrum file from VNMR.

When you open your file you may not see all your spectrum files. Therefore click on 'all files' then you will be able to see all your files.

For each of the above type select particular file from your spectrum EXCEPT FOR SPECTRUM file.

After you fill in all the file names click on GO. Then you will see some series of messages in gCVT window, which shows you the progress of your processing. (It's very fast..) Then it will give you a message

“ generating spectrum by unweighted FFT

Phasing will probably be incorrect.” $\quad$ Click OK.

This will open up your gSPG program and you will see your spectrum but with phasing totally wrong. You will have to phase this spectrum.

Now we are in gSPG program:

1. Try clicking on Spectrum menu and then Auto phase. This might be helpful but not for sure.

2. If the spectrum doesn't look good then Click on Spectrum Menu and then Phase. One window will open up. In this window it's usually good idea to Select - Quick phasing option. Then by clicking on 

i) Left Phase
ii) Right phase
iii) Pivot phase

Adjust your spectrum till it looks as good as a normal NMR spectrum in VNMR. When you are satisfied Click on Done.

Now if you are planning to simulate Whole Spectrum then at this stage you will have to SAVE this spectrum. Be sure to SAVE your spectrum after you think it's perfect for processing in gNMR.

As I am more interested in only methylene protons (i.e. 4.2-5.5 ppm) I will do the following:

i) Put the cursor on $4.2 \mathrm{ppm}$

ii) Drag the cursor till $5.5 \mathrm{ppm}$

iii) Click anywhere in the middle, this will show now only 4.2-5.5 ppm of the spectrum

iv) Click on Spectrum Menu, Reduce followed by Sub spectrum, this will now kind of enlarge your spectrum

v) If you want your spectrum in this enlarged form for the processing in gNMR then you will have to save this spectrum. I usually save this as same name which I used to save spectra in gSPG with added name as $*_{\text {-sub }}$ 
Now you are ready to process this spectrum in gNMR to get,

i) theoretical and experimental spectrum comparison

ii) rate of exchange process.

Repeat all of the above procedure for all your spectra at each temperature.

All these things are usually done as follows:

Open gNMR program now.

1. gNMR program opens up with a small window with title Welcome to gNMR inside the main big window.

2. Click on "start with an empty molecule".

3. GO to gSPG program where you have your experimental spectrum and note down the ppm values for the two peaks.(note: you can put the cursor on the peak and it's ppm value will appear in the left corner of the spectrum window)

4. In gNMR in document1-molecule1 window.

5. Enter approximate ppm value for $1^{\text {st }}$ peak in column for $\delta$ (The one, which you noted down in step 3 above). Press Enter. Also remember to keep this ppm value as variable. To do this, type any alphabetical letter in the window exactly below your ppm value window.

6. Enter the approximate ppm value for $2^{\text {nd }}$ peak in column for $\delta$ (the one which you noted down in step 3 above). Press Enter. Also remember to keep this ppm value as variable. To do this, type any $\underline{\text { (must be different from the letter which }}$ you used in step 5) alphabetical letter in the window which exactly below your ppm value window. 
7. Click on Spectrum. Spectrum with 2 peaks (sharp) will appear.

8. Click on Settings - Spectrum, a small window will appear inside which you will see all the spectrum parameters like,
i) Line width
ii) Scale
iii) From ppm
iv) To ppm
v) Verticle size

9. In from-ppm put $4.2 \mathrm{ppm}$ (this comes from the spectrum which you saved in your gSPG program) REFER to page 2 above.

10. In to -ppm put $5.5 \mathrm{ppm}$ (this comes from the spectrum which you saved in your gSPG program) REFER to page 2 above.

\section{Click on OK}

12. New spectrum window appears in which you have to scroll horizontally to see your $2^{\text {nd }}$ peak. Therefore close this spectrum window by clicking on $\mathbf{X}$ Again in document 1-molecule1 window click on spectrum. Now you will see your spectrum with 2 peaks in one window without doing scroll. (The height of the 2 peaks might be different but don't worry!)

13. Now go to Settings - Spectrum - Iteration your Left hand side you will see that Iteration is in blue.

14. In right hand side it shows Spectrum: None 
15. Click on File. Go and find out your corresponding gSPG spectrum which you saved as *-sub file above.

16. Click OK. A message will appear saying "Spectrometer frequency mismatch. Adjust automatically?" say OK to this.

17. You will now see that your experimental spectrum is now on TOP and theoretical Spectrum is now on BOTTOM in your spectrum 1 window.

18. In Spectrum -Settings window in Display choose option as Overlapping. Click on Apply Check "full line shape iteration" and click OK. You will see in Spectrum window that one spectrum is in Red color.

19. Next to your UNDO-REDO icons there is one icon, which is for exchange click on that icon and one exchange window opens up.

Rate: type any number ( I usually type 100)

Iter? : incompl

$1-2: 2-1$

$2-1: 1-2$

After you type all these things the $2^{\text {nd }}$ option Iter? Will change from Incoml to Fixed.

Change that option by scrolling down to variable. Then, Click on Recalculate.

20. Now you will see that spectrum window has changed and you will see 2 sets of peaks not overlapping exactly.

21. IMPORTANT: In your left hand corner you will see a minimized window which is a LOG window open that window and adjust it's size so that you can clearly see your spectrum iteration.

22. IMPORTANT: you will see that in the spectrum window 
RED color spectrum: theoretical Spectrum has a base line UP certain height from your experimental BLACK Spectrum there for to fix that problem go to SettingsSpectrum-Baseline and you will see 3 options:

Phase(0):

Phase (1):

Height :

In any height window $1^{\text {st }}$ type very small number say 0.01 and click Apply.

Now you will see that in spectrum window both the peaks RED and BLACK have

nearly same Baseline Height. If you still see a very large difference in base line

height then try typing very small number in Phase(0) and then in Phase (1) in same

order. Try one at a time. Sometimes you have to type number in all of the above 3

windows. IMPORTANT: also be sure to check Iterate on Baseline in Settings-

Spectrum-Iteration option. This is below "full line shape iteration", which you checked

earlier. Click on APPLY — OK

23. Now you are all set to do iteration. Click on Iterate-GO you will see movement in two spectrums to match exactly with each other. At the same time you will see something is going on in LOG window and also in Exchange window. At the end of the matching you will now see that two spectrums match exactly and in exchange window you will see the exchange rate of this process. Take down this value and remember this value is for particular spectrum, which was recorded at particular temperature.

\section{SAVE your Results in gNMR.}

Sometimes if you see in gNMR Log window you will see that Error in Rate measurement is very high. To minimize the error you can do one thing. Go to your Molecule window where you put your ppm value initially and remove the alphabetical 
letters which have put in there at the beginning and then again click on Iterate-GO. Again same calculations will be performed but this time you will see that the error in $\mathrm{K}$ measurement is very less. Till today I haven't been able to find out the reasoning behind this but this is just my observation.

24. Repeat ALL these steps (1-23) for all of your recorded spectrums in VNMR to get exchange rate at all that temperature.

25. Plot graph of Ink vs $1 / T$ in excel and calculate $\Delta G$ value from slope. 


\section{Step by step guide to perform PM3 Semi-empirical calculations using Gaussian ${ }^{\mathrm{TM}}$}

The following steps were followed to do the semi-empirical PM3 molecular orbital calculations on all of the isoindolines (1-3, Chapter 4).

1. Draw the structure in HyperChem ${ }^{\mathrm{TM}} \mathrm{v} 6.02$.

2. Import the structure in Gaussian ${ }^{\mathrm{TM}} 1998$.

3. Optimize the structure using PM3 semi-empirical molecular orbital calculation method.

4. Define the z-matrix of the structure using z-mat editor in molden (program used to see the structure of the molecule).

5. Identify the two dihedral angles which you would like to study.

6. In gaussian, do the geometry optimization calculations by holding each of the dihedral angle at its fixed value and allowing rest of the molecule to relax to its minimum energy conformation. For example, rotate one dihedral angle from 0 to $360^{\circ}$ while holding the other dihedral angle at any particular fixed value between 0 to $360^{\circ}$. Energies in the form of eigenvalues (in atmic units) would be provided during these calculations in the ".log" file of each of these calculations.

7. Plot a 3D surface plot (preferably in MathCad ${ }^{\mathrm{TM}}$ ) of the energy values obtained at each of the data point versus two dihedral angle values at the same data point.

8. Locate the minimum (on either side of the maxima) and maximum energy conformation on the surface plot (using the obtained energy values) and further 
single point frequency calculations on each of these points were performed. This is necessary as the calculations performed in PM3 are the gas phase calculations.

9. From the energy valus obtained from the frequency calculations rotational barrier was evaluated, after converting atomic units into $\mathrm{kcal} / \mathrm{mol}$.

10. Identical calculations were performed in a study, which showed correlated nitrogen pyramidalization and $\mathrm{N}-\mathrm{Ar}$ rotation by making the only change in one dihedral angle. Instead of using S-Ar angle as one dihedral angle and N-Ar as the other in 1-3, virtual dihedral angle was used along with $\mathrm{N}-\mathrm{Ar}$ as the second dihedral angle. 